Universidade de São Paulo

Instituto de Física

\title{
O Modelo de Landau-Lifshitz e a Integrabilidade em Teoria de Cordas
}

Gabriel Weber

Orientador: Prof. Dr. Victor de Oliveira Rivelles

Tese de doutorado apresentada ao Instituto de Física para a obtenção do título de Doutor em Ciências

Comissão examinadora:

Prof. Dr. Victor de Oliveira Rivelles (IFUSP)

Prof. Dr. Diego Trancanelli (IFUSP)

Prof. Dr. Marcelo Otávio Caminha Gomes (IFUSP)

Prof. Dr. Andrei Mikhailov (IFT/UNESP)

Prof. Dr. Itzhak Roditi (CBPF)

São Paulo 


\section{FICHA CATALOGRÁFICA}

\section{Preparada pelo Serviço de Biblioteca e Informação}

do Instituto de Física da Universidade de São Paulo

Weber, Gabriel

O Modelo de Landau-Lifshitz e a Integrabilidade em

Teoria de Cordas - São Paulo, 2011

Tese (Doutorado) - Universidade de São Paulo.

Instituto de Física - Depto. Física Matemática.

Orientador: Prof. Dr. Victor de Oliveira Rivelles

Área de Concentração: Física Matemática

Unitermos: 1. Teoria das Cordas; 2. Teoria Quântica de

Campos; 3. Sistemas Dinâmicos (Física Matemática) 
"Don't just read it; fight it! Ask your own questions, look for your own examples, discover your own proofs. Is the hypothesis necessary? Is the converse true? What happens in the classical special case? What about the degenerate cases? Where does the proof use the hypothesis?

Paul R. Halmos 



\section{Agradecimentos}

"I don't know half of you half as well as I should like; and I like less than half of you half as well as you deserve."

J.R.R.Tolkien, The Lord of the Rings - The Fellowship of the Ring

À minha família, em particular aos meus pais, meus irmãos e, principalmente, à minha vó por terem propiciados as condições iniciais e de contorno para que eu pudesse ser quem hoje sou.

À Rebeca e à minha segunda família.

Ao professor Luiz Antonio Peresi.

Ao meu orientador Victor Rivelles.

Aos meus colaboradores e amigos Arsen Melikyan e Aleksandr Pinzul.

Aos meus irmãos de sina Eliane, Leandro, Rennan e Sérgio.

Aos meus amigos Guilherme, Márcio, Nícolas, Renato e Thomaz.

Aos meus irmãos em armas Fernando e Yuri e ao meu mestre Min.

Aos tensores, em particular, Áttis, Caio, Chico, Daniel, Dudu, Elisa, Elton, Leonardo, Manu, Pivo, Quel, Rafael, Renato, Timpa e Wilson.

Ao Rone, à Laura e à Marcela por tornarem a sala 337 um ambiente produtivo e aconchegante.

Finalmente, à FAPESP e ao CNPq pelo apoio financeiro. 



\section{Resumo}

Nesta tese, estudamos a integrabilidade quântica de modelos contínuos relevantes no contexto da quantização da supercorda do tipo IIB em $A d S_{5} \times S^{5}$, e, conseqüentemente, de interesse para a demonstração e uma melhor compreensão da correspondência AdS/CFT. Para os modelos de Landau-Lifshitz e de Alday-Arutyunov-Frolov, calculamos as amplitudes de espalhamento para três partículas e mostramos a fatorabilidade de suas matrizes $S$ em primeira ordem não-trivial. Propomos também um novo método para a quantização de sistemas integráveis contínuos no exemplo do modelo de Landau-Lifshitz $\mathfrak{s u}(1,1)$. Nosso método fornece uma solução alternativa para o problema do ordenamento operatorial, bem como uma prescrição para a dedução das identidades de traço e do espectro das cargas quânticas conservadas. Ademais, mostramos que, por ser baseado em um processo de regularização e renormalização operatorial, concomitante à construção das extensões auto-adjuntas, a integrabilidade é preservada durante a quantização. 



\section{Abstract}

In this thesis, we study the quantum integrability of continuous models which arise from consistent truncations of type IIB superstring theory on $A d S_{5} \times S^{5}$, and, therefore are relevant for improving our current understanding of the AdS/CFT correspondence. For the Landau-Lifshitz and the Alday-Arutyunov-Frolov models, we compute the three-particle scattering amplitude and show the factorizability of the corresponding $S$ matrices at the first non-trivial order. We also propose a new method for quantizing continuous integrable systems and apply it to the $\mathfrak{s u}(1,1)$ Landau-Lifshitz model. Our method provides an alternative solution to the longstanding operator ordering problem and gives a prescription to obtain the quantum trace identities, and the spectrum for the higher-order local charges. Moreover, since it is based on operator regularization and renormalization, as well as on the construction of the self-adjoint extensions, the integrability is preserved during the quantization process. 



\section{Sumário}

$\begin{array}{ll}\text { Agradecimentos } & \mathbf{v}\end{array}$

$\begin{array}{lc}\text { Resumo } & \text { vii }\end{array}$

$\begin{array}{ll}\text { Abstract } & \text { ix }\end{array}$

$\begin{array}{lc}\text { Sumário } & \text { xi }\end{array}$

Lista de Figuras $\quad$ xv

$\begin{array}{llr}\text { I Introdução } & 1\end{array}$

1 Motivação $\quad 3$

2 Integrabilidade Clássica $\quad 13$

2.1 Formalismo Hamiltoniano . . . . . . . . . . . . . . . . . . . . . . . . . . . . . . . . 13

2.2 Geometria Simplética . . . . . . . . . . . . . . . . . . . . . . . . 15

2.3 Integrabilidade de Liouville . . . . . . . . . . . . . . . . . . . . . . . . . . . . . . . . 18

2.4 Integrabilidade na Teoria Clássica de Campos . . . . . . . . . . . . . . . . . . 23

2.4.1 O Modelo de Schrödinger não-linear - Um Exemplo Representativo . . . . . 25

2.5 A Representação de Lax . . . . . . . . . . . . . . . . . . . . . . . . . . . . . . . . . . 28

2.6 Quantidades Conservadas . . . . . . . . . . . . . . . . . . . . . . . 31

2.6.1 Identidades de Traço para o Modelo NLS . . . . . . . . . . . . . . . . 35

2.7 A Matriz r Clássica . . . . . . . . . . . . . . . . . . . . . . . . . . . . . . . . . 41

2.8 O Método do Espalhamento Inverso, o Problema de Riemann-Hilbert e a Linearização das Equações de Movimento . . . . . . . . . . . . . . . . . . . . . . . . . . . . 51

3 Integrabilidade Quântica $\quad \mathbf{5 9}$

3.1 ○ Ansatz de Bethe Coordenado . . . . . . . . . . . . . . . . . . . . . . . . . . 60

3.2 O Método do Espalhamento Inverso Quântico . . . . . . . . . . . . . . . . . . . . 68

3.2.1 Sistemas Integráveis Clássicos Discretos - Uma Pequena Digressão . . . . . 69

3.2.2 Sistemas Discretos Quânticos . . . . . . . . . . . . . . . . . . . 71

3.3 ○ Ansatz de Bethe Algébrico . . . . . . . . . . . . . . . . . . . . . . . . . . . 75 
3.4 Quantização de Sistemas Contínuos . . . . . . . . . . . . . . . . . . . . . . . . 80

3.4.1 As Relações de Comutação e a Matriz R . . . . . . . . . . . . . . . . . . . 81

3.4.2 Coordenadas de Ângulo e Ação . . . . . . . . . . . . . . . . . . . . . . . 87

3.5 A Equação de Yang-Baxter . . . . . . . . . . . . . . . . . . . . . . . . . . . . . . . 90

3.5.1 Espalhamento em 1+1 Dimensões . . . . . . . . . . . . . . . . . . . . 91

3.6 Fatorabilidade da Matriz S para o NLS via Diagramas de Feynman . . . . . . . . . . 97

3.6.1 Somando Todos os Diagramas . . . . . . . . . . . . . . . . . . . . . 98

$\begin{array}{ll}\text { II O Modelo de Landau-Lifshitz } & 107\end{array}$

4 O Modelo de Landau-Lifshitz $\quad 109$

4.1 O Modelo de LL Clássico . . . . . . . . . . . . . . . . . . . . . . . . . . . . . . . . 110

4.2 O Método do Espalhamento Inverso Clássico . . . . . . . . . . . . . . . . . . . . 113

4.2.1 Cargas Conservadas Locais . . . . . . . . . . . . . . . . . . . . . . 115

4.3 A Matriz r para o LL . . . . . . . . . . . . . . . . . . . . . . . 119

5 Fatorabilidade da Matriz S 121

5.1 O Modelo de LL como uma Teoria Quântica de Campos . . . . . . . . . . . . . . . 123

5.1 Quantização do Campo Livre . . . . . . . . . . . . . . . . . . . . . 126

5.2 Espalhamento de Duas Partículas . . . . . . . . . . . . . . . . . . . . . . . . . 129

5.3 Espalhamento de Três Partículas . . . . . . . . . . . . . . . . . . . . . . . . . 132

5.3.1 Cálculos Diagramáticos . . . . . . . . . . . . . . . . . . . . . . 133

5.4 Fatoração da Matriz S . . . . . . . . . . . . . . . . . . . . . . . . . 138

6 Cargas Conservadas e Identidades de Traço 143

6.1 Quantização . . . . . . . . . . . . . . . . . . . . . . . 146

6.1 .1 A Hierarquia dos Operadores $\mathcal{M}_{m} \ldots \ldots \ldots$. . . . . . . . . . . 152

6.2 Regularização dos Operadores . . . . . . . . . . . . . . . . . . . . . . . 153

6.2 .1 Operador de Casimir . . . . . . . . . . . . . . . . . . 156

6.2.2 Hamiltoniano $\mathcal{F}$-regularizado . . . . . . . . . . . . . . . . . . . . . . . 158

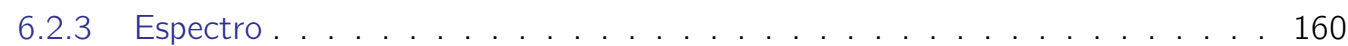

6.2.4 Cargas Conservadas Superiores . . . . . . . . . . . . . . . . . . . . . . 161

6.3 Extensões Auto-Adjuntas . . . . . . . . . . . . . . . . . . . . . . . . 163

$\begin{array}{lll}\text { III O Modelo de Alday-Arutyunov-Frolov } & 167\end{array}$

7 O Modelo de Alday-Arutyunov-Frolov $\quad 169$

7.1 A Supercorda em $A d S_{5} \times S^{5}$ como um Modelo Sigma . . . . . . . . . . . . . . . . . 169

7.1 .1 A Álgebra Superconforme . . . . . . . . . . . . . . . . . . . . . . 170

7.1 .2 Graduação $\mathbb{Z}_{4} \ldots \ldots \ldots \ldots \ldots \ldots 17 \ldots \ldots \ldots$

7.1.3 A Supercorda de Green-Schwarz como um Conjunto Quociente . . . . . . . 173 
7.1.4 Parametrização do Conjunto Quociente . . . . . . . . . . . . . . . . . . . . 174

7.1 .5 Simetrias Globais . . . . . . . . . . . . . . . . . . . . . . . . 176

7.2 O Setor $\mathfrak{s u}(1 \mid 1)$ da Teoria de Supercordas . . . . . . . . . . . . . . . . . . . . . . 179

7.2.1 A Lagrangeana do Modelo de Alday-Arutyunov-Frolov . . . . . . . . . . . . . 181

7.2.2 A Hamiltoniana e a Estrutura de Poisson do Modelo de Alday-Arutyunov-Frolov184

7.2 .3 A Conexão de Lax . . . . . . . . . . . . . . . . . . . . . . . . . . . 185

8 Fatorabilidade da Matriz S 189

8.1 O Modelo de AAF como uma Teoria Quântica de Campos . . . . . . . . . . . . . . 190

8.1.1 Quantização do Campo de Dirac . . . . . . . . . . . . . . . . . . . . . 194

8.1.2 Espalhamento de Pseudopartículas no Modelo de AAF . . . . . . . . . . . . 198

8.2 Espalhamento de Duas Pseudopartículas . . . . . . . . . . . . . . . . . . . . . 199

8.3 Espalhamento de Três Pseudopartículas . . . . . . . . . . . . . . . . . . . . . . . . . 202

8.3 .1 Cálculo Diagramáticos . . . . . . . . . . . . . . . . 203

8.4 Continuidade das Amplitudes de Espalhamento . . . . . . . . . . . . . . . . . . . . . 207

8.5 Fatoração da Matriz S . . . . . . . . . . . . . . . . . . . . . . . 209

8.6 Comentários Finais . . . . . . . . . . . . . . . . . . . . . . 212

$\begin{array}{ll}\text { Conclusão } & 216\end{array}$

$\begin{array}{ll}\text { IV Apêndices } & 217\end{array}$

$\begin{array}{lr}\text { A Algumas Demonstrações } & 219\end{array}$

A.1 Demonstração do Teorema de Darboux . . . . . . . . . . . . . . . . . . . . . . . . 219

A.2 Propriedades Analíticas da Matriz de Transição para o Modelo NLS . . . . . . . . . 221

A.3 Admissibilidade do Funcional Gerador das Quantidades Conservadas para o Modelo de Schrödinger Não-Linear . . . . . . . . . . . . . . . . . . . . . . . . 227

B Matrizes Gama $\quad 231$

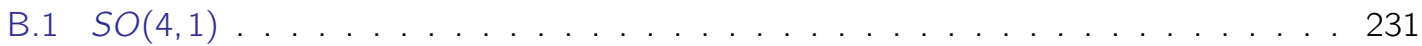

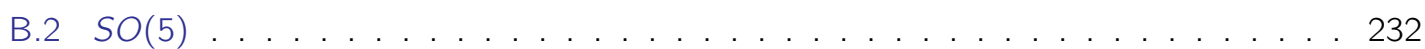

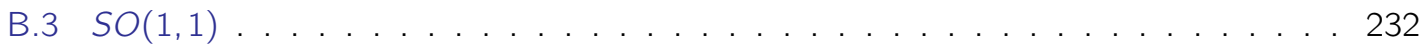

C Equivalência de Calibre entre o LL e o NLS 235

D Integrais Relevantes para o Modelo de Landau-Lifshitz 239

D.1 Integrais para o Espalhamento de Duas Partículas: Detalhes Computacionais . . . . 240

D.2 Integrais para o Espalhamento de Três Partículas: Detalhes Computacionais . . . . . 242

E Diagramas de Feynman Adicionais para o Modelo de Landau-Lifshitz 245 
F Equação de Dirac em Duas Dimensões 249

F.1 Soluções de Onda Plana . . . . . . . . . . . . . . . . . . . . . . . . . . . . . . . . 250

F.2 Relações de Ortonormalização e Completeza . . . . . . . . . . . . . . . . . . . . 250

G Integrais Relevantes para o Modelo de Alday-Arutyunov-Frolov

G.1 Integrais para o Espalhamento de Duas Pseudopartículas: Detalhes Computacionais 254

G.2 Integrais para o Espalhamento de Três Pseudopartículas: Detalhes Computacionais . 256

H Expressões Relevantes para a Demonstração da Fatorabilidade da Matriz $S$ no Modelo de Alday-Arutyunov-Frolov

Referências Bibliográficas

Índice Remissivo 


\section{Lista de Figuras}

1.1 Caráter dual das D-branas: (a) hiperplanos onde as cordas abertas se prendem; (b) fonte para cordas fechadas. . . . . . . . . . . . . . . . . . . . 5

1.2 Equivalência entre as estruturas integráveis no contexto da correspondência AdS/CFT: pelo lado da teoria de calibre, temos cadeias de spin integráveis, enquanto que pelo lado da teoria de cordas, temos o modelo sigma para uma corda rodando com alto momento angular. . . . . . . . . . . . . . . . . . . . .

2.1 Superfície de nível. . . . . . . . . . . . . . . . . . . . . . . . . . . . 15

2.2 Uma folha $\Gamma_{2 n}^{f}$ no espaço de fase. . . . . . . . . . . . . . . . . . . . . . . . . . 21

2.3 Caminho fechado de integração $\gamma=\cup_{i=1}^{4} \gamma_{i}$. . . . . . . . . . . . . . . . . . . . . . . 34

3.1 Representação gráfica da matriz $S$ para o espalhamento entre duas partículas em uma teoria relativística. . . . . . . . . . . . . . . . . . . . . . . . . . . 94

3.2 Usando o operador $e^{i \xi Q_{s}}$ para deslocar uma partícula. . . . . . . . . . . . . . . . . . . 94

3.3 Três trajetórias possíveis para o espalhamento de $3 \rightarrow 3$ partículas. A igualdade das amplitudes de espalhamento em (a), (b) e (c) equivale à equação de Yang-Baxter

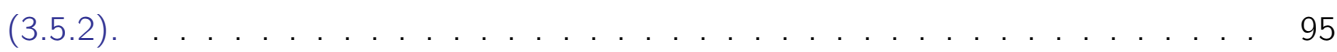

3.4 Diagrama com 1 laço para o espalhamento de duas partículas no modelo NLS. . . . 97

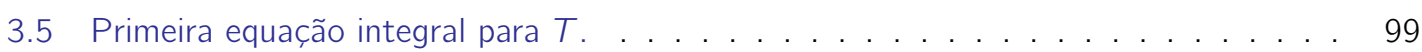

3.6 Segunda equação integral para T. . . . . . . . . . . . . . . . . . . . . . . . . . 100

3.7 Terceira equação integral para T. . . . . . . . . . . . . . . . . . . . . . . 101

5.1 Interpretação geométrica do termo de Wess-Zumino. . . . . . . . . . . . . . . . . . 124

5.2 Prescrição de pólos para o propagador do modelo de LL. . . . . . . . . . . . . . . . . 126

5.3 Qualquer ciclo fechado com propagadores orientados na mesma direção se anula identicamente. . . . . . . . . . . . . . . . . . . . . 127

5.4 (a) Correções para a energia do estado fundamental; (b) Correções para a função de Green. . . . . . . . . . . . . . . . . . . . . . . . . 128

5.5 Diagramas do tipo bolha para o espalhamento de duas partículas. . . . . . . . . . . 129

5.6 Vértice de interação na representação dos momentos, para a ação truncada até a quarta ordem nos campos. 
5.7 Vértices de interação na representação dos momentos adicionais para a ação truncada até a sexta ordem nos campos. . . . . . . . . . . . . . . . . . . . . 133

5.8 Os diagramas de Feynman para o nível de árvore para o espalhamento de três partículas no modelo de LL. . . . . . . . . . . . . . . . . . . . . . . . . . . . . . . 134

5.9 Representação diagramática do vértice de interação no espaço dos momentos. . . . 134

5.10 Representação diagramática da amplitude de espalhamento em segunda ordem. . . . 135

5.11 Diagramas de Feynman correspondentes à equação (5.3.8a) . . . . . . . . . . . . . . 136

5.12 Diagramas de Feynman para o primeiro termo das equações (5.3.8a) e (5.3.10) com as derivadas explicitamente colocadas. . . . . . . . . . . . . . . . . . . . . 140

7.1 Definição do setor $\mathfrak{s u}(1 \mid 1)$ na teoria de Supercordas a partir do setor $\mathfrak{s u}(1 \mid 1)$ na teoria de calibre dual. . . . . . . . . . . . . . . . . . . . . . . 179

8.1 Vértices de interação na representação dos momentos. . . . . . . . . . . . . . . . 193

8.2 Prescrição de pólos para o propagador do modelo de AAF. . . . . . . . . . . . . . . 198

8.3 Diagrama tipo bolha para o espalhamento de duas pseudopartículas. . . . . . . . . . 199

8.4 Diagramas de Feynman para a amplitude de espalhamento a 1 -laço $V_{1}^{(2)}(\mathbf{k}, \mathbf{p})$. O primeiro gráfico corresponde ao valor principal da contribuição oriunda dos termos com apenas uma contração, enquanto que o segundo, à soma das contribuições das funções delta dos termos com apenas uma contração com as contribuições advindas do termo com duas contrações.

8.5 Gráficos de Feynman correspondentes às amplitudes de 1-laço: (a) $V_{2}^{(2)}(\mathbf{k}, \mathbf{p})$ e

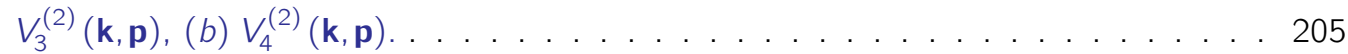

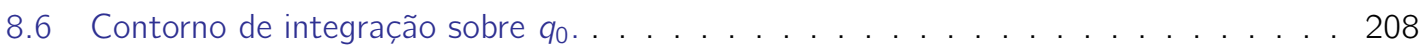

A.1 Folheação do espaço de fase pelas hipersuperfícies $\Sigma_{t}$ e $\Phi_{s}$. . . . . . . . . . . . . 220

D.1 Disposição dos pólos em $q^{0}$ de (D.1.1) e contorno de integração. . . . . . . . . . 241

D.2 Caminho de integração $\gamma$ para a integral radial. . . . . . . . . . . . . . . . . . . 243

E.1 Diagramas de Feynman para o segundo termo da equação (5.3.8a) com as derivadas explicitamente colocadas. . . . . . . . . . . . . . . . . . . 245

E.2 Diagramas de Feynman proporcionais a $g_{2}^{2}$, correspondendo à equação (5.3.8b). . . 246

E.3 Diagramas de Feynman proporcionais a $g_{3}^{2}$, correspondendo à equação (5.3.8c). . . . 246

E.4 Diagramas de Feynman proporcionais a $g_{1} g_{2}$, correspondendo à equação (5.3.8d). Por simplicidade omitimos os diagramas obtidos por reversão temporal. . . . . . . . 246

E.5 Diagramas de Feynman proporcionais a $g_{1} g_{3}$, correspondendo à equação (5.3.8e). Por simplicidade omitimos os diagramas obtidos por reversão temporal. . . . . . . . . 247

E.6 Diagramas de Feynman proporcionais a $g_{2} g_{3}$, correspondendo à equação (5.3.8f). Por simplicidade omitimos os diagramas obtidos por reversão temporal. . . . . . . . . 247

G.1 Disposição dos pólos em $q_{0}$ de (G.1.1) e contorno de integração. . . . . . . . . . . 255

G.2 Caminho de integração $\Gamma$ para o primeiro termo de (G.2.3). . . . . . . . . . . . . . 257 


\section{Parte I}

\section{Introdução}





\section{Capítulo 1}

\section{Motivação}

"Physics is like sex. Sure, it may give some practical results, but that's not why we do it."

Richard Phillips Feynman

Dois dos mais desafiadores e importantes problemas da física teórica contemporânea consistem em compreender apropriadamente a gravitação quântica e as interações fortes no regime nãoperturbativo. A teoria de cordas permeia esse contexto de uma forma particularmente interessante e não-trivial. De fato, foi inicialmente idealizada, no final da década de sessenta, como uma tentativa de descrever a força nuclear forte. Sua formulação, baseada em objetos fundamentais unidimensionais estendidos, fornecia uma explicação qualitativa de vários aspectos intrigantes da interação forte obtidos experimentalmente, como as trajetórias de Regge, de uma forma surpreendentemente elegante [1]. O motivo de tal apelo era o caráter unificado da descrição de uma miríade de hádrons distintos como apenas diferentes modos vibracionais de um único objeto fundamental: a corda. No entanto, com o advento da cromodinâmica quântica (QCD) no início da década de setenta, aliado à constatação de que a teoria de cordas sofria de alguns problemas técnicos, bem como era incapaz de incorporar o comportamento partônico, evidenciado por novos dados experimentais e explicado prontamente pela QCD, fez com que a motivação original por trás dos modelos de cordas ruísse e o interesse por eles desvanecesse.

Contudo, algumas dessas dificuldades técnicas que assolavam a teoria de cordas, como a presença de partículas sem massa adicionais em seu espectro e a necessidade de um espaço-tempo com dimensionalidade maior do que quatro para a sua consistência, e ajudaram a eliminá-la como uma possível candidata para a descrição das interações fortes, foram também responsáveis por impedir o seu completo esquecimento. A presença de uma partícula sem massa e com spin dois no espectro da corda fechada indicou a possibilidade da teoria de cordas fornecer uma teoria para a gravitação quântica. De fato, a teoria de supercordas fornece, atualmente, o único arcabouço teórico capaz de incorporar tanto a gravidade quanto a mecânica quântica de uma forma consistente e livre de anomalias [1-5]. Ademais, a necessidade de dimensões extras fomentou a hipótese de que a teoria de cordas não seria apenas uma teoria de gravitação quântica, mas uma teoria unificada de todas as interações e partículas. Esse alumbramento, ocorrido em meados da década de oitenta, é usualmente 
conhecida como a "primeira revolução das supercordas".

Por outro lado, ainda na década de setenta, ficou claro que as ferramentas teóricas disponíveis dificilmente seriam capazes de fornecer uma solução para a QCD. Por ser uma teoria assintoticamente livre, ou seja, cuja constante de acoplamento efetiva decresce com o aumento da energia, é fortemente acoplada a baixas energias, impossibilitando, pois, uma abordagem perturbativa nesse regime. Foi sugerido por 't Hooft [6] que a QCD, uma teoria de Yang-Mills com grupo de calibre SU(3), poderia ser significativamente simplificada caso o posto do grupo calibre ${ }^{1} N$ fosse suficientemente grande, de forma a possibilitar uma expansão em potências de $1 / N$. Notadamente, essa expansão permite escrever a função de partição da teoria de calibre como uma soma sobre superfícies com característica de Euler $\chi$ :

$$
Z_{\text {calibre }}=\sum_{\chi} N^{\chi} F_{\chi}(\lambda) \quad \text { com } \quad \lambda=g_{Y M}^{2} N
$$

onde $g_{Y M}$ é a constante de acoplamento da teoria de calibre e $\lambda$ é denominada a constante de 't Hooft. Considerando o limite de 't Hooft, que corresponde a tomar $N \rightarrow \infty \operatorname{com} \lambda$ fixo, verifica-se que os diagramas não-planares² são suprimidos.

Surpreendentemente, uma expansão similar também aparece naturalmente no contexto da teoria de cordas, pois a interação entre cordas, que consiste em juntá-las ou separá-las, pode ser interpretada como a adição ou remoção de buracos ou alças ${ }^{3}$ à sua folha-mundo. Mais explicitamente,

$$
Z_{\text {cordas }}=\sum_{\chi} g_{s}^{-\chi} F_{\chi}(\lambda) \quad \text { com } g_{s}=e^{\langle\phi\rangle}
$$

onde $\phi$ representa o diláton. A analogia com a expansão na teoria de calibre se completa ao identificarmos o fator $1 / N$ com a constante de acoplamento da teoria de cordas $g_{s}$. Essa coincidência entre as expansões perturbativas fornece uma das primeiras evidências em favor de uma dualidade entre teorias de cordas e de calibre.

A descoberta das $D$-branas, em meados da década de noventa, motivou o estudo de efeitos não-perturbativos e das dualidades entre as diversas teorias de cordas, levando, assim, a um meIhor entendimento da relação com a teoria de calibre e geometrias associadas a buracos negros. Toda essa atividade culminou na unificação das cinco teorias de cordas existentes, no contexto da recém proposta teoria $M$. Esse período é normalmente conhecido como a "segunda revolução das supercordas". As D-branas são sólitons da teoria de supercordas e, do ponto de vista da teoria de perturbações, podem ser entendidas simplesmente como as superfícies onde as pontas das cordas abertas se prendem [7]. Assim, ao considerarmos um conjunto de $N$ branas coincidentes, as cordas abertas podem começar e terminar em branas distintas, carregando, pois, dois índices que vão de 1 a $N$. Conseqüentemente, a dinâmica de baixas energias é descrita por uma teoria de calibre com grupo $U(N)$ [8]. Essas branas se acoplam aos campos de Ramond-Ramond, que pertencem ao multipleto da supergravidade, e, portanto, carregam as respectivas cargas. De forma que, quando adicionamos as $D$-branas, elas geram um fluxo que contribui para o tensor de energia-momento, e

\footnotetext{
${ }^{1}$ Claramente, para o caso da QCD N=3.

${ }^{2}$ Aqueles que não podem ser desenhados sobre um plano ou uma esfera.

${ }^{3} \mathrm{~A}$ característica de Euler de uma superfície está relacionada com o seu gênero $g$, o número de alças, e o número de fronteiras (ou buracos) $b$, através da seguinte relação: $\chi=2-2 g-b$.
} 


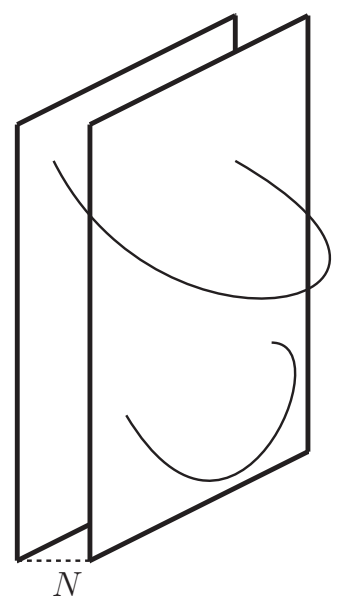

(a)

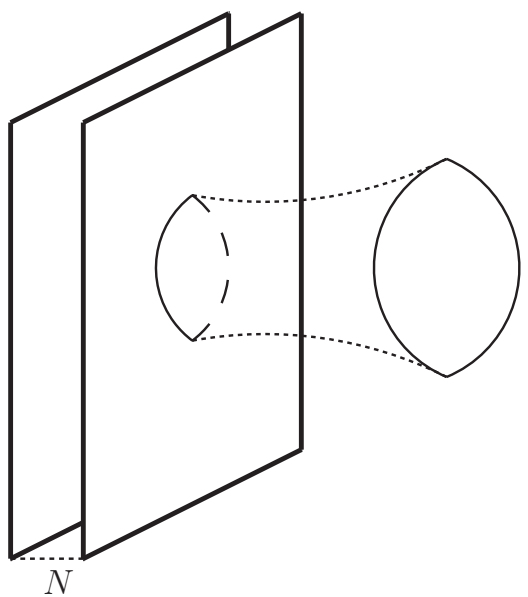

(b)

Figura 1.1: Caráter dual das D-branas: (a) hiperplanos onde as cordas abertas se prendem; (b) fonte para cordas fechadas.

portanto, curvam a geometria. As soluções das equações da supergravidade dotadas de tais fluxos são significativamente similares às soluções de buracos-negros carregados extremos na relatividade geral.

O passo seguinte foi dado por Maldacena em 1997 com a proposição da correspondência AdS/CFT, que conjectura a equivalência exata entre a teoria maximalmente supersimétrica de Yang-Mills $(\mathcal{N}=4$ SYM) quadridimensional com grupo de calibre $S U(N)$ e a teoria de supercordas do tipo IIB em $\operatorname{AdS}_{5} \times S^{5}[9]^{4}$. A motivação para tal dualidade surge ao considerarmos cordas em uma geometria criada por N D3-branas coincidentes, figura 1.1. Nesse caso, a geometria próxima ao horizonte é $A d S_{5} \times S^{5}$, enquanto que a dinâmica de baixas energias no volume-mundo quadridimensional das branas é descrita por uma teoria de calibre com grupo $U(N)$ e supersimetria $\mathcal{N}=4$. Essa descrição dual admite duas representações perturbativas válidas em regimes distintos. Para $g_{s} N$ pequeno ${ }^{5}$, a descrição em termos de uma teoria de campos perturbativa é válida, ao passo que, para $g_{s} N$ grande, a descrição gravitacional perturbativa é aplicável, pois o raio de curvatura é muito maior do que a escala da teoria de cordas. Dessa forma, somos levados naturalmente a supor que a teoria de Yang-Mills (no regime de acoplamento forte) descreve a região próxima do horizonte de uma brana, cuja geometria é $A d S_{5} \times S^{5}$.

A correspondência AdS/CFT é o mais notável exemplo da implementação do princípio holográfico, por meio do qual uma teoria de gravidade quântica pode ser descrita através de uma teoria quântica de campos, esta última definida na fronteira do espaço onde vive a teoria de gravitação [14]. Nesse caso, o espaço quadridimensional da teoria de Yang-Mills corresponde à fronteira do espaço $\operatorname{AdS}^{5}$, enquanto que a esfera está associada à simetria interna dos campos de calibre. A idéia de holografia

\footnotetext{
${ }^{4}$ Para algumas revisões vide [10-13].

${ }^{5}$ Quando existem N D-branas coincidentes, o parâmetro efetivo de expansão em laços para cordas abertas é, de fato, $g_{s} N$, em vez de simplesmente $g_{s}$.
} 
surgiu originalmente do estudo das propriedades termodinâmicas de buracos negros, em particular do limite de Bekenstein, que afirma que a entropia de um buraco negro é proporcional à sua área, e não ao seu volume [15].

A conjectura de Maldacena propõem uma relação muito profunda entre as teorias de calibre $\left(\mathcal{N}=4\right.$ SYM) e a gravitação quântica (supercordas do tipo IIB em $A d S_{5} \times S^{5}$ ), que proporcionou uma forma revolucionária de encarar esses dois problemas fundamentais da física teórica contemporânea. Ela estabelece um mapa concreto entre diversos objetos das duas teorias, como, por exemplo, seus parâmetros livres. Pelo lado da teoria de calibre os dois parâmetros livres necessários são: a constante de acoplamento $g_{Y M}$ e o número de $\operatorname{cores}^{6} \mathrm{~N}$, que podem ser combinados na constante de 't Hooft, $\lambda=g_{Y M}^{2} N$. Já pelo lado da teoria de cordas, temos apenas um único parâmetro fundamental ${ }^{7}$ : $\alpha^{\prime}=l_{s}^{2}$, onde $l_{s}$ é o comprimento característico da corda. A relação explícita entre tais parâmetros é a seguinte:

$$
4 \pi g_{s}=g_{Y M}^{2}=\frac{\lambda}{N} \quad \text { e }\left(\frac{R^{2}}{\alpha^{\prime}}\right)^{2}=\lambda
$$

onde $R$ é o raio tanto de $S^{5}$ quanto de $A d S_{5}$.

A $\mathcal{N}=4$ SYM possui uma função $\beta$ nula, e por esse motivo constitui uma teoria conforme em quatro dimensões. A álgebra das transformações conformes, $\mathfrak{s o}(4,2)$, inclui, além dos geradores de Poincaré, os geradores das transformações de escala (dilatações) e os "boosts" conformes. A supersimetria estende a álgebra conforme, para a superconforme, $\mathfrak{p s u}(2,2 \mid 4)$, que contém adicionalmente os geradores de $\mathfrak{s u}(4) \sim \mathfrak{s o}(6)$, e corresponde à simetria $R$. Já a teoria de supercordas do tipo IIB em $\operatorname{AdS}_{5} \times S^{5}$ pode ser formulada como um modelo sigma não-linear com espaço alvo dado pelo conjunto quociente [16]:

$$
\frac{\operatorname{PSU}(2,2 \mid 4)}{S O(4,1) \times S O(5)}
$$

Assim, do ponto de vista das simetrias, ambas as teorias são invariantes sob o mesmo supergrupo de Lie $\mathfrak{p s u}(2,2 \mid 4)$, e, conquanto não seja uma condição suficiente para a sua equivalência, é uma condição necessária.

É importante salientar que apesar de possuir uma estrutura muito semelhante com a QCD, afinal ambas são teorias de calibre quadridimensionais renormalizáveis, baseadas nos mesmos tipos de partículas e interações, a $\mathcal{N}=4$ SYM é uma teoria muito mais simétrica. Em particular, apresenta supersimetria maximal e simetria conforme, ausentes na QCD. Embora teorias conformes sejam necessariamente não massivas, existe uma analogia entre os operadores locais da $\mathcal{N}=4$ SYM e as partículas massivas da QCD. Tais operadores locais são compostos pelos campos fundamentais da $\mathcal{N}=4$ SYM atuando sobre um mesmo ponto do espaço-tempo e, da mesma forma que na QCD, suas cargas de cor são balanceadas, tornando-os, pois, objetos invariantes de calibre. Nesse caso, a quantidade fisicamente relevante, que substitui a massa, é a dimensão de escala dos operadores. Classicamente, ela é obtida a partir da soma das dimensões dos campos constituintes, e, assim como a massa, recebe correções quânticas, as dimensões anômalas. A despeito de todas essas diferenças, a correspondência AdS/CFT, por meio da $\mathcal{N}=4$ SYM, tem fornecido novas ferramentas que levaram

\footnotetext{
${ }^{6}$ Posto do grupo de calibre.

${ }^{7}$ Lembre-se que a constante de acoplamento $g_{s}$ é o valor esperado do dílaton no vácuo, e, por isso não constitui um parâmetro fundamental.
} 
a compreensão de fenômenos fundamentais que permeiam tais sistemas fortemente acoplados, mas que não dependem muito dos detalhes da teoria específica.

Nesse contexto, o operador de dilatação assume um papel proeminente, correspondendo ao Hamiltoniano na quantização radial ${ }^{8}$. Os autovalores do operador de dilatação fornecem as dimensões de escala dos operadores locais de uma teoria com invariância conforme, e são os únicos rótulos de uma representação (super-)conforme a poderem depender continuamente dos parâmetros do modelo. Pelo lado da teoria de cordas, as quantidades de interesse físicos são as energias dos diversos estados. De acordo com a versão mais geral da dualidade AdS/CFT os estados físicos das teorias de cordas e calibre estão organizados no mesmo conjunto de multipletos de $\mathfrak{p} \mathfrak{s u}(2,2 \mid 4)$, de forma que as dimensões de escala dos operadores na teoria de calibre devem coincidir exatamente com as energias dos estados correspondentes na teoria de cordas.

O grande poder preditivo da conjectura AdS/CFT advém do fato de que ela relaciona o limite de acoplamento fraco de uma teoria com o limite de acoplamento forte da outra. Portanto, torna-se possível utilizar métodos perturbativos em um dos lados da dualidade para se obter informações sobre o regime não-perturbativo do outro. Desse ponto de vista, a correspondência proporciona duas descrições complementares de um mesmo sistema. Conseqüentemente, problemas difíceis de se estudar em uma descrição são, às vezes, fáceis de se abordar na outra. Contudo, essa complementaridade de descrições introduz uma grande dificuldade na verificação da validade da conjectura, dado que uma comparação direta entre os resultados obtidos em ambos os lados não é geralmente possível, em virtude das limitações impostas pela natureza perturbativa dos métodos disponíveis. Dessa forma, grande parte da pesquisa inicial concentrou-se no estudo de estados especiais (estados BPS ${ }^{9}$ ), que por não receberem correções quânticas, em virtude da supersimetria, podiam ser diretamente computados em ambos os lados, fornecendo os primeiros testes da validade da dualidade.

Em 2002, Berenstein, Maldacena e Nastase [17] consideraram excitações ao redor de uma configuração de cordas em forma de ponto que se movia com grande momento angular sobre um equador de $S^{5}$. Nesse limite (limite de BMN), o fundo sentido pela corda corresponde a uma geometria de ondas planas, que permite uma quantização exata da teoria. Assim, com o conhecimento do espectro exato da teoria de cordas, foi possível inferir as dimensões de escala dos operadores na teoria de calibre. Esses resultados foram confirmados, através do cálculo direto das dimensões de escala nas primeiras ordens [18]. Todavia, um progresso considerável tanto na demonstração da validade de tal conjectura, quanto no entendimento das teorias de cordas e de calibre, foi apenas obtido com o descobrimento de estruturas integráveis em ambos os lados da dualidade ${ }^{10}$.

Sistemas integráveis [20-23] formam uma das mais fascinantes áreas da física teórica e da matemática aplicada, o crescente interesse em sistemas completamente solúveis proporcionou um rápido desenvolvimento e uma vasta aplicabilidade de seus métodos. Partindo do estudo de ondas solitárias (sólitons) em águas rasas e aspectos clássicos de equações diferenciais parciais não-lineares integráveis, a área se expandiu, e hoje permeia muitos campos da física como, para citar apenas alguns, a física da matéria condensada e a física de partículas elementares. Em suma, o conceito de integrabi-

\footnotetext{
${ }^{8} \mathrm{O}$ esquema de quantização no qual os parênteses de Poisson são calculados na hipersuperfície de mesma coordenada radial.

${ }^{9}$ Bogomol'nyi?Prasad?Sommerfield.

${ }^{10}$ Para uma revisão compreensiva da extensa (e crescente) literatura a respeito da aplicação da integrabilidade no contexto da correspondência AdS/CFT, vide [19]
} 
lidade corresponde às condições necessárias para que um dado sistema dinâmico seja analiticamente solúvel, incluindo também métodos para obter tais soluções e suas propriedades.

Modelos integráveis são, em geral, caracterizados pela existência de infinitas quantidades conservadas. Na mecânica clássica, por exemplo, a existência de um número ${ }^{11}$ suficiente de integrais de movimento permite a introdução das variáveis de ângulo e ação, e conseqüente solução das equações de movimento por meio de quadraturas. Já no contexto quântico, para teorias em $(1+1)$ dimensões, a existência de infinitas leis de conservação restringe severamente a forma da matriz $S$ [24,25]. Em particular, o espalhamento em teorias integráveis quânticas é fatorável, ou seja, a matriz $S$ para o espalhamento de $n$ corpos é dada simplesmente pelo produto de matrizes $S$ para o espalhamento de duas partículas. Além do mais, no decorrer de tal espalhamento não há produção nem aniquilação de partículas, e o conjunto inicial de momentos coincide com o final. Dessa forma, não é surpreendente que se possa calcular a matriz $S$ exatamente, bem como obter o espectro das excitações, através da diagonalização simultânea de todas as cargas conservadas.

Pelo lado da teoria de calibre, a integrabilidade forneceu métodos para o cálculo da dimensão anômala de operadores locais no limite planar da $\mathcal{N}=4$ SYM, posto que, nesse caso, o operador de dilatação pode ser mapeado na Hamiltoniana de uma cadeia de spins integrável [26, 27]. Enquanto que pelo lado da teoria de cordas, a estrutura integrável relevante surgiu do modelo sigma para uma corda rodando com grande momento angular [28-30]. Impressionantemente, uma equivalência detalhada entre tais estruturas foi encontrada, apesar de se ter um modelo quântico discreto em um dos lados e um modelo contínuo clássico do outro [31], vide figura 1.2. O trabalho de Bena, Polchinski e Roiban [32] revelou a presença de simetrias yangianas, relacionadas com a existência de um número infinito de correntes conservadas, e, por consegüinte estabeleceu a integrabilidade clássica do modelo sigma de cordas. Ao se perceber que a integrabilidade se estende a todos os operadores da $\mathcal{N}=4$ SYM em primeira ordem [27], bem como tal resultado permanece válido para as duas ordens seguintes [18], foi proposto que a integrabilidade deveria ser uma propriedade exata. Ademais, as estruturas integráveis descobertas perturbativamente em ambos os lados da dualidade seriam manifestações de um mesmo objeto não-perturbativo.

Logo, para um melhor entendimento da correspondência AdS/CFT é necessário quantizar a teoria de supercordas em $A d S_{5} \times S^{5}$, a qual, conquanto um modelo classicamente integrável, resistiu a todas as tentativas de quantização através dos métodos convencionais desenvolvidos na teoria de sistemas integráveis quânticos, em particular no contexto do método do espalhamento inverso quântico [33, 34]. Notamos, contudo, que a integrabilidade quântica da teoria de cordas em $\operatorname{AdS}_{5} \times S^{5}$ tem sido uma hipótese corriqueira para permitir diversos avanços como o cálculo das relações de dispersão para excitações elementares e da matriz $S$ descrevendo o seu espalhamento $[13,35]$. Tais dificuldades não são exclusividade da teoria de cordas, aparecendo também em vários outros modelos relevantes, como, por exemplo, o modelo principal quiral, o modelo de sine-Gordon e o modelo de LandauLifshitz. Dentre elas, uma das mais críticas é relativa à presença de termos não-ultralocais, ou seja, proporcionais a,

$$
\partial_{\sigma}\left(\sigma-\sigma^{\prime}\right)
$$

\footnotetext{
${ }^{11}$ Esse número corresponde aos graus de liberdade do sistema, caso ele seja finito.
} 

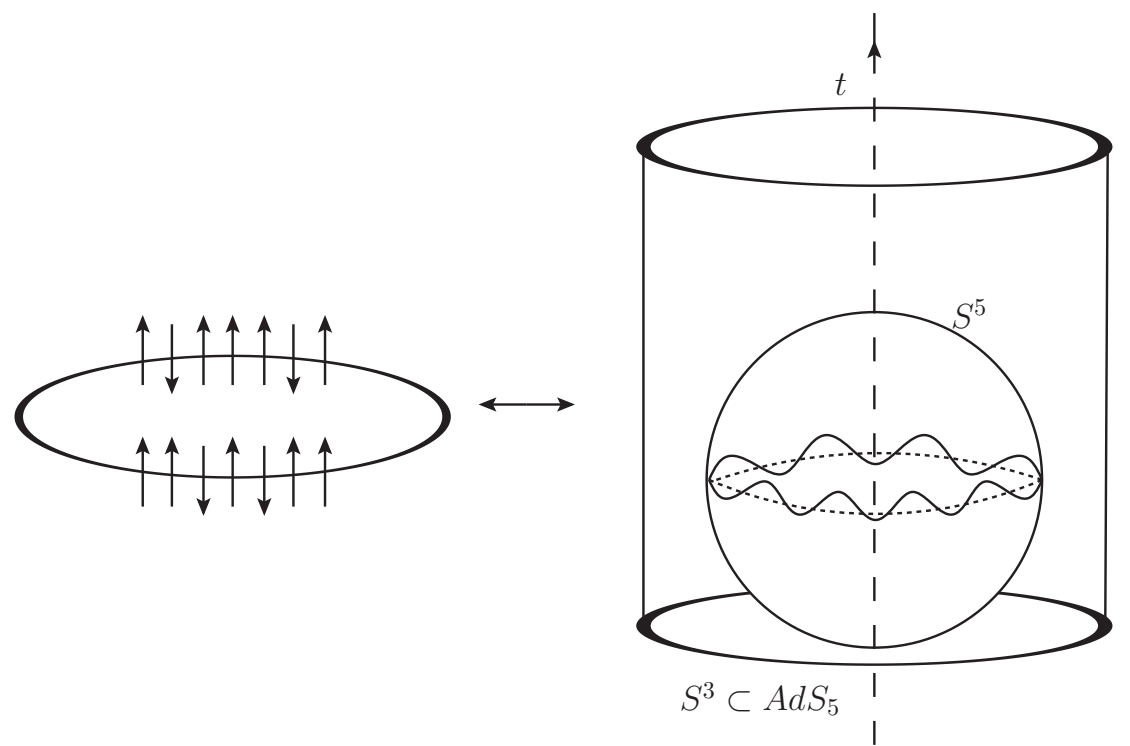

Figura 1.2: Equivalência entre as estruturas integráveis no contexto da correspondência AdS/CFT: pelo lado da teoria de calibre, temos cadeias de spin integráveis, enquanto que pelo lado da teoria de cordas, temos o modelo sigma para uma corda rodando com alto momento angular.

que, em geral, impossibilitam a aplicação do método do espalhamento inverso [20-23].

É bem sabido que modelos contínuos proporcionam um problema de quantização severamente mais complicado do que suas contrapartes discretas. Isso se deve à presença de divergências advindas de um produto mal definido de operadores atuando sobre um mesmo ponto, que no caso de sistemas discretos são suavizadas pelo espaçamento da rede. Apesar de existirem diversas técnicas de discretização, as quais podem não ser diretamente aplicáveis ao já mais complexo modelo sigma de cordas, elas, em geral, levam a resultados sensivelmente complicados [36], envolvendo operadores não-locais. Portanto, se estamos interessados em estender a correspondência entre as estruturas integráveis no contexto da dualidade AdS/CFT além do limite de cordas com grande momento angular, precisamos aprender a lidar diretamente com modelos integráveis quânticos contínuos. Um primeiro passo nessa direção foi dado em [37], onde propusemos um novo método para a quantização de modelos integráveis contínuos no exemplo representativo do modelo isotrópico $\mathfrak{s u}(1,1)$ de Landau-Lifshitz (LL), sem recorrer a nenhum esquema de discretização.

Alternativamente, é possível estudar a integrabilidade quântica de um dado modelo, ao considerálo como uma teoria de campos bidimensional e testar a fatorabilidade de sua matriz $S$ por meio de cálculos perturbativos. A fatorabilidade da matriz $S[24,25]$ é uma propriedade fundamental dos modelos integráveis quânticos, e um dos princípios que permitem preservar a integrabilidade de uma teoria durante a sua quantização. Sua relação com o método do espalhamento inverso quântico é bem conhecida [38], contudo até recentemente, tenha sido apenas verificada para o caso mais simples do modelo não-linear de Schrödinger (NLS) [39]. Não obstante, essa técnica tem sido amplamente utilizada no contexto da dualidade AdS/CFT para a obtenção da matriz $S$ e das equações de Bethe a partir da hipótese de integrabilidade quântica $[35,40]$. Dessa forma, torna-se, pois, imperativa 
a verificação da fatorabilidade da matriz $S$ para tais modelos. Interessantemente, a demonstração dessa propriedade requer um intrincado mecanismo de cancelamentos entre amplitudes advindas de diferentes ordens $[41,42]$.

Uma oportunidade particularmente interessante de se abordar a quantização da teoria de supercordas em $\operatorname{AdS}_{5} \times S^{5}$ consiste em estudar os modelos oriundos de seus truncamentos consistentes a subsetores que sejam duais a setores fechados da teoria de calibre [35, 41, 42]. Espera-se que tais modelos percam algumas das propriedades interessantes da teoria completa, como a invariância conforme e a renormalizabilidade, contudo a integrabilidade clássica deve persistir. Dessa forma, os modelos resultantes, apesar de mais simples, devem ainda propiciar exemplos representativos das dificuldades associadas à quantização da teoria de cordas. Nesse contexto dois modelos sobressaem: o modelo $\mathfrak{s u}(2)$ de LL e o modelo de Alday-Arutyunov-Frolov (AAF) [43], que surgem, respectivamente, dos truncamentos consistentes aos setores $\mathfrak{s u}(2)$ e $\mathfrak{s u}(1 \mid 1)$.

Tanto o modelo $\mathfrak{s u}(2)$ de LL quanto o de AAF constituem exemplos formidáveis das dificuldades associadas à quantização de sistemas integráveis contínuos. Notadamente, a formulação do método do espalhamento inverso quântico exige uma definição cuidadosa dos operadores quânticos, de forma a evitar as singularidades inerentes às álgebras operatoriais de tais modelos, bem como a correta reconstrução dos espaços de Hilbert $[37,44]$. Além disso, é interessante ressaltar que o modelo de AAF é o primeiro exemplo de um modelo integrável fermiônico não-trivial, que apresenta uma estrutura não-linear e singular.

Esta tese está organizada da seguinte maneira. No restante da parte I, fazemos uma revisão dos conceitos básicos relacionados à integrabilidade clássica e quântica. No capítulo 2, consideramos a integrabilidade clássica, inicialmente no contexto da mecânica clássica, em sistemas com um número ( $n$ ) finito de graus de liberdade, introduzimos o conceito de integrabilidade de Liouville e demonstramos que suas equações de movimento são solúveis por quadraturas, se existirem $n$ quantidades conservadas e independentes em involução. Ademais estabelecemos a relação entre essas quantidades e as variáveis de ângulo e ação. A seguir, generalizamos o conceito de integrabilidade para sistemas com um número infinito de graus de liberdade, de forma a podermos analisar teorias de campos clássicas. Apresentamos o modelo de Schrödinger não-linear (NLS), a teoria de campos clássica mais completamente estudada, com a qual ilustramos todos os conceitos importantes. A partir da representação de Lax, construímos a matriz de transição e mostramos como as infinitas cargas conservadas podem ser extraídas ao considerarmos o seu traço. Em particular, para o modelo NLS obtemos, explicitamente, as identidades de traço. Para verificar se as integrais de movimento assim obtidas estão em involução empregamos o formalismo da matriz $r$. Finalizamos esse capítulo com uma discussão sobre a existência de um mapa bijetor responsável por linearizar as equações de movimento e introduzimos os conceitos essenciais do método do espalhamento inverso clássico (MEIC).

No capítulo 3 abordamos a difícil questão de estender o conceito de integrabilidade ao contexto quântico. Começamos discutindo o Ansatz de Bethe coordenado ( $A B C)$, que usamos para diagonalizar o Hamiltoniano da versão quântica do modelo NLS e assim inferir o seu espectro. A seguir, desenvolvemos o método do espalhamento inverso quântico (MEIQ), que combina idéias do MEIC com as do $A B C$ para fornecer uma abordagem mais sistemática para se obter as autofunções e seus 
respectivos autovalores. Inicialmente, consideramos sistemas discretos, para os quais desenvolvemos o Ansatz de Bethe algébrico (ABA). E posteriormente para modelos contínuos, no exemplo do modelo NLS, exibimos as construções necessárias para se obter o equivalente quântico das coordenadas de ângulo e ação e as quantidades conservadas. Comentamos as dificuldades associadas à dedução das cargas conservadas de ordem superior e sua relação com a incapacidade do ordenamento de Wick em fornecer uma solução para o problema do ordenamento de operadores. Discutimos também o papel desempenhado pela equação de Yang-Baxter no contexto do espalhamento de partículas em teorias de campos em $1+1$ dimensões. Finalmente, baseados nessas idéias estabelecemos a integrabilidade quântica do modelo NLS, ao demonstrarmos a fatorabilidade de sua matriz $S$, com o uso de uma abordagem perturbativa baseada em diagramas de Feynman.

Nas partes II e III apresentamos material original, baseados nos artigos [41], [37] e [42].

Na parte II, estudamos o modelo de Landau-Lifshitz (LL), que descreve a cadeia contínua de spins de Heisenberg, e, mais recentemente, surgiu no contexto da correspondência AdS/CFT como uma das primeiras evidências da estrutura integrável subjacente. Estamos particularmente interessados na versão que aparece no subsetor $\mathbb{R} \times S^{3}$ da supercordas em $A d S_{5} \times S^{5}$ no limite de momento angular grande. No capítulo 4, apresentamos o modelo de LL clássico em suas diferentes versões: anisotrópica e isotrópica, $\mathfrak{s u}(2)$ e $\mathfrak{s u}(1,1)$. Aplicamos o MEIC para as duas versões isotrópicas: $\mathfrak{s u}(2)$ e $\mathfrak{s u}(1,1)$ e comentamos sobre a equivalência de calibre entre o LL e o NLS. No capítulo 5, baseado na publicação [41], consideramos o modelo de LL isotrópico $\mathfrak{s u}(2)$ como uma teoria de campos, conforme obtido a partir da teoria de cordas. Examinamos a quantização do campo livre e constatamos propriedades interessantes relacionadas com o caráter não-relativístico do modelo, que permitem o cálculo exato da matriz $S$ para o espalhamento da duas partículas. A seguir, com o intuito de sondar a integrabilidade quântica do modelo, estudamos o espalhamento de três partículas e concluímos que, devido a um intrincado esquema de cancelamentos, a matriz $S$ é fatorável na primeira ordem não-trivial.

No capítulo 6, baseado em [37], consideramos em detalhe a quantização do modelo de LL. Consideramos apenas a versão hiperbólica $(\mathfrak{s u}(1,1))$ do modelo de $L L$, uma vez que só ela admite uma quantização consistente com respeito ao vácuo ferromagnético. Discutimos minuciosamente o procedimento de regularização e renormalização dos campos necessário para definir consistentemente o produto de operadores atuando sobre um mesmo ponto, bem como a estrutura algébrica resultante e demonstramos a integrabilidade quântica do modelo. Com os operadores quânticos devidamente regularizados podemos prosseguir com a construção das cargas conservadas, sua conseqüente diagonalização e a dedução das identidades de traço. Explicamos em que sentido o nosso método pode ser considerado como uma alternativa ao processo de discretização e estabelecemos uma conexão explícita com o MEIQ. Finalmente, consideramos a construção das extensões auto-adjuntas das cargas conservadas.

Na parte III, analisamos o modelo de Alday-Arutyunov-Frolov (AAF). No capítulo 7, fazemos uma breve revisão de alguns fatos relevantes sobre a formulação da teoria de supercordas do tipo IIB em $A d S_{5} \times S^{5}$ como um modelo sigma. De forma a podermos proceder com a redução ao setor $\mathfrak{s u}(1 \mid 1)$ para obtermos o modelo de AAF. Discutimos tanto a sua formulação Lagrangeana, quanto Hamiltoniana. Finalmente, exibimos a representação de Lax para o modelo de AAF, obtida por um processo 
de redução semelhante, a partir do par de Lax para o modelo de cordas, e, assim, estabelecemos a sua integrabilidade clássica. No capítulo 8, baseado na publicação [42], discutimos o modelo de AAF como uma teoria de campos quântica e preparamos todo o ferramental necessário para o cálculo das amplitudes de espalhamento de duas e três partículas. Fornecemos uma dedução baseada em métodos tradicionais da matriz $S$ para os setores com duas e três partículas e demonstramos a sua fatorabilidade na primeira ordem não trivial.

$\mathrm{Na}$ conclusão, coletamos os principais resultados obtidos. Relegamos aos apêndices A-H todos os detalhes técnicos pertinentes que não foram exibidos no texto principal. 


\title{
Capítulo 2
}

\section{Integrabilidade Clássica}

\author{
"Begin at the beginning," the King said gravely, "and go on till you come to the end: \\ then stop." \\ Lewis Carroll, Alice's Adventures in Wonderland
}

No contexto de sistemas dinâmicos, o conceito de integrabilidade está relacionado com a existência de folheações invariantes e regulares da variedade na qual o sistema está definido. Uma $n$-folheação de uma variedade $m$-dimensional $M$ pode ser entendida como uma coleção $M_{i}$ de subvariedades $n$-dimensionais de $M$, conexas e disjuntas aos pares, denominadas folhas da folheação de $M$, de forma que, para cada ponto $x \in M$, exista uma carta $(U, \phi)$, com $x \in U$ e tal que para cada folha $M_{i}$ a intersecção $M_{i} \cap U$ seja vazia ou uma coleção contável de subespaços n-dimensionais afins, cujas últimas $m-n$ coordenadas sejam constantes. Assim, uma folheação regular e invariante é aquela cujas folhas são variedades imersas de menor dimensão possível que são invariantes sob o fluxo.

Para o caso particular de sistemas Hamiltonianos, o conceito de integrabilidade admite um refinamento relacionado ao teorema de Liouville, a saber, podemos relacionar a existência de folheações invariantes e regulares com a existência de um conjunto maximal de invariantes em involução com respeito a álgebra de Poisson do sistema. Tais invariantes em involução, são fisicamente mais relevantes, uma vez que correspondem às integrais de movimento do sistema, como por exemplo a energia e o momento. Com a ajuda delas é possível, através de quadraturas, encontrar soluções explícitas das equações de movimento. No que se segue, vamos expor um tratamento simplificado e sucinto que permite ao leitor vislumbrar essa conexão.

\subsection{Formalismo Hamiltoniano}

Na mecânica clássica, o movimento de um sistema é descrito por uma trajetória imersa no espaço de fase, que é, em geral, um espaço de dimensão par, descrito por coordenadas de posição e momento. Por concreteza, consideramos um sistema com $n$ graus de liberdade ( $n$ finito) descrito por um espaço de fase $\Gamma_{2 n}$, com coordenadas locais:

$$
\xi=\left(p_{i}, q_{i}\right), i=1, \ldots, n .
$$


Dessa forma, o espaço de fase corresponde, localmente, a um conjunto aberto de $\mathbb{R}^{2 n}$, mas que globalmente pode apresentar uma topologia não-trivial, como por exemplo, a de uma esfera, ou a de um toro. As variáveis dinâmicas são funções $f: \Gamma_{2 n} \times \mathbb{R} \rightarrow \mathbb{R}$, de forma que $f=f(p, q, t)$, onde $t$ é um parâmetro especial, denominado tempo. Sejam duas tais funções, $f, g: \Gamma_{2 n} \times \mathbb{R} \rightarrow \mathbb{R}$, introduzimos os parênteses de Poisson de $f$ e $g$ como a seguinte função:

$$
\{f, g\}:=\sum_{k=1}^{n} \frac{\partial f}{\partial p_{k}} \frac{\partial g}{\partial q_{k}}-\frac{\partial f}{\partial q_{k}} \frac{\partial g}{\partial p_{k}} .
$$

Claramente, os parênteses de Poisson (2.1.1) são antissimétricos:

$$
\{f, g\}=-\{g, f\}
$$

e satisfazem a identidade de Jacobi:

$$
\{f,\{g, h\}\}+\{g,\{h, f\}\}+\{h,\{f, g\}\}=0 .
$$

Ademais, dizemos que duas funções $f$ e $g$ estão em involução, se satisfazem:

$$
\{f, g\}=0
$$

Claramente, as coordenadas locais $\xi$ satisfazem os parênteses de Poisson canônicos:

$$
\left\{p_{i}, p_{j}\right\}=0, \quad\left\{q_{i}, q_{j}\right\}=0, \quad\left\{p_{i}, q_{j}\right\}=\delta_{i j}
$$

Dada uma Hamiltoniana $H(p, q, t)$, que, em geral, independe do tempo, a dinâmica do sistema é determinada pela seguinte equação diferencial:

$$
\frac{d f}{d t}=\frac{\partial f}{\partial t}+\{H, f\}, \quad \forall f=f(p, q, t)
$$

Fixando $f=p_{i}$ ou $f=q_{i}$, obtemos as equações de Hamilton

$$
\dot{q}_{i}=\frac{\partial H}{\partial p_{i}}, \quad \dot{p}_{i}=-\frac{\partial H}{\partial q_{i}},
$$

que compõem um sistema de equações diferenciais de primeira ordem ${ }^{1}$. O sistema (2.1.4) é determinístico, uma vez que as coordenadas generalizadas a um tempo arbitrário $\xi(t)$ são univocamente determinadas pelas $2 n$ condições iniciais $\xi(0)$. Além disso, os elementos de volume no espaço de fase são conservados.

Definição 2.1.1. Uma função $f: \Gamma_{2 n} \times \mathbb{R} \rightarrow \mathbb{R}$ é dita ser uma constante do movimento ${ }^{2}$ se satisfizer $\dot{f}=0$, quando as equações de Hamilton (2.1.4) forem válidas. Equivalentemente, $f(p(t), q(t), t)=$ $c$, onde c é uma constante, se $p(t)$ e $q(t)$ forem soluções de (2.1.4).

Naturalmente, quando encontramos um sistema de equações diferenciais como (2.1.4), perguntamo-

\footnotetext{
${ }^{1}$ Onde denotamos as derivadas temporais por um ponto.

${ }^{2}$ Ou uma carga conservada, ou uma primeira integral, ou uma integral do movimento,...
} 


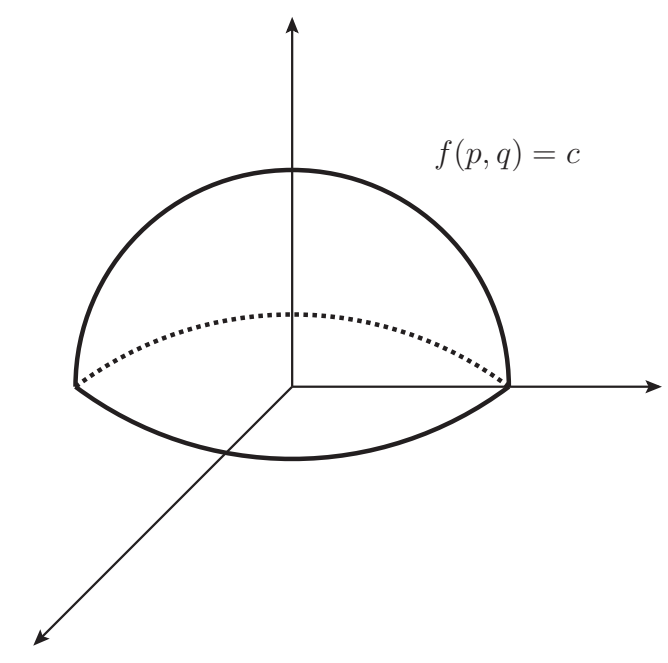

Figura 2.1: Superfície de nível.

nos se existem soluções, se sim, quantas e como construí-las. Em geral, o sistema (2.1.4) é solúvel se existe um número suficiente de integrais de movimento, pois cada uma elimina uma equação diferencial. Suponha, por exemplo, que exista uma carga conservada $f$ que não dependa explicitamente do tempo e que defina uma hipersuperfície $f(p, q)=c$ em $\Gamma_{2 n}$, com $c$ constante, vide figura 2.1. Duas hipersuperfícies correspondentes a cargas conservadas independentes se interceptam em uma superfície com codimensão $2 \mathrm{em} \Gamma_{2 n}$. De maneira geral, a trajetória é descrita sobre uma superfície com dimensão $2 n-L$, onde $L$ é o número de integrais do movimento independentes, se $L=2 n-1$ essa superfície é uma curva e uma solução de (2.1.4).

Assim, o problema de construir uma solução de (2.1.4) é reduzido à construção dessas hipersuperfícies, mas para isso é necessário encontrar um número correspondente de cargas conservadas. De fato, dadas duas integrais de movimento independentes, que não dependam explicitamente do tempo, sempre podemos usar seus parênteses de Poisson para definir uma terceira constante do movimento. O teorema de Noether, por sua vez, fornece algumas primeiras integrais, relacionadas às simetrias do sistema. Contudo, as cargas conservadas obtidas dessa maneira não são, em geral, suficientes para prosseguirmos.

\subsection{Geometria Simplética}

Existe uma forma natural de se estender o formalismo Hamiltoniano ao se generalizar o conceito de parênteses de Poisson (2.1.1). Uma abordagem mais geométrica da mecânica clássica, fornecida pela geometria simplética $[45,46]$, permite uma melhor compreensão dos sistemas integráveis. Considere, então, o conjunto das funções diferenciáveis no espaço de fase $\Gamma_{2 n}$ e denote por $\mathcal{F}\left(\Gamma_{2 n}\right)$, a álgebra de tais funções. Definimos, pois, o conceito de parênteses de Poisson:

Definição 2.2.1. Os parênteses de Poisson são uma derivação bilinear e antissimétrica da álgebra $\mathcal{F}\left(\Gamma_{2 n}\right):\{\quad, \quad\}: \mathcal{F}\left(\Gamma_{2 n}\right) \times \mathcal{F}\left(\Gamma_{2 n}\right) \rightarrow \mathcal{F}\left(\Gamma_{2 n}\right)$ tal que: 
Antissimetria: $\left\{f_{1}, f_{2}\right\}=-\left\{f_{2}, f_{1}\right\}$;

Linearidade: $\left\{f_{1}, a f_{2}+b f_{3}\right\}=a\left\{f_{1}, f_{2}\right\}+b\left\{f_{1}, f_{3}\right\}$, com $a, b$ constantes;

Regra de Leibnitz: $\left\{f_{1}, f_{2} f_{3}\right\}=\left\{f_{1}, f_{2}\right\} f_{3}+f_{2}\left\{f_{1}, f_{3}\right\}$;

Identidade de Jacobi: $\left\{f_{1},\left\{f_{2}, f_{3}\right\}\right\}+\left\{f_{2},\left\{f_{3}, f_{1}\right\}\right\}+\left\{f_{3},\left\{f_{1}, f_{2}\right\}\right\}=0$.

Dado que os parênteses de Poisson são lineares em suas entradas e satisfazem a regra de Leibnitz, para cada função $H \in \mathcal{F}\left(\Gamma_{2 n}\right)$ podemos associar um campo vetorial $X_{H} f=\{H, f\}$ em $\Gamma_{2 n}$. Se $H$ for a Hamiltoniana do sistema, esse campo define a evolução temporal de $f$ :

$$
\dot{f}=X_{H} f=\{H, f\}, \quad \forall f \in \mathcal{F}\left(\Gamma_{2 n}\right) .
$$

Assim, podemos definir o conceito de variedade de Poisson.

Definição 2.2.2. Uma variedade $\Gamma_{2 n}$ na qual um parênteses de Poisson está definido é dita uma variedade de Poisson.

Uma das mais importantes propriedades dos parênteses de Poisson no contexto da mecânica clássica é que se $f_{1}$ e $f_{2}$ forem quantidades conservadas, ou seja,

$$
\left\{H, f_{1}\right\}=0 \quad \text { e } \quad\left\{H, f_{2}\right\}=0,
$$

então $\left\{f_{1}, f_{2}\right\}$ também é conservada em virtude da identidade de Jacobi. Em geral, os parênteses de Poisson são degenerados, ou seja, existem funções $f_{i} \in \mathcal{F}\left(\Gamma_{2 n}\right)$ tais que $\left\{f_{i}, g\right\}=0$ para qualquer $g \in \mathcal{F}\left(\Gamma_{2 n}\right)$. O conjunto dessas funções $f_{i}$ é denominado centro da álgebra de Poisson. Se o centro for não-trivial, isto é, se pelo menos uma dessas $f_{i}$ não for uma função constante, é possível reduzir o sistema dinâmico em questão, ao impor que todas as funções do centro sejam iguais a valores constantes. Esse procedimento define uma folheação de $\Gamma_{2 n}$ e os parênteses de Poisson em cada folha são não-degenerados.

Para um dado sistema local de coordenadas $x^{i}$ de $\Gamma_{2 n}$ podemos escrever:

$$
\left\{f_{1}, f_{2}\right\}_{\Gamma_{2 n}}=P^{i j}(x) \frac{\partial f_{1}}{\partial x^{i}} \frac{\partial f_{2}}{\partial x^{j}} .
$$

A antissimetria dos parênteses de Poisson impõem que:

$$
P^{i j}(x)=-P^{j i}(x)
$$

enquanto que a identidade de Jacobi assume a seguinte forma:

$$
P^{i s} \partial_{s} P^{j k}+P^{k s} \partial_{s} P^{i j}+P^{j s} \partial_{s} P^{k i}=0
$$

Um caso de especial interesse ocorre quando $P^{i j}$ é inversível, implicando, em particular, que o centro da álgebra de Poisson é trivial e que a dimensão da variedade seja par. Denotando a inversa de $P^{i j}$ 
por $\left(P^{-1}\right)_{i j}$, temos que $\partial_{s} P^{i j}=-P^{i a} \partial_{s}\left(P^{-1}\right)_{a b} P^{b j}$, que inserido na identidade de Jacobi fornece:

$$
\begin{array}{r}
P^{i s} P^{j a} P^{k b}\left(\partial_{s}\left(P^{-1}\right)_{a b}+\partial_{b}\left(P^{-1}\right)_{s a}+\partial_{a}\left(P^{-1}\right)_{b s}\right)=0 \\
\Rightarrow \partial_{s}\left(P^{-1}\right)_{a b}+\partial_{b}\left(P^{-1}\right)_{s a}+\partial_{a}\left(P^{-1}\right)_{b s}=0
\end{array}
$$

onde usamos o fato de que $P^{i j}$ é inversível. Essas condições garantem a existência de uma 2-forma fechada:

$$
\omega=-\frac{1}{2}\left(P^{-1}\right)_{i j} d x^{i} \wedge d x^{j},
$$

ou seja, tal que $d \omega=0$. Essa 2-forma é invariante sob transformações de coordenadas, e por isso globalmente definida em $\Gamma_{2 n}$.

Definição 2.2.3. Seja $\Gamma_{2 n}$ uma variedade diferenciável com dimensão par. Uma estrutura simplética em $\Gamma_{2 n}$ é dada por uma 2-forma diferencial fechada e não-degenerada $\omega$ :

$$
d \omega=0 \quad \text { e } \quad \forall \zeta \neq 0 \quad \exists \eta: \omega(\zeta, \eta) \neq 0, \quad \zeta, \eta \in T_{x} \Gamma_{2 n} .
$$

O par $\left(\Gamma_{2 n}, \omega\right)$ é denominado uma variedade simplética.

No contexto de variedades simpléticas existe um isomorfismo natural entre campos vetoriais e 1-formas estabelecido pela estrutura simplética. Sejam $\mathcal{V}\left(\Gamma_{2 n}\right)$ e $\Omega^{r}\left(\Gamma_{2 n}\right)$, o conjunto de todos os campos vetoriais e o espaço vetorial de todas as $r$-formas diferenciais, respectivamente, em $\Gamma_{2 n}$, e considere o produto interior associado a um vetor arbitrário $\zeta \in \mathcal{V}\left(\Gamma_{2 n}\right), i_{\zeta}: \Omega^{r}\left(\Gamma_{2 n}\right) \rightarrow \Omega^{r-1}\left(\Gamma_{2 n}\right)$. Assim, para cada vetor $\zeta \in \mathcal{V}\left(\Gamma_{2 n}\right)$ podemos associar a seguinte 1-forma através da estrutura simplética:

$$
\zeta \mapsto i_{\zeta} \omega=\omega(\zeta, \cdot)
$$

Portanto, estabelecemos um isomorfismo entre $\mathcal{V}\left(\Gamma_{2 n}\right) \leftrightarrow \Omega^{1}\left(\Gamma_{2 n}\right)$. Com esse isomorfismo podemos introduzir o campo vetorial Hamiltoniano associado à função $f \in \mathcal{F}\left(\Gamma_{2 n}\right), X_{f}$, implicitamente por:

$$
i_{X_{f}} \omega=\omega\left(X_{f}, \cdot\right)=-d f
$$

Em termos das coordenadas locais $x^{i}$ em $\Gamma_{2 n}$, temos:

$$
\begin{aligned}
\omega & =\frac{1}{2} \omega_{i j} d x^{i} \wedge d x^{j}, \\
X_{f} & =X_{f}^{i} \partial_{i}=\omega^{i j} \partial_{j} f,
\end{aligned}
$$

com $\omega^{i j}=\left(\omega_{i j}\right)^{-1}$. Conseqüentemente, com o conhecimento da forma simplética $\omega$, podemos reconstruir os parênteses de Poisson:

$$
\left\{f_{1}, f_{2}\right\}=X_{f_{1}}\left(f_{2}\right)=-X_{f_{2}}\left(f_{1}\right)=\omega\left(X_{f_{1}}, X_{f_{2}}\right),
$$

ou, em componentes:

$$
\left\{f_{1}, f_{2}\right\}=-\omega^{i j} \partial_{i} f_{1} \partial_{j} f_{2}
$$

Outro conceito importante que pode ser construído em um espaço simplético é o de transforma- 
ções simpléticas, para isso considere uma bijeção $\gamma: \Gamma_{2 n} \rightarrow \Gamma_{2 n}$, a transformação da forma simplética $\omega$ sob $\gamma$ é:

$$
\left(\gamma^{*} \omega\right)_{m}(V, W)=\omega_{\gamma(m)}\left(\gamma_{*} V, \gamma_{*} W\right)
$$

onde $m \in \Gamma_{2 n}$ e $\gamma_{*}$ é o diferencial de $\gamma$, levando um vetor tangente em $m$ em um vetor tangente em $\gamma(m)$. Diz-se que a transformação $\gamma$ é simplética se $\gamma^{*} \omega=\omega$, isto é, uma transformação simplética é aquela que preserva a forma simplética. Para uma transformação infinitesimal, $\gamma$ é especificada por um vetor $X$ em $\Gamma_{2 n}$, e essa condição é equivalente a $\mathcal{L}_{X} \omega=0$, com $\mathcal{L}_{X}=d i_{X}+i_{X} d$ sendo a derivada de Lie. Com respeito aos parênteses de Poisson a condição de simpleticidade de uma transformação adquire a seguinte forma:

$$
\gamma\left\{f_{1}, f_{2}\right\}=\left\{\gamma f_{1}, \gamma f_{2}\right\}
$$

Proposição 2.2.4. Qualquer fluxo Hamiltoniano é uma transformação simplética.

Demonstração: Seja $H$ uma hamiltoniana e $X_{H}$ o campo vetorial associado, tal que $i_{X_{H}} \omega=-d H$, então:

$$
\mathcal{L}_{X_{H}} \omega=\left(i_{X_{H}} d+d i_{X_{H}}\right) \omega=d\left(i_{X_{H}} \omega\right)=-d^{2} H=0
$$

Exemplo 2.2.5. O exemplo mais simples de um espaço simplético é dado por $\Gamma_{2 n}=\mathbb{R}^{2 n}$ com as coordenadas canônicas $\xi=\left(p_{i}, q_{i}\right)$ e a seguinte forma simplética:

$$
\omega=d p^{i} \wedge d q_{i}
$$

De forma que para duas funções quaisquer $f\left(p_{i}, q_{i}\right)$ e $g\left(p_{i}, q_{i}\right)$ definidas no espaço de fase, os parênteses de Poisson podem ser escritos em termos das coordenadas canônicas como (2.1.1). Por sua vez, o campo vetorial Hamiltoniano associado a uma função $f \in \mathcal{F}\left(\Gamma_{2 n}\right)$ é dado por:

$$
X_{f}=-\frac{\partial f}{\partial q_{i}} \partial_{p_{i}}+\frac{\partial f}{\partial p_{i}} \partial_{q_{i}}
$$

De fato, esse exemplo é bem geral, uma vez que, pelo menos localmente, o teorema de Darboux garante tal construção.

Teorema 2.2.6. Em qualquer variedade simplética $\left(\Gamma_{2 n}, \omega\right)$ é possível introduzir, localmente ao redor de um ponto $m_{0}$, coordenadas canônicas $\xi=\left(p_{i}, q_{i}\right)$ tais que $\omega=d p^{i} \wedge d q_{i}$. Além do mais, é possível escolher $p_{1}$ como qualquer função $H$ em $\Gamma_{2 n}$ tal que $d H\left(m_{0}\right) \neq 0$.

A demonstração desse teorema se encontra no apêndice A.1.

\subsection{Integrabilidade de Liouville}

Em virtude da equação (2.2.1) fica claro que a Hamiltoniana é uma quantidade conservada sob a evolução temporal, de forma que o movimento ocorre em uma subvariedade do espaço de fase definida por $H=E$, onde $E$ é uma constante. Sistemas dinâmicos descritos pelas (2.1.4) não são exatamente solúveis em geral, contudo para uma certa classe desses sistemas, conhecidos 
como sistemas integráveis no sentido de Liouville, soluções explícitas podem ser obtidas por meio de quadraturas. Antes de formularmos precisamente esses conceitos, é instrutivo considerar um exemplo ilustrativo de modo a melhor compreender a geometria da situação.

Exemplo 2.3.1. O Oscilador Harmônico: Considere o espaço de fase bidimensional parametrizado pelas coordenadas canônicas $\xi=(p, q)$, de modo que a Hamiltoniana assume a seguinte forma:

$$
H=\frac{1}{2}\left(p^{2}+\omega^{2} q^{2}\right)
$$

com os parênteses de Poisson dados por:

$$
\{p, q\}=1
$$

$O$ espaço de fase é fibrado em elipses $H=E$, com exceção do ponto $(0,0)$, que é estacionário. Um outro sistema de coordenadas melhor adaptado ao oscilador harmônico é $(\rho, \theta)$ :

$$
p=\rho \cos \theta \quad \text { e } \quad q=\frac{\rho}{\omega} \sin \theta
$$

com os seguintes parênteses de Poisson não-nulos:

$$
\{\rho, \theta\}=\frac{\omega}{\rho} .
$$

Nessas coordenadas podemos escrever o fluxo como:

$$
\rho=\sqrt{2 E}, \quad \theta=\omega t+\theta_{0}
$$

ou seja, o fluxo ocorre nas elipses descritas acima.

Não é complicado generalizar esse resultado para a soma direta de $n$ osciladores harmônicos, cuja Hamiltoniana é dada por:

$$
H=\frac{1}{2} \sum_{i=1}^{n}\left(p_{i}^{2}+\omega_{i}^{2} q_{i}^{2}\right)
$$

com parênteses de Poisson dados pela equação (2.1.2). Agora, existem n quantidades conservadas em involução, $F_{i}=\frac{1}{2}\left(p_{i}^{2}+\omega_{i}^{2} q_{i}^{2}\right)$, e a variedade de nível $\Gamma_{2 n}^{f}=\left\{\xi \in \Gamma_{2 n}: F_{i}=f_{i}\right\}$ é um toro real n-dimensional. O movimento ocorre nesses toros, que folheiam o espaço de fase. É também possivel introduzir $n$ ângulos $\theta_{i}$, que assim como no caso unidimensional, evoluem linearmente no tempo com freqüências $\omega_{i}$.

Inspirados pelo exemplo 2.3.1 introduzimos o conceito de integrabilidade de Liouville:

Definição 2.3.2. Um sistema dinâmico Hamiltoniano definido em uma variedade simplética $\left(\Gamma_{2 n}, \omega\right)$ com Hamiltoniana $H$ é integrável no sentido de Liouville, se existirem n funções independentes $F_{i}$, $i=1, \ldots, n$ tais que:

1. $\left\{H, F_{i}\right\}=0$;

2. $\left\{F_{i}, F_{j}\right\}=0$. 
A condição 1 da definição 2.3 .2 implica que as funções $F_{i}$ são conservadas com respeito ao fluxo do campo vetorial Hamiltoniano, enquanto que a condição 2 demanda que tais funções estejam em involução. Já a independência dessas funções significa que, para um ponto qualquer do espaço de fase, os $d F_{i}$ são linearmente independentes, o que equivale ao fato de que o espaço tangente à superfície $F_{i}=f_{i}$ existe para todos os pontos e sua dimensão é $n$. Notamos que não podem existir mais do que $n$ quantidades conservadas independentes em involução, pois caso contrário os parênteses de Poisson seriam degenerados, o que é incompatível com a condição de que o espaço de fase seja uma variedade simplética. A Hamiltoniana $H$ deve depender das coordenadas do sistema, que em virtude do teorema de Darboux, podem ser tomadas, ao menos localmente, como $\xi=\left(p_{i}, q_{i}\right)$, somente através das integrais de movimento $F_{i}$.

Como imediata consequiência da definição 2.3 .2 podemos demonstrar que as soluções das equações de movimento de um sistema integrável no sentido de Liouville podem ser obtidas por quadraturas, ou seja, através de um número finito de integrações e operações algébricas. Esse é o conteúdo do teorema de Liouville.

Teorema 2.3.3. Seja um sistema dinâmico Hamiltoniano integrável no sentido de Liouville, então as suas equações de movimento são integráveis por quadraturas.

Demonstração: Considere a variedade simplética $\left(\Gamma_{2 n}, \omega\right)$ onde o sistema dinâmico Hamiltoniano integrável está definido. Uma vez que a forma simplética $\omega$ é fechada, pelo lema de Poincaré ${ }^{3}$, temos que, ao menos localmente, ela é exata. Assim, podemos introduzir a 1-forma canônica $\alpha=p^{i} d q_{i}$ de forma que $\omega=d \alpha$. Queremos construir uma transformação canônica (simplética) $\left(p_{i}, q_{i}\right) \rightarrow\left(F_{i}, \theta_{i}\right)$, tal que as quantidades conservadas $F_{i}$ estejam dentre as novas coordenadas:

$$
\omega=d p_{i} \wedge d q^{i}=d F_{i} \wedge d \theta^{i}
$$

Para isso, seja a variedade de nível $\Gamma_{2 n}^{f}=\left\{(p, q) \in \Gamma_{2 n}: F_{i}(p, q)=f_{i}\right\}$. Pelo teorema da função implícita, podemos resolver para os $p_{i}$ em $\Gamma_{2 n}^{f}, p_{i}=p_{i}(f, q)$. Considere, então, a seguinte função:

$$
S(F, q) \equiv \int_{m_{0}}^{m} \alpha=\int_{q_{0}}^{q} p_{i}(f, q) d q^{i}
$$

com o caminho de integração em $\Gamma_{2 n}^{f}$ indo do ponto com coordenadas $\left(p\left(f, q_{0}\right), q_{0}\right)$ ao ponto $(p(f, q), q)$, onde $q_{0}$ é apenas um valor de referência, vide figura 2.2. Se essa função existir, ou seja, se a integração não depender do caminho entre $m_{0}$ e $m$, então:

$$
p_{i}=\frac{\partial S}{\partial q_{i}}
$$

e definindo $\theta_{i}$ por:

$$
\theta_{i}=\frac{\partial S}{\partial F_{i}}
$$

temos que:

$$
d S=\theta_{i} d F^{i}+p_{i} d q^{i} .
$$

\footnotetext{
${ }^{3}$ Lema de Poincaré: "Se uma vizinhança $U$ de uma variedade $M$ é contrátil a um ponto $p \in M$, então qualquer $r$-forma fechada é exata em U".
} 


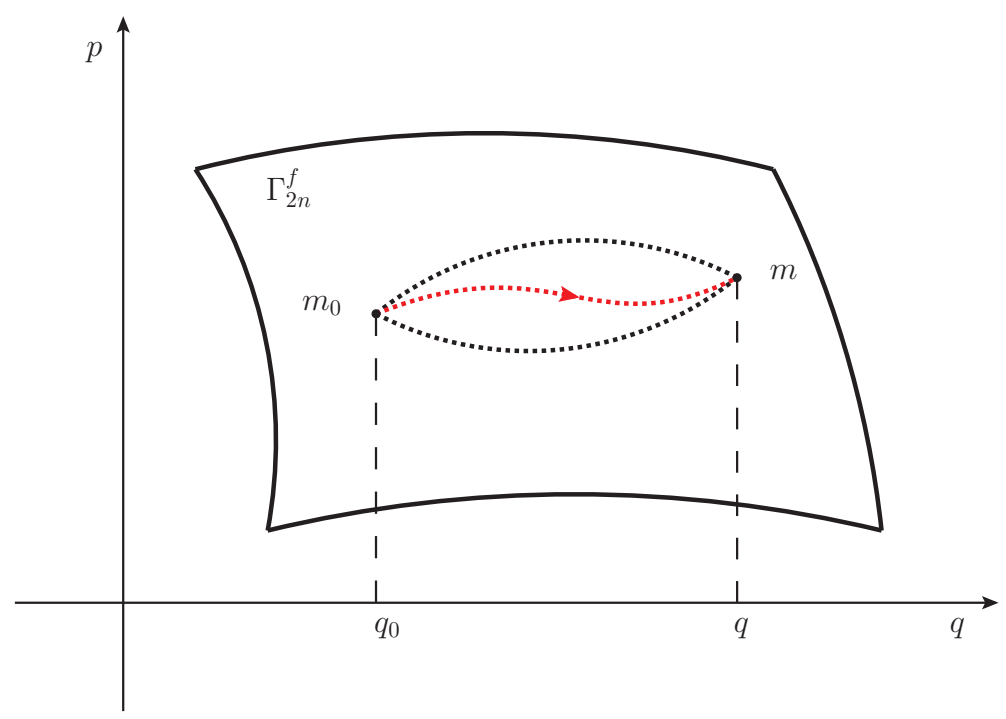

Figura 2.2: Uma folha $\Gamma_{2 n}^{f}$ no espaço de fase.

Finalmente, como $d^{2} S=0$ concluímos que a equação (2.3.2) é verdadeira. Assim, se $S$ for uma função bem definida, temos que a transformação é canônica, pois $0=d^{2} S=-\mathcal{L}_{X_{S}}$. Agora, para mostrarmos que $S$ existe, devemos mostrar que ela é independente da escolha do caminho de integração. Pelo teorema de Stokes, é suficiente provarmos que:

$$
\left.d \alpha\right|_{\Gamma_{2 n}^{f}}=\left.\omega\right|_{\Gamma_{2 n}^{f}}=0
$$

Seja $X_{i}$ o campo vetorial Hamiltoniano associado com $F_{i}$ :

$$
X_{i}=\frac{\partial F_{i}}{\partial q^{k}} \frac{\partial}{\partial p_{k}}-\frac{\partial F_{i}}{\partial p^{k}} \frac{\partial}{\partial q_{k}},
$$

esses campos vetoriais são tangentes à variedade de nível $\Gamma_{2 n}^{f}$, uma vez que estão em involução,

$$
X_{i}\left(F_{j}\right)=\left\{F_{i}, F_{j}\right\}=0
$$

Como por hipótese os $F_{i}$ formam um conjunto de funções independentes, temos que o espaço tangente à subvariedade $\Gamma_{2 n}^{f}$ é gerado em cada ponto $m \in \Gamma_{2 n}$ pelos vetores $\left.X_{i}\right|_{m}(i=1, \ldots, n)$. Mas então, $\omega\left(X_{i}, X_{j}\right)=-d F_{i}\left(X_{j}\right)=0$, de forma que demonstramos que $\left.\omega\right|_{\Gamma_{2 n}^{f}}=0$. Logo, $S$ existe.

Conforme provamos anteriormente, a existência de $S$ implica a existência da transformação canônica $\left(p_{i}, q_{i}\right) \rightarrow\left(F_{i}, \theta_{i}\right)$, com $F_{i}$ sendo as cargas conservadas, portanto as equações de movimento se 
tornam triviais:

$$
\begin{aligned}
& \dot{F}_{i}=\left\{H, F_{i}\right\}=0, \\
& \dot{\theta}_{i}=\left\{H, \theta_{i}\right\}=\frac{\partial H}{\partial F_{i}}=\Omega_{i},
\end{aligned}
$$

onde os $\Omega_{i}$ dependem apenas de $F$ e conseqüentemente são constantes no tempo. Nessas coordenadas, a solução das equações de movimento assume a seguinte forma:

$$
F_{i}(t)=F_{i}(0) \quad, \quad \theta_{i}(t)=\theta_{i}(0)+\Omega_{i} t
$$

Assim, construímos uma solução explícita das equações de movimento de um sistema dinâmico Hamiltoniano, ao supormos a existência de quantidades conservadas em involução. As integrais de movimento $F_{i}$, por sua vez, introduzem superfícies $n$-dimensionais, as variedades de nível $\Gamma_{2 n}^{f}$ a partir da condição $F_{i}=f_{i}$, que fornecem uma folheação invariante e regular do espaço de fase.

A partir das coordenadas que introduzimos para demonstrar o teorema 2.3.3 podemos construir as famigeradas coordenadas de ângulo e ação. Para isso, notamos que da condição de $\alpha$ ser fechada em $\Gamma_{2 n}^{f}$, concluímos que a função $S$ é invariante sob deformações contínuas do caminho $\left(m_{0}, m\right)$. Porém, se $\Gamma_{2 n}^{f}$ possuir ciclos não-triviais, $S$ se torna uma função multivalorada definida em uma vizinhança de $\Gamma_{2 n}^{f}$. A variação sob um ciclo dada por:

$$
\Delta_{\text {ciclo }} S=\int_{\text {ciclo }} \alpha
$$

é uma função apenas de $F$. Assim, temos que as variáveis $\theta_{i}$ também assumem vários valores

$$
\Delta_{\text {ciclo }} \theta_{i}=\frac{\partial}{\partial F_{i}} \Delta_{\text {ciclo }} S
$$

Dessa forma, podemos escolher coordenadas especiais em $\Gamma_{2 n}^{f}$, que sejam duais a esses $n$ ciclos fundamentais $C_{i}$. Inicialmente, definimos as coordenadas de ação $l_{j}$ como as integrais da 1 -forma canônica sobre os ciclos $C_{j}$,

$$
l_{j}=\frac{1}{2 \pi} \int_{C_{j}} \alpha
$$

Os $l_{j}$ são funções apenas das constantes de movimento $F_{j}$ e supomos que sejam independentes, de forma que se soubermos os valores de $l_{j}$, então $\Gamma_{2 n}^{f}$ estará determinada. Consideramos a função geradora (2.3.3) com os $F_{j}$ substituídos pelos $l_{j}$ e denotamos por $\varphi_{j}$ as coordenadas conjugadas a $l_{j}$. A transformação canônica gerada por $S$ é definida por:

$$
p_{j}=\frac{\partial S}{\partial q_{j}} \quad \text { e } \quad \varphi_{j}=\frac{\partial S}{\partial l_{j}}
$$

Assim,

$$
\int_{C_{j}} d \varphi_{k}=\frac{\partial}{\partial I_{k}} \int_{C_{j}} d S=\frac{\partial}{\partial I_{k}} \int_{C_{j}}\left(p^{i} d q_{i}+\varphi^{i} d I_{i}\right)=\frac{\partial}{\partial I_{k}} \int_{C_{j}} \frac{\partial S}{\partial q_{i}} d q_{i}=\frac{\partial}{\partial I_{k}} \int_{C_{j}} \alpha=2 \pi \delta_{j k},
$$


onde usamos que sobre a variedade de nível $\Gamma_{2 n}^{f}, d l_{i}=0$. Mostramos, pois, que as coordenadas $\varphi_{j}$ podem ser entendidas como coordenadas angulares normalizadas. A existência dessas coordenadas angulares indica que sob certas circunstâncias a topologia do espaço de fase deve ser similar a de um n-toro.

Notamos também que ao usarmos as funções em involução $F_{i}{ }^{4}$, é possível resolver simultaneamente as $n$ equações de evolução temporal:

$$
\frac{d F}{d t_{i}}=\left\{F_{i}, F\right\}
$$

pois

$$
\partial_{t_{i}} \partial_{t_{j}} F-\partial_{t_{j}} \partial_{t_{i}} F=\left\{F_{i},\left\{F_{j}, F\right\}\right\}-\left\{F_{j},\left\{F_{i}, F\right\}\right\}=\left\{\left\{F_{i}, F_{j}\right\}, F\right\}=0 .
$$

Uma vez que os campos vetoriais Hamiltonianos estão bem definidos e são linearmente independentes em todos os pontos, os fluxos definem uma ação localmente livre ${ }^{5}$ e transitiva $^{6}$ de um pequeno conjunto aberto de $\mathbb{R}^{n}$ sobre a superfície $\Gamma_{2 n}^{f}$. Supondo que $\Gamma_{2 n}^{f}$ seja compacta e conexa, esses fluxos se estendem para todos os valores de tempo $t_{i}$ e preenchem toda a superfície $\Gamma_{2 n}^{f}$, temos, pois, uma ação sobrejetora de $\mathbb{R}^{n}$ em $\Gamma_{2 n}^{f}$. O estabilizador de um ponto é um subgrupo Abeliano discreto de $\mathbb{R}^{n}$, dado que a ação é localmente livre, ele deve ser da forma $\mathbb{Z}^{n}$. Portanto, $\Gamma_{2 n}^{f}$ surge como o quociente de $\mathbb{R}^{n}$ por $\mathbb{Z}^{n}$, ou seja, como um toro. Esse refinamento do teorema de Liouville é conhecido como teorema de Arnold-Liouville.

Teorema 2.3.4. Seja um sistema dinâmico Hamiltoniano integrável no sentido de Liouville, então:

1. $\Gamma_{2 n}^{f}$ é uma variedade suave e invariante sob o fluxo de fase com Hamiltoniana $H=F_{1}$;

2. O fluxo de fase com Hamiltoniana $H$ determina um movimento condicionalmente periódico em $\Gamma_{2 n}^{f}$, isto é, em coordenadas angulares $\varphi_{i}, i=1, \ldots, n$ temos que:

$$
\frac{d \varphi_{i}}{d t}=\Omega_{i}, \quad \Omega=\Omega(f)
$$

3. As equações canônicas com Hamiltoniana H podem ser integradas por quadraturas;

4. Se a variedade $\Gamma_{2 n}^{f}$ for compacta e conexa, então é difeomórfica a um toro n-dimensional $T^{n}=\left\{\left(\varphi_{1}, \ldots, \varphi_{n}\right) \bmod 2 \pi\right\}$.

A demonstração desse teorema pode ser encontrada em [45].

\subsection{Integrabilidade na Teoria Clássica de Campos}

Até então consideramos apenas sistemas com um número finito de graus de liberdade, desenvolvendo nesse contexto um critério objetivo para determinar se um dado sistema é integrável ou não. Contudo, desejamos estudar modelos integráveis em teorias de campos, e para isso precisamos

\footnotetext{
${ }^{4}$ Equivalentemente, podemos utilizar as variáveis de ação $I_{i}$.

${ }^{5}$ Sem pontos fixos.

${ }^{6}$ Para todos os pontos.
} 
estender o conceito de integrabilidade para sistemas com um número infinito de graus de liberdade. Tal generalização envolve o desenvolvimento do método do espalhamento inverso clássico (MEIC), que fornece um algoritmo para se encontrar soluções de equações diferenciais parciais não-lineares integráveis.

O conceito de integrabilidade de Liouville, fornecido pela definição 2.3.2, admite uma extensão imediata para o caso de teorias de campos, que consiste em permitir que o número de graus de liberdade $n$ seja infinito. Assim, para que uma teoria de campos seja integrável, ela deve possuir infinitas quantidades conservadas em involução. Entretanto, essa definição esconde algumas sutilezas, a mais notável delas está relacionada com o fato de que um dado sistema pode não ser integrável mesmo possuindo infinitas cargas conservadas em involução. Isso é devido ao fato de que precisamos de uma integral do movimento para cada grau de liberdade, de forma que mesmo um número infinito pode não ser suficiente. Portanto, para podermos abordar essa questão de um modo mais rigoroso, é necessário que se construa um mapa bijetor entre os graus de liberdade do sistema e suas primeiras integrais. Esse mapa está diretamente relacionado com a construção das coordenadas de ângulo e ação.

A estrutura de Poisson, que introduzimos na seção 2.2, é útil no estabelecimento do formalismo Hamiltoniano para sistemas com infinitos graus de liberdade. Formalmente, podemos substituir as coordenadas $\xi_{i}(t)$ pela variável dinâmica $\Psi(x, t)$, de forma que o índice discreto $i$ se torna a variável contínua e independente $x$. O espaço de fase $\Gamma_{2 n}$, antes localmente isomórfico a $\mathbb{R}^{2 n}$, é substituído por um espaço de funções suaves sobre um subconjunto $D \subset \mathbb{R}$ com condições de contorno adequadas. De uma forma bem esquemática temos que fazer as seguintes substituições:

$$
\begin{aligned}
\text { Equações Diferenciais Ordinárias } & \longrightarrow \text { Equações Diferenciais Parciais, } \\
\xi_{i}(t), i=1, \ldots, n & \longrightarrow \psi(x, t), x \in D, \\
\sum_{i} & \longrightarrow \int_{D} d x, \\
\text { Funções } f(\xi) & \longrightarrow \text { Funcionais } F[\psi(x, t)] \\
\frac{\partial}{\partial \xi_{i}} & \longrightarrow \frac{\delta}{\delta \psi} .
\end{aligned}
$$

Os funcionais são dados por integrais da seguinte forma:

$$
F[\Psi(x, t)]=\int_{D} d x f\left(\Psi, \partial_{x} \Psi, \partial_{x}^{2} \Psi, \ldots\right)
$$

enquanto que a derivada funcional é dada por:

$$
\frac{\delta F}{\delta \Psi(x, t)}=\frac{\partial f}{\partial \Psi}-\frac{\partial}{\partial x} \frac{\partial f}{\partial \partial_{x} \Psi}+\left(\frac{\partial}{\partial x}\right)^{2} \frac{\partial f}{\partial\left(\partial_{x}^{2} \Psi\right)}+\cdots, \quad \operatorname{com} \frac{\delta \Psi(x, t)}{\delta \Psi(y, t)}=\delta(x-y)
$$

De uma forma análoga a (2.2.2) podemos definir os parênteses de Poisson:

$$
\{F, G\}=\int_{D^{2}} d x d y \omega(x, y, \Psi) \frac{\delta F}{\delta \Psi(x, t)} \frac{\delta G}{\delta \Psi(y, t)}
$$


onde a estrutura de Poisson $\omega(x, y, \psi)$ deve garantir a antissimetria de (2.4.1) e a validade da identidade de Jacobi. A escolha canônica corresponde a:

$$
\omega(x, y, \Psi)=-\frac{1}{2} \frac{\partial}{\partial x} \delta(x-y)+\frac{1}{2} \frac{\partial}{\partial y} \delta(x-y),
$$

com a qual encontramos:

$$
\{F, G\}=\frac{1}{2} \int_{D} d x\left[\frac{\partial}{\partial x} \frac{\delta F}{\delta \Psi(x)} \frac{\delta G}{\delta \Psi(x)}-\frac{\delta F}{\delta \Psi(x)} \frac{\partial}{\partial x} \frac{\delta G}{\delta \Psi(x)}\right]
$$

que é o equivalente em dimensão infinita de (2.1.1). Note que para obtermos (2.4.3) a partir de (2.4.2) precisamos usar que o operador de diferenciação $\partial_{x}$ é anti-auto-adjunto com respeito ao produto interno usual:

$$
(\Psi, \Phi)=\int_{D} d x \Psi(x) \Phi(x) .
$$

Nesse caso, as equações de Hamilton adquirem a seguinte forma:

$$
\frac{\partial \Psi}{\partial t}=\{H[\Psi], \Psi\}=\int_{D} d y \frac{\partial}{\partial y}\left(\frac{\delta H}{\delta \Psi(y)}\right) \frac{\delta \Psi(x)}{\delta \Psi(y)}=\frac{\partial}{\partial x} \frac{\delta H}{\delta \Psi(x)} .
$$

No que se segue, faremos uma consideração geral, porém, naturalmente incompleta ${ }^{7}$, do método do espalhamento inverso clássico utilizando uma teoria de campos clássica em duas dimensões definida em um cilindro com coordenadas espacial $\sigma \in[-L, L]$ e temporal $\tau \in(-\infty,+\infty)$, cuja Hamiltoniana $H$ fornece uma equação não-linear integrável de evolução Hamiltoniana. Finalmente, é importante ressaltar que todas as manipulações formais que efetuamos podem ser justificadas e fundamentadas no contexto da análise funcional.

\subsubsection{O Modelo de Schrödinger não-linear - Um Exemplo Representativo}

Ilustramos nossas considerações acerca do MEIC através do exemplo mais simples, contudo representativo, de sua aplicação: o modelo de Schrödinger não-linear (NLS). O sistema dinâmico considerado é gerado pela seguinte equação não-linear:

$$
i \frac{\partial \psi}{\partial t}=-\frac{\partial^{2} \psi}{\partial x^{2}}+2 g|\psi|^{2} \psi
$$

com condições iniciais

$$
\left.\psi(x, t)\right|_{t=0}=\psi(x)
$$

onde $\psi(x, t)$ é uma função com valores complexos, o campo clássico carregado, e $|\psi|^{2}=\psi \bar{\psi}$, com a barra denotando a conjugação complexa. O domínio da variável $x$ é a reta real inteira, $-\infty<$ $x<\infty$ e o valor inicial $\psi(x)$ é suposto suficientemente suave. O parâmetro real $g$ é a constante de acoplamento, no limite linear, $g=0$, a equação (2.4.5) se reduz à equação de Schrödinger para a função de onda de uma partícula com massa $m=\frac{1}{2}$ em uma dimensão. Por esse motivo, a equação

\footnotetext{
${ }^{7}$ Em virtude da natureza do texto, que impõem severas limitações na quantidade de tópicos abordados, só consideraremos aqueles que julgamos essenciais à compreensão do conceito de integrabilidade e que sejam úteis no contexto quântico. Para uma abordagem mais completa recomendamos as monografias $[20,46,47]$.
} 
(2.4.5) é comumente referida como equação de Schrödinger não-linear.

Ao problema de valor inicial determinado por (2.4.5) e (2.4.6) devem ser adicionadas condições de contorno. Consideramos o caso mais geral com condições de contorno quasiperiódicas:

$$
\psi(x+2 L, t)=e^{i \theta} \psi(x, t)
$$

onde $0 \leq \theta<2 \pi$ e $\theta$ não depende de $t$. Claramente, em virtude das condições de contorno (2.4.7), precisamos considerar em (2.4.5) apenas o domínio fundamental do grupo de translações gerado pela transformação: $x \mapsto x+2 L$, que, por concreteza, escolhemos como $-L \leq x<L$. Um limite interessante das condições de contorno (2.4.7) é obtido no caso em que $L \rightarrow \infty$ e

$$
\psi(x, t) \stackrel{|x| \rightarrow \infty}{\longrightarrow} 0,
$$

com $\psi \in \mathscr{S}\left(\mathbb{R}^{1}\right)$.

As coordenadas do espaço de fase $\Gamma_{L, \theta}$ são fornecidas pelo par de funções suaves $\psi(x)$ e $\bar{\psi}(x)$ sujeitas à condição (2.4.7). Naturalmente, os funcionais em $\Gamma_{L, \theta}$ dependem apenas dos valores de $\psi(x)$ e $\bar{\psi}(x)$ no domínio fundamental e são da forma:

$$
\begin{aligned}
F(\psi, \bar{\psi}) & =c+\sum_{\substack{n, m=0 \\
(n, m) \neq(0,0)}}^{\infty} \int_{-L}^{L} \ldots \int_{-L}^{L} d y_{1} \ldots d y_{n} d z_{1} \ldots d z_{m} c_{n m}\left(y_{1}, \ldots, y_{n} \mid z_{1}, \ldots, z_{m}\right) \\
& \cdot \psi\left(y_{1}\right) \cdots \psi\left(y_{n}\right) \bar{\psi}\left(z_{1}\right) \cdots \bar{\psi}\left(z_{m}\right)
\end{aligned}
$$

onde os $c_{n m}\left(y_{1}, \ldots, y_{n} \mid z_{1}, \ldots, z_{m}\right)$ são funções generalizadas e temperadas de $\mathbb{R}^{n+m}$, simétricas tanto nos $y_{j}$, quanto nos $z_{k}, \operatorname{com} j=1, \ldots, n$ e $k=1, \ldots, m$ e satisfazendo a condição de realidade

$$
c_{n m}\left(y_{1}, \ldots, y_{n} \mid z_{1}, \ldots, z_{m}\right)=\overline{c_{n m}\left(y_{1}, \ldots, y_{n} \mid z_{1}, \ldots, z_{m}\right)},
$$

e as condições de quasiperiodicidade:

$$
\begin{aligned}
c_{n m}\left(y_{1}, \ldots, y_{j}+2 L, \ldots, y_{n} \mid z_{1}, \ldots, z_{m}\right) & =e^{-i \theta} c_{n m}\left(y_{1}, \ldots, y_{j}, \ldots, y_{n} \mid z_{1}, \ldots, z_{m}\right), \\
c_{n m}\left(y_{1}, \ldots, y_{n} \mid z_{1}, \ldots, z_{k}+2 L, \ldots z_{m}\right) & =e^{i \theta} c_{n m}\left(y_{1}, \ldots, y_{n} \mid z_{1}, \ldots, z_{k}, \ldots z_{m}\right),
\end{aligned}
$$

de forma que os integrandos em (2.4.9) são periódicos em cada variável separadamente e a integração não depende da escolha do domínio fundamental. Tais funcionais são usualmente denominados funcionais reais-analíticos quasiperiódicos. Em termos das derivadas funcionais, as condições de quasiperiodicidade (2.4.11) podem ser escritas como:

$$
\frac{\delta F}{\delta \psi(x)}=\left.e^{i \theta} \frac{\delta F}{\delta \psi(y)}\right|_{y=x+2 L} \quad \text { e } \frac{\delta F}{\delta \bar{\psi}(x)}=\left.e^{-i \theta} \frac{\delta F}{\delta \bar{\psi}(y)}\right|_{y=x+2 L} .
$$

Funcionais reais-analíticos quasiperiódicos suaves constituem a álgebra de observáveis do espaço 
de fase $\Gamma_{L, \theta}$, nesse caso os parênteses de Poisson entre dois observáveis:

$$
\{F, G\}=i \int_{-L}^{L} d x\left(\frac{\delta F}{\delta \psi(x)} \frac{\delta G}{\delta \bar{\psi}(x)}-\frac{\delta F}{\delta \bar{\psi}(x)} \frac{\delta G}{\delta \psi(x)}\right)
$$

estão bem definidos e são não-degenerados. Formalmente, temos que para as coordenadas $\psi(x)$ e $\bar{\psi}(x)$ :

$$
\begin{aligned}
& \{\psi(x), \psi(y)\}=0 \\
& \{\bar{\psi}(x), \bar{\psi}(y)\}=0 \\
& \{\psi(x), \bar{\psi}(y)\}=i \delta_{L, \theta}(x-y)
\end{aligned}
$$

onde $\delta_{L, \theta}$ é uma função delta média,

$$
\delta_{L, \theta}(x)=\sum_{n=-\infty}^{\infty} e^{i \theta n} \delta(x-2 n L)
$$

que satisfaz a quasiperiodicidade em $x$.

A Hamiltoniana do modelo NLS é:

$$
H=\int_{-L}^{L} d x\left(\left|\frac{\partial \psi}{\partial x}\right|^{2}+g|\psi|^{4}\right)
$$

Outros dois funcionais importantes são a carga (número de partículas) $Q$ e o momento $P$ :

$$
\begin{aligned}
& Q=\int_{-L}^{L} d x|\psi|^{2}, \\
& P=\frac{1}{2 i} \int_{-L}^{L} d x\left(\frac{\partial \psi}{\partial x} \bar{\psi}-\frac{\partial \bar{\psi}}{\partial x} \psi\right) .
\end{aligned}
$$

Esses funcionais são integrais do movimento em involução:

$$
\{H, Q\}=\{H, P\}=0 \quad \text { e } \quad\{P, Q\}=0 .
$$

Além dos funcionais associados aos observáveis acima, consideramos também funcionais com suporte compacto. Por um funcional com suporte compacto entendemos que os coeficientes de (2.4.9), $c_{n m}\left(y_{1}, \ldots, y_{n} \mid z_{1}, \ldots, z_{m}\right)$, possuem suporte no domínio fundamental $-L \leq x \leq L$ e que suas derivadas funcionais são funções contínuas dentro do suporte. Para tais funcionais, os parênteses de Poisson (2.4.12) estão bem definidos e são não-degenerados. Notamos que a álgebra dos funcionais admissíveis é o completamento do conjunto dos funcionais com suporte compacto após a imposição da condição de quasiperiodicidade, e os parênteses de Poisson para os observáveis são obtidos pelo mesmo limite, a partir dos parênteses de Poisson para os funcionais com suporte compacto. 


\subsection{A Representação de Lax}

O método do espalhamento inverso clássico é baseado na observação de que uma certa classe de equações diferenciais parciais em duas dimensões pode ser representada a partir da condição de compatibilidade para o seguinte sistema sobredeterminado de equações diferenciais ordinárias:

$$
\begin{aligned}
& \frac{\partial \Psi}{\partial \sigma}=L_{\sigma}(\sigma, \tau ; \lambda) \Psi \\
& \frac{\partial \Psi}{\partial \tau}=L_{\tau}(\sigma, \tau ; \lambda) \Psi
\end{aligned}
$$

também conhecido como o problema linear fundamental, onde $\Psi=\Psi(\sigma, \tau ; \lambda)$ é um vetor de posto $k$ e o par $L_{\sigma}=L_{\sigma}(\sigma, \tau ; \lambda)$ e $L_{\tau}=L_{\tau}(\sigma, \tau ; \lambda)$ consiste de matrizes quadradas $k \times k$. Esse par de matrizes, assim como o inteiro $k$ dependem da equação que se deseja representar. Tanto $\Psi$ quanto $L_{\sigma}$ e $L_{\tau}$ dependem de um parâmetro complexo adicional, o parâmetro espectral $\lambda$.

A condição de consistência para o sistema (2.5.1) surge da seguinte consideração:

$$
\begin{aligned}
\partial_{\tau} \partial_{\sigma} \psi & =\partial_{\tau}\left(L_{\sigma} \psi\right)=\partial_{\tau} L_{\sigma} \psi+L_{\sigma} \partial_{\tau} \Psi=\partial_{\tau} L_{\sigma} \psi+L_{\sigma} L_{\tau} \Psi \\
& =\left(\partial_{\tau} L_{\sigma}+L_{\sigma} L_{\tau}\right) \Psi \\
\partial_{\sigma} \partial_{\tau} \Psi & =\partial_{\sigma}\left(L_{\tau} \Psi\right)=\partial_{\sigma} L_{\tau} \Psi+L_{\tau} \partial_{\sigma} \Psi=\partial_{\sigma} L_{\tau} \Psi+L_{\tau} L_{\sigma} \Psi \\
& =\left(\partial_{\sigma} L_{\tau}+L_{\tau} L_{\sigma}\right) \Psi
\end{aligned}
$$

Supondo ao menos que $\Psi \in \mathscr{C}^{2}\left(\mathbb{R}^{2}\right)$, temos que $\partial_{\tau} \partial_{\sigma} \Psi=\partial_{\sigma} \partial_{\tau} \psi$, logo:

$$
\begin{aligned}
0 & =\left(\partial_{\sigma} L_{\tau}-\partial_{\tau} L_{\sigma}+L_{\tau} L_{\sigma}-L_{\sigma} L_{\tau}\right) \Psi \\
& \Rightarrow\left(\partial_{\sigma} L_{\tau}-\partial_{\tau} L_{\sigma}+\left[L_{\tau}, L_{\sigma}\right]\right) \Psi=0 \\
& \Rightarrow\left(\partial_{\tau}-L_{\tau}\right) \partial_{\sigma} \Psi-\partial_{\sigma}\left(\partial_{\tau}-L_{\tau}\right) \Psi-\left(\partial_{\tau}-L_{\tau}\right) L_{\sigma} \Psi+L_{\sigma}\left(\partial_{\tau}-L_{\tau}\right) \Psi=0 \\
& \Rightarrow\left[\partial_{\tau}-L_{\tau}, \partial_{\sigma}\right] \Psi-\left[\partial_{\tau}-L_{\tau}, L_{\sigma}\right] \Psi=0 \\
& \Rightarrow\left[\partial_{\tau}-L_{\tau}, \partial_{\sigma}-L_{\sigma}\right] \Psi=0 .
\end{aligned}
$$

Uma vez que $\Psi$ é arbitrário, concluímos que a condição de compatibilidade para o sistema (2.5.1) pode ser escrita a partir da equação (2.5.3) da seguinte forma:

$$
[M, L]=0, \quad \text { com } \quad M:=\partial_{\tau}-L_{\tau} \quad \text { e } \quad L:=\partial_{\sigma}-L_{\sigma}
$$

O par de operadores diferenciais ${ }^{8} L$ e $M$ é denominado par de Lax. Uma condição análoga pode ser obtida a partir da equação (2.5.2):

$$
\partial_{\sigma} L_{\tau}-\partial_{\tau} L_{\sigma}+\left[L_{\tau}, L_{\sigma}\right]=0
$$

As equações (2.5.1) e sua condição de compatibilidade (2.5.5) possuem uma interpretação geométrica. De fato, podemos considerar $L_{\tau}$ e $L_{\sigma}$ como as componentes de uma conexão local nãoabeliana bidimensional $L_{\alpha}$ no fibrado vetorial trivial $\mathbb{R}^{2} \times V$, onde $\mathbb{R}^{2}$ é o espaço base e $V$ é a fibra

\footnotetext{
${ }^{8}$ Derivadas covariantes.
} 
onde o campo vetorial $\Psi(\sigma, \tau ; \lambda)$ está definido. As equações (2.5.1) mostram que $\Psi$ é um vetor covariantemente constante, enquanto que a condição de compatibilidade implica a curvatura nula da conexão $L_{\alpha}$,

$$
\partial_{\alpha} L_{\beta}-\partial_{\beta} L_{\alpha}-\left[L_{\alpha}, L_{\beta}\right]=0
$$

Por esse motivo a representação de uma equação não-linear na forma (2.5.6) é também denominada representação de curvatura nula.

O par de matrizes $L_{\tau}$ e $L_{\sigma}$ deve ser escolhido de forma que tanto a condição de curvatura nula, quanto o par de Lax, $M$ e $N$, através da equação (2.5.4), impliquem que a equação parcial diferencial não-linear original seja satisfeita para quaisquer valores do parâmetro espectral $\lambda$. É importante salientar que para uma dada equação parcial diferencial não-linear integrável a conexão de Lax (e conseqüentemente, o par de Lax) não é de forma alguma única, nem mesmo o é o posto $k$ das matrizes quadradas $L_{\tau}$ e $L_{\sigma}$.

As componentes da conexão $L_{\alpha}$ são afetadas por uma mudança linear de referencial na fibra, a saber, a transformação

$$
\Psi(\sigma, \tau ; \lambda) \rightarrow h(\sigma, \tau ; \lambda) \Psi(\sigma, \tau ; \lambda)
$$

induzida pela mudança de referencial fornecida pela matriz $h(\sigma, \tau ; \lambda)$ incute a seguinte transformação de calibre a $L_{\alpha}$ :

$$
L_{\alpha} \rightarrow L_{\alpha}^{\prime}=h L_{\alpha} h^{-1}+\partial_{\alpha} h h^{-1}
$$

Claramente, a condição de curvatura nula (2.5.6) é invariante sob transformações de calibre, pois

$$
\begin{aligned}
\partial_{\alpha} L_{\beta}^{\prime} & =\partial_{\alpha} h L_{\beta} h^{-1}+h \partial_{\alpha} L_{\beta} h^{-1}-h L_{\beta} h^{-1} \partial_{\alpha} h h^{-1}+\partial_{\alpha} \partial_{\beta} h h^{-1}-\partial_{\beta} h h^{-1} \partial_{\alpha} h h^{-1}, \\
\partial_{\beta} L_{\alpha}^{\prime} & =\partial_{\beta} h L_{\alpha} h^{-1}+h \partial_{\beta} L_{\alpha} h^{-1}-h L_{\alpha} h^{-1} \partial_{\beta} h h^{-1}+\partial_{\beta} \partial_{\alpha} h h^{-1}-\partial_{\alpha} h h^{-1} \partial_{\beta} h h^{-1}, \\
{\left[L_{\alpha}^{\prime}, L_{\beta}^{\prime}\right] } & =h\left[L_{\alpha}, L_{\beta}\right] h^{-1}+h L_{\alpha} h^{-1} \partial_{\beta} h h^{-1}-h L_{\beta} h^{-1} \partial_{\alpha} h h^{-1}+\partial_{\alpha} h L_{\beta} h^{-1}-\partial_{\beta} h L_{\alpha} h^{-1}+ \\
& +\partial_{\alpha} h h^{-1} \partial_{\beta} h h^{-1}-\partial_{\beta} h h^{-1} \partial_{\alpha} h h^{-1}
\end{aligned}
$$

e conseqüentemente, temos que

$$
\begin{aligned}
\partial_{\alpha} L_{\beta}^{\prime}-\partial_{\beta} L_{\alpha}^{\prime}-\left[L_{\alpha}^{\prime}, L_{\beta}^{\prime}\right] & =h \partial_{\alpha} L_{\beta} h^{-1}-h \partial_{\beta} L_{\alpha} h^{-1}-h\left[L_{\alpha}, L_{\beta}\right] h^{-1} \\
& =h\left\{\partial_{\alpha} L_{\beta}-\partial_{\beta} L_{\alpha}-\left[L_{\alpha}, L_{\beta}\right]\right\} h^{-1} \\
& =0 .
\end{aligned}
$$

Portanto, a representação da equação diferencial parcial não-linear original em termos da condição de compatibilidade (2.5.6) é válida para toda a classe de conexões equivalentes por transformações de calibre.

Apliquemos, pois, o conceito de par de Lax ao NLS. 
Exemplo 2.5.1. Um possível par de Lax para o modelo NLS é dado por:

$$
\begin{aligned}
& M=\partial_{t}-L_{t}, \quad L_{t}=\frac{i \lambda^{2}}{2} \gamma^{3}+\lambda \Omega(x)+i \gamma^{3}\left(\partial_{x} \Omega(x)+g \bar{\psi} \psi\right), \\
& L=\partial_{x}-L_{x}, \quad L_{x}=\frac{-i \lambda}{2} \gamma^{3}-\Omega(x),
\end{aligned}
$$

onde:

$$
\gamma^{3}=\left(\begin{array}{cc}
1 & 0 \\
0 & -1
\end{array}\right) \quad \text { e } \Omega(x)=\left(\begin{array}{cc}
0 & i \sqrt{g} \bar{\psi}(x) \\
-i \sqrt{g} \psi(x) & 0
\end{array}\right)
$$

com $L_{\alpha}$ definindo uma conexão local sobre o fibrado vetorial trivial $\mathbb{R}^{2} \times \mathbb{C}^{2}$. Mostremos então que o par de Lax (2.5.9) implica a equação de movimento original (2.4.5) ao utilizarmos a condição (2.5.4):

$$
[M, L]=0 \quad \Leftrightarrow \quad \partial_{x} L_{t}-\partial_{t} L_{x}+\left[L_{t}, L_{x}\right]=0 .
$$

Como:

$$
\begin{aligned}
\partial_{x} L_{t} & =\lambda \partial_{x} \Omega+i \gamma^{3}\left(\partial_{x}^{2} \Omega+g \partial_{x} \bar{\psi} \psi+g \bar{\psi} \partial_{x} \psi\right), \\
\partial_{t} L_{x} & =-\partial_{t} \Omega, \\
{\left[L_{t}, L_{x}\right] } & =\frac{\lambda}{2}\left(\gamma^{3} \partial_{x} \Omega \gamma^{3}-\gamma^{32} \partial_{x} \Omega\right)-i \gamma^{3} \partial_{x} \Omega \Omega+i \Omega \gamma^{3} \partial_{x} \Omega-i g \bar{\psi} \psi\left[\gamma^{3}, \Omega\right],
\end{aligned}
$$

podemos escrever que:

$$
\begin{aligned}
{[M, L] } & =i \gamma^{3} \partial_{x}^{2} \Omega+\partial_{t} \Omega-i g \bar{\psi} \psi\left[\gamma^{3}, \Omega\right]+i\left\{\gamma^{3} g\left(\partial_{x} \bar{\psi} \psi+\bar{\psi} \partial_{x} \psi\right)-\gamma^{3} \partial_{x} \Omega \Omega+\Omega \gamma^{3} \partial \Omega\right\}+ \\
& +\frac{\lambda}{2}\left(\partial_{x} \Omega+\gamma^{3} \partial_{x} \Omega \gamma^{3}\right)
\end{aligned}
$$

Usando as seguintes identidades:

$$
\begin{gathered}
{\left[\gamma^{3}, \Omega\right]=2 i \sqrt{g}\left(\begin{array}{cc}
0 & \bar{\psi} \\
\psi & 0
\end{array}\right) \quad, \quad \gamma^{3} \partial_{x}^{2} \Omega=i \sqrt{g}\left(\begin{array}{cc}
0 & \partial_{x}^{2} \bar{\psi} \\
\partial_{x}^{2} \psi & 0
\end{array}\right),} \\
\gamma^{3} \partial_{x} \Omega \Omega=g\left(\begin{array}{cc}
\partial_{x} \bar{\psi} \psi & 0 \\
0 & -\partial_{x} \psi \bar{\psi}
\end{array}\right) \quad, \quad \Omega \gamma^{3} \partial_{x} \Omega=-g\left(\begin{array}{cc}
\bar{\psi} \partial_{x} \psi & 0 \\
0 & -\psi \partial_{x} \bar{\psi}
\end{array}\right), \\
\gamma^{3} \partial_{x} \Omega \gamma^{3}=-\partial_{x} \Omega,
\end{gathered}
$$

concluímos que:

$$
\begin{aligned}
\gamma^{3} g\left(\partial_{x} \bar{\psi} \psi+\bar{\psi} \partial_{x} \psi\right)-\gamma^{3} \partial_{x} \Omega \Omega+\Omega \gamma^{3} \partial_{x} \Omega & =0 \\
\partial_{x} \Omega+\gamma^{3} \partial_{x} \Omega \gamma^{3} & =0
\end{aligned}
$$

Finalmente,

$$
[M, L]=i \gamma^{3} \partial_{x}^{2} \Omega+\partial_{t} \Omega-i g \psi \bar{\psi}\left[\gamma^{3}, \Omega\right]
$$




$$
=\sqrt{g}\left(\begin{array}{cc}
0 & -\partial_{x}^{2} \bar{\psi}+i \partial_{t} \bar{\psi}+2 g|\psi|^{2} \bar{\psi} \\
-\partial_{x}^{2} \psi-i \partial_{t} \psi+2 g|\psi|^{2} \psi & 0
\end{array}\right)
$$

Portanto, ao impormos que os operadores $L$ e $M$ constituam um par de Lax, temos que a equação (2.5.10) é identicamente nula, implicando que tanto a equação de movimento para o NLS (2.4.5), quanto sua complexa conjugada são satisfeitas.

Analogamente, ao exemplo 2.5.1, podemos mostrar que a validade da equação de movimento para o NLS (2.4.5) garante a existência do par de Lax (2.5.9). Assim, fica claro que o par de Lax, ou a condição de curvatura nula são duas formas equivalentes de se representar uma equação diferencial parcial não-linear integrável em duas dimensões.

\subsection{Quantidades Conservadas}

A grande vantagem de empregar a representação de Lax no tratamento de modelos integráveis reside no fato de que tal formalismo fornece um método canônico para a construção das integrais de movimento, o qual é, em geral, o primeiro passo na obtenção de soluções explícitas da equação de movimento em questão. Para isso consideramos uma translação do sistema (2.5.1), $\Psi(\sigma, \tau ; \lambda)$, ao longo da direção $\sigma$, para um tempo fixo:

$$
\Psi\left(\sigma_{2}, \tau ; \lambda\right)=T\left(\sigma_{2}, \sigma_{1} ; \lambda\right) \Psi\left(\sigma_{1}, \tau ; \lambda\right)
$$

A matriz $T\left(\sigma_{2}, \sigma_{1} ; \lambda\right)$, denominada matriz de transição, é uma matriz quadrada de posto $k$ definida no intervalo $\left[\sigma_{1}, \sigma_{2}\right]$, pelas seguintes condições, também conhecidas como problema linear auxiliar:

$$
\left[\partial_{\sigma_{2}}-L_{\sigma}\left(\sigma_{2}, \tau ; \lambda\right)\right] T\left(\sigma_{2}, \sigma_{1} ; \lambda\right)=0 \quad \text { com } \quad T\left(\sigma_{1}, \sigma_{1} ; \lambda\right)=\mathbb{1}_{k},
$$

que são facilmente obtidas do problema linear fundamental (2.5.1), usando o Ansatz (2.6.1).

$$
\begin{aligned}
\partial_{\sigma_{2}} \Psi\left(\sigma_{2}, \tau ; \lambda\right) & =\partial_{\sigma_{2}} T\left(\sigma_{2}, \sigma_{1} ; \lambda\right) \Psi\left(\sigma_{1}, \tau ; \lambda\right)+T\left(\sigma_{2}, \sigma_{1} ; \lambda\right) \partial_{\sigma_{2}} \Psi\left(\sigma_{1}, \tau ; \lambda\right) \\
& \Rightarrow L_{\sigma}\left(\sigma_{2}, \tau ; \lambda\right) \Psi\left(\sigma_{2}, \tau ; \lambda\right)=\partial_{\sigma_{2}} T\left(\sigma_{2}, \sigma_{1} ; \lambda\right) \Psi\left(\sigma_{1}, \tau ; \lambda\right) \\
& \Rightarrow L_{\sigma}\left(\sigma_{2}, \tau ; \lambda\right) T\left(\sigma_{2}, \sigma_{1} ; \lambda\right) \Psi\left(\sigma_{1}, \tau ; \lambda\right)-\partial_{\sigma_{2}} T\left(\sigma_{2}, \sigma_{1} ; \lambda\right) \Psi\left(\sigma_{1}, \tau ; \lambda\right)=0 \\
& \Rightarrow\left[\partial_{\sigma_{2}}-L_{\sigma}\left(\sigma_{2}, \tau ; \lambda\right)\right] T\left(\sigma_{2}, \sigma_{1} ; \lambda\right)=0 .
\end{aligned}
$$

Já para a condição inicial temos claramente que:

$$
\Psi\left(\sigma_{1}, \tau ; \lambda\right)=T\left(\sigma_{1}, \sigma_{1} ; \lambda\right) \Psi\left(\sigma_{1}, \tau ; \lambda\right) \Rightarrow T\left(\sigma_{1}, \sigma_{1} ; \lambda\right)=\mathbb{1}_{k}
$$

A matriz de transição também exibe a propriedade de superposição:

$$
T\left(\sigma_{2}, \tilde{\sigma} ; \lambda\right) T\left(\tilde{\sigma}, \sigma_{1} ; \lambda\right)=T\left(\sigma_{2}, \sigma_{1} ; \lambda\right)
$$


que, em particular, demanda que:

$$
T\left(\sigma_{2}, \sigma_{1} ; \lambda\right)=T^{-1}\left(\sigma_{1}, \sigma_{2} ; \lambda\right)
$$

consistente com a equação diferencial para $T\left(\sigma_{2}, \sigma_{1} ; \lambda\right)$ com respeito a $\sigma_{1}$ :

$$
\partial_{\sigma_{1}} T\left(\sigma_{2}, \sigma_{1} ; \lambda\right)+T\left(\sigma_{2}, \sigma_{1} ; \lambda\right) L_{\sigma}\left(\sigma_{1}, \tau ; \lambda\right)=0
$$

A matriz de transição para o intervalo fundamental completo $T(L,-L ; \lambda) \equiv T_{L}(\lambda)$ é denominada matriz de monodromia e exerce um papel central na construção das cargas conservadas. É conveniente considerar a seguinte solução formal de (2.6.2):

$$
T\left(\sigma_{2}, \sigma_{1} ; \lambda\right)=P \exp \left\{\int_{\sigma_{1}}^{\sigma_{2}} d \sigma L_{\sigma}(\sigma, \tau ; \lambda)\right\}
$$

onde $P$ denota o ordenamento por caminho para fatores não-comutativos.

Exemplo 2.6.1. A matriz de transição para o modelo NLS é dada por:

$$
T(x, y ; \lambda)=P \exp \left\{\int_{y}^{x} d z L_{x}(z, t ; \lambda)\right\}=P \exp \left(\begin{array}{cc}
\frac{-i \lambda}{2}(x-y) & -i \sqrt{g} \int_{y}^{x} d z \bar{\psi}(z) \\
i \sqrt{g} \int_{y}^{x} d z \psi(z) & \frac{i \lambda}{2}(x-y)
\end{array}\right)
$$

Usando que: $\operatorname{det}\left(e^{A}\right)=e^{\operatorname{tr} A}$, podemos facilmente concluir que:

$$
\operatorname{det} T(x, y ; \lambda)=P \exp \left\{\frac{-i \lambda}{2}(x-y)+\frac{i \lambda}{2}(x-y)\right\}=1
$$

A matriz de transição também satisfaz:

$$
\gamma^{0} \bar{T}(x, y ; \bar{\lambda}) \gamma^{0}=T(x, y ; \lambda)
$$

com a matriz $\gamma^{0}$ definida no apêndice B. Para demonstrar essa propriedade escrevemos:

$$
T(x, y ; \lambda)=P e^{M}=P \sum_{n=0}^{\infty} \frac{M^{n}}{n !},
$$

usando que $\left(\gamma^{0}\right)^{2}=\mathbb{1}_{2}$, notamos que o n-ésimo termo da série para a matriz de transição pode ser escrito como:

$$
\frac{M^{n}}{n !}=\frac{1}{n !} M \gamma^{0} \gamma^{0} M \gamma^{0} \cdots \gamma^{0} M \gamma^{0} \gamma^{0} M
$$

de forma que basta mostrarmos que $\gamma^{0} \bar{M} \gamma^{0}=M$, de fato:

$$
\gamma^{0} \bar{M} \gamma^{0}=\left(\begin{array}{ll}
0 & 1 \\
1 & 0
\end{array}\right)\left[\begin{array}{cc}
i \bar{A}(\bar{\lambda}) & i B(\bar{\lambda}) \\
-i \bar{B}(\bar{\lambda}) & -i A(\bar{\lambda})
\end{array}\right]\left(\begin{array}{ll}
0 & 1 \\
1 & 0
\end{array}\right)=\left[\begin{array}{cc}
-i A(\lambda) & -i \bar{B}(\bar{\lambda}) \\
i B(\lambda) & i \bar{A}(\bar{\lambda})
\end{array}\right]=M,
$$

$\operatorname{com} A:=\frac{\lambda}{2}(x-y)$ e $B:=\sqrt{g} \int_{y}^{x} d z \psi(z)$.

Analogamente, podemos introduzir o objeto que translada a solução do sistema (2.5.1) ao longo 
da direção $\tau$ com $\sigma$ fixo:

$$
\Psi\left(\sigma, \tau_{2} ; \lambda\right)=S\left(\tau_{2}, \tau_{1} ; \lambda\right) \Psi\left(\sigma, \tau_{1} ; \lambda\right)
$$

De forma que,

$$
S\left(\tau_{2}, \tau_{1} ; \lambda\right)=P \exp \left\{\int_{\tau_{1}}^{\tau_{2}} L_{\tau}(\sigma, \tau ; \lambda) d \tau\right\}
$$

Em geral, devemos considerar uma translação arbitrária da solução de (2.5.1) ao longo do plano $\tau \times \sigma$. Seja, pois, uma curva $\gamma$ em $\mathbb{R}^{2}$, então o transporte paralelo ao longo de $\gamma$ é descrito pela matriz:

$$
\Omega_{\gamma}=P \exp \left\{\int_{\gamma} A^{\mu} d x_{\mu}\right\}=P \exp \left\{\int_{\gamma} L_{\sigma} d \sigma+L_{\tau} d \tau\right\} .
$$

De uma forma mais precisa, seja uma partição de $\gamma$ em $N$ segmentos adjacentes $\gamma_{1}, \ldots, \gamma_{N}$ e considere:

$$
\Omega_{N}=P \prod_{n=1}^{N} L_{n}=L_{N} \cdots L_{1}, \quad \text { com } \quad L_{n}=\mathbb{1}_{k}+\int_{\gamma_{n}} A_{\mu} d x^{\mu} .
$$

Portanto, $\Omega_{\gamma}$ é obtido a partir do limite de uma partição infinitamente densa de $\Omega_{N}$. Naturalmente, o transporte de um vetor $\psi$ ao longo de $\gamma, \psi_{\gamma}=\Omega_{\gamma} \psi$, define um campo vetorial $\psi_{\gamma}$ covariantemente constante em $\gamma$. Em virtude da condição de curvatura nula satisfeita pela conexão de Lax, temos que a exponencial ordenada por caminhos é independente do caminho escolhido e, em particular, se a curva $\gamma$ for fechada podemos escrever:

$$
\Omega_{\gamma}=P \exp \left\{\oint_{\gamma} A^{\mu} d x_{\mu}\right\}=\mathbb{1}_{k}+F_{\mu \nu} d x^{\mu} d x^{\nu}+O\left(F^{2}\right)=\mathbb{1}_{k}
$$

De posse desses fatos podemos construir as quantidades conservadas.

Proposição 2.6.2. Seja uma equação diferencial parcial não-linear que admite a representação de Lax. Se os campos $\Psi(\sigma, \tau ; \lambda)$ forem quasiperiódicos em $\sigma$ com período $2 L$, então as quantidades:

$$
\zeta^{(n)}(\lambda)=\operatorname{tr}\left[\left(T_{L}(\tau ; \lambda) Q(\theta)\right)^{n}\right]
$$

onde $T_{L}(\tau ; \lambda)$ é a matriz de monodromia e $Q(\theta)$, a matriz que implementa as condições quasiperiódicas, são independentes do tempo. Logo, $\zeta(\lambda)$ é o funcional gerador das quantidades conservadas.

Demonstração: Como a equação diferencial em questão admite a representação de Lax, a conexão de Lax associada satisfaz a condição de curvatura nula e, conseqüentemente, a quantidade $\Omega_{\gamma}$ independe do caminho $\gamma$. Tomemos, pois, a curva $\gamma$ como a borda do retângulo $-L \leq \sigma \leq L, \tau_{1} \leq \tau \leq \tau_{2}$, vide figura 2.3. Assim, podemos utilizar a equação (2.6.12) para escrever ${ }^{9}$ :

$$
\mathbb{1}_{k}=\Omega_{\gamma}=\Omega_{\gamma_{4}} \Omega_{\gamma_{3}} \Omega_{\gamma_{2}} \Omega_{\gamma_{1}}=S^{-1}(-L) T_{L}^{-1}\left(\tau_{2}\right) S(L) T_{L}\left(\tau_{1}\right) \Rightarrow T_{L}\left(\tau_{2}\right) S(-L)=S(L) T_{L}\left(\tau_{1}\right) .
$$

Como os campos são quasiperiódicos em $\sigma$, isto é, $Q(\theta) \Psi(\sigma, \tau)=\Psi(\sigma+2 L, \tau)$, com $Q(\theta)$ sendo

\footnotetext{
${ }^{9}$ Onde omitimos a dependência em $\lambda$
} 


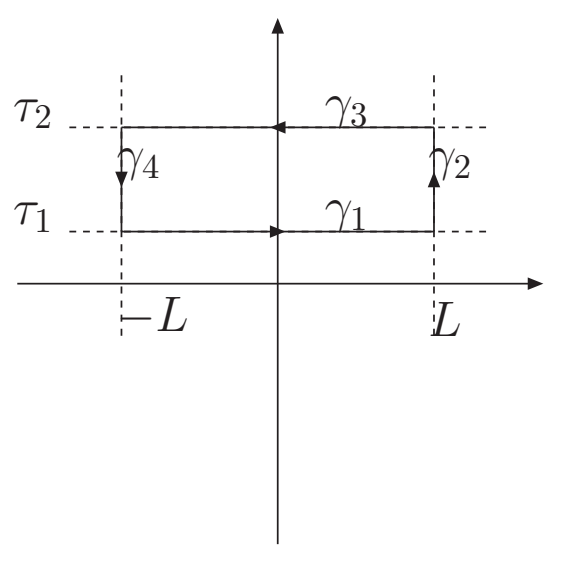

Figura 2.3: Caminho fechado de integração $\gamma=\cup_{i=1}^{4} \gamma_{i}$.

uma matriz $k \times k$ que independe de $\sigma$ e $\tau$, temos que

$$
L_{\alpha}(\sigma+2 L, \tau ; \lambda)=Q^{-1}(\theta) L_{\alpha}(\sigma, \tau ; \lambda) Q(\theta)
$$

Em particular, a quasiperiodicidade implica que $S(L)$ é conjugado a $S(-L)$,

$$
S(-L)=Q(\theta) S(L) Q^{-1}(\theta) .
$$

Portanto,

$$
\begin{aligned}
T_{L}\left(\tau_{2}\right) Q(\theta) S(L) Q^{-1}(\theta)=S(L) T_{L}\left(\tau_{1}\right) Q(\theta) & \Rightarrow T_{L}\left(\tau_{2}\right) Q(\theta)=S(L) T_{L}\left(\tau_{1}\right) Q(\theta) S^{-1}(L) \\
& \Rightarrow\left(T_{L}\left(\tau_{2}\right) Q(\theta)\right)^{n}=S(L)\left(T_{L}\left(\tau_{1}\right) Q(\theta)\right)^{n} S^{-1}(L) \\
& \Rightarrow \operatorname{tr}\left[\left(T_{L}\left(\tau_{2}\right) Q(\theta)\right)^{n}\right]=\operatorname{tr}\left[\left(T_{L}\left(\tau_{1}\right) Q(\theta)\right)^{n}\right] .
\end{aligned}
$$

Logo, $\zeta^{(n)}$ é uma constante sob a evolução temporal. Expandindo em $\lambda$ obtemos um conjunto infinito de quantidades conservadas.

Claramente, se os campos forem periódicos, ou seja, $\Psi(L)=\Psi(-L), Q(\theta)=\mathbb{1}_{k}$, o funcional gerador das quantidades conservadas simplifica-se:

$$
\zeta^{(n)}(\lambda)=\operatorname{tr}\left[\left(T_{L}(\tau ; \lambda)\right)^{n}\right] .
$$

A proposição 2.6.2 afirma que as propriedades espectrais de um modelo integrável estão codificadas na matriz de monodromia, de forma que ao expandirmos o traço dessa matriz com respeito ao parâmetro espectral $\lambda$ devemos ser capazes de obter explicitamente as cargas conservadas. As equações (2.6.13) e (2.6.14) são conhecidas como identidades de traço. 


\subsubsection{Identidades de Traço para o Modelo NLS}

Nesta seção ${ }^{10}$ estudamos as identidades de traço (2.6.13) para o modelo NLS com condições de contorno quasiperiódicas, e desenvolvemos um algoritmo para obter as integrais de movimento como um funcional local no espaço de fase $\Gamma_{L, \theta}$, isto é,

$$
F(\psi, \bar{\psi})=\int_{-L}^{L} d x P(x)
$$

onde $P(x)$ é um polinômio em $\psi(x)$ e $\bar{\psi}(x)$ e suas derivadas espaciais. Para isso precisamos de algumas propriedades analíticas da matriz de transição para o modelo NLS.

Proposição 2.6.3. Seja o modelo NLS com condições de contorno quasiperiódicas, se $\psi(x), \bar{\psi}(x) \in$ $\mathscr{C}^{\infty}(\mathbb{R})$, então a matriz de transição $T(x, y ; \lambda)$ é uma função inteira e admite a seguinte expansão assintótica para $\lambda \in \mathbb{R}, \lambda \gg 0$,

$$
T(x, y ; \lambda)=E(x-y ; \lambda)+\sum_{n=1}^{\infty} \frac{T_{n}(x, y)}{\lambda^{n}} E(x-y ; \lambda)+\sum_{n=1}^{\infty} \frac{\tilde{T}_{n}(x, y)}{\lambda^{n}} E(y-x ; \lambda)+O\left(|\lambda|^{-\infty}\right),
$$

$\operatorname{com} E(x ; \lambda)=e^{\lambda L_{x}^{1} x}$.

O fator $O\left(|\lambda|^{-\infty}\right)$ denota uma função cuja expansão assintótica em $\lambda^{-1}$ se anula identicamente. É conveniente denotarmos a conexão de Lax espacial da seguinte forma:

$$
L_{x}(x ; \lambda)=L_{x}^{0}(x)+\lambda L_{x}^{1}, \quad \text { com } \quad L_{x}^{0}(x)=-\Omega(x)=i \sqrt{g}\left[\psi(x) \gamma^{+}-\bar{\psi}(x) \gamma^{-}\right] \quad \text { e } \quad L_{x}^{1}=\frac{\gamma^{3}}{2 i},
$$

onde $\gamma^{ \pm}=\frac{\gamma^{0} \pm \gamma^{1}}{2}$. Em particular, se utilizarmos que a matriz de monodromia pode ser escrita como ${ }^{11}$ :

$$
T_{L}(\lambda)=\left(\begin{array}{cc}
a_{L}(\lambda) & \epsilon \bar{b}_{L}(\bar{\lambda}) \\
b_{L}(\lambda) & \bar{a}_{L}(\bar{\lambda})
\end{array}\right),
$$

com $\epsilon=\operatorname{sign}(g)$; a proposição 2.6.3 implica o seguinte corolário:

Corolário 2.6.4. Seja o modelo NLS com condições de contorno quasiperiódicas, se $\psi(x), \bar{\psi}(x) \in$ $\mathscr{C}^{\infty}(\mathbb{R})$, então $a_{L}(\lambda)$ e $b_{L}(\lambda)$ são funções inteiras com as seguintes expansões assintóticas para $\lambda \in \mathbb{R}$, $\lambda \gg 0$,

$$
\begin{aligned}
& a_{L}(\lambda)=e^{-i \lambda L}+e^{-i \lambda L} \sum_{n=1}^{\infty} \frac{a_{n}}{\lambda^{n}}+e^{i \lambda L} \sum_{n=1}^{\infty} \frac{\tilde{a}_{n}}{\lambda^{n}}+O\left(|\lambda|^{-\infty}\right), \\
& b_{L}(\lambda)=e^{-i \lambda L} \sum_{n=1}^{\infty} \frac{b_{n}}{\lambda^{n}}+e^{i \lambda L} \sum_{n=1}^{\infty} \frac{\tilde{b}_{n}}{\lambda^{n}}+O\left(|\lambda|^{-\infty}\right) .
\end{aligned}
$$

A demonstração da proposição 2.6.3 e da corolário 2.6.4 encontram-se no apêndice A.2. Denominamos $a_{L}(\lambda)$ e $b_{L}(\lambda)$ de coeficientes de transição; eles satisfazem a seguinte condição de normalização:

$$
\left|a_{L}(\lambda)\right|^{2}-\epsilon\left|b_{L}(\lambda)\right|^{2}=1,
$$

\footnotetext{
${ }^{10}$ Dedicada a Eliane Pereira.

${ }^{11}$ Vide exemplo 2.6.1.
} 
uma conseqüência imediata da unimodularidade da matriz de monodromia. Com esses resultados podemos demonstrar a seguinte proposição:

Proposição 2.6.5. Existe uma transformação $G(x ; \lambda)$ que diagonaliza a matriz de transição do modelo NLS, i.e.,

$$
T(x, y ; \lambda)=G(x ; \lambda) D(x, y ; \lambda) G^{-1}(y ; \lambda),
$$

onde $G(x ; \lambda)=\mathbb{1}_{2}+W(x ; \lambda)$ e $D(x, y ; \lambda)=\exp Z(x, y ; \lambda) \operatorname{com} W(x ; \lambda)$ sendo uma matriz antidiagonal e $Z(x, y ; \lambda)$, uma matriz diagonal, que admitem as seguintes expansões assintóticas:

$$
\begin{aligned}
W(x ; \lambda) & =\sum_{n=1}^{\infty} \frac{W_{n}(x)}{\lambda^{n}}+O\left(|\lambda|^{-\infty}\right), \\
Z(x, y ; \lambda) & =\lambda(x-y) L_{x}^{1}+\sum_{n=1}^{\infty} \frac{Z_{n}(x, y)}{\lambda^{n}}+O\left(|\lambda|^{-\infty}\right) .
\end{aligned}
$$

Demonstração: A proposição 2.6.3 garante que a expansão do lado direito de (2.6.20) deve possuir a mesma estrutura de (2.6.16), portanto para provarmos (2.6.20) basta mostrarmos que os coeficientes $W_{n}(x)$ e $Z_{n}(x, y)$ são univocamente determinados por $T(x, y ; \lambda)$. Para fazer isso, consideramos a equação diferencial satisfeita pela matriz e transição (2.6.2):

$$
\partial_{x} T(x, y ; \lambda)=L_{x}(x ; \lambda) T(x, y ; \lambda) \quad \text { com }\left.T(x, y ; \lambda)\right|_{x=y}=\mathbb{1}_{2} .
$$

Substituindo (2.6.20) na equação diferencial acima, obtemos

$$
\partial_{x} G(x ; \lambda) D(x, y ; \lambda)+G(x ; \lambda) \partial_{x} D(x, y ; \lambda)-L_{x}(x ; \lambda) G(x ; \lambda) D(x, y ; \lambda)=0 .
$$

Notando que $G(x ; \lambda)=\mathbb{1}_{2}+W(x ; \lambda), D(x, y ; \lambda)=\exp Z(x, y ; \lambda)$ e $L_{x}(x ; \lambda)=L_{x}^{0}(x)+\lambda L_{x}^{1}$, separamos a equação diferencial nas partes diagonal e antidiagonal:

$$
\begin{aligned}
\partial_{x} W(x ; \lambda)+W(x ; \lambda) \partial_{x} Z(x, y ; \lambda) & =L_{x}^{0}(x)+\lambda L_{x}^{1} W(x ; \lambda), \\
\partial_{x} Z(x, y ; \lambda) & =L_{x}^{0}(x) W(x ; \lambda)+\lambda L_{x}^{1} .
\end{aligned}
$$

Usando a equação (2.6.22b) para eliminar a dependência em $\partial_{x} Z(x, y ; \lambda)$ de (2.6.22a), bem como o fato de $W(x ; \lambda)$ anticomutar com $L_{x}^{1}$, obtemos uma equação não-linear de Ricatti para $W(x ; \lambda)$ :

$$
\partial_{x} W+i \lambda \gamma^{3} W+W L_{x}^{0} W-L_{x}^{0}=0
$$

A equação (2.6.22b) é facilmente integrada,

$$
Z(x, y ; \lambda)=\frac{\lambda}{2 i} \gamma^{3}(x-y)+\int_{y}^{x} d z L_{x}^{0}(z) W(z ; \lambda),
$$

onde usamos a condição inicial $\left.Z(x, y ; \lambda)\right|_{x=y}=0$ obtida da condição inicial para a matriz de transição. Dessa forma, se a expansão (2.6.21a) para $W(x ; \lambda)$ for válida, temos que $Z(x, y ; \lambda)$ admite a expansão assintótica (2.6.21b) com

$$
Z_{n}(x, y)=\int_{y}^{x} d z L_{x}^{0}(z) W_{n}(z) .
$$


Assim, resta provar (2.6.21a), para isso consideramos sua substituição em (2.6.23), obtendo:

$$
\sum_{n=1}^{\infty}\left[\frac{\partial_{x} W_{n}(x)}{\lambda^{n}}+\sum_{k=1}^{n-1} \frac{W_{k}(x) L_{x}^{0}(x) W_{n-k}(x)}{\lambda^{n}}+i \gamma^{3} \frac{W_{n+1(x)}}{\lambda^{n}}\right]+i \gamma^{3} W_{1}(x)-L_{x}^{0}=0 .
$$

Concluímos, pois, que os $W_{n}(x)$ satisfazem a seguinte relação de recursão:

$$
W_{n+1}(x)=i \gamma^{3}\left[\partial_{x} W_{n}(x)+\sum_{k=1}^{n-1} W_{k}(x) L_{x}^{0}(x) W_{n-k}(x)\right],
$$

sujeita à condição inicial

$$
W_{1}(x)=-\sqrt{g}\left[\psi(x) \gamma^{+}+\bar{\psi}(x) \gamma^{-}\right] .
$$

Portanto, os coeficientes $W_{n}(x)$ são univocamente determinados por (2.6.26) e podem ser expressos em termos de $L_{x}^{0}(x)$ e de suas derivadas de uma forma local.

No que se segue caracterizamos a matriz $W(x ; \lambda)$ definida pela série $(2.6 .21 a)$.

Proposição 2.6.6. A expansão assintótica de $W(x ; \lambda)$ satisfaz a relação de involução

$$
\gamma^{i} \bar{W}(x, \bar{\lambda}) \gamma^{i}=W(x ; \lambda), \quad \text { com } \quad i=0,1
$$

Demonstração: Basta que demonstremos que a relação de involução é válida para cada uma das componentes $W_{n}(x)$ da expansão (2.6.21a). Façamos isso por indução. Claramente, para $n=1$ temos que:

$$
\begin{aligned}
& \gamma^{0} \bar{W}_{1}(x) \gamma^{0}=-\sqrt{g}\left[\psi(x) \gamma^{+}+\bar{\psi}(x) \gamma^{-}\right]=W_{1}(x) \\
& \gamma^{1} \bar{W}_{1}(x) \gamma^{1}=-\sqrt{g}\left[\psi(x) \gamma^{+}+\bar{\psi}(x) \gamma^{-}\right]=W_{1}(x)
\end{aligned}
$$

A seguir, assumimos a hipótese de indução e notamos que

$$
\gamma^{i} \bar{L}_{x}^{0} \gamma^{i}=L_{x}^{0}, \quad i=0,1
$$

bem como que $\gamma^{0}$ e $\gamma^{1}$ anticomutam com $\gamma^{3}$, para mostrar que para $n+1$ temos que:

$$
\gamma^{i} \bar{W}_{n+1} \gamma^{i}=i \gamma^{3}\left[\partial_{x}\left(\gamma^{i} W_{n}(x) \gamma^{i}\right)+\sum_{k=1}^{n-1} \gamma^{i} W_{k}(x) \gamma^{i} \gamma^{i} L_{x}^{0}(x) \gamma^{i} \gamma^{i} W_{n-k}(x) \gamma^{i}\right]=W_{n+1}(x) .
$$

A matriz $Q(\theta)$ que implementa a condição de quasiperiodicidade para o modelo NLS possui a seguinte forma:

$$
Q(\theta)=\left(\begin{array}{cc}
e^{\frac{i \theta}{2}} & 0 \\
0 & e^{-\frac{i \theta}{2}}
\end{array}\right)=\exp \left[\frac{i \theta}{2} \gamma^{3}\right]
$$

Claramente, temos que:

$$
L_{x}(x+2 L ; \lambda)=Q^{-1}(\theta) L_{x}(x ; \lambda) Q(\theta) .
$$


Dessa forma, podemos mostrar a seguinte proposição.

Proposição 2.6.7. A expansão assintótica de $W(x ; \lambda)$ é quasiperiódica.

Demonstração: Novamente, é suficiente mostrarmos por indução que os coeficientes $W_{n}(x)$ são quasiperiódicos. Para $n=1$ temos que:

$$
\begin{aligned}
W_{1}(x+2 L) & =-\sqrt{g}\left(\begin{array}{cc}
0 & \bar{\psi}(x+2 L) \\
\psi(x+2 L) & 0
\end{array}\right)=-\sqrt{g}\left(\begin{array}{cc}
0 & e^{-i \theta} \bar{\psi}(x) \\
e^{i \theta} \psi(x) & 0
\end{array}\right) \\
& =-\sqrt{g} \exp \left[\frac{-i \theta}{2} \gamma^{3}\right]\left(\begin{array}{cc}
0 & \bar{\psi}(x) \\
\psi(x) & 0
\end{array}\right) \exp \left[\frac{i \theta}{2} \gamma^{3}\right] \\
& =Q^{-1}(\theta) W_{1}(x) Q(\theta) .
\end{aligned}
$$

Assumindo a hipótese de indução, temos que para $n+1$

$$
\begin{aligned}
W_{n+1}(x+2 L) & =i \gamma^{3}\left[Q^{-1}(\theta) \partial_{x} W_{n}(x) Q(\theta)+\right. \\
& \left.+\sum_{k=1}^{n-1} Q^{-1}(\theta) W_{k}(x) Q(\theta) Q^{-1}(\theta) L_{x}^{0}(x) Q(\theta) Q^{-1}(\theta) W_{n-k}(x) Q(\theta)\right] \\
& =Q^{-1}(\theta) W_{n+1}(x) Q(\theta) .
\end{aligned}
$$

De posse desses resultados podemos demonstrar a seguinte e importante proposição:

Proposição 2.6.8. A expansão assintótica de $W(x ; \lambda)$ admite a seguinte decomposição:

$$
W(x ; \lambda)=-\sqrt{g}\left[\omega(x ; \lambda) \gamma^{+}+\bar{\omega}(x ; \bar{\lambda}) \gamma^{-}\right]
$$

com os coeficientes

$$
\omega(x ; \lambda)=\sum_{n=1}^{\infty} \frac{\omega_{n}(x)}{\lambda^{n}}
$$

quasiperiódicos.

Demonstração: Primeiramente, mostremos por indução que os coeficientes $W_{n}(x)$ admitem uma decomposição da forma (2.6.28). Claramente, para $n=1$ essa decomposição é verdadeira em virtude da equação (2.6.26b). Em seguida, consideramos a hipótese de indução, a saber,

$$
W_{n}(x)=-\sqrt{g}\left[\omega_{n}(x) \gamma^{+}+\bar{\omega}_{n}(x) \gamma^{-}\right]
$$


e verificamos sua validade para $n+1$.

$$
\begin{aligned}
W_{n+1}(x) & =i \gamma^{3}\left\{-\sqrt{g}\left[\partial_{x} \omega_{n}(x) \gamma^{+}+\partial_{x} \bar{\omega}_{n}(x) \gamma^{-}\right]+\right. \\
& \left.+i g \sqrt{g} \sum_{k=1}^{n-1}\left[\omega_{k}(x) \gamma^{+}+\bar{\omega}_{k}(x) \gamma^{-}\right]\left[\psi(x) \gamma^{+}-\bar{\psi}(x) \gamma^{-}\right]\left[\omega_{n-k}(x) \gamma^{+}+\bar{\omega}_{n-k}(x) \gamma^{-}\right]\right\} \\
& =i \sqrt{g}\left\{\left[\partial_{x} \omega_{n}(x) \gamma^{+}+\partial_{x} \bar{\omega}_{n}(x) \gamma^{-}\right]+\right. \\
& \left.+i g \sum_{k=1}^{n-1}\left[\bar{\psi}(x) \omega_{k}(x) \omega_{n-k}(x) \gamma^{+}+\psi(x) \bar{\omega}_{k}(x) \bar{\omega}_{n-k}(x) \gamma^{-}\right]\right\} \\
& =-\sqrt{g}\left[\omega_{n+1}(x) \gamma^{+}+\bar{\omega}_{n+1}(x) \gamma^{-}\right],
\end{aligned}
$$

onde introduzimos:

$$
\omega_{n+1}(x)=-i \partial_{x} \omega_{n}(x)+g \bar{\psi}(x) \sum_{k=1}^{n-1} \omega_{k}(x) \omega_{n-k}(x) \quad \text { com } \quad \omega_{1}(x)=\psi(x) .
$$

Em virtude da proposição 2.6 .5 concluímos a validade da expansão (2.6.29).

Para mostrarmos a quasiperiodicidade dos coeficientes $\omega_{n}(x)$, consideramos:

$$
\begin{aligned}
W(x+2 L ; \lambda) & =Q^{-1}(\theta) W(x ; \lambda) Q(\theta) \\
\Rightarrow-\sqrt{g}\left[\omega(x+2 L ; \lambda) \gamma^{+}+\bar{\omega}(x+2 L ; \bar{\lambda}) \gamma^{-}\right] & =-\sqrt{g} \exp \left[\frac{\theta}{2 i} \gamma^{3}\right]\left[\omega(x ; \lambda) \gamma^{+}+\bar{\omega}(x ; \bar{\lambda}) \gamma^{-}\right] \exp \left[\frac{i \theta}{2} \gamma^{3}\right] \\
& =-\sqrt{g}\left[\omega(x ; \lambda) e^{i \theta} \gamma^{+}+\bar{\omega}(x ; \bar{\lambda}) e^{-i \theta} \gamma^{-}\right] \\
\Rightarrow \omega(x+2 L ; \lambda) & =e^{i \theta} \omega(x ; \lambda) \\
\Rightarrow \omega_{n}(x+2 L ; \lambda) & =e^{i \theta} \omega_{n}(x ; \lambda) .
\end{aligned}
$$

Para completarmos a discussão sobre a matriz de transição, notamos que a matriz diagonal $L_{x}^{0}(x) W(x ; \lambda)$ que aparece na representação de $Z(x, y ; \lambda)$, equação (2.6.24), pode ser escrita como:

$$
L_{x}^{0}(x) W(x ; \lambda)=i g\left(\begin{array}{cc}
\bar{\psi}(x) \omega(x ; \lambda) & 0 \\
0 & -\psi(x) \bar{\omega}(x ; \bar{\lambda})
\end{array}\right) .
$$

Claramente,

$$
\bar{\psi}(x+2 L) \omega(x+2 L ; \lambda)=e^{-i \theta} \bar{\psi}(x) e^{i \theta} \omega(x ; \lambda)=\bar{\psi}(x) \omega(x ; \lambda),
$$

logo, as séries assintóticas $\bar{\psi}(x) \omega(x ; \lambda)$ e $\psi(x) \bar{\omega}(x ; \bar{\lambda})$ são periódicas em $x$.

Especializando a nossa discussão para o caso da matriz de monodromia, obtemos naturalmente a partir de (2.6.20) a seguinte representação:

$$
\begin{aligned}
T_{L}(\lambda) & =T(L,-L ; \lambda)=G(L ; \lambda) D(L,-L ; \lambda) G^{-1}(-L ; \lambda) \\
& =\left[\mathbb{1}_{2}+W(L ; \lambda)\right] \exp Z_{L}(\lambda)\left[\mathbb{1}_{2}+W(-L ; \lambda)\right]^{-1},
\end{aligned}
$$


com

$$
Z_{L}(\lambda)=Z(L,-L ; \lambda)=-i \lambda L \gamma^{3}+\int_{-L}^{L} d x L_{x}^{0}(x) W(x ; \lambda)
$$

É interessante notar que, como $L_{x}^{0}(x) W(x ; \lambda)$ é uma função periódica em $x$, a integral (2.6.33) não depende da escolha do domínio fundamental. Ao considerarmos o produto das expansões assintóticas para $\left[\mathbb{1}_{2}+W(L ; \lambda)\right]$ e $Z_{L}(\lambda)$, obtemos a partir de (2.6.32) uma representação assintótica para $T_{L}(\lambda)$ que possui a mesma forma que (2.6.18). Ademais, podemos desenvolver um algoritmo para calcular os coeficientes $a_{n}, \tilde{a}_{n}, b_{n}$ e $\tilde{b}_{n}$ presentes em (2.6.18).

Em particular, tais cálculos se simplificam significativamente para o caso da função geradora das integrais de movimento,

$$
T_{L}(\lambda) Q(\theta)=\left[\mathbb{1}_{2}+W(L ; \lambda)\right] \exp \left[Z_{L}(\lambda)\right] Q(\theta)\left[\mathbb{1}_{2}+W(-L ; \lambda)\right]^{-1}
$$

Assim, ao tomarmos o traço,

$$
\zeta(\lambda)=\operatorname{tr}\left[T_{L}(\lambda) Q(\theta)\right]=\operatorname{tr}\left[\exp \left(Z_{L}(\lambda)+\frac{i \theta^{3}}{\gamma}\right)\right]
$$

Uma vez que $T_{L}(\lambda) Q(\theta)$ é unimodular, temos que

$$
\begin{aligned}
1 & =\operatorname{det}\left[T_{L}(\lambda) Q(\theta)\right]=\operatorname{det}\left[\exp \left(Z_{L}(\lambda)+\frac{i \theta^{3}}{\gamma}\right)\right]=\exp \left[\operatorname{tr}\left(Z_{L}(\lambda)+\frac{i \theta^{3}}{\gamma}\right)\right]=\exp \left[\operatorname{tr} Z_{L}(\lambda)\right] \\
& \Rightarrow \operatorname{tr} Z_{L}(\lambda)=O\left(|\lambda|^{-\infty}\right)
\end{aligned}
$$

De (2.6.31) e (2.6.35) concluímos que:

$$
Z_{L}(\lambda)=-i \lambda L \gamma^{3}+\left(\begin{array}{cc}
i \varphi_{L}(\lambda) & 0 \\
0 & -i \bar{\varphi}_{L}(\bar{\lambda})
\end{array}\right) \quad \operatorname{com} \quad \varphi_{L}(\lambda)=g \int_{-L}^{L} d x \bar{\psi}(x) \omega(x ; \lambda)
$$

sendo uma série assintótica com coeficientes reais, $\varphi_{L}(\lambda)=\bar{\varphi}_{L}(\bar{\lambda})$, devido a (2.6.36). Considerando a expansão assintótica de $\omega(x ; \lambda)(2.6 .29)$ obtemos:

com

$$
\varphi_{L}(\lambda)=g \sum_{n=1}^{\infty} \frac{I_{n}}{\lambda^{n}}+O\left(|\lambda|^{-\infty}\right)
$$

$$
I_{n}(\psi, \bar{\psi})=\int_{-L}^{L} d x P_{n}(x)
$$

onde

$$
P_{n}(x)=\bar{\psi}(x) \omega_{n}(x)
$$

é um polinômio em $\psi(x)$ e $\bar{\psi}(x)$ e suas derivadas espaciais. A periodicidade de $P_{n}(x)$ implica que $I_{n}$ é um funcional admissível ${ }^{12}$ em $\Gamma_{L, \theta}$.

\footnotetext{
${ }^{12} \mathrm{~A}$ admissibilidade dos funcionais $I_{n}$ é uma conseqüência imediata da admissibilidade de $\zeta(\lambda)$, que demonstramos no apêndice A.3.
} 
Conseqüentemente,

$$
\zeta(\lambda)=\operatorname{tr} \exp \left[i \gamma^{3}\left(\varphi_{L}(\lambda)+\frac{\theta}{2}-\lambda L\right)\right]=2 \cos \left[\varphi_{L}(\lambda)+\frac{\theta}{2}-\lambda L\right] .
$$

Logo, é natural introduzirmos a quantidade

$$
p_{L}(\lambda)=\arccos \left(\frac{\zeta(\lambda)}{2}\right)=\varphi_{L}(\lambda)+\frac{\theta}{2}-\lambda L
$$

e considerarmos a expansão (2.6.38), para deduzir:

$$
p_{L}(\lambda)=-\lambda L+\frac{\theta}{2}+g \sum_{n=1}^{\infty} \frac{I_{n}}{\lambda^{n}}+O\left(|\lambda|^{-\infty}\right) .
$$

Os funcionais $I_{n}$ são as integrais de movimento para o modelo NLS com condições de contorno quasiperiódicas. Em particular, os três primeiros funcionais, obtidos a partir das densidades:

$$
\begin{aligned}
& P_{1}(x)=|\psi(x)|^{2}, \\
& P_{2}(x)=-i \bar{\psi}(x) \partial_{x} \psi(x), \\
& P_{3}(x)=-\bar{\psi}(x) \partial_{x}^{2} \psi(x)+g|\psi(x)|^{4}
\end{aligned}
$$

coincidem com os funcionais para a carga $Q$, momento $P$ e Hamiltoniana $H$. Já a quarta densidade possui a seguinte forma:

$$
P_{4}(x)=i \bar{\psi} \partial_{x}^{3} \psi(x)-i g|\psi(x)|^{2}\left[\psi \partial_{x} \bar{\psi}(x)+4 \bar{\psi} \partial_{x} \psi\right] .
$$

Esse exemplo ilustra claramente que as (infinitas) quantidades conservadas, e, em particular, a Hamiltoniana, de um sistema integrável estão codificadas no traço da matriz de monodromia, notamos contudo, que a expansão (2.6.41), apesar de ter sido deduzida para o caso particular do modelo NLS com condições de contorno quasiperiódicas, é válida em geral. É importante ressaltar que embora tenhamos obtido um conjunto de infinitas cargas conservadas, não fica claro por esse argumento que há um número suficiente delas para se garantir a integrabilidade do modelo, a solução desse problema encontra-se na construção de um mapa bijetor que linearize a equação diferencial parcial.

\subsection{A Matriz $r$ Clássica}

Verificamos na última seção que podemos construir as infinitas cargas conservadas de um sistema dinâmico Hamiltoniano bidimensional com infinitos graus de liberdade, se a sua equação de movimento admitir a representação de Lax. Porém, para verificarmos a integrabilidade desse sistema, ainda é necessário averiguar se as integrais de movimento estão em involução. Para tanto, precisamos calcular os parênteses de Poisson entre os elementos da matriz de monodromia. Um método eficiente para se executar tal tarefa é baseado na existência da matriz $r$. Ademais, é possível mostrar que a representação dos parênteses de Poisson em termos da matriz $r$ substitui a condição de curvatura nula. 
É conveniente, nesse estágio, introduzir a seguinte notação para o produto tensorial de duas matrizes $k \times k$. Se $A$ e $B$ são matrizes $k \times k$, o seu produto tensorial $A \otimes B$, uma matriz $k^{2} \times k^{2}$, pode ser representado como:

$$
(A \otimes B)_{i k, j l}=A_{i j} B_{k l}
$$

Por exemplo, nessa notação, o produto tensorial de duas matrizes $2 \times 2$ assume a seguinte forma ${ }^{13}$ :

$$
A \otimes B=\left(\begin{array}{ll}
A_{11} & A_{12} \\
A_{21} & A_{22}
\end{array}\right) \otimes\left(\begin{array}{ll}
B_{11} & B_{12} \\
B_{21} & B_{22}
\end{array}\right)=\left(\begin{array}{llll}
A_{11} B_{11} & A_{11} B_{12} & A_{12} B_{11} & A_{12} B_{12} \\
A_{11} B_{21} & A_{11} B_{22} & A_{12} B_{21} & A_{12} B_{22} \\
A_{21} B_{11} & A_{21} B_{12} & A_{22} B_{11} & A_{22} B_{12} \\
A_{21} B_{21} & A_{21} B_{22} & A_{22} B_{21} & A_{22} B_{22}
\end{array}\right)
$$

Duas matrizes importantes nesse contexto são a identidade $E=\mathbb{1}_{k} \otimes \mathbb{1}_{k}$ e a matriz de permutação $\Pi$, ambas matrizes $k^{2} \times k^{2}$, cujas componentes são respectivamente:

$$
E_{i j, I m}=\delta_{i l} \delta_{j m} \quad \text { e } \quad \Pi_{i j, I m}=\delta_{i m} \delta_{j l}
$$

A matriz de permutação satisfaz as seguintes propriedades:

$$
\Pi^{2}=E \quad \text { e } \Pi(A \otimes B) \Pi=B \otimes A
$$

e em duas dimensões, isto é, para $k=2$, admite uma representação em termos das matrizes gama (vide apêndice B):

$$
\Pi=\frac{1}{2}\left(\mathbb{1}_{k} \otimes \mathbb{1}_{k}+\gamma^{0} \otimes \gamma^{0}-\gamma^{1} \otimes \gamma^{1}+\gamma^{3} \otimes \gamma^{3}\right)=\left(\begin{array}{cccc}
1 & 0 & 0 & 0 \\
0 & 0 & 1 & 0 \\
0 & 1 & 0 & 0 \\
0 & 0 & 0 & 1
\end{array}\right) .
$$

Assim, podemos introduzir a seguinte definição para os parênteses de Poisson:

Definição 2.7.1. Sejam A e B dois funcionais matriciais com suporte compacto, isto é, duas matrizes $k \times k$, cujos elementos são funcionais de $\Psi(\sigma)$ e $\bar{\psi}(\sigma)$ com suporte compacto para $-L<\sigma<L$. Os parênteses de Poisson do produto tensorial:

$$
\{A \stackrel{\otimes}{, B}\}=i \int_{-L}^{L} d \sigma\left[\frac{\partial A}{\partial \psi} \otimes \frac{\partial B}{\partial \bar{\psi}}-\frac{\partial A}{\partial \bar{\psi}} \otimes \frac{\partial B}{\partial \psi}\right]
$$

são definidos como a matriz $k^{2} \times k^{2}$, cujos elementos são os parênteses de Poisson dos elementos das matrizes $A$ e $B$. Em termos das coordenadas:

$$
\{A \stackrel{\otimes}{,} B\}_{i j, l m}=\left\{A_{i l}, B_{j m}\right\} .
$$

\footnotetext{
${ }^{13}$ Outra notação usual consiste em escrever $A \otimes B=A_{1} B_{2}$ onde $A_{1}=A \otimes \mathbb{1}_{k}$ e $B_{2}=\mathbb{1}_{k} \otimes B$.
} 
Em particular, as propriedades básicas dos parênteses de Poisson adquirem a seguinte forma:

Antissimetria: $\{A \stackrel{\otimes}{,} B\}=-\Pi\{B \stackrel{\otimes}{,} A\} \Pi$,

Regra de Leibnitz: $\quad\{A \stackrel{\otimes}{, B C}\}=\{A \stackrel{\otimes}{, B}\}\left(\mathbb{1}_{k} \otimes C\right)+\left(\mathbb{1}_{k} \otimes B\right)\{A \stackrel{\otimes}{,} C\}$,

Identidade de Jacobi: $\{A \stackrel{\otimes}{,}\{B \stackrel{\otimes}{,}, C\}\}+\Pi_{13} \Pi_{23}\{C \stackrel{\otimes}{,}\{A \stackrel{\otimes}{,} B\}\} \Pi_{23} \Pi_{13}+$

$$
+\Pi_{13} \Pi_{12}\{B \stackrel{\otimes}{,}\{C \stackrel{\otimes}{,} A\}\} \Pi_{12} \Pi_{13}=0 .
$$

Em virtude da definição 2.7.1 fica claro que a operação $\{\cdot \stackrel{\otimes}{,} \cdot\}$ está definida para matrizes com dimensão arbitrária ${ }^{14}$, de forma que $\{A \stackrel{\otimes}{,}\{B \stackrel{\otimes}{,}, C\}\} \in \mathbb{C}^{k} \otimes \mathbb{C}^{k} \otimes \mathbb{C}^{k}$ e $\Pi_{12}\left(\Pi_{13}\right.$ e $\left.\Pi_{23}\right)$ denota uma matriz que é a unidade no terceiro espaço (respectivamente, no segundo e no primeiro) e coincide com $\Pi$ no produto dos espaços remanescentes.

Introduzimos a matriz $r$ através da seguinte definição:

Definição 2.7.2. A matriz $r$ é uma matriz $k^{2} \times k^{2}$ definida de forma a satisfazer os seguintes parênteses de Poisson:

$$
\left\{L_{\sigma}\left(\sigma_{1}, \tau ; \lambda\right) \stackrel{\otimes}{,} L_{\sigma}\left(\sigma_{2}, \tau ; \mu\right)\right\}=\left[r(\lambda-\mu), L_{\sigma}\left(\sigma_{1}, \tau ; \lambda\right) \otimes \mathbb{1}_{k}+\mathbb{1}_{k} \otimes L_{\sigma}\left(\sigma_{2}, \tau ; \mu\right)\right] \delta\left(\sigma_{1}-\sigma_{2}\right)
$$

A relação (2.7.5) é denominada parênteses de Poisson fundamentais (PPF).

Os PPF desempenham um papel central, pois permitem que demonstremos a involução das cargas conservadas. No que se segue, consideramos apenas matrizes $r$ não-dinâmicas, isto é, aquelas que não dependem explicitamente das variáveis dinâmicas do sistema, no caso, os campos $\Psi(x)$ e $\bar{\psi}(x)$. Diz-se que a equação (2.7.5) é ultralocal devido a presença apenas de $\delta\left(\sigma_{1}-\sigma_{2}\right)$, mas não de suas derivadas. A ultralocalidade é uma propriedade importante do potencial $L_{x}(\sigma, \tau ; \lambda)$ do problema linear auxiliar, notamos, contudo, que apesar de essas hipóteses sobre a forma dos PPF parecerem bem restritivas, elas, de fato, contemplam uma ampla classe de modelos integráveis em teorias de campos. No próximo exemplo calculamos a matriz $r$ para o NLS.

Exemplo 2.7.3. Rescrevendo a conexão de Lax, $L_{x}$ (2.5.9) da seguinte forma:

$$
L_{x}(x, t ; \lambda)=\frac{\lambda}{2 i} \gamma^{3}+L_{x}^{0}(x)=\frac{\lambda}{2 i} \gamma^{3}+i \sqrt{g}\left(\psi(x) \gamma^{+}-\bar{\psi}(x) \gamma^{-}\right)
$$

com $\gamma^{ \pm}=\frac{\gamma^{0} \pm \gamma^{1}}{2}$, calculamos a partir da definição, os PPF:

$$
\left\{L_{x}(x, t ; \lambda) \stackrel{\otimes}{,} L_{x}(y, t ; \mu)\right\}=i g\left(\gamma^{+} \otimes \gamma^{-}-\gamma^{-} \otimes \gamma^{+}\right) \delta(x-y) .
$$

Nesse estágio, já é claro que os PPF para o modelo NLS são ultralocais. Usando as seguintes relações de comutação entre as matrizes gama:

$$
\left[\gamma^{-}, \gamma^{+}\right]=\gamma^{3}, \quad\left[\gamma^{3}, \gamma^{-}\right]=2 \gamma^{-}, \quad\left[\gamma^{3}, \gamma^{+}\right]=-2 \gamma^{+}
$$

\footnotetext{
${ }^{14}$ Porém, finita.
} 
podemos escrever:

$$
\begin{aligned}
{\left[\Pi, \gamma^{3} \otimes \mathbb{1}_{2}\right] } & =\frac{1}{2}\left[\mathbb{1}_{2} \otimes \mathbb{1}_{2}+\gamma^{0} \otimes \gamma^{0}-\gamma^{1} \otimes \gamma^{1}+\gamma^{3} \otimes \gamma^{3}, \gamma^{3} \otimes \mathbb{1}_{2}\right] \\
& =\frac{1}{2}\left[2 \gamma^{-} \otimes \gamma^{+}+2 \gamma^{+} \otimes \gamma^{-}+\gamma^{3} \otimes \gamma^{3}, \gamma^{3} \otimes \mathbb{1}_{2}\right] \\
& =\left[\gamma^{-}, \gamma^{3}\right] \otimes \gamma^{+}+\left[\gamma^{+}, \gamma^{3}\right] \otimes \gamma^{-} \\
& =2\left(\gamma^{+} \otimes \gamma^{-}-\gamma^{-} \otimes \gamma^{+}\right) .
\end{aligned}
$$

As propriedades da matriz de permutação $\Pi$ implicam:

$$
\left[\Pi, \mathbb{1}_{2} \otimes \gamma^{3}\right]=-2\left(\gamma^{+} \otimes \gamma^{-}-\gamma^{-} \otimes \gamma^{+}\right)
$$

Logo,

$$
\frac{g}{\lambda-\mu}\left[\Pi, \frac{i \lambda}{2} \gamma^{3} \otimes \mathbb{1}_{2}+\frac{i \mu}{2} \mathbb{1}_{2} \otimes \gamma^{3}\right]=i g\left(\gamma^{+} \otimes \gamma^{-}-\gamma^{-} \otimes \gamma^{+}\right) .
$$

Devido a (2.7.1), temos que $\Pi$ comuta com $L_{x}^{0}(x) \otimes \mathbb{1}_{2}+\mathbb{1}_{2} \otimes L_{x}^{0}(x)$, portanto, ao combinarmos esse fato com as equações (2.7.6) e (2.7.9), concluímos finalmente que:

$$
\left\{L_{x}(x, t ; \lambda), L_{x}(y, t ; \mu)\right\}=-\left[\frac{g \sqcap}{\lambda-\mu}, L_{x}(x, t ; \lambda) \otimes \mathbb{1}_{2}+\mathbb{1}_{2} \otimes L_{x}(y, t ; \mu)\right] \delta(x-y),
$$

de onde deduzimos que a matriz r para o NLS é:

$$
r(\lambda-\mu)=-\frac{g \sqcap}{\lambda-\mu} .
$$

Ressaltamos que essa matriz $r$ não depende das variáveis dinâmicas. Além do mais, notamos que o lado direito de (2.7.10) contém uma singularidade aparente em $\lambda=\mu$, entretanto, como $\Pi$ comuta com $L_{x}(x, t ; \lambda) \otimes \mathbb{1}_{2}+\mathbb{1}_{2} \otimes L_{x}(x, t ; \lambda)$, temos que o numerador do lado direito de (2.7.10) também se anula em $\lambda=\mu$ e a singularidade desaparece.

É importante deixar claro que não é qualquer matriz $k^{2} \times k^{2}$ que pode assumir o papel da matriz r. Condições suficientes para que os PPF (2.7.5) sejam consistentes com a antissimetria (2.7.4a) e a identidade de Jacobi (2.7.4c) são respectivamente:

$$
\begin{array}{r}
r(-\lambda)=-\Pi r(\lambda) \Pi \\
{\left[r_{13}(\lambda-\mu), r_{23}(\mu-\nu)\right]+\left[r_{12}(\lambda-\mu), r_{13}(\lambda-\nu)\right]+\left[r_{12}(\lambda-\mu), r_{23}(\mu-\nu)\right]=0 .}
\end{array}
$$

Naturalmente, essas condições são válidas para a matriz $r$ (2.7.11). Por outro lado, se (2.7.12) são satisfeitas, então (2.7.5) define uma estrutura de Poisson no espaço dos funcionais dos elementos de matriz de $L_{\sigma}(\sigma, \tau ; \lambda)$. A equação (2.7.12b) é denominada equação de Yang-Baxter clássica.

Para demonstrarmos os dois resultados centrais dessa seção são necessários os dois lemas seguintes.

Lema 2.7.4. Sejam $L_{\sigma}(\sigma, \tau ; \lambda)$ uma conexão de $\operatorname{Lax}$ e $T\left(\sigma_{2}, \sigma_{1} ; \lambda\right)$ a matriz de transição associada, 
$\operatorname{com} \sigma, \sigma_{1}, \sigma_{2} \in[-L ; L]$ e $\sigma_{2}>\sigma_{1}$, então os seguintes parênteses de Poisson são válidos:

$$
\left\{T\left(\sigma_{2}, \sigma_{1} ; \lambda\right) \stackrel{\otimes}{,} L_{\sigma}(\sigma, \tau ; \mu)\right\}=\int_{\sigma_{1}}^{\sigma_{2}} d \xi T\left(\sigma_{2}, \xi ; \lambda\right) \otimes \mathbb{1}_{k}\left\{L_{\sigma}(\xi, \tau ; \lambda) \stackrel{\otimes}{,} L_{\sigma}(\sigma, \tau ; \mu)\right\} T\left(\xi, \sigma_{1} ; \lambda\right) \otimes \mathbb{1}_{k} .
$$

Demonstração: Como a matriz de transição $T\left(\sigma_{2}, \sigma_{1} ; \lambda\right)$ satisfaz (2.6.2):

$$
\left[\partial_{\sigma_{2}}-L_{\sigma}\left(\sigma_{2}, \tau ; \lambda\right)\right] T\left(\sigma_{2}, \sigma_{1} ; \lambda\right)=0 \quad \text { com } \quad T\left(\sigma_{1}, \sigma_{1} ; \lambda\right)=1
$$

podemos escrever que:

$$
T\left(\sigma_{2}, \sigma_{1} ; \lambda\right)=P \exp \int_{\sigma_{1}}^{\sigma_{2}} d \sigma L_{\sigma}(\sigma, \tau ; \lambda)
$$

Dividindo o intervalo $\left[\sigma_{1} ; \sigma_{2}\right]$ em subintervalos infinitesimais $\Delta_{i}$, a matriz de transição pode ser escrita como:

$$
T\left(\sigma_{1}, \sigma_{2} ; \lambda\right)=\lim _{N \rightarrow \infty} P \prod_{i=1}^{N} T_{i}(\lambda)=\lim _{N \rightarrow \infty} T_{N} T_{N-1} \cdots T_{1}
$$

com

$$
T_{i}(\lambda):=P \exp \int_{\Delta_{i}} d \sigma_{i} L_{\sigma}\left(\sigma_{i}, \tau ; \lambda\right)=\mathbb{1}_{k}+\int_{\Delta_{i}} d \sigma_{i} L_{\sigma}\left(\sigma_{i}, \tau ; \lambda\right)+O\left(\Delta^{2}\right),
$$

onde $\left\{T_{i}(\lambda) \stackrel{\otimes}{,} T_{j}(\mu)\right\}=0$ se $i \neq j$. Logo, temos que:

$$
\left\{T\left(\sigma_{2}, \sigma_{1} ; \lambda\right) \stackrel{\otimes}{,} L_{\sigma}(\sigma, \tau ; \mu)\right\}=\lim _{N \rightarrow \infty}\left\{P \prod_{i=1}^{N} T_{i}(\lambda) \stackrel{\otimes}{,} L_{\sigma}(\sigma, \tau ; \mu)\right\} .
$$

Uma vez que os parênteses de Poisson satisfazem a regra de Leibnitz, podemos mostrar que:

$$
\left\{P \prod_{i=1}^{N} T_{i}(\lambda) \stackrel{\otimes}{,} L_{\sigma}(\sigma, \tau ; \mu)\right\}=P \sum_{i=1}^{N} \prod_{j=i+1}^{N}\left[T_{j}(\lambda) \otimes \mathbb{1}_{k}\right]\left\{T_{i}(\lambda) \stackrel{\otimes}{,} L_{\sigma}(\sigma, \tau ; \mu)\right\} \prod_{l=1}^{i-1}\left[T_{l}(\lambda) \otimes \mathbb{1}_{k}\right] .
$$

Façamos, isso por indução finita em $N$. Claramente, para $N=1$ temos que $\left\{T_{1}(\lambda) \stackrel{\otimes}{,} L_{\sigma}(\sigma, \tau ; \mu)\right\}=$ $\left\{T_{1}(\lambda) \stackrel{\otimes}{,} L_{\sigma}(\sigma, \tau ; \mu)\right\}$. Supomos, pois, que a tese é verdadeira e provamos para $N+1$ :

$$
\begin{aligned}
\left\{P \prod_{i=1}^{N+1} T_{i}, L_{\sigma}\right\} & =\left\{T_{N+1} P \prod_{i=1}^{N} T_{i}, L_{\sigma}\right\}=T_{N+1} \otimes \mathbb{1}_{k}\left\{P \prod_{i=1}^{N} T_{i}, L_{\sigma}\right\}+\left\{T_{N+1}, \otimes L_{\sigma}\right\} P \prod_{i=1}^{N} T_{i} \otimes \mathbb{1}_{k} \\
& =P T_{N+1} \otimes \mathbb{1}_{k} \sum_{i=1}^{N} \prod_{j=i+1}^{N}\left(T_{j} \otimes \mathbb{1}_{k}\right)\left\{T_{i}, L_{\sigma}\right\} \prod_{l=1}^{i-1}\left(T_{l} \otimes \mathbb{1}_{k}\right)+\left\{T_{N+1}, \stackrel{\otimes}{,} L_{\sigma}\right\} P \prod_{i=1}^{N} T_{i} \otimes \mathbb{1}_{k} \\
& =P \sum_{i=1}^{N} \prod_{j=i+1}^{N+1}\left(T_{j} \otimes \mathbb{1}_{k}\right)\left\{T_{i}, L_{\sigma}\right\} \prod_{l=1}^{i-1}\left(T_{l} \otimes \mathbb{1}_{k}\right)+\left\{T_{N+1}, \stackrel{\otimes}{,} L_{\sigma}\right\} P \prod_{i=1}^{N} T_{i} \otimes \mathbb{1}_{k} \\
& =P \sum_{i=1}^{N+1} \prod_{j=i+1}^{N+1}\left(T_{j} \otimes \mathbb{1}_{k}\right)\left\{T_{i}, L_{\sigma}\right\} \prod_{l=1}^{i-1}\left(T_{l} \otimes \mathbb{1}_{k}\right) .
\end{aligned}
$$

Conseqüentemente,

$$
\left\{T\left(\sigma_{2}, \sigma_{1} ; \lambda\right) \stackrel{\otimes}{,} L_{\sigma}(\sigma, \tau ; \mu)\right\}=\lim _{N \rightarrow \infty} P \sum_{i=1}^{N} \prod_{j=i+1}^{N}\left[T_{j}(\lambda) \otimes \mathbb{1}_{k}\right]\left\{T_{i}(\lambda) \stackrel{\otimes}{,} L_{\sigma}(\sigma, \tau ; \mu)\right\} \prod_{l=1}^{i-1}\left[T_{l}(\lambda) \otimes \mathbb{1}_{k}\right]
$$




$$
\begin{aligned}
& =\lim _{N \rightarrow \infty} P \sum_{i=1}^{N} \prod_{j=i+1}^{N}\left[T_{j}(\lambda) \otimes \mathbb{1}_{k}\right]\left\{\mathbb{1}_{k}+\int_{\Delta_{i}} d \sigma_{i} L_{\sigma}\left(\sigma_{i}, \tau ; \lambda\right) \stackrel{\otimes}{,} L_{\sigma}(\sigma, \tau ; \mu)\right\} \prod_{l=1}^{i-1}\left[T_{l}(\lambda) \otimes \mathbb{1}_{k}\right] \\
& =\lim _{N \rightarrow \infty} P \sum_{i=1}^{N} \prod_{j=i+1}^{N}\left[T_{j}(\lambda) \otimes \mathbb{1}_{k}\right] \int_{\Delta_{i}} d \sigma_{i}\left\{L_{\sigma}\left(\sigma_{i}, \tau ; \lambda\right) \stackrel{\otimes}{,} L_{\sigma}(\sigma, \tau ; \mu)\right\} \prod_{l=1}^{i-1}\left[T_{l}(\lambda) \otimes \mathbb{1}_{k}\right] \\
& =\int_{\sigma_{1}}^{\sigma_{2}} d \xi T\left(\sigma_{2}, \xi ; \lambda\right) \otimes \mathbb{1}_{k}\left\{L_{\sigma}(\xi, \tau ; \lambda) \stackrel{\otimes}{,} L_{\sigma}(\sigma, \tau ; \mu)\right\} T\left(\xi, \sigma_{1} ; \lambda\right) \otimes \mathbb{1}_{k} .
\end{aligned}
$$

Lema 2.7.5. Nas mesmas condições do lema 2.7.4, os seguintes parênteses de Poisson são verdadeiros:

$$
\begin{aligned}
\left\{T\left(\sigma_{2}, \sigma_{1} ; \lambda\right) \stackrel{\otimes}{,} T\left(\sigma_{2}, \sigma_{1} ; \mu\right)\right\}=\int_{\sigma_{1}}^{\sigma_{2}} d \xi_{1} \int_{\sigma_{1}}^{\sigma_{2}} d \xi_{2} T\left(\sigma_{2}, \xi_{1} ; \lambda\right) \otimes T\left(\sigma_{2}, \xi_{2} ; \mu\right) . \\
\cdot\left\{L_{\sigma}\left(\xi_{1}, \tau ; \lambda\right) \stackrel{\otimes}{,} L_{\sigma}\left(\xi_{2}, \tau ; \mu\right)\right\} T\left(\xi_{1}, \sigma_{1} ; \lambda\right) \otimes T\left(\xi_{2}, \sigma_{1} ; \mu\right) .
\end{aligned}
$$

Demonstração: Procedendo de forma análoga à demonstração do lema 2.7.4, escrevemos,

$$
\begin{aligned}
\left\{T\left(\sigma_{2}, \sigma_{1} ; \lambda\right) \stackrel{\otimes}{,} T\left(\sigma_{2}, \sigma_{1} ; \mu\right)\right\}=\lim _{N \rightarrow \infty}\left\{T\left(\sigma_{2}, \sigma_{1} ; \lambda\right) \stackrel{\otimes}{,} P \prod_{i=1}^{N} T_{i}(\mu)\right\} \\
=\lim _{N \rightarrow \infty} P \sum_{i=1}^{N} \prod_{j=i+1}^{N} \mathbb{1}_{k} \otimes T_{j}(\mu)\left\{T\left(\sigma_{2}, \sigma_{1} ; \lambda\right) \stackrel{\otimes}{,} T_{i}(\mu)\right\} \prod_{l=1}^{i-1} \mathbb{1}_{k} \otimes T_{l}(\mu) \\
=\lim _{N \rightarrow \infty} P \sum_{i=1}^{N} \prod_{j=i+1}^{N} \mathbb{1}_{k} \otimes T_{j}(\mu) \int_{\Delta_{i}} d \sigma_{i}\left\{T\left(\sigma_{2}, \sigma_{1} ; \lambda\right) \stackrel{\otimes}{,} L_{\sigma}\left(\sigma_{i}, \tau ; \mu\right)\right\} \prod_{l=1}^{i-1} \mathbb{1}_{k} \otimes T_{l}(\mu) \\
=\int_{\sigma_{1}}^{\sigma_{2}} d \xi_{2} \mathbb{1}_{k} \otimes T\left(\sigma_{2}, \xi_{2} ; \mu\right)\left\{T\left(\sigma_{2}, \sigma_{1} ; \lambda\right) \stackrel{\otimes}{,} L_{\sigma}\left(\xi_{2}, \tau ; \mu\right)\right\} \mathbb{1}_{k} \otimes T\left(\xi_{2}, \sigma_{1} ; \mu\right) .
\end{aligned}
$$

Finalmente, ao utilizarmos o lema 2.7.4 obtemos a tese.

De posse lema 2.7.5 podemos demonstrar o seguinte teorema.

Teorema 2.7.6. Se os parênteses de Poisson entre os elementos da conexão de $\operatorname{Lax} L_{\sigma}(\sigma, \tau ; \lambda)$ admitem a representação em termos da matriz $r$, equação (2.7.5), então os parênteses de Poisson dos elementos da matriz de transição são dados por ${ }^{15}$ :

$$
\left\{T\left(\sigma_{2}, \sigma_{1} ; \lambda\right) \stackrel{\otimes}{,} T\left(\sigma_{2}, \sigma_{1} ; \mu\right)\right\}=\left[r(\lambda-\mu), T\left(\sigma_{2}, \sigma_{1} ; \lambda\right) \otimes T\left(\sigma_{2}, \sigma_{1} ; \mu\right)\right] .
$$

Conseqüentemente, os funcionais $\zeta^{(n)}(\lambda)=\operatorname{tr}\left[\left(T_{L}(\tau ; \lambda) Q(\theta)\right)^{n}\right]$ geram as quantidades conservadas em involução:

$$
\left\{\zeta^{(n)}(\lambda), \zeta^{(m)}(\mu)\right\}=0
$$

\footnotetext{
${ }^{15}$ Ou por:

$$
\left\{T_{1}\left(\sigma_{2}, \sigma_{1} ; \lambda\right), T_{2}\left(\sigma_{2}, \sigma_{1} ; \mu\right)\right\}=\left[r_{12}(\lambda-\mu), T_{1}\left(\sigma_{2}, \sigma_{1} ; \lambda\right) T_{2}\left(\sigma_{2}, \sigma_{1} ; \mu\right)\right] .
$$
}


Demonstração: Começamos demonstrando a validade da equação (2.7.13), para isso lembramos que a matriz de transição $T\left(\sigma_{2}, \sigma_{1} ; \lambda\right)$ satisfaz as seguintes equações diferenciais:

$$
\begin{aligned}
& \partial_{\sigma_{1}} T\left(\sigma_{2}, \sigma_{1} ; \lambda\right)+T\left(\sigma_{2}, \sigma_{1} ; \lambda\right) L_{\sigma}\left(\sigma_{1}, \tau ; \lambda\right)=0 \\
& \partial_{\sigma_{2}} T\left(\sigma_{2}, \sigma_{1} ; \lambda\right)-L_{\sigma}\left(\sigma_{2}, \tau ; \lambda\right) T\left(\sigma_{2}, \sigma_{1} ; \lambda\right)=0
\end{aligned}
$$

com a condição $T(\sigma, \sigma ; \lambda)=1$. Substituindo os PPF, equação (2.7.5) no resultado do lema 2.7.5 e usando as equações diferenciais satisfeitas pela matriz de transição, obtemos:

$$
\begin{aligned}
\left\{T\left(\sigma_{2}, \sigma_{1} ; \lambda\right) \stackrel{\otimes}{,} T\left(\sigma_{2}, \sigma_{1} ; \mu\right)\right\}=\int_{\sigma_{1}}^{\sigma_{2}} d \xi_{1} \int_{\sigma_{1}}^{\sigma_{2}} d \xi_{2}\left[\left[T\left(\sigma_{2}, \xi_{1} ; \lambda\right) \otimes T\left(\sigma_{2}, \xi_{2} ; \mu\right)\right] r(\lambda-\mu) .\right. \\
\cdot\left(\partial_{\xi_{1}}+\partial_{\xi_{2}}\right)\left[T\left(\xi_{1}, \sigma_{1} ; \lambda\right) \otimes T\left(\xi_{2}, \sigma_{1} ; \mu\right)\right]+ \\
\left.+\left(\partial_{\xi_{1}}+\partial_{\xi_{2}}\right)\left[T\left(\sigma_{2}, \xi_{1} ; \lambda\right) \otimes T\left(\sigma_{2}, \xi_{2} ; \mu\right)\right] r(\lambda-\mu)\left[T\left(\xi_{1}, \sigma_{1} ; \lambda\right) \otimes T\left(\xi_{2}, \sigma_{1} ; \mu\right)\right]\right] \delta\left(\xi_{1}-\xi_{2}\right) \\
=\int_{\sigma_{1}}^{\sigma_{2}} d \xi \partial_{\xi}\left[\left[T\left(\sigma_{2}, \xi ; \lambda\right) \otimes T\left(\sigma_{2}, \xi ; \mu\right)\right] r(\lambda-\mu)\left[T\left(\xi, \sigma_{1} ; \lambda\right) \otimes T\left(\xi, \sigma_{1} ; \mu\right)\right]\right] .
\end{aligned}
$$

Integrando essa derivada total, obtemos a relação (2.7.13).

Em seguida, mostramos que os funcionais $\zeta^{(n)}(\lambda)$ geram as quantidades conservadas em involução. Para isso, consideramos a equação (2.7.13) para $-L<\sigma_{1}<\sigma_{2}<L$ e a multiplicamos por $Q(\theta) \otimes Q(\theta)$ pela direita. Ao notarmos que

$$
[r(\lambda) ; Q(\theta) \otimes Q(\theta)]=0
$$

constatamos que (2.7.13) continua válida quando $T\left(\sigma_{2}, \sigma_{1} ; \lambda\right)$ e $T\left(\sigma_{2}, \sigma_{1} ; \mu\right)$ são substituídos por $T\left(\sigma_{2}, \sigma_{1} ; \lambda\right) Q(\theta)$ e $T\left(\sigma_{2}, \sigma_{1} ; \mu\right) Q(\theta)$, respectivamente. Em particular,

$$
\begin{aligned}
\left\{\left[T\left(\sigma_{2}, \sigma_{1} ; \lambda\right) Q(\theta)\right]^{n} \stackrel{\otimes}{,}\left[T\left(\sigma_{2}, \sigma_{1} ; \mu\right) Q(\theta)\right]^{m}\right\}= \\
=\left[r(\lambda-\mu),\left[T\left(\sigma_{2}, \sigma_{1} ; \lambda\right) Q(\theta)\right]^{n} \otimes\left[T\left(\sigma_{2}, \sigma_{1} ; \mu\right) Q(\theta)\right]^{m}\right] .
\end{aligned}
$$

Tomando o traço da equação resultante e usando o fato de que

$$
\operatorname{tr}(A \otimes B)=\operatorname{tr}(A) \operatorname{tr}(B)
$$

bem como que o traço de um comutador se anula identicamente, inferimos:

$$
\left\{\operatorname{tr}\left[T\left(\sigma_{2}, \sigma_{1} ; \lambda\right) Q(\theta)\right]^{n}, \operatorname{tr}\left[T\left(\sigma_{2}, \sigma_{1} ; \mu\right) Q(\theta)\right]^{m}\right\}=0 .
$$

Finalmente, ao considerarmos o limite $\sigma_{2} \rightarrow L$ e $\sigma_{1} \rightarrow-L$, obtemos (2.7.14).

O teorema 2.7.6 não só afirma que as cargas conservadas estão em involução, mas também que para cada carga conservada existe um fluxo Hamiltoniano correspondente. Para sermos mais 
explícitos, consideramos o exemplo do modelo NLS, e expandimos $p_{L}(\lambda)=\arccos \left(\frac{\zeta(\lambda)}{2}\right)(2.6 .41)$, de (2.7.14) deduzimos:

$$
\partial_{t} \psi=\left\{I_{n}, \psi\right\} \quad \text { e } \partial_{t} \bar{\psi}=\left\{I_{n}, \bar{\psi}\right\},
$$

ou seja, que para cada $I_{n}$ em $\Gamma_{L, \theta}$ há um fluxo Hamiltoniano associado. Para $n=1,2$ (2.6.42), tais fluxos possuem interpretações físicas simples e para $n=3$ recuperamos a equação NLS, contudo para $n>3$ as equações resultantes não possuem uma interpretação trivial, sendo denominadas equações não-lineares de Schrödinger de ordem mais alta. Mostramos, em seguida, que as correspondentes equações de movimento assumem a forma de uma condição de curvatura nula.

Teorema 2.7.7. Tomando $\zeta(\mu)$ como a Hamiltoniana, temos que:

$$
\partial_{\tau} L_{\sigma}(\sigma, \tau ; \lambda):=\left\{\zeta(\mu), L_{\sigma}(\sigma, \tau ; \lambda)\right\}=\partial_{\sigma} M(\sigma ; \mu, \lambda)+\left[M(\sigma ; \mu, \lambda), L_{\sigma}(\sigma, \tau ; \lambda)\right]
$$

onde

$$
M(\sigma ; \mu, \lambda)=\operatorname{tr}_{1}\left[\left(T(L, \sigma ; \mu) \otimes \mathbb{1}_{k}\right) r(\mu-\lambda)\left(T(\sigma,-L ; \mu) \otimes \mathbb{1}_{k}\right)\left(Q(\theta) \otimes \mathbb{1}_{k}\right)\right],
$$

proporcionando equações de movimento para uma hierarquia de tempos ao expandirmos em $\mu$.

Demonstração: Para provarmos a equação (2.7.16) utilizamos o lema 2.7.4 para calcular a seguinte quantidade:

$$
\begin{aligned}
& \left\{T(L,-L ; \mu) \stackrel{\otimes}{,} L_{\sigma}(\sigma, \tau ; \lambda)\right\}=\int_{-L}^{L} d \xi\left(T(L, \xi ; \mu) \otimes \mathbb{1}_{k}\right)\left\{L_{\sigma}(\xi, \tau ; \mu) \stackrel{\otimes}{,} L_{\sigma}(\sigma, \tau ; \lambda)\right\}\left(T(\xi,-L ; \mu) \otimes \mathbb{1}_{k}\right) \\
& \quad=\int_{-L}^{L} d \xi\left(T(L, \xi ; \mu) \otimes \mathbb{1}_{k}\right)\left[r(\mu-\lambda), L_{\sigma}(\xi, \tau ; \mu) \otimes \mathbb{1}_{k}+\mathbb{1}_{k} \otimes L_{\sigma}(\sigma, \tau ; \lambda)\right]\left(T(\xi,-L ; \mu) \otimes \mathbb{1}_{k}\right) \delta(\mu-\lambda) \\
& \quad=\left(T(L, \sigma ; \mu) \otimes \mathbb{1}_{k}\right) r(\mu-\lambda)\left[\left(L_{\sigma}(\sigma, \tau ; \mu) T(\sigma,-L ; \mu)\right) \otimes \mathbb{1}_{k}\right]- \\
& \quad-\left[\left(T(L, \sigma ; \mu) L_{\sigma}(\sigma, \tau ; \mu)\right) \otimes \mathbb{1}_{k}\right] r(\mu-\lambda)\left(T(\sigma,-L ; \mu) \otimes \mathbb{1}_{k}\right)+ \\
& \quad+\left(T(L, \sigma ; \mu) \otimes \mathbb{1}_{k}\right) r(\mu-\lambda)\left(T(\sigma,-L ; \mu) \otimes L_{\sigma}(\sigma, \tau ; \lambda)\right)- \\
& \quad-\left(T(L, \sigma ; \mu) \otimes L_{\sigma}(\sigma, \tau ; \lambda)\right) r(\mu-\lambda)\left(T(\sigma,-L ; \mu) \otimes \mathbb{1}_{k}\right) .
\end{aligned}
$$

Usando as equações diferenciais para a matriz de transição (2.6.2) podemos expressar os dois primeiros termos da última passagem como uma derivada em $\sigma$ de

$$
V(\sigma ; \mu, \lambda):=\left[T(L, \sigma ; \mu) \otimes \mathbb{1}_{k}\right] r(\mu-\lambda)\left[T(\sigma,-L ; \mu) \otimes \mathbb{1}_{k}\right] .
$$

De forma que

$$
\left\{T(L,-L ; \mu) \stackrel{\otimes}{,} L_{\sigma}(\sigma, \tau ; \lambda)\right\}=\partial_{\sigma} V(\sigma ; \mu, \lambda)+\left[V(\sigma ; \mu, \lambda), \mathbb{1}_{k} \otimes L_{\sigma}(\sigma, \tau ; \lambda)\right] .
$$

Multiplicando ambos os lados por $Q(\theta) \otimes \mathbb{1}_{k}$ pela direita e tomando o traço sobre o primeiro espaço 
concluímos que

$$
\left\{\zeta(\mu), L_{\sigma}(\sigma, \tau ; \lambda)\right\}=\partial_{\sigma} M(\sigma ; \mu, \lambda)+\left[M(\sigma ; \mu, \lambda), L_{\sigma}(\sigma, \tau ; \lambda)\right]
$$

onde

$$
M(\sigma ; \mu, \lambda)=\operatorname{tr}_{1}\left[V(\sigma ; \mu, \lambda)\left(Q(\theta) \otimes \mathbb{1}_{k}\right)\right]
$$

Exemplo 2.7.8. Utilizamos o teorema 2.7.7 para mostrar como, no contexto do modelo NLS, é possível a partir da matriz $r$ e da conexão de $L_{a x} L_{x}(x, t ; \lambda)$ construir uma seqüência de matrizes $V_{n}(x ; \lambda)$ fornecendo a representação de curvatura nula para as equações de Schrödinger não-lineares de ordem mais alta. Primeiramente, computemos a matriz $M(x ; \mu, \lambda)$ para o modelo NLS, substituindo a expressão da matriz r (2.7.11) em (2.7.17).

$$
\begin{aligned}
M(x ; \mu, \lambda) & =\frac{g}{\lambda-\mu} \operatorname{tr}_{1}\left[\left(T(L, x ; \mu) \otimes \mathbb{1}_{2}\right) \Pi\left(T(x,-L ; \mu) Q(\theta) \otimes \mathbb{1}_{2}\right)\right] \\
& =\frac{g}{\lambda-\mu} \operatorname{tr}_{1}\left[\Pi\left(T(x,-L ; \mu) Q(\theta) T(L, x ; \mu) \otimes \mathbb{1}_{k}\right)\right] \\
& =\frac{g}{\lambda-\mu} \operatorname{tr}_{1}\left[\left(\mathbb{1}_{k} \otimes T(x,-L ; \mu) Q(\theta) T(L, x ; \mu)\right) \Pi\right] \\
& =\frac{g}{\lambda-\mu} T(x,-L ; \mu) Q(\theta) T(L, x ; \mu) \operatorname{tr}_{1} \Pi \\
& =\frac{g}{\lambda-\mu} T(x,-L ; \mu) Q(\theta) T(L, x ; \mu)
\end{aligned}
$$

onde usamos a ciclicidade do traço na primeira posição

$$
\operatorname{tr}_{1}\left[\left(A \otimes \mathbb{1}_{k}\right) X\right]=\operatorname{tr}_{1}(X)\left(A \otimes \mathbb{1}_{k}\right),
$$

com A sendo uma matriz $k \times k$ e $X$ uma matriz $k^{2} \times k^{2}$, a propriedade (2.7.1) da matriz de permutação, que

$$
\operatorname{tr}_{1}\left[\left(\mathbb{1}_{k} \otimes A\right) X\right]=A \operatorname{tr}_{1} X
$$

e finalmente que $\operatorname{tr}_{1} \Pi=\mathbb{1}_{2}$. Claramente, a expressão para $M(x ; \mu, \lambda)$ é quasiperiódica, pois:

$$
\left.\begin{array}{l}
M(L ; \mu, \lambda)=\frac{g}{\lambda-\mu} T_{L}(\mu) Q(\theta) \\
M(-L ; \mu, \lambda)=\frac{g}{\lambda-\mu} Q(\theta) T_{L}(\mu)
\end{array}\right\} \Rightarrow M(L ; \mu, \lambda)=Q^{-1}(\theta) M(-L ; \mu, \lambda) Q(\theta) .
$$

A seguir, consideramos a representação com caráter assintótico (2.6.20) para a matriz de transição:

$$
T(x, y ; \mu)=\left[\mathbb{1}_{k}+W(x ; \mu)\right] e^{Z(x, y ; \mu)}\left[\mathbb{1}_{k}+W(x ; \mu)\right]^{-1},
$$

e a substituímos em (2.7.18). Usando a quasiperiodicidade de $W(x ; \mu)$,

$$
W(x+2 L ; \mu)=Q^{-1}(\theta) W(x ; \mu) Q(\theta) \Rightarrow \mathbb{1}_{k}+W(L ; \mu)=Q^{-1}(\theta)\left[\mathbb{1}_{k} W(-L ; \mu)\right] Q(\theta),
$$


e o fato de que $Z(x, y ; \mu)$ é diagonal, obtemos:

$$
M(x ; \mu, \lambda)=\frac{g}{\lambda-\mu}\left[\mathbb{1}_{k}+W(x ; \mu)\right] e^{z_{L}(\mu)} Q(\theta)\left[\mathbb{1}_{k}+W(x ; \mu)\right]^{-1} .
$$

Por outro lado (2.7.19) juntamente com a quasiperiodicidade de $W(x ; \mu)$ permitem escrever:

$$
T_{L}(\mu) Q(\theta)=\left[\mathbb{1}_{k}+W(L ; \mu)\right] e^{Z_{L}(\mu)} Q(\theta)\left[\mathbb{1}_{k}+W(L ; \mu)\right]^{-1} .
$$

A unimodularidade de $T_{L}(\mu) Q(\theta)$ juntamente com a definição

$$
\operatorname{tr}\left[T_{L}(\mu) Q(\theta)\right]=2 \cos \left[p_{L}(\mu)\right]
$$

implicam

$$
e^{Z_{L}(\mu)} Q(\theta)=\cos \left[p_{L}(\mu)\right] \mathbb{1}_{2}+i \sin \left[p_{L}(\mu)\right] \gamma^{3},
$$

cuja substituição em (2.7.20) leva a:

$$
M(x ; \mu, \lambda)=\frac{g}{\lambda-\mu} \cos \left[p_{L}(\mu)\right] \mathbb{1}_{2}-2 \sin \left[p_{L}(\mu)\right] V(x ; \mu, \lambda),
$$

com

$$
V(x ; \mu, \lambda)=\frac{g}{2 i(\lambda-\mu)}\left[\mathbb{1}_{k}+W(x ; \mu)\right] \gamma^{3}\left[\mathbb{1}_{k}+W(x ; \mu)\right]^{-1} .
$$

Notamos que o primeiro termo de (2.7.21) não depende de $x$ e é proporcional a $\mathbb{1}_{2}$, portanto não contribui para (2.7.16). Conseqüentemente, sem perda de generalidade, podemos desprezá-lo na definição da matriz $M(x ; \mu, \lambda)$ :

$$
M(x ; \mu, \lambda)=-2 \sin \left[p_{L}(\mu)\right] V(x ; \mu, \lambda) .
$$

Finalmente, calculamos

$$
\begin{aligned}
\partial_{t} L_{x}(x ; \lambda) & =\left\{p_{L}(\mu), L_{x}(x ; \lambda)\right\}=\left\{\arccos \left(\frac{\zeta(\mu)}{2}\right), L_{x}(x ; \lambda)\right\} \\
& =-\frac{1}{2} \frac{1}{\sqrt{1-\left(\frac{\zeta(\mu)}{2}\right)^{2}}}\left\{\zeta(\mu), L_{x}(x ; \lambda)\right\} \\
& =-\frac{1}{2 \sin \left[p_{L}(\mu)\right]}\left\{-2 \sin \left[p_{L}(\mu)\right]\left\{\partial_{x} V(x ; \mu, \lambda)+\left[V(x ; \mu, \lambda), L_{x}(x ; \lambda)\right]\right\}\right\} \\
& =\partial_{x} V(x ; \mu, \lambda)+\left[V(x ; \mu, \lambda), L_{x}(x ; \lambda)\right]
\end{aligned}
$$

onde utilizamos o teorema 2.7.7 em conjunto com a expressão para a matriz $M(x ; \mu, \lambda)$ (2.7.23). Claramente, a equação (2.7.24) fornece a representação de curvatura ${ }^{16}$ nula que procurávamos. Substituindo a expansão assintótica de $W(x ; \mu)(2.6 .21 a)$

$$
W(x ; \mu)=\sum_{n=1}^{\infty} \frac{W_{n}(x)}{\mu^{n}}+O\left(|\mu|^{-\infty}\right),
$$

\footnotetext{
${ }^{16}$ Note que essa representação deve ser entendida de maneira assintótica, isto é, válida até $O\left(|\mu|^{-\infty}\right)$.
} 
em (2.7.22) e expandindo o fator $\frac{1}{\lambda-\mu}$ em potências de $\mu^{-1}$ inferimos

$$
V(x ; \mu, \lambda)=g \sum_{n=1}^{\infty} \frac{V_{n}(x ; \lambda)}{\mu^{n}}+O\left(|\mu|^{-\infty}\right)
$$

onde os coeficientes $V_{n}(x ; \lambda)$, que constituem a seqüência de matrizes que aparece na representação de curvatura nula para as equações de Schrödinger não-lineares de ordem mais alta, são polinômios em $\lambda, \psi(x), \bar{\psi}(x)$ e suas derivadas espaciais, cujo grau em $\lambda$ é $n-1$. Em particular, temos que:

$$
V_{1}(x ; \lambda)=\frac{i}{2} \gamma^{3}, \quad V_{2}(x ; \lambda)=-L_{x}(x ; \lambda) \quad \text { e } \quad V_{3}(x ; \lambda)=L_{t}(x ; \lambda)
$$

Ao considerarmos a expansão (2.6.41) em (2.7.24) verificamos que as equações de Schrödinger não-lineares de ordem mais alta,

$$
\partial_{t} \psi=\left\{I_{n}, \psi\right\} \quad \text { e } \partial_{t} \bar{\psi}=\left\{I_{n}, \bar{\psi}\right\} \quad \text { com } n \in \mathbb{N}^{*}
$$

admitem uma representação de curvatura nula com matrizes $L_{x}(x ; \lambda)$ e $V_{n}(x ; \lambda)$.

Esse exemplo ilustra de uma maneira concreta que a existência dos PPF fornece uma alternativa à condição de curvatura nula.

\subsection{O Método do Espalhamento Inverso, o Problema de Riemann- Hilbert e a Linearização das Equações de Movimento}

Para mostrarmos a integrabilidade completa de um dado modelo precisamos contemplar as seguintes questões:

1. Existem cargas conservadas?

2. As cargas conservadas estão em involução?

3. Existe um número suficiente de cargas conservadas?

Para o caso onde o número de graus de liberdade é finito, o critério de Liouville (definição 2.3.2) estabelece que o número de integrais de movimento necessárias corresponde ao número de graus de liberdade do sistema. De forma que ao satisfazermos essas condições podemos utilizar o teorema de Arnold-Liouville (teorema 2.3.4) para construir as coordenadas de ângulo e ação e assim integrar as equações de movimento. Contudo para o caso de teorias de campos, com um número infinito de graus de liberdade, a situação é severamente mais complicada. Conseguimos responder afirmativamente às duas primeiras questões para um sistema cuja equação diferencial parcial admite a representação de Lax, ao mostrarmos que a partir da matriz de monodromia podemos gerar infinitas cargas conservadas, e com o auxílio da matriz $r$ provar que elas estão em involução. No entanto, é uma tarefa difícil contar adequadamente o número de integrais do movimento, quando há infinitas delas. A solução usual consiste em encontrar um mapa bijetor que linearize as equações do 
movimento, introduzindo as coordenadas de ângulo e ação, de tal maneira a possibilitar a integração explícita das equações de movimento.

A construção desse mapa constitui uma tarefa essencial, porém significativamente complicada, em parte porque depende explicitamente de características particulares de cada modelo, como o par de Lax e a matriz $r$. Ademais, como tal construção não será efetivamente utilizada no restante desta tese, optamos por somente ilustrá-la no caso mais simples do modelo NLS no limite $L \rightarrow \infty$ com condições de contorno correspondendo ao decaimento rápido no infinito (2.4.8). Para maiores detalhes e as devidas demonstrações, vide [20].

Começamos analisando algumas propriedades da matriz de transição $T(x, y ; \lambda)$ definida na reta real, isto é, com $-\infty<x, y<\infty$. Em consonância com a condição de contorno (2.4.8), supomos que $\psi(x), \bar{\psi}(x) \in L_{1}(\mathbb{R})$, de forma que

$$
\int_{-\infty}^{\infty} d x\left\|L_{x}^{0}(x)\right\|<\infty
$$

Sob essas condições os limites

$$
T_{ \pm}(x ; \lambda)=\lim _{y \rightarrow \pm \infty} T(x, y ; \lambda) E(y ; \lambda)
$$

existem para $\lambda \in \mathbb{R}$. E assim como $T(x, y ; \lambda)$, satisfazem o problema linear auxiliar (2.6.2), que, em particular, assume a forma de um problema de autovalores:

$$
\mathscr{D} F=\frac{\lambda}{2} F \quad \text { onde } \quad \mathscr{D}=i \gamma^{3} \partial_{x}-\sqrt{g}\left[\psi(x) \gamma^{+}+\bar{\psi}(x) \gamma^{-}\right]
$$

com as condições iniciais substituídas pelas seguintes condições assintóticas:

$$
T_{ \pm}(x ; \lambda) \stackrel{x \rightarrow \pm \infty}{\longrightarrow} E(x ; \lambda)+o(1)
$$

As matrizes $T_{ \pm}(x ; \lambda)$, conhecidas como soluções de Jost, desempenham um papel central na discussão que se segue. Introduzindo a notação para as colunas de $T_{ \pm}(x ; \lambda)$ :

$$
T_{ \pm}(x ; \lambda)=\left(T_{ \pm}^{(1)}(x ; \lambda), T_{ \pm}^{(2)}(x ; \lambda)\right)
$$

verificamos que $T_{-}^{(1)}(x ; \lambda)$ e $T_{+}^{(2)}(x ; \lambda)$ admitem uma continuação analítica para $\Im(\lambda)>0$, enquanto que $T_{+}^{(1)}(x ; \lambda)$ e $T_{-}^{(2)}(x ; \lambda)$ para $\Im(\lambda)<0$.

Outro limite interessante da matriz de transição é

$$
T(\lambda)=\lim _{\substack{x \rightarrow+\infty \\ y \rightarrow-\infty}} E(-x ; \lambda) T(x, y ; \lambda) E(y ; \lambda),
$$

denominado matriz de monodromia reduzida, que pode ser considerado como o limite para um intervalo infinito da matriz de monodromia periódica $T_{L}(\lambda)$. Em particular, a matriz de monodromia reduzida pode ser expressa em termos das soluções de Jost:

$$
T(\lambda)=T_{+}^{-1}(x ; \lambda) T_{-}(x ; \lambda)
$$




\subsection{O Método do Espalhamento Inverso, o Problema de Riemann-Hilbert e a Linearização} das Equações de Movimento

Além disso, como a matriz de monodromia reduzida apresenta as mesmas propriedades de involução que $T_{L}(\lambda)$, podemos escrevê-la na seguinte forma:

$$
T(\lambda)=\left(\begin{array}{cc}
a(\lambda) & \epsilon \bar{b}(\lambda) \\
b(\lambda) & \bar{a}(\lambda)
\end{array}\right) \operatorname{com}\left\{\begin{array}{l}
a(\lambda)=\lim _{L \rightarrow \infty} e^{i \lambda L} a_{L}(\lambda)=\operatorname{det}\left(T_{-}^{(1)}(x ; \lambda), T_{+}^{(2)}(x ; \lambda)\right) \\
b(\lambda)=\lim _{L \rightarrow \infty} b_{L}(\lambda)=\operatorname{det}\left(T_{+}^{(1)}(x ; \lambda), T_{-}^{(1)}(x ; \lambda)\right) .
\end{array}\right.
$$

Também denominamos $a(\lambda)$ e $b(\lambda)$ coeficientes de transição, em virtude de sua definição em termos de $a_{L}(\lambda)$ e $b_{L}(\lambda)$, eles herdam a condição de normalização:

$$
|a(\lambda)|^{2}-\epsilon|b(\lambda)|^{2}=1
$$

As propriedades analíticas das colunas de $T_{ \pm}(x ; \lambda)$ implicam que $a(\lambda)$ possui uma continuação analítica no semiplano complexo superior, $\Im \lambda \geq 0$, enquanto que $b(\lambda)$ não admite nenhuma continuação além da reta real.

É importante notar que o coeficiente de transição $a(\lambda)$ pode se anular se as soluções $T_{-}^{(1)}(x ; \lambda)$ e $T_{+}^{(2)}(x ; \lambda)$ forem linearmente dependentes, isso acontece quando $\lambda$ é um autovalor discreto do problema linear auxiliar. Para $g>0$, o operador $\mathscr{D}$ é auto-adjunto e, conseqüentemente, não há espectro discreto, contudo para $g<0, \mathscr{D}$ não é mais auto-adjunto e $a(\lambda)$ pode possuir zeros. A análise desse segundo caso é notadamente mais complicada e por isso focamos apenas no caso com $g>0$ para ilustrar a construção do mapa bijetor. Em particular, as propriedades analíticas de $a(\lambda)$ e a relação de normalização permitem que expressemos $a(\lambda)$ em termos de $b(\lambda)$ :

$$
a(\lambda)=\exp \left\{\frac{1}{2 \pi i} \int_{-\infty}^{\infty} d \mu \frac{\log \left(1+|b(\mu)|^{2}\right)}{\mu-\lambda-i \varepsilon}\right\}
$$

Com isso, definimos um mapa

$$
\mathscr{F}:(\psi(x), \bar{\psi}(x)) \rightarrow(b(\lambda), \bar{b}(\lambda))
$$

com $\psi(x), \bar{\psi}(x) \in L_{1}(\mathbb{R})$ e $b(\lambda), \bar{b}(\lambda) \in \mathfrak{R}_{0}$, o anel das transformadas de Fourrier de funções em $L_{1}(\mathbb{R})$.

Queremos mostrar que o mapa $\mathscr{F}$ lineariza as equações de movimento, para isso consideramos a evolução temporal da matriz de transição, cuja equação diferencial é obtida ao derivarmos o problema linear auxiliar (2.6.2) com respeito ao tempo e utilizarmos a condição de curvatura nula (2.5.6):

$$
\begin{aligned}
\partial_{t} \partial_{x} T & =L_{x} \partial_{t} T+\partial_{t} L_{x} T=\left(\partial_{x} L_{t}+\left[L_{t}, L_{x}\right]\right) T+L_{x} \partial_{t} T=\partial_{x} L_{t} T+L_{t} \partial_{x} T-L_{x} L_{t} T+L_{x} \partial_{t} T \\
& =\partial_{x}\left(L_{t} T\right)+L_{x}\left(\partial_{t} T-L_{t} T\right) \\
& \Rightarrow \partial_{x}\left(\partial_{t} T-L_{t} T\right)=L_{x}\left(\partial_{t} T-L_{t} T\right) \Rightarrow \partial_{t} T(x, y)=L_{t}(x) T(x, y)+T(x, y) C
\end{aligned}
$$


onde a matriz $C$ independe de $x$. Usando a condição inicial, inferimos que $C=-L_{t}(y)$ e obtemos a equação de evolução temporal para a matriz de transição:

$$
\partial_{t} T(x, y ; \lambda)=L_{t}(x ; \lambda) T(x, y ; \lambda)-T(x, y ; \lambda) L_{t}(y ; \lambda) .
$$

Multiplicando (2.8.12) por $E(y ; \lambda)$ pela direita podemos então tomar o limite $y \rightarrow \pm \infty$ para obter

$$
\partial_{t} T_{ \pm}(x ; \lambda)=L_{t}(x ; \lambda) T_{ \pm}(x ; \lambda)-\frac{i \lambda^{2}}{2} T_{ \pm}(x ; \lambda) \gamma^{3}
$$

onde usamos que no limite $|x| \rightarrow \infty, L_{t}(x ; \lambda) \rightarrow \frac{i \lambda^{2}}{2} \gamma^{3}$. Repetindo o mesmo argumento com respeito a $x$, obtemos uma equação de evolução temporal para a matriz de monodromia reduzida ${ }^{17}$ :

$$
\partial_{t} T(t ; \lambda)=\frac{i \lambda^{2}}{2}\left[\gamma^{3}, T(t ; \lambda)\right]
$$

Uma característica peculiar da equação (2.8.14) é que a dependência nos campos originais $\psi(x)$ e $\bar{\psi}(x)$ foi completamente eliminada. Em particular, podemos a partir de (2.8.14) inferir a equação de evolução temporal para os coeficientes de transição:

$$
\partial_{t} a(t ; \lambda)=0 \quad \text { e } \quad \partial_{t} b(t ; \lambda)=-i \lambda^{2} b(t ; \lambda)
$$

Claramente, $a(\lambda)$ é independente do tempo para $\lambda \in \mathbb{R}$, ademais, como $a(\lambda)$ admite uma continuação analítica para $\Im(\lambda)>0$, é possível mostrar que essa independência temporal pode ser estendida para $\Im(\lambda)>0$. Dessa forma, $a(\lambda)$ é a função geradora das quantidades conservadas para o modelo NLS com os campos se anulando rapidamente no infinito. A equação para $b(t ; \lambda)$ também pode ser facilmente resolvida, obtemos, pois, que:

$$
a(t ; \lambda)=a(t=0 ; \lambda) \quad \text { e } \quad b(t ; \lambda)=e^{-i \lambda^{2} t} b(t=0 ; \lambda) .
$$

Assim, estabelecemos que o mapa $\mathscr{F}$ de fato lineariza as equações de movimento. Nesse estágio é evidente a semelhança entre as equações de movimento para os coeficientes de transição (2.8.15) e para as coordenadas de ângulo e ação $F_{i}$ e $\theta_{i}$ do caso com graus de liberdade finitos (2.3.4), de forma que fica claro que o mapa $\mathscr{F}$ fornece a transformação para as coordenadas de ângulo e ação, $a(t, \lambda)$ e $b(t, \lambda)$.

Podemos agora obter as identidades de traço a partir dos resultados deduzidos na seção 2.6.1. Fixando $\theta=0$ e utilizando a definição da matriz de monodromia reduzida, bem como (2.8.8), escrevemos para $\lambda \in \mathbb{R}$

$$
\operatorname{tr} T_{L}(\lambda)=e^{-i \lambda L} a(\lambda)+e^{i \lambda L} \bar{a}(\lambda)+o(1)=2|a(\lambda)| \cos (\arg a(\lambda)-\lambda L)+o(1),
$$

\footnotetext{
${ }^{17}$ Nesse ponto é conveniente reintroduzir a dependência explícita em $t$ da matriz de monodromia reduzida.
} 


\subsection{Método do Espalhamento Inverso, o Problema de Riemann-Hilbert e a Linearização} das Equações de Movimento

conforme $L \rightarrow \infty$. Usando o comportamento assintótico de $a(\lambda)$, temos que $|a(\lambda)|=1+O\left(|\lambda|^{-\infty}\right)$, de forma que:

$$
p_{L}(\lambda)=\arccos \left(\frac{\operatorname{tr} T_{L}(\lambda)}{2}\right)=-\lambda L+\arg a(\lambda)+o(1)=-\lambda L-i \log a(\lambda)+o(1)
$$

onde usamos outra conseqüência do comportamento assintótico de $a(\lambda), \log a(\lambda)=i \arg a(\lambda)+$ $O\left(|\lambda|^{-\infty}\right)$. Finalmente, tomando o limite $L \rightarrow \infty$, inferimos:

$$
\log a(\lambda)=i \lim _{L \rightarrow \infty}\left[p_{L}(\lambda)+\lambda L\right]=i g \sum_{n=1}^{\infty} \frac{I_{n}}{\lambda^{n}}+O\left(|\lambda|^{-\infty}\right)
$$

cujos coeficientes podem ser determinados ao expandirmos (2.8.10):

$$
I_{n}=\int_{-\infty}^{\infty} d x P_{n}(x)=\frac{1}{2 \pi g} \int_{-\infty}^{\infty} d \mu \log \left(1+|b(\lambda)|^{2}\right) \lambda^{n-1}
$$

com os $P_{n}(x)$ definidos por (2.6.38).

Finalmente, investigamos a injetividade e a inversibilidade do mapa $\mathscr{F}$. A estratégia ${ }^{18}$ consiste em reduzir o problema de espalhamento inverso a um problema de Riemann-Hilbert, que para o caso em questão assume a seguinte forma:

O Problema de Riemann-Hilbert: Seja $G(\lambda)$ uma matriz $k \times k$ não-degenerada definida na reta real, $\lambda \in \mathbb{R}$, e normalizada a $\mathbb{1}_{k}$ no limite $|\lambda| \rightarrow \infty$. O problema de Riemann-Hilbert consiste em encontrar duas matrizes $k \times k$ não-degeneradas $G_{+}(\lambda)$ e $G_{-}(\lambda)$, normalizadas a $\mathbb{1}_{k}$ no limite $|\lambda| \rightarrow \infty$, que possam ser estendidas analiticamente aos semiplanos complexos superior e inferior, respectivamente, e que forneçam uma fatoração de $G(\lambda)$ :

$$
G(\lambda)=G_{+}(\lambda) G_{-}(\lambda)
$$

O problema de Riemann-Hilbert possui uma solução única, com $G_{ \pm}(\lambda)$ satisfazendo as condições acima, se a matriz $G(\lambda)+G^{\dagger}(\lambda)$ for positiva definida.

O nosso ponto de partida é a relação entre as soluções de Jost e a matriz de monodromia reduzida, equação (2.8.7), que apesar de parecer com um problema de Riemann-Hilbert, ainda não constitui um, pois as colunas de $T_{ \pm}$são analíticas em diferentes semiplanos complexos. Essa dificuldade não é contudo fatal, visto que podemos introduzir as matrizes

$$
S_{+}(x ; \lambda)=\left(T_{-}^{(1)}(x ; \lambda), T_{+}^{(2)}(x ; \lambda)\right) \quad \text { e } \quad S_{-}(x ; \lambda)=\left(T_{+}^{(1)}(x ; \lambda), T_{-}^{(2)}(x ; \lambda)\right)
$$

que também são soluções do problema linear auxiliar, mas que possuem as extensões analíticas corretas. Em particular, em termos das matrizes $S_{ \pm}(x ; \lambda)$ podemos rescrever (2.8.7):

$$
S_{-}(x ; \lambda)=S_{+}(x ; \lambda) S(\lambda)
$$

\footnotetext{
${ }^{18}$ Uma abordagem alternativa mais tradicional é baseada na equação de Gelfand-Levitan-Marchenko.
} 
com

$$
S(\lambda)=\frac{1}{a(\lambda)}\left(\begin{array}{cc}
1 & \bar{b}(\lambda) \\
-b(\lambda) & 1
\end{array}\right)
$$

assumindo o papel da matriz de espalhamento para o problema linear auxiliar. Como escolhemos tratar apenas do caso $g>0$, para o qual $a(\lambda) \neq 0$, podemos simplesmente absorvê-lo na definição do fator $G_{+}(x ; \lambda)$ :

$$
G_{+}(x ; \lambda)=a(\lambda) E(x ; \lambda) S_{+}^{-1}(x ; \lambda) \quad \text { e } \quad G_{-}(x ; \lambda)=S_{-}(x ; \lambda) E^{-1}(x ; \lambda) .
$$

Assim, tais matrizes $G_{ \pm}(x ; \lambda)$ resolvem o problema de Riemann-Hilbert (2.8.21) com a normalização usual:

$$
G_{ \pm}(x ; \lambda) \stackrel{|\lambda| \rightarrow \infty}{\longrightarrow} \mathbb{1}_{k}+o(1) \quad \text { e } \quad G(x ; \lambda) \stackrel{|\lambda| \rightarrow \infty}{\longrightarrow} \mathbb{1}_{k}+o(1)
$$

pois

$$
G(x ; \lambda)=G_{+}(x ; \lambda) G_{-}(x ; \lambda)=E(x ; \lambda) G(x) E(-x ; \lambda)=\left(\begin{array}{cc}
1 & \bar{b}(\lambda) e^{-i \lambda x} \\
-b(\lambda) e^{i \lambda x} & 1
\end{array}\right),
$$

de forma que $G(x ; \lambda)+G^{\dagger}(x ; \lambda)=2 \cdot \mathbb{1}_{2}$ é positiva definida. Portanto, mostramos que as matrizes $G(x ; \lambda)$ e $G_{ \pm}(x ; \lambda)$ estão relacionadas de uma maneira injetora e única. Em particular, se considerarmos a evolução temporal dos coeficientes de transição (2.8.16) podemos rescrever (2.8.21) incluindo a dependência em $t$ :

$$
G(x, t ; \lambda)=G_{+}(x, t ; \lambda) G_{-}(x, t ; \lambda)
$$

com

$$
G(x, t ; \lambda)=E^{-1}\left(t ; \lambda^{2}\right) G(x ; \lambda) E(t ; \lambda), \quad \text { onde } \quad E\left(t ; \lambda^{2}\right)=\exp \left\{\frac{\lambda^{2} t}{2 i} \gamma^{3}\right\}
$$

Conseqüentemente, podemos mostrar que as funções

$$
\begin{aligned}
& F_{+}(x, t ; \lambda)=G_{+}^{-1}(x, t ; \lambda) E(x ; \lambda) E^{-1}\left(t ; \lambda^{2}\right) \\
& F_{-}(x, t ; \lambda)=G_{-}(x, t ; \lambda) E(x ; \lambda) E^{-1}\left(t ; \lambda^{2}\right)
\end{aligned}
$$

satisfazem o problema linear fundamental (2.5.1) com a conexão de Lax dada por (2.5.9).

Logo, a partir dos coeficientes de transição $b(t ; \lambda)$ e $\bar{b}(t ; \lambda)$ construímos

$$
G(t ; \lambda)=\left(\begin{array}{cc}
1 & \bar{b}(t ; \lambda) \\
b(t ; \lambda) & 1
\end{array}\right)
$$

e usamos o problema de Riemann-Hilbert para obter as matrizes $G_{ \pm}(x, t ; \lambda)$ e, em seguida, as matrizes $F_{ \pm}(x, t ; \lambda)$, que permitem a reconstrução da conexão de $\operatorname{Lax}_{\alpha}(x, t ; \lambda), \alpha=t, x$ satisfazendo a condição de curvatura nula (2.5.6), de onde, finalmente, extraímos os campos $\psi(x, t)$ e $\bar{\psi}(x, t)$ satisfazendo a equação NLS (2.4.5). Estabelecemos, pois, que os coeficientes de transição $b(\lambda)$ e $\bar{b}(\lambda)$ estão em correspondência biunívoca com os campos iniciais $\psi(x)$ e $\bar{\psi}(x)$, de sorte que o mapa 
2.8 O Método do Espalhamento Inverso, o Problema de Riemann-Hilbert e a Linearização das Equações de Movimento

$\mathscr{F}$, que fornece a transformação não-linear de variáveis responsável por linearizar as equações de movimento do modelo NLS, é inversível.

O método que exibimos para resolver o problema de valores iniciais para a equação NLS pode ser representado sucintamente através do seguinte diagrama comutativo:

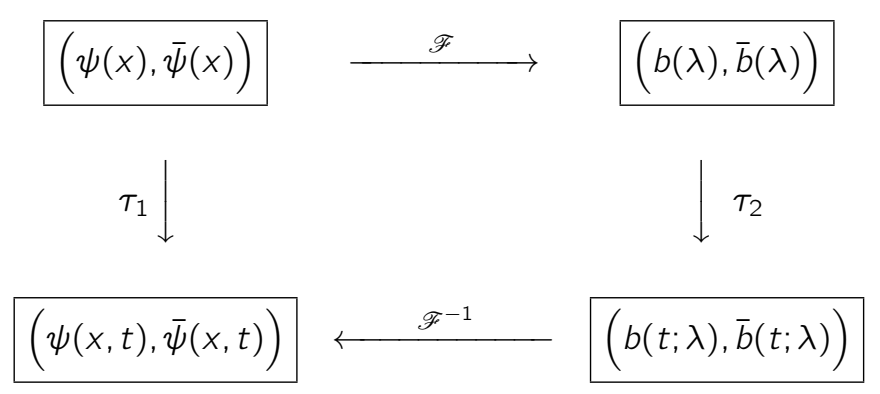

onde $\tau_{1}$ é o deslocamento temporal implementado pela equação NLS (2.4.5), enquanto $\tau_{2}$ é a evolução temporal dada explicitamente por (2.8.16). 



\section{Capítulo 3}

\section{Integrabilidade Quântica}

There is a theory which states that if ever anyone discovers exactly what the Universe is for and why it is here, it will instantly disappear and be replaced by something even more bizarre and inexplicable. There is another theory which states that this has already happened.

Douglas Adams, The Hitchhiker's Guide to the Galaxy

Um dos conceitos mais poderosos para o estudo de sistemas dinâmicos clássicos, conforme discutimos no capítulo anterior, é o de integrabilidade. Seja o número de graus de liberdade do sistema finito ou infinito, se ele possuir um determinado número ${ }^{1}$ de cargas conservadas em involução é possível construir as variáveis de ângulo e ação, e assim integrar explicitamente as equações de movimento. Dessa forma o significado da integrabilidade de um dado sistema clássico é claro, e, em particular, separa os modelos clássicos em classes com propriedades bem distintas: as soluções das equações de movimento integráveis descrevem movimentos periódicos em toros que folheiam o espaço de fase, enquanto que para o caso de modelos não-integráveis, as soluções preenchem o espaço de fase de uma forma densa.

Com o sucesso da aplicação do método do espalhamento inverso clássico (MEIC) para a demonstração da integrabilidade clássica de diversos modelos, como por exemplo os modelos de Schrödinger não-linear, de Thirring, de sine-Gordon e de Landau-Lifshitz, questionou-se se as suas contrapartes quânticas também não seriam integráveis. Surpreendentemente, estender o conceito de integrabilidade ao contexto quântico se mostrou severamente complicado, e persiste até hoje como uma questão em aberto e motivo de intenso debate [48-50].

Uma das principais dificuldades na extensão do conceito de integrabilidade ao contexto quântico está atrelada à noção de grau de liberdade, essencial na formulação do teorema de Arnold-Liouville. Na mecânica clássica, a contagem de graus de liberdade é um procedimento trivial, pois associamos um grau de liberdade a cada par de variáveis conjugadas do espaço de fase, necessárias para descrever as configurações de um dado sistema. Já na mecânica quântica, o simples conceito de grau de liberdade não está matematicamente bem definido. O motivo está relacionado com o fato de que todos os espaços de Hilbert com dimensão infinita são isometricamente isomórficos, e, por isso, as

\footnotetext{
${ }^{1}$ Relacionado com o número de graus de liberdade do sistema.
} 
álgebras $\mathbb{C}^{*}$ de operadores limitados atuando sobre tais espaços de Hilbert são isomórficas. Dessa forma, é impossível distinguir o espaço de Hilbert dos estados de uma única partícula, do espaço de Hilbert para os estados de muitas partículas. Em particular, um teorema de von Neumann ${ }^{2}$ arruína a idéia de utilizar um conjunto maximal de operadores comutantes para mimetizar o conjunto maximal de cargas clássicas conservadas em involução, uma vez que podemos encontrar um operador quântico, a partir do qual podemos expressar todos os outros como suas funções.

Uma abordagem mais pragmática à integrabilidade quântica, e em consonância com o questionamento inicial, consiste em reconhecer que, quando lidamos com sistemas quânticos concretos, existe um conjunto preferencial de observáveis desde o princípio $^{3}$, de forma que o espaço de Hilbert não é o objeto fundamental inicial. Notadamente, o espaço de Hilbert é apenas o portador da representação de tais variáveis preferenciais. Assim, dado um operador Hamiltoniano $H$ procuramos por um grupo $G$ e uma álgebra de Lie $\mathfrak{g}$, de forma que exista uma representação unitária de $G$ no espaço de Hilbert, tal que o Hamiltoniano $H$ possa ser identificado com a ação de um elemento da álgebra universal envelopante $U(\mathfrak{g})$. Informações sobre o espectro e sobre as autofunções de $H$ podem, pois, ser obtidas a partir da estrutura das representações unitárias de $G$ e de sua decomposição em representações irredutíveis.

Claramente, proceder como delineado acima para uma situação geral não é uma tarefa fácil, portanto começamos a nossa análise de sistemas quânticos "integráveis", estudando uma maneira particular de se resolver certos modelos quânticos. Diferentemente do caso clássico, onde, em geral, estamos interessados em obter a evolução temporal dos campos iniciais (ou, no contexto do MEIC, dos coeficientes de transição), na mecânica quântica a abordagem estacionária é mais usual, na qual o interesse predominante está na dedução do espectro dos operadores relevantes. O Ansatz de Bethe coordenado $(A B C)$ fornece um método para se obter tais soluções, ao propiciar a construção do conjunto completo das autofunções e dos autovalores de um dado operador Hamiltoniano. Ao combinar idéias advindas do $\mathrm{ABC}$ com as do MEIC desenvolvemos o método do espalhamento inverso quântico (MEIQ), que proporciona um ponto de vista unificado da solubilidade de modelos clássicos e quânticos. A matriz $R$ quântica satisfaz a equação de Yang-Baxter, que assume um papel central no estudo do espalhamento em teorias em $(1+1)$-dimensões. Finalmente, a noção de espalhamento fatorável permite a formulação de um critério objetivo para a integrabilidade quântica.

\subsection{O Ansatz de Bethe Coordenado}

O Ansatz de Bethe coordenado é um método analítico para a resolução exata de alguns problemas de muitos corpos em $(1+1)$-dimensões na mecânica quântica. Foi desenvolvido por Hans Bethe em 1931 [51] para o cálculo dos autovalores e autovetores do ferromagneto de Heisenberg. O Ansatz de Bethe prescreve uma parametrização dos autovetores para a solução do problema de autovalores fornecido pela equação de Schrödinger. Diferentemente de Bethe, ilustraremos o seu Ansatz na versão quântica de nosso exemplo corriqueiro, o modelo de Schrödinger não-linear (NLS), que quanticamente também é conhecido como o modelo unidimensional para o gás de Bose com interações pontuais.

\footnotetext{
${ }^{2}$ Teorema:" Seja um espaço de Hilbert $\mathscr{H}$ e considere dois observáveis comutantes, $A$ e $B$, então existe um terceiro observável C, tal que tanto A quanto B são funções de C."

${ }^{3}$ Operadores de momento e posição de uma partícula, operadores de spin para magnetos, etc.
} 
Seguimos a exposição de [22].

O Hamiltoniano do modelo é o seguinte:

$$
H=\int_{-L}^{L} d x\left[\partial_{x} \psi^{\dagger} \partial_{x} \psi+g \psi^{\dagger} \psi^{\dagger} \psi \psi\right]
$$

onde $\psi(x)$ e $\psi^{\dagger}(x)$ são dois operadores bosônicos autoadjuntos, periódicos ${ }^{4}$ em $x$, com período igual a $2 L$, que satisfazem as seguintes relações de comutação canônicas:

$$
\begin{aligned}
& {\left[\Psi(x), \Psi^{\dagger}(y)\right]=\delta(x-y)} \\
& {[\Psi(x), \Psi(y)]=0} \\
& {\left[\Psi^{\dagger}(x), \Psi^{\dagger}(y)\right]=0}
\end{aligned}
$$

Claramente, a equação de movimento correspondente é (2.4.5), com os campos clássicos substituídos pelos operadores quânticos,

$$
i \partial_{t} \psi=-\partial_{x}^{2} \psi+2 g \psi^{\dagger} \psi \psi
$$

Consideramos o caso com $g>0$, para o qual o estado fundamental em temperatura nula é uma esfera de Fermi. Definimos o vácuo de Fock, ou pseudovácuo, por

$$
\psi(x)|0\rangle=0=\langle 0| \psi^{\dagger}(x) \quad \text { com } \quad x \in \mathbb{R} \quad \text { e } \quad\langle 0 \mid 0\rangle=1 .
$$

É importante notar que o pseudovácuo e o vácuo físico são estados distintos, o último, em particular, é o estado fundamental do Hamiltoniano.

Os operadores número e momento são obtidos a partir da quantização direta de suas contrapartes clássicas (2.4.16) e (2.4.17):

$$
\begin{aligned}
& Q=\int_{-L}^{L} d x \psi^{\dagger}(x) \psi(x), \\
& P=-\frac{i}{2} \int_{-L}^{L} d x\left(\psi^{\dagger}(x) \partial_{x} \psi(x)-\partial_{x} \psi^{\dagger}(x) \psi(x)\right) .
\end{aligned}
$$

Tais operadores Hermiteanos são integrais do movimento comutantes:

$$
[H, Q]=[H, P]=[Q, P]=0 .
$$

Assim, podemos procurar por autofunções simultâneas dos operadores $Q, P$ e $H$ :

$$
\left|\psi_{N}\right\rangle=\frac{1}{\sqrt{N !}} \int_{-L}^{L} d^{N} z f_{N}\left(z_{1}, \ldots, z_{N}\right) \prod_{n=1}^{N} \psi^{\dagger}\left(z_{n}\right)|0\rangle,
$$

com as funções complexas $f_{N}$, simétricas em suas $N$ entradas reais e de quadrado integrável, satisfazendo as equações de autovalores:

$$
Q\left|\psi_{N}\right\rangle=N\left|\psi_{N}\right\rangle, \quad P\left|\psi_{N}\right\rangle=p_{N}\left|\psi_{N}\right\rangle \quad \text { e } \quad H\left|\psi_{N}\right\rangle=E_{N}\left|\psi_{N}\right\rangle .
$$

\footnotetext{
${ }^{4}$ Também consideramos o limite $L \rightarrow \infty$, nesse caso os operadores $\psi(x)$ e $\psi^{\dagger}(x)$ se anulam rapidamente no infinito.
} 
O espaço de Hilbert dos estados do sistema consiste, pois, no espaço de Fock $\mathcal{F}$ para bósons unidimensionais, cujos elementos podem ser representados em termos da base de autovalores:

$$
|f\rangle=\sum_{N=0}^{\infty} f_{N}\left|\psi_{N}\right\rangle \quad \text { com } \quad\left|\psi_{0}\right\rangle=|0\rangle
$$

e escritos convenientemente sob a forma de vetores coluna:

$$
|f\rangle=\left(\begin{array}{l}
f_{0} \\
f_{1}\left(z_{1}\right) \\
\vdots \\
f_{N}\left(z_{1}, \ldots, z_{N}\right) \\
\vdots
\end{array}\right) \text { com }|0\rangle=\left(\begin{array}{c}
1 \\
0 \\
\vdots \\
0 \\
\vdots
\end{array}\right)
$$

Introduzimos o seguinte produto escalar em $\mathcal{F}$ :

$$
\langle f \mid g\rangle=\bar{f}_{0} g_{0}+\sum_{N=1}^{\infty} \int_{-L}^{L} d^{N} z \bar{f}_{N}\left(z_{1}, \ldots, z_{N}\right) g\left(z_{1}, \ldots, z_{N}\right)
$$

Dessa forma, o espaço de Fock é decomposto na soma direta de $N$ subespaços parciais $\mathcal{F}_{N}$,

$$
\mathcal{F}=\bigoplus_{N=1}^{\infty} \mathcal{F}_{N} \quad \text { com } \quad f_{N}\left(z_{1}, \ldots, z_{N}\right) \in \mathcal{F}_{N}
$$

A partir das equações de autovalores (3.1.8) podemos encontrar as expressões para o Hamiltoniano quântico e para o operador de momento quântico, ou seja, os operadores $\mathcal{H}_{N}$ e $\mathcal{P}_{N}$ que atuam sobre os vetores $f_{N}\left(z_{1}, \ldots, z_{N}\right) \in \mathcal{F}_{N}$. Para isso, consideremos a ação dos operadores $Q, P$ e $H$ nos estados $\operatorname{com} N=1$ :

$$
\begin{aligned}
& Q\left|\psi_{1}\right\rangle=\int_{-L}^{L} d z f_{1}(z) \psi^{\dagger}(z)|0\rangle=1\left|\psi_{1}\right\rangle \\
& P\left|\psi_{1}\right\rangle=\left.\frac{i}{2} f_{1}(z) \psi^{\dagger}(z)\right|_{z=-L} ^{z=L}|0\rangle+\int_{-L}^{L} d z\left(-i \partial_{z}\right) f_{1}(z) \psi^{\dagger}(z)|0\rangle \\
& H\left|\psi_{1}\right\rangle=\left.\partial_{z} f_{1}(z) \psi^{\dagger}(z)\right|_{z=-L} ^{z=L}|0\rangle+\int_{-L}^{L} d z\left(-\partial_{z}^{2}\right) f_{1}(z) \psi^{\dagger}(z)|0\rangle
\end{aligned}
$$

e $N=2$ :

$$
\begin{aligned}
Q\left|\psi_{2}\right\rangle & =2 \int_{-L}^{L} d z_{1} d z_{2} f_{2}\left(z_{1}, z_{2}\right) \psi^{\dagger}\left(z_{1}\right) \psi^{\dagger}\left(z_{2}\right)|0\rangle=2\left|\psi_{2}\right\rangle, \\
P\left|\psi_{2}\right\rangle & =\left.\frac{i}{2} \int_{-L}^{L} d z\left[f_{2}(z, x)+f_{2}(x, z)\right] \psi^{\dagger}(z) \psi^{\dagger}(x)\right|_{x=-L} ^{x=L}|0\rangle+ \\
& +\int_{-L}^{L} d z_{1} d z_{2}\left(-i \partial_{z_{1}}-i \partial_{z_{2}}\right) f_{2}\left(z_{1}, z_{2}\right) \psi^{\dagger}\left(z_{1}\right) \psi^{\dagger}\left(z_{2}\right)|0\rangle,
\end{aligned}
$$




$$
\begin{aligned}
H\left|\psi_{2}\right\rangle & =\left.\int_{-L}^{L} d z \partial_{z}\left[f_{2}(z, x)+f_{2}(x, z)\right] \psi^{\dagger}(z) \psi^{\dagger}(x)\right|_{x=-L} ^{x=L}|0\rangle+ \\
& +\int_{-L}^{L} d z_{1} d z_{2}\left(-\partial_{z_{1}}^{2}-\partial_{z_{2}}^{2}+2 g \delta\left(z_{1}-z_{2}\right)\right) f_{2}\left(z_{1}, z_{2}\right) \psi^{\dagger}\left(z_{1}\right) \psi^{\dagger}\left(z_{2}\right)|0\rangle
\end{aligned}
$$

Com a aplicação das condições de contorno para os campos $\psi(x)$ e $\psi^{\dagger}(x)$, concluímos que as funções $f_{N} \in \mathcal{F}_{N}$ são autofunções de $\mathcal{P}_{N}$ e $\mathcal{H}_{N}$ :

$$
\mathcal{P}_{N}=\sum_{j=1}^{N}\left(-i \partial_{z_{j}}\right) \quad \text { e } \quad \mathcal{H}_{N}=\sum_{j=1}^{N}\left(-\partial_{z_{j}}^{2}\right)+2 g \sum_{1 \leq k<j \leq N} \delta\left(z_{j}-z_{k}\right)
$$

com autovalores $p_{N}$ e $E_{N}$. É importante ressaltar que o Hamiltoniano quântico $\mathcal{H}_{N}$ atuando sobre $f_{N}$ como o operador diferencial introduzido em (3.1.13) possui a forma de um operador de Schrödinger multiparcial com interação pontual. Dessa forma, reformulamos o problema originalmente no contexto da teoria quântica de campos como um problema de mecânica quântica.

Podemos dar um significado preciso ao potencial singular $\delta\left(z_{j}-z_{k}\right)$ presente em (3.1.13), substituindoo por condições de contorno adequadas. Em virtude da simetria de $f_{N}$ em todas as suas entradas $z_{j}$, precisamos considerar, sem perda de generalidade, apenas o seguinte domínio:

$$
-L \leq z_{1}<z_{2}<\cdots<z_{N} \leq L
$$

Ao impormos que $f_{N}$ seja uma autofunção do Hamiltoniano quântico livre:

$$
\mathcal{H}_{N}^{0}=-\sum_{j=1}^{N} \partial_{z_{j}}^{2}, \quad \text { i.e., } \quad \mathcal{H}_{N}^{0} f_{N}=E_{N} f_{N}
$$

obtemos, para o caso $N=2(3.1 .12 \mathrm{c})$ que:

$$
\left.\int_{-L}^{L} d z \partial_{z}\left[f_{2}(z, x)+f_{2}(x, z)\right] \psi^{\dagger}(z) \psi^{\dagger}(x)\right|_{x=-L} ^{x=L}+2 g \int_{-L}^{L} d z_{1} d z_{2} \delta\left(z_{1}-z_{2}\right) \psi^{\dagger}\left(z_{1}\right) \psi^{\dagger}\left(z_{2}\right)=0 .
$$

Notando que (no limite $\epsilon \rightarrow 0$ ):

$$
\begin{aligned}
\left.\int_{-L}^{L} d z \partial_{z} f_{2}(z, x) \psi^{\dagger}(z) \psi^{\dagger}(x)\right|_{x=-L} ^{x=L} & =\int_{-L}^{L} d z \partial_{z} f_{2}(z) \psi^{\dagger}(z) \psi^{\dagger}(x)\left[\left.\right|_{x=-L} ^{x=z-\epsilon}+\left.\right|_{x=z+\epsilon} ^{x=L}\right] \\
& =\left.\int_{-L}^{L} d z \partial_{z} f_{2}(z) \psi^{\dagger}(z) \psi^{\dagger}(x)\right|_{x=z+\epsilon} ^{x=z-\epsilon} \\
& =-\int_{-L}^{L} d z\left(\partial_{z+\epsilon}-\partial_{z}\right) f_{2}(z+\epsilon, z) \psi^{\dagger}(z+\epsilon) \psi^{\dagger}(z)
\end{aligned}
$$

inferimos de (3.1.15) a seguinte condição de contorno:

$$
\left.\left(\partial_{z_{2}}-\partial_{z_{1}}-g\right) f_{2}\left(z_{1}, z_{2}\right)\right|_{z_{2}=z_{1}+\epsilon}=0
$$


que pode ser facilmente generalizada para um valor $N$ arbitrário:

$$
\left.\left(\partial_{z_{j+1}}-\partial_{z_{j}}-g\right) f_{N}\left(z_{1}, \ldots, z_{N}\right)\right|_{z_{j+1}=z_{j}+\epsilon}=0
$$

Portanto, estabelecemos que a equação (3.1.14) suplementada pelas condições de contorno (3.1.17) é equivalente ao problema de autovalores $\mathcal{H}_{N} f_{N}=E_{N} f_{N}$, com $\mathcal{H}_{N}$ dada por (3.1.13).

A próxima etapa consiste em construir as funções $f_{N}$ propriamente, é nesse ponto que utilizamos o famigerado Ansatz de Bethe, que fornece uma expressão hipotética para tais funções. Consideramos inicialmente o caso com $N=2$ para maior claridade do argumento, onde supomos que:

$$
f_{2}\left(z_{1}, z_{2}\right)=f_{2}\left(z_{1}, z_{2} \mid \lambda_{1}, \lambda_{2}\right)=A_{1}\left(\lambda_{1}, \lambda_{2}\right) e^{i \lambda_{1} z_{1}+i \lambda_{2} z_{2}}+A_{2}\left(\lambda_{1}, \lambda_{2}\right) e^{i \lambda_{2} z_{1}+i \lambda_{1} z_{2}}
$$

correspondendo a uma superposição de duas ondas planas, cada uma multiplicada por uma amplitude dependente apenas das variáveis $\lambda_{1}$ e $\lambda_{2}$. Resta então verificar se a solução tentativa (3.1.18) é de fato uma solução da equação de autovalores (3.1.14) com condições de contorno (3.1.17). É fácil constatar que tal $f_{2}\left(z_{1}, z_{2} \mid \lambda_{1}, \lambda_{2}\right)$ é uma autofunção do Hamiltoniano livre com autovalor $\lambda_{1}^{2}+\lambda_{2}^{2}$ :

$$
\mathcal{H}_{2}^{0} f_{2}\left(z_{1}, z_{2} \mid \lambda_{1}, \lambda_{2}\right)=\left(\lambda_{1}^{2}+\lambda_{2}^{2}\right) f_{2}\left(z_{1}, z_{2} \mid \lambda_{1}, \lambda_{2}\right)
$$

Já a condição de contorno (3.1.17):

$$
0=\left.\left(\partial_{z_{2}}-\partial_{z_{1}}-g\right) f_{2}\left(z_{1}, z_{2} \mid \lambda_{1}, \lambda_{2}\right)\right|_{z_{2}=z_{1}+\epsilon}=\left[\left(i \lambda_{2}-i \lambda_{1}-g\right) A_{1}+\left(i \lambda_{1}-i \lambda_{2}-g\right) A_{2}\right] e^{i z_{2}\left(\lambda_{1}+\lambda_{2}\right)},
$$

fornece um vínculo entre $A_{1}\left(\lambda_{1}, \lambda_{2}\right)$ e $A_{2}\left(\lambda_{1}, \lambda_{2}\right)$ :

$$
\frac{A_{2}\left(\lambda_{1}, \lambda_{2}\right)}{A_{1}\left(\lambda_{1}, \lambda_{2}\right)}=\frac{\lambda_{2}-\lambda_{1}+i g}{\lambda_{2}-\lambda_{1}-i g} \equiv-e^{-i \theta\left(\lambda_{2}-\lambda_{1}\right)}
$$

Assim, podemos rescrever $f_{2}\left(z_{1}, z_{2} \mid \lambda_{1}, \lambda_{2}\right)$ :

$$
f_{2}\left(z_{1}, z_{2} \mid \lambda_{1}, \lambda_{2}\right)=(\text { const })\left[e^{i \lambda_{1} z_{1}+i \lambda_{2} z_{2}}-e^{-i \theta\left(\lambda_{2}-\lambda_{1}\right)} e^{i \lambda_{2} z_{1}+i \lambda_{1} z_{2}}\right]
$$

de forma a interpretarmos o Ansatz de Bethe como uma superposição de uma onda plana incidente com uma espalhada, multiplicada por uma fase $e^{-i \theta\left(\lambda_{2}-\lambda_{1}\right)}$, que assume o papel de uma matriz S. Portanto, se a condição (3.1.20) for satisfeita a função $f_{2}\left(z_{1}, z_{2}\right)$ definida por (3.1.18) é uma solução do problema de autovalores (3.1.14) com condições de contorno (3.1.17). Além do mais, é fácil verificar que tal $f_{2}\left(z_{1}, z_{2}\right)$ é também uma autofunção do operador de momento com autovalor $\lambda_{1}+\lambda_{2}$. Finalmente, ao impormos as condições de periodicidade na função de onda $f_{2}\left(z_{1}, z_{2}\right)$, isto é, $f_{2}\left(z_{1}-L, z_{2}\right)=f_{2}\left(z_{1}+L, z_{2}\right)$ e usarmos a simetria de $f_{2}$ em suas entradas deduzimos o seguinte sistema de equações para os valores permitidos ${ }^{5}$ de $\lambda_{1}$ e $\lambda_{2}$ :

$$
e^{2 i L \lambda_{1}}=e^{-i \theta\left(\lambda_{1}-\lambda_{2}\right)} \quad e \quad e^{2 i L \lambda_{2}}=e^{-i \theta\left(\lambda_{2}-\lambda_{1}\right)}
$$

\footnotetext{
${ }^{5}$ Condições de quantização para os momentos de partículas em uma caixa de comprimento $2 L$.
} 
conhecidas como equações de Bethe.

Uma generalização de (3.1.18) para $N$ arbitrário pode ser inferida ao tomarmos, em consonância com (3.1.20),

$$
A_{1}\left(\lambda_{1}, \lambda_{2}\right)=i \lambda_{2}-i \lambda_{1}+g \quad \text { e } \quad A_{2}\left(\lambda_{1}, \lambda_{2}\right)=-\left(i \lambda_{1}-i \lambda_{2}+g\right)
$$

e rescrevermos $f_{2}\left(z_{1}, z_{2} \mid \lambda_{1}, \lambda_{2}\right)$ como:

$$
\begin{aligned}
f_{2}\left(z_{1}, z_{2} \mid \lambda_{1}, \lambda_{2}\right) & =\left(i \lambda_{2}-i \lambda_{1}+g\right) e^{i \lambda_{1} z_{1}+i \lambda_{2} z_{2}}-\left(i \lambda_{1}-i \lambda_{2}+g\right) e^{i \lambda_{2} z_{1}+i \lambda_{1} z_{2}} \\
& =\left(\partial_{z_{2}}-\partial_{z_{1}}+g\right)\left[e^{i \lambda_{1} z_{1}+i \lambda_{2} z_{2}}-e^{i \lambda_{2} z_{1}+i \lambda_{1} z_{2}}\right] \\
& =\left(\partial_{z_{2}}-\partial_{z_{1}}+g\right) \operatorname{det}\left[\exp \left(i \lambda_{j} z_{k}\right)\right],
\end{aligned}
$$

com a matriz $2 \times 2$ :

$$
\exp \left(i \lambda_{j} z_{k}\right)=\left(\begin{array}{ll}
e^{i \lambda_{1} z_{1}} & e^{i \lambda_{1} z_{2}} \\
e^{i \lambda_{2} z_{1}} & e^{i \lambda_{2} z_{2}}
\end{array}\right) .
$$

Logo, o Ansatz de Bethe para $N$ arbitrário assume a seguinte forma:

$$
f_{N}\left(z_{1}, \ldots, z_{N} \mid \lambda_{1}, \ldots, \lambda_{N}\right)=\text { (const) }\left[\prod_{1 \leq k<j \leq N}\left(\partial_{z_{j}}-\partial_{z_{k}}+g\right)\right] \operatorname{det}\left[\exp \left(i \lambda_{j} z_{k}\right)\right],
$$

onde $\exp \left(i \lambda_{j} z_{k}\right)$ é uma matriz $N \times N$. É fácil verificar que a função $f_{N}$ acima satisfaz as condições de contorno (3.1.17), mostremos isso explicitamente para o caso $j=1$. Para tanto, escreva $f_{N}$ da seguinte forma:

$$
\begin{aligned}
& f_{N}=\left(\partial_{z_{2}}-\partial_{z_{1}}+g\right) \tilde{f}_{N} \text { com } \\
& \tilde{f}_{N}=(\text { const }) \prod_{j=3}^{N}\left[\left(\partial_{z_{j}}-\partial_{z_{1}}+g\right)\left(\partial_{z_{j}}-\partial_{z_{2}}+g\right)\right] \prod_{3 \leq k<1 \leq N}\left(\partial_{z_{l}}-\partial_{z_{k}}+g\right) \operatorname{det}\left[\exp \left(i \lambda_{j} z_{k}\right)\right]
\end{aligned}
$$

Claramente, $\tilde{f}_{N}$ é antissimétrica com respeito a troca $z_{1} \leftrightarrow z_{2}$ e se anula quando $z_{1}=z_{2}$, conseqüentemente,

$$
\left.\left[\left(\partial_{z_{2}}-\partial_{z_{1}}\right)^{2}-g^{2}\right] \tilde{f}_{N}\right|_{z_{2}=z_{1}}=0
$$

As outras condições de contorno podem ser verificadas da mesma maneira. Portanto, (3.1.25) fornece as autofunções dos operadores $H, P$ e $Q$, cujos respectivos autovalores são:

$$
E_{N}=\sum_{j=1}^{N} \lambda_{j}^{2}, \quad p_{N}=\sum_{j=1}^{N} \lambda_{j} \quad \text { e } \quad q_{N}=N
$$

As autofunções de Bethe (3.1.25) podem ser rescritas de uma forma mais conveniente ao usarmos que o determinante pode ser escrito como uma soma sobre as permutações $\mathcal{P}$ de $(1,2, \ldots, N)$ :

$$
\operatorname{det}\left[\exp \left(i \lambda_{j} z_{k}\right)\right]=\sum_{\mathcal{P}}(-1)^{[\mathcal{P}]} \exp \left(i \sum_{n=1}^{N} z_{n} \lambda_{\mathcal{P}_{n}}\right)
$$


onde $[\mathcal{P}]$ denota a paridade da permutação, de forma que obtemos [22]:

$$
\begin{aligned}
f_{N}\left(z_{1}, \ldots, z_{N} \mid \lambda_{1}, \ldots, \lambda_{N}\right) & =\frac{(-i)^{\frac{N(N-1)}{2}}}{\sqrt{N !}}\left[\prod_{1 \leq k<j \leq N} \operatorname{sign}\left(z_{j}-z_{k}\right)\right] \sum_{\mathcal{P}}\left\{(-1)^{[\mathcal{P}]} \exp \left(i \sum_{l=1}^{N} z_{l} \lambda_{\mathcal{P}_{l}}\right) .\right. \\
& \left.\cdot \exp \left[\frac{i}{2} \sum_{1 \leq l<m \leq N} \operatorname{sign}\left(z_{m}-z_{l}\right) \theta\left(\lambda_{\mathcal{P}_{m}}-\lambda_{\mathcal{P}_{l}}\right)\right]\right\}
\end{aligned}
$$

onde

$$
\theta(\lambda-\mu)=i \log \left(\frac{i g+\lambda-\mu}{i g-\lambda+\mu}\right)
$$

de acordo com (3.1.20). Tais funções constituem um conjunto completo e ortonormalizado ( [22, 52, 53]).

A fórmula (3.1.27) determina a função de onda de Bethe, que é simétrica e contínua com respeito as variáveis $z_{j}, j=1, \ldots, N$. Em contrapartida, $f_{N}$ é uma função antissimétrica nas variáveis $\lambda_{j}$, e conseqüentemente se anula caso $\lambda_{j}=\lambda_{k}$ para $k \neq j$. Esse fato é de notável importância para sistemas em $(1+1)$-dimensões ${ }^{6}$, pois indica uma dualidade entre férmions e bósons, e, em particular, alguns modelos bosônicos são equivalentes a modelos fermiônicos, por exemplo o modelo para o gás de Bose com $g \rightarrow \infty$ é equivalente ao modelo para um férmion livre, enquanto que o modelo de sine-Gordon corresponde ao modelo de Thirring massivo. Outra propriedade fundamental, e que desempenhará um papel central na definição do conceito de integrabilidade quântica, codificada em (3.1.27) é que a dinâmica do modelo depende apenas da interação entre dois corpos, de forma que o espalhamento de $n$ partículas pode ser reduzido a uma seqüência de colisões entre apenas duas partículas, isto é, o espalhamento é fatorável ${ }^{7}$.

Prosseguindo de maneira semelhante ao caso com $N=2$, consideramos o sistema definido em um intervalo finito e periódico com comprimento $2 L$. Ao impormos condições de periodicidade nas funções de Bethe $f_{N}$ em cada uma de suas entradas independentemente

$$
f_{N}\left(z_{1}, \ldots, z_{j}-L, \ldots, z_{N} \mid \lambda_{1}, \ldots, \lambda_{N}\right)=f_{N}\left(z_{1}, \ldots, z_{j}+L, \ldots, z_{N} \mid \lambda_{1}, \ldots, \lambda_{N}\right),
$$

encontramos as equações de Bethe

$$
e^{2 i L \lambda_{j}}=-\prod_{k=1}^{N} \frac{\lambda_{j}-\lambda_{k}+i g}{\lambda_{j}-\lambda_{k}-i g}, \quad j=1, \ldots, N,
$$

que determinam os valores permitidos para os momentos $\lambda_{j}$. Por consegüinte, as equações de Bethe podem ser interpretadas em termos de um espalhamento fatorado para as partículas de Bethe, que não necessariamente coincidem com os graus de liberdade físicos da teoria. Desse ponto de vista, as equações de Bethe são apenas as condições de quantização para o momento de partículas contidas em uma caixa de comprimento $2 L$, onde $\theta\left(\lambda_{j}-\lambda_{k}\right)$ é a mudança de fase sentida pela partícula $j$ ao colidir com a partícula $k$. É claro, pois, que o espectro é completamente determinado apenas pelo espalhamento de duas partículas e pela relação de dispersão, não existindo interações genuínas entre muitas partículas.

\footnotetext{
${ }^{6}$ Notamos que o teorema da conexão spin-estatística não é válido em $(1+1)$-dimensões.

${ }^{7}$ Ou não-difrativo.
} 
No resto desta seção estudamos algumas propriedades das equações de Bethe (3.1.29) e de suas soluções (cuja existência demonstramos). Começamos estabelecendo a realidade de tais soluções.

Teorema 3.1.1. Seja $\left\{\lambda_{j}\right\}$ o conjunto das soluções das equações de Bethe (3.1.29), então $\lambda_{j} \in \mathbb{R}$.

Demonstração: Seja $\lambda_{\max } \in\left\{\lambda_{j}\right\}$ a solução $\lambda_{j}$ com parte imaginária maximal, isto é,

$$
\Im\left(\lambda_{\max }\right) \geq \Im\left(\lambda_{j}\right), \quad j=1, \ldots, N,
$$

e considere o módulo de (3.1.29) para $\lambda_{j}=\lambda_{\max }$, então

$$
\left|e^{2 i L \lambda_{\max }}\right|=\left|\prod_{k=1}^{N} \frac{\lambda_{\max }-\lambda_{k}+i g}{\lambda_{\max }-\lambda_{k}-i g}\right| \geq 1,
$$

onde usamos

$$
\left|\frac{\lambda+i g}{\lambda-i g}\right| \gtreqless 1 \quad \text { se } \quad \Im(\lambda) \gtreqless 0 .
$$

Ademais, devido a

$$
\left|e^{2 i L \lambda}\right| \lesseqgtr 1 \text { se } \Im(\lambda) \gtreqless 0,
$$

inferimos que $\Im\left(\lambda_{\max }\right) \leq 0$, porém $\lambda_{\max }$ é maximal, logo

$$
\Im\left(\lambda_{j}\right) \leq 0, \quad j=1, \ldots, N .
$$

Similarmente, podemos definir um $\lambda_{j}$ minimal,

$$
\Im\left(\lambda_{\min }\right) \leq \Im\left(\lambda_{j}\right), \quad j=1, \ldots, N,
$$

e mostrar que

$$
0 \leq \Im\left(\lambda_{\min }\right) \leq \Im\left(\lambda_{j}\right), \quad j=1, \ldots, N .
$$

Portanto, pelo teorema do confronto somos levados à conclusão: $\Im\left(\lambda_{j}\right)=0, j=1, \ldots, N$.

Para demonstrarmos a existência de soluções para o sistema de equações (3.1.29), é conveniente que o rescrevamos em forma logarítmica:

$$
\varphi_{j}=2 L \lambda_{j}+\sum_{\substack{k=1 \\ k \neq j}} \varphi\left(\lambda_{j}-\lambda_{k}\right)
$$

onde os $\varphi_{j}=2 \pi \tilde{n}_{j}, j=1, \ldots, N$ com $\tilde{n}_{j} \in \mathbb{Z} \mathrm{e}$

$$
\varphi(\lambda)=i \log \left(\frac{\lambda+i g}{\lambda-i g}\right), \quad-2 \pi<\varphi(\lambda)<0 \quad \text { e } \quad \Im(\lambda)=0 .
$$

Claramente podemos expressar $\varphi(\lambda)$ em termos de $\theta(\lambda)$, (3.1.28), $\theta(\lambda)=\varphi(\lambda)+\pi$. De forma, que obtemos:

$$
2 L \lambda_{j}+\sum_{k=1}^{N} \theta\left(\lambda_{j}-\lambda_{k}\right)=2 \pi n_{j} \quad \text { com } \quad n_{j}=\tilde{n}_{j}+\frac{N-1}{2} .
$$


Teorema 3.1.2. Existem soluções para as equações de Bethe (3.1.30), e elas podem ser unicamente parametrizadas por um conjunto de inteiros (ou semi-inteiros) $\left\{n_{j}\right\}$.

Demonstração: Seja a ação de Yang-Yang [52]

$$
S=2 L \sum_{j=1}^{N} \lambda_{j}^{2}-2 \pi \sum_{j=1}^{N} n_{j} \lambda_{j}+\frac{1}{2} \sum_{j, k}^{N} \theta_{1}\left(\lambda_{j}-\lambda_{k}\right) \quad \operatorname{com} \quad \theta_{1}(\lambda)=\int_{0}^{\lambda} d \mu \theta(\mu) .
$$

As equações de Bethe (3.1.30) surgem como condições de extremo para a ação $S, \frac{\partial S}{\partial \lambda_{j}}=0$. Mostremos que esse extremo é de fato um mínimo:

$$
\begin{array}{r}
\frac{\partial^{2} S}{\partial \lambda_{j} \partial \lambda_{l}}=\delta_{j l}\left[2 L+\sum_{m=1}^{N} K\left(\lambda_{j}, \lambda_{m}\right)\right]-K\left(\lambda_{j}, \lambda_{l}\right) \quad \text { onde } K(\lambda, \mu)=\theta^{\prime}(\lambda-\mu)=\frac{2 g}{g^{2}+(\lambda-\mu)^{2}} \\
\Rightarrow \sum_{j, l}^{N} \frac{\partial^{2} S}{\partial \lambda_{j} \partial \lambda_{l}} v_{j} v_{l}=2 L \sum_{j=1}^{N} v_{j}^{2}+\sum_{j>l=1}^{N} K\left(\lambda_{j}, \lambda_{l}\right)\left(v_{j}-v_{l}\right)^{2} \geq 2 L \sum_{j=1}^{N} v_{j}^{2}>0
\end{array}
$$

para qualquer vetor $v_{j}$ com componentes reais. Logo, a matriz das segundas derivadas é positiva definida e a ação é convexa. Portanto, $S$ possui um único mínimo que define as soluções das equações de Bethe.

\subsection{O Método do Espalhamento Inverso Quântico}

O Ansatz de Bethe coordenado, que estudamos na última seção, constitui um método direto para se diagonalizar um Hamiltoniano (bem como todas as outras cargas conservadas), e assim obter seu espectro, porém de uma maneira extremamente dependente de uma hipótese inicial sobre a forma das autofunções. Essa dependência excessiva obscurece as características fundamentais que permitem a diagonalização de um dado Hamiltoniano. O método do espalhamento inverso quântico (MEIQ) proporciona uma abordagem alternativa e mais sistemática de se lidar com tais problemas de autovalores, ao combinar elementos oriundos do MEIC com idéias advindas do ABC. Em particular, parte-se da contraparte quântica da conexão de Lax para, com a ajuda da versão quântica do problema linear auxiliar, diagonalizar a sua solução, a matriz de transição, e conseqüentemente reproduzir os resultados do $A B C$. A abordagem do Ansatz de Bethe no contexto do MEIQ é denominada Ansatz de Bethe algébrico (ABA).

Diferentemente do caso clássico, é mais fácil iniciarmos a discussão do MEIQ estudando sistemas discretos. Ademais, a discretização de diversos modelos contínuos é, muitas vezes, essencial para o correto tratamento das divergências ultravioletas que assolam inúmeros sistemas no contexto da teoria quântica de campos. Dessa forma, uma pequena digressão sobre sistemas integráveis clássicos discretos torna-se imperativa. 


\subsubsection{Sistemas Integráveis Clássicos Discretos - Uma Pequena Digressão}

Nesta seção de caráter auxiliar, introduzimos a representação de Lax, bem como outros conceitos relacionados à integrabilidade, como, por exemplo, a matriz $r$, para sistemas discretos. Considere, pois, uma rede periódica de comprimento $L$ com $M$ sítios e espaçamento $\Delta$, nessas condições o problema linear fundamental (2.5.1) assume a seguinte forma:

$$
\begin{aligned}
\partial_{\tau} \Psi(n, \tau ; \lambda) & =U(n, \tau ; \lambda) \Psi(n, \tau ; \lambda), \\
\Psi(n+1, \tau ; \lambda) & =L(n, \tau ; \lambda) \Psi(n, \tau ; \lambda),
\end{aligned}
$$

onde denotamos por $n$ o número do sítio na rede. $L$ e $U$ são duas matrizes $k \times k$ que dependem tanto do parâmetro espectral $\lambda$, quanto das variáveis dinâmicas do modelo. Novamente, a condição de consistência para o sistema (3.2.1) fornece a representação de Lax:

$$
\partial_{t} L(n, \tau ; \lambda)=U(n+1, \tau ; \lambda) L(n, \tau ; \lambda)-L(n, \tau ; \lambda) U(n, \tau ; \lambda)
$$

A conexão com os modelos contínuos aparece ao considerarmos uma rede infinitesimal, $\Delta \rightarrow 0$, dessa forma, a coordenada do $n$-ésimo sítio da rede é dada por $\sigma_{n}=n \Delta,\{n \in \mathbb{N}: n=0 \equiv n=M\}$ com $M=\frac{L}{\Delta}$. Para essa rede temos que:

$$
L(n, \tau ; \lambda)=\mathbb{1}_{k}+L_{\sigma}\left(\sigma_{n}, \tau ; \lambda\right)+O\left(\Delta^{2}\right)
$$

A matriz de transição discreta do $m$-ésimo sítio para o $(n+1)$-ésimo sítio, com $n \geq m$ pode ser representada como o produto ordenado das seguintes $(n-m+1)$ matrizes:

$$
T(n, m ; \lambda)=\prod_{i=m}^{n} L(i, \tau ; \lambda):=L(n, \tau ; \lambda) L(n-1, \tau ; \lambda) \cdots L(m, \tau ; \lambda),
$$

onde $L(i, \tau ; \lambda) \equiv T(i, i ; \lambda)$ é a matriz de transição elementar para um sítio da rede, também denominada operador $L$ (ou de Lax). A matriz de monodromia é definida de forma análoga ao caso contínuo, como a matriz de transição para o comprimento completo da rede, $T_{L}(\lambda):=T(M, 1 ; \lambda)$. Assim como no caso contínuo, o traço das potências da matriz de monodromia $\zeta^{(n)}(\lambda)=\operatorname{tr}\left[T_{L}^{n}(\tau ; \lambda)\right]$ gera as quantidades conservadas.

A matriz $r$ para o caso discreto é introduzida de uma forma similar:

Definição 3.2.1. A matriz $r$ é uma matriz $k^{2} \times k^{2}$ definida de forma a satisfazer os seguintes parênteses de Poisson:

$$
\{L(i, \tau ; \lambda) \stackrel{\otimes}{,} L(j, \tau ; \mu)\}=\delta_{i j}[r(\lambda-\mu), L(i, \tau ; \lambda) \otimes L(j, \tau ; \mu)] .
$$

A relação (3.2.5) é denominada analogamente parênteses de Poisson fundamentais discretos.

Com isso podemos demonstrar a versão discreta do teorema 2.7.6.

Teorema 3.2.2. Se os parênteses de Poisson entre os elementos da matriz de transição elementar $L(i, \tau ; \lambda)$ admitem a representação em termos da matriz $r$, equação (3.2.5), então os parênteses de 
Poisson dos elementos da matriz de transição são dados por:

$$
\{T(n, m ; \lambda) \stackrel{\otimes}{,} T(n, m ; \mu)\}=[r(\lambda-\mu), T(n, m ; \lambda) \otimes T(n, m ; \mu)] .
$$

Conseqüentemente, os traços das potências da matriz de monodromia $\zeta^{(n)}(\lambda)=\operatorname{tr}\left(T_{L}^{n}(\tau ; \lambda)\right)$ geram as quantidades conservadas em involução:

$$
\left\{\zeta^{(n)}(\lambda), \zeta^{(m)}(\mu)\right\}=0
$$

Demonstração: A primeira parte do teorema é demonstrada por indução em $i \operatorname{com} n=m-1+i$, lembramos também que $n \geq m$ e $i \in \mathbb{N}$. Omitindo a dependência temporal para maior clareza, temos trivialmente para $i=1$ que:

$$
\begin{aligned}
\{T(m, m ; \lambda) \stackrel{\otimes}{,} T(m, m ; \mu)\} & =\{L(m ; \lambda) \stackrel{\otimes}{,} L(m ; \mu)\} \\
& =[r(\lambda-\mu), L(m ; \lambda) \otimes L(m ; \mu)] \\
& =[r(\lambda-\mu), T(m, m ; \lambda) \otimes T(m, m ; \mu)] .
\end{aligned}
$$

Tomando a expressão para $i=n-m+1$, equação (3.2.6), como verdadeira, mostramos para $i=$ $n-m+2$ :

$$
\begin{aligned}
& \{T(n+1, m ; \lambda) \stackrel{\otimes}{,} T(n+1, m ; \mu)\}=\{L(n+1 ; \lambda) T(n, m ; \lambda) \stackrel{\otimes}{,} L(n+1 ; \mu) T(n, m ; \mu)\} \\
& =L(n+1 ; \lambda) \otimes L(n+1 ; \mu)\{T(n, m ; \mu) \stackrel{\otimes}{,} T(n, m ; \mu)\}+ \\
& +L(n+1 ; \lambda) \otimes \mathbb{1}_{k}\{T(n, m ; \mu) \stackrel{\otimes}{,} L(n+1 ; \mu)\} \mathbb{1}_{k} \otimes T(n, m ; \mu)+ \\
& +\mathbb{1}_{k} \otimes L(n+1 ; \mu)\{L(n+1 ; \lambda) \stackrel{\otimes}{,} T(n, m ; \mu)\} T(n, m ; \lambda) \otimes \mathbb{1}_{k}+ \\
& +\{L(n+1 ; \lambda) \stackrel{\otimes}{,} L(n+1 ; \mu)\} T(n, m ; \lambda) \otimes T(n, m ; \mu) \\
& =L(n+1 ; \lambda) \otimes L(n+1 ; \mu)[r(\lambda-\mu), T(n, m ; \lambda) \otimes T(n, m ; \mu)]+ \\
& +[r(\lambda-\mu), L(n+1 ; \lambda) \otimes L(n+1 ; \mu)] T(n, m ; \lambda) \otimes L(n, m ; \mu) .
\end{aligned}
$$

Na última passagem usamos a hipótese de indução no primeiro termo e os parênteses de Poisson fundamentais discretos no quarto termo, enquanto que o segundo e o terceiro termos se anularam identicamente, pois a equação (3.2.5) implica que $\{L(n+1 ; \lambda) \stackrel{\otimes}{,} T(n, m ; \mu)\}=0$. Abrindo os comutadores restantes, obtemos:

$$
\begin{aligned}
& =r(\lambda-\mu)(L(n+1 ; \lambda) \otimes L(n+1 ; \mu))(T(n, m ; \lambda) \otimes T(n, m ; \mu))- \\
& -(L(n+1 ; \lambda) \otimes L(n+1 ; \mu))(T(n, m ; \lambda) \otimes T(n, m ; \mu)) r(\lambda-\mu) .
\end{aligned}
$$

Agrupando os termos restantes em um comutador, obtemos a equação (3.2.6). Para mostramos a segunda parte, tomamos $n=M$ e $m=1$ na equação (3.2.6), de forma a estabelecermos:

$$
\left\{T_{L}^{n}(\lambda) \stackrel{\otimes}{,} T_{L}^{m}(\mu)\right\}=\left[r(\lambda-\mu), T_{L}^{n}(\lambda) \otimes T_{L}^{m}(\mu)\right] .
$$


Finalmente, ao tomarmos o traço dessa relação concluímos a veracidade da tese.

Em virtude do teorema 3.2.2 temos que a família de sistemas dinâmicos definidos por:

$$
L(i, \tau ; \lambda)=\left\{\operatorname{tr} T_{L}(\mu), L(i, \tau ; \lambda)\right\}
$$

possui um número infinito de cargas conservadas em involução.

Uma vez que a matriz $r$ determina a estrutura das variáveis de ângulo e ação, e a sua forma explícita não deve mudar ao discretizarmos o operador de $\operatorname{Lax} L(\sigma, \tau ; \lambda)=\partial_{\sigma}-L_{\sigma}(\sigma, \tau ; \lambda)$ para obtermos a matriz de transição elementar $L(i, \tau ; \lambda)$, devemos esperar que a estrutura das variáveis de ângulo e ação permaneça inalterada na transição do contínuo para o discreto. Dessa forma, a condensação da rede, isto é, o limite para o contínuo $(\Delta \rightarrow 0)$ deve ocorrer de forma suave.

\subsubsection{Sistemas Discretos Quânticos}

Com a introdução da versão discreta do MEIC na última seção, podemos prosseguir com a sua quantização. Seja $\eta_{i}$ o espaço de Hilbert em cada sítio da rede, de forma que o espaço de Hilbert do sistema completo seja dado por:

$$
\mathcal{H}=\bigotimes_{i=1}^{M} \eta_{i}
$$

com todos os $\eta_{i}$ idênticos. A Hamiltoniana $H$ e os elementos das matrizes $U(n, \tau ; \lambda)$ e $L(n, \tau ; \lambda)$ são agora operadores quânticos dependentes das variáveis dinâmicas do sistema, $X_{i}^{\alpha}$; o índice $i$ denota o sítio da rede sobre o qual o observável atua, enquanto que $\alpha$ representa algum índice de simetria interna. A álgebra de observáveis $\mathcal{A}$ é definida ao fixarmos as regras de comutação satisfeitas pelos $X_{i}^{\alpha}$. Estaremos particularmente interessados em relações de comutação ultralocais, isto é, tais que $X_{i}^{\alpha}$ comuta com $X_{j}^{\beta}$ se $i \neq j$. Notamos que para sistemas relativísticos algum esquema de regularização se faz necessário devido à presença de divergências ultravioletas, de sorte que nesse contexto a formulação do modelo na rede se torna interessante, pois o inverso do espaçamento entre os sítios $\Delta^{-1}$ pode ser interpretado como um cut off ultravioleta.

Assim como no MEIC, o operador de Lax, $L(i, \tau ; \lambda)$, assume um papel central no formalismo do MEIQ. Sua definição, contudo, envolve além do espaço de Hilbert local $\eta_{i}$, um espaço auxiliar $V$, de modo que a ação do operador $L(i, \tau ; \lambda)$ seja sobre o produto tensorial $\eta_{i} \otimes V$. Esses operadores podem, em geral, ser obtidos através da quantização direta das correspondentes expressões clássicas, dessa forma é possível enxergar o operador de Lax como uma matriz no espaço $V$, cujos elementos são operadores quânticos atuando sobre $\eta_{i}$. Conseqüentemente, é necessário cuidado ao manipular os elementos de $L(i, \tau ; \lambda)$, pois por serem operadores, eles não necessariamente comutam entre si. A matriz de transição é definida da mesma forma que no caso clássico:

$$
T(n, m ; \lambda)=\prod_{i=m}^{n} L(i, \tau ; \lambda):=L(n, \tau ; \lambda) L(n-1, \tau ; \lambda) \cdots L(m, \tau ; \lambda) .
$$

A ultralocalidade da álgebra de observáveis $\mathcal{A}$ garante que os elementos do operador de Lax comutam 
em diferentes sítios da rede:

$$
\left[L_{i j}(m, \tau ; \lambda), L_{k l}(n, \tau ; \mu)\right]=0, \quad \text { se } \quad m \neq n,
$$

de forma que a matriz de transição e, em particular, a matriz de monodromia, definida como a matriz de transição para a rede inteira, $T_{L}(\lambda):=T(M, 1 ; \lambda)$, estejam bem definidas.

Introduzimos a versão quântica da matriz $r$ através da seguinte definição.

Definição 3.2.3. A matriz $R$ é um operador quântico independente das variáveis dinâmicas do sistema, atuando sobre o produto tensorial de dois espaços auxiliares $V_{1} \otimes V_{2}$, e definido de forma que as relações de comutação entre os elementos do operador de Lax assumam a seguinte representação:

$$
R(\lambda-\mu)[L(i, \tau ; \lambda) \otimes L(i, \tau ; \mu)]=[L(i, \tau ; \mu) \otimes L(i, \tau ; \lambda)] R(\lambda-\mu) .
$$

A relação (3.2.12) é denominada relação de comutação fundamental (RCF).

Com isso podemos obter as relações de comutação para os elementos da matriz de transição:

Teorema 3.2.4. Seja um operador de Lax ultralocal cujos elementos satisfaçam a RCF (3.2.12), então as relações de comutação entre os elementos da matriz de transição $T(n, m ; \lambda)$ adquirem a seguinte forma:

$$
R(\lambda-\mu)[T(n, m ; \lambda) \otimes T(n, m ; \mu)]=[T(n, m ; \mu) \otimes T(n, m ; \lambda)] R(\lambda-\mu) .
$$

Demonstração: Essa demonstração é facilmente feita por indução em $n$ com $m$ fixo. Não é difícil ver que ao tomarmos $n=m$ a expressão (3.2.13) se reduz à equação (3.2.12), o que prova a sua veracidade. Resta-nos, pois, ao tomar (3.2.13) como verdadeira para $n=j$, mostrar a sua validade para $n=j+1$. De fato,

$$
\begin{aligned}
R(\lambda-\mu)[T(j+1, m ; \lambda) \otimes & T(j+1, m ; \mu)]=R(\lambda-\mu)[L(j+1, \tau ; \lambda) \otimes L(j+1, \tau ; \mu)] . \\
& \cdot R^{-1}(\lambda-\mu) R(\lambda-\mu)[T(j, m ; \lambda) \otimes T(j, m ; \mu)] R^{-1}(\lambda-\mu) R(\lambda-\mu) \\
& =[L(j+1, \tau ; \mu) \otimes L(j+1, \tau ; \lambda)][T(j, m ; \mu) \otimes T(j, m ; \lambda)] R(\lambda-\mu) \\
& =[T(j+1, m ; \mu) \otimes T(j+1, m ; \lambda)] R(\lambda, \mu) .
\end{aligned}
$$

As relações (3.2.12) e (3.2.13) são por muitas vezes denominadas relações bilineares (ou de entrelaçamento), diz-se que tanto o operador de Lax quanto a matriz de transição são entrelaçados pela mesma matriz $R$. Uma outra e útil forma de se escrever a equação (3.2.13) pode ser obtida multiplicando-se tal relação pela esquerda pela matriz de permutação $\Pi$ (2.7.1).

$$
\begin{aligned}
\widetilde{R}(\lambda-\mu)\left[T(n, m ; \lambda) \otimes \mathbb{1}_{k}\right]\left[\mathbb{1}_{k} \otimes T(n, m ; \mu)\right] & =\left[\mathbb{1}_{k} \otimes T(n, m ; \mu)\right]\left[T(n, m ; \lambda) \otimes \mathbb{1}_{k}\right] \widetilde{R}(\lambda-\mu) \\
\widetilde{R}_{12}(\lambda-\mu) T_{1}(n, m ; \lambda) T_{2}(n, m ; \mu) & =T_{2}(n, m ; \mu) T_{1}(n, m ; \lambda) \widetilde{R}_{12}(\lambda-\mu),
\end{aligned}
$$


com $\widetilde{R}(\lambda-\mu):=\Pi R(\lambda-\mu)$. Claramente, ao tomarmos $n=M$ e $m=1$ na equação (3.2.14) obtemos uma expressão para a matriz de monodromia:

$$
\widetilde{R}_{12}(\lambda-\mu) T_{L 1}(\lambda) T_{L 2}(\mu)=T_{L 2}(\mu) T_{L 1}(\lambda) \widetilde{R}_{12}(\lambda-\mu) .
$$

Com essa notação, fica claro que a matriz $k \times k, T_{L}(\lambda)$, atua somente sobre o primeiro espaço vetorial auxiliar $V_{1}$, enquanto que a matriz $T_{L}(\mu)$ atua apenas no segundo espaço vetorial $V_{2}$. Já a matriz $\widetilde{R}$ atua no produto tensorial desses espaços, $V_{1} \otimes V_{2}$.

Uma notável consequiência da relação de entrelaçamento para a matriz de transição (3.2.13) é a existência de uma família quantidades comutantes, $\zeta(\lambda)=\operatorname{tr} T_{L}(\lambda)$,

$$
[\zeta(\lambda), \zeta(\mu)]=0
$$

Para provarmos essa relação, rescrevemos (3.2.13) da seguinte forma:

$$
R(\lambda-\mu) T_{L}(\lambda) \otimes T_{L}(\mu) R^{-1}(\lambda-\mu)=T_{L}(\mu) \otimes T_{L}(\lambda)
$$

e tomamos o traço no espaço $V_{1} \otimes V_{2}$.

Assim como no caso clássico, o Hamiltoniano de um modelo integrável (bem como as outras quantidades conservadas) pode ser obtido a partir do traço da matriz de monodromia, $\zeta(\lambda)$,

$$
H=\left.\sum_{k, l} c_{k l} \frac{d^{k}}{d \lambda^{k}} \log \zeta(\lambda)\right|_{\lambda=\nu_{l}}
$$

onde os $C_{k l}$ são apenas coeficientes e os pontos $\nu_{l}$ são escolhidos de forma a tornar o Hamiltoniano local. Dessa forma, a família de quantidades comutantes se torna uma família de quantidades conservadas comutantes, pois da equação (3.2.16) segue que:

$$
[\log \zeta(\lambda), \zeta(\mu)]=0
$$

ao derivarmos essa expressão com respeito a $\lambda$ e usarmos a equação (3.2.17), concluímos que:

$$
[H, \zeta(\mu)]=0 .
$$

Finalmente expandindo em $\mu$, encontramos uma família de quantidades conservadas. Notamos que no limite de rede infinita, $M \rightarrow \infty$, obtemos um conjunto infinito de leis de conservação.

Exemplo 3.2.5. Neste exemplo obtemos a matriz $R$ para o modelo NLS discreto. A versão discreta do operador de Lax pode ser obtida através das equações (3.2.3) e (2.5.9):

$$
L(n, \tau ; \lambda)=\left(\begin{array}{cc}
1-i \frac{\lambda \Delta}{2} & -i \sqrt{g} \psi_{n}^{\dagger} \Delta \\
i \sqrt{g} \psi_{n} \Delta & 1+i \frac{\lambda \Delta}{2}
\end{array}\right)+O\left(\Delta^{2}\right),
$$

com os operadores:

$$
\psi_{n}=\frac{1}{\Delta} \int_{x_{n}-1}^{x_{n}} d x \psi(x) \quad \text { e } \quad \psi_{n}^{\dagger}=\frac{1}{\Delta} \int_{x_{n}-1}^{x_{n}} d x \psi^{\dagger}(x)
$$


satisfazendo

$$
\left[\psi_{n}, \psi_{m}^{\dagger}\right]=\frac{\delta_{m n}}{\Delta}
$$

Nessas condições, a relação bilinear (3.2.12) é válida para a seguinte matriz $R$ :

$$
R(\lambda-\mu)=\Pi-\frac{i g}{\lambda-\mu} \mathbb{1}_{4} \quad \text { ou } \quad \tilde{R}(\lambda-\mu)=\Pi R(\lambda-\mu)=\mathbb{1}_{4}-\frac{i g}{\lambda-\mu} \Pi,
$$

A existência da matriz $R$ também implica que a equação não-linear admite uma representação de Lax.

Teorema 3.2.6. A seguinte representação de Lax existe:

$$
i\left[\frac{d}{d \mu} \log \zeta(\mu), L(n, \tau ; \lambda)\right]=U(n+1 ; \lambda, \mu) L(n, \tau ; \lambda)-L(n, \tau ; \lambda) U(n ; \lambda, \mu) .
$$

Com o funcional gerador dos operadores de evolução temporal, $U(n ; \lambda, \mu)$, dado por:

$$
U(n ; \lambda, \mu)=i \frac{d}{d \mu} \log \zeta(\mu) \mathbb{1}_{k}-i q_{2}^{-1}(n ; \lambda, \mu) \frac{d}{d \mu} q_{2}(n ; \lambda, \mu),
$$

onde $q_{2}(n ; \lambda, \mu)$ é a seguinte matriz $k \times k$ :

$$
q_{2}(n ; \lambda, \mu)=\operatorname{tr}_{1}\left[T_{1}(M, n ; \mu) \widetilde{R}_{12}(\mu-\lambda) T_{1}(n-1,1 ; \mu)\right] .
$$

Demonstração: Começamos a demonstração obtendo a relação de comutação entre $L(n, \tau ; \lambda)$ e $q_{2}(n ; \lambda, \mu)$.

$$
\begin{aligned}
q_{2}(n+1 ; \lambda, \mu) L(n ; \lambda) & =\operatorname{tr}_{1}\left[T_{1}(M, n+1 ; \mu) \widetilde{R}_{12}(\mu-\lambda) T_{1}(n, 1 ; \mu)\right] L(n ; \lambda) \\
& =\operatorname{tr}_{1}\left[T_{1}(M, n+1 ; \mu) \widetilde{R}_{12}(\mu-\lambda) L_{1}(n ; \mu) T_{1}(n-1,1 ; \mu) L_{2}(n ; \lambda)\right] \\
& =\operatorname{tr}_{1}\left[T_{1}(M, n+1 ; \mu) \widetilde{R}_{12}(\mu-\lambda) L_{1}(n ; \mu) L_{2}(n ; \lambda) T_{1}(n-1,1 ; \mu)\right] \\
& =\operatorname{tr}_{1}\left[T_{1}(M, n+1 ; \mu) L_{2}(n ; \lambda) L_{1}(n ; \mu) \widetilde{R}_{12}(\mu-\lambda) T_{1}(n-1,1 ; \mu)\right] \\
& =L(n ; \lambda) \operatorname{tr}_{1}\left[T_{1}(M, n ; \mu) \widetilde{R}_{12}(\mu-\lambda) T_{1}(n-1,1 ; \mu)\right] \\
& =L(n, \lambda) q_{2}(n ; \lambda, \mu) .
\end{aligned}
$$

Na passagem da segunda para a terceira linha e da quarta para a quinta, usamos a ultralocalidade dos operadores de Lax, enquanto que na passagem da terceira para a quarta linha usamos a equação (3.2.12) escrita em termos de $\widetilde{R}_{12}$, isto é:

$$
\widetilde{R}_{12}(\lambda-\mu) L_{1}(k ; \lambda) L_{2}(k ; \mu)=L_{2}(k ; \mu) L_{1}(k ; \lambda) \widetilde{R}_{12}(\lambda-\mu) .
$$

Ao diferenciarmos a equação (3.2.23) com respeito a $\mu$ e multiplicarmos ambos os lados pela esquerda por $q_{2}^{-1}(n+1 ; \lambda, \mu)$, obtemos:

$$
q_{2}^{-1}(n+1 ; \lambda, \mu) \frac{d}{d \mu} q_{2}(n+1 ; \lambda, \mu) L(n ; \lambda)=q_{2}^{-1}(n+1 ; \lambda, \mu) L(n, \lambda) \frac{d}{d \mu} q_{2}(n ; \lambda, \mu) .
$$


Podemos rearranjar (3.2.23) da seguinte forma:

$$
L(n ; \lambda) q_{2}^{-1}(n ; \lambda, \mu)=q_{2}^{-1}(n+1 ; \lambda, \mu) L(n ; \lambda),
$$

e ao substituí-la em (3.2.24) concluímos que:

$$
q_{2}^{-1}(n+1 ; \lambda, \mu) \frac{d}{d \mu} q_{2}(n+1 ; \lambda, \mu) L(n ; \lambda)=L(n ; \lambda) q_{2}^{-1}(n ; \lambda, \mu) \frac{d}{d \mu} q_{2}(n ; \lambda, \mu) .
$$

Finalmente ao somarmos e subtraímos a quantidade:

$$
\frac{d}{d \mu}[\log \zeta(\mu)] L(n ; \lambda)
$$

à equação (3.2.25), obtemos a tese.

Através das identidades de traço, podemos rescrever o lado esquerdo de (3.2.20) de forma a obtermos o comutador de $L$ com a Hamiltoniana,

$$
\partial_{\tau} L(n, \tau ; \lambda)=i[H, L(n, \tau ; \lambda)]
$$

com o operador de evolução temporal $U(n ; \lambda)$ para a Hamiltoniana (3.2.17) dado pela seguinte combinação linear:

$$
U(n ; \lambda)=\left.\sum_{l, a} c_{l a} \frac{d^{l-1}}{d \mu^{l-1}} U(n ; \lambda, \mu)\right|_{\mu=\nu_{a}} .
$$

Além do mais, a condição de curvatura nula assume a seguinte forma:

$$
\partial_{\tau} L(n, \tau ; \lambda)=i[H, L(n, \tau ; \lambda)]=U(n+1 ; \lambda) L(n, \tau ; \lambda)-L(n, \tau ; \lambda) U(n ; \lambda) .
$$

Notamos que, apesar de todas as considerações dessa seção terem sido feitas para modelos na rede, elas continuam válidas para os modelos contínuos, podendo ser obtidas ao considerarmos o limite contínuo formal dos sistemas discretos.

\subsection{O Ansatz de Bethe Algébrico}

O Ansatz de Bethe algébrico (ABA) é uma das mais importantes construções no contexto do $M E I Q$, fornecendo uma generalização do $A B C$. O ABA proporciona um método para a construção dos autovetores do Hamiltoniano, e conseqüente diagonalização de todas as cargas conservadas comutantes, por meio da ação de operadores de criação e aniquilação sobre um pseudovácuo. Nesse processo, a matriz $R$ e a RFC (3.2.12) assumem um papel central. Desenvolvemos o ABA tendo em vista os exemplos mais simples, para os quais a matriz de monodromia é uma matriz $2 \times 2$, da forma:

$$
T_{L}(\lambda)=\prod_{n=1}^{M} L(n, \tau ; \lambda)=\prod_{n=1}^{M}\left(\begin{array}{cc}
A_{n}(\lambda) & B_{n}(\lambda) \\
C_{n}(\lambda) & D_{n}(\lambda)
\end{array}\right)=\left(\begin{array}{cc}
A(\lambda) & B(\lambda) \\
C(\lambda) & D(\lambda)
\end{array}\right),
$$


e as relações de comutação, obtidas a partir da relação bilinear (3.2.13) com

$$
R(\lambda)=\left(\begin{array}{cccc}
f(\lambda) & 0 & 0 & 0 \\
0 & g(\lambda) & 1 & 0 \\
0 & 1 & g(\lambda) & 0 \\
0 & 0 & 0 & f(\lambda)
\end{array}\right)
$$

onde $f(\lambda)$ e $g(\lambda)$ são funções do parâmetro espectral $\lambda \in \mathbb{C}$. Para o caso do modelo NLS ${ }^{8}$,

$$
f(\lambda)=1-\frac{i c}{\lambda} \quad \text { e } g(\lambda)=-\frac{i c}{\lambda} .
$$

Um outro modelo que admite uma matriz $R$ da forma (3.3.2) é o modelo de sine-Gordon, para o qual

$$
f(\lambda)=\frac{\sinh (\lambda-2 i \eta)}{\sinh (\lambda)} \text { e } \frac{\sin 2 \eta}{i \sinh (\lambda)}, \quad \text { onde } \quad \eta=-\frac{\beta^{2}}{16} .
$$

Substituindo a forma geral da matriz $R$ (3.3.2) e a expressão explícita para

$$
T_{L}(\lambda) \otimes T_{L}(\mu)=\left(\begin{array}{llll}
A(\lambda) A(\mu) & A(\lambda) B(\mu) & B(\lambda) A(\mu) & B(\lambda) B(\mu) \\
A(\lambda) C(\mu) & A(\lambda) D(\mu) & B(\lambda) C(\mu) & B(\lambda) D(\mu) \\
C(\lambda) A(\mu) & C(\lambda) B(\mu) & D(\lambda) A(\mu) & D(\lambda) B(\mu) \\
C(\lambda) C(\mu) & C(\lambda) D(\mu) & D(\lambda) C(\mu) & D(\lambda) D(\mu)
\end{array}\right)
$$

em

$$
R(\lambda-\mu) T_{L}(\lambda) \otimes T_{L}(\mu)=T_{L}(\mu) \otimes T_{L}(\lambda) R(\lambda-\mu),
$$

obtemos explicitamente as relações de comutação entre os diversos elementos da matriz de monodromia:

$$
\begin{aligned}
{[A(\lambda), A(\mu)] } & =0, \quad[B(\lambda), B(\mu)]=0, \quad[C(\lambda), C(\mu)]=0, \quad[D(\lambda), D(\mu)]=0, \\
A(\mu) B(\lambda) & =f(\lambda-\mu) B(\lambda) A(\mu)+g(\mu-\lambda) B(\mu) A(\lambda) \\
D(\mu) B(\lambda) & =f(\mu-\lambda) B(\lambda) D(\mu)+g(\lambda-\mu) B(\mu) D(\lambda) \\
C(\lambda) A(\mu) & =f(\lambda-\mu) A(\mu) C(\lambda)+g(\mu-\lambda) A(\lambda) C(\mu) \\
C(\lambda) D(\mu) & =f(\mu-\lambda) D(\mu) C(\lambda)+g(\lambda-\mu) D(\lambda) C(\mu) \\
B(\mu) A(\lambda) & =f(\lambda-\mu) A(\lambda) B(\mu)+g(\mu-\lambda) A(\mu) B(\lambda) \\
B(\lambda) D(\mu) & =f(\lambda-\mu) D(\mu) B(\lambda)+g(\mu-\lambda) D(\lambda) B(\mu) \\
D(\mu) C(\lambda) & =f(\lambda-\mu) C(\lambda) D(\mu)+g(\mu-\lambda) C(\mu) D(\lambda) \\
A(\lambda) C(\mu) & =f(\lambda-\mu) C(\mu) A(\lambda)+g(\mu-\lambda) C(\lambda) A(\mu) \\
{[D(\lambda), A(\mu)] } & =g(\mu-\lambda)(B(\lambda) C(\mu)-B(\mu) C(\lambda)) \\
{[A(\lambda), D(\mu)] } & =g(\mu-\lambda)(C(\lambda) B(\mu)-C(\mu) B(\lambda)) \\
{[C(\lambda), B(\mu)] } & =g(\mu-\lambda)(A(\lambda) D(\mu)-A(\mu) D(\lambda))
\end{aligned}
$$

\footnotetext{
${ }^{8}$ Vide exemplo 3.2.5.
} 


$$
[B(\lambda), C(\mu)]=g(\mu-\lambda)(D(\lambda) A(\mu)-D(\mu) A(\lambda)) .
$$

Das identidades de traço (3.2.16) e do fato de que o Hamiltoniano está presente nessa família de quantidades comutantes (3.2.17), segue que o Hamiltoniano e o traço da matriz de monodromia possuem o mesmo conjunto de autofunções. Sua construção requer a introdução um estado de referência $|0\rangle$, o pseudovácuo, definido como o estado aniquilado pelo operador $C(\lambda)$. Notamos que se existir um estado $|0\rangle_{n}$, tal que ${ }^{9}$

$$
L(n, \tau ; \lambda)|0\rangle_{n}=\left(\begin{array}{cc}
a_{n}(\lambda) & \star \\
0 & d_{n}(\lambda)
\end{array}\right),
$$

com $a_{n}(\lambda)$ e $d_{n}(\lambda)$ sendo os autovalores de $A_{n}(\lambda)$ e $D_{n}(\lambda)$, respectivamente, podemos construir tal pseudovácuo como

$$
|0\rangle=\bigotimes_{n=1}^{M}|0\rangle_{n}
$$

de forma que

$$
\begin{aligned}
& A(\lambda)|0\rangle=a(\lambda)|0\rangle \quad \text { com } \quad a(\lambda)=\prod_{n=1}^{M} a_{n}(\lambda) \\
& D(\lambda)|0\rangle=d(\lambda)|0\rangle \quad \text { com } \quad d(\lambda)=\prod_{n=1}^{M} d_{n}(\lambda) \\
& C(\lambda)|0\rangle=0 .
\end{aligned}
$$

Claramente, $|0\rangle$ é um autovetor simultâneo de $A(\lambda), D(\lambda)$ e, em particular, de $\zeta(\lambda)=\operatorname{tr} T_{L}(\lambda)=$ $A(\lambda)+D(\lambda)$.

É importante ressaltar que a mera existência de uma matriz $R$ não garante a existência de um pseudovácuo. Dessa forma é imprescindível que se estabeleça a sua existência para cada modelo em particular. Notamos, contudo, que tal construção é possível para uma grande classe de modelos integráveis, da qual o modelo NLS faz parte, pois, conforme (3.1.4), podemos definir o pseudovácuo como o vácuo de Fock, $\psi(x)|0\rangle=0$, e em virtude de (3.2.18) constatar que

$$
\psi_{n}|0\rangle=0 \quad \Rightarrow \quad C_{n}(\lambda)|0\rangle=i \sqrt{g} \psi_{n} \Delta|0\rangle=0
$$

Nesse caso,

$$
a_{n}(\lambda)=1-\frac{i \lambda \Delta}{2} \quad \text { e } \quad b_{n}(\lambda)=1+\frac{i \lambda \Delta}{2} .
$$

O próximo passo consiste em considerar o seguinte Ansatz (de Bethe) para um estado geral:

$$
\left|\left\{\lambda_{j}\right\}_{N}\right\rangle=\prod_{j=1}^{N} B\left(\lambda_{j}\right)|0\rangle \quad \text { com } \quad \lambda_{j} \neq \lambda_{k} \quad \text { se } \quad j \neq k
$$

Claramente, a comutatividade dos operadores $B\left(\lambda_{j}\right)$ (3.3.4a) implica a simetria de $\left|\left\{\lambda_{j}\right\}_{N}\right\rangle$ no conjunto $\left\{\lambda_{j}\right\}_{N}$, além do mais, vetores linearmente independentes $\left|\left\{\lambda_{j}\right\}_{N}\right\rangle$ correspondem a conjuntos

\footnotetext{
${ }^{9}$ Usamos o símbolo * para denotar uma expressão, cuja forma explícita não é de nosso interesse.
} 
$\left\{\lambda_{j}\right\}_{N}$ diferentes. Ao impormos que $\left|\left\{\lambda_{j}\right\}_{N}\right\rangle$ seja um autovetor da família de quantidades conservadas comutantes gerada por $\zeta(\lambda)$, deduzimos um sistema de equações algébricas (equações de Bethe) relacionando os parâmetros $\lambda_{j}, j=1, \ldots, N$. Usando as relações de comutação (3.3.4) podemos calcular a ação dos elementos da matriz de monodromia em (3.3.8).

A relação (3.3.4b) permite que movamos o operador $A(\mu)$ atuando à esquerda de $\left|\left\{\lambda_{j}\right\}_{N}\right\rangle$ através dos $N$ operadores $B\left(\lambda_{j}\right)$. Notando que o primeiro termo de (3.3.4b) preserva a ordem dos argumentos dos operadores, enquanto que o segundo a troca, inferimos:

$$
\begin{aligned}
& A(\mu)\left|\left\{\lambda_{j}\right\}_{N}\right\rangle=\Lambda \prod_{j=1}^{N} B\left(\lambda_{j}\right)|0\rangle+\sum_{n=1}^{N} \Lambda_{n} B(\mu) \prod_{\substack{j=1 \\
j \neq n}}^{N} B\left(\lambda_{j}\right)|0\rangle, \\
& \text { onde } \quad \Lambda=a(\mu) \prod_{j=1}^{N} f\left(\lambda_{j}-\mu\right) \quad \text { e } \wedge_{n}=a\left(\lambda_{n}\right) g\left(\mu-\lambda_{n}\right) \prod_{\substack{j=1 \\
j \neq n}}^{N} f\left(\lambda_{j}-\lambda_{n}\right) .
\end{aligned}
$$

A expressão para $\Lambda$ é facilmente obtida usando apenas o primeiro termo do lado direito de (3.3.4b). Já a fórmula para $\Lambda_{n}$ surge ao coletarmos todos os outros $2^{N-1}$ termos com pelo menos uma contribuição do segundo termo de (3.3.4b). Calcular todos os coeficientes $\Lambda_{n}$ explicitamente pode ser deveras trabalhoso, pois para obter cada $\Lambda_{n}$ precisamos empregar alguma das várias expressões seguintes:

$$
\begin{aligned}
f\left(\lambda_{1}-\lambda_{2}\right) g\left(\mu-\lambda_{2}\right) & =f\left(\lambda_{1}-\mu\right) g\left(\mu-\lambda_{2}\right)+g\left(\mu-\lambda_{1}\right) g\left(\lambda_{1}-\lambda_{2}\right), \\
f\left(\lambda_{1}-\lambda_{3}\right) f\left(\lambda_{2}-\lambda_{3}\right) g\left(\mu-\lambda_{3}\right) & =f\left(\lambda_{1}-\mu\right) f\left(\lambda_{2}-\mu\right) g\left(\mu-\lambda_{3}\right)+f\left(\lambda_{2}-\lambda_{1}\right) g\left(\mu-\lambda_{1}\right) g\left(\lambda_{1}-\lambda_{3}\right)+ \\
& +f\left(\lambda_{1}-\lambda_{2}\right) g\left(\mu-\lambda_{2}\right) g\left(\lambda_{2}-\lambda_{3}\right)
\end{aligned}
$$

Uma forma mais direta de se convencer da validade da expressão para $\Lambda_{n}$ consistem em usar (3.3.4a) para escrever:

$$
\left|\left\{\lambda_{j}\right\}_{N}\right\rangle=B\left(\lambda_{n}\right) \prod_{\substack{j=1 \\ j \neq n}}^{N} B\left(\lambda_{j}\right)|0\rangle,
$$

e usar o segundo termo de (3.3.4b) para mover $A(\mu)$ através de $B\left(\lambda_{n}\right)$, obtendo

$$
g\left(\mu-\lambda_{n}\right) B(\mu) A\left(\lambda_{n}\right) \prod_{\substack{j=1 \\ j \neq n}}^{N} B\left(\lambda_{j}\right)|0\rangle .
$$

A seguir, usamos apenas o primeiro termo de (3.3.4b) para comutar $A\left(\lambda_{n}\right)$ com $B\left(\lambda_{j}\right)$, e assim obter a expressão para $\Lambda_{n}$.

De uma forma análoga podemos obter a partir de (3.3.4c) a seguinte expressão para a ação de 
$D(\lambda)$ sobre $\left|\left\{\lambda_{j}\right\}_{N}\right\rangle:$

$$
\begin{aligned}
& D(\mu)\left|\left\{\lambda_{j}\right\}_{N}\right\rangle=\tilde{\Lambda} \prod_{j=1}^{N} B\left(\lambda_{j}\right)|0\rangle+\sum_{n=1}^{N} \tilde{\Lambda}_{n} B(\mu) \prod_{\substack{j=1 \\
j \neq n}}^{N} B\left(\lambda_{j}\right)|0\rangle \\
& \text { onde } \tilde{\Lambda}=d(\mu) \prod_{j=1}^{N} f\left(\mu-\lambda_{j}\right) \quad \text { e } \quad \tilde{\Lambda}_{n}=d\left(\lambda_{n}\right) g\left(\lambda_{n}-\mu\right) \prod_{\substack{j=1 \\
j \neq n}}^{N} f\left(\lambda_{n}-\lambda_{j}\right) .
\end{aligned}
$$

Com um pouco mais de trabalho podemos obter a ação dos operadores $C(\mu)$ em (3.3.8):

$$
\begin{aligned}
& C(\mu)\left|\left\{\lambda_{j}\right\}_{N}\right\rangle=\sum_{n=1}^{N} M_{n} \prod_{\substack{j=1 \\
j \neq n}}^{N} B\left(\lambda_{j}\right)|0\rangle+\sum_{k>n}^{N} M_{k n} B(\mu) \prod_{\substack{j=1 \\
j \neq k, n}}^{N} B\left(\lambda_{j}\right)|0\rangle, \quad \text { onde } \\
& M_{n}=g\left(\lambda_{n}-\mu\right) a(\mu) d\left(\lambda_{n}\right) \prod_{\substack{j=1 \\
j \neq n}}^{N} f\left(\lambda_{n}-\lambda_{j}\right) f\left(\lambda_{j}-\mu\right)+g\left(\mu-\lambda_{n}\right) a\left(\lambda_{n}\right) d(\mu) \prod_{\substack{j=1 \\
j \neq n}}^{N} f\left(\mu-\lambda_{j}\right) f\left(\lambda_{j}-\lambda_{n}\right) \\
& \text { e } M_{k n}=d\left(\lambda_{k}\right) a\left(\lambda_{n}\right) g\left(\lambda_{k}-\mu\right) g\left(\mu-\lambda_{n}\right) f\left(\lambda_{k}-\lambda_{n}\right) \prod_{\substack{j=1 \\
j \neq k, n}}^{N} f\left(\lambda_{k}-\lambda_{j}\right) f\left(\lambda_{j}-\lambda_{n}\right)+ \\
& +d\left(\lambda_{n}\right) a\left(\lambda_{k}\right) g\left(\lambda_{n}-\mu\right) g\left(\mu-\lambda_{k}\right) f\left(\lambda_{n}-\lambda_{k}\right) \prod_{\substack{j=1 \\
j \neq k, n}}^{N} f\left(\lambda_{n}-\lambda_{j}\right) f\left(\lambda_{j}-\lambda_{k}\right) .
\end{aligned}
$$

Em virtude da forma da ação de $A(\mu)$ e $D(\mu)$ (3.3.9 e 3.3.10) sobre os estados de Bethe (3.3.8), para que $\left|\left\{\lambda_{j}\right\}_{N}\right\rangle$ seja um auto-estado de $\zeta(\lambda)$ uma condição necessária e suficiente é a imposição de que $\Lambda_{n}+\tilde{\Lambda}_{n}=0$, que nos leva às equações de Bethe $^{10}$ :

$$
r\left(\lambda_{n}\right) \prod_{\substack{j=1 \\ j \neq n}}^{N} \frac{f\left(\lambda_{j}-\lambda_{n}\right)}{f\left(\lambda_{n}-\lambda_{j}\right)}=1, \quad n=1, \ldots, N, \quad \operatorname{com} \quad r(\lambda):=\frac{a(\lambda)}{d(\lambda)} .
$$

De forma que,

$$
\zeta(\lambda)\left|\left\{\lambda_{j}\right\}_{N}\right\rangle=\left[a(\lambda) \prod_{j=1}^{N} f\left(\lambda_{j}-\lambda\right)+d(\lambda) \prod_{j=1}^{N} f\left(\lambda-\lambda_{j}\right)\right]\left|\left\{\lambda_{j}\right\}_{N}\right\rangle .
$$

Observamos que as equações (3.3.12) implicam que os pólos superficiais do autovalor de $\zeta(\lambda)$ se cancelam, garantido que ele seja um polinômio em $\lambda$ de grau $N$, conforme esperávamos. Portanto, somos levados a pensar que apenas as soluções $\left\{\lambda_{j}\right\}_{N}$ com $\lambda_{j} \neq \lambda_{k}, j \neq k$ são relevantes, uma vez que se $\lambda_{j}=\lambda_{k}$ indica a existência de pólos espúrios nas equações de Bethe. Em particular, pode-se mostrar para o modelo NLS [22] que ao relaxarmos essa condição, permitindo, por exemplo, que $\lambda_{1}=\lambda_{2}$, que não existem autofunções de $\zeta(\lambda)$ com dois momentos iguais.

\footnotetext{
${ }^{10}$ Não é difícil de verificar que ao substituirmos as expressões de $f(\lambda), a(\lambda)$ e $d(\lambda)$ para o modelo NLS, recuperamos equações da forma (3.1.29).
} 


\subsection{Quantização de Sistemas Contínuos}

Nas últimas seções desenvolvemos o MEIQ para sistemas discretos, cujo tratamento é significativamente mais simples do que de suas contrapartes contínuas, uma vez que a rede introduz uma regularização natural para o, em geral, mal definido produto de operadores em um mesmo ponto, bem como uma solução para o problema de ordenamento dos operadores. Contudo, nem sempre é desejável ter que recorrer à discretização de um modelo contínuo para estudar suas propriedades, posto que a versão discreta de um dado modelo nem sempre é conhecida, e as técnicas para obtê-las levam normalmente a resultados complicados [22,36,54-57]. Nesta seção, expomos o MEIQ aplicado diretamente a um modelo contínuo, no exemplo do modelo NLS.

A primeira questão crítica a surgir na análise de modelo contínuos é a seguinte: como para um dado funcional das variáveis clássicas canônicas $\psi(x)$ e $\bar{\psi}(x)$ associamos um operador quântico? De uma forma geral (e um tanto quanto formal) existem várias formas de se proceder com essa associação, a mais usual consiste no ordenamento normal (ou de Wick). De antemão, já ressaltamos que essa abordagem apresenta algumas limitações no que tange a obtenção das identidades de traço e a, conseqüente, construção das cargas conservadas.

Denotando a quantização de Wick por : :, introduzimos a matriz de transição quântica a partir do ordenamento normal de sua contraparte clássica (2.6.5):

$$
\mathbb{T}\left(x_{2}, x_{1} ; \lambda\right)=: T\left(x_{2}, x_{1} ; \lambda\right):
$$

A propriedade de superposição exibida pela matriz de transição clássica (2.6.3) pode ser estendida à sua contraparte quântica, em virtude da comutatividade dos operadores $\psi(x)$ e $\psi^{\dagger}(x)$ em intervalos disjuntos:

$$
\mathbb{T}\left(x_{3}, x_{1} ; \lambda\right)=\mathbb{T}\left(x_{3}, x_{2} ; \lambda\right), \mathbb{T}\left(x_{2}, x_{1} ; \lambda\right) \quad \text { para } \quad x_{1}<x_{2}<x_{3} \quad \text { ou } \quad x_{1}>x_{2}>x_{3}
$$

Assim como a sua versão clássica, a matriz de transição quântica admite a seguinte representação ${ }^{11}$ :

$$
\begin{aligned}
\mathbb{T}\left(x_{2}, x_{1} ; \lambda\right) & =\left(\begin{array}{cc}
A\left(x_{2}, x_{1} ; \lambda\right) & \epsilon B^{\dagger}\left(x_{2}, x_{1} ; \lambda\right) \\
B\left(x_{2}, x_{1} ; \lambda\right) & A^{\dagger}\left(x_{2}, x_{1} ; \lambda\right)
\end{array}\right) \quad \text { com } \epsilon=\text { sign } g, \\
A\left(x_{2}, x_{1} ; \lambda\right) & =: a\left(x_{2}, x_{1} ; \lambda\right):, \quad B\left(x_{2}, x_{1} ; \lambda\right)=: b\left(x_{2}, x_{1} ; \lambda\right): \\
A^{\dagger}\left(x_{2}, x_{1} ; \lambda\right) & =\left(A\left(x_{2}, x_{1} ; \bar{\lambda}\right)\right)^{\dagger} \quad \text { e } \quad B^{\dagger}\left(x_{2}, x_{1} ; \lambda\right)=\left(B\left(x_{2}, x_{1} ; \bar{\lambda}\right)\right)^{\dagger}
\end{aligned}
$$

Já o problema linear auxiliar (2.6.2) assume a seguinte forma:

$$
\begin{aligned}
\partial_{x_{2}} \mathbb{T}\left(x_{2}, x_{1} ; \lambda\right) & =: L\left(x_{2}, t ; \lambda\right) T\left(x_{2}, x_{1} ; \lambda\right): \\
& =\left(\frac{\lambda}{2 i} \gamma^{3}-i \sqrt{g} \gamma^{-} \psi^{\dagger}\left(x_{2}\right)\right) \mathbb{T}\left(x_{2}, x_{1} ; \lambda\right)+i \sqrt{g} \gamma^{+} \mathbb{T}\left(x_{2}, x_{1} ; \lambda\right) \psi\left(x_{2}\right), \\
\partial_{x_{1}} \mathbb{T}\left(x_{2}, x_{1} ; \lambda\right) & =-: T\left(x_{2}, x_{1} ; \lambda\right) L\left(x_{1}, t ; \lambda\right): \\
& =i \sqrt{g} \psi^{\dagger}\left(x_{1}\right) \mathbb{T}\left(x_{2}, x_{1} ; \lambda\right) \gamma^{-}-\mathbb{T}\left(x_{2}, x_{1} ; \lambda\right)\left(\frac{\lambda}{2 i} \gamma^{3}+i \sqrt{g} \gamma^{+} \psi\left(x_{1}\right)\right),
\end{aligned}
$$

\footnotetext{
${ }^{11}$ Vide (2.6.17), considerando-a para um subintervalo do intervalo fundamental completo.
} 


$$
\left.\operatorname{com} \mathbb{T}\left(x_{2}, x_{1} ; \lambda\right)\right|_{x_{1}=x_{2}}=\mathbb{1}_{2} .
$$

Emprestamos de [23] o sinal : : que denota o rearranjo normal de fatores operacionais, que quando aplicado ao produto de vários operadores (incluindo $\psi$ e $\psi^{\dagger}$ ), rearranja-os de forma que todos os $\psi^{\dagger}$ sejam levados à extrema esquerda, enquanto todos os $\psi$, à extrema direita, sem afetar, contudo, a ordem dos demais fatores. Por exemplo,

$$
\vdots X \psi \psi^{\dagger} Y:=\psi^{\dagger} X Y \psi
$$

Com essa notação, o problema linear fundamental quântico (3.4.4) pode ser rescrito da seguinte forma:

$$
\begin{aligned}
& \partial_{x_{2}} \mathbb{T}\left(x_{2}, x_{1} ; \lambda\right)=: \mathbb{L}\left(x_{2}, t ; \lambda\right) \mathbb{T}\left(x_{2}, x_{1} ; \lambda\right) \vdots, \\
& \partial_{x_{1}} \mathbb{T}\left(x_{2}, x_{1} ; \lambda\right)=-: \mathbb{T}\left(x_{2}, x_{1} ; \lambda\right) \mathbb{L}\left(x_{1}, t ; \lambda\right) \\
& \operatorname{com} \quad \mathbb{L}(x, t ; \lambda)=: L(x, t ; \lambda):=\frac{\lambda}{2 i} \gamma^{3}+i \sqrt{g}\left[\gamma^{+} \psi(x)-\gamma^{-} \psi^{\dagger}(x)\right] .
\end{aligned}
$$

\subsubsection{As Relações de Comutação e a Matriz R}

Com a matriz de transição bem definida para um intervalo finito através do problema linear auxiliar (3.4.5), podemos nos preocupar com a obtenção das relações de comutação entre seus elementos. Nossa meta é obter a versão contínua da relação bilinear (3.2.14), para isso precisamos do resultado auxiliar seguinte:

Lema 3.4.1. Os produtos $\mathbb{T}_{1}\left(x_{2}, x_{1} ; \lambda\right) \mathbb{T}_{2}\left(x_{2}, x_{1} ; \mu\right) e \mathbb{T}_{2}\left(x_{2}, x_{1} ; \mu\right) \mathbb{T}_{1}\left(x_{2}, x_{1} ; \lambda\right)$ satisfazem as seguintes equações diferenciais parciais:

$$
\begin{aligned}
& \partial_{x_{2}}\left(\mathbb{T}_{1}\left(x_{2}, x_{1} ; \lambda\right) \mathbb{T}_{2}\left(x_{2}, x_{1} ; \mu\right)\right)=\vdots \mathscr{L}\left(x_{2} ; \lambda, \mu\right) \mathbb{T}_{1}\left(x_{2}, x_{1} ; \lambda\right) \mathbb{T}_{2}\left(x_{2}, x_{1} ; \mu\right) \\
& \partial_{x_{2}}\left(\mathbb{T}_{2}\left(x_{2}, x_{1} ; \mu\right) \mathbb{T}_{1}\left(x_{2}, x_{1} ; \lambda\right)\right)=\vdots \mathscr{L}^{\prime}\left(x_{2} ; \lambda, \mu\right) \mathbb{T}_{2}\left(x_{2}, x_{1} ; \mu\right) \mathbb{T}_{1}\left(x_{2}, x_{1} ; \lambda\right)
\end{aligned}
$$

com a condição inicial

$$
\left.\mathbb{T}_{1}\left(x_{2}, x_{1} ; \lambda\right) \mathbb{T}_{2}\left(x_{2}, x_{1} ; \mu\right)\right|_{x_{1}=x_{2}}=\left.\mathbb{T}_{2}\left(x_{2}, x_{1} ; \mu\right) \mathbb{T}_{1}\left(x_{2}, x_{1} ; \lambda\right)\right|_{x_{1}=x_{2}}=\mathbb{1}_{4}
$$

e os operadores:

$$
\begin{aligned}
& \mathscr{L}(x ; \lambda, \mu)=\mathbb{L}_{1}(x, t ; \lambda)+\mathbb{L}_{2}(x, t ; \lambda)+g \gamma_{1}^{+} \gamma_{2}^{-} \\
& \mathscr{L}^{\prime}(x ; \lambda, \mu)=\mathbb{L}_{1}(x, t ; \lambda)+\mathbb{L}_{2}(x, t ; \lambda)+g \gamma_{1}^{-} \gamma_{2}^{+} .
\end{aligned}
$$

Demonstração: Seguimos a segunda demonstração apresentada em [23]. Notamos inicialmente que 
o problema linear auxiliar (3.4.5) é equivalente à seguinte equação integral:

$$
\mathbb{T}\left(x_{2}, x_{1} ; \lambda\right)=\mathbb{1}_{2}+\int_{x_{1}}^{x_{2}} d x: \mathbb{L}(x, t ; \lambda) \mathbb{T}(x, x ; \lambda) \vdots
$$

de forma que podemos escrever o produto:

$$
\begin{aligned}
\mathbb{T}_{1}\left(x_{2}, x_{1} ; \lambda\right) \mathbb{T}_{2}\left(x_{2}, x_{1} ; \mu\right) & \left.=\left[\mathbb{1}_{2}+\int_{x_{1}}^{x_{2}} d \xi \vdots \mathbb{L}(\xi, t ; \lambda) \mathbb{T}(\xi, x ; \lambda) \vdots\right]_{1} \mathbb{1}_{2}+\int_{x_{1}}^{x_{2}} d \eta: \mathbb{L}(\xi, t ; \mu) \mathbb{T}(\eta, x ; \mu) \vdots\right]_{2} \\
& =\mathbb{1}_{4}+\int_{x_{1}}^{x_{2}} d \xi \vdots \mathbb{L}_{1}(\xi, t ; \lambda) \mathbb{T}_{1}\left(\xi, x_{1} ; \lambda\right) \vdots+\int_{x_{1}}^{x_{2}} d \eta \vdots \mathbb{L}_{2}(\eta, t ; \mu) \mathbb{T}_{2}\left(\eta, x_{1} ; \mu\right) \vdots \\
& +\int_{x_{1}}^{x_{1}} d \xi \int_{x_{1}}^{x_{1}} d \eta \vdots \mathbb{L}_{1}(\xi, t ; \lambda) \mathbb{T}_{1}\left(\xi, x_{1} ; \lambda\right): \vdots \mathbb{L}_{2}(\eta, t ; \mu) \mathbb{T}_{2}\left(\eta, x_{1} ; \mu\right) \vdots
\end{aligned}
$$

onde o índice denotando o espaço vetorial sobre o qual uma dada matriz atua não deve ser confundido com o índice representando a dimensão da unidade.

Trabalhamos inicialmente apenas o quarto termo de (3.4.8), onde podemos substituir a expressão explícita de $\mathbb{L}$, bem como utilizar as relações de comutação (3.1.2).

$$
\begin{aligned}
\int_{x_{1}}^{x_{2}} d \xi \int_{x_{1}}^{x_{2}} d \eta\{ & {\left[\left(\frac{\lambda}{2 i} \gamma_{1}^{3}-i \sqrt{g} \gamma_{1}^{-} \psi^{\dagger}(\xi)\right) \mathbb{T}_{1}\left(\xi, x_{1} ; \lambda\right)+i \sqrt{g} \gamma_{1}^{+} \mathbb{T}_{1}\left(\xi, x_{1} ; \lambda\right) \psi(\xi)\right] } \\
& {\left.\left[\left(\frac{\mu}{2 i} \gamma_{2}^{3}-i \sqrt{g} \gamma_{2}^{-} \psi^{\dagger}(\eta)\right) \mathbb{T}_{2}\left(\eta, x_{1} ; \mu\right)+i \sqrt{g} \gamma_{2}^{+} \mathbb{T}_{2}\left(\eta, x_{1} ; \mu\right) \psi(\eta)\right]\right\} } \\
=\int_{x_{1}}^{x_{2}} d \xi \int_{x_{1}}^{x_{2}} d \eta & \left\{\left(\frac{\lambda}{2 i} \gamma_{1}^{3}-i \sqrt{g} \gamma_{1}^{-} \psi^{\dagger}(\xi)\right) \mathbb{T}_{1}\left(\xi, x_{1} ; \lambda\right)\left(\frac{\mu}{2 i} \gamma_{2}^{3}-i \sqrt{g} \gamma_{2}^{-} \psi^{\dagger}(\eta)\right) \mathbb{T}_{2}\left(\eta, x_{1} ; \mu\right)+\right. \\
+ & \left(\frac{\lambda}{2 i} \gamma_{1}^{3}-i \sqrt{g} \gamma_{1}^{-} \psi^{\dagger}(\xi)\right) i \sqrt{g} \gamma_{2}^{+} \mathbb{T}_{1}\left(\xi, x_{1} ; \lambda\right) \mathbb{T}_{2}\left(\eta, x_{1} ; \mu\right) \psi(\eta)- \\
& -g \gamma_{1}^{+} \gamma_{2}^{+} \mathbb{T}_{1}\left(\xi, x_{1} ; \lambda\right) \psi(\xi) \mathbb{T}_{2}\left(\eta, x_{1} ; \mu\right) \psi(\eta)+ \\
& +i \sqrt{g} \gamma_{1}^{+} \mathbb{T}_{1}\left(\xi, x_{1} ; \lambda\right)\left(\frac{\mu}{2 i} \gamma_{2}^{3}-i \sqrt{g} \gamma_{2}^{-} \psi^{\dagger}(\eta)\right) \psi(\xi) \mathbb{T}_{2}\left(\eta, x_{1} ; \mu\right)+ \\
& \left.+g \gamma_{1}^{+} \gamma_{2}^{-} \delta(\eta-\xi) \mathbb{T}_{1}\left(\xi, x_{1} ; \lambda\right) \mathbb{T}_{2}\left(\eta, x_{1} ; \mu\right)\right\}
\end{aligned}
$$

A seguir dividimos o domínio de integração em duas partes:

$$
x_{1}<\xi<\eta<x_{2} \quad \text { e } \quad x_{1}<\eta<\xi<x_{2}
$$

Para o primeiro intervalo, onde $\xi<\eta$, podemos utilizar o fato de que $\psi^{\dagger}(\eta)$ comuta com $\mathbb{T}_{1}\left(\xi, x_{1} ; \lambda\right)$, para levar o fator $\psi^{\dagger}(\eta)$ à extrema esquerda no primeiro e no quarto termos acima. Similarmente, para $\xi>\eta$, carregamos o fator $\psi(\xi)$ para à direita nos terceiro e quarto termos. Ademais, utilizamos a função delta presente no quinto termo para efetuar uma das integrações ${ }^{12}$.

$$
=\int_{x_{1}}^{x_{2}} d \eta \int_{x_{1}}^{\eta} d \xi\left\{\left(\frac{\mu}{2 i} \gamma_{2}^{3}-i \sqrt{g} \gamma_{2}^{-} \psi^{\dagger}(\eta)\right)\left[\left(\frac{\lambda}{2 i} \gamma_{1}^{3}-i \sqrt{g} \gamma_{1}^{-} \psi^{\dagger}(\xi)\right) \mathbb{T}_{1}+i \sqrt{g} \gamma_{1}^{+} \mathbb{T}_{1} \psi(\xi)\right] \mathbb{T}_{2}+\right.
$$

\footnotetext{
${ }^{12}$ Para uma notação mais concisa, omitimos a dependência explícita de $\mathbb{T}$, uma vez que o índice do espaço vetorial as determina univocamente, ou seja, $\mathbb{T}_{1} \equiv \mathbb{T}_{1}\left(\xi, x_{1} ; \lambda\right)$ e $\mathbb{T}_{2} \equiv \mathbb{T}_{2}\left(\eta, x_{1} ; \mu\right)$.
} 


$$
\begin{aligned}
& \left.+i \sqrt{g} \gamma_{2}^{+}\left[\left(\frac{\lambda}{2 i} \gamma_{1}^{3}-i \sqrt{g} \gamma_{1}^{-} \psi^{\dagger}(\xi)\right) \mathbb{T}_{1}+i \sqrt{g} \gamma_{1}^{+} \mathbb{T}_{1} \psi(\xi)\right] \mathbb{T}_{2} \psi(\eta)\right\}+ \\
& +\int_{x_{1}}^{x_{2}} d \xi \int_{x_{1}}^{\xi} d \eta\left\{\left(\frac{\lambda}{2 i} \gamma_{1}^{3}-i \sqrt{g} \gamma_{1}^{-} \psi^{\dagger}(\xi)\right) \mathbb{T}_{1}\left[\left(\frac{\mu}{2 i} \gamma_{2}^{3}-i \sqrt{g} \gamma_{2}^{-} \psi^{\dagger}(\eta)\right) \mathbb{T}_{2}+i \sqrt{g} \gamma_{2}^{+} \mathbb{T}_{2} \psi(\eta)\right]\right\}+ \\
& \left.+i \sqrt{g} \gamma_{1}^{+} \mathbb{T}_{1}\left[\left(\frac{\mu}{2 i} \gamma_{2}^{3}-i \sqrt{g} \gamma_{2}^{-} \psi^{\dagger}(\eta)\right) \mathbb{T}_{2}+i \sqrt{g} \gamma_{2}^{+} \mathbb{T}_{2} \psi(\eta)\right] \psi(\xi)\right\}+ \\
& +\int_{x_{1}}^{x_{2}} g \gamma_{1}^{+} \gamma_{2}^{-} \mathbb{T}_{1}\left(x, x_{1} ; \lambda\right) \mathbb{T}_{2}\left(x, x_{1} ; \mu\right)
\end{aligned}
$$

Utilizando o problema linear auxiliar em sua versão diferencial (3.4.4), obtemos:

$$
\begin{aligned}
& =\int_{x_{1}}^{x_{2}} d \eta \int_{x_{1}}^{\eta} d \xi\left\{\left(\frac{\mu}{2 i} \gamma_{2}^{3}-i \sqrt{g} \gamma_{2}^{-} \psi^{\dagger}(\eta)\right) \partial_{\xi} \mathbb{T}_{1} \mathbb{T}_{2}+i \sqrt{g} \gamma_{2}^{+} \partial_{\xi} \mathbb{T}_{1} \mathbb{T}_{2} \psi(\eta)\right\}+ \\
& +\int_{x_{1}}^{x_{2}} d \xi \int_{x_{1}}^{\xi} d \eta\left\{\left(\frac{\lambda}{2 i} \gamma_{1}^{3}-i \sqrt{g} \gamma_{1}^{-} \psi^{\dagger}(\xi)\right) \mathbb{T}_{1} \partial_{\eta} \mathbb{T}_{2}+i \sqrt{g} \gamma_{1}^{+} \mathbb{T}_{1} \partial_{\eta} \mathbb{T}_{2} \psi(\xi)\right\}+ \\
& +\int_{x_{1}}^{x_{2}} g \gamma_{1}^{+} \gamma_{2}^{-} \mathbb{T}_{1}\left(x, x_{1} ; \lambda\right) \mathbb{T}_{2}\left(x, x_{1} ; \mu\right) .
\end{aligned}
$$

Ao integrarmos as derivadas totais, notamos que os termos oriundos dos limites $\xi=x_{1}$ e $\eta=x_{1}$ cancelam, respectivamente, o terceiro e o segundo termos de (3.4.8). Conseqüentemente, ao substituirmos tal resultado de volta em (3.4.8), concluímos:

$$
\mathbb{T}_{1}\left(x_{2}, x_{1} ; \lambda\right) \mathbb{T}_{2}\left(x_{2}, x_{1} ; \mu\right)=\mathbb{1}_{4}+\int_{x_{1}}^{x_{2}} d x:\left(\mathbb{L}_{1}(x, t ; \lambda)+\mathbb{L}_{2}(x, t ; \mu)+g \gamma_{1}^{+} \gamma_{2}^{-}\right) \mathbb{T}_{1}\left(x, x_{1} ; \lambda\right) \mathbb{T}_{2}\left(x, x_{1} ; \mu\right):,
$$

que equivale ao problema de Cauchy definido por (3.4.6a) com (3.4.6c). A demonstração de (3.4.6b) é feita exatamente da mesma maneira.

Teorema 3.4.2. As relações de comutação entre os elementos da matriz de transição para um intervalo finito no modelo NLS contínuo estão codificadas na seguinte relação de entrelaçamento:

$$
\widetilde{R}(\lambda-\mu) \mathbb{T}_{1}\left(x_{1}, x_{2} ; \lambda\right) \mathbb{T}_{2}\left(x_{2}, x_{1} ; \mu\right)=\mathbb{T}_{2}\left(x_{2}, x_{1} ; \mu\right) \mathbb{T}_{1}\left(x_{2}, x_{1} ; \lambda\right) \widetilde{R}(\lambda-\mu),
$$

com

$$
\widetilde{R}(\lambda)=\mathbb{1}_{4}-\frac{i g}{\lambda} \Pi
$$

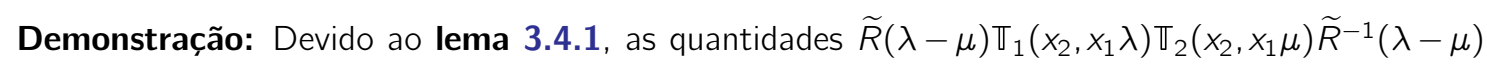
e $\mathbb{T}_{2}\left(x_{2}, x_{1} \mu\right) \mathbb{T}_{1}\left(x_{2}, x_{1} \lambda\right)$ satisfazem a mesma equação diferencial com a mesma condição inicial se

$$
\widetilde{R}(\lambda-\mu) \mathscr{L}(x ; \lambda, \mu)=\mathscr{L}^{\prime}(x ; \lambda, \mu) \widetilde{R}(\lambda-\mu),
$$

uma vez que a matriz $R$ é uma matriz constante cujos elementos comutam com os elementos de $\mathbb{T}\left(x_{2}, x_{1} ; \lambda\right)$. Já a equação (3.4.11) pode ser facilmente verificada de maneira explícita para a matriz $R(3.4 .10)$. 
Nesse estágio as diferenças entre a quantização de sistemas integráveis contínuos e discretos já começam a aparecer, pois, ao compararmos as relações bilineares (3.4.11) contínuas com as discretas (3.2.12) e suas contrapartes clássicas, verificamos a existência de termos adicionais, $g \gamma_{1}^{+} \gamma_{2}^{-}$e $g \gamma_{1}^{+} \gamma_{2}^{-}$, oriundas da não-comutatividade dos operadores quânticos. O próximo passo consiste em estender o resultado do teorema 3.4.2 para o intervalo infinito. Em analogia ao caso clássico, introduzimos os limites da matriz de transição para os intervalos semi-infinitos e infinito:

$$
\begin{array}{r}
\mathbb{T}_{-}(x ; \lambda):=: T_{-}(x ; \lambda): \text { e } \mathbb{T}_{+}(x ; \lambda):=: T_{+}^{-1}(x ; \lambda): \\
\mathbb{T}(\lambda):=: T(x):=\mathbb{T}_{+}(x ; \lambda) \mathbb{T}_{-}(x ; \lambda) .
\end{array}
$$

Teorema 3.4.3. As relações de comutação entre os elementos das matrizes de transição $\mathbb{T}_{ \pm}(x ; \lambda)$ e $\mathbb{T}(\lambda)$, para $\lambda, \mu \in \mathbb{R}$ assumem a seguinte forma:

$$
\begin{aligned}
& \widetilde{R}(\lambda-\mu)\left[\mathbb{T}_{-}(x ; \lambda)\right]_{1}\left[\mathbb{T}_{-}(x ; \mu)\right]_{2}\left(1-\frac{i g \gamma_{1}^{+} \gamma_{2}^{-}}{\lambda-\mu+i \epsilon}\right)=\left[\mathbb{T}_{-}(x ; \mu)\right]_{2}\left[\mathbb{T}_{-}(x ; \lambda)\right]_{1}\left(1+\frac{i g \gamma_{1}^{-} \gamma_{2}^{+}}{\lambda-\mu-i \epsilon}\right) \widetilde{R}(\lambda-\mu), \\
& \widetilde{R}(\lambda-\mu)\left(1+\frac{i g \gamma_{1}^{+} \gamma_{2}^{-}}{\lambda-\mu-i \epsilon}\right)\left[\mathbb{T}_{+}(x ; \lambda)\right]_{1}\left[\mathbb{T}_{+}(x ; \mu)\right]_{2}=\left(1-\frac{i g \gamma_{1}^{-} \gamma_{2}^{+}}{\lambda-\mu+i \epsilon}\right)\left[\mathbb{T}_{+}(x ; \mu)\right]_{2}\left[\mathbb{T}_{+}(x ; \lambda)\right]_{1} \widetilde{R}(\lambda-\mu), \\
& \widetilde{R}(\lambda-\mu)\left(1+\frac{i g \gamma_{1}^{+} \gamma_{2}^{-}}{\lambda-\mu-i \epsilon}\right) \mathbb{T}_{1}(\lambda) \mathbb{T}_{2}(\mu)\left(1-\frac{i g \gamma_{1}^{+} \gamma_{2}^{-}}{\lambda-\mu+i \epsilon}\right)=\left(1-\frac{i g \gamma_{1}^{-} \gamma_{2}^{+}}{\lambda-\mu+i \epsilon}\right) \mathbb{T}_{2}(\mu) \mathbb{T}_{1}(\lambda)\left(1+\frac{i g \gamma_{1}^{-} \gamma_{2}^{+}}{\lambda-\mu-i \epsilon}\right) \widetilde{R}(\lambda-\mu) \text {. }
\end{aligned}
$$

Demonstração: Mostramos a validade algébrico-formal da equação (3.4.13a) baseados na análise feita originalmente por [23]. A idéia dessa demonstração é estudar o comportamento assintótico dos produtos $\mathbb{T}_{1}\left(x_{2}, x_{1} ; \lambda\right) \mathbb{T}_{2}\left(x_{2}, x_{1} ; \mu\right)$ e $\mathbb{T}_{2}\left(x_{2}, x_{1} ; \mu\right) \mathbb{T}_{1}\left(x_{2}, x_{1} ; \lambda\right)$ conforme $x_{1} \rightarrow-\infty$. Para tanto é conveniente introduzir as matrizes:

$$
\begin{array}{rlrl}
\mathscr{E}(x ; \lambda, \mu) & =e^{\mathscr{L}_{0}(\lambda, \mu) x}, & \text { com } \quad \mathscr{L}_{0}(\lambda, \mu) & =\frac{\lambda}{2 i} \gamma_{2}^{3}+\frac{\mu}{2 i} \gamma_{2}^{3}+g \gamma_{1}^{+} \gamma_{2}^{-}, \\
\mathscr{E}^{\prime}(x ; \lambda, \mu) & =e^{\mathscr{L}_{0}^{\prime}(\lambda, \mu) x}, & \text { com } \quad \mathscr{L}_{0}^{\prime}(\lambda, \mu)=\frac{\lambda}{2 i} \gamma_{2}^{3}+\frac{\mu}{2 i} \gamma_{2}^{3}+g \gamma_{1}^{-} \gamma_{2}^{+},
\end{array}
$$

onde $\mathscr{L}_{0}(\lambda, \mu)$ e $\mathscr{L}_{0}^{\prime}(\lambda, \mu)$ representam o comportamento assintótico (conforme $|x| \rightarrow \infty$ ) dos operadores $\mathcal{L}(x ; \lambda, \mu)$ e $\mathcal{L}^{\prime}(x ; \lambda, \mu)$, respectivamente. Da relação de entrelaçamento (3.4.11) temos claramente que

$$
\widetilde{R}(\lambda-\mu) \mathscr{L}_{0}(\lambda, \mu)=\mathscr{L}_{0}^{\prime}(\lambda, \mu) \widetilde{R}(\lambda-\mu) \quad \Rightarrow \quad \widetilde{R}(\lambda-\mu) \mathscr{E}(x ; \lambda, \mu)=\mathscr{E}^{\prime}(x ; \lambda, \mu) \widetilde{R}(\lambda-\mu)
$$

Começamos estudando o comportamento assintótico de $\mathbb{T}_{1}\left(x_{2}, x_{1} ; \lambda\right) \mathbb{T}_{2}\left(x_{2}, x_{1} ; \mu\right)$, quando $x_{1} \rightarrow$ $-\infty$. Rescrevemos a equação diferencial (3.4.6a) da seguinte forma:

$$
\partial_{x_{2}}\left(\mathbb{T}_{1}\left(x_{2}, x_{1} ; \lambda\right) \mathbb{T}_{2}\left(x_{2}, x_{1} ; \mu\right)\right)=\vdots\left(\mathscr{L}_{0}(\lambda, \mu)+\mathbb{V}_{1}\left(x_{2}\right)+\mathbb{V}_{2}\left(x_{2}\right)\right)\left(\mathbb{T}_{1}\left(x_{2}, x_{1} ; \lambda\right) \mathbb{T}_{2}\left(x_{2}, x_{1} ; \mu\right)\right) \vdots
$$


que, sob a condição inicial (3.4.6a), equivale a uma equação integral de Volterra:

$$
\begin{aligned}
\mathbb{T}_{1}\left(x_{2}, x_{1} ; \lambda\right) \mathbb{T}_{2}\left(x_{2}, x_{1} ; \mu\right) & =\mathscr{E}\left(x_{2}-x_{1} ; \lambda, \mu\right)+ \\
& +\int_{x_{1}}^{x_{2}} d x: \mathbb{T}_{1}\left(x_{2}, x ; \lambda\right) \mathbb{T}_{2}\left(x_{2}, x ; \mu\right)\left[\mathbb{V}_{1}(x)+\mathbb{V}_{2}(x)\right]: \mathscr{E}\left(x-x_{1} ; \lambda, \mu\right)
\end{aligned}
$$

onde introduzimos a notação auxiliar: $\mathbb{V}(x)=: L_{x}^{0}(x):=i \sqrt{g}\left[\psi(x) \gamma^{+}-\psi^{\dagger}(x) \gamma^{-}\right]$. Ao consideramos o limite:

$$
\mathscr{T}(x ; \lambda, \mu)=\lim _{x_{1} \rightarrow-\infty} \mathbb{T}_{1}\left(x, x_{1} ; \lambda\right) \mathbb{T}_{2}\left(x, x_{1} ; \mu\right) \mathscr{E}\left(x_{1} ; \lambda, \mu\right),
$$

inferimos

$$
\mathscr{T}(x ; \lambda, \mu)=\mathscr{E}(x ; \lambda, \mu)+\int_{-\infty}^{x} d \eta \mathbb{\mathbb { T }}_{1}(x, \eta ; \lambda) \mathbb{T}_{2}(x, \eta ; \mu)\left[\mathbb{V}_{1}(\eta)+\mathbb{V}_{2}(\eta)\right]: \mathscr{E}(\eta ; \lambda, \mu),
$$

ou, equivalentemente,

$$
\partial_{x} \mathscr{T}(x ; \lambda, \mu)=\vdots \mathscr{L}(x ; \lambda, \mu) \mathscr{T}(x ; \lambda, \mu)
$$

Por outro lado, podemos também considerar o produto

$$
\mathscr{T}_{-}(x ; \lambda, \mu)=\left[\mathbb{T}_{-}(x ; \lambda)\right]_{1}\left[\mathbb{T}_{-}(x ; \mu)\right]_{2},
$$

que por uma argumentação semelhante à empregada na demonstração do lema 3.4.1 satisfaz

$$
\partial_{x} \mathscr{T}_{-}(x ; \lambda, \mu)=\vdots \mathscr{L}(x ; \lambda, \mu) \mathscr{T}_{-}(x ; \lambda, \mu) \vdots
$$

Comparando (3.4.18) com (3.4.19) fica claro que as quantidades $\mathscr{T}(x ; \lambda, \mu)$ e $\mathscr{T}_{-}(x ; \lambda, \mu)$ podem diferir apenas por uma fator multiplicativo matricial $\mathbb{E}(\lambda, \mu)$, ou seja

$$
\mathscr{T}_{-}(x ; \lambda, \mu)=\mathscr{T}(x ; \lambda, \mu) \mathbb{F}(\lambda, \mu) .
$$

Determinamos tal matriz ao compararmos o comportamento assintótico de $\mathscr{T}(x ; \lambda, \mu)$ com $\mathscr{T}_{-}(x ; \lambda, \mu)$, conforme $x \rightarrow-\infty$.

O comportamento assintótico de $\mathscr{T}(x ; \lambda, \mu)$ pode ser facilmente inferido a partir de sua representação integral (3.4.17):

$$
\mathscr{T}(x ; \lambda, \mu) \stackrel{x \rightarrow-\infty}{\longrightarrow} \mathscr{E}(x ; \lambda, \mu) .
$$

Já para obtermos o comportamento assintótico de $\mathscr{T}_{-}(x ; \lambda, \mu)$, precisamos considerar a representação integral para $\mathbb{T}_{-}(x ; \lambda)$ (equivalente a (A.2.1)):

$$
\mathbb{T}_{-}(x ; \lambda)=E(x ; \lambda)+\int_{-\infty}^{x} d \eta \vdots \mathbb{T}(x, \eta ; \lambda) \mathbb{V}(\eta) \vdots E(\eta ; \lambda),
$$


onde relembramos a definição de $E(x ; \lambda)=e^{\lambda L_{x}^{1} x}=e^{\frac{\lambda x}{2 i} \gamma^{3}}$, para escrever os fatores presentes em $\mathscr{T}_{-}(x ; \lambda, \mu)$. Logo,

$$
\begin{aligned}
\mathscr{T}_{-}(x ; \lambda, \mu) & =\mathbb{E}(x ; \lambda, \mu)+\int_{-\infty}^{x} d \eta \mathbb{\mathbb { T }}_{1}(x, \eta ; \lambda) \mathbb{V}_{1}(\eta) \vdots E_{1}(\eta ; \lambda) E_{2}(x ; \mu)+ \\
& +\int_{-\infty}^{x} d \eta \vdots \mathbb{T}_{2}(x, \eta ; \mu) \mathbb{V}_{2}(\eta) \vdots E_{1}(x ; \lambda) E_{2}(\eta ; \mu)- \\
& -g \int_{-\infty}^{x} d \eta_{1} \int_{-\infty}^{x} d \eta_{2}\left[\mathbb{T}_{1}\left(x, \eta_{1} ; \lambda\right) \gamma_{1}^{+} \psi\left(\eta_{1}\right)-\psi^{\dagger}\left(\eta_{1}\right) \mathbb{T}_{1}\left(x, \eta_{1} ; \lambda\right) \gamma_{1}^{-}\right] . \\
& \cdot\left[\mathbb{T}_{2}\left(x, \eta_{2} ; \mu\right) \gamma_{2}^{+} \psi\left(\eta_{2}\right)-\psi^{\dagger}\left(\eta_{2}\right) \mathbb{T}_{2}\left(x, \eta_{2} ; \mu\right) \gamma_{2}^{-}\right] E_{1}\left(\eta_{1} ; \lambda\right) E_{2}\left(\eta_{2} ; \mu\right),
\end{aligned}
$$

onde introduzimos a notação

$$
\mathbb{E}(x ; \lambda, \mu)=E_{1}(x ; \lambda) E_{2}(x ; \mu) .
$$

O quarto termo de (3.4.22) pode ser tratado exatamente da mesma forma que o quarto termo de (3.4.8). Assim, sem mais delonga, exibimos o resultado final:

$$
\mathscr{T}_{-}(x ; \lambda, \mu) \mathbb{E}(x ; \lambda, \mu)+\int_{-\infty}^{x} d \eta \mathbb{\mathbb { T }}_{1}(x, \eta ; \lambda) \mathbb{T}_{2}(x, \eta ; \mu)\left[\mathbb{V}_{1}(\eta)+\mathbb{V}_{2}(\eta)+g \gamma_{1}^{+} \gamma_{2}^{-}\right]: \mathbb{E}(\eta ; \lambda, \mu) .
$$

Notando que de (3.4.16) podemos extrair

$$
\mathbb{T}_{1}(x, \eta ; \lambda) \mathbb{T}_{2}(x, \eta ; \mu) \stackrel{x, \eta \rightarrow-\infty}{\longrightarrow} \mathscr{E}(x-\eta ; \lambda, \mu),
$$

chegamos a seguinte conclusão:

$$
\begin{aligned}
\mathscr{T}_{-}(x ; \lambda, \mu) & \stackrel{x \rightarrow-\infty}{\longrightarrow} \mathbb{E}(x ; \lambda, \mu)+\int_{-\infty}^{x} d \eta \mathscr{E}(x-\eta ; \lambda, \mu) g \gamma_{1}^{+} \gamma_{2}^{-} \mathbb{E}(\eta ; \lambda, \mu) \\
& \stackrel{x \rightarrow-\infty}{\longrightarrow} \mathbb{E}(x ; \lambda, \mu)+\frac{i g}{\lambda-\mu+i \epsilon} e^{\frac{\lambda-\mu}{2 i} x} \gamma_{1}^{+} \gamma_{2}^{-} \\
& \stackrel{x \rightarrow-\infty}{\longrightarrow} \mathscr{E}(x ; \lambda, \mu) \mathbb{E}(\lambda, \mu) \quad \operatorname{com} \quad \mathbb{E}(\lambda, \mu)=\mathbb{1}_{4}+\frac{i g}{\lambda-\mu+i \epsilon} \gamma_{1}^{+} \gamma_{2}^{-} .
\end{aligned}
$$

Dessa forma, podemos rescrever (3.4.20)

$$
\mathscr{T}(x ; \lambda, \mu)=\mathscr{T}_{-}(x ; \lambda, \mu) \mathbb{F}^{-1}(\lambda, \mu) \quad \operatorname{com} \quad \mathbb{F}^{-1}(\lambda, \mu)=\mathbb{1}_{4}-\frac{i g}{\lambda-\mu-i \epsilon} \gamma_{1}^{+} \gamma_{2}^{-},
$$

e utilizar as definições de $\mathscr{T}(x ; \lambda, \mu)$ e $\mathscr{T}_{-}(x ; \lambda, \mu)$ para concluir:

$$
\lim _{x_{1} \rightarrow-\infty}\left[\mathbb{T}_{1}\left(x, x_{1} ; \lambda\right) \mathbb{T}_{2}\left(x, x_{1} ; \mu\right)\right] \mathscr{E}(x ; \lambda, \mu)=\left[\mathbb{T}_{-}(x ; \lambda)\right]_{1}\left[\mathbb{T}_{-}(x ; \mu)\right]_{2}\left(\mathbb{1}_{4}-\frac{i g}{\lambda-\mu+i \epsilon} \gamma_{1}^{+} \gamma_{2}^{-}\right) .
$$

Analogamente ${ }^{13}$, obtemos:

$$
\lim _{x_{1} \rightarrow-\infty}\left[\mathbb{T}_{2}\left(x, x_{1} ; \mu\right) \mathbb{T}_{1}\left(x, x_{1} ; \lambda\right)\right] \mathscr{E}^{\prime}(x ; \lambda, \mu)=\left[\mathbb{T}_{-}(x ; \mu)\right]_{2}\left[\mathbb{T}_{-}(x ; \lambda)\right]_{1}\left(\mathbb{1}_{4}+\frac{i g}{\lambda-\mu-i \epsilon} \gamma_{1}^{-} \gamma_{2}^{+}\right) .
$$

\footnotetext{
${ }^{13} \mathrm{Ou}$ mais simplesmente, basta que consideremos a troca entre os espaços auxiliares, isto é, $1 \leftrightarrow 2$ concomitante com a troca dos parâmetros espectrais $\lambda \leftrightarrow \mu$ em (3.4.23a).
} 
A seguir multiplicamos a relação de entrelaçamento (3.4.9) pela direita por $\mathscr{E}(x ; \lambda, \mu)$ e usamos (3.4.15) para obter:

$$
\widetilde{R}(\lambda-\mu) \mathbb{T}_{1}\left(x, x_{1} ; \lambda\right) \mathbb{T}_{2}\left(x, x_{1} ; \mu\right) \mathscr{E}\left(x_{1} ; \lambda, \mu\right)=\mathbb{T}_{2}\left(x, x_{1} ; \mu\right) \mathbb{T}_{1}\left(x, x_{1} ; \lambda\right) \mathscr{E}^{\prime}(x ; \lambda, \mu) \widetilde{R}(\lambda-\mu) .
$$

Finalmente, tomando o limite $x_{1} \rightarrow-\infty$ e usando (3.4.23) estabelecemos a veracidade de (3.4.13a). A equação (3.4.13b) pode ser demonstrada do mesmo modo. Para verificarmos a validade de (3.4.13c), basta que combinemos os resultados (3.4.13a) e (3.4.13b) de acordo com a relação (3.4.12b).

A partir do teorema 3.4.3 podemos obter as relações de comutação entre os diversos elementos da matriz de transição para os intervalos semi-infinitos $(-\infty, x]$ e $[x, \infty)$ e para o intervalo infinito $(-\infty, \infty)$, que exibimos abaixo:

$$
\begin{aligned}
A(\lambda) A(\mu) & =A(\mu) A(\lambda) \\
A(\lambda) A^{\dagger}(\mu) & =A^{\dagger}(\mu) A(\lambda) \\
B(\mu) A(\lambda) & =\left(1+\frac{i g}{\lambda-\mu+i \epsilon}\right) A(\lambda) B(\mu) \\
A(\lambda) B^{\dagger}(\mu) & =\left(1+\frac{i g}{\lambda-\mu+i \epsilon}\right) B^{\dagger}(\mu) A(\lambda) \\
B(\lambda) B(\mu) & =B(\mu) B(\lambda) \\
B(\lambda) B^{\dagger}(\mu) & =\left(1+\frac{i g}{\lambda-\mu+i \epsilon}\right)\left(1-\frac{i g}{\lambda-\mu-i \epsilon}\right) B^{\dagger}(\mu) B(\lambda)+2 \pi A^{\dagger}(\mu) A(\lambda) \delta(\lambda-\mu)
\end{aligned}
$$

Para as relações de comutação entre os elementos de $\mathbb{T}_{ \pm}(x ; \lambda)$ vide [23].

\subsubsection{Coordenadas de Ângulo e Ação}

É importante ressaltar que a relação (3.4.24f) possui um produto de funções generalizadas:

$$
\frac{1}{\lambda-\mu+i \epsilon} \frac{1}{\lambda-\mu-i \epsilon}
$$

que, de uma forma geral, não é um objeto bem definido, e indica o caráter altamente singular dos operadores $B(\lambda)$ e $B^{\dagger}(\lambda)$. Entretanto, é possível remediar tal situação, ao regularizarmos esses operadores:

$$
\Phi(\lambda)=\left[2 \pi A^{\dagger}(\lambda) A\right]^{-\frac{1}{2}} B(\lambda) \quad \text { e } \quad \Phi^{\dagger}(\lambda)=B^{\dagger}(\lambda)\left[2 \pi A^{\dagger}(\lambda) A\right]^{-\frac{1}{2}} .
$$

Motivemos essa regularização. Em primeiro lugar, notamos que da relação (3.4.24d), em conjunto com a comutatividade entre os operadores $A(\lambda)$ para diferentes valores do parâmetro espectral, (3.4.24a), inferimos que para qualquer polinômio $f(\lambda)$ :

$$
f(A(\lambda)) B^{\dagger}(\mu)=B^{\dagger}(\mu) f\left[\left(1+\frac{i g}{\lambda-\mu+i \epsilon}\right) A(\lambda)\right] .
$$


Podemos formalmente estender (3.4.26a) para funções analíticas e, em particular, para funções da forma $f(\lambda)=\lambda^{-\frac{1}{2}}$, por meio de uma decomposição em série potências ao redor do ponto $\lambda=\lambda_{0} \neq 0$. Analogamente, de (3.4.24c), deduzimos:

$$
B(\lambda) f(A(\mu))=f\left[\left(1-\frac{i g}{\lambda-\mu-i \epsilon}\right) A(\mu)\right] B(\lambda) .
$$

Conseqüentemente, tais relações em conjunto com a comutatividade entre $A(\lambda)$ e $A^{\dagger}(\mu)(3.4 .24 b)$ garantem que, ao menos de uma maneira formal, as expressões para $\Phi(\lambda)$ e $\Phi^{\dagger}(\lambda)$ estão bem definidas. Ademais a partir de (3.4.26) podemos mostrar que:

$$
\begin{aligned}
{[\Phi(\lambda), \Phi(\mu)] } & =0, \\
{\left[\Phi^{\dagger}(\lambda), \Phi^{\dagger}(\mu)\right] } & =0, \\
{\left[\Phi(\lambda), \Phi^{\dagger}(\mu)\right] } & =\delta(\lambda-\mu),
\end{aligned}
$$

conferindo um caráter de coordenadas de ângulo e ação aos operadores (3.4.25).

Em conexão com o ABA, esperaríamos que o estado de $N$-partículas fosse descrito por:

$$
\left|\left\{\lambda_{j}\right\}_{N}\right\rangle_{B}=\prod_{j=1}^{N} B^{\dagger}\left(\lambda_{j}\right)|0\rangle
$$

De fato, tal estado está diretamente relacionado com o estado de $N$-partículas que obtivemos diretamente pelo $\mathrm{ABC},\left|\left\{\lambda_{j}\right\}_{N}\right\rangle_{A B C}$, associado à função de onda normalizada $f_{N}\left(z_{1}, \ldots, z_{N} \mid \lambda_{1}, \ldots, \lambda_{N}\right)$ (3.1.27):

$$
\left|\left\{\lambda_{j}\right\}_{N}\right\rangle_{B} \sim \prod_{k<j}\left|\frac{\lambda_{k}-\lambda_{j}+i g}{\lambda_{k}-\lambda_{j}}\right|\left|\left\{\lambda_{j}\right\}_{N}\right\rangle_{A B C} .
$$

Contudo, a presença dos fatores $\left(\lambda_{k}-\lambda_{j}\right)^{-1}$ torna a normalização dessas funções tão singular que não podemos sequer considerar os operadores $B^{\dagger}(\lambda)$ como funções generalizadas. Por outro lado, se utilizarmos as regularizações (3.4.25):

$$
\left|\left\{\lambda_{j}\right\}_{N}\right\rangle=\prod_{j=1}^{N} \Phi^{\dagger}\left(\lambda_{j}\right)|0\rangle
$$

concluímos que

$$
\left|\left\{\lambda_{j}\right\}_{N}\right\rangle=\left|\left\{\lambda_{j}\right\}_{N}\right\rangle_{A B C}
$$

Logo, podemos considerar os operadores $\Phi^{\dagger}(\lambda)$ como operadores de criação normalizados para as autofunções do Hamiltoniano (3.1.1).

Finalmente, discutimos as integrais de movimento quânticas, que, assim como no caso clássico, são geradas pelo $\log A(\lambda)$. Para vermos isso, notamos que da relação de comutação (3.4.24a) obtemos que:

$$
[\log A(\lambda), \log A(\mu)]=0
$$

Para que possamos atuar com $\log A(\lambda)$ sobre um estado de $N$-partículas, $\left|\left\{\lambda_{j}\right\}_{N}\right\rangle$, consideramos: 
$f(\lambda)=\log \lambda$ em (3.4.26a), para inferir:

$$
\begin{gathered}
{[\log A(\lambda)] B^{\dagger}(\mu)=B^{\dagger}(\mu)\left[\log A(\lambda)+\log \left(1+\frac{i g}{\lambda-\mu+i \epsilon}\right)\right]} \\
\Rightarrow \quad\left[\log A(\lambda), \Phi^{\dagger}(\mu)\right]=\Phi^{\dagger}(\mu) \log \left(1+\frac{i g}{\lambda-\mu+i \epsilon}\right) .
\end{gathered}
$$

De modo que,

$$
\log A(\lambda)\left|\left\{\lambda_{j}\right\}_{N}\right\rangle=\sum_{l}^{N} \log \left(1+\frac{i g}{\lambda-\mu+i \epsilon}\right)\left|\left\{\lambda_{j}\right\}_{N}\right\rangle
$$

onde usamos ${ }^{14}$ que

$$
A(\lambda)|0\rangle=|0\rangle \quad \Rightarrow \quad \log A(\lambda)|0\rangle=0
$$

Podemos decompor ambos os lados de (3.4.29) em séries em $\lambda^{-1}$

$$
\begin{array}{r}
\log A(\lambda)=i g \sum_{j=1}^{\infty} \frac{A_{j}}{\lambda^{j}} \text { e } \log \left(1+\frac{i g}{\lambda-\lambda_{k}}\right)=i g \sum_{j=1}^{\infty} \frac{c_{j}\left(\lambda_{k}\right)}{\lambda^{j}} \\
\operatorname{com} \quad c_{j}\left(\lambda_{k}\right)=\frac{\lambda_{k}^{j}-\left(\lambda_{k}-i g\right)^{j}}{i j g}=\sum_{m=1}^{j} \frac{(j-1) !}{m !(j-m) !}(-i g)^{m-1} \lambda_{k}^{j-m+1} .
\end{array}
$$

e, então concluir que os estados $\left|\left\{\lambda_{j}\right\}_{N}\right\rangle$ também são autoestados dos operadores $A_{j}$ com autovalores $c_{j}\left(\lambda_{k}\right)$ :

$$
A_{j}\left|\left\{\lambda_{l}\right\}_{N}\right\rangle=\sum_{k=1}^{N} c_{j}\left(\lambda_{k}\right)\left|\left\{\lambda_{l}\right\}_{N}\right\rangle
$$

Diferentemente do caso clássico, onde existe um algoritmo geral para se determinar os funcionais $I_{n}$ (2.6.38) diretamente a partir de uma relação de recursão fechada (2.6.30), inexiste no contexto quântico ${ }^{15}$ um método geral para se obter as identidades de traço e assim expressar as quantidades conservadas em termos de integrais de densidades locais. Dessa forma, é necessário utilizar algum artifício independente do MEIQ para construir as integrais do movimento. Para o caso do modelo NLS é possível efetuar a quantização das três primeiras integrais do movimento $Q, P$ e $H$ através do ordenamento normal, de forma a garantir que os operadores resultantes sejam auto-adjuntos no espaço de Fock $\mathcal{F}$ [23]. Assim

$$
\square_{i}=: I_{n}: \text { para } i=1,2,3 .
$$

com $I_{n}$ definido por (2.6.38) e (2.6.42).

Os autovalores de $\mathbb{a}_{n}$ sobre os estados $\left|\left\{\lambda_{l}\right\}_{N}\right\rangle$ são:

$$
\mathbb{\square}_{n}\left|\left\{\lambda_{l}\right\}_{N}\right\rangle=\sum_{k=1}^{N} \lambda_{k}^{n}\left|\left\{\lambda_{l}\right\}_{N}\right\rangle \quad \text { para } \quad i=1,2,3
$$

\footnotetext{
${ }^{14} \mathrm{~A}$ validade da primeira afirmação abaixo pode ser facilmente estabelecida ao iterarmos as equações integrais de Volterra, equivalentes ao problema linear auxiliar (3.4.5), para obter uma expansão de $A(\lambda) \sim 1+\sum_{j=1}^{N} \mathscr{I}_{n}$ onde os $\mathscr{I}_{n}$ são integrais $2 N$-dimensionais de integrandos proporcionais a $:\left(\psi^{\dagger} \psi\right)^{n}:$, que conseqüentemente se anulam ao atuar sobre o pseudovácuo $|0\rangle$.

${ }^{15}$ Para modelos contínuos.
} 
Comparando (3.4.30) com (3.4.32) obtemos:

$$
\begin{aligned}
& A_{1}=\square_{1} \quad \Rightarrow \quad Q=A_{1}, \\
& A_{2}=\square_{2}-\frac{i g}{2} \square_{1} \quad \Rightarrow \quad P=A_{2}+\frac{i g}{2} A_{1}, \\
& A_{3}=\square_{3}-i g \square_{2}-\frac{g^{2}}{3} \square_{1} \quad \Rightarrow \quad H=A_{3}+i g A_{2}-\frac{g^{2}}{6} A_{1} .
\end{aligned}
$$

Dessa forma fica claro que, em termos dos operadores $A_{j}$, mesmo para a segunda e a terceira cargas conservadas, correções quânticas são necessárias. Notamos, contudo, que tais correções são implementadas por operadores que já pertenciam ao conjunto dos geradores da subálgebra de operadores comutantes.

Apesar de a prescrição do ordenamento normal fornecer um método para a obtenção das três primeiras integrais do movimento quânticas a partir de suas contrapartes clássicas, ele falha quando aplicado a próxima quantidade conservada. De fato, o operador obtido a partir do ordenamento de Wick de $I_{4}$ não corresponde à versão segundoquantizada do operador diferencial ${ }^{16} \mathrm{com}$ autovalor $\sum_{j=1}^{N} \lambda_{j}^{3}$ [58]. Para $n \geq 5$, o problema persiste, e as quantidades conservadas quânticas $\mathbb{a}_{n}$ também não podem ser obtidas a partir do ordenamento normal de suas contrapartes clássicas [58]. Isso torna a obtenção das identidades de traço uma tarefa extremamente não trivial mesmo para o caso do simple modelo NLS.

O fato de que as quantidades conservadas de maior ordem não são ordenadas normalmente, é equivalente ao seguinte e surpreendente resultado:

$$
\sum_{j=1} \frac{A_{j}}{\lambda^{j}} \neq \sum_{j=1} \frac{A_{j}^{\text {clássico }}}{\lambda^{j}}
$$

embora $A(\lambda)$ seja corretamente definido como : $A^{\text {clássico }}(\lambda)$ :. A lição que devemos tirar disso é que o "resultado folclórico", que afirma que a versão quântica dos parênteses de Poisson entre observáveis clássicos (por exemplo, uma lei de conservação) corresponde ao ordenamento normal do funcional clássico, não é necessariamente um requisito (nem uma garantia) para a integrabilidade de uma dada teoria quântica. Em outras palavras, a noção amplamente aceita ${ }^{17}$ de que a prescrição de Wick resolve o problema de ordenamento operatorial não é de fato correta.

\subsection{A Equação de Yang-Baxter}

Assim como no caso clássico, existem alguns vínculos que a matriz $R$ deve satisfazer para poder codificar as relações de comutação de um certo sistema integrável. Um deles, a equação de Yang-Baxter, pode ser obtido ao considerarmos o produto tensorial triplo ${ }^{18} T(n, m ; \lambda) \otimes T(n, m ; \mu) \otimes$ $T(n, m ; \nu)$ e usarmos a relação bilinear (3.2.13) para reduzi-lo ao seguinte produto tensorial triplo $T(n, m ; \nu) \otimes T(n, m ; \mu) \otimes T(n, m ; \lambda)$, o que pode ser feito de duas formas distintas. Por simplicidade

\footnotetext{
${ }^{16}$ Da mesma forma que (3.1.13).

${ }^{17}$ Quase um inconsciente coletivo.

${ }^{18}$ Fazemos isso para o caso discreto, devido a maior transparência do argumento. Além do mais, o resultado análogo para o caso contínuo pode ser obtido a partir da condensação da rede.
} 
deixaremos de denotar os sítios sobre o qual a matriz de transição atua, bem como trabalharemos com $\widetilde{R}_{12}(\lambda-\mu)$, utilizando a relação de entrelaçamento (3.2.14). Por um lado temos que:

$$
\begin{aligned}
& T_{1}(\lambda) T_{2}(\mu) T_{3}(\nu)=\widetilde{R}_{23}^{-1}(\mu-\nu) T_{1}(\lambda) T_{3}(\nu) T_{2}(\mu) \widetilde{R}_{23}(\mu-\nu) \\
& =\widetilde{R}_{23}^{-1}(\mu-\nu) \widetilde{R}_{13}^{-1}(\lambda-\nu) T_{3}(\nu) T_{1}(\lambda) T_{2}(\mu) \widetilde{R}_{13}(\lambda-\nu) \widetilde{R}_{23}(\mu-\nu) \\
& =\widetilde{R}_{23}^{-1}(\mu-\nu) \widetilde{R}_{13}^{-1}(\lambda-\nu) \widetilde{R}_{12}^{-1}(\lambda-\mu) T_{3}(\nu) T_{2}(\mu) T_{1}(\lambda) \widetilde{R}_{12}(\lambda-\mu) \widetilde{R}_{13}(\lambda-\nu) \widetilde{R}_{23}(\mu-\nu),
\end{aligned}
$$

enquanto que pelo outro:

$$
\begin{aligned}
& T_{1}(\lambda) T_{2}(\mu) T_{3}(\nu)=\widetilde{R}_{12}^{-1}(\lambda-\mu) T_{2}(\mu) T_{1}(\lambda) T_{3}(\nu) \widetilde{R}_{12}(\lambda-\mu) \\
& =\widetilde{R}_{12}^{-1}(\lambda-\mu) \widetilde{R}_{13}^{-1}(\lambda-\nu) T_{2}(\mu) T_{3}(\nu) T_{1}(\lambda) \widetilde{R}_{13}(\lambda-\nu) \widetilde{R}_{12}(\lambda-\mu) \\
& =\widetilde{R}_{12}^{-1}(\lambda-\mu) \widetilde{R}_{13}^{-1}(\lambda-\nu) \widetilde{R}_{23}^{-1}(\mu-\nu) T_{3}(\nu) T_{2}(\mu) T_{1}(\lambda) \widetilde{R}_{23}(\mu-\nu) \widetilde{R}_{13}(\lambda-\nu) \widetilde{R}_{12}(\lambda-\mu) .
\end{aligned}
$$

Comparando (3.5.1a) e (3.5.1b) obtemos a equação de Yang Baxter:

$$
\widetilde{R}_{12}(\lambda-\mu) \widetilde{R}_{13}(\lambda-\nu) \widetilde{R}_{23}(\mu-\nu)=\widetilde{R}_{23}(\mu-\nu) \widetilde{R}_{13}(\lambda-\nu) \widetilde{R}_{12}(\lambda-\mu) .
$$

Alternativamente, podemos escrevê-la em termos da matriz $R(\lambda-\mu)$ :

$$
\left[\mathbb{1}_{k} \otimes R(\lambda-\mu)\right]\left[R(\lambda-\nu) \otimes \mathbb{1}_{k}\right]\left[\mathbb{1}_{k} \otimes R(\mu-\nu)\right]=\left[R(\mu-\nu) \otimes \mathbb{1}_{k}\right]\left[\mathbb{1}_{k} \otimes R(\lambda-\nu)\right]\left[R(\lambda-\mu) \otimes \mathbb{1}_{k}\right] .
$$

\subsubsection{Espalhamento em $1+1$ Dimensões}

A equação de Yang-Baxter desempenha um papel muito importante no estudo do espalhamento em teorias de campos integráveis em $1+1$ dimensões, ao relacionar a existência de infinitas cargas conservadas em involução com a fatorabilidade do processo de espalhamento.

Definição 3.5.1. Seja um espalhamento envolvendo M partículas, ele é dito fatorável, ou nãodifrativo, se:

1. Não há produção, nem aniquilação de partículas, de forma que o número de partículas nos estados final e inicial é o mesmo;

2. O conjunto de momentos que descreve os estados final e inicial é o mesmo, o máximo que pode acontecer no decorrer do espalhamento é uma redistribuição dos momentos entre as partículas;

3. A matriz $S$ para o espalhamento de $M$ partículas pode ser descrita como o produto de $\frac{M(M-1)}{2}$ matrizes $S$ para o espalhamento de 2 partículas.

Conforme discutimos anteriormente, no contexto do ABC para o modelo NLS, a forma das funções de onda (3.1.27) indica que a dinâmica do NLS depende apenas da interação entre dois corpos, de sorte as condições da definição 3.5.1 podem ser explicitamente verificadas. O modelo NLS não é o único modelo em teoria de campos em $1+1$ dimensões solúvel pelo Ansatz de Bethe a apresentar um espalhamento fatorável, o mesmo ocorre, por exemplo, para o modelo de sine-Gordon. É natural, pois, que nos perguntemos qual é a característica fundamental comum a esses modelos 
que permite a fatorabilidade do espalhamento. No que se segue argumentamos que a existência de infinitas cargas conservadas em involução é tal propriedade.

Considere o espalhamento de $M$ partículas idênticas no contexto de uma teoria relativística, de sorte que é possível parametrizar a energia e o momento de tais partículas pela sua rapidez $\theta_{i}$, $i=1, \ldots, M$ :

$$
\left\{\begin{array} { l } 
{ p _ { 0 } ^ { i } = m \operatorname { c o s h } \theta _ { i } , } \\
{ p _ { 1 } ^ { i } = m \operatorname { s i n h } \theta _ { i } , }
\end{array} \Longleftrightarrow \left\{\begin{array}{l}
p^{i}=p_{0}^{i}+i p_{1}^{i}=m e^{\theta_{i}} \\
\bar{p}^{i}=p_{0}^{i}-i p_{1}^{i}=m e^{-\theta_{i}}
\end{array}\right.\right.
$$

Denotemos um estado de $M$ partículas por:

$$
\left|\left\{A_{i}\left(\theta_{i}\right)\right\}_{M}\right\rangle=\left|A_{1}\left(\theta_{1}\right) \cdots A_{M}\left(\theta_{M}\right)\right\rangle
$$

onde é conveniente atribuir um caráter não-comutativo aos símbolos $A_{i}\left(\theta_{i}\right)$. Dessa forma, ao considerarmos um estado assintótico inicial, ou seja, para o qual não existem interações conforme $t \rightarrow-\infty$, temos que a partícula mais rápida deve estar na extrema esquerda, enquanto que a mais devagar na extrema direita e o ordenamento dos fatores $A_{i}\left(\theta_{i}\right)$ reflete o ordenamento espacial das partículas que representam:

$$
\left|\left\{A_{i}\left(\theta_{i}\right)\right\}_{M}\right\rangle_{i}=\left|A_{1}\left(\theta_{1}\right) A_{2}\left(\theta_{2}\right) \cdots A_{M}\left(\theta_{M}\right)\right\rangle \quad \text { com } \quad \theta_{1}>\theta_{2}>\cdots>\theta_{M}
$$

Similarmente, um estado final não apresenta mais interações quando $t \rightarrow \infty$, portanto as partículas mais rápidas devem estar à direita das mais lentas, isto é,

$$
\left|\left\{A_{i}\left(\theta_{i}\right)\right\}_{M}\right\rangle_{f}=\left|A_{1}\left(\theta_{1}\right) A_{2}\left(\theta_{2}\right) \cdots A_{M}\left(\theta_{M}\right)\right\rangle \quad \text { com } \quad \theta_{1}<\theta_{2}<\cdots<\theta_{M}
$$

Diferentes ordenamentos podem ser vistos como representantes de estados intermediários, para os quais as partículas estejam devidamente separadas, de modo a garantir o caráter assintótico do estado.

Sob um "boost" de Lorentz: $\theta \rightarrow \theta+\alpha$, temos que $p \rightarrow p e^{\alpha}$ e $\bar{p} \rightarrow \bar{p} e^{-\alpha}$, de forma que para o estado de uma partícula, obtemos a seguinte ação do operador momento:

$$
P\left|A_{i}\left(\theta_{i}\right)\right\rangle=p^{i}\left|A_{i}\left(\theta_{i}\right)\right\rangle \quad \text { e } \quad \bar{P}\left|A_{i}\left(\theta_{i}\right)\right\rangle=\bar{p}^{i}\left|A_{i}\left(\theta_{i}\right)\right\rangle \text {. }
$$

Já, ao considerarmos o caso mais geral de um tensor de spin $s$, temos que sob esse mesmo "boost", $q_{s} \rightarrow q_{s} e^{s \alpha}$ e $\bar{q}_{s} \rightarrow \bar{q}_{s} e^{-s \alpha}$, e escrevemos a ação de um operador em uma representação de ordem maior do grupo de Lorentz sobre o estado de uma partícula como:

$$
Q_{s}\left|A_{i}\left(\theta_{i}\right)\right\rangle=q_{s}^{(i)}(p)\left|A_{i}\left(\theta_{i}\right)\right\rangle \quad \text { e } \quad \bar{Q}_{s}\left|A_{i}\left(\theta_{i}\right)\right\rangle=\bar{q}_{s}^{(i)}(p)\left|A_{i}\left(\theta_{i}\right)\right\rangle
$$

onde $q_{s}^{(i)}(p)=q_{s}\left(p^{i}\right) e^{s \theta_{i}}$ e, em particular, para $s=1$ devemos recuperar (3.5.4a), de forma que $q_{s=1}\left(p^{i}\right)=m$.

Os operadores $Q_{s}$ são as cargas conservadas em involução que foram diagonalizadas simultaneamente na base de estados assintóticos $\left|\left\{A_{i}\left(\theta_{i}\right)\right\}_{M}\right\rangle$ e, portanto devem possuir autovalores 
$q_{s}(p) \sim p^{s}$ [25]. Uma hipótese importante sobre a forma das quantidades conservadas é que elas possam ser expressas como integrais de densidades locais

$$
Q_{s}=\int_{-\infty}^{\infty} d x \rho_{s+1}(x)
$$

uma vez que isso garante a aditividade de sua ação sobre estados com várias partículas:

$$
Q_{s}\left|\left\{A_{i}\left(\theta_{i}\right)\right\}_{M}\right\rangle=\left[\sum_{i=1}^{M} q_{s}^{(i)}(p)\right]\left|\left\{A_{i}\left(\theta_{i}\right)\right\}_{M}\right\rangle
$$

Seja um espalhamento de $M \rightarrow M^{\prime}$ partículas, então, se uma carga $Q_{s}$ é conservada, tem-se que um dado autoestado inicial de $Q_{s}$ com seu respectivo autovalor deve evoluir para uma superposição de estados, todos compartilhando o mesmo autovalor,

$$
\sum_{i=1}^{M} q_{s}^{(i)}(p)=\sum_{i=1}^{M^{\prime}} q_{s}^{(i)}\left(p^{\prime}\right) \Rightarrow q_{s}\left(p^{1}\right) e^{s \theta_{1}}+\cdots+q_{s}\left(p^{M}\right) e^{s \theta_{M}}=q_{s}\left(p^{1^{\prime}}\right) e^{s \theta_{1}^{\prime}}+\cdots+q_{s}\left(p^{M^{\prime \prime}}\right) e^{s \theta_{M^{\prime}}} .
$$

Para $s=1$ a equação (3.5.6) representa simplesmente a conservação de energia e momento. Agora, se existem infinitas cargas conservadas $Q_{s}$, para um par de estados inicial e final arbitrários, existem infinitas equações algébricas a serem satisfeitas. A única solução nesse caso é a trivial com $M=M^{\prime}$ e

$$
\left\{p_{\mu}^{i}, i=1, \ldots, M\right\}=\left\{p_{\mu}^{i \prime}, i=1, \ldots, M^{\prime}\right\} .
$$

Logo, estabelecemos a validade das condições 1. e 2. da definição 3.5.1 para uma teoria relativística com infinitas cargas conservadas em involução.

Uma propriedade importante de teorias relativísticas é que suas amplitudes de espalhamento só podem depender da diferença entre rapidezes. Uma justificativa para tal comportamento pode ser encontrada ao considerarmos o produto entre os momentos $p_{i}$ e $p_{j}$ de duas partículas arbitrárias $A_{i}$ e $A_{j}$, respectivamente:

$$
\eta_{\mu \nu} p_{\mu}^{i} p_{\nu}^{j}=m_{i} m_{j} \cosh \theta_{i j} \quad \operatorname{com} \quad \theta_{i j}=\theta_{i}-\theta_{j},
$$

onde $\eta=\operatorname{diag}(1,-1)$ é a métrica plana de Minkowski bidimensional. Em particular, a matriz $S$ que proporciona um mapa entre os estados inicial e final, também só deve depender da diferença entre rapidezes. De forma que podemos escrever para o espalhamento de $2 \rightarrow 2$ partículas:

$$
\left|A_{i}\left(\theta_{1}\right) A_{j}\left(\theta_{2}\right)\right\rangle_{i}=\sum_{k, I} S_{i j}^{k I}\left(\theta_{12}\right)\left|A_{k}\left(\theta_{2}\right) A_{l}\left(\theta_{1}\right)\right\rangle_{f}
$$

Graficamente podemos representar a matriz $S$ presente em (3.5.11) pela figura 3.1.

Estamos agora em posição de estabelecer a validade da propriedade 3. da definição 3.5 .1 e a conexão da fatorabilidade do espalhamento com a equação de Yang-Baxter (3.5.2). Para isso vamos considerar o efeito da ação das cargas conservadas em pacotes de onda localizados [24]. Seja o 


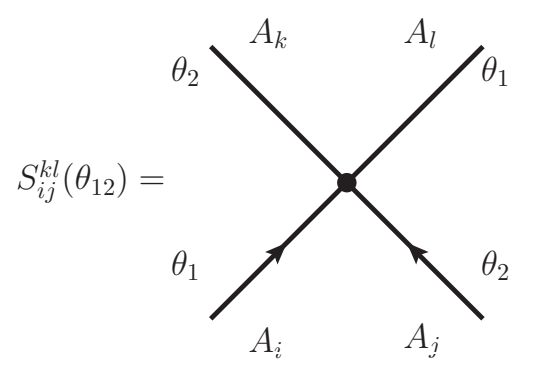

Figura 3.1: Representação gráfica da matriz $S$ para o espalhamento entre duas partículas em uma teoria relativística.

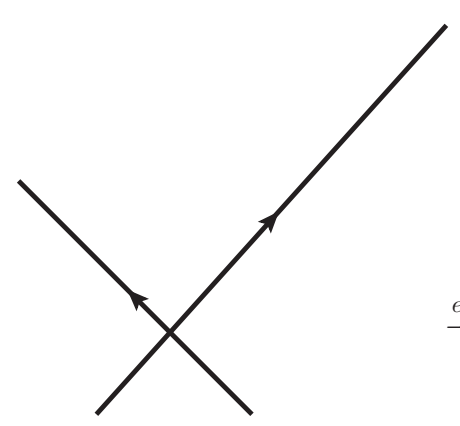

$\left|A_{i}\right\rangle$
$\left|A_{j}\right\rangle$

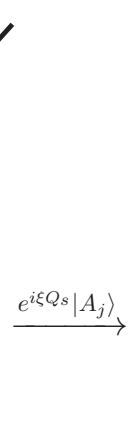

$\stackrel{{ }_{i \xi Q s}\left|A_{j}\right\rangle}{\longrightarrow}$

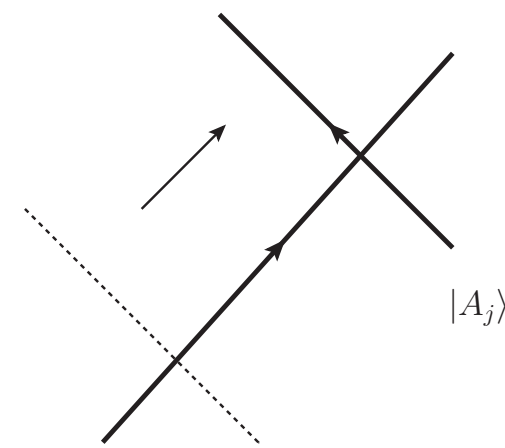

$\left|A_{i}\right\rangle$

Figura 3.2: Usando o operador $e^{i \xi Q_{s}}$ para deslocar uma partícula.

estado de uma partícula, representado pela função de onda no espaço das posições:

$$
\psi(x) \sim \int_{-\infty}^{\infty} d p e^{-a^{2}\left(p-p_{1}\right)^{2}} e^{i p\left(x-x_{1}\right)} .
$$

A equação (3.5.8) descreve uma partícula aproximadamente em $x_{1}$ com a componente espacial do momento aproximadamente igual a $p_{1}$. Atuando sobre essa partícula com um operador fornecendo uma fase dependente do momento $e^{-i \varphi(p)}$, obtemos:

$$
\tilde{\psi}(x) \sim \int_{-\infty}^{\infty} d p e^{-a^{2}\left(p-p_{1}\right)^{2}} e^{i p\left(x-x_{1}\right)} e^{-i \varphi(p)} .
$$

A maior parte da contribuição da integral acima advém de $p \approx p_{1}$. Podemos então expandir $\varphi(p)$ em potências de $\left(p-p_{1}\right)$ para encontrarmos os novos valores da posição e da componente espacial do momento:

$$
\tilde{x}_{1}=x_{1}+\varphi^{\prime}\left(p_{1}\right) \text { e } \quad \tilde{p}_{1}=p_{1}
$$

Para descrevermos um estado com várias partículas devidamente separadas, podemos empregar o produto de funções de onda para uma partícula. De forma que, nesse estado $\left|p_{i} p_{j} \cdots\right\rangle$, a ação 


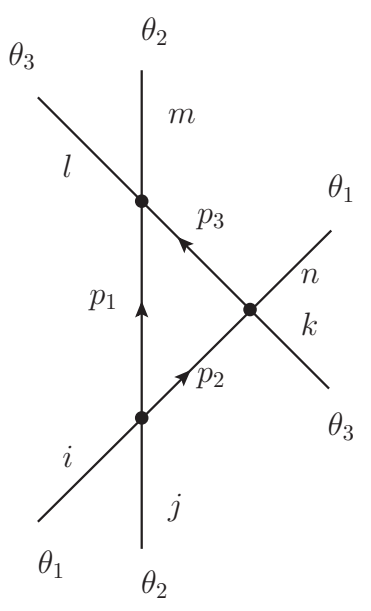

$(a)$

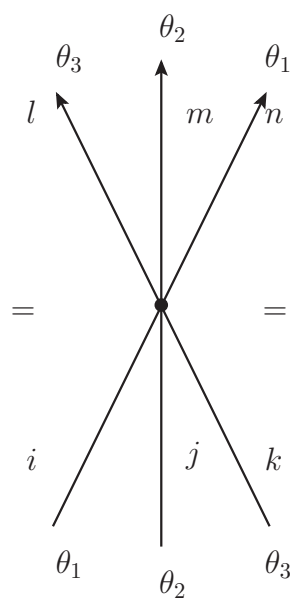

(b)

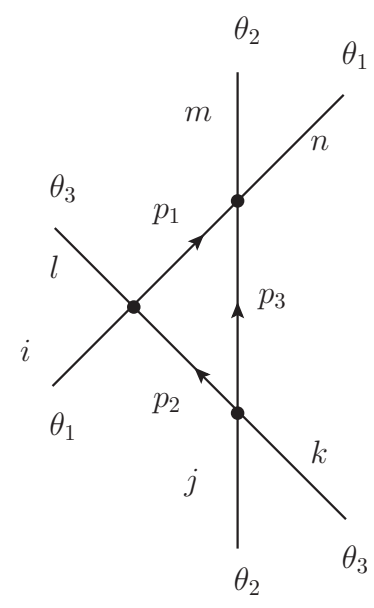

$(c)$

Figura 3.3: Três trajetórias possíveis para o espalhamento de $3 \rightarrow 3$ partículas. A igualdade das amplitudes de espalhamento em (a), (b) e (c) equivale à equação de Yang-Baxter (3.5.2).

do operador definido acima corresponde simplesmente a deslocar a $i$-ésima partícula por $\varphi^{\prime}\left(p_{i}\right)$. Tomando tais operadores como $e^{i \xi Q_{s}}$, o fator de fase é dado simplesmente por $\varphi_{s}(p)=-\xi p^{s}$, e, conseqüentemente, uma partícula com momento $p_{i}$ terá sua posição deslocada por $-s \xi p_{i}^{s-1}$. Para o caso do operador de momento, $s=1$, todas as partículas são deslocadas pela mesma quantidade $\xi$, porém, para $s>1$, partículas com momentos diferentes são transladadas diferentemente, e podem, pois, ser deslocadas relativamente uma às outras. De fato, como $Q_{s}$ é uma carga conservada, deve comutar com a matriz $S$, e portanto

$$
\langle\text { final }|S| \text { inicial }\rangle=\left\langle\text { final }\left|e^{i \xi Q_{s}} S e^{-i \xi Q_{s}}\right| \text { inicial }\right\rangle .
$$

Logo, podemos utilizar o operador $e^{i \xi Q_{s}}$ para rearranjar as configurações iniciais e finais sem alterar as amplitudes, como na figura 3.2.

Dessa forma podemos considerar o espalhamento $3 \rightarrow 3$ partículas:

$$
\left|A_{i}\left(\theta_{1}\right) A_{j}\left(\theta_{2}\right) A_{k}\left(\theta_{3}\right)\right\rangle_{i}=\sum_{l, m, n} S_{i j k}^{l m n}\left(\theta_{m n}\right)\left|A_{l}\left(\theta_{3}\right) A_{m}\left(\theta_{2}\right) A_{n}\left(\theta_{1}\right)\right\rangle_{f},
$$

que pode ocorrer de três maneiras distintas, vide figura 3.3, dependendo das posições dos pacotes de onda incidentes. Na figura (3.3), observamos que o processo de espalhamento ocorre através de interações envolvendo apenas duas partículas tanto em (a), quanto em (c); ao passo que em (b) verificamos uma genuína colisão de três corpos. Notamos porém, que de acordo com a discussão acima, é possível atuar sobre o estado inicial com $\exp \left(i \xi Q_{N}\right)$, gerando um deslocamento de cada partícula proporcional ao seu momento. Ao permitirmos que o parâmetro real $\xi$ torne-se arbitrariamente grande, podemos gerar uma mudança arbitrária na posição relativa de duas partículas. Assim, ao deslocarmos a linha 1 paralelamente a ela mesma, relativamente a linha 2 ou a linha 3, podemos ir do 
diagrama em (a) para o diagrama em (c) continuamente. Uma vez que $Q_{N}$ é uma carga conservada, ela comuta com a Hamiltoniana e por isso deixa a matriz $S$ invariante. Logo, a amplitude para os três processos descritos na figura (3.3) deve ser a mesma, denotando-as por:

$$
\begin{aligned}
& \mathcal{A}_{(a)}=\sum_{p_{1}, p_{2}, p_{3}} S_{i j}^{p_{1} p_{2}}\left(\theta_{12}\right) S_{p_{2} k}^{p_{3} n}\left(\theta_{13}\right) S_{p_{1} p_{3}}^{I m}\left(\theta_{23}\right), \\
& \mathcal{A}_{(b)}=S_{i j k}^{I m n}\left(\theta_{1}, \theta_{2}, \theta_{3}\right), \\
& \mathcal{A}_{(c)}=\sum_{p_{1}, p_{2}, p_{3}} S_{j k}^{p_{2} p_{3}}\left(\theta_{23}\right) S_{i p_{2}}^{I p_{1}}\left(\theta_{13}\right) S_{p_{1} p_{3}}^{m n}\left(\theta_{12}\right),
\end{aligned}
$$

inferimos

$$
\begin{aligned}
S_{i j k}^{I m n}\left(\theta_{1}, \theta_{2}, \theta_{3}\right) & =\sum_{p_{1}, p_{2}, p_{3}} S_{i j}^{p_{1} p_{2}}\left(\theta_{12}\right) S_{p_{2} k}^{p_{3} n}\left(\theta_{13}\right) S_{p_{1} p_{3}}^{I m}\left(\theta_{23}\right), \\
& =\sum_{p_{1}, p_{2}, p_{3}} S_{j k}^{p_{2} p_{3}}\left(\theta_{23}\right) S_{i p_{2}}^{I p_{1}}\left(\theta_{13}\right) S_{p_{1} p_{3}}^{m n}\left(\theta_{12}\right),
\end{aligned}
$$

que equivale a equação de Yang-Baxter (3.5.2) para a matriz S:

$$
S_{12}\left(\theta_{12}\right) S_{13}\left(\theta_{13}\right) S_{23}\left(\theta_{23}\right)=S_{23}\left(\theta_{23}\right) S_{13}\left(\theta_{13}\right) S_{12}\left(\theta_{12}\right)
$$

Portanto, temos que tal espalhamento pode ser descrito em termos do produto de matrizes $S$ para apenas duas partículas, e a condição 3. da definição 3.5.1 está estabelecida para $M=3$. É possível estender indutivamente esse argumento, mostrando que um conjunto de $M$ partículas incidentes sempre pode ser rearranjado de modo a garantir que o espalhamento ocorra como uma seqüência de eventos, nos quais apenas $M-1$ partículas participam. A equação de Yang-Baxter aparece como uma condição necessária para fatorabilidade do espalhamento [25].

É importante ressaltar que o argumento que utilizamos para relacionar a existência de infinitas cargas conservadas com a fatorabilidade do espalhamento, depende fortemente da hipótese que fizemos sobre a forma das quantidades conservadas, isto é, que elas devem ser expressas como integrais de uma densidade local. Conseqüentemente, não devemos esperar que qualquer modelo a possuir um conjunto infinito de cargas conservadas apresente um espalhamento fatorável. Naturalmente, podemos tornar precisas tais considerações sobre a fatorabilidade do espalhamento e a sua conexão com a existência de integrais do movimento para o caso não-relativístico. Sua viabilidade está intimamente relacionada com a forma das funções de onda, obtida a partir do Ansatz de Bethe, conforme discussão na seção 3.1. Com isso podemos enunciar a definição de Sutherland para integrabilidade quântica [59], que utilizaremos para nortear todas as futuras discussões.

Definição 3.5.2. Um sistema é quanticamente integrável se o espalhamento por ele suportado for fatorável. 


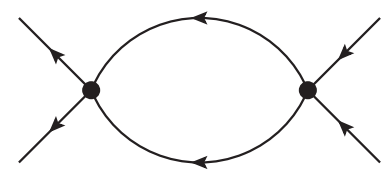

Figura 3.4: Diagrama com 1 laço para o espalhamento de duas partículas no modelo NLS.

\subsection{Fatorabilidade da Matriz $S$ para o NLS via Diagramas de Feynman}

Nesta seção abordamos a questão da integrabilidade quântica do modelo NLS do ponto de vista da fatorabilidade de sua matriz $S$, que pode ser calculada a partir de amplitudes de espalhamento obtidas da soma de diagramas de Feynman. Essa conexão com o formalismo usual da teoria quântica de campos fornece uma ferramenta poderosa para se sondar a integrabilidade quântica de um dado modelo, pois podemos através de métodos perturbativos (a soma de diagramas de Feynman) obter informações claramente não-perturbativas.

A matriz S para o NLS foi originalmente obtida por Yang [60] ao empregar o Ansatz de Bethe, uma característica importante de seu resultado é a sua analiticidade quando a constante de acoplamento se aproxima de zero. Logo, deve ser possível obter o mesmo resultado por intermédio de técnicas usuais no contexto de teorias quânticas de campos, ao considerarmos a soma dos diagramas de Feynman para o espalhamento de $N$ partículas. De fato, para $N=2$ todos os gráficos são obtidos das potências do diagrama com 1 laço (figura 3.4). De forma que a série perturbativa resultante $\sum_{n=0}^{\infty} A^{n}$ pode ser explicitamente calculada, fornecendo o resultado de Yang. Contudo, para o espalhamento envolvendo três ou mais partículas, os gráficos de Feynman são, em geral, difíceis de se caracterizar e os fatores combinatórios, substancialmente mais complicados. No que se segue, consideramos o espalhamento de três partículas e obtemos a respectiva matriz $S$, demonstrando, pois, a sua fatorabilidade [39].

Ao Hamiltoniano quântico no setor de $N$-partículas (3.1.1), corresponde a seguinte densidade Lagrangeana:

$$
\mathscr{L}=\frac{i}{2}\left[\psi^{\dagger} \partial_{t} \psi-\partial_{t} \psi^{\dagger} \psi\right]-\left|\partial_{x} \psi\right|^{2}-g|\psi|^{4},
$$

da qual podemos facilmente ler as regras de Feynman da teoria:

(a.1) Uma integração sobre o momento e a energia $\int \frac{d^{2} q}{4 \pi^{2}}$ para cada ciclo fechado;

(a.2) Um propagador $\frac{i}{q^{0}-q^{2}+i \epsilon}$ para cada linha interna;

(a.3) Um fator de $(-4 i g)$ para cada vértice.

A integral ainda deve ser multiplicada pelos fatores de simetria usuais para linhas idênticas e por um fator convencional de $(-i)$.

É conveniente simplificar essas regras de Feynman, ao efetuarmos todas as integrais de energia. Uma vez que o integrando é sempre um produto de pólos simples, podemos simplesmente integrar 
usando o teorema dos resíduos. Assim para cada diagrama introduzimos cortes, linhas que separam dois vértices adjacentes, de forma que, para um diagrama com $n$ vértices temos $n-1$ cortes. Conseqüentemente, as regras de Feynman simplificadas são:

(b.1) Uma integração sobre o momento $\int \frac{d p}{2 \pi}$ para cada ciclo fechado;

(b.2) Um fator $\frac{i}{E-\sum_{l} E_{l}+i \epsilon}$ para cada corte, onde $E$ é a energia inicial total e $\sum_{l} E_{l}$ é a soma das energias das linhas cortadas, sendo que todas as linhas internas devem ser tomadas na camada de massa $E_{l}=q_{l}^{2}$;

(b.3) Um fator de (-4ig) para cada vértice;

(b.4) Se houver mais do que uma forma para se cortar um diagrama em particular, o valor do diagrama é dado pela soma de todas as possíveis formas de cortá-lo.

Esse último item é relevante apenas para gráficos nos quais a ordenação temporal dos vértices não é fixada pela topologia do mesmo. Entretanto, situações nas quais tal regra é necessária só ocorrem para diagramas descrevendo o espalhamento de quatro ou mais partículas, que não serão encontrados no presente cálculo.

Existe ainda uma pequena sutileza ao utilizarmos as regras $(b)$ para deduzirmos equações integrais, no que tange a diferença entre a continuação de amplitudes com partículas fora da camada de massa, mas com a energia total conservada entre os estados inicial e final, correspondendo ao conjunto de regras (a) e, a continuação de uma amplitude fora da camada de energia, mas com as partículas na camada de massa, regras $(b)$. No contexto da formulação dependente do tempo da teoria de perturbações, todas as amplitudes conservam a energia total e as integrações tiram as partículas da camada de massa. Entretanto, as equações integrais consideradas a seguir, no espírito da formulação independente do tempo, envolvem integrações sobre amplitudes para as quais as partículas permanecem na camada de massa, enquanto que a energia total não. Tais amplitudes são definidas pelo conjunto de regras (b), ou equivalentemente, por

$$
-2 \pi \delta\left(p_{f}-p_{i}\right) T_{f ; i}=\left\langle\text { final }\left|H_{1} U(0,-\infty)\right| \text { inicial }\right\rangle
$$

onde |final $\rangle$ e |inicial $\rangle$ são autoestados de $H_{0}$ e $U$ é o operador de evolução temporal usual na representação de interação. A conexão entre esses dois tratamentos da amplitude é dado pela integração da energia que fizemos para deduzir as regras (b) a partir das regras (a). Assim, ao considerarmos o conjunto de regras (b) podemos resolver as equações integrais para (3.6.2) mesmo se $E_{f} \neq E_{i}$.

\subsubsection{Somando Todos os Diagramas}

Ao empregarmos o conjunto de regras $(b)$, resolvemos todas as integrais sobre as energias trivialmente, entretanto, as integrais restantes, sobre os momentos, envolvem denominadores quadráticos, de forma que para gráficos individuais, seu cômputo se torna proibitivo além das ordens mais baixas na teoria de perturbação. Simplificações interessantes, no entanto, são introduzidas quando um certo conjunto de gráficos são somados. Para isso consideramos o conjunto de equações integrais para a 
amplitude de espalhamento completa de três partículas, doravante denotada por $T$, escrito graficamente nas figuras 3.5, 3.6 e 3.7. É importante notar que tais diagramas, de fato, representam uma soma sobre gráficos com a mesma topologia, porém distintas permutações dos momentos externos, os quais são tomados, de agora em diante, na camada de massa.
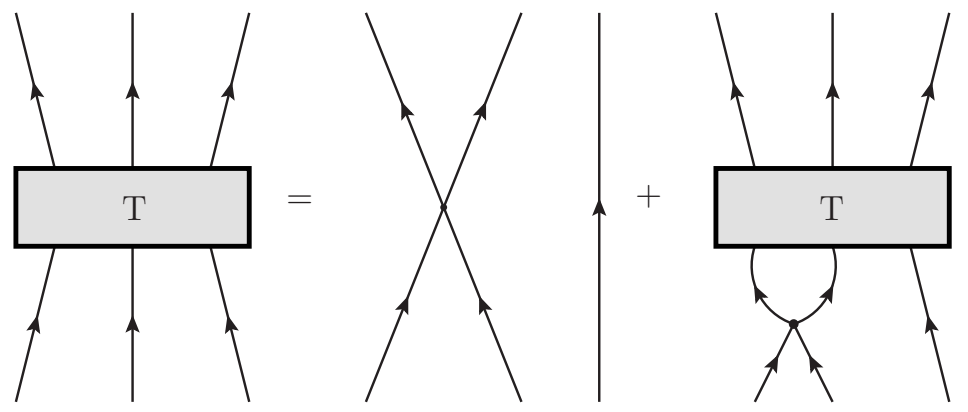

Figura 3.5: Primeira equação integral para $T$.

A idéia do cálculo que se segue é primeiramente obter as expressões analíticas das equações integrais representadas nas figuras 3.5, 3.6 e 3.7 utilizando o conjunto (b) de regras Feynman, em seguida usamos as duas primeiras equações como identidades para eliminar as integrações na terceira equação e assim obter uma equação algébrica para $T$. É interessante notar, nesse estágio, que as equações integrais que consideramos são obtidas a partir de expansões da amplitude de espalhamento completa em termos dos diagramas de Feynman até a $n$-ésima ordem, somados aos gráficos dessa ordem multiplicados pela amplitude de espalhamento completa.

É interessante considerar os momentos das partículas no decorrer do espalhamento como as componentes de um vetor de $\mathbb{R}^{3}$, de sorte que $T(\mathbf{p} ; \mathbf{k})$ denota a amplitude de espalhamento de um estado com os momentos $\left(k_{1}, k_{2}, k_{3}\right)$ indo para um estado com os momentos $\left(p_{1}, p_{2}, p_{3}\right)$. Outra notação conveniente no atual contexto é fornecida pelo operador de simetrização sobre os momentos, também denominado simetrizador e definido como:

$$
\mathcal{S}_{p}[f(\mathbf{p})]=\frac{1}{3 !} \sum_{A} f(A \mathbf{p})
$$

onde o vetor $A \mathbf{p}=\left(p_{A_{1}}, p_{A_{2}}, p_{A_{3}}\right)$ e a soma é sobre todas as possíveis permutações $\left(A_{1}, A_{2}, A_{3}\right)$ de $(1,2,3)$.

A expressão analítica correspondente à primeira equação integral, descrita na figura 3.5 é:

$$
T(\mathbf{p} ; \mathbf{k})=T^{(1)}(\mathbf{p} ; \mathbf{k})+\frac{1}{2}(-4 i g) 3 \mathcal{S}_{k} \int \frac{d q}{2 \pi} \frac{i T\left(\mathbf{p} ;\left(q, k_{1}+k_{2}-q, k_{3}\right)\right)}{k_{1}^{2}+k_{2}^{2}-q^{2}-\left(k_{1}+k_{2}-q\right)^{2}+i \epsilon},
$$

onde $T^{(1)}$ representa a soma de todos os gráficos de primeira ordem, isto é,

$$
T^{(1)}(\mathbf{p}, \mathbf{k})=(12 i g) 2 \pi i \mathcal{S}_{p}\left[\delta\left(p_{1}-k_{1}\right)+\delta\left(p_{2}-k_{2}\right)+\delta\left(p_{3}-k_{3}\right)\right] .
$$

O fator de $\frac{1}{2}$ que aparece multiplicando a integral em (3.6.4) é simplesmente o fator de simetria 
requerido para os gráficos nos quais os dois primeiros vértices formam um laço fechado. Porém, diagramas para os quais esse não é o caso, são contados duas vezes quando a equação é iterada e, portanto esse fator de $\frac{1}{2}$ aparece como um fator global. Podemos introduzir algumas funções delta na equação (3.6.4) de modo a escrevê-la de uma forma mais simétrica nos momentos intermediários integrados:

$$
T(\mathbf{p} ; \mathbf{k})=T^{(1)}(\mathbf{p} ; \mathbf{k})-\frac{1}{6(2 \pi)^{2}} \int d^{3} q \delta\left(\sum q-\sum k\right) T(\mathbf{p} ; \mathbf{q}) \frac{1}{\sum k^{2}-\sum q^{2}+i \epsilon} T^{(1)}(\mathbf{q} ; \mathbf{k}),
$$

onde por simplicidade omitimos os limites das somas. Doravante denotamos $\sum f:=\sum_{i=1}^{3} f_{i}$, exceto quando explicitamente escrito de outra forma. Um procedimento análogo nos permite escrever as expressões analíticas para a segunda equação integral, descrita na figura 3.6:

$$
\begin{aligned}
T(\mathbf{p} ; \mathbf{k}) & =T^{(1)}(\mathbf{p}, \mathbf{k})+T^{(2)}(\mathbf{p}, \mathbf{k})- \\
& -\frac{1}{6(2 \pi)^{2}} \int d^{3} q \delta\left(\sum q-\sum k\right) T(\mathbf{p} ; \mathbf{q}) \frac{1}{\sum k^{2}-\sum q^{2}+i \epsilon} T^{(2)}(\mathbf{q} ; \mathbf{k}),
\end{aligned}
$$

e para a terceira equação integral descrita na figura 3.7:
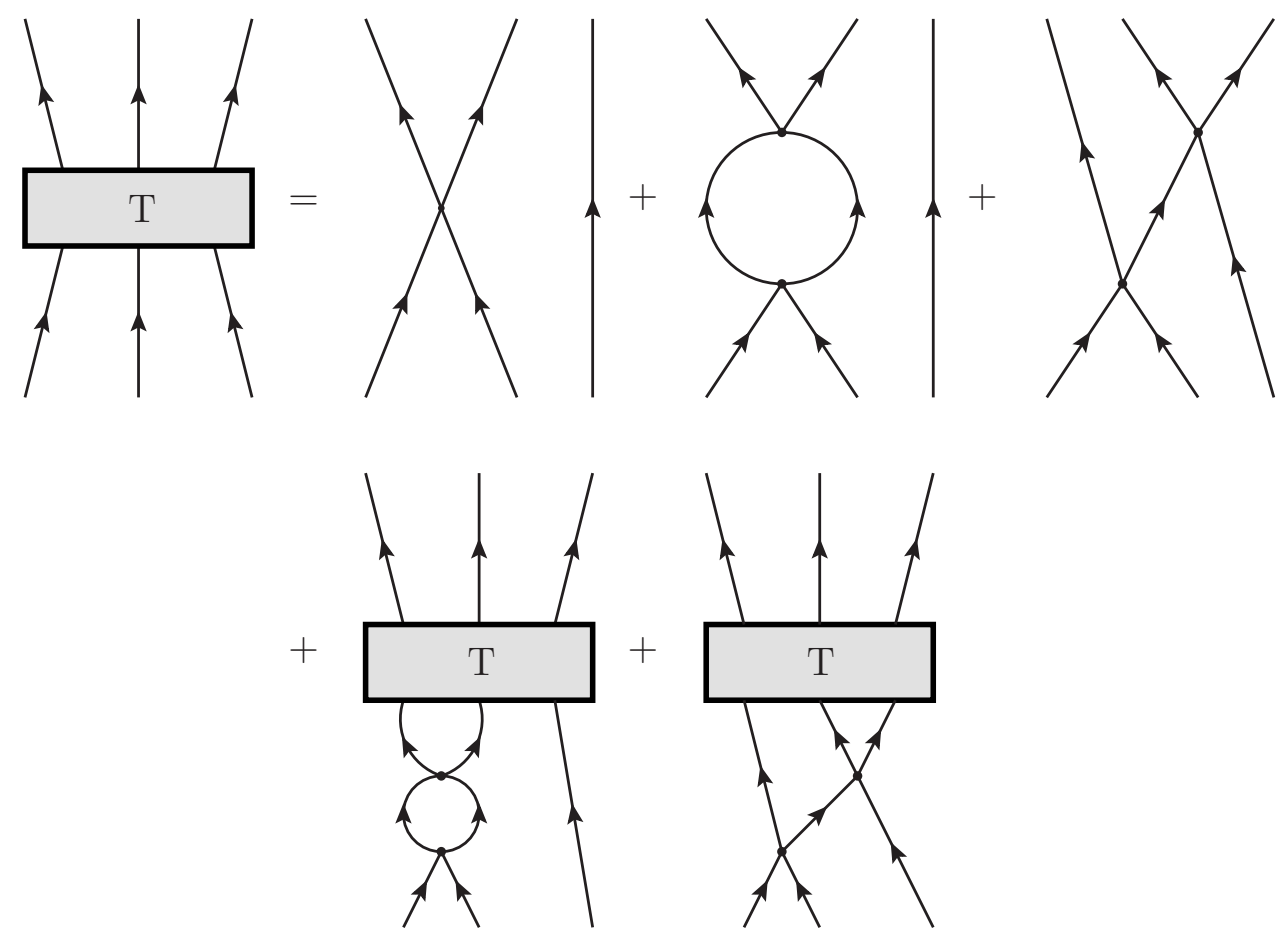

Figura 3.6: Segunda equação integral para $T$.

$$
\begin{aligned}
T(\mathbf{p} ; \mathbf{k}) & =T^{(1)}(\mathbf{p}, \mathbf{k})+T^{(2)}(\mathbf{p}, \mathbf{k})+T^{(3)}(\mathbf{p}, \mathbf{k}) \\
& -\frac{1}{6(2 \pi)^{2}} \int d^{3} q \delta\left(\sum q-\sum k\right) T(\mathbf{p} ; \mathbf{q}) \frac{1}{\sum k^{2}-\sum q^{2}+i \epsilon} T^{(3)}(\mathbf{q} ; \mathbf{k})
\end{aligned}
$$



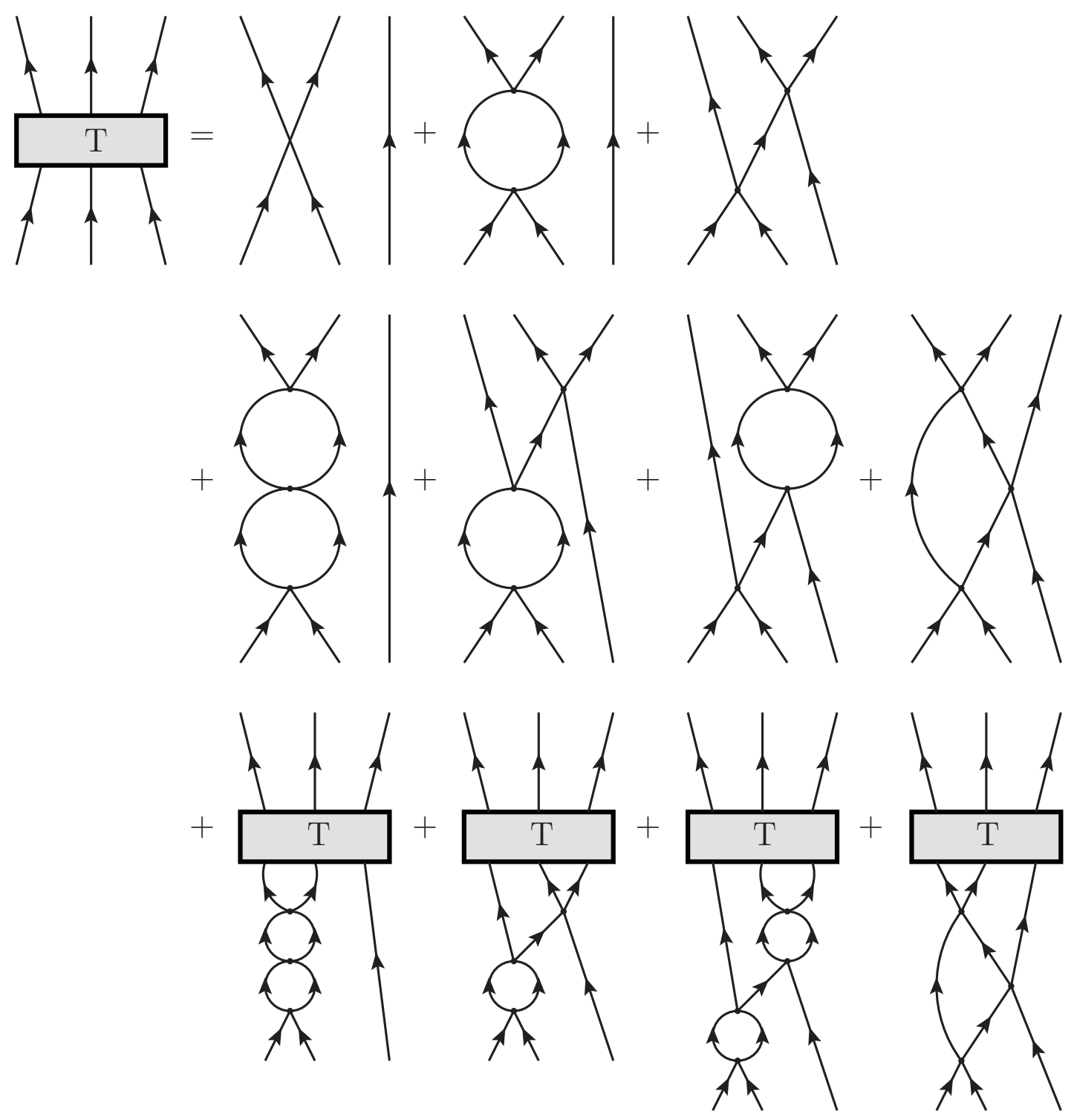

Figura 3.7: Terceira equação integral para $T$.

É conveniente trabalhar separadamente as partes conexas e desconexas das amplitudes de espaIhamento $T^{(2)}$ e $T^{(3)}$, que, em termos dessa decomposição, são escritas como: $T^{(n)}=T_{C}^{(n)}+T_{D}^{(n)}$. Os diagramas, que compõem o termo desconexo de segunda ordem, são trivialmente calculados ao efetuarmos a integral sobre o laço fechado, podemos sem perda alguma de generalidade ordenar os momentos incidentes, de forma que $k_{1}<k_{2}<k_{3}$. Obtemos, pois,

$$
T_{D}^{(2)}(\mathbf{p} ; \mathbf{k})=(12 i g)(2 \pi i) \mathcal{S}_{p}\left[x_{12} \delta\left(p_{3}-k_{3}\right)+x_{13} \delta\left(p_{2}-k_{2}\right)+x_{23} \delta\left(p_{1}-k_{1}\right)\right],
$$


onde introduzimos:

$$
x_{i j}=\frac{i g}{k_{i}-k_{j}} .
$$

Já a soma dos gráficos conexos fornece:

$$
T_{C}^{(2)}(\mathbf{p} ; \mathbf{k})=-i(-4 i g)^{2} 9 \mathcal{S}_{p, k}\left[\frac{1}{k_{1}^{2}+k_{2}^{2}-p_{1}^{2}-\left(k_{1}+k_{2}-p_{1}\right)^{2}+i \epsilon}\right] .
$$

Para a nossa escolha do ordenamento dos momentos incidentes, podemos verificar facilmente a validade da identidade seguinte:

$$
\frac{i g}{k_{1}^{2}+k_{2}^{2}-p_{1}^{2}-\left(k_{1}+k_{2}-p_{1}\right)^{2}+i \epsilon}=\frac{x_{12}}{2}\left(\frac{1}{p_{1}-k_{2}-i \epsilon}+\frac{1}{k_{1}-p_{1}-i \epsilon}\right) .
$$

A identidade (3.6.10) juntamente com suas permutações permite que rescrevamos (3.6.9) ao considerarmos explicitamente todas as permutações sobre $k$,

$$
\begin{aligned}
T_{C}^{(2)}(\mathbf{p} ; \mathbf{k}) & =24 i g \mathcal{S}_{p}\left[x_{12}\left(\frac{1}{p_{1}-k_{2}-i \epsilon}+\frac{1}{k_{1}-p_{1}-i \epsilon}\right)+x_{13}\left(\frac{1}{p_{1}-k_{3}-i \epsilon}+\frac{1}{k_{1}-p_{1}-i \epsilon}\right)+\right. \\
& \left.+x_{23}\left(\frac{1}{p_{1}-k_{3}-i \epsilon}+\frac{1}{k_{2}-p_{1}-i \epsilon}\right)\right] .
\end{aligned}
$$

A parte desconexa da amplitude de espalhamento de terceira ordem é calculada ao computarmos as integrais sobre os laços fechados, fornecendo basicamente a expressão de $T_{D}^{(2)}$ acrescida de um fator $x_{i j}$ correspondente ao laço fechado adicional,

$$
T_{D}^{(3)}(\mathbf{p} ; \mathbf{k})=(12 i g)(2 \pi i) \mathcal{S}_{p}\left[x_{12}^{2} \delta\left(p_{3}-k_{3}\right)+x_{13}^{2} \delta\left(p_{2}-k_{2}\right)+x_{23}^{2} \delta\left(p_{1}-k_{1}\right)\right] .
$$

Entretanto, a contribuição dos gráficos conexos de terceira ordem é bem mais complexa, uma vez que envolve a soma de três diagramas distintos. Para simplificar um pouco, consideramos a contribuição de cada diagrama separadamente, $T_{C}^{(3)}=T_{C_{1}}^{(3)}+T_{C_{2}}^{(3)}+T_{C_{3}}^{(3)}$, onde os três termos correspondem aos gráficos representados pelo quinto, sexto e sétimo diagramas na figura 3.7, respectivamente. A expressão para $T_{C_{1}}^{(3)}$ é obtida da mesma forma que $T_{D}^{(2)}$, com uma única diferença, a presença de um fator adicional de $x_{i j}$ oriundo do laço extra presente no diagrama, novamente podemos usar a identidade (3.6.10) para escrever:

$$
\begin{aligned}
T_{C_{1}}^{(3)}(\mathbf{p} ; \mathbf{k}) & =24 i g \mathcal{S}_{p}\left[x_{12}^{2}\left(\frac{1}{p_{1}-k_{2}-i \epsilon}+\frac{1}{k_{1}-p_{1}-i \epsilon}\right)+x_{13}^{2}\left(\frac{1}{p_{1}-k_{3}-i \epsilon}+\frac{1}{k_{1}-p_{1}-i \epsilon}\right)+\right. \\
& \left.+x_{23}^{2}\left(\frac{1}{p_{1}-k_{3}-i \epsilon}+\frac{1}{k_{2}-p_{1}-i \epsilon}\right)\right] .
\end{aligned}
$$

É conveniente combinarmos as expressões analíticas de $T_{C_{2}}^{(3)}$ e $T_{C_{3}}^{(3)}$ antes de calcularmos as integrais sobre os laços fechados. Para $T_{C_{2}}^{(3)}$ podemos usar a identidade (3.6.10) para fatorar o denominador que aparece fora da integral, enquanto que deixamos intocado o denominador que 
aparece sob o símbolo de integração,

$$
\begin{aligned}
& T_{C_{2}}^{(3)}(\mathbf{p} ; \mathbf{k})=\left(16 i g^{2}\right) 3 \mathcal{S}_{p}\left\{\left[x_{12}\left(\frac{1}{p_{1}-k_{2}-i \epsilon}+\frac{1}{k_{1}-p_{1}-i \epsilon}\right)+x_{13}\left(\frac{1}{p_{1}-k_{3}-i \epsilon}+\frac{1}{k_{1}-p_{1}-i \epsilon}\right)+\right.\right. \\
& \left.\left.\quad+x_{23}\left(\frac{1}{p_{1}-k_{3}-i \epsilon}+\frac{1}{k_{2}-p_{1}-i \epsilon}\right)\right] \int \frac{d q}{2 \pi} \frac{1}{\sum k^{2}-p_{1}^{2}-q^{2}-\left(p_{2}+p_{3}-q\right)^{2}+i \epsilon}\right\} .
\end{aligned}
$$

Já para $T_{C_{3}}^{(3)}$ ambos os denominadores estão integrados, conquanto, ainda é conveniente fatorar um deles,

$$
\begin{aligned}
T_{C_{3}}^{(3)}(\mathbf{p} ; \mathbf{k}) & =\left(32 i g^{2}\right) 3 \mathcal{S}_{p}\left\{\int \frac { d q } { 2 \pi } \left[x_{12}\left(\frac{1}{q-k_{2}-i \epsilon}+\frac{1}{k_{1}-q-i \epsilon}\right)+x_{13}\left(\frac{1}{q-k_{3}-i \epsilon}+\frac{1}{k_{1}-q-i \epsilon}\right)+\right.\right. \\
& \left.\left.+x_{23}\left(\frac{1}{q-k_{3}-i \epsilon}+\frac{1}{k_{2}-q-i \epsilon}\right)\right] \frac{1}{\sum k^{2}-p_{1}^{2}-q^{2}-\left(p_{2}+p_{3}-q\right)^{2}+i \epsilon}\right\}
\end{aligned}
$$

Podemos, agora, somar as equações (3.6.14) e (3.6.15), ao fazermos isso, notamos que a soma dos termos entre colchetes pode ser substituída pela simetrização sobre o vetor $q=\left(p_{1}, q, p_{2}+p_{3}-q\right)$, de forma a obtermos,

$$
\begin{aligned}
T_{C_{2}}^{(3)}+T_{C_{3}}^{(3)} & =9\left(16 i g^{2}\right) \mathcal{S}_{p}\left\{\int \frac { d ^ { 3 } q } { 2 \pi } \frac { \delta ( q _ { 1 } - p _ { 1 } ) \delta ( \sum q - \sum p ) } { \sum k ^ { 2 } - \sum q ^ { 2 } + i \epsilon } \mathcal { S } _ { q } \left[x_{12}\left(\frac{1}{q_{1}-k_{2}-i \epsilon}+\frac{1}{k_{1}-q_{1}-i \epsilon}\right)+\right.\right. \\
& \left.\left.+x_{13}\left(\frac{1}{q_{1}-k_{3}-i \epsilon}+\frac{1}{k_{1}-q_{1}-i \epsilon}\right)+x_{23}\left(\frac{1}{q_{1}-k_{3}-i \epsilon}+\frac{1}{k_{2}-q_{1}-i \epsilon}\right)\right]\right\} .
\end{aligned}
$$

O denominador restante em (3.6.16) não pode ser fatorado com o auxílio da identidade (3.6.10), entretanto, devido à conservação do momento total e à igualdade $\sum q=\sum p$ introduzida por uma função delta em (3.6.16) podemos escrever a seguinte identidade:

$$
\mathcal{S}_{q}\left[\frac{k_{1}-k_{2}}{q_{3}-k_{3}-i \epsilon}+\frac{k_{2}-k_{3}}{k_{1}-q_{1}-i \epsilon}\right]=\mathcal{S}_{q}\left[\frac{\sum k^{2}-\sum q^{2}+i \epsilon\left(k_{3}-k_{1}\right)}{\left(q_{3}-k_{3}-i \epsilon\right)\left(k_{1}-q_{1}-i \epsilon\right)}\right]
$$

que, juntamente com outras duas obtidas a partir das permutações: $\left(k_{1}, k_{2}, k_{3}\right) \rightarrow\left(k_{1}, k_{3}, k_{2}\right)$ e $\left(k_{1}, k_{2}, k_{3}\right) \rightarrow\left(k_{2}, k_{1}, k_{3}\right)$, permite que usemos o seguinte fato:

$$
\begin{aligned}
\int \frac{d^{3} q}{2 \pi} \delta\left(q_{1}-p_{1}\right) \delta\left(\sum q-\sum p\right) & \mathcal{S}_{q}\left[\frac{1}{\left(k_{1}-q_{1}-i \epsilon\right)\left(q_{3}-k_{3}-i \epsilon\right)}\right] \\
& =\frac{i}{6}\left(\frac{1}{k_{1}-p_{1}-i \epsilon}+\frac{1}{p_{1}-k_{3}-i \epsilon}\right) .
\end{aligned}
$$

para efetuarmos todas as integrais restantes e podermos expressar (3.6.16) como:

$$
\begin{aligned}
T_{C_{2}}^{(3)}+T_{C_{3}}^{(3)} & =24 i g \mathcal{S}_{p}\left[x_{12} x_{13}\left(\frac{2}{k_{1}-p_{1}-i \epsilon}+\frac{1}{p_{1}-k_{2}-i \epsilon}+\frac{1}{p_{1}-k_{3}-i \epsilon}\right)+\right. \\
& +x_{13} x_{23}\left(\frac{1}{k_{1}-p_{1}-i \epsilon}+\frac{1}{k_{2}-p_{1}-i \epsilon}+\frac{2}{p_{3}-k_{3}-i \epsilon}\right)+ \\
& \left.+x_{12} x_{23}\left(\frac{1}{k_{1}-p_{1}-i \epsilon}+\frac{1}{p_{1}-k_{3}-i \epsilon}\right)\right] .
\end{aligned}
$$

Podemos sem dificuldade somar (3.6.19) com (3.6.13) para obter amplitude de espalhamento 
conexa de terceira ordem,

$$
\begin{aligned}
T_{C}^{(3)}(\mathbf{p} ; \mathbf{k})=24 i g \mathcal{S}_{p} & {\left[\frac{\left(x_{12}+x_{13}\right)\left(x_{12}+x_{13}+x_{23}\right)}{k_{1}-p_{1}-i \epsilon}+\frac{x_{12}\left(x_{12}+x_{13}\right)}{p_{1}-k_{2}-i \epsilon}+\right.} \\
& \left.+\frac{x_{23}\left(x_{13}+x_{23}\right)}{k_{2}-p_{1}-i \epsilon}+\frac{\left(x_{13}+x_{23}\right)\left(x_{12}+x_{13}+x_{23}\right)}{p_{1}-k_{3}-i \epsilon}\right] .
\end{aligned}
$$

Ao compararmos essa expressão com a equação (3.6.13) verificamos uma grande similaridade entre as amplitudes de espalhamento conexas de segunda e terceira ordem, o que nos permite escrever,

$$
T_{C}^{(3)}(\mathbf{p} ; \mathbf{k})=\left(x_{12}+x_{13}+x_{23}\right) T_{C}^{(2)}-24 i g x_{12} x_{23} \mathcal{S}_{p}\left[2 \pi i \delta\left(p_{2}-k_{2}\right)\right]
$$

Uma relação semelhante entre as amplitudes desconexas de segunda e terceira ordem é providenciada pelas equações (3.6.5), (3.6.7) e (3.6.12),

$$
\begin{aligned}
T_{D}^{(3)}(\mathbf{p} ; \mathbf{k}) & =\left(x_{12}+x_{13}+x_{23}\right) T_{D}^{(2)}(\mathbf{p} ; \mathbf{k})-\left(x_{12} x_{13}+x_{12} x_{23}+x_{13} x_{23}\right) T^{(1)}(\mathbf{p} ; \mathbf{k})+ \\
& +12 i g(2 \pi i) \mathcal{S}_{p}\left[x_{12} x_{13} \delta\left(p_{1}-k_{1}\right)+x_{12} x_{23} \delta\left(p_{2}-k_{2}\right)+x_{13} x_{23} \delta\left(p_{3}-k_{3}\right)\right] .
\end{aligned}
$$

Finalmente, ao adicionarmos (3.6.21) a (3.6.22) obtemos a amplitude de espalhamento de terceira ordem completa,

$$
\begin{aligned}
T^{(3)}(\mathbf{p} ; \mathbf{k}) & =\left(x_{12}+x_{13}+x_{23}\right) T^{(2)}(\mathbf{p} ; \mathbf{k})-\left(x_{12} x_{13}+x_{12} x_{23}+x_{13} x_{23}\right) T^{(1)}(\mathbf{p} ; \mathbf{k})+ \\
& +12 i g(2 \pi i) \mathcal{S}_{p}\left[x_{12} x_{13} \delta\left(p_{1}-k_{1}\right)+x_{12} x_{23} \delta\left(p_{2}-k_{2}\right)+x_{13} x_{23} \delta\left(p_{3}-k_{3}\right)\right] .
\end{aligned}
$$

A equação (3.6.23) juntamente com as equações (3.6.6a) e (3.6.6b) são suficientes para eliminar a integral da equação (3.6.6c), pois, ao substituirmos a equação (3.6.23) no integrando de (3.6.6c), os dois primeiros termos fornecem as mesmas integrais que aparecem em (3.6.6a) e (3.6.6b). O conjunto de equações integrais (3.6.6) pode ser escrito em uma forma mais sucinta como:

$$
\begin{aligned}
& T=T^{(1)}+I_{1}, \\
& T=T^{(1)}+T^{(2)}+I_{2}, \\
& T=T^{(1)}+T^{(2)}+T^{(3)}+I_{3},
\end{aligned}
$$

enquanto que a equação (3.6.23) assume a seguinte forma:

$$
I_{3}=\phi_{1}(x) I_{2}-\phi_{2}(x) I_{1}+J_{3}
$$

onde os $\phi_{j}(x)$ são polinômios simétricos nas variáveis $x$, definidos por:

$$
\begin{aligned}
& \phi_{1}(x):=x_{12}+x_{13}+x_{23} \\
& \phi_{2}(x):=x_{12} x_{13}+x_{12} x_{23}+x_{13} x_{23} \\
& \phi_{3}(x):=x_{12} x_{13} x_{23}
\end{aligned}
$$

e $J_{3}$ é a integral obtida ao se substituir o último termo de (3.6.25) no integrando de (3.6.6c), se 
também fatorarmos o denominador com o auxílio da identidade (3.6.10) obtemos:

$$
J_{3}=-\frac{i}{\pi} \phi_{3}(x) \int d^{3} q \delta\left(\sum q-\sum k\right) T(\mathbf{p} ; \mathbf{k}) h(\mathbf{q} ; \mathbf{k}),
$$

onde introduzimos:

$$
h(\mathbf{q} ; \mathbf{k}):=\mathcal{S}_{q}\left[\frac{\delta\left(q_{1}-k_{1}\right)}{q_{2}-k_{3}-i \epsilon}-\frac{\delta\left(q_{2}-k_{2}\right)}{q_{3}-k_{3}-i \epsilon}+\frac{\delta\left(q_{3}-k_{3}\right)}{q_{1}-k_{2}-i \epsilon}\right] .
$$

Calcular a integral em (3.6.27) é possível graças a uma propriedade de $T(\mathbf{p}, \mathbf{q})$, exibida por uma versão generalizada da equação integral (3.6.6a). Para isso precisamos considerar uma continuação da amplitude de espalhamento na energia inicial, $T_{z}(\mathbf{p} ; \mathbf{q})$, que é calculada através das regras $(b)$, mas com o denominador de energia, (b.2), substituído por $\frac{i}{z-\sum_{l} E_{l}}$. A amplitude de espalhamento desejada é então obtida ao nos aproximarmos do eixo real por cima,

$$
T(\mathbf{p} ; \mathbf{q})=\left.T_{z}(\mathbf{p} ; \mathbf{q})\right|_{z=\sum k^{2}+i \epsilon}
$$

Essa versão generalizada da amplitude de espalhamento, $T_{z}$, satisfaz uma equação integral da mesma forma que as equações (3.6.6). Em particular, a equação (3.6.6a) se torna

$$
T_{z}(\mathbf{p} ; \mathbf{q})=\int d^{3} r f_{z}(\mathbf{p} ; \mathbf{r}) \delta\left(\sum r-\sum q\right) T^{(1)}(\mathbf{r} ; \mathbf{q}),
$$

com

$$
f_{z}(\mathbf{p} ; \mathbf{r}):=-\frac{1}{6(2 \pi)^{2}}\left[\frac{T_{z}(\mathbf{p} ; \mathbf{r})}{z-\sum r^{2}+i \epsilon}-6(2 \pi)^{2} \delta\left(p_{1}-r_{1}\right) \delta\left(p_{2}-r_{2}\right)\right] .
$$

Agora, definimos uma versão generalizada da equação (3.6.27), ao substituirmos a expressão de $T$, pela de $T_{z}$

$$
J_{3 z}:=-\frac{i}{\pi} \phi_{3}(x) \int d^{3} q \delta\left(\sum q-\sum k\right) T_{z}(\mathbf{p} ; \mathbf{q}) h(\mathbf{q} ; \mathbf{k}),
$$

de forma que recuperamos $J_{3}$ ao tomarmos o limite $z \rightarrow \sum k^{2}+i \epsilon$ na expressão acima. Ao substituirmos (3.6.29) em (3.6.30), podemos efetuar explicitamente as integrações sobre $q$ utilizando (3.6.5) para $T^{(1)}$; o resultado que obtemos é:

$$
\int d^{3} q \delta\left(\sum q-\sum k\right) \delta\left(\sum r-\sum q\right) T^{(1)}(\mathbf{r} ; \mathbf{q}) h(\mathbf{q} ; \mathbf{k})=i \pi \delta\left(\sum r-\sum k\right) T^{(1)}(\mathbf{r}, \mathbf{k}) .
$$

Conseqüentemente, (3.6.30) assume a seguinte forma:

$$
J_{3 z}=\phi_{3}(x) \int d^{3} r f_{z}(\mathbf{p} ; \mathbf{r}) T^{(1)}(\mathbf{r} ; \mathbf{k}) \delta\left(\sum r-\sum k\right) \stackrel{z \rightarrow \sum k^{2}+i \epsilon}{\longrightarrow} J_{3}=\phi_{3}(x) T(\mathbf{p} ; \mathbf{k}) .
$$

Finalmente, usando as equações (3.6.6a), (3.6.6b), (3.6.25) e (3.6.32), reduzimos a equação integral (3.6.6c) a uma equação algébrica:

$$
\left[1-\phi_{1}+\phi_{2}-\phi_{3}\right] T(\mathbf{p} ; \mathbf{k})=\left[1-\phi_{1}+\phi_{2}\right] T^{(1)}(\mathbf{p} ; \mathbf{k})+\left[1-\phi_{1}\right] T^{(2)}(\mathbf{p} ; \mathbf{k})+T^{(3)}(\mathbf{p} ; \mathbf{k}) .
$$

A solução da equação (3.6.33) é válida tanto na camada de energia como fora dela, uma vez que 
a amplitude $T$, definida por (3.6.2), satisfaz as equações (3.6.6) mesmo se $\sum p^{2} \neq \sum k^{2}$. A expressão para a matriz $S$ pode ser obtida a partir de sua relação com a amplitude de espalhamento:

$$
S(\mathbf{p} ; \mathbf{k})=\langle\mathbf{p}|\hat{S}| \mathbf{k}\rangle=\langle\mathbf{p} \mid \mathbf{k}\rangle+2 \pi i \delta\left(\sum p^{2}-\sum k^{2}\right) \delta\left(\sum p-\sum k\right) T(\mathbf{p} ; \mathbf{k})
$$

onde

$$
\langle\mathbf{p} \mid \mathbf{k}\rangle=3 !(2 \pi)^{3} \mathcal{S}_{p}\left[\delta\left(p_{1}-k_{1}\right) \delta\left(p_{2}-k_{2}\right) \delta\left(p_{3}-k_{3}\right)\right] .
$$

Assim, para calcularmos $S$ (a partir de $T$ ) é necessário que o segundo termo de (3.6.34) seja reduzido a algo proporcional a $\mathcal{S}_{p}\left[\delta\left(p_{1}-k_{1}\right) \delta\left(p_{2}-k_{2}\right) \delta\left(p_{3}-k_{3}\right)\right]$, o que basicamente corresponde a mostrarmos que $T$ é proporcional a uma soma de funções delta. Para nossa sorte, todos os termos a exceção de $T_{C}^{(2)}$ já se encontram nessa forma, restando-nos, pois, apenas reduzir tal termo à soma de funções delta. Para fazer isso invocamos o teorema de Sokhotsky-Weierstrass, que afirma que:

$$
\lim _{\epsilon \rightarrow 0^{+}} \frac{f(x)}{x \pm i \epsilon}=\mp i \pi f(x) \delta(x)+\text { v.p. }\left(\frac{f(x)}{x}\right) .
$$

Entretanto, o valor principal (v.p.) de $T_{C}^{(2)}$ anula-se trivialmente, demonstrando-se assim que esse termo também é proporcional a uma soma de funções delta. Portanto,

$$
S(\mathbf{p} ; \mathbf{k})=\left(\frac{1+x_{12}}{1-x_{12}}\right)\left(\frac{1+x_{13}}{1-x_{13}}\right)\left(\frac{1+x_{23}}{1-x_{23}}\right)
$$

que claramente exibe a propriedade de fatoração. 
Parte II

O Modelo de Landau-Lifshitz 



\section{Capítulo 4}

\section{O Modelo de Landau-Lifshitz}

"And now for something completely different"

Newsreader [John Cleese], Monty Python's Flying Circus

O modelo de Landau-Lifshitz (LL) descreve a dinâmica da cadeia contínua de spins de Heisenberg anisotrópica. Versões de sua contraparte isotrópica surgiram na última década tanto em teorias de calibre quanto de cordas, fornecendo as primeiras evidências em favor da existência de uma estrutura integrável subjacente à correspondência AdS/CFT [28-30,61-68]. Pelo lado da teoria de cordas em $A d S_{5} \times S^{5}$, o modelo de $L L$ aparece no subsetor $\mathbb{R} \times S^{3}$ no limite de grande momento angular [28,69-74]. Alternativamente, o LL também pode ser obtido a partir do limite de baixas energias do modelo de Faddeev-Reshetikhin (FR) [33,35]; este último sendo utilizado para resolver as dificuldades encontradas no processo de quantização inerente a todos os modelos sigma [20,21,34,75-80]. Já no contexto da $\mathcal{N}=4$ SYM, o modelo de LL emerge como o limite termodinâmico da cadeia de spins (de Heisenberg, na aproximação de 1-laço) correspondente ao setor $\operatorname{SU}(2)[26,69,70]$. Mais recentemente, o modelo de LL também apareceu no subsetor $S U(2) \times S U(2)$ da teoria de cordas em $A d S_{4} \times \mathbb{C} P^{3}$ [81], no contexto da dualidade entre a teoria de Chern-Simons superconforme com $\mathcal{N}=6$ e a teoria de cordas em $A d S_{4} \times \mathbb{C} P^{3}$ no limite de t'Hooft [82].

Apesar de ter sido alvo de intensa investigação durante muitos anos, apenas a teoria clássica para o modelo de LL foi devidamente estabelecida, em particular, a sua estrutura integrável clássica já foi amplamente discutida na literatura sob diversos pontos de vista. As soluções solitônicas clássicas foram encontradas e discutidas extensivamente em [20,83-87]. Uma análise clássica mais completa tornou-se possível com o estabelecimento da integrabilidade clássica, primeiramente para o caso isotrópico [87, 88], enquanto que para o caso mais geral, o anisotrópico, só foi verificada em [20, 89] ${ }^{1}$. As variáveis canônicas de ângulo e ação foram construídas em [85]. Em [90], mostrou-se a equivalência clássica entre o modelo de LL isotrópico e o modelo NLS com a construção de uma transformação de calibre relacionando as conexões de Lax para ambos os modelos. O espectro quasi-clássico foi analisado em [86, 91].

Já no contexto quântico, as propriedades integráveis desses modelos são notadamente diferentes, por exemplo, em [92], Sklyanin mostrou a existência de inúmeras sutilezas no processo de quantização

\footnotetext{
${ }^{1}$ Agradeço a A. Melikyan por compartilhar a referência [89].
} 
do LL, que estavam ausentes no classicamente equivalente NLS, ao considerar em detalhe o método do espalhamento inverso quântico para o LL $(\mathfrak{s u}(1,1))$. Uma das dificuldades mais surpreendentes foi a impossibilidade de se obter a equação de Yang-Baxter a partir de sua contraparte clássica para o LL anisotrópico. A álgebra de observáveis, assim como a conexão de Lax, devem ser modificadas à mão de forma a se obter a matriz $R$ quântica.

Outra conseqüência notável do trabalho de Sklyanin reside na dificuldade da construção das integrais de movimento na teoria quântica, que permeiam a integrabilidade quântica do modelo. Em particular, tal problema, que está relacionado com o fato de o produto de operadores em um mesmo ponto não estar matematicamente bem definindo [37,44], já aparece na construção da Hamiltoniana local para o setor de duas partículas. Notamos que o Hamiltoniano quântico para o setor de $n$ partículas só foi obtido recentemente em [44], enquanto que as outras cargas quânticas conservadas, apenas em [37]. Um método canônico para evitar esse tipo de complicação consiste em discretizar o modelo [36, 93], que conquanto aplicável tanto para o FR, quanto para o LL $[22,33,94]$, permitindo a posterior utilização do Ansatz de Bethe para a dedução dos respectivos espectros, leva a resultados complicados, em geral, envolvendo Hamiltonianos não locais. Ademais, a teoria de cordas completa em $A d S_{5} \times S^{5}$ é significativamente mais complexa, de forma que mesmo se for possível discretizá-la, não se espera que os resultados sejam muito esclarecedores. Portanto, é necessário lidar diretamente com a quantização de modelos contínuos, e o modelo de LL, apesar de sua aparente simplicidade, é um interessante modelo representativo das dificuldades associadas com o método do espalhamento inverso quântico contínuo.

Neste capítulo, apresentamos o modelo de LL e discutimos as suas principais propriedades no contexto clássico, focando a nossa exposição no limite isotrópico. Deixamos o desenvolvimento da teoria quântica para os capítulos 5 e 6 . O modelo de LL pode ser definido tanto sobre uma esfera quanto sobre um hiperbolóide, e, estudando-os simultaneamente, explicitamos não só as suas diferenças, como, principalmente, as suas semelhanças. A equivalência clássica com o modelo NLS serve como um guia, bem como propicia muitas das justificativas, para o desenvolvimento do método do espalhamento inverso clássico. Nesse âmbito obtemos o conjunto completo das integrais de movimento, e, através do formalismo da matriz $r$, mostramos sua involução, estabelecendo, pois, a integrabilidade clássica do modelo.

\subsection{O Modelo de LL Clássico}

O espaço de fase do modelo de LL consiste de funções reais

$$
\begin{aligned}
\mathbf{S}: & \mathbb{R} \rightarrow \mathbb{R}^{3} \\
x & \mapsto \mathbf{S}(x)=\left(S^{1}(x), S^{2}(x), S^{3}(x)\right),
\end{aligned}
$$

dotado do seguinte produto escalar:

$$
\mathbf{S}^{2}(x)=\eta_{i j} S^{i}(x) S^{j}(x)=-\varepsilon\left(S^{1}(x)\right)^{2}-\varepsilon\left(S^{2}(x)\right)^{2}+\left(S^{3}(x)\right)^{2},
$$


onde $\eta=\operatorname{diag}(-\varepsilon,-\varepsilon, 1)$, e sujeito ao vínculo

$$
\mathbf{S}^{2}(x)=1
$$

O vínculo (4.1.2) em conjunto com o sinal de $\varepsilon$ determinam se o modelo de LL está definido sobre a esfera $\mathfrak{s u}(2)(\varepsilon=-1)$, ou sobre o hiperbolóide $\mathfrak{s u}(1,1)(\varepsilon=1)$. As equações de movimento são:

$$
\partial_{t} \mathbf{S}=\mathbf{S} \wedge \partial^{2} \mathbf{S}+\mathbf{S} \wedge \sqrt{ } \mathbf{S}
$$

onde $\mathbb{J}(\mathbf{S})$ é a forma quadrática associada a uma matriz constante $\mathbb{J}$, que, sem perda de generalidade, tomamos como diagonal, de forma que

$$
\mathbb{J}(\mathbf{S})=-\varepsilon J_{1}\left(S^{1}(x)\right)^{2}-\varepsilon J_{2}\left(S^{2}(x)\right)^{2}+J_{3}\left(S^{3}(x)\right)^{2}, \quad J_{1} \leq J_{2} \leq J_{3} .
$$

As equações (4.1.3) descrevem um magneto anisotrópico e, foram originalmente derivadas fenomenologicamente por Landau e Lifshitz em 1935. Claramente, elas preservam o vínculo (4.1.2), de forma que o modelo é invariante por transformações de $O(3)$ se $\varepsilon=-1$, ou $O(1,2)$ se $\varepsilon=1$.

As equações de movimento (4.1.3) precisam ser suplementadas por condições de contorno adequadas,

$$
\lim _{|x| \rightarrow \infty} \mathbf{S}(x)=\mathbf{S}_{0}
$$

onde supomos que os valores limítrofes são alcançados de modo suficientemente rápido, como, por exemplo, no sentido de Schwartz. Além disso, em virtude da invariância do modelo por transformações de $O(3)$ ou de $O(1,2)$, podemos fixar, sem perda de generalidade, a forma do vetor $\mathbb{S}_{0}$ :

$$
\mathbf{S}_{0}=(0,0,1)
$$

A estrutura de Poisson no espaço de fase é fornecida pelos parênteses de Poisson seguintes:

$$
\left\{S^{i}(x), S^{j}(y)\right\}=\varepsilon \epsilon^{i j k} S_{k}(y) \delta(x-y),
$$

onde $\epsilon^{i j k}$ é o tensor totalmente antissimétrico de posto 3 , normalizado a $\epsilon^{123}=1$. As equações de movimento (4.1.3) podem, alternativamente, ser obtidas por intermédio do formalismo Hamiltoniano,

$$
\partial_{t} \mathbf{S}=\{H, \mathbf{S}\}
$$

com a Hamiltoniana anisotrópica dada por:

$$
H=-\frac{\varepsilon}{2} \int d x\left[\eta_{i j} \partial_{x} S^{i}(x) \partial_{x} S^{j}(x)-\left(\mathbb{J}(\mathbf{S})-\mathbb{J}\left(\mathbf{S}_{0}\right)\right)\right],
$$

onde omitimos os limites de integração, $-\infty<x<\infty$.

Mesmo no contexto clássico, o modelo de LL anisotrópico já se mostra severamente mais complicado que o modelo NLS, que estudamos nos capítulos anteriores. As principais dificuldades são introduzidas pela presença de três campos vinculados, de forma que para uma primeira análise é 
conveniente atenuar a anisotropia do modelo, seja ao considerar o modelo com apenas anisotropia unaxial , $J_{1}=J_{2}=0$ e $J_{3}=4 \gamma^{2}$, onde $\gamma$ é o parâmetro de anisotropia, ou simplesmente o limite isotrópico $\rrbracket \rightarrow 0$. Na discussão que se segue, consideramos apenas o caso isotrópico. Por consegüinte, a Hamiltoniana (4.1.8) e as equações de movimento (4.1.3) são significativamente simplificadas:

$$
\begin{aligned}
H & =-\frac{\varepsilon}{2} \int d x \eta_{i j} \partial_{x} S^{i}(x) \partial_{x} S^{j}(x), \\
\partial_{t} S^{i} & =\epsilon^{i j k} S_{j} \partial_{x}^{2} S_{k} .
\end{aligned}
$$

É conveniente introduzir o seguinte campo matricial:

$$
\mathbb{S}=S^{i} \sigma_{i}
$$

onde $\sigma_{i}, i=1,2,3$, são as matrizes de Pauli, que satisfazem:

$$
\begin{aligned}
{\left[\sigma^{i}, \sigma^{j}\right] } & =-2 i \varepsilon \epsilon^{i j k} \sigma_{k}, \\
\left\{\sigma^{i}, \sigma^{j}\right\} & =2 \eta^{i j} .
\end{aligned}
$$

Empregamos as representações usuais para:

$$
\begin{gathered}
\mathfrak{s u}(2): \sigma_{1}=\gamma^{0}, \quad \sigma_{2}=i \gamma^{1} \text { e } \quad \sigma_{3}=\gamma^{3}, \\
\mathfrak{s u}(1,1): \sigma_{1}=i \gamma^{0}, \quad \sigma_{2}=-\gamma^{1} \text { e } \quad \sigma_{3}=\gamma^{3}
\end{gathered}
$$

vide apêndice $\mathrm{B}$ para as definições de $\gamma^{i}, i=0,1,3$. Dessa forma temos que:

$$
\mathbb{S}^{\mathfrak{s u}(2)}=\left(\begin{array}{cc}
S^{3} & S^{1}-i S^{2} \\
S^{1}+i S^{2} & -S^{3}
\end{array}\right) \quad \text { e } \mathbb{S}^{\mathfrak{s u}(1,1)}=\left(\begin{array}{cc}
S^{3} & i S^{1}+S^{2} \\
i S^{1}-S^{2} & -S^{3}
\end{array}\right) .
$$

Claramente, S é uma matriz ${ }^{2}$ com traço nulo, tal que o vínculo (4.1.2) é respeitado:

$$
\mathbb{S}^{2}=\mathbb{1}_{2}
$$

Em termos de $\mathbb{S}$ as equações de movimento isotrópicas (4.1.9b) adquirem a seguinte forma:

$$
\partial_{t} \mathbb{S}=\frac{i \varepsilon}{2}\left[\mathbb{S}, \partial_{x}^{2} \mathbb{S}\right]
$$

As equações (4.1.14) admitem uma representação de curvatura nula (2.5.6), em termos da seguinte conexão de Lax:

$$
L_{x}=\frac{\lambda}{2 i} \mathbb{S} \quad \text { e } \quad L_{t}=-\varepsilon\left[\frac{i \lambda^{2}}{2} \mathbb{S}+\frac{\lambda}{2}\left(\partial_{x} \mathbb{S}\right) \mathbb{S}\right]
$$

\footnotetext{
${ }^{2}$ Hermiteana, apenas no caso $\mathfrak{s u}(2)$
} 
Verifiquemos, isso:

$$
\partial_{x} L_{t}-\partial_{t} L_{x}+\left[L_{t}, L_{x}\right]=0 \Rightarrow\left\{\begin{array} { r l } 
{ - \varepsilon \frac { \lambda ^ { 2 } } { 2 } \partial _ { x } \mathbb { S } - \varepsilon \frac { \lambda ^ { 2 } } { 4 i } [ ( \partial _ { x } \mathbb { S } ) , \mathbb { S } ] = 0 } \\
{ - \varepsilon \frac { \lambda } { 2 } [ ( \partial _ { x } ^ { 2 } \mathbb { S } ) \mathbb { S } + \partial _ { x } \mathbb { S } \partial _ { x } \mathbb { S } ] - \frac { \lambda } { 2 i } \partial _ { t } \mathbb { S } = 0 }
\end{array} \Rightarrow \left\{\begin{array}{rl}
\mathbb{S}^{2} & =\mathbb{1}_{2} \\
\partial_{t} \mathbb{S} & =\frac{i \varepsilon}{2}\left[\mathbb{S}, \partial_{x}^{2} \mathbb{S}\right]
\end{array}\right.\right.
$$

\subsection{O Método do Espalhamento Inverso Clássico}

Ao obtermos a equação de movimento para o LL a partir da condição de curvatura nula para a conexão de Lax (4.1.15), estamos em condição de formular o problema linear fundamental (2.5.1):

$$
\begin{aligned}
& \partial_{x} \psi=\frac{\lambda}{2 i} \mathbb{S} \psi, \\
& \partial_{t} \psi=-\varepsilon\left[\frac{i \lambda^{2}}{2} \mathbb{S}+\frac{\lambda}{2}\left(\partial_{x} \mathbb{S}\right) \mathbb{S}\right] \psi
\end{aligned}
$$

As condições de contorno (4.1.5) assumem a seguinte forma:

$$
\lim _{|x| \rightarrow \infty} \mathbb{S}(x)=\sigma_{3}
$$

É importante notar que o problema espectral auxiliar, fornecido pela equação (4.2.1a), bem como as condições de contorno (4.2.2) não dependem de $\varepsilon$. Logo, ambos os conjuntos de invariantes, para o caso esférico e hiperbólico, devem apresentar exatamente a mesma forma, se escritos de uma maneira covariante, ou seja, usando a métrica $\eta=\operatorname{diag}(-\varepsilon,-\varepsilon, 1)$ para contrair os índices. Por outro lado, o modelo de LL $\mathfrak{s u ( 2 ) ~ e ́ ~ e q u i v a l e n t e ~ a o ~ m o d e l o ~ N L S , ~ v i d e ~ a p e ̂ n d i c e ~} C$, por intermédio da transformação de calibre:

$$
\tilde{\psi}(x, \lambda)=\Omega(x) \psi(x, \lambda) .
$$

Assim, nesse caso, o problema espectral auxiliar é reduzido a

$$
\partial_{x} \tilde{\psi}=\left(\frac{\lambda}{2 i} \sigma_{3}+L_{x}^{0}\right) \tilde{\psi} \quad \text { com } \quad L_{x}^{0}=\partial_{x} \Omega \Omega^{-1}
$$

onde a matriz $\Omega(x)$ é determinada a partir de $\mathbb{S}$, através da relação

$$
\mathbb{S}(x)=\Omega^{-1}(x) \sigma_{3} \Omega(x)
$$

e da condição que a diagonal principal de $L_{x}^{0}$ seja nula,

$$
L_{x}^{0}=i \sqrt{g}\left(\psi \sigma_{-}-\bar{\psi} \sigma_{+}\right),
$$

que corresponde ao modelo NLS com condições de contorno correspondendo ao decaimento rápido no infinito, que estudamos extensivamente no capítulo 2. Conseqüentemente, todas as informações relevantes sobre o problema linear auxiliar (4.2.1) para o caso esférico podem ser obtidas diretamente da análise do problema (4.2.3).

Já a dependência temporal da solução é controlada por (4.2.1b) e depende explicitamente de $\varepsilon$. Contudo, tal dependência pode ser eliminada através de uma redefinição do tempo $t \rightarrow \tilde{t}=\varepsilon t$. 
Assim, em termos de $\tilde{t}$, a evolução temporal de ambos os casos possui a mesma forma. Em particular, as cargas conservadas são as mesmas. Dessa forma, fica claro que, apesar de termos estabelecido apenas a equivalência de calibre entre o modelo NLS e o LL esférico, podemos também estabelecer vários fatos importantes sobre o caso hiperbólico. Entretanto, para uma maior clareza da presente exposição, esboçamos os passos principais para a construção do conjunto infinito de integrais do movimento a partir de (4.2.1).

A matriz de transição $T(x, y ; \lambda)$ é definida como a solução da equação diferencial (4.2.1a) suplementada pela condição inicial

$$
\left.T(x, y ; \lambda)\right|_{x=y}=\mathbb{1}_{2},
$$

e pode ser escrita como:

$$
T(x, y ; \lambda)=P \exp \left\{\frac{\lambda}{2 i} \int_{y}^{x} d z \mathbb{S}(z)\right\} .
$$

Para introduzirmos as soluções de Jost, precisamos considerar o problema espectral auxiliar (4.2.1a) no limite $|x| \rightarrow \infty$ :

$$
\partial_{x} E(x ; \lambda)=\frac{\lambda}{2 i} \sigma_{3} E(x ; \lambda),
$$

que, em particular, pode ser resolvido explicitamente,

$$
E(x ; \lambda)=e^{\frac{\lambda x}{2 i} \sigma_{3}} .
$$

Dessa forma, as soluções de Jost $T_{ \pm}(x ; \lambda)$, para $\lambda \in \mathbb{R}$ são definidas a partir dos limites:

$$
T_{ \pm}(x ; \lambda)=\lim _{y \rightarrow \pm} T(x, y ; \lambda) E(y ; \lambda) .
$$

Assim como a matriz de transição, as matrizes $T_{ \pm}(x ; \lambda)$ são unimodulares e satisfazem a equação (4.2.1a). Além disso, possuem o seguinte comportamento assintótico:

$$
T_{ \pm}(x ; \lambda) \stackrel{x \rightarrow \pm \infty}{\longrightarrow} E(x ; \lambda) .
$$

A matriz de monodromia reduzida $T(\lambda)$ é definida a partir da seguinte razão

$$
T(\lambda)=T_{+}^{-1}(x ; \lambda) T_{-}(x ; \lambda)
$$

fornecendo, pois, o operador que conecta as duas soluções de Jost. Por outro lado, ela pode ser escrita como o limite:

$$
T(\lambda)=\lim _{\substack{x \rightarrow+\infty \\ y \rightarrow-\infty}} E(-x ; \lambda) T(x, y ; \lambda) E(y ; \lambda) .
$$

Utilizando (4.2.1b) podemos inferir facilmente a dependência temporal de $T(\lambda)$ :

$$
\partial_{t} T(\lambda, t)=-\varepsilon \frac{i \lambda^{2}}{2}\left[\sigma_{3}, T(\lambda, t)\right] \quad \Rightarrow \quad T(\lambda, t)=e^{-\varepsilon \frac{i \lambda^{2}}{2} \sigma_{3} t} T(\lambda, 0) e^{\varepsilon \frac{i \lambda^{2}}{2} \sigma_{3} t} .
$$

É bem sabido que as variáveis canônicas de ângulo e ação são construídas a partir dos elementos da matriz de monodromia reduzida, levando a um conjunto de infinitas cargas conservadas. Em 
particular, o elemento diagonal de $T(\lambda, t), a(\lambda, t)$, é independente do tempo, de forma que é possível obter tal conjunto de integrais do movimento a partir dos coeficientes da expansão assintótica de $\Im[\log a(\lambda)]$.

\subsubsection{Cargas Conservadas Locais}

Nesta seção, desenvolvemos um método ${ }^{3}$ para obter uma família de integrais do movimento locais $[20,85,86]$, isto é, que são funcionais com a seguinte forma:

$$
F=\int_{-\infty}^{\infty} d x f(x)
$$

onde a densidade $f(x)$ é um polinômio dos elementos de matriz de $\mathbb{S}(x)$ e suas derivadas espaciais. Começamos considerando a expansão assintótica da matriz de transição conforme $|\lambda| \rightarrow \infty$,

$$
T(x, y ; \lambda)=\left(\mathbb{1}_{2}+W(x ; \lambda)\right) e^{Z(x, y ; \lambda)}\left(\mathbb{1}_{2}+W(y ; \lambda)\right)^{-1},
$$

onde $W(x ; \lambda)$ é uma matriz antidiagonal, e $Z(x, y ; \lambda)$ uma matriz diagonal, tal que

$$
\left.Z(x, y ; \lambda)\right|_{x=y}=0
$$

Por conveniência notacional, no que se segue, omitimos termos da ordem $O\left(|\lambda|^{-\infty}\right)$, nas expansões assintóticas.

Substituindo (4.2.14) no problema espectral auxiliar (4.2.1a), obtemos:

$$
\partial_{x} W+\partial_{x} Z+W \partial_{x} Z=\frac{\lambda}{2 i}(\mathbb{S}+\mathbb{S} W)
$$

que pode ser separada nas suas partes diagonal e antidiagonal

$$
\begin{aligned}
\partial_{x} Z & =\frac{\lambda}{2 i}\left[S^{3} \sigma_{3}+\left(S^{1} \sigma_{1}+S^{2} \sigma_{2}\right) W\right], \\
\partial_{x} W+W \partial_{x} Z & =\frac{\lambda}{2 i}\left[S^{1} \sigma_{1}+S^{2} \sigma_{2}+S^{3} \sigma_{3} W\right] .
\end{aligned}
$$

A primeira pode ser integrada trivialmente,

$$
Z(x, y ; \lambda)=\frac{\lambda}{2 i} \int_{y}^{x} d x^{\prime}\left[S^{3}\left(x^{\prime}\right) \sigma_{3}+\left(S^{1}\left(x^{\prime}\right) \sigma_{1}+S^{2}\left(x^{\prime}\right) \sigma_{2}\right) W\left(x^{\prime} ; \lambda\right)\right],
$$

enquanto que a segunda fornece uma equação de Ricatti para $W(x ; \lambda)$ :

$$
\partial_{x} W+i \lambda S^{3} \sigma_{3} W+\frac{i \lambda}{2}\left(S^{1} \sigma_{1}+S^{2} \sigma_{2}\right)-\frac{i \lambda}{2} W\left(S^{1} \sigma_{1}+S^{2} \sigma_{2}\right) W .
$$

Diferentemente do modelo NLS, a expansão assintótica de $W(x ; \lambda)$ em potências de $\lambda$ possui um

\footnotetext{
${ }^{3}$ Similar ao empregado na seção 2.6.1 no caso do modelo NLS
} 
termo constante, logo, devemos escrever:

$$
W(x ; \lambda)=\sum_{n=0}^{\infty} \frac{W_{n}(x)}{\lambda^{n}}
$$

Substituindo-a em (4.2.18) e rearranjando as somatórias, inferimos a seguinte relação de recursão para os coeficientes $W_{n}(x), n \geq 1$ :

$$
\begin{array}{r}
i S^{3} \sigma_{3} W_{n+1}-\frac{i}{2} W_{0}\left(S^{1} \sigma_{1}+S^{2} \sigma_{2}\right) W_{n+1}-\frac{i}{2} W_{n+1}\left(S^{1} \sigma_{1}+S^{2} \sigma_{2}\right) W_{0} \\
=-\partial_{x} W_{n}+\frac{i}{2} \sum_{k=1}^{n} W_{k}\left(S^{1} \sigma_{1}+S^{2} \sigma_{2}\right) W_{n-k+1}
\end{array}
$$

com o termo inicial $W_{0}(x)$ determinado pela equação:

$$
2 S^{3} \sigma_{3} W_{0}-W_{0}\left(S^{1} \sigma_{1}+S^{2} \sigma_{2}\right) W_{0}+\left(S^{1} \sigma_{1}+S^{2} \sigma_{2}\right)=0 .
$$

Introduzindo a matriz diagonal

$$
Q=\left(S^{1} \sigma_{1}+S^{2} \sigma_{2}\right) W_{0}
$$

podemos rescrever (4.2.20b) como

$$
-2 S^{3} \sigma_{3} Q-Q^{2}+\left(S^{1} \sigma_{1}+S^{2} \sigma_{2}\right)^{2}=0 .
$$

Ao notarmos que

$$
\left(S^{1} \sigma_{1}+S^{2} \sigma_{2}\right)^{2}=\left(S^{1} \sigma_{1}\right)^{2}+\left(S^{2} \sigma_{2}\right)^{2}=\left\{\begin{array}{ll}
\left(S^{1}\right)^{2}+\left(S^{2}\right)^{2}, & \mathfrak{s u}(2) \\
-\left(S^{1}\right)^{2}-\left(S^{2}\right)^{2}, & \mathfrak{s u}(1,1)
\end{array}\right\}=1-\left(S^{3}\right)^{2}
$$

concluímos

$$
Q^{2}+2 S^{3} \sigma_{3} Q+\left[\left(S^{3}\right)^{2}-1\right] \mathbb{1}_{2}=0 \Rightarrow\left(Q+S^{3} \sigma_{3}\right)^{2}=\mathbb{1}_{2},
$$

pois $Q \sigma_{3}=\sigma_{3} Q$. A equação (4.2.22) possui quatro raízes, a que nos interessa é

$$
Q+S^{3} \sigma_{3}=\sigma_{3}
$$

A partir de (4.2.23) determinamos univocamente $W_{0}$ :

$$
W_{0}=i \frac{S^{2} \sigma_{1}-S^{1} \sigma_{2}}{1+S^{3}}
$$

Dessa forma, a relação de recursão (4.2.20a) pode ser escrita como

$$
i S^{3} \sigma_{3} W_{n+1}+\frac{i}{2}\left(1-S^{3}\right)\left[\sigma_{3} W_{n+1}-W_{n+1} \sigma_{3}\right]=-\partial_{x} W_{n}+\frac{i}{2} \sum_{k=1}^{n} W_{k}\left(S^{1} \sigma_{1}+S^{2} \sigma_{2}\right) W_{n+1-k}
$$


posto que $W_{n}(x)$ é uma matriz antidiagonal, anticomuta com $\sigma_{3}$, e, conseqüentemente, obtemos:

$$
W_{n+1}=i \sigma_{3} \partial_{x} W_{n}-\frac{i}{2} \sum_{k=1}^{n} W_{k}\left(S^{1} \sigma_{2}-S^{2} \sigma_{1}\right) W_{n-k+1}
$$

empregando a expressão explícita das matrizes de Pauli, obtemos para o $\mathfrak{s u}(2)$ :

$$
W_{n+1}=\left(\begin{array}{cc}
i & 0 \\
0 & -i
\end{array}\right) \partial_{x} W_{n}+\frac{1}{2} \sum_{k=1}^{n} W_{k}\left(\begin{array}{cc}
0 & -S^{1}+i S^{2} \\
S^{1}+i S^{2} & 0
\end{array}\right) W_{n-k+1}
$$

enquanto que, para $0 \mathfrak{s u}(1,1)$ :

$$
W_{n+1}=\left(\begin{array}{cc}
i & 0 \\
0 & -i
\end{array}\right) \partial_{x} W_{n}+\frac{1}{2} \sum_{k=1}^{n} W_{k}\left(\begin{array}{cc}
0 & S^{2}-i S^{1} \\
S^{2}+i S^{1} & 0
\end{array}\right) W_{n-k+1}
$$

Portanto, para o caso esférico os coeficientes $W_{n}(x)$ são matrizes anti-hermiteanas,

$$
W_{n}(x)=\left(\begin{array}{cc}
0 & -\bar{w}_{n}(x) \\
w_{n}(x) & 0
\end{array}\right)
$$

de sorte que, em termos das componentes $w_{n}(x)$ a relação de recursão (4.2.25b) assume a seguinte forma:

$$
w_{n+1}(x)=-i \partial_{x} w_{n}(x)-\frac{S^{1}(x)-i S^{2}(x)}{2} \sum_{k=1}^{n} w_{k}(x) w_{n-k+1}(x), \quad n \in \mathbb{N},
$$

com

$$
w_{0}(x)=\frac{S^{1}(x)+i S^{2}(x)}{1+S^{3}(x)} .
$$

Já para o caso hiperbólico os coeficientes $W_{n}(x)$ são matrizes Hermiteanas,

$$
W_{n}(x)=\left(\begin{array}{cc}
0 & \bar{W}_{n}(x) \\
W_{n}(x) & 0
\end{array}\right)
$$

de sorte que, em termos das componentes $w_{n}(x)$ a relação de recursão (4.2.25b) assume a seguinte forma:

$$
w_{n+1}(x)=-i \partial_{x} w_{n}(x)+\frac{S^{2}(x)-i S^{1}(x)}{2} \sum_{k=1}^{n} w_{k}(x) w_{n-k+1}(x), \quad n \in \mathbb{N},
$$

com

$$
w_{0}(x)=\frac{-S^{2}(x)+i S^{1}(x)}{1+S^{3}(x)} .
$$

É importante ressaltar que as expressões para os casos $\mathfrak{s u}(2)$ e $\mathfrak{s u}(1,1)$, contidas em (4.2.26) e (4.2.27), respectivamente, diferem entre si apenas porque utilizamos as formas explícitas para as respectivas matrizes de Pauli.

Estamos em posição de obter a expansão assintótica para $Z(x, y ; \lambda)$. Posto que as expressões (4.2.26) e (4.2.27) não coincidem, precisamos considerar separadamente o caso esférico do hiper- 
bólico, porém, após efetuarmos as devidas substituições e simplificações, constatamos que ambos os casos admitem a mesma forma para a expressão de $Z(x, y ; \lambda)$ :

$$
Z(x, y ; \lambda)=\frac{\lambda}{2 i}(x-y) \sigma_{3}+\sum_{n=0}^{\infty} \frac{Z_{n}(x, y)}{\lambda^{n}}
$$

com

$$
Z_{n}(x, y)=\left(\begin{array}{cc}
z_{n}(x, y) & 0 \\
0 & -\bar{z}_{n}(x, y)
\end{array}\right)
$$

diferindo apenas na forma dos coeficientes $z_{n}(x, y)$. Para o caso esférico, temos:

$$
z_{n}(x, y)=\frac{1}{2 i} \int_{y}^{x} d x^{\prime}\left[S^{1}\left(x^{\prime}\right)-i S^{2}\left(x^{\prime}\right)\right] w_{n+1}\left(x^{\prime}\right)
$$

enquanto que para o hiperbólico:

$$
z_{n}(x, y)=\frac{1}{2 i} \int_{y}^{x} d x^{\prime}\left[i S^{1}\left(x^{\prime}\right)+S^{2}\left(x^{\prime}\right)\right] w_{n+1}\left(x^{\prime}\right)
$$

Finalmente, podemos considerar o limite (4.2.11) para obter a expansão assintótica, conforme $|\lambda| \rightarrow \infty$, da matriz de monodromia reduzida. Claramente, as condições de contorno (4.2.2) demandam que $W(x ; \lambda) \stackrel{|x| \rightarrow \infty}{\longrightarrow} 0$. Logo,

$$
T(\lambda)=e^{P(\lambda)}+O\left(|\lambda|^{-\infty}\right), \quad \operatorname{com} \quad P(\lambda)=i\left(\begin{array}{cc}
p(\lambda) & 0 \\
0 & -\bar{p}(\lambda)
\end{array}\right),
$$

onde

$$
p(\lambda)=\sum_{n=0}^{\infty} \frac{I_{n}}{\lambda^{n}} \quad \text { com } \quad I_{n}=\lim _{\substack{x \rightarrow+\infty \\ y \rightarrow-\infty}} \frac{z_{n}(x, y)}{i}
$$

Notando que

$$
z_{n}^{\mathfrak{s u}(1,1)}=i z_{n}^{\mathfrak{s u}(2)} \text { e } \quad \sigma_{j}^{\mathfrak{s u}(1,1)}=i \sigma_{j}^{\mathfrak{s u}(2)}, \quad \text { para } \quad j=1,2 ;
$$

concluímos que os coeficientes $I_{n}$ devem possuir exatamente a mesma forma em ambos os casos, se escritos de uma maneira covariante. Ademais, da unimodularidade de $T(\lambda)$, temos que $\operatorname{tr} P(\lambda)=0$ e, conseqüentemente, os coeficientes $I_{n} \in \mathbb{R}$. Portanto, estabelecemos a expansão assintótica:

$$
-\Im[\log a(\lambda)]=\frac{1}{i} \log a(\lambda)=\sum_{n=0}^{\infty} \frac{I_{n}}{\lambda^{n}}, \quad|\lambda| \rightarrow \infty
$$

onde os coeficientes reais $I_{n}$ fornecem as integrais de movimento para os modelos de LL esférico e hiperbólico. É importante notar que os funcionais $I_{n}$ são da forma (4.2.13). Em particular, os primeiros funcionais fornecem:

$$
\begin{aligned}
& I_{0}=-\frac{1}{2} \int d x \frac{S^{1} \partial_{x} S^{2}-S^{2} \partial_{x} S^{1}}{1+S^{3}}=-\frac{1}{2} P \\
& I_{1}=-\frac{1}{2} H
\end{aligned}
$$

onde $P$ é o momento e $H$ a Hamiltoniana. 
Empregando a técnica de redução descrita em [91], introduzimos o seguinte conjunto de vetores ortonormais:

$$
e_{1}^{i}=\frac{\partial_{x} S^{i}}{\sqrt{2 \mathcal{H}}}, \quad e_{2}^{i}=\frac{\epsilon^{i j k} S_{j} \partial_{x} S_{k}}{\sqrt{2 \mathcal{H}}}, \quad e_{3}^{i}=S^{1} \quad \text { com } \quad \mathcal{H}=\frac{1}{2} \eta_{i j} \partial_{x} S^{i} \partial_{x} S^{j} \quad \text { e tal que } \quad \eta_{i j} e_{a}^{i} e_{b}^{j}=\delta_{a b}
$$

Naturalmente, um vetor arbitrário dos campos $S^{i}(x), i=1,2,3$ e de suas derivadas espaciais pode ser expandido na base acima, com componentes invariantes sob $O(3)$, para o caso esférico, e sob $O(1,2)$ para o caso hiperbólico. Dessa forma, na teoria clássica, qualquer invariante de $O(3)$ ou de $O(1,2)$ pode ser escrito em termos dos seguinte conjunto de invariantes fundamentais e de suas derivadas espaciais:

$$
\mathcal{C}(x)=S^{i} S_{i}, \quad \mathcal{H}(x)=\frac{1}{2} \partial_{x} S^{i} \partial_{x} S_{i} \quad \text { e } \quad \mathcal{Q}_{3}(x)=\epsilon^{i j k} S_{i} \partial_{x} S_{j} \partial_{x}^{2} S_{k}
$$

Nesse caso, $\mathcal{C}(x), \mathcal{H}(x)$, and $\mathcal{Q}_{3}(x)$ correspondem às densidades de Casimir, Hamiltoniana e da carga conservada cúbica. Por exemplo, a carga conservada quártica:

$$
Q_{4}=\int d x\left[\partial_{x}^{2} S^{i} \partial_{x}^{2} S_{i}+\frac{5}{4}\left(\partial_{x} S^{i} \partial_{x} S_{i}\right)^{2}\right]
$$

pode ser escrita como a seguinte função não-linear de $\mathcal{H}$ e $\mathcal{Q}_{3}$ [91]:

$$
Q_{4}=\int d x\left[9 \mathcal{H}^{2}+\frac{\left(\partial_{x} \mathcal{H}\right)^{2}+\mathcal{Q}_{3}^{2}}{2 \mathcal{H}}\right] .
$$

\subsection{A Matriz $r$ para o LL}

Nesta seção, mostramos que a estrutura de Poisson do modelo de LL (tanto no caso esférico quanto no hiperbólico) admite a formulação em termos de uma matriz $r$. Em virtude da definição 2.7.2, precisamos rescrever os parênteses de Poisson (4.1.6) de forma a envolver explicitamente a conexão de Lax (4.1.15). Claramente, em termos da matriz $\mathbb{S}=\eta_{i j} S^{i}(x) \sigma^{j}$, os parênteses de Poisson (4.1.6) assumem a seguinte forma:

$$
\{\mathbb{S}(x) \stackrel{\otimes}{,}(y)\}=\varepsilon \epsilon^{i j k} S_{k}(x)\left(\sigma_{i} \otimes \sigma_{j}\right) \delta(x-y) .
$$

Para eliminarmos o termo $S_{k}(x)$ presente em seu lado direito, usamos a fórmula para multiplicação de matrizes de Pauli, com $\varepsilon=-1$ para o caso esférico e $\varepsilon=1$ para o caso hiperbólico:

$$
\sigma_{i} \sigma_{j}=\eta_{i j} \mathbb{1}_{2}-\varepsilon i \epsilon_{i j k} \sigma^{k}
$$

e a definição da matriz de permutação:

$$
\Pi=\frac{1}{2}\left(\mathbb{1}_{4}+\eta^{i j} \sigma_{i} \otimes \sigma_{j}\right)
$$


Dessa forma podemos expressar o produto tensorial de matrizes de Pauli presentes no lado direito de (4.3.1) por intermédio da relação:

$$
\sigma_{i} \otimes \sigma_{j}-\sigma_{j} \otimes \sigma_{i}=-i \varepsilon \epsilon_{i j k} \Pi\left(\mathbb{1}_{2} \otimes \sigma^{k}-\sigma^{k} \otimes \mathbb{1}_{2}\right) .
$$

Conseqüentemente, obtemos:

$$
\{\mathbb{S}(x) \stackrel{\otimes}{S}(y)\}=i \varepsilon^{2} \Pi\left(\mathbb{S} \otimes \mathbb{1}_{2}-\mathbb{1}_{2} \otimes \mathbb{S}\right) \delta(x-y)=i\left[\Pi, \mathbb{S} \otimes \mathbb{1}_{2}\right] \delta(x-y) .
$$

Finalmente, para obtermos $L_{x}(x ; \lambda)=\frac{\lambda}{2 i} S(x)$ em (4.3.5), multiplicamos-a por $\frac{i^{2} \lambda \mu}{4}$ e transformamos seu lado direito da seguinte maneira:

$$
\begin{aligned}
\frac{\lambda \mu}{4 i}\left[\Pi, \mathbb{S} \otimes \mathbb{1}_{2}\right] & =\frac{\lambda \mu}{2(\lambda-\mu)}\left[\Pi, L_{x}(x ; \lambda) \otimes \mathbb{1}_{2}-L_{x}(x ; \mu) \otimes \mathbb{1}_{2}\right] \\
& =\frac{\lambda \mu}{2(\lambda-\mu)}\left[\Pi, L_{x}(x ; \lambda) \otimes \mathbb{1}_{2}+\mathbb{1}_{2} \otimes L_{x}(x ; \mu)\right] .
\end{aligned}
$$

De sorte que a expressão (4.3.5) fornece os parênteses de Poisson fundamentais:

$$
\left\{L_{x}(x ; \lambda) \stackrel{\otimes}{,} L_{x}(y ; \mu)\right\}=\left[r(\lambda, \mu), L_{x}(x ; \lambda) \otimes \mathbb{1}_{2}+\mathbb{1}_{2} \otimes L_{x}(x ; \mu)\right] \delta(x-y),
$$

com a matriz $r$ dada por:

$$
r(\lambda, \mu)=\frac{\lambda \mu}{2(\lambda-\mu)} \Pi
$$

É interessante notar que

$$
r^{L L}(\lambda, \mu)=-\frac{1}{2} r^{N L S}\left(\frac{1}{\lambda}-\frac{1}{\mu}\right)
$$

onde $r^{N L S}$ é a matriz $r$ para o modelo NLS (2.7.11). Assim, se considerarmos $\lambda^{-1}$ e $\mu^{-1}$ como variáveis independentes, a matriz $r$ para o LL depende apenas da diferença entre seus argumentos.

Procedendo de uma maneira similar a seção 2.7, podemos usar (4.3.7) para inferir os parênteses de Poisson para a matriz de transição:

$$
\{T(x, y ; \lambda) \stackrel{\otimes}{,} T(x, y ; \mu)\}=[r(\lambda, \mu), T(x, y ; \lambda) \otimes T(x, y ; \mu)] \quad \text { para } \quad y<x
$$

Uma conseqüência notável de (4.3.9) é que, ao considerarmos o limite da matriz de transição reduzida, concluímos que as integrais de movimento $I_{n}(4.2 .29 b)$ estão em involução:

$$
\left\{I_{n}, I_{m}\right\}=0
$$

Dessa forma, levando em consideração a discussão ao final da seção 4.2.1, em conjunto com a equivalência clássica entre o modelo de LL e o modelo NLS, inferimos a existência de um conjunto completo de cargas conservadas em involução, e estabelecemos a integrabilidade clássica do LL. 


\title{
Capítulo 5
}

\section{Fatorabilidade da Matriz S}

\author{
"You can't always get what you want \\ But if you try sometimes, you just might find \\ You get what you need"
}

The Rolling Stones - You Can't Always Get What You Want

Os modelos de Landau-Lifshitz (LL) e Faddeev-Reshetkhin (FR), bem como outros ${ }^{1}$ que surgem de limites da teoria de cordas completa em $A d S_{5} \times S^{5}$, têm sido recentemente estudados como teorias de campos bidimensionais, e suas matrizes $S$ foram obtidas por meio de cálculos perturbativos [35, 40-42, 95-97]. A idéia central desse método, assim como a sua relação com o método do espalhamento inverso, está bem ilustrada para o caso do simples modelo NLS em [38]. De uma forma sucinta, ele se utiliza da integrabilidade, que se supõem válida também no nível quântico, e, em particular, da propriedade de fatoração da matriz $S$ [24, 25,41,98-103], que permite escrever a matriz $S$ para o espalhamento de $N$ partículas como um produto de matrizes $S$ para o espalhamento de duas partículas. A verificação direta dessa propriedade, via cálculo de diagramas de Feynman, só havia sido feita para o modelo NLS em $[39,104]$. Neste capítulo, expomos o resultado originalmente obtido em [41], onde demonstramos a fatorabilidade da matriz $S$ para o modelo de LL em primeira ordem não-trivial.

Consideramos o espalhamento de três partículas no modelo de $L L$ isotrópico e esférico ${ }^{2}$ e, ao computarmos os diagramas de Feynman necessários até a segunda ordem, mostramos que esse é fatorável na primeira ordem não-trivial. Existem diversos fatores conceituais que tornam o cálculo da matriz $S$ para o modelo de LL uma tarefa extremamente não-trivial. Primeiro, como notamos anteriormente, o modelo de LL é o limite de baixas energias do modelo de FR, e conforme foi mostrado em [80], a Hamiltoniana para o modelo de FR não é diagonalizável na classe da representação canônica para o setor de duas partículas:

$$
\left|p_{1} p_{2}\right\rangle=\iint_{x_{1} \neq x_{2}} d x_{1} d x_{2}\left[e^{p_{1} x_{1}+p_{2} x_{2}}+S e^{p_{1} x_{2}+p_{2} x_{1}}\right] \phi^{\dagger}\left(x_{1}\right) \phi^{\dagger}\left(x_{2}\right)|0\rangle .
$$

\footnotetext{
${ }^{1}$ Como, por exemplo, o modelo de de Alday-Arutyunov-Frolov (AAF), que consideramos nos capítulos 7 e 8.

${ }^{2}$ Conforme ficará claro mais adiante, consideramos apenas tal caso, pois esse é o que surge naturalmente no contexto da correspondência AdS/CFT. Dessa forma, no decorrer deste capítulo, toda vez que nos referirmos ao modelo de LL, temos em mente somente o caso isotrópico e esférico.
} 
Essa representação está no cerne do Ansatz de Bethe. O primeiro e o segundo termos dessa equação podem ser interpretados, respectivamente, como ondas incidentes e espalhadas, onde a matriz $S$, $S\left(p_{1}, p_{2}\right)=e^{i \Delta\left(p_{1}, p_{2}\right)}$, representa apenas uma mudança de fase devido ao espalhamento. Para uma maior clareza da exposição, supomos sem perda de generalidade que $p_{1}>p_{2}$. Procedendo de um modo similar a [80], não é difícil mostrar que a Hamiltoniana para o modelo de LL também não pode ser diagonalizada nessa classe de funções (5.0.1), contudo a matriz $S$ exata para o espalhamento de duas partículas no modelo de FR pode ser obtida através de cálculos perturbativos [35]. Isso pode ser visto de uma forma alternativa por intermédio do método do espalhamento inverso quântico (MEIQ) [92], onde se mostrou que a função de onda do estado de duas partículas adquire um termo adicional ao exibido em (5.0.1). Um dos motivos pelo qual isso acontece é a natureza extremamente singular da interação presente no modelo de LL [92]. De fato, não é difícil de ver que a interação do ponto de vista da mecânica quântica corresponde a uma segunda derivada da função delta de Dirac, cujo tratamento está longe de ser uma tarefa simples. Para escrevermos esse termo extra é necessária a introdução de um novo operador de criação, que para o estado de duas partículas corresponderia a um estado ligado. Naturalmente, o número de tais termos aumenta conforme mais partículas participam do processo de espalhamento. Por exemplo, para o espalhamento de três partículas, existem duas contribuições adicionais, correspondendo a estados ligados de duas e três partículas.

Um ponto importante é que a forma (5.0.1) sugere que as partículas criadas pelos campos $\phi^{\dagger}(x)$ correspondem às partículas de Bethe, e assim, todas as conseqüências da integrabilidade, tal como a fatoração da matriz $S$, seriam verdadeiras em função do argumento apresentado em $[25,98,100]$. Esse é de fato o caso para um grande número de modelos, como, por exemplo, o modelo NLS, onde (5.0.1) é realmente um estado de duas partículas, e as partículas $\phi^{\dagger}(x)|0\rangle$ representam as partículas de Bethe, para as quais é possível obter a matriz $S$ tanto por meio de cálculos perturbativos, como pela diagonalização direta da Hamiltoniana. Entretanto, a priori, esse não é o caso geral, e as partículas criadas pelos campos $\phi^{\dagger}(x)$ podem não corresponder às partículas de Bethe. O modelo de LL se enquadra nesse caso mais geral, no qual as partículas de Bethe não coincidem com $\phi^{\dagger}(x)|0\rangle$, exigindo, pois, considerações mais minuciosas.

Enfatizamos que ao examinarmos um processo de espalhamento, a fatoração da matriz $S$ só faz sentido em termos das partículas de Bethe. Assim, o único método confiável para a diagonalização simultânea de todas as quantidades conservadas locais, incluindo a Hamiltoniana, e, como conseqüência, a construção de todos os auto-estados correspondentes às partículas de Bethe, é o MEIQ. Porém, como mencionamos anteriormente, essa construção falha miseravelmente para o modelo de $L L^{3}$, e as quantidades conservadas não podem ser obtidas por intermédio das identidades de traço, devido à singularidade das expressões envolvidas. Conseqüentemente, testar a integrabilidade quântica, por exemplo, a fatoração da matriz $S$, bem como estabelecer uma conexão com a construção fornecida pelo MEIQ, usando métodos usuais de teoria de campos consiste em uma tarefa de fundamental importância, que deve se mostrar de grande relevância ao se considerar modelos mais complexos do que o LL.

\footnotetext{
${ }^{3}$ No capítulo 6, consideramos a quantização dos modelos de LL isotrópicos esférico e hiperbólico e construímos as identidades de traço para o caso hiperbólico, porém tal construção não se aplica diretamente ao caso esférico, que ainda constitui um problema em aberto.
} 
Existem outras sutilezas que tornam a fatorabilidade da matriz $S$ para o modelo de LL uma característica altamente não-trivial; por exemplo a teoria de campos bidimensional não-vinculada na folha-mundo que corresponde ao modelo de LL contém um número infinito de vértices, o que contrasta em muito com o modelo NLS, onde existe apenas um vértice, tornando os cálculos de ordem mais alta no NLS muito mais fáceis. No modelo de LL, é necessário considerar novos tipos de vértices conforme a ordem do cálculo aumenta. Ressaltamos que para se calcular a matriz $S$ para o espalhamento de duas partículas precisamos apenas considerar termos de até quarta ordem na Lagrangeana. A função dos demais termos é a de preservar a integrabilidade no nível quântico, a saber, para a matriz $S$ para o espalhamento de três partículas é necessário manter termos de até sexta ordem nos campos.

A integrabilidade quântica se manifesta através da fatoração da matriz $S$ para o espalhamento de três partículas. Apesar de a análise combinatória, devido ao número infinito de vértices, tornar o cálculo proibitivo em ordens mais altas, é possível mostrar explicitamente, na primeira ordem nãotrivial, que os vértices de ordem mais alta são exatamente da forma necessária para que a matriz $S$ seja fatorável, isto é, termos de ordem maior cancelam termos indesejáveis de ordem menor, de forma a garantir a fatoração. Além desse mecanismo não-trivial, mostramos explicitamente que não existe nem criação, nem aniquilação de partículas no decorrer do processo de espalhamento, bem como que o conjunto de momentos antes e depois do espalhamento coincidem, o que está em perfeito consenso com o nosso entendimento de integrabilidade.

\subsection{O Modelo de LL como uma Teoria Quântica de Campos}

O modelo de LL é a teoria efetiva de baixas energias do ferromagneto de Heisenberg, cuja Hamiltoniana:

$$
H=\frac{\lambda}{16 \pi^{2}} \sum_{l=1}^{J}\left(1-\mathbf{S}_{l} \cdot \mathbf{S}_{l+1}\right),
$$

surge no contexto da correspondência AdS/CFT pelo lado da teoria de calibre como a matriz de mistura a um laço dos operadores escalares compostos do setor fechado $S U(2), \operatorname{tr}\left(\sum_{\text {permutações }} Z^{J-M} W^{M}\right)$ [26]. As equações de movimento (4.1.9b) podem ser obtidas a partir de (5.1.1) ao considerarmos o limite contínuo $J \rightarrow \infty$ e substituirmos os operadores de spin: $\mathbb{S}_{I}(t) \rightarrow \mathbb{S}(x, t), \operatorname{com} x=\frac{2 \pi l}{J}$. Pelo lado da teoria de cordas em $A d S_{5} \times S^{5}$, o modelo de LL surge no setor $S U(2)$ composto por estados com duas das componentes do momento angular grandes e cujo movimento está restrito à esfera $S^{3} \subset S^{5}$. Para obtermos a ação efetiva, precisamos isolar uma coordenada "rápida" $\alpha$ associada ao momento $p_{\alpha}$, que é grande dentre a classe de configurações de cordas que consideramos. Fixando o calibre $t \sim \tau$ e $p_{\alpha} \sim J$ e expandindo a ação em derivadas das coordenadas "lentas" (ou transversais), que identificamos com $\mathbf{S}$, obtemos $[69,70]$ :

$$
S=\frac{J}{2 \pi} \int d \tau \int_{0}^{2 \pi} d \sigma\left[C_{\tau}(\mathbf{S})-\frac{\lambda}{8 J^{2}}\left(\partial_{\sigma} \mathbf{S}\right)^{2}\right]
$$




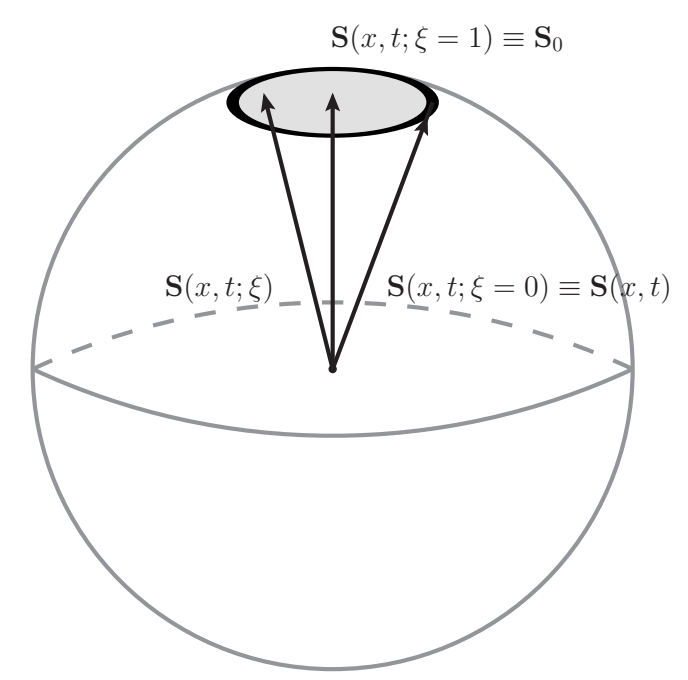

Figura 5.1: Interpretação geométrica do termo de Wess-Zumino.

onde $\lambda$ é a constante de 't Hooft e $C_{q}(\mathbf{S})$ denota o termo de Wess-Zumino:

$$
C_{q}(\mathbf{S})=-\frac{1}{2} \int_{0}^{1} d \xi \epsilon^{i j k} S_{i} \partial_{\xi} S_{j} \partial_{q} S_{k} .
$$

Alternativamente, a ação (5.1.2) pode ser obtida através da abordagem de integral de caminhos para estados coerentes do modelo de Heisenberg no limite contínuo $[69,70]$.

Fazendo as transformações de escala:

$$
\sigma=\frac{2 \pi}{J} x \quad \text { e } \quad \tau=\frac{8 \pi^{2}}{\lambda} t
$$

obtemos a ação de Landau-Lifshitz:

$$
S=\int d^{2} x\left[C_{t}(\mathbf{S})-\frac{1}{4}\left(\partial_{x} \mathbf{S}\right)^{2}\right],
$$

da qual podemos extrair as equações de movimento (4.1.9b) através do princípio variacional. Claramente o campo $\mathbf{S}(x)$ deve satisfazer o vínculo (4.1.2) para o caso esférico (com simetria $\mathfrak{s u}(2)$ e $\varepsilon=-1)$ :

$$
\mathbf{S}^{2}=\left(S_{1}\right)^{2}+\left(S_{2}\right)^{2}+\left(S_{3}\right)^{2}=1 .
$$

A ação (5.1.4) contém o termo não-local de Wess-Zumino (5.1.3), no qual é introduzida a dependência do vetor $\mathbf{S}$ no parâmetro adicional $\xi$, de forma que as seguintes condições de contorno são satisfeitas:

$$
\left\{\begin{array}{l}
\mathbf{S}(x, t ; \xi=1)=\mathbf{S}_{0}, \\
\mathbf{S}(x, t ; \xi=0)=\mathbf{S}(x, t),
\end{array}\right.
$$

onde $\mathbf{S}_{0} \in \mathbb{R}^{3}$ é algum vetor constante. Essa dependência em $\xi$ é introduzida de forma a descrever o vetor $\mathbf{S}$ conforme ele varre uma superfície na esfera de raio unitário, vide figura 5.1. Assim, o termo 
de Wess-Zumino corresponde à área coberta por esse vetor, conforme $\xi$ vai de 0 a 1.

Para podermos desenvolver a teoria de perturbações, precisamos rescrever o termo de WessZumino em uma forma local,

$W Z[\mathbf{S}]=\int d t C_{t}(\mathbf{S})=-\frac{1}{4} \iint \epsilon^{i j k} S_{i}\left[\partial_{\xi} S_{j} d \xi+\partial_{t} S_{j} d t\right] \wedge\left[\partial_{\xi} S_{k} d \xi+\partial_{t} S_{k} d t\right]=-\frac{1}{4} \iint \epsilon^{i j k} S_{i} d S_{j} d S_{k}$,

onde $d \mathbf{S}=\partial_{t} \mathbf{S} d t+\partial_{\xi} \mathbf{S} d \xi$, mas, uma vez que:

$$
\begin{aligned}
& \frac{1}{2} \epsilon^{i j k} S_{i} d S_{j} \wedge d S_{k}=\left(S_{1} d S_{2}-S_{2} d S_{1}\right) \wedge d S_{3}+S_{3} d S_{1} \wedge d S_{2} \quad \text { e } \\
& \mathbf{S}^{2}=1 \quad \Rightarrow \quad S^{i} d S_{i}=0 \quad \Rightarrow \quad d S_{3}=-\frac{1}{S_{3}}\left(S_{1} d S_{1}+S_{2} d S_{2}\right)
\end{aligned}
$$

são verdadeiras, podemos escrever que:

$$
\frac{1}{2} \epsilon^{i j k} S_{i} d S_{j} \wedge d S_{k}=\frac{d S_{1} \wedge d S_{2}}{S_{3}}=\left[\frac{2}{1+S_{3}}+\frac{S_{1}^{2}+S_{2}^{2}}{S_{3}\left(1+S_{3}\right)^{2}}\right] d S_{1} \wedge d S_{2}
$$

Além disso, se notarmos que:

$$
d\left(\frac{S_{1} d S_{2}}{1+S_{3}}\right)=\left(\frac{1}{1+S_{3}}+\frac{S_{1}^{2}}{S_{3}\left(1+S_{3}\right)^{2}}\right) d S_{1} \wedge d S_{2}
$$

com uma expressão análoga para o termo $\frac{S_{2} d S_{1}}{1+S_{3}}$, concluímos que:

$$
\frac{d S_{1} \wedge d S_{2}}{S_{3}}=d\left(\frac{S_{1} d S_{2}-S_{2} d S_{1}}{1+S_{3}}\right)
$$

Logo, podemos rescrever o termo de Wess-Zumino, da seguinte forma:

$$
W Z[\mathbf{S}]=-\frac{1}{2} \iint d\left(\frac{S_{1} d S_{2}-S_{2} d S_{1}}{1+S_{3}}\right)=\frac{1}{2} \int \frac{S_{2} d S_{1}-S_{1} d S_{2}}{1+S_{3}}=\frac{1}{2} \int d t \frac{\dot{S}_{1} S_{2}-S_{1} \dot{S}_{2}}{1+S_{3}}
$$

onde por um ponto denotamos uma derivada temporal. Assim, fomos capazes de expressar o termo de Wess-Zumino em uma forma local, contudo deixamos de ter a simetria SO(3) manifesta.

Nosso próximo passo consiste na resolução do vínculo introduzido pelo fato de $\mathbf{S}$ ser um vetor de norma fixa, ao expressarmos a componente $S_{3}$ em termos das outras duas componentes: $S_{1}$ e $S_{2}$. Nesse estágio, também é conveniente fazer uma redefinição dos campos de forma a nos livrarmos das não-linearidades no termo cinético e combinar $S_{1}$ e $S_{2}$ em um único campo escalar complexo [35].

$$
\varphi=\frac{S_{1}+i S_{2}}{\sqrt{2+2 S_{3}}} \quad \text { e } \quad S_{3}=1-2|\varphi|^{2} .
$$

A ação seguindo de (5.1.4), (5.1.7) e (5.1.8) pode, pois, ser escrita como:

$$
\begin{aligned}
S=\int d^{2} x & \left\{\frac{i}{2}\left(\varphi^{*} \partial_{t} \varphi-\partial_{t} \varphi^{*} \varphi\right)-\left|\partial_{x} \varphi\right|^{2}-\right. \\
& \left.-\frac{1}{4} \frac{2-|\varphi|^{2}}{1-|\varphi|^{2}}\left[\left(\varphi^{*} \partial_{x} \varphi\right)^{2}+\left(\partial_{x} \varphi^{*} \varphi\right)^{2}\right]-\frac{1}{2} \frac{|\varphi|^{4}\left|\partial_{x} \varphi\right|^{2}}{1-|\varphi|^{2}}\right\} .
\end{aligned}
$$




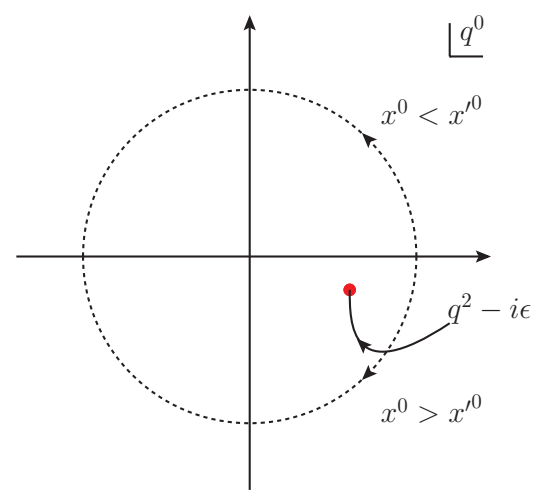

Figura 5.2: Prescrição de pólos para o propagador do modelo de LL.

Essa ação descreve a teoria de um campo escalar interagente. É importante notar o caráter altamente não-linear da interação em (5.1.9). Tal não-linearidade tem sua origem na resolução do vínculo (4.1.2) e introduz sérias complicações na análise perturbativa.

\subsubsection{Quantização do Campo Livre}

O caráter não-relativístico do modelo de LL leva a algumas simplificações no processo de quantização do campo livre, que é bem parecido com o do campo escalar complexo usual, salvo às diferenças introduzidas pelo fato de as equações de movimento (4.1.9b) serem de primeira ordem nas derivadas temporais e de segunda ordem nas espaciais. Considerando a Lagrangeana livre,

$$
\mathscr{L}_{\text {livre }}=\frac{i}{2}\left(\varphi^{*} \partial_{t} \varphi-\partial_{t} \varphi^{*} \varphi\right)-\left|\partial_{x} \varphi\right|^{2}
$$

cuja variação com respeito aos campos livres $\varphi(x, t)$ e $\varphi^{*}(x, t)$ implica as seguintes equações de movimento:

$$
\left(i \partial_{t}-\partial_{x}^{2}\right) \varphi^{*}=0 \quad \text { e }\left(i \partial_{t}+\partial_{x}^{2}\right) \varphi=0,
$$

concluímos que os campos podem ser expandidos somente em termos dos modos de freqüência negativa na representação de interação:

$$
\varphi(x)=\int \frac{d p}{2 \pi} e^{-i p \cdot x} a_{p} \quad \text { e } \quad \varphi^{*}(x)=\int \frac{d p}{2 \pi} e^{i p \cdot x} a_{p}^{\dagger}
$$

onde empregamos a notação:

$$
p \cdot x=p^{2} x^{0}-p x \quad \text { com } \quad x^{0}=t \quad \text { e } \quad x^{1}=x .
$$

Os operadores $a_{p}$ e $a_{p}^{\dagger}$ criam e aniquilam, respectivamente, partículas com momento $p$ e energia $p^{2}$; assim como obedecem à relação canônica de comutação:

$$
\left[a_{p^{\prime}}, a_{p}^{\dagger}\right]=2 \pi \delta\left(p-p^{\prime}\right) .
$$




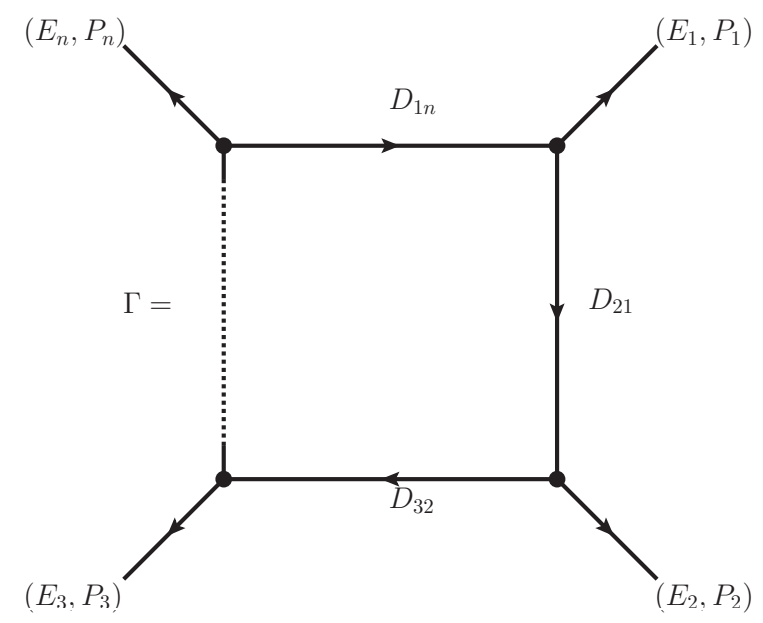

Figura 5.3: Qualquer ciclo fechado com propagadores orientados na mesma direção se anula identicamente.

Definindo o estado fundamental da maneira usual, isto é, como o estado aniquilado por $a_{p}$, verificamos que, em virtude da forma de $\varphi(x)$ em (5.1.12),

$$
\varphi(x)|0\rangle=0 .
$$

O fato do estado fundamental ser aniquilado pelo operador de campo $\varphi(x, t)$ significa que as partículas não viajam para trás no tempo e, conseqüentemente, o propagador deve possuir apenas um pólo na representação dos momentos, vide figura 5.2:

$$
\begin{aligned}
D\left(x-x^{\prime}\right) & =\left\langle 0\left|T \varphi(x) \varphi^{*}\left(x^{\prime}\right)\right| 0\right\rangle=\theta\left(t-t^{\prime}\right)\left\langle 0\left|\varphi(x) \varphi^{*}\left(x^{\prime}\right)\right| 0\right\rangle+\theta\left(t^{\prime}-t\right)\left\langle 0\left|\varphi^{*}\left(x^{\prime}\right) \varphi(x)\right| 0\right\rangle \\
& =\int \frac{d^{2} q}{4 \pi^{2}} \frac{i e^{-i q \cdot\left(x-x^{\prime}\right)}}{q^{0}-q^{2}+i \epsilon}
\end{aligned}
$$

com $d^{2} q=d q^{0} d q^{1}$. Alternativamente, a expressão para o propagador pode ser obtida ao resolvermos a equação diferencial:

$$
\left(i \partial_{t}+\partial_{x}^{2}\right) D(x)=i \delta(x),
$$

que leva à seguinte expressão no espaço das coordenadas:

$$
D(x)=\theta(t) \sqrt{\frac{\pi}{i t}} e^{\frac{i x^{2}}{4 t}} .
$$

O fato de o propagador ser puramente retardado implica o importante resultado:

Teorema 5.1.1. Qualquer ciclo fechado de propagadores orientados na mesma direção se anula identicamente.

Demonstração: Denote o propagado entre dois pontos $x_{m}$ e $x_{n}$ por $D_{m n}=D\left(x_{m}-x_{n}\right)$ e considere, 


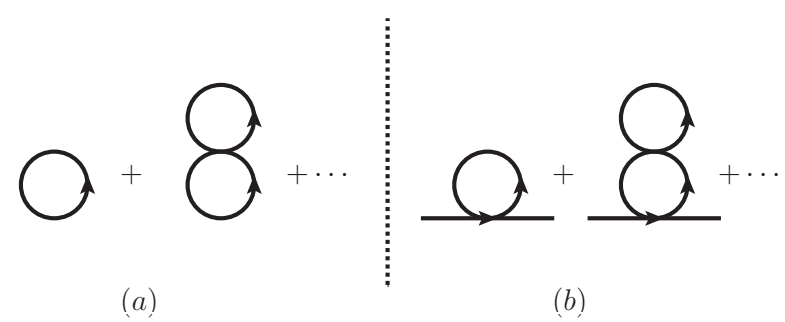

Figura 5.4: (a) Correções para a energia do estado fundamental; (b) Correções para a função de Green.

sem perda de generalidade, o propagador descrito pela figura 5.3, então:

$$
\begin{aligned}
\Gamma & =D\left(x_{2}-x_{1}\right) D\left(x_{3}-x_{2}\right) \ldots D\left(x_{n}-x_{n-1}\right) D\left(x_{1}-x_{n}\right) \\
& =\prod_{j=1}^{n}\left[\sqrt{\frac{\pi}{i t_{j}}} e^{\frac{i x_{j}^{2}}{4 t_{j}}}\right] \theta\left(t_{2}-t_{1}\right) \theta\left(t_{3}-t_{2}\right) \ldots \theta\left(t_{n}-t_{n-1}\right) \theta\left(t_{1}-t_{n}\right) .
\end{aligned}
$$

Como se trata de um ciclo fechado, apenas uma das seguintes condições pode ser verdadeira: $t_{1}<$ $t_{2}<\cdots<t_{n}$ ou $t_{1}>t_{2}>\cdots>t_{n}$, porém, ambas implicam que pelo menos uma das funções $\theta(t)$ terá seu argumento negativo. Logo, tal expressão é identicamente nula.

O teorema 5.1.1 possui três consequiências importantes:

1. O estado fundamental não é renormalizado. Posto que as correções quânticas à energia do vácuo devem ser da forma descrita pela figura 5.4 (a), o teorema 5.1.1 implica que a contribuição de tais diagramas é nula. Logo, $E_{\text {vácuo }}=0$.

2. A função de Green de uma partícula não é renormalizada. As correções radiativas, descritas na figura 5.4 (b), são suprimidas em virtude do teorema 5.1.1. Conseqüentemente, a relação de dispersão $E=p^{2}$ não recebe nenhuma correção quântica.

3. A matriz $S$ para o espalhamento de duas partículas é dada pela soma de diagramas, vide figura 5.5. Para constatarmos a validade dessa propriedade, basta que consideremos um diagrama genérico para o espalhamento de duas partículas e notemos que devido à conservação da carga o número de propagadores orientados para o futuro e para o passado deve ser constante. $O$ fato de que o número de propagadores orientados para o passado é nulo implica que o número de propagadores em qualquer instante temporal é igual ao número de pernas entrando ou saindo do diagrama. Assim, para a matriz $\mathrm{S}$ descrevendo o espalhamento de duas partículas só podem existir duas linhas em cada momento, de forma, que podemos concluir que o diagrama deve ser a soma de diagramas tipo bolha.

É interessante notar que o diagrama descrito na figura 5.5 é exatamente o mesmo que aparece ao considerarmos o espalhamento no contexto do modelo NLS, vide figura 3.4. Embora para o caso 


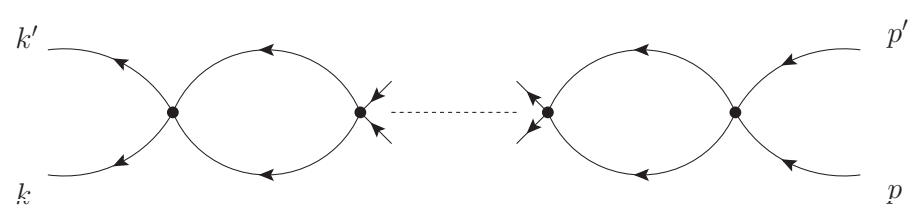

Figura 5.5: Diagramas do tipo bolha para o espalhamento de duas partículas.

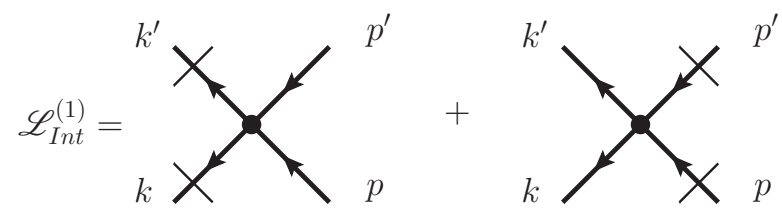

Figura 5.6: Vértice de interação na representação dos momentos, para a ação truncada até a quarta ordem nos campos.

do LL seja necessário levar em conta a presença de derivadas nos termos de interação, resultando, pois, em quatro tipos distintos de vértices, que dependem da posição das derivadas com respeito às linhas internas ou externas do diagrama. De toda forma, os cálculos ainda são factíveis.

\subsection{Espalhamento de Duas Partículas}

Para podermos estudar o espalhamento entre duas partículas no contexto do modelo de LL, precisamos considerar os termos de interação altamente não-lineares presentes na ação (5.1.9). Essa tarefa que é aparentemente impraticável, torna-se possível, uma vez que as únicas contribuições para a amplitude de espalhamento de duas partículas são dadas por diagramas como o da figura 5.5, e, para calcular a matriz $S$ para o espalhamento de duas partículas podemos, pois, truncar a ação complicada (5.1.9), mantendo apenas termos até a quarta ordem nos campos. Notando que:

$$
\frac{2-|\varphi|^{2}}{1-|\varphi|^{2}}=1+\frac{1}{1-|\varphi|^{2}}=1+\sum_{n=0}^{\infty}\left(|\varphi|^{2}\right)^{n}
$$

onde utilizamos uma expansão em série de Taylor, que se justifica pois $|\varphi|^{2}=\varphi \varphi^{*}=\frac{\left(1-S_{3}\right)}{2}$ e sabemos que $-1 \leq S_{3} \leq 1$, de forma que $|\varphi|^{2}<1^{4}$. Assim, a ação (5.1.9) assume a seguinte forma:

$$
S=\int d^{2} \times \frac{i}{2}\left(\varphi^{*} \partial_{t} \varphi-\partial_{t} \varphi^{*} \varphi\right)-\left|\partial_{x} \varphi\right|^{2}-\frac{g_{1}}{2}\left[\left(\varphi^{*} \partial_{x} \varphi\right)^{2}+\left(\partial_{x} \varphi^{*} \varphi\right)^{2}\right]+O\left(\varphi^{6}\right) .
$$

O vértice de interação na representação dos momentos está esboçado na figura 5.6, onde denotamos as derivadas espaciais por cortes sobre as linhas-mundo de uma dada partícula. É importante notar que cada gráfico denota uma soma sobre todos os diagramas de mesma topologia, mas com diferentes permutações dos momentos externos.

Introduzimos na expansão (5.2.1) o parâmetro formal $g_{1}$, de forma a manter o controle sobre a

\footnotetext{
${ }^{4}$ Para que essa afirmação seja realmente verdadeira precisamos excluir o caso em que $S_{3}=-1$, o que corresponde a não considerarmos o vetor $S=(0,0,-1) \in \mathbb{R}^{3}$.
} 
ordem da expansão perturbativa, que, ao final dos cálculos, deve ser tomado igual a unidade. Notamos que isso é muito importante para a fatoração da amplitude de espalhamento de três partículas, onde, de uma forma similar, vários parâmetros formais serão necessários para contabilizar a ordem dos diferentes vértices de interação, que surgem quando expandimos a ação (5.1.9) até a sexta ordem nos campos. Uma questão que surge naturalmente nesse ponto, versa sobre a consistência entre as propriedades da renormalização do modelo de LL e esse limite que devemos impor sobre tais parâmetros.

Tomando sem nenhuma perda de generalidade o seguinte ordenamento para os momentos incidentes: $p>p^{\prime}$, de forma a garantir que o espalhamento de fato ocorra, determinamos a matriz $S$ no setor de duas partículas através da relação:

$$
\left\langle k k^{\prime}|\hat{S}| p p^{\prime}\right\rangle=S\left(p, p^{\prime}\right) \delta_{+}^{(2)}\left(p, p^{\prime} ; k, k^{\prime}\right)
$$

onde denotamos:

$$
\delta_{ \pm}^{(2)}\left(p, p^{\prime} ; k, k^{\prime}\right)=(2 \pi)^{2}\left[\delta(p-k) \delta\left(p^{\prime}-k^{\prime}\right) \pm \delta\left(p-k^{\prime}\right) \delta\left(p^{\prime}-k\right)\right] .
$$

A amplitude de espalhamento, dada por:

$$
\begin{aligned}
\left\langle k k^{\prime}|\hat{S}| p p^{\prime}\right\rangle & =\left\langle k k^{\prime}\right| T e^{-i \int d t H_{l n t}\left|p p^{\prime}\right\rangle} \\
& =\left\langle k k^{\prime} \mid p p^{\prime}\right\rangle-i\left\langle k k^{\prime}\left|T \int d t H_{\text {Int }}\right| p p^{\prime}\right\rangle-\frac{1}{2}\left\langle k k^{\prime}\left|T\left(\int d t H_{l n t}\right)^{2}\right| p p^{\prime}\right\rangle+\cdots,
\end{aligned}
$$

é facilmente computada com

$$
H_{\text {Int }}=\int d x \mathscr{L}_{\text {Int }}^{(1)}=\frac{g_{1}}{2} \int d x\left[\left(\varphi^{*} \partial_{x} \varphi\right)^{2}+\left(\partial_{x} \varphi^{*} \varphi\right)^{2}\right]
$$

em cada ordem. A contribuição do termo de não-espalhamento e do nível de árvore são trivialmente calculadas e possuem, respectivamente, as seguintes expressões:

$$
\left.\left\langle k k^{\prime}|\hat{S}| p p^{\prime}\right\rangle\right|_{g_{1}^{0}}=\left\langle k k^{\prime} \mid p p^{\prime}\right\rangle=\delta_{+}^{(2)}\left(p, p^{\prime} ; k, k^{\prime}\right),
$$

e

$$
\left.\left\langle k k^{\prime}|\hat{S}| p p^{\prime}\right\rangle\right|_{g_{1}}=-i\left\langle k k^{\prime}\left|T \int d t H_{\mid n t}\right| p p^{\prime}\right\rangle=2 i g_{1} \frac{p p^{\prime}}{p-p^{\prime}} \delta_{+}^{(2)}\left(p, p^{\prime} ; k, k^{\prime}\right)
$$

Para evitar a complicada análise combinatória de [35], associada à devida colocação das derivadas nas linhas internas e externas, preferimos calcular as amplitudes de espalhamento utilizando o vértice de interação completo, que na representação dos momentos assume a seguinte forma:

$$
V\left(k, k^{\prime} ; p, p^{\prime}\right)=2 i g_{1}\left[p p^{\prime}+k k^{\prime}\right] 4 \pi^{2} \delta^{(2)}\left(p+p^{\prime}-k-k^{\prime}\right)
$$

onde $\delta^{(2)}(q)=\delta\left(q^{0}\right) \delta\left(q^{1}\right)$. Dessa forma, para calcularmos a amplitude de espalhamento a 1-laço, 
basta considerarmos o produto de dois vértices (5.2.7), ou seja,

$$
\begin{aligned}
\left.\left\langle k k^{\prime}|\hat{S}| p p^{\prime}\right\rangle\right|_{g_{1}^{2}} & =-\frac{1}{2}\left\langle k k^{\prime}\left|T\left(\int H_{i n t} d t\right)^{2}\right| p p^{\prime}\right\rangle \\
& =\frac{1}{2} \int \frac{d^{2} q}{4 \pi^{2}} \int \frac{d^{2} q^{\prime}}{4 \pi^{2}} V\left(k, k^{\prime} ; q, q^{\prime}\right) D(q) D\left(q^{\prime}\right) V\left(q, q^{\prime} ; p, p^{\prime}\right) \\
& =2 g_{1}^{2} \int \frac{d^{2} q}{4 \pi^{2}} \frac{\left[p p^{\prime}+q\left(p+p^{\prime}-q\right)\right]\left[k k^{\prime}+q\left(k+k^{\prime}-q\right)\right]}{\left[q^{0}-q^{2}+i \epsilon\right]\left[p^{0}+p^{\prime 0}-q^{0}-\left(p+p^{\prime}-q\right)^{2}+i \epsilon\right]} 4 \pi^{2} \delta^{(2)}\left(p+p^{\prime}-k-k^{\prime}\right) \\
& =2\left(i g_{1}\right)^{2}\left[p p^{\prime} k k^{\prime} I_{0}\left(p, p^{\prime}\right)-\left(p p^{\prime}+k k^{\prime}\right) I_{1}\left(p, p^{\prime}\right)+I_{2}\left(p, p^{\prime}\right)\right] 4 \pi^{2} \delta^{(2)}\left(p+p^{\prime}-k-k^{\prime}\right),
\end{aligned}
$$

onde as integrais $I_{i}\left(p, p^{\prime}\right), i=0,1,2$ estão definidas no apêndice $\mathrm{D}$ pelas equações (D.0.2). Após algumas manipulações obtemos:

$$
\begin{aligned}
\left.\left\langle k k^{\prime}|\hat{S}| p p^{\prime}\right\rangle\right|_{g_{1}^{2}} & =\frac{\left(i g_{1}\right)^{2}}{8} 4 \pi^{2} \delta^{(2)}\left(p+p^{\prime}-k-k^{\prime}\right)\left[\left(p^{0}+p^{\prime 0}-p^{2}-p^{\prime 2}\right)-4 p p^{\prime}\right] . \\
& \cdot \frac{2\left(p^{0}+p^{\prime 0}-p^{2}-p^{\prime 2}\right)+\left(p-p^{\prime}\right)^{2}-\left(k-k^{\prime}\right)^{2}-8 k k^{\prime}}{\sqrt{\left(p-p^{\prime}\right)^{2}+2\left(p^{0}+p^{\prime 0}-p^{2}-p^{\prime 2}\right)}} .
\end{aligned}
$$

Se impusermos que as partículas incidentes, isto é, aquelas com momento $p$ e $p^{\prime}$ estejam na camada de massa:

$$
p^{0}=p^{2} \quad \text { e } \quad p^{\prime 0}=p^{\prime 2},
$$

simplificamos drasticamente a expressão (5.2.8),

$$
\begin{aligned}
\left.\left\langle k k^{\prime}|\hat{S}| p p^{\prime}\right\rangle\right|_{g_{1}^{2}} & =i g_{1} \frac{p p^{\prime}}{\left|p-p^{\prime}\right|}\left[2 i g_{1}\left(p p^{\prime}+k k^{\prime}\right) 4 \pi^{2} \delta^{(2)}\left(p+p^{\prime}-k-k^{\prime}\right)\right] \\
& =i g_{1} \frac{p p^{\prime}}{p-p^{\prime}} V\left(k, k^{\prime} ; p, p^{\prime}\right),
\end{aligned}
$$

onde na última passagem reconhecemos a expressão do vértice de interação completo na representação dos momentos (5.2.7) e invocamos o ordenamento para os momentos iniciais: $p>p^{\prime}$.

A equação (5.2.9) já inclui a soma sobre todos os diagramas com 1-laço, levando em consideração todas as possíveis configurações das derivadas atuando sobre as linhas internas e externas, com os devidos fatores combinatórios. Ao notarmos a presença do vértice de interação (5.2.7) em (5.2.9), podemos considerar a amplitude de espalhamento com 1-laço como o vértice de interação com os momentos $p$ e $p^{\prime}$ na camada de massa multiplicada por uma função desse par de momentos e do parâmetro de expansão $g_{1}$. Conseqüentemente, a amplitude de espalhamento com $n$-laços corresponde ao produto de $n$ vértices de interação,

$$
\left.\left\langle k k^{\prime}|\hat{S}| p p^{\prime}\right\rangle\right|_{g_{1}^{n+1}}=\left(i g \frac{p p^{\prime}}{p-p^{\prime}}\right)^{n}\left[2 i g_{1}\left(p p^{\prime}+k k^{\prime}\right) 4 \pi^{2} \delta^{(2)}\left(p+p^{\prime}-k-k^{\prime}\right)\right] .
$$

Ao colocarmos $k$ e $k^{\prime}$ também na camada de massa, e utilizarmos a relação:

$$
4 \pi^{2} \delta^{(2)}\left(p+p^{\prime}-k-k^{\prime}\right)=\frac{1}{2\left(p-p^{\prime}\right)} \delta_{+}^{(2)}\left(p, p^{\prime} ; k, k^{\prime}\right),
$$


concluímos que:

$$
\left.\left\langle k k^{\prime}|\hat{S}| p p^{\prime}\right\rangle\right|_{g_{1}^{n+1}}=2\left(i g_{1} \frac{p p^{\prime}}{p-p^{\prime}}\right)^{n+1} \delta_{+}^{(2)}\left(p, p^{\prime} ; k, k^{\prime}\right) .
$$

Logo, a matriz $S$ para o espalhamento de duas partículas assume a forma [35]:

$$
S\left(p, p^{\prime}\right)=1+2 \sum_{n=1}^{\infty}\left(i g_{1}\right)^{n}\left(\frac{p p^{\prime}}{p-p^{\prime}}\right)^{n}=\frac{\frac{1}{p}-\frac{1}{p^{\prime}}-i g_{1}}{\frac{1}{p}-\frac{1}{p^{\prime}}+i g_{1}} \stackrel{g_{1} \rightarrow 1}{\longrightarrow} \frac{\frac{1}{p}-\frac{1}{p^{\prime}}-i}{\frac{1}{p}-\frac{1}{p^{\prime}}+i} .
$$

\subsection{Espalhamento de Três Partículas}

A verificação direta da fatorabilidade da matriz $S$ através de cálculos perturbativos é significativamente complicada de se efetuar, notadamente, devido à intrincada análise diagramática necessária. Dessa forma, não é surpreendente que ela tenha sido verificada explicitamente ${ }^{5}$ somente para o caso mais simples do modelo NLS em [39, 104], conforme explicamos na seção 3.6. Antes de apresentarmos os cálculos para o modelo de LL, gostaríamos de ressaltar que existe uma diferença significativa entre os modelos NLS e de LL, que é responsável pela maior dificuldade em se lidar com o segundo. De fato, se considerarmos a Lagrangeana do modelo NLS (3.6.1),

$$
\mathscr{L}=\frac{i}{2}\left[\psi^{\dagger} \partial_{t} \psi-\partial_{t} \psi^{\dagger} \psi\right]-\left|\partial_{x} \psi\right|^{2}-g|\psi|^{4}
$$

verificamos que, ao calcularmos a amplitude de espalhamento de três partículas, precisamos lidar com apenas um tipo de vértice. Ainda mais importante é o fato de que isso contínua verdade para o caso geral envolvendo o espalhamento de $N$ partículas, ou seja, não surgem novos vértices de interação conforme mais partículas participam do espalhamento. Entretanto, para o modelo de LL a situação é drasticamente diferente e, para se obter a amplitude de espalhamento no setor de três partículas, precisamos truncar a ação (5.1.9), mantendo termos até a sexta ordem nos campos, o que acarreta na introdução de dois novos vértices de interação. Fica claro, pois, que no caso do modelo de LL, conforme envolvemos mais partículas no processo de espalhamento, mais vértices de interação precisam ser considerados. Essa peculiaridade do modelo de $L L$ torna a análise perturbativa impraticável para o espalhamento de $N$ partículas. Não obstante, tomamos o primeiro passo nessa direção ao mostrarmos a fatorabilidade da matriz $S$ para o espalhamento de três partículas em primeira ordem perturbativa.

Restringindo nossa análise ao setor com três partículas, podemos parar a expansão perturbativa da ação (5.1.9) na sexta ordem nos campos, pois a conservação da carga, em conjunção com o fato de o propagador ser puramente retardado, implica que os vértices advindos de ordens superiores não contribuem [35]. A Lagrangeana resultante após o truncamento é a seguinte:

$$
\begin{aligned}
\mathscr{L}=\frac{i}{2}\left(\varphi^{*} \partial_{t} \varphi-\partial_{t} \varphi^{*} \varphi\right)-\left|\partial_{x} \varphi\right|^{2} & -\frac{g_{1}}{2}\left[\left(\varphi^{*} \partial_{x} \varphi\right)^{2}+\left(\partial_{x} \varphi^{*} \varphi\right)^{2}\right]- \\
& -\frac{g_{2}}{4}|\varphi|^{2}\left[\left(\varphi^{*} \partial_{x} \varphi\right)^{2}+\left(\partial_{x} \varphi^{*} \varphi\right)^{2}\right]-\frac{g_{3}}{2}|\varphi|^{4}\left|\partial_{x} \varphi\right|^{2},
\end{aligned}
$$

onde introduzimos, analogamente ao cálculo perturbativo da matriz $S$ para o setor de duas partículas,

\footnotetext{
${ }^{5}$ Considerando todas as ordens em teoria de perturbações.
} 


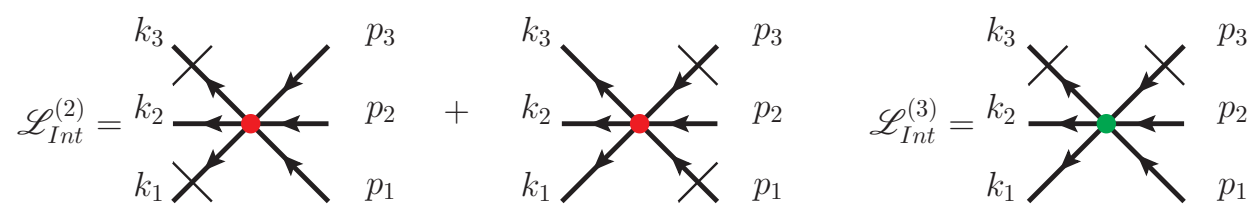

Figura 5.7: Vértices de interação na representação dos momentos adicionais para a ação truncada até a sexta ordem nos campos.

constantes de acoplamento arbitrárias $g_{1}, g_{2}$ e $g_{3}$ para cada um dos vértices, de forma a manter um controle sobre a ordem dos cálculos perturbativos. Assim, como no caso do espalhamento de duas partículas, devemos ao final das contas considerar o limite $g_{i} \rightarrow 1, i=1,2,3$. Como mostramos adiante, esse é um ponto crucial para se obter a fatoração da matriz $S$. Os vértices de interação na representação dos momentos estão esboçados nas figuras 5.6 e 5.7 .

\subsubsection{Cálculos Diagramáticos}

Ao supormos a analiticidade ${ }^{6}$ da amplitude de espalhamento no setor de três partículas nas constantes de acoplamento $g_{i}, i=1,2,3$, podemos escrevê-la como:

$$
\langle\mathbf{k}|\hat{S}| \mathbf{p}\rangle=\langle\mathbf{k} \mid \mathbf{p}\rangle+\left.\langle\mathbf{k}|\hat{S}| \mathbf{p}\rangle\right|_{g}+\left.\langle\mathbf{k}|\hat{S}| \mathbf{p}\rangle\right|_{g^{2}}+\cdots
$$

onde $g$ representa algum dos parâmetros $g_{i}$ e agrupamos os momentos das partículas no decorrer do espalhamento, de forma a considerá-las como as componentes de um vetor em $\mathbb{R}^{3}$ :

$$
\mathbf{k}=\left(k_{1}, k_{2}, k_{3}\right) \quad \text { e } \quad \mathbf{p}=\left(p_{1}, p_{2}, p_{3}\right) .
$$

Sem perda de generalidade, demandamos que as componentes de $\mathbf{p}$ estejam arranjadas na seguinte ordem: $p_{1}>p_{2}>p_{3}$.

Assim como no caso do espalhamento de três partículas no contexto do modelo NLS (seção 3.6), é conveniente considerarmos o operador de simetrização, definido por (3.6.3). Com isso é fácil mostrar que o termo de não-espalhamento assume a seguinte forma:

$$
\langle\mathbf{p} \mid \mathbf{k}\rangle=3 !(2 \pi)^{3} \mathcal{S}_{p}\left[\delta\left(p_{1}-k_{1}\right) \delta\left(p_{2}-k_{2}\right) \delta\left(p_{3}-k_{3}\right)\right],
$$

enquanto que o termo do nível de árvore:

$$
\left.\langle\mathbf{k}|\hat{S}| \mathbf{p}\rangle\right|_{g}=9 i(2 \pi)^{2} \mathcal{S}_{k, p}\left\{2 g_{1}\left(k_{1} k_{2}+p_{1} p_{2}\right) 2 \pi \delta\left(k_{3}-p_{3}\right)+g_{2}\left(k_{1} k_{2}+p_{1} p_{2}\right)-2 g_{3} k_{1} p_{1}\right\} \delta E \delta P,
$$

onde denotamos por $\delta E$ e $\delta P$ as funções delta correspondentes à conservação da energia e momento

\footnotetext{
${ }^{6} \mathrm{~A}$ analiticidade da matriz $S$ para o caso do modelo NLS pode ser verificada explicitamente através das considerações do Ansatz de Bethe coordenado feitas por Yang em [60,105], dessa forma, para o caso do modelo de LL, trata-se de uma hipótese natural.
} 


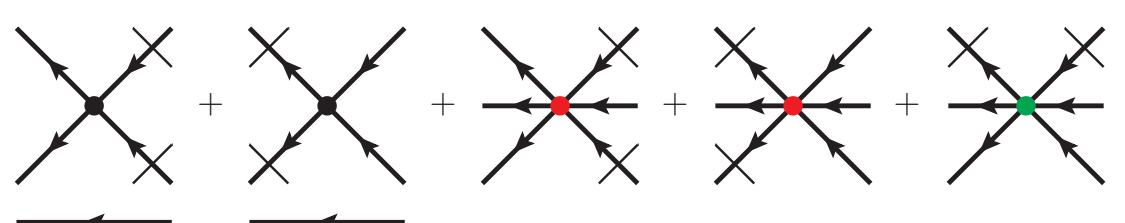

Figura 5.8: Os diagramas de Feynman para o nível de árvore para o espalhamento de três partículas no modelo de LL.

totais,

$$
\delta E=\delta\left(\sum_{i=1}^{3}\left(k_{i}^{0}-p_{i}^{0}\right)\right) \quad \text { e } \delta P=\delta\left(\sum_{i=1}^{3}\left(k_{i}-p_{i}\right)\right) .
$$

Exibimos os gráficos de Feynman para o nível de árvore (conexos e desconexos) na figura 5.8.

A expressão para o nível de árvore (5.3.3b) define o vértice de interação na representação dos momentos, vide figura 5.9. Usando tal vértice somos capazes de calcular a amplitude de espalhamento

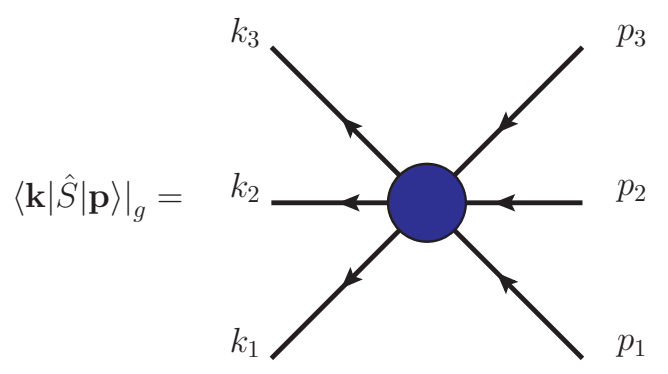

Figura 5.9: Representação diagramática do vértice de interação no espaço dos momentos.

em segunda ordem, conforme descrito pela figura 5.10, cuja expressão analítica é:

$$
\begin{aligned}
\left.\langle\mathbf{k}|\hat{S}| \mathbf{p}\rangle\right|_{g^{2}} & =\frac{1}{6} \iiint \prod_{j=1}^{3}\left[\frac{d^{2} q_{j}}{4 \pi^{2}} D\left(q_{j}\right)\right] 9 i(2 \pi)^{2} \mathcal{S}_{k, q}\left\{2 g_{1}\left(k_{1} k_{2}+q_{1} q_{2}\right) 2 \pi \delta\left(k_{3}-q_{3}\right)+g_{2}\left(k_{1} k_{2}+q_{1} q_{2}\right)-\right. \\
& \left.-2 g_{3} k_{1} q_{1}\right\} 9 i(2 \pi)^{2} \mathcal{S}_{q, p}\left\{2 g_{1}\left(q_{1} q_{2}+p_{1} p_{2}\right) 2 \pi \delta\left(q_{3}-p_{3}\right)+g_{2}\left(q_{1} q_{2}+p_{1} p_{2}\right)-2 g_{3} q_{1} p_{1}\right\} . \\
& \cdot \delta\left(\sum_{i=1}^{3}\left(k_{i}^{0}-q_{i}^{0}\right)\right) \delta\left(\sum_{i=1}^{3}\left(k_{i}-q_{i}\right)\right) \delta\left(\sum_{i=1}^{3}\left(q_{i}^{0}-p_{i}^{0}\right)\right) \delta\left(\sum_{i=1}^{3}\left(q_{i}-p_{i}\right)\right) .
\end{aligned}
$$

As funções delta presentes em (5.3.5) podem ser usadas para integrarmos sobre $q_{3}^{0}$ e $q_{3}$, já as integrais sobre $q_{1}^{0}$ e $q_{2}^{0}$ podem ser efetuadas ao fecharmos o contorno no semiplano inferior. Aproveitamos também para atuar com os simetrizadores sobre $\mathbf{q}$, de forma que a expressão dependa 


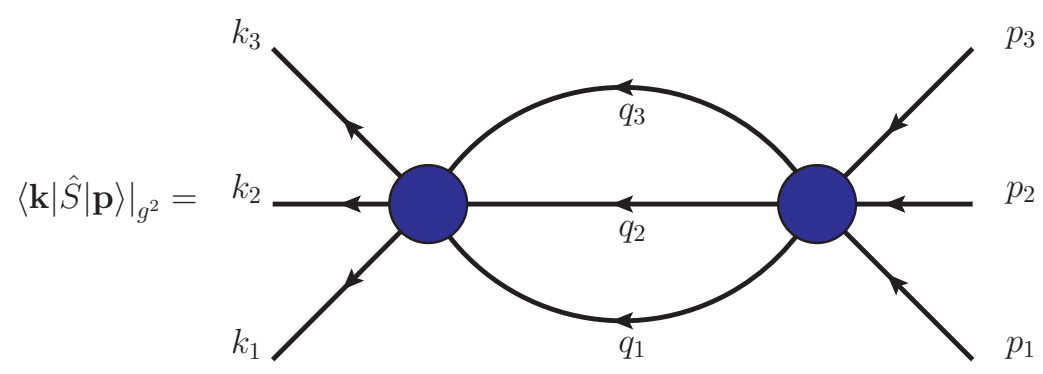

Figura 5.10: Representação diagramática da amplitude de espalhamento em segunda ordem.

apenas dos momentos externos simetrizados,

$$
\begin{aligned}
\left.\langle\mathbf{k}|\hat{S}| \mathbf{p}\rangle\right|_{g^{2}} & =\frac{3}{2 i} \delta E \delta P \mathcal{S}_{p, k}\left\{\int \int \frac { d q _ { 1 } d q _ { 2 } } { \sum p ^ { 0 } - q _ { 1 } ^ { 2 } - q _ { 2 } ^ { 2 } - ( \sum p - q _ { 1 } - q _ { 2 } ) ^ { 2 } + i \epsilon } \left[16 \pi^{2} g_{1}^{2} f_{1}(\mathbf{k}, \mathbf{q}) f_{1}(\mathbf{p}, \mathbf{q})+\right.\right. \\
& +g_{2}^{2} f_{2}(\mathbf{k}, \mathbf{q}) f_{2}(\mathbf{p}, \mathbf{q})+4 g_{3}^{2} k_{1} p_{1} \sum k \sum p+4 \pi g_{1} g_{2}\left(f_{1}(\mathbf{k}, \mathbf{q}) f_{2}(\mathbf{p}, \mathbf{q})+f_{2}(\mathbf{k}, \mathbf{q}) f_{1}(\mathbf{p}, \mathbf{q})\right)- \\
& \left.\left.-8 \pi g_{1} g_{3}\left(p_{1} \sum p f_{1}(\mathbf{k}, \mathbf{q})+k_{1} \sum k f_{1}(\mathbf{p}, \mathbf{q})\right)-2 g_{2} g_{3}\left(p_{1} \sum p f_{2}(\mathbf{k}, \mathbf{q})+k_{1} \sum k f_{2}(\mathbf{p}, \mathbf{q})\right)\right]\right\},
\end{aligned}
$$

onde introduzimos as seguintes funções:

$$
\begin{aligned}
f_{1}(\mathbf{x}, \mathbf{q}) & =\left(x_{1} x_{2}+q_{1} q_{2}\right) \delta\left(x_{1}+x_{2}-q_{1}-q_{2}\right)+\left[x_{1} x_{2}+q_{1}\left(\sum x-q_{1}-q_{2}\right)\right] \delta\left(x_{3}-q_{2}\right)+ \\
& +\left[x_{1} x_{2}+q_{2}\left(\sum x-q_{1}-q_{2}\right)\right] \delta\left(x_{3}-q_{1}\right) \\
f_{2}(\mathbf{x}, \mathbf{q}) & =3 x_{1} x_{2}+\left(q_{1}+q_{2}\right) \sum x-q_{1}^{2}-q_{2}^{2}-q_{1} q_{2}
\end{aligned}
$$

Devido a já enorme quantidade de índices, simplificamos a notação ao deixar de escrever os índices de soma, isto é, passamos a denotar $\sum x \equiv \sum_{i=1}^{3} x_{i}$. É mais simples calcular cada termo da equação (5.3.6) separadamente.

(a) Termo proporcional a $g_{1}^{2}$ : A maior parte das integrais é trivialmente calculada com o emprego das funções delta presentes em $f_{1}(\mathbf{x}, \mathbf{q})$, enquanto que as funções delta remanescentes, correspondendo às conservações de energia e momento totais, permitem que escrevamos os termos resultantes como uma soma das seguintes contribuições: (i) a parte finita, que é uma função dos momentos externos; e (ii) o termo proporcional a $\delta\left(p_{3}-k_{3}\right)$. A expressão resultante 


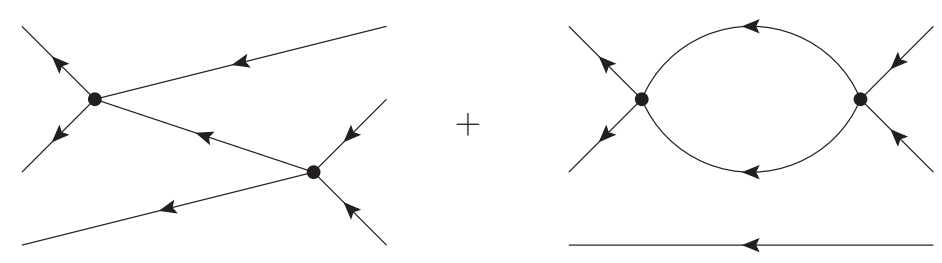

Figura 5.11: Diagramas de Feynman correspondentes à equação (5.3.8a).

completa é a seguinte:

$$
\begin{aligned}
& 16 \pi^{2} g_{1}^{2} \iint \frac{f_{1}(\mathbf{k}, \mathbf{q}) f_{1}(\mathbf{p}, \mathbf{q}) d q_{1} d q_{2}}{\sum p^{0}-q_{1}^{2}-q_{2}^{2}-\left(\sum p-q_{1}-q_{2}\right)^{2}+i \epsilon} \\
& \quad=96 \pi^{2} g_{1}^{2} \frac{\left[k_{1} k_{2}+p_{3}\left(k_{1}+k_{2}-p_{3}\right)\right]\left[p_{1} p_{2}+k_{3}\left(p_{1}+p_{2}-k_{3}\right)\right]}{\sum p^{0}-p_{3}^{2}-k_{3}^{2}-\left(p_{1}+p_{2}-k_{3}\right)^{2}+i \epsilon}- \\
& \quad-24 \pi^{2} g_{1}^{2} \int d q \frac{\left(s-2 p_{1} p_{2}\right)\left[s+\frac{1}{4}\left(p_{1}-p_{2}\right)^{2}-\frac{1}{4}\left(k_{1}-k_{2}\right)^{2}-2 k_{1} k_{2}\right]}{q^{2}-\frac{1}{4}\left(p_{1}-p_{2}\right)^{2}-s-i \epsilon} \delta\left(k_{3}-p_{3}\right),
\end{aligned}
$$

onde denotamos $s \equiv \frac{1}{2}\left(\sum p^{0}-\sum p^{2}\right)$ e mudamos a variável de integração $q \rightarrow q+\frac{1}{2}\left(k_{1}+k_{2}\right)$. Ademais, supomos que a parte divergente, que não escrevemos explicitamente, pode ser apropriadamente incorporada por algum esquema de regularização (como, por exemplo, o de PauliVillars). Os diagramas correspondentes estão exibidos na figura 5.11. Mais uma vez ressaltamos que cada diagrama corresponde, de fato, a uma soma sobre todos os gráficos com a mesma topologia, porém, com distintas permutações dos momentos externos e com as derivadas atuando sobre linhas diferentes. O primeiro diagrama na figura 5.11 representa o primeiro termo na equação (5.3.8a), enquanto que o segundo termo fornece a parte finita do segundo diagrama. Exibimos os diagramas correspondentes à figura 5.11, mas com as derivadas explicitamente distribuídas, nas figuras 5.12 e $\mathrm{E} .1$, respectivamente.

(b) Termo proporcional a $g_{2}^{2}$ : Após algumas manipulações algébricas, esse termo pode ser escrito na seguinte forma:

$$
g_{2}^{2} \iint \frac{d q_{1} d q_{2} f_{2}(\mathbf{k}, \mathbf{q}) f_{2}(\mathbf{p}, \mathbf{q})}{\sum p^{0}-q_{1}^{2}-q_{2}^{2}-\left(\sum p-q_{1}-q_{2}\right)^{2}+i \epsilon}=-\frac{g_{2}^{2}}{2} \iint \frac{d q_{1} d q_{2}\left[3 p_{1} p_{2}-s\right]\left[3 k_{1} k_{2}-s\right]}{q_{1}^{2}+q_{2}^{2}+q_{1} q_{2}-\left(q_{1}+q_{2}\right) \sum p-s-i \epsilon} .
$$

Os gráficos de Feynman correspondentes estão representados na figura E.2.

(c) Termo proporcional a $g_{3}^{2}$ : Simples transformações levam à seguinte expressão:

$$
4 g_{3}^{2} \iint \frac{d q_{1} d q_{2} k_{1} p_{1} \sum k \sum p}{\sum p^{0}-q_{1}^{2}-q_{2}^{2}-\left(\sum p-q_{1}-q_{2}\right)^{2}+i \epsilon}=-2 g_{3}^{2} \iint \frac{d q_{1} d q_{2} k_{1} p_{1} \sum k \sum p}{q_{1}^{2}+q_{2}^{2}+q_{1} q_{2}-\left(q_{1}+q_{2}\right) \sum p-s-i \epsilon} .
$$


Os diagramas de Feynman correspondentes estão representados na figura E.3.

(d) Termo proporcional a $g_{1} g_{2}$ : Uma das integrais nesse termo é trivialmente calculada usando a função delta presente em $f_{1}(\mathbf{x}, \mathbf{q})$, enquanto que a integral restante, depois de algumas manipulações algébricas e da mudança de variável de integração

$$
q_{1} \rightarrow q_{1}+\frac{1}{2}\left(k_{1}+k_{2}\right) \quad \text { e } \quad q_{2} \rightarrow q_{2}+\frac{1}{2}\left(p_{1}+p_{2}\right)
$$

fornece:

$$
\begin{aligned}
4 \pi g_{1} g_{2} \iint d q_{1} d q_{2} \frac{f_{1}(\mathbf{k}, \mathbf{q}) f_{2}(\mathbf{p}, \mathbf{q})+f_{2}(\mathbf{k}, \mathbf{q}) f_{1}(\mathbf{p}, \mathbf{q})}{\sum p^{0}-q_{1}^{2}-q_{2}^{2}-\left(\sum p-q_{1}-q_{2}\right)^{2}+i \epsilon} \\
=-6 \pi g_{1} g_{2} \int d q \frac{\left[k_{3}\left(k_{1}+k_{2}\right)-k_{1} k_{2}+s\right]\left(s-3 p_{1} p_{2}\right)}{q^{2}-\frac{1}{4}\left(k_{1}+k_{2}\right)^{2}-k_{3}\left(k_{1}+k_{2}\right)-s-i \epsilon}- \\
-6 \pi g_{1} g_{2} \int d q \frac{\left[p_{3}\left(p_{1}+p_{2}\right)-p_{1} p_{2}+s\right]\left(s-3 k_{1} k_{2}\right)}{q^{2}-\frac{1}{4}\left(p_{1}+p_{2}\right)^{2}-p_{3}\left(p_{1}+p_{2}\right)-s-i \epsilon} .
\end{aligned}
$$

Os diagramas de Feynman correspondentes estão representados na figura E.4.

(e) Termo proporcional a $g_{1} g_{3}$ : Mais uma vez as funções delta em $f_{1}(\mathbf{x}, \mathbf{q})$ permitem efetuar trivialmente uma das integrações, de sorte que a expressão resultante adquire a seguinte forma:

$$
\begin{aligned}
-8 \pi g_{1} g_{3} \iint d q_{1} d q_{2} \frac{p_{1} \sum p f_{1}(\mathbf{k}, \mathbf{q})+k_{1} \sum k f_{1}(\mathbf{p}, \mathbf{q})}{\sum p^{0}-q_{1}^{2}-q_{2}^{2}-\left(\sum p-q_{1}-q_{2}\right)^{2}+i \epsilon} \\
=-12 \pi g_{1} g_{3} \int d q\left[p_{1} \sum p+k_{1} \sum k\right]- \\
-12 \pi g_{1} g_{3} \int d q \frac{k_{1} \sum k\left[p_{3}\left(p_{1}+p_{2}\right)-p_{1} p_{2}+s\right]}{q^{2}-\frac{1}{4}\left(p_{1}+p_{2}\right)^{2}-p_{3}\left(p_{1}+p_{2}\right)-s-i \epsilon}- \\
-12 \pi g_{1} g_{3} \int d q \frac{p_{1} \sum p\left[k_{3}\left(k_{1}+k_{2}\right)-k_{1} k_{2}+s\right]}{q^{2}-\frac{1}{4}\left(k_{1}+k_{2}\right)^{2}-k_{3}\left(k_{1}+k_{2}\right)-s-i \epsilon}
\end{aligned}
$$

Os diagramas de Feynman correspondentes estão representados na figura E.5.

(f) Termo proporcional a $g_{2} g_{3}$ : Nesse termo inexistem funções delta, assim após algumas manipulações algébricas, obtemos:

$$
\begin{aligned}
& -2 g_{2} g_{3} \iint d q_{1} d q_{2} \frac{p_{1} \sum p f_{2}(\mathbf{k}, \mathbf{q})+k_{1} \sum k f_{2}(\mathbf{p}, \mathbf{q})}{\sum p^{0}-q_{1}^{2}-q_{2}^{2}-\left(\sum p-q_{1}-q_{2}\right)^{2}+i \epsilon} \\
& \quad=-g_{2} g_{3} \iint d q_{1} d q_{2} \frac{\left(p_{1} \sum p+k_{1} \sum k\right) s-3 k_{1} k_{2} p_{1} \sum p-3 k_{1} p_{1} p_{2} \sum k}{q_{1}^{2}+q_{2}^{2}+q_{1} q_{2}-\left(q_{1}+q_{2}\right) \sum p-s-i \epsilon} .
\end{aligned}
$$

Os diagramas de Feynman correspondentes estão representados na figura E.6.

Ressaltamos que nas equações (5.3.8) desprezamos as divergências em virtude de sua incorporação em um método de regularização, de forma a só trabalharmos com as respectivas partes finitas. Note também que essas fórmulas foram obtidas para o caso geral, onde todas as partículas estão fora 
da camada de massa, contudo simplificações consideráveis são obtidas ao colocarmos os momentos externos $p_{i}^{0}=p_{i}^{2}$ e $k_{i}^{0}=k_{i}^{2}$, com $i=1,2,3$, na camada de massa, devido as seguintes identidades:

$$
\begin{aligned}
\sum p^{0}-\sum p^{2} & =\sum k^{0}-\sum k^{2}=0, \\
\sum p-\left(\sum p\right)^{2} & =-2\left(p_{1} p_{2}+p_{1} p_{3}+p_{2} p_{3}\right), \\
\sum k-\left(\sum k\right)^{2} & =-2\left(k_{1} k_{2}+k_{1} k_{3}+k_{2} k_{3}\right), \\
\sum p-\left(\sum p\right)^{2} & =\sum k-\left(\sum k\right)^{2} \Rightarrow p_{1} p_{2}+p_{1} p_{3}+p_{2} p_{3}=k_{1} k_{2}+k_{1} k_{3}+k_{2} k_{3} .
\end{aligned}
$$

Notamos que a integral remanescente no segundo termo de (5.3.8a) é simplesmente $I_{a}\left(p_{1}, p_{2}\right)$, dada por (D.0.5a), enquanto que em (5.3.8d) e (5.3.8e) resta apenas computar, respectivamente, $I_{b}\left(k_{1}, k_{2}, k_{3}\right)$ e $I_{b}\left(p_{1}, p_{2}, p_{3}\right)$, cujo resultado exibimos em (D.0.5b). Finalmente, a integral dupla presente em (5.3.8b), (5.3.8c) e (5.3.8f) é $I_{c}\left(p_{1}, p_{2}, p_{3}\right)$ (D.0.5c). Assim, se coletarmos todas as contribuições (5.3.8) em (5.3.6), substituindo os resultados (na camada de massa) das integrais ${ }^{7}$ (D.0.5) inferimos a seguinte expressão para a amplitude de espalhamento em segunda ordem:

$$
\begin{aligned}
\left.\langle\mathbf{k}|\hat{S}| \mathbf{p}\rangle\right|_{g^{2}}= & -\frac{3}{2} \mathcal{S}_{p, k}\left\{9 6 \pi ^ { 2 } g _ { 1 } ^ { 2 } \left[\frac{\left[k_{1} k_{2}+p_{3}\left(k_{1}+k_{2}-p_{3}\right)\right]\left[p_{1} p_{2}+k_{3}\left(p_{1}+p_{2}-k_{3}\right)\right]}{p_{1}^{2}+p_{2}^{2}-k_{3}^{2}-\left(p_{1}+p_{2}-k_{3}\right)^{2}+i \epsilon}-\right.\right. \\
& \left.-\frac{k_{1} k_{2} p_{1} p_{2}}{p_{1}-p_{2}} 2 \pi i \delta\left(p_{3}-k_{3}\right)\right]+k_{1} p_{1}\left[48 \pi^{2} i g_{1} g_{3}\left(\frac{k_{1} p_{2}}{p_{1}-p_{2}}+\frac{k_{2} p_{1}}{\left|k_{1}-k_{2}\right|}\right)+\right. \\
& \left.+\frac{4 \pi}{\sqrt{3}}\left(i \pi-\log Q^{2}\right)\left[\left(k_{1} p_{2}+k_{2} p_{1}\right) g_{3}\left(2 g_{3}-3 g_{2}\right)+g_{3}^{2} k_{1} p_{1}\right]\right]+ \\
& +k_{1} k_{2} p_{1} p_{2}\left[48 \pi^{2} i\left(\frac{1}{p_{1}-p_{2}}+\frac{1}{\left|k_{1}-k_{2}\right|}\right) g_{1}\left(2 g_{3}-3 g_{2}\right)+\right. \\
& \left.\left.+\frac{4 \pi}{\sqrt{3}}\left(i \pi-\log Q^{2}\right)\left(2 g_{3}-3 g_{2}\right)^{2}\right]\right\} \delta E \delta P .
\end{aligned}
$$

\subsection{Fatoração da Matriz S}

Tivéssemos suposto a integrabilidade quântica do modelo de LL, poderíamos ter obtido a matriz $S$ para o espalhamento de três partículas através da relação:

$$
\langle\mathbf{k}|\hat{S}| \mathbf{p}\rangle=S(\mathbf{p}) \delta_{+}^{(3)}(\mathbf{p}, \mathbf{k}) \quad \text { onde } \quad \delta_{+}^{(3)}(\mathbf{p}, \mathbf{k})=\langle\mathbf{p} \mid \mathbf{k}\rangle .
$$

Essa relação, análoga a (5.2.2), indica a impossibilidade da aniquilação ou da criação de partículas durante o processo de espalhamento, por consegüinte os conjuntos de momentos inicial e final devem ser o mesmo. Isso não é óbvio do ponto de vista dos cálculos efetuados na seção anterior. De fato, apesar de o termo de não-espalhamento claramente possuir a forma (5.4.1), nem o termo de árvore (5.3.3b), muito menos os termos de segunda ordem (5.3.10) estão manifestamente nessa forma. Contudo, mostraremos que esse é o caso, e, portanto será possível deduzir expressões para o nível de árvore (5.3.3b) e para a segunda ordem (5.3.10) que sejam proporcionais a $\delta_{+}^{(3)}(\mathbf{p}, \mathbf{k})$.

Foquemos inicialmente no termo oriundo do nível de árvore (5.3.3b). Expandindo explicitamente

\footnotetext{
${ }^{7}$ Mantemos apenas a parte finita da integral $I_{c}\left(p_{1}, p_{2}, p_{3}\right)(D .0 .5 c)$.
} 
o operador de simetrização $\mathcal{S}_{k, p}$, obtemos:

$$
\begin{aligned}
\left.\langle\mathbf{k}|\hat{S}| \mathbf{p}\rangle\right|_{g} & =2 i g_{1}\left[\frac{p_{1} p_{2}}{p_{1}-p_{2}}+\frac{p_{1} p_{3}}{p_{1}-p_{3}}+\frac{p_{2} p_{3}}{p_{2}-p_{3}}\right] \delta_{+}^{(3)}(\mathbf{p}, \mathbf{k})+ \\
& +2 i(2 \pi)^{2}\left[\left(p_{1} p_{2}+p_{1} p_{3}+p_{2} p_{3}\right)\left(3 g_{2}-2 g_{3}\right)-g_{3}\left(p_{1}^{2}+p_{2}^{2}+p_{3}^{2}\right)\right] \delta E \delta P .
\end{aligned}
$$

Notamos de imediato que os dois termos presentes nessa expressão são consideravelmente distintos. Pois, enquanto que o primeiro termo de (5.4.2) já se encontra na forma necessária para que a matriz $S$ satisfaça (5.4.1), o segundo termo não contempla tal condição. Logo, se tivéssemos nos restringido apenas a essa ordem, a condição (5.4.1), que é necessária para se ter a integrabilidade quântica do modelo, não seria satisfeita. Similarmente, não é difícil de ver que a segunda ordem da amplitude de espalhamento também contém termos que não são proporcionais a $\delta_{+}^{(3)}(\mathbf{p}, \mathbf{k})$, tais termos se encontram no segundo e terceiro colchetes da equação (5.3.10).

Entretanto, se a integrabilidade quântica tivesse sido estabelecida e, conseqüentemente, houvesse a fatorização da matriz $S$, a matriz $S$ para o espalhamento de três partículas assumiria a seguinte forma exata:

$$
\begin{aligned}
S^{(3)}(\mathbf{p}, \mathbf{k}) & =S^{(2)}\left(p_{1}, p_{2}\right) S^{(2)}\left(p_{1}, p_{3}\right) S^{(2)}\left(p_{2}, p_{3}\right) \\
& =\left(\frac{\frac{1}{p_{1}}-\frac{1}{p_{2}}-i g}{\frac{1}{p_{1}}-\frac{1}{p_{2}}+i g}\right)\left(\frac{\frac{1}{p_{1}}-\frac{1}{p_{3}}-i g}{\frac{1}{p_{1}}-\frac{1}{p_{3}}+i g}\right)\left(\frac{\frac{1}{p_{2}}-\frac{1}{p_{3}}-i g}{\frac{1}{p_{2}}-\frac{1}{p_{3}}+i g}\right) \\
& =1+2 \sum_{n=1}^{2}\left[i g\left(\frac{p_{1} p_{2}}{p_{1}-p_{2}}+\frac{p_{1} p_{3}}{p_{1}-p_{3}}+\frac{p_{2} p_{3}}{p_{2}-p_{3}}\right)\right]^{n}+O\left(g^{3}\right),
\end{aligned}
$$

na última linha, expandimos a expressão exata até a segunda ordem em $g$. Comparando as equações (5.4.2) e (5.4.3), verificamos que a unidade em (5.4.3) corresponde ao termo de não-espalhamento $\langle\mathbf{p} \mid \mathbf{k}\rangle$, enquanto que o primeiro termo em (5.4.2) equivale ao termo de primeira ordem em (5.4.3). Demonstraremos, agora, que o segundo termo da equação (5.4.2) é tal que ele pode ser cancelado exatamente por um termo da contribuição de segunda ordem (5.3.10).

Uma expressão muito similar para o primeiro termo de (5.3.10) aparece nas considerações do modelo NLS (vide a expressão (3.6.9) para a soma dos gráficos conexos na seção 3.6). Nesse caso, o uso do teorema de Sokhotsky-Weierstrass (3.6.36):

$$
\frac{1}{x \pm i 0}=\mp i \pi \delta(x)+\text { v.p. }\left(\frac{1}{x}\right)
$$

provou-se essencial para a demonstração da fatoração. Esperamos, pois, que essa fórmula assuma um papel semelhante para o caso em consideração. No entanto, enquanto que a contribuição do valor principal para o caso do modelo NLS era nula, no modelo de LL, ela adquire um papel altamente não-trivial e induz os cancelamentos que tornam a fatoração possível. Os gráficos de Feynman para esse termo estão expostos na figura 5.12, onde somente a soma sobre diagramas topologicamente equivalentes com diferentes permutações dos momentos externos está implícita. Assim, existem quatro diagramas, que correspondem a todas as possíveis distribuições das derivadas.

Lembramos que escolhemos uma ordenação particular dos momentos incidentes: $p_{1}>p_{2}>p_{3}$, 


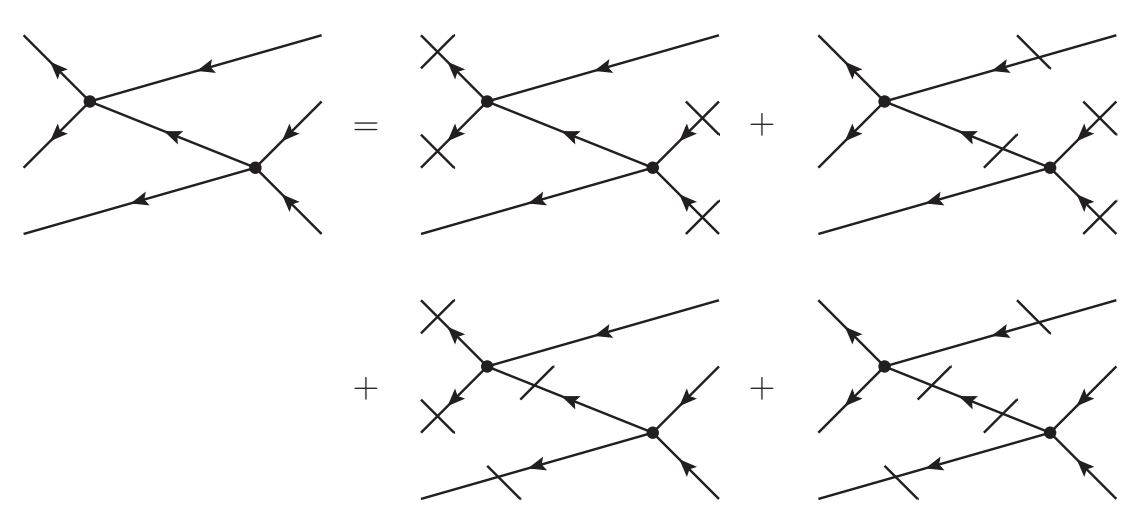

Figura 5.12: Diagramas de Feynman para o primeiro termo das equações (5.3.8a) e (5.3.10) com as derivadas explicitamente colocadas.

de forma a garantir que o espalhamento, de fato, ocorra. O valor principal do primeiro termo da equação (5.3.10) pode ser calculado se tomarmos $k_{i} \neq p_{j}, i, j \in\{1,2,3\}$ e utilizarmos a identidade:

$$
\frac{i}{k_{i}^{2}+k_{j}^{2}-p_{l}^{2}-\left(k_{i}+k_{j}-p_{l}\right)^{2}+i \epsilon}=\frac{1}{2} \frac{i}{k_{i}-k_{j}}\left(\frac{1}{p_{l}-k_{j}-i \epsilon}+\frac{1}{k_{i}-p_{l}-i \epsilon}\right)
$$

bem como levarmos em consideração as funções delta para a conservação da energia e do momento. Notamos que somente o primeiro e o último diagrama no lado direito da figura 5.12 contribuem para o valor principal, enquanto que os outros dois se anulam identicamente devido à conservação do momento. Após longas e tediosas transformações, obtemos:

$$
\begin{aligned}
& \text { v.p. }\left\{\mathcal{S}_{p, k}\left[\frac{\left[k_{1} k_{2}+p_{3}\left(k_{1}+k_{2}-p_{3}\right)\right]\left[p_{1} p_{2}+k_{3}\left(p_{1}+p_{2}-k_{3}\right)\right]}{p_{1}^{2}+p_{2}^{2}-k_{3}^{2}-\left(p_{1}+p_{2}-k_{3}\right)^{2}+i \epsilon}\right]\right\}= \\
& \quad=\frac{1}{18}\left(-p_{1}^{2}-p_{2}^{2}-p_{3}^{2}+p_{1} p_{2}+p_{1} p_{3}+p_{2} p_{3}\right) .
\end{aligned}
$$

A parte proporcional a função delta de (5.4.4) fornece:

$$
\delta\left[p_{1}^{2}+p_{2}^{2}-k_{3}^{2}-\left(p_{1}+p_{2}-k_{3}\right)\right]=\frac{1}{2\left(p_{1}-p_{2}\right)}\left[\delta\left(p_{1}-k_{3}\right)+\delta\left(p_{2}-k_{3}\right)\right] .
$$

Substituindo os resultados (5.4.6) de volta em (5.3.10), concluímos:

$$
\begin{aligned}
\left.\langle\mathbf{k}|\hat{S}| \mathbf{p}\rangle\right|_{g^{2}} & =8 i \pi^{2} g_{1}^{2}\left(p_{1}^{2}+p_{2}^{2}+p_{3}^{2}-p_{1} p_{2}-p_{1} p_{3}-p_{2} p_{3}\right) \delta E \delta P- \\
& -\frac{3 i}{2} \delta E \delta P \mathcal{S}_{p, k}\left\{-96 \pi^{2} g_{1}^{2} \frac{k_{1} k_{2} p_{1} p_{2}}{p_{1}-p_{2}} 2 \pi i\left[\delta\left(k_{3}-p_{1}\right)+\delta\left(k_{3}-p_{2}\right)+\delta\left(k_{3}-p_{3}\right)\right]+\right. \\
& +k_{1} p_{1}\left[48 \pi^{2} i g_{1} g_{3}\left(\frac{k_{1} p_{2}}{p_{1}-p_{2}}+\frac{k_{2} p_{1}}{\left|k_{1}-k_{2}\right|}\right)+\right. \\
& \left.+\frac{4 \pi}{\sqrt{3}}\left(i \pi-\log Q^{2}\right)\left[\left(k_{1} p_{2}+k_{2} p_{1}\right) g_{3}\left(2 g_{3}-3 g_{2}\right)+g_{3}^{2} k_{1} p_{1}\right]\right]+
\end{aligned}
$$




$$
\begin{aligned}
& +k_{1} k_{2} p_{1} p_{2}\left[48 \pi^{2} i\left(\frac{1}{p_{1}-p_{2}}+\frac{1}{\left|k_{1}-k_{2}\right|}\right) g_{1}\left(2 g_{3}-3 g_{2}\right)\right. \\
& \left.\left.+\frac{4 \pi}{\sqrt{3}}\left(i \pi-\log Q^{2}\right)\left(2 g_{3}-3 g_{2}\right)^{2}\right]\right\} .
\end{aligned}
$$

Finalmente, ao expandirmos explicitamente o simetrizador $\mathcal{S}_{k, p}$, obtemos a seguinte expressão para a segunda ordem da amplitude de espalhamento:

$$
\begin{aligned}
\left.\langle\mathbf{k}|\hat{S}| \mathbf{p}\rangle\right|_{g^{2}} & =8 i \pi^{2} g_{1}^{2}\left(p_{1}^{2}+p_{2}^{2}+p_{3}^{2}-p_{1} p_{2}-p_{1} p_{3}-p_{2} p_{3}\right) \delta E \delta P+ \\
& +2 i^{2} g_{1}^{2}\left[\frac{p_{1} p_{2}}{p_{1}-p_{2}}+\frac{p_{1} p_{3}}{p_{1}-p_{3}}+\frac{p_{2} p_{3}}{p_{2}-p_{3}}\right]^{2} \delta_{+}^{(3)}(\mathbf{p}, \mathbf{k})-\frac{3 i}{2} \mathcal{R}\left(g^{2}\right) \delta E \delta P,
\end{aligned}
$$

onde separamos a contribuição de 1-laço:

$$
\begin{aligned}
\mathcal{R}\left(g^{2}\right) & =\frac{16}{3 i} \pi^{2} g_{1}\left\{\left[\frac{p_{1} p_{2}}{p_{1}-p_{2}}+\frac{p_{1} p_{3}}{p_{1}-p_{3}}+\frac{p_{2} p_{3}}{p_{2}-p_{3}}\right] f_{3}(\mathbf{k})+\left[\frac{k_{1} k_{2}}{\left|k_{1}-k_{2}\right|}+\frac{k_{1} k_{3}}{\left|k_{1}-k_{3}\right|}+\frac{k_{2} k_{3}}{\left|k_{2}-k_{3}\right|}\right] f_{3}(\mathbf{p})\right\}+ \\
& +\frac{4 \pi}{\sqrt{3}}\left(i \pi-\log Q^{2}\right) f_{3}(\mathbf{p}) f_{3}(\mathbf{k}),
\end{aligned}
$$

onde introduzimos a função auxiliar:

$$
f_{3}(\mathbf{q})=\left(3 g_{2}-2 g_{3}\right)\left(q_{1} q_{2}+q_{1} q_{3}+q_{2} q_{3}\right)-g_{3}\left(q_{1}^{2}+q_{2}^{2}+q_{3}^{2}\right) .
$$

Notamos que no limite $g_{1}=g_{2}=g_{3}=1$, o primeiro termo de (5.4.8a) coincide com o oposto do segundo termo de (5.4.2). Assim, o termo espúrio do nível de árvore é cancelado, permitindo, pois, que os termos remanescentes se combinem da seguinte maneira:

$$
\langle\mathbf{k}|\hat{S}| \mathbf{p}\rangle=\left\{1+2 \sum_{n=1}^{2}\left[i\left(\frac{p_{1} p_{2}}{p_{1}-p_{2}}+\frac{p_{1} p_{3}}{p_{1}-p_{3}}+\frac{p_{2} p_{3}}{p_{2}-p_{3}}\right)\right]^{n}\right\} \delta_{+}^{(3)}(\mathbf{p}, \mathbf{k})-\frac{3 i}{2} \mathcal{R}\left(g^{2}\right) \delta E \delta P+O\left(g^{3}\right) .
$$

É fácil constatar que o primeiro termo de (5.4.9) fornece a matriz $S$ para o espalhamento de três partículas em segunda ordem, consistente com a relação (5.4.3) obtida através do argumento de fatorabilidade. Logo, concluímos que para termos a fatoração da matriz $S$ mesmo nas ordens mais baixas, a contribuição de termos de ordens superiores deve ser levada em consideração, de forma a cancelarmos os termos espúrios. Diagramaticamente, ao utilizarmos a relação (5.4.4), verificamos que o valor principal dos gráficos de Feynman descritos na figura 5.12 cancela os termos, presentes na contribuição de primeira ordem, que impediam a fatoração da matriz $S$ nessa ordem, enquanto que a contribuição do termo proporcional a função delta em (5.4.4), quando adicionado ao termo representado na figura E.1, leva à contribuição fatorável de segunda ordem à matriz $S$. Apesar de termos desprezado a contribuição de $\mathcal{R}\left(g^{2}\right)$ em (5.4.9), tal cálculo fornece um esquema notável pelo qual contribuições de ordens distintas se cancelam de forma a garantir a fatorabilidade da matriz $S$.

Não é difícil de ver que os diagramas, que tivemos que somar para obtermos a matriz $S$ fatorável para o espalhamento de três partículas em primeira ordem, são proporcionais a $\hbar^{0}$, uma vez que nem os diagramas do nível de árvore, nem os gráficos presentes na figura 5.12 possuem algum laço. Claramente, o cancelamento em ordens mais altas deve ocorrer apenas entre diagramas de mesma 
ordem em $\hbar$. É importante ressaltar que a despeito de nosso sucesso em demonstrar a fatorabilidade em primeira ordem, a complexidade da análise diagramática e combinatória torna o cálculo das ordens superiores praticamente impossível de se efetuar, de sorte que uma nova metodologia precisa ser empregada. 


\title{
Capítulo 6
}

\section{Cargas Conservadas e Identidades de Traço}

\author{
"Once three friends \\ Sweet in sadness \\ Now part of their past. \\ In the end \\ Full of gladness \\ Went from class to class."
}

Gentle Giant - Three Friends

É bem sabido que a quantização direta de modelos contínuos proporciona um problema muito mais complicado do que de suas contrapartes discretas. O modelo de Landau-Lifshitz (LL) não é uma exceção, de fato, o desenvolvimento de sua teoria quântica foi afetado por uma série de sutilezas e nuances, que levaram a um processo de quantização errôneo, como notado por [92]. Alguns poucos modelos à parte, como o modelo de Schrödinger não-linear (NLS) e o modelo fermiônico de Thirring, que podem ser quantizados diretamente tanto através do método do espalhamento inverso quântico (MEIQ), quanto pelo Ansatz de Bethe coordenado, o procedimento usual consiste em considerar primeiramente a quantização da versão discretizada de um dado modelo contínuo. Com a introdução da rede, os problemas inerentes à quantização de modelos contínuos: as divergências ultravioletas e o produto de operadores em um mesmo ponto, são evitados. Dessa forma, a Hamiltoniana quântica, bem como as outras cargas conservadas podem, em princípio, ser obtidas através das identidades de traço.

Por outro lado, mesmo quando o processo de discretização de um dado modelo contínuo é conhecido, as técnicas usuais levam normalmente a resultados bem complicados [22,36, 54-57]. De fato, para um dado modelo contínuo existem, em geral, diversas discretizaçõs possíveis, e apesar de a integrabilidade restringir a escolha da rede correspondente, ela não a elimina completamente. Em [36] foi desenvolvido um método sistemático para a obtenção das contrapartes discretas de teorias quânticas de campos bidimensionais, que foi prontamente aplicado aos exemplos dos modelos NLS e de sine-Gordon (SG). Essa construção mostrou-se significativamente não-trivial, mesmo para o 
simples modelo NLS, levando a uma Hamiltoniana quântica com um termo de interação envolvendo oito vizinhos mais próximos. Para o modelo de SG, e para outros modelos contínuos, em geral, a Hamiltoniana é não-local, isto é, cuja interação depende de todos os sítios da rede. A rede correspondendo ao modelo de LL é conhecida, e pode ser obtida a partir da cadeia de spins $X X X$ para o caso isotrópico e $X Y Z$ para o caso geral. Contudo, a não-localidade da Hamiltoniana impõem uma séria barreira em potencial para o estudo de suas propriedades. Dessa forma, é altamente desejável que se desenvolva um método para se quantizar sistemas contínuos diretamente, sem a necessidade de considerar uma rede (e todas as complicações inerentes) em passos intermediários.

Esse programa, no contexto do modelo de LL, foi iniciado em [92] e revelou uma série de sutilezas relacionadas à quantização de modelos contínuos em geral. Uma das principais dificuldades envolve a construção das integrais do movimento e, em particular, da Hamiltoniana, uma vez que o uso formal das identidades de traço leva a resultados incorretos [106]. É importante ressaltar que apesar de tais manipulações formais (envolvendo objetos altamente singulares) levarem aos resultados corretos para os modelos NLS e de Thirring, a demonstração da integrabilidade quântica em tais modelos, conforme enfatizado em $[37,44]$, não é matematicamente rigorosa, assim, não é de todo surpreendente que falhem em situações mais complicadas como no modelo de LL. A forma matematicamente correta de se lidar com tais modelos foi obtida originalmente em [44], onde no exemplo do modelo de LL, mostrou-se que não somente é incorreto fazer manipulações formais com potenciais singulares como $\delta^{\prime \prime}(x)$, mas também é necessário que se construa extensões auto-adjuntas e se considere um produto operatorial regularizado.

As consequiências físicas de tais operações não são desprezíveis, por exemplo, as propriedades termodinâmicas do modelo de LL, consideradas em [107], são altamente não-triviais e permitem a demonstração da estabilidade do sistema. Outra e ainda mais notável consequêencia é que a noção amplamente aceita de que a função de onda para $n$ partículas pode ser representada na forma fatorada ${ }^{1}$ não é correta em geral. De fato, ela é incorreta para os modelos de LL, de Alday-ArutyunovFrolov [43] e para o modelo principal quiral $[33,35,80]$, embora nesse último o potencial não seja tão singular quanto $\delta^{\prime \prime}(x)$. É interessante notar que tais modelos integráveis surgem em diferentes subsetores da teoria de supercordas em $A_{d} S_{5} \times S^{5}$ e requerem extensões auto-adjuntas distintas. Não obstante, mesmo no caso da interação do tipo $\delta(x)$ é necessária a construção de extensões auto-adjuntas. Fica claro, pois, que o tratamento de modelo contínuos requer um maior cuidado matemático.

Em [44], foram dados os primeiros passos nessa direção com a construção da Hamiltoniana quântica para o modelo de $\operatorname{LL} \mathfrak{s u}(1,1)$ isotrópico. A questão de como estender esse método para a obtenção das demais cargas conservadas, originalmente abordada em [37], constitui o tema do presente capítulo, onde abordamos tal problema e desenvolvemos um método geral, e que deve ser aplicável a uma grande classe de modelos integráveis, para se obter as identidades de traço quânticas. É importante lembrar que, conforme discutido na seção 3.4², mesmo para o modelo NLS, deduzir as identidades de traço não é uma tarefa simples. Em particular, a noção amplamente aceita de que o ordenamento normal com respeito aos campos bosônicos $\psi(x)$ e $\psi^{\dagger}(x)$ resolve o problema do ordenamento de operadores não é estritamente correta. De fato, para o modelo NLS, apesar

\footnotetext{
${ }^{1}$ Vide (5.0.1) para o exemplo com $n=2$.

${ }^{2}$ Vide também [22, 36, 54-56, 108-110].
} 
de ser possível extrair as três primeiras cargas das identidades de traço quânticas, a quarta já não corresponde ao ordenamento normal usual $[22,58,111]$. Para o modelo de $L L$, a situação é a inda mais complicada, porque nesse caso existem três campos $\mathbf{S}$ sujeitos ao vínculo hiperbólico (ou esférico). Não é claro, a priori, como devemos escolher o ordenamento operatorial de um modo consistente. Em [92], Sklyanin optou por resolver o vínculo, e usar o ordenamento normal para os dois campos remanescentes. Entretanto, essa abordagem apresenta algumas limitações sérias, como, por exemplo, a impossibilidade de se obter a Hamiltoniana quântica em termos dos campos não vinculados, conquanto a Hamiltoniana em termos dos campos originais $\mathbf{S}$ admite uma forma razoavelmente simples $[37,44]$.

Esse problema se torna ainda mais crucial para outros modelos contínuos, para os quais resolver os vínculos pode não ser sequer possível. Assim, o primeiro problema que abordamos é sobre como lidar com o produto operatorial nesse caso. Fazemos isso por meio de um procedimento de regularização e renormalização, que mostramos implicar a relação de entrelaçamento para a matriz de monodromia, fundamental para a integrabilidade quântica e, conseqüentemente, para as identidades de traço. A regularização e renormalização que usamos difere profundamente da empregada por Sklyanin em [92], que foi introduzida apenas para satisfazer a relação de entrelaçamento, regularizando, pois, apenas as singularidade de um dado tipo. Contudo, como mostramos mais adiante, ela não é suficiente para quantizar o modelo corretamente, e por isso, consideramos um esquema de regularização e renormalização operatorial mais geral, que nos permite lidar com todas as singularidades ao mesmo tempo. A nossa abordagem possui algumas propriedade e consequiências interessantes. Dentre elas duas são mais proeminentes: primeiramente a álgebra regularizada e renormalizada que obtemos é altamente não-local mesmo para o modelo de LL isotrópico ${ }^{3}$, tornando a análise subseqüente significativamente mais intrincada. Em segundo lugar, ressaltamos que, diferentemente do procedimento usual, a nossa prescrição não utiliza nenhuma versão discreta do modelo em nenhum passo intermediário, bem como só precisamos empregar representações integráveis da álgebra ${ }^{4}$.

O segundo passo consiste em verificar se cada um dos operadores associados a cada carga conservada são auto-adjuntos, o que por sua vez requer a construção das extensões auto-adjuntas e leva a uma descrição acurada do formalismo quântico [44]. Na terceira etapa, utilizamos o formalismo do operador $\mathcal{M}$, que estabelece uma conexão entre o nosso método e o $M E I Q$, e fornece uma dedução concreta do espectro de cada operador, sem a necessidade de considerar o limite clássico para conjectura-lo, como feito por Sklyanin em [92]. Discutimos em detalhe também outros pontos importantes, como a regularização e renormalização do vínculo, obtendo a sua contraparte quântica. Ademais, mostramos que ao removermos a regularização, o operador de Casimir resultante corresponde a representações integráveis, consistentes com as condições de contorno e o fato dos operadores serem auto-adjuntos. Finalmente, ao compararmos nossos resultados com os resultados clássicos, usamos a simetria do modelo para argumentar que qualquer outra carga quântica conservada pode ser escrita em termos de três operadores fundamentais: o operador de Casimir $\mathcal{C}$, o Hamiltoniano quântico $\mathcal{H}$ e a terceira carga conservada quântica $\mathcal{Q}_{3}$. Dessa forma resolvemos essen-

\footnotetext{
${ }^{3}$ Note que tais álgebras não-locais (as álgebras de Sklyanin) surgem originalmente apenas no contexto do modelo de LL anisotrópico [92,112,113]. Mostramos, contudo, que é necessário introduzir uma álgebra análoga a de Sklyanin, mesmo para o caso isotrópico.

${ }^{4}$ Notamos que ao considerar o procedimento usual de discretização, em geral, é necessária a introdução de representações não-integráveis.
} 
cialmente o problema da obtenção das identidades de traço quânticas para o modelo de $\operatorname{LL} \mathfrak{s u}(1,1)$ contínuo.

\subsection{Quantização}

Nesta seção consideramos a quantização do modelo de LL isotrópico, cujo primeiro passo consiste em promover os campos clássicos $S^{i}, i=1,2,3$, a operadores quânticos que satisfazem as relações canônicas de comutação,

$$
\begin{aligned}
{\left[S^{3}(x) ; S^{ \pm}(y)\right] } & = \pm S^{ \pm}(x) \delta(x-y) \\
{\left[S^{-}(x) ; S^{+}(y)\right] } & =2 \varepsilon S^{3}(x) \delta(x-y)
\end{aligned}
$$

que são obtidas a partir de (4.1.6) com

$$
S^{ \pm}=S^{1} \pm i S^{2}
$$

Além disso, impomos as seguintes condições de conjugação:

$$
\left[S^{3}(x)\right]^{*}=S^{3}(x) \text { e }\left[S^{ \pm}(x)\right]^{*}=S^{\mp}(x) .
$$

O passo seguinte é a construção de representações da álgebra (6.1.1) com respeito ao vácuo ferromagnético:

$$
S^{3}(x)|0\rangle=\varepsilon|0\rangle \quad \text { e } \quad S^{-}(x)|0\rangle=0 .
$$

Podemos proceder como em outros modelos integráveis contínuos [22] e introduzir os vetores:

$$
\left|f_{n}\right\rangle=\int \prod_{i=1}^{n} d x_{i} f_{n}\left(x_{1}, \ldots, x_{n}\right) \prod_{j=1}^{n} S^{+}\left(x_{j}\right)|0\rangle,
$$

onde as funções $f_{n}\left(x_{1}, \ldots, x_{n}\right)$ são contínuas, simétricas com respeito ao conjunto $\left\{x_{n}\right\}$ e decrescem suficientemente rápido, de forma que a integral (6.1.3) esteja bem definida. Portanto, o espaço de representação coincide com o espaço vetorial gerado pelos vetores $\left|f_{n}\right\rangle, n=1,2, \ldots$

A seguir, consideramos o produto escalar no subespaço de $n$-partículas:

$$
\left\langle g_{n} \mid f_{n}\right\rangle=\int \prod_{i=1}^{n} d y_{i} \prod_{j=1}^{n} d x_{j} g_{n}^{*}\left(y_{1}, \ldots, y_{n}\right) f_{n}\left(x_{1}, \ldots, x_{n}\right)\left\langle 0\left|\prod_{k=1}^{n} S^{-}\left(y_{k}\right) \prod_{l=1}^{n} S^{+}\left(x_{l}\right)\right| 0\right\rangle .
$$

Ao levarmos todos os operadores $S^{-}\left(y_{k}\right)$ para a direita utilizando as relações de comutação canônicas (6.1.1), obtemos uma soma sobre as partições $P$ do conjunto $\left\{x_{j}\right\}_{j=1}^{n}$ em subconjuntos disjuntos $X_{m}^{P}$, ou seja,

$$
\left\{x_{j}\right\}_{j=1}^{n}=\bigcup_{m=1}^{M_{P}} X_{m}^{P} \quad \text { tal que } \quad X_{m_{j}}^{P} \cap X_{m_{k}}^{P}=\emptyset \text { se } j \neq k,
$$


a saber,

$$
\left\langle g_{n} \mid f_{n}\right\rangle=\sum_{P} \varepsilon^{n-M_{P}} C_{P} \int\left\{d \xi_{m}\right\}_{m=1}^{M_{P}}\left\{\int\left\{d x_{j}\right\}_{j=1}^{n} g_{n}^{*}\left(x_{1}, \ldots, x_{n}\right) f_{n}\left(x_{1}, \ldots, x_{n}\right) \prod_{m=1}^{M_{P}} \prod_{x_{j} \in X_{m}^{P}} \delta\left(\xi_{m}-x_{j}\right)\right\},
$$

onde os coeficientes $C_{P}$ são inteiros positivos. Fica claro a partir de (6.1.5) que o produto escalar (6.1.4) só é positivo definido para $\varepsilon=1$, que corresponde ao caso hiperbólico, ao passo que é indefinido para o caso esférico, onde $\varepsilon=-1$ [92]. Logo, somente no caso não-compacto é possível construir representações fisicamente apreciáveis da álgebra (6.1.1) sobre o vácuo ferromagnético, sendo, pois, imperativo que fixemos $\varepsilon=1$. Dessa forma, pelo resto do presente capítulo sempre que nos referimos ao modelo de $L L$, consideramos apenas o caso $\mathfrak{s u}(1,1)$ no limite isotrópico. Como um último adendo ao caso esférico, gostaríamos de ressaltar que, embora não exista nenhum produto escalar positivo definido para o caso $\mathfrak{s u}(2)$ com respeito ao vácuo ferromagnético, é possível construir uma representação com $\varepsilon=-1$ sobre um vácuo não-ferromagnético [114].

O operador de Lax quântico:

$$
\mathcal{L}(\lambda, x)=\frac{i}{\lambda}\left(\begin{array}{cc}
S^{3}(x) & -S^{+}(x) \\
S^{-}(x) & -S^{3}(x)
\end{array}\right)
$$

pode ser obtido a partir de sua versão clássica (4.1.15)

$$
L_{x}=\frac{\lambda}{2 i} \mathbb{S}^{\mathfrak{s u}(1,1)}=\frac{\lambda}{2 i}\left(\begin{array}{cc}
S^{3}(x) & i S^{-}(x) \\
i S^{+}(x) & -S^{3}(x)
\end{array}\right)
$$

através da transformação de calibre:

$$
\partial_{x} \phi=\mathcal{L}_{x}(x ; \lambda) \phi \quad \text { onde } \quad \phi=\Lambda \psi \quad \text { e } \quad \mathcal{L}_{x}(x ; \lambda)=\Lambda L_{x} \Lambda^{-1} \quad \text { com } \quad \Lambda=\left(\begin{array}{cc}
0 & 1 \\
i & 0
\end{array}\right)
$$

concomitante com a transformação do parâmetro espectral: $\lambda \rightarrow \frac{2}{\lambda}$. A forma $\mathcal{L}_{x}(x ; \lambda)$ é mais conveniente no caso quântico, pois a matriz $R$ quântica (6.1.8b) possui a forma canônica (como no caso $\mathfrak{s u}(2))$. $O$ fato de a transformação $\wedge$ não ser unitária não representa nenhum problema, uma vez que nem $\psi$, nem $\phi$ possuem alguma interpretação probabilística.

A matriz de monodromia para o intervalo $\left[x_{-}, x_{+}\right]$é definida da maneira usual:

$$
T_{x_{-}}^{x_{+}}(\lambda)=P \exp \int_{x_{-}}^{x_{+}} d x \mathcal{L}(\lambda, x) \equiv\left(\begin{array}{ll}
A_{x_{-}}^{x_{+}}(\lambda) & B_{x_{-}}^{x_{+}}(\lambda) \\
C_{x_{-}}^{x_{+}}(\lambda) & D_{x_{-}}^{x_{+}}(\lambda)
\end{array}\right)
$$

Nesse caso, a relação bilinear,

$$
R\left(\lambda_{1}-\lambda_{2}\right) \stackrel{(1)}{T_{X_{-}}^{X_{+}}}\left(\lambda_{1}\right) \stackrel{(2)}{T_{x_{-}}^{x_{+}}}\left(\lambda_{2}\right)=\stackrel{(2)}{T_{x_{-}}^{x_{+}}}\left(\lambda_{2}\right) \stackrel{(1)}{T_{x_{-}}^{X_{+}}}\left(\lambda_{1}\right) R\left(\lambda_{1}-\lambda_{2}\right)
$$


onde introduzimos a seguinte notação alternativa para o produto tensorial:

$$
\stackrel{(1)}{A} \equiv A \otimes \mathbb{1} \quad \text { e } \stackrel{(2)}{A} \equiv \mathbb{1} \otimes A,
$$

é válida com a seguinte matriz $R$ quântica: ${ }^{5}$

$$
R(\lambda)=\sum_{a=0}^{3} w_{a}(\lambda) \sigma_{a} \otimes \sigma_{a} \quad \text { com } \quad w_{0}(\lambda)=\lambda-\frac{i}{2} \quad \text { e } \quad w_{j}(\lambda)=-\frac{i}{2}, \quad j=1,2,3,
$$

se a relação de entrelaçamento,

$$
\begin{aligned}
R\left(\lambda_{1}-\lambda_{2}\right) & {\left[\stackrel{(1)}{\mathcal{L}}\left(\lambda_{1}, x\right)+\stackrel{(2)}{\mathcal{L}}\left(\lambda_{2}, x\right)+\stackrel{(1)}{\mathcal{L}}\left(\lambda_{1}, x\right) \circ \stackrel{(2)}{\mathcal{L}}\left(\lambda_{2}, x\right)\right]=} \\
= & {\left[\stackrel{(1)}{\mathcal{L}}\left(\lambda_{1}, x\right)+\stackrel{(2)}{\mathcal{L}}\left(\lambda_{2}, x\right)+\stackrel{(2)}{\mathcal{L}}\left(\lambda_{2}, x\right) \circ \stackrel{(1)}{\mathcal{L}}\left(\lambda_{1}, x\right)\right] R\left(\lambda_{1}-\lambda_{2}\right), }
\end{aligned}
$$

for satisfeita pelo operador de Lax quântico. O produto "o" foi originalmente introduzido em [92] com o intuito de resolver os problemas inerentes à definição do produto de dois operadores atuando sobre o mesmo ponto, sendo definido para um par de campos locais $A(x)$ e $B(x)$ quaisquer como:

$$
A(x) \circ B(x) \equiv \lim _{\Delta \rightarrow 0} \frac{1}{\Delta} \int_{x}^{x+\Delta} d \xi_{1} \int_{x}^{x+\Delta} d \xi_{2} A\left(\xi_{1}\right) B\left(\xi_{2}\right) .
$$

É importante notar que, embora o produto de Sklyanin (6.1.9) seja suficiente para tornar a relação bilinear (6.1.8c) válida, ele não constitui uma boa prescrição em geral, pois somente regulariza o tipo de singularidade presente em (6.1.8c). Uma rápida inspeção de (6.1.9) permite constatar que sua ação corresponde formalmente a dividir o produto de operadores atuando sobre um mesmo ponto por $\delta(0)$. De fato, é fácil verificar que o produto de dois operadores satisfazendo a álgebra (6.1.1) é regularizado por (6.1.9) da seguinte forma:

$$
\left[S^{3}(x), S^{+}(x)\right]=S^{+}(x) \delta(0) \stackrel{(6.1 .9)}{\longrightarrow}\left[S^{3}(x) \text {; } S^{+}(x)\right]=S^{+}(x) .
$$

Ressaltamos que a validade de (6.1.8c) depende somente da estrutura algébrica fornecida pela segunda expressão presente em (6.1.10). Assim, fica claro que a prescrição de Sklyanin (6.1.9) não é somente inadequada para lidar com o produto de operadores regulares, como também com o produto de operadores acometidos por singularidades mais severas. Por exemplo, se considerarmos $A(x)=B(x)=\mathbb{1}$, o produto de Sklyanin (6.1.9) implica que $\mathbb{1} \circ \mathbb{1}=0$. Portanto, (6.1.9) não constitui propriamente uma regularização, mas sim um procedimento muito específico que demanda um cuidado especial. Mais adiante, na seção 6.2, mostraremos que esse problema pode ser abordado adequadamente ao introduzirmos operadores regularizados associados aos campos fundamentais $S^{i}(x)$. Enfatizamos que a validade de (6.1.8a) e, conseqüentemente do MEIQ [21-23] depende do produto (6.1.9).

Após considerarmos o limite para um intervalo infinito, constatamos que os elementos da matriz de monodromia satisfazem as relações usuais de comutação, enquanto que as quantidades obtidas

\footnotetext{
${ }^{5}$ No que se segue empregamos as matrizes de Pauli usuais para $\mathfrak{s u}(2): \sigma_{a}=\left(\mathbb{1}, \sigma_{i}\right)$, vide (4.1.12a).
} 
a partir do $\operatorname{tr}[T(\lambda)]$ formam uma família de operadores comutantes, que podem ser interpretados como as integrais de movimento para o modelo de LL. Suas autofunções adquirem à seguinte forma:

$$
\left|\lambda_{1} \ldots \lambda_{n}\right\rangle=\prod_{i=1}^{n} B\left(\lambda_{i}\right)|0\rangle
$$

com os autovalores correspondentes dados por:

$$
\sum_{i=1}^{n} \log \left[A\left(\lambda_{i}\right)\right]
$$

É nesse ponto que nos deparamos com o problema crucial de se obter as identidades de traço quânticas, a saber, de se extrair do $\operatorname{tr}[T(\lambda)]$ as quantidades quânticas conservadas sob a forma de integrais de densidades locais, como em (4.2.30) e (4.2.31) para o caso clássico. O principal motivo dessa dificuldade reside na presença do produto de operadores atuando sobre um mesmo ponto nas cargas locais, tornando-as, pois, quantidades extremamente mal definidas. Em particular, não podemos proceder como no caso clássico, e simplesmente decompor a série:

$$
\operatorname{tr}[T(\lambda)]=\sum_{n=0}^{\infty} I_{n} \lambda^{n}
$$

para obter as cargas conservadas locais $I_{n}$. Logo, a construção das integrais de movimento locais proporciona um problema altamente não-trivial no contexto de modelos quânticos contínuos, que, do ponto de vista da teoria quântica de campos, corresponde ao procedimento de renormalização ${ }^{6}$.

Em [92] somente as duas primeiras cargas foram devidamente construídas: o operador número $\mathcal{N}$ e o operador momento $\mathcal{P}$. Para escreve-las foi necessária a introdução de um novo conjunto de operadores bosônicos $\Psi_{n}(x)$, correspondendo aos agrupamentos de $n$ partículas, que aniquilam o vácuo ferromagnético, ou seja, $\Psi_{n}(x)|0\rangle=0$, e satisfazem a seguinte álgebra:

$$
\left[\Psi_{m}(x) ; \Psi_{n}^{\dagger}(y)\right]=\delta_{m n} \delta(x-y)
$$

Em termos desses novos campos $\psi_{n}(x)$, podemos rescrever os operadores associados aos campos originais da seguinte forma:

$$
\begin{aligned}
& S^{3}(x)=s_{0}^{3}+\sum_{n=1}^{\infty} s_{n}^{3} \Psi_{n}^{\dagger}(x) \Psi_{n}(x), \\
& S^{+}(x)=s_{0}^{+} \Psi_{1}^{\dagger}(x)+\sum_{n=1}^{\infty} s_{n}^{+} \Psi_{n+1}^{\dagger}(x) \Psi_{n}(x), \\
& S^{-}(x)=s_{0}^{+} \Psi_{1}(x)+\sum_{n=1}^{\infty} s_{n}^{+} \Psi_{n}^{\dagger}(x) \Psi_{n+1}(x),
\end{aligned}
$$

onde $s_{0}^{3}=1, s_{0}^{+}=\sqrt{2}, s_{n}^{3}=n$ e $s_{n}^{+}=\sqrt{n(n+1)}$ para $n \geq 1$. De forma que os operadores número e

\footnotetext{
${ }^{6}$ Vide, por exemplo, [115].
} 
momento admitem as seguintes expressões:

$$
\begin{aligned}
& \mathcal{N}=\sum_{n=1}^{\infty} n \int d x \Psi_{n}^{\dagger}(x) \Psi_{n}(x) \\
& \mathcal{P}=\sum_{n=1}^{\infty} \frac{i}{2} \int d x\left[\partial_{x} \Psi_{n}^{\dagger}(x) \Psi_{n}(x)-\Psi_{n}^{\dagger}(x) \partial_{x} \Psi_{n}(x)\right]
\end{aligned}
$$

Contudo, não foi possível deduzir a forma do Hamiltoniano quântico, apenas a sua ação operatorial sobre os estados com uma e duas partículas, conjecturada. Mais recentemente, em [44], mostrou-se que para se obter um Hamiltoniano local para o setor geral de $n$ partículas, é necessário que se regularize o produto mal definido de operadores atuando sobre um mesmo ponto simultaneamente à construção das devidas extensões auto-adjuntas. Seguindo a metodologia empregada em [44], primeiramente precisamos considerar uma regularização para o Hamiltoniano quântico, como a obtida através do método da separação de pontos ${ }^{7}$ :

$$
\begin{aligned}
H_{Q}=\frac{1}{2} \lim _{\epsilon \rightarrow 0} \int d x d y F_{\epsilon}(x, y) & \left\{-\partial_{x} S^{3}(x) \partial_{y} S^{3}(y)+\partial_{x} S^{+}(x) \partial_{y} S^{-}(y)+\right. \\
+ & \left.\partial_{x} \partial_{y}\left[S^{3}(x) \delta(x-y)\right]-\partial_{x} \partial_{y} \delta(x-y)\right\}
\end{aligned}
$$

onde $F_{\epsilon}(x, y)$ é alguma função suave, simétrica em $(x, y)$ e que decresce suficientemente rápido no infinito, de forma a tornar a integração presente em (6.1.14) bem definida. A dependência no parâmetro $\epsilon$ é introduzida para que no limite de $\epsilon \rightarrow 0$, tenhamos:

$$
\lim _{\epsilon \rightarrow 0} F_{\epsilon}(x, y)=\delta(x-y)
$$

É claro da definição (6.1.14) que $H_{Q}$ aniquila o vácuo ferromagnético:

$$
H_{Q}|0\rangle=0
$$

Também não é difícil calcular a ação do Hamiltoniano regularizado sobre o estado geral com $n$ partículas:

$$
\begin{aligned}
H_{Q}\left|f_{n}\right\rangle & =-\int d \mathbf{x}\left[\triangle_{2} f_{n}(\mathbf{x})\right] \prod_{i=1}^{n} S^{+}\left(x_{i}\right)|0\rangle \\
& +\left.\sum_{i>j} \int \prod_{k \neq j} d x_{k}\left\{\left[\partial_{i} f_{n}(\mathbf{x})-\partial_{j} f_{n}(\mathbf{x})\right]_{x_{i}=x_{j}+\epsilon}^{x_{i}=x_{j}-\epsilon}+\left.\partial_{j} \partial_{j} f_{n}(\mathbf{x})\right|_{x_{i}=x_{j}}\right\} \prod_{i=1}^{n} S^{+}\left(x_{i}\right)\right|_{x_{i}=x_{j}}|0\rangle,
\end{aligned}
$$

onde $\triangle_{2}=\sum_{i=1}^{n} \partial_{i}^{2}$ representa o Laplaceano em $n$ dimensões. Portanto, para que $\left|f_{n}\right\rangle$ seja um autoestado de $H_{Q}$, precisamos impor as seguintes condições de compatibilidade:

$$
\left[\partial_{i} f_{n}(\mathbf{x})-\partial_{j} f_{n}(\mathbf{x})\right]_{x_{i}=x_{j}+\epsilon}^{x_{i}=x_{j}-\epsilon}+\left.\partial_{i} \partial_{j} f_{n}(\mathbf{x})\right|_{x_{i}=x_{j}}=0, \quad \forall i>j .
$$

\footnotetext{
${ }^{7}$ Point-splitting regularizarion method.
} 
Logo, a partir de (6.1.15) podemos inferir:

$$
-\triangle_{2} f_{n}(\mathbf{x})=E_{n} f_{n}(\mathbf{x}) \quad \Leftrightarrow \quad H_{Q}\left|f_{n}\right\rangle=E_{n}\left|f_{n}\right\rangle
$$

onde $E_{n}$ é a energia do estado com $n$ partículas. Uma conseqüência deveras importante das condições de compatibilidade (6.1.16), conforme mostrado em [44], é a fatorabilidade da matriz $S$.

De posse de um Hamiltoniano regular, o passo seguinte consiste na construção das extensões auto-adjuntas. Por simplicidade, consideramos somente o setor com duas partículas, posto que ele já exibe todas as idéias importantes, e as complicações inerentes ao caso geral são apenas de caráter técnico ${ }^{8}$. Para tanto, introduzimos o espaço vetorial $V$ gerado pelos vetores da forma:

$$
\psi=\left(\begin{array}{c}
f_{1}(x) \\
f_{2}(x, y)
\end{array}\right)
$$

onde $f_{1}(x) \in \mathscr{L}^{2}(\mathbb{R}, d x), f_{2}(x, y) \in \mathscr{L}^{2}\left(\mathbb{R}^{2} \backslash\{x=y\}, d x d y\right)$ e $\left.f_{2}(x, y)\right|_{x=y}=f_{1}(x) \in \mathscr{C}^{0}(\mathbb{R})$. Um produto escalar ${ }^{9}$ em $V$ pode ser definido para qualquer par $\psi, \Phi \in V$ como:

$$
\langle\Phi \mid \Psi\rangle=\frac{1}{2} \int d x g_{1}^{*}(x) f_{1}(x)+\int_{x \neq y} d x d y g_{2}^{*}(x, y) f(x, y) .
$$

Assim, podemos definir o operador $\hat{H}: V \rightarrow V$ pelas duas condições seguintes:

1. A ação de $\hat{H}$ sobre $f_{2}(x, y)$ é simplesmente o Laplaceano $-\triangle_{2}$ para qualquer $(x, y) \in \mathbb{R}^{2} \backslash\{x=$ $y\}$, isto é,

$$
\hat{H} \Psi=\left(\begin{array}{c}
\hat{h} f_{1}(x) \\
-\triangle_{2} f_{2}(x, y)
\end{array}\right)
$$

2. $\hat{H}$ é Hermiteano em $V$ com respeito ao produto escalar (6.1.19), ou seja,

$$
\langle\hat{H} \Phi \mid \Psi\rangle=\langle\Phi \mid \hat{H} \Psi\rangle
$$

Essas condições em conjunto com o fato de $V$ ser fechado sob a ação de $\hat{H}$, isto é,

$$
\hat{H} \Psi \in \Psi, \quad \forall \Psi \in V,
$$

fixam a forma de $\hat{h}$ e permitem a dedução da ação do Hamiltoniano:

$$
H_{Q}\left|f_{2}\right\rangle=\left(\begin{array}{c}
\left.2\left(\partial_{x}-\partial_{y}\right) f_{2}(x, y)\right|_{y=x-\epsilon} ^{y=x+\epsilon}-\partial_{x}^{2} f_{2}(x, x) \\
-\triangle_{2} f_{2}(x, y)
\end{array}\right),
$$

que coincide com a forma conjecturada originalmente por Sklyanin em [92] e está de acordo com (6.1.16).

\footnotetext{
${ }^{8}$ Encorajamos o leitor interessado a consultar a referência [44] para os detalhes da construção das extensões autoadjuntas para o setor de $n$ partículas.

${ }^{9}$ Note que esse produto escalar é equivalente ao produto escalar que introduzimos anteriormente (6.1.5) com $n=2$.
} 


\subsubsection{A Hierarquia dos Operadores $\mathcal{M}_{m}$}

Conforme discutimos ao final da seção $2.7^{10}$, para qualquer modelo integrável clássico, a evolução temporal de um campo $\Phi(x, t)$ :

$$
\partial_{t} \Phi(x, t)=\left\{I_{m}, \Phi(x, t)\right\},
$$

onde uma carga conservada arbitrária $I_{m}$ é tomada como a Hamiltoniana, é equivalente à hierarquia de operadores $\mathcal{M}_{m}^{(c l)}(x ; \lambda)$, satisfazendo a condição de compatibilidade ${ }^{11}$ [22]:

$$
\partial_{t} \mathcal{L}^{(\mathrm{cl})}(x ; \lambda)=\partial_{x} \mathcal{M}_{m}^{(\mathrm{cl})}(x ; \lambda)+\left[\mathcal{M}_{m}^{(\mathrm{cl})}(x ; \lambda), \mathcal{L}_{x}^{(\mathrm{cl})}(x ; \lambda)\right],
$$

onde $\mathcal{L}_{x}^{(\mathrm{cl})}(x ; \lambda)$ é o limite clássico do operador de Lax (6.1.6). Por exemplo, o par de Lax da teoria clássica pode ser obtido a partir de $\left(\mathcal{L}_{x}^{(\mathrm{cl})}(x ; \lambda), \mathcal{M}_{2}^{(\mathrm{cl})}(x ; \lambda)\right)$. Para um sistema definido no intervalo $\left[x_{1}, x_{2}\right]$ com condições de contorno periódicas, a solução possui a forma [20,23]:

$$
\left\{\operatorname{tr} T_{x_{1}}^{x_{2}}(\lambda), \mathcal{L}_{x}^{(\mathrm{cl})}(x ; \mu)\right\}=\partial_{x} \mathcal{M}_{x_{1}}^{x_{2}}(x ; \lambda, \mu)+\left[\mathcal{M}_{x_{1}}^{x_{2}}(x ; \lambda, \mu), \mathcal{L}_{x}^{(\mathrm{cl})}(x ; \mu)\right]
$$

onde

$$
\mathcal{M}_{x_{1}}^{x_{2}}(x ; \lambda, \mu)=\stackrel{(1)}{\operatorname{tr}}\left(\stackrel{(1)}{T_{x}^{\times}}(\lambda) r(\lambda-\mu) \stackrel{(1)}{T_{x_{1}}^{\times}}(\lambda)\right)
$$

e $r(\lambda-\mu)$ é a matriz $r$ clássica ${ }^{12}$, enquanto que a operação $\stackrel{(1)}{\operatorname{tr}}$ representa o traço operatorial sobre o primeiro espaço em $\mathbb{C}^{2} \otimes \mathbb{C}^{2}$ :

$$
\operatorname{tr}(A \otimes B)=\stackrel{(1)}{\operatorname{tr}}(\stackrel{(1)}{A} \cdot \stackrel{(2)}{B})=(\operatorname{tr} A) B
$$

No limite do intervalo infinito, o operador $\mathcal{M}_{m}^{(c)}(\lambda, x)$ correspondente à carga conservada $I_{m}$ pode ser obtido a partir da série:

$$
\lim _{\substack{x_{2} \rightarrow+\infty \\ x_{1} \rightarrow-\infty}} \mathcal{M}_{x_{1}}^{x_{2}}(x ; \lambda, \mu)=\sum_{m=0}^{\infty} \mu^{-m} \mathcal{M}_{m}^{(\mathrm{cl})}(\lambda, x)
$$

Estamos, de fato, interessados nos operadores $\mathcal{M}_{m}(\lambda, x)$ quânticos, que descrevem a evolução temporal da matriz de transição quântica $T_{x_{1}}^{x_{2}}(\lambda)$. Para o modelo NLS tais relações, bem como os detalhes envolvidos na sua dedução, podem ser encontrados em [23]. Já, para o modelo de LL, devido à dificuldade em se extrair as integrais de movimento locais, só foi possível obter os operadores $\mathcal{M}_{m}(x ; \lambda)$ :

$$
i\left[I_{n}, \mathcal{L}(x ; \lambda)\right]=\partial_{x} \mathcal{M}_{n}(x ; \lambda)+:\left[\mathcal{M}_{n}(x ; \lambda), \mathcal{L}(x ; \lambda)\right]:
$$

\footnotetext{
${ }^{10}$ Vide teorema 2.7 .7 e a discussão subseqüente.

${ }^{11}$ No que se segue, omitimos a dependência temporal.

${ }^{12}$ Vide 6.2.40.
} 
para as duas primeiras cargas

$$
\begin{aligned}
& I_{0}=\mathcal{N} \quad \longrightarrow \quad \mathcal{M}_{0}(x ; \lambda)=\frac{i \sigma_{3}}{2}, \\
& I_{1}=\mathcal{P} \quad \longrightarrow \quad \mathcal{M}_{1}(x ; \lambda)=-\mathcal{L}(x ; \lambda),
\end{aligned}
$$

onde denotamos o ordenamento normal com respeito aos campos $\Psi_{n}(x)$ da maneira usual : $\cdots:$ [92]. A relação (6.1.23) implica:

$$
i\left[I_{n}, T_{x_{-}}^{x_{+}}(\lambda)\right]=: \mathcal{M}_{n}\left(x_{+} ; \lambda\right) T_{x_{-}}^{x_{+}}(\lambda)-T_{x_{-}}^{x_{+}}(\lambda) \mathcal{M}_{n}\left(x_{-} ; \lambda\right):
$$

da qual inferimos as relações de comutação ${ }^{13}$ :

$$
\begin{array}{lll}
{[\mathcal{N}, \log A(\lambda)]=0} & \text { e } & {[\mathcal{N}, B(\lambda)]=B(\lambda)} \\
{[\mathcal{P}, \log A(\lambda)]=0} & \text { e } & {[\mathcal{P}, B(\lambda)]=-\frac{2}{\lambda} B(\lambda)}
\end{array}
$$

Em [92] a ação do Hamiltoniano quântico sobre o estado de duas partículas, bem como as relações de comutação (no limite do intervalo infinito):

$$
[\mathcal{H}, \log A(\lambda)]=0 \quad \text { e } \quad[\mathcal{H}, B(\lambda)]=\frac{4}{\lambda^{2}} B(\lambda)
$$

que levam ao espectro, foram conjecturadas de forma a reproduzir o limite clássico correto. Na próxima seção, abordamos esse quesito em detalhe, deduzindo as relações (6.1.27) apropriadamente. Portanto, a importância da hierarquia dos operadores $\mathcal{M}$ é estabelecer uma conexão entre o o MEIQ, a diagonalização direta e a construção das extensões auto-adjuntas das cargas conservadas quânticas.

\subsection{Regularização dos Operadores}

Nesta seção, propomos um método para lidar com as singularidades associadas ao produto de operadores atuando sobre um mesmo ponto, e consideramos suas conseqüências, demonstrando alguns resultados interessantes. Em particular, obtemos as contrapartes quânticas dos três invariantes fundamentais clássicos (4.2.32), deduzimos seus espectros e os operadores $\mathcal{M}_{m}(x, t ; \lambda)$ correspondentes. Ademais, explicamos em que sentido o nosso método pode ser visto como uma alternativa à discretização.

Conforme discutimos anteriormente, o problema mais desafiador no contexto do modelo de LL, e de outros modelos contínuos integráveis, reside na dificuldade em se expressar as quantidades conservadas comutantes na forma de integrais de densidades locais. A origem desse problema é a presença do produto de operadores atuando sobre um mesmo ponto nas expressões definidoras de tais cargas locais, o que faz com que as supracitadas integrais sejam quantidades extremamente mal definidas. A nossa solução para esse problema consiste simplesmente em lidar desde o começo

\footnotetext{
${ }^{13}$ Consideramos o limite isotrópico das relações (29) de [92]
} 
somente com um conjunto de campos regularizados:

$$
S_{\mathcal{F}}^{i}(x):=\int d \xi \mathcal{F}_{\epsilon}(x, \xi) S^{i}(\xi), \quad i=+,-, 3,
$$

onde $\mathcal{F}_{\epsilon}(x, y)$ é uma função suave, simétrica em $(x, y)$, dependente de um parâmetro $\epsilon$ e que decresce rapidamente no infinito de forma a tornar a integral (6.2.1) uma quantidade bem definida. Outras condições sobre a função $\mathcal{F}_{\epsilon}(x, y)$ serão impostas mais adiante. Nosso objetivo é reformular completamente a teoria singular (que envolve os campos despidos $S^{i}(x)$ ) em termos dos campos regularizados $S_{\mathcal{F}}^{i}(x)$.

É importante enfatizar que a introdução de tal função vem ao encontro da ideologia por trás da função $F_{\epsilon}(x, y)$ introduzida por [44] ${ }^{14} \mathrm{com}$ o intuito de regularizar a Hamiltoniana quântica, e difere, em sua essência, da abordagem empregada por Sklyanin em [92], onde o produto operatorial foi modificado, com a introdução do produto de Sklyanin (6.1.9). Ressaltamos que em nossa consideração o produto operatorial permanece inalterado, ou seja, é simplesmente o produto usual entre operadores, enquanto que os operadores são regularizados de acordo com (6.2.1), e, como ficará claro mais adiante, também renormalizados. O nosso objetivo inicial é mostrar que a introdução de tal regularização permite que a relação bilinear (6.1.8a) seja satisfeita, sem precisarmos empregar um subterfúgio como o fornecido pelo produto (6.1.9).

Como um primeiro passo nessa direção, deduzimos a álgebra satisfeita pelos operadores regularizados $S_{\mathcal{F}}^{i}(x)$. Invertendo a relação (6.2.1),

$$
S^{i}(\xi)=\int d z \mathcal{G}_{\epsilon}(\xi, z) S_{\mathcal{F}}^{i}(z)
$$

introduzimos $\mathcal{G}_{\epsilon}(x, y)$ outra função suave, simétrica em $(x, y)$, dependente do parâmetro $\epsilon$ e que decresce suficientemente rápido no infinito, mas tal que:

$$
S_{\mathcal{F}}^{i}(x)=\int d \xi d z \mathcal{F}_{\epsilon}(x, \xi) \mathcal{G}_{\epsilon}(\xi, z) S_{\mathcal{F}}^{i}(z) \Leftrightarrow \int d \xi \mathcal{F}_{\epsilon}(x, \xi) \mathcal{G}_{\epsilon}(\xi, z)=\delta(x-z) .
$$

Então, com o uso da álgebra satisfeita pelos operadores despidos (6.1.1), é fácil mostrar que os operadores regularizados $S_{\mathcal{F}}$ satisfazem às seguintes relações de comutação:

$$
\begin{aligned}
& {\left[S_{\mathcal{F}}^{3}(x) ; S_{\mathcal{F}}^{ \pm}(y)\right]= \pm \int d z \mathcal{K}_{\epsilon}(x, y, z) S_{\mathcal{F}}^{ \pm}(z),} \\
& {\left[S_{\mathcal{F}}^{-}(x) ; S_{\mathcal{F}}^{+}(y)\right]=2 \int d z \mathcal{K}_{\epsilon}(x, y, z) S_{\mathcal{F}}^{3}(z),}
\end{aligned}
$$

\footnotetext{
${ }^{14}$ Embora a função $F_{\epsilon}$ introduzida em [44] difira da função $\mathcal{F}_{\epsilon}(x, y)$, que utilizamos na definição de (6.2.1), existe naturalmente uma conexão entre as duas, que é dada por:

$$
F_{\epsilon}(x, y) \sim \int d \alpha \mathcal{F}_{\epsilon}(x, \alpha) \mathcal{F}_{\epsilon}(\alpha, y)
$$

Apesar de não ter sido importante para os propósitos de [44], ficará claro que a introdução de $\mathcal{F}_{\epsilon}(x, y)$ aqui é necessária para lidar com as singularidades presentes na álgebra e na relação de entrelaçamento. Naturalmente, isso afeta a normalização dos operadores, e resulta na representação correta de $\mathfrak{s u}(1,1)$.
} 
onde o núcleo $\mathcal{K}_{\epsilon}(x, y, z)$ é a seguinte função simétrica em $(x, y)$ :

$$
\mathcal{K}_{\epsilon}(x, y, z) \equiv \int d \xi \mathcal{F}_{\epsilon}(x, \xi) \mathcal{F}_{\epsilon}(y, \xi) \mathcal{G}_{\epsilon}(z, \xi)
$$

Notemos que, contudo a álgebra (6.2.4) satisfeita pelos operadores regularizados seja não-local, ao removermos a regularização, isto é, ao tomarmos o limite $\epsilon \rightarrow 0$ (vide equação (6.2.9), mais adiante), verificamos que a localidade é recuperada.

De posse da álgebra (6.2.4), podemos considerar a relação de entrelaçamento (6.1.8a). Para podermos escrevê-la em termos dos campos regularizados $S_{\mathcal{F}}^{i}$, lembramos que as condições necessárias para garantir a veracidade de $(6.1 .8 \mathrm{c})^{15}$ são:

$$
\left[S_{\mathcal{F}}^{3}(x) ; S_{\mathcal{F}}^{ \pm}(x)\right]= \pm S_{\mathcal{F}}^{ \pm}(x) \quad \text { e } \quad\left[S_{\mathcal{F}}^{-}(x) ; S_{\mathcal{F}}^{+}(x)\right]=2 S_{\mathcal{F}}^{3}(x)
$$

Assim, enquanto a álgebra (6.2.6) for satisfeita, a relação (6.1.8a) é válida. É fácil demonstrar que esse requisito impõem a seguinte condição sobre a função $\mathcal{F}_{\epsilon}(x, y)$ :

$$
\lim _{\epsilon \rightarrow 0}\left[\mathcal{F}_{\epsilon}(x)\right]^{2}=\lim _{\epsilon \rightarrow 0} \mathcal{F}_{\epsilon}(x)
$$

Podemos, pois, concluir que com essa restrição sobre a função $\mathcal{F}_{\epsilon}(x, y)$ no limite $\epsilon \rightarrow 0$, a álgebra (6.2.4) se reduz às condições (6.2.6) no limite $x=y$ e $\epsilon \rightarrow 0$. Portanto, a relação (6.1.8c) é válida no limite $\epsilon \rightarrow 0$, com o produto o tomado como o produto operatorial usual, mas com o operador de Lax, $\mathcal{L}(x ; \lambda)$, definido em termos dos campos regularizados, $S_{\mathcal{F}}(x)$, ou seja,

$$
\begin{aligned}
\lim _{\epsilon \rightarrow 0} & \left\{R\left(\lambda_{1}-\lambda_{2}\right)\left[\stackrel{(1)}{\mathcal{L}}_{\mathcal{F}}\left(x ; \lambda_{1}\right)+\stackrel{(2)}{\mathcal{L}}_{\mathcal{F}}\left(x ; \lambda_{2}\right)+\stackrel{(1)}{\mathcal{L}}_{\mathcal{F}}\left(x ; \lambda_{1}\right) \cdot \stackrel{(2)}{\mathcal{L}}_{\mathcal{F}}\left(x ; \lambda_{2}\right)\right]\right\}= \\
= & \lim _{\epsilon \rightarrow 0}\left\{\left[\stackrel{(1)}{\mathcal{L}}_{\mathcal{F}}\left(x ; \lambda_{1}\right)+\stackrel{(2)}{\mathcal{L}}_{\mathcal{F}}\left(x ; \lambda_{2}\right)+\stackrel{(2)}{\mathcal{L}}_{\mathcal{F}}\left(x ; \lambda_{2}\right) \cdot \stackrel{(1)}{\mathcal{L}}_{\mathcal{F}}\left(x ; \lambda_{1}\right)\right] R\left(\lambda_{1}-\lambda_{2}\right)\right\},
\end{aligned}
$$

e

$$
\mathcal{L}_{\mathcal{F}}(\lambda, x)=\frac{i}{\lambda}\left(\begin{array}{ll}
S_{\mathcal{F}}^{3}(x) & -S_{\mathcal{F}}^{+}(x) \\
S_{\mathcal{F}}^{-}(x) & -S_{\mathcal{F}}^{3}(x)
\end{array}\right)
$$

Mas agora podemos utilizar (6.2.8a) para garantir a validade da relação bilinear (6.1.8a) no limite $\epsilon \rightarrow 0$, e com a matriz de monodromia (6.1.7) definida com respeito ao operador de Lax regularizado. Conforme explicado anteriormente, a equação (6.1.8a) é fundamental para a aplicabilidade do MEIQ.

Finalmente, gostaríamos de comentar que, apesar de a condição (6.2.7) não possuir nenhuma solução não-trivial dentre as funções usuais, ela, de fato, possui uma solução na classe das funções generalizadas, que pode ser escrita como:

$$
\mathcal{F}_{\epsilon}(x, y)=\frac{\delta_{\epsilon}(x-y)}{\delta_{\epsilon}(0)}
$$

onde $\delta_{\epsilon}(x)$ é alguma regularização da função $\delta$, de sorte que $\delta_{\epsilon}(0)$ é uma constante bem definida antes de tomarmos o limite $\epsilon \rightarrow 0$. É deste ponto de vista que a função $\mathcal{F}_{\epsilon}(x, y)$ não constitui apenas uma regularização, mas também uma renormalização. Portanto, para o caso do produto de dois

\footnotetext{
${ }^{15}$ Que por sua vez é um requisito para a validade de (6.1.8a).
} 
operadores, fornecerá um produtos como (6.1.9), porém sem o comportamento problemático com respeito aos campos regulares.

\subsubsection{Operador de Casimir}

Uma questão que surge naturalmente neste contexto, é sobre o que acontece na teoria quântica com o vínculo clássico (4.1.2):

$$
\mathcal{C} \equiv S^{3}(x) S^{3}(x)-S^{1}(x) S^{1}(x)-S^{2}(x) S^{2}(x)=S^{3}(x) S^{3}(x)-S^{+}(x) S^{-}(x)=1
$$

Não é difícil de constatar que o operador quântico definido por $\mathcal{C}$ é formalmente um operador de Casimir da álgebra (6.1.1), porém, novamente, devido à presença de produtos de operadores atuando sobre um mesmo ponto, é claro que a expressão (6.2.10) não fornece um operador bem definido. Existem duas formas de se contornar esse problema. Por um lado, é possível considerar os operadores despidos e substituir o produto usual pelo de Sklyanin (6.1.9), enquanto que pelo outro podemos simplesmente rescrever (6.2.10) em termos dos campos $\mathcal{F}$-regularizados e manter o produto operatorial usual. Todavia já tenhamos enumerado algumas das vantagens da segunda abordagem em comparação com a primeira, gostaríamos de elaborar esse tópico em maior detalhe para o produto (6.1.9), de forma a explicitar e clarificar um dos problemas inerentes ao uso do produto de Sklyanin.

Já vimos ${ }^{16}$ que $\mathbb{1} \circ \mathbb{1}=0$, queremos agora exibir um exemplo mostrando uma inconsistência ainda maior do produto de Sklyanin. Ao notarmos que:

$$
\begin{aligned}
S^{3}(x) \circ S^{3}(x)|0\rangle & =\lim _{\Delta \rightarrow 0} \frac{1}{\Delta} \int_{x}^{x+\Delta} d \xi_{1} \int_{x}^{x+\Delta} d \xi_{2} S^{3}\left(\xi_{1}\right) S^{3}\left(\xi_{2}\right)|0\rangle \\
& =\lim _{\Delta \rightarrow 0} \frac{1}{\Delta} \int_{x}^{x+\Delta} d \xi_{1} \int_{x}^{x+\Delta} d \xi_{2}|0\rangle=\lim _{\Delta \rightarrow 0} \frac{\Delta^{2}}{\Delta}|0\rangle=0,
\end{aligned}
$$

e usarmos (6.2.10), lembrando que o vácuo ferromagnético é aniquilado por $S^{-}(x)$, concluímos um resultado absurdo. Portanto, verificamos explicitamente que lidando diretamente com os operadores despidos, mesmo se empregarmos o produto (6.1.9), não é possível efetuar uma regularização completamente consistente do modelo.

Resta-nos, pois, a segunda opção: usar os operadores regularizados $S_{\mathcal{F}}^{i}$. Consideremos, inicialmente, a seguinte expressão geral:

$$
\mathcal{C}_{\mathcal{F}}=S_{\mathcal{F}}^{3}(x) S_{\mathcal{F}}^{3}(x)-S_{\mathcal{F}}^{+}(x) S_{\mathcal{F}}^{-}(x)+\gamma_{1} S_{\mathcal{F}}^{3}=\kappa_{\epsilon},
$$

como o nosso candidato ao operador de Casimir. Os parâmetros $\gamma_{1}$ e $\kappa_{\epsilon}$ foram introduzidos com o intuito de refletir a não comutatividade entre os operadores quânticos $S^{+}(x)$ e $S^{-}(x)$. É importante ressaltar que a expressão (6.2.11) depende do parâmetro de regularização $\epsilon$ em ambos os lados, e que, embora não seja mais um operador de Casimir da álgebra (6.2.4), podemos verificar que, ao tomarmos o limite $\epsilon \rightarrow 0$, torna-se novamente um. Conseqüentemente, um maior cuidado é necessário ao se lidar com o vínculo quântico regularizado (6.2.11), e, em geral, não podemos supor que $\mathcal{C}=1$,

\footnotetext{
${ }^{16}$ Vide discussão após (6.1.10).
} 
como no caso clássico, pois empregamos operadores renormalizados. Ademais, mostraremos que, no limite $\epsilon \rightarrow 0$, as representações construídas a partir dos campos $S_{\mathcal{F}}^{i}$ possuem, de fato, spin $s=1 / 2$.

A seguir, determinamos o parâmetro $\gamma_{1}$, o parâmetro $\kappa_{\epsilon}$ será considerado em detalhe nas seções subseqüentes. Usando o operador de Lax (6.2.8b) e a álgebra (6.2.4), inferimos, no limite $\epsilon \rightarrow 0$ que:

$$
\left(\mathcal{L}_{\mathcal{F}}\right)^{2}=\frac{i}{\lambda}-\mathbb{1}\left[\kappa_{\epsilon}-\left(\gamma_{1}+1\right) S_{\mathcal{F}}^{3}\right]
$$

Então, para que $\left(\mathcal{L}_{\mathcal{F}}\right)^{3}$ seja univocamente definido, isto é, de forma que

$$
\left(\mathcal{L}_{\mathcal{F}}\right)^{3}=\left(\mathcal{L}_{\mathcal{F}}\right) \cdot\left(\mathcal{L}_{\mathcal{F}}\right)^{2}=\left(\mathcal{L}_{\mathcal{F}}\right)^{2} \cdot\left(\mathcal{L}_{\mathcal{F}}\right)
$$

precisamos impor a condição:

$$
\left[\mathcal{L}_{\mathcal{F}}, S_{\mathcal{F}}^{3}\right]=0
$$

que leva à determinação do parâmetro $\gamma_{1}$ :

$$
\gamma_{1}=-1
$$

Logo, o vínculo regularizado (6.2.11) assume a seguinte forma:

$$
\mathcal{C}_{\mathcal{F}}=S_{\mathcal{F}}^{3}(x) S_{\mathcal{F}}^{3}(x)-\frac{1}{2}\left[S_{\mathcal{F}}^{+}(x) S_{\mathcal{F}}^{-}(x)+S_{\mathcal{F}}^{-}(x) S_{\mathcal{F}}^{+}(x)\right]=\kappa_{\epsilon}
$$

Uma outra expressão útil que segue de (6.2.12), e que será utilizada mais adiante, quando deduzirmos os respectivos operadores $\mathcal{M}_{n}(x ; \lambda)$, é:

$$
\left[\left[\mathcal{L}_{\mathcal{F}}, \partial_{x} \mathcal{L}_{\mathcal{F}}\right], \mathcal{L}_{\mathcal{F}}\right]=\partial_{x}\left[\frac{1+4 \kappa_{\epsilon}}{\lambda^{2}} \mathcal{L}_{\mathcal{F}}\right]
$$

Finalmente, consideramos a renormalização do vácuo ferromagnético, para isso definimos, como anteriormente, o vácuo ferromagnético com respeito aos operadores despidos,

$$
S^{3}|0\rangle=a_{0}|0\rangle \quad \text { e } \quad S^{-}|0\rangle=0
$$

o que pode ser escrito, em termos dos operadores regularizados, $S_{\mathcal{F}}^{i}$ da seguinte forma:

$$
S_{\mathcal{F}}^{3}|0\rangle=\zeta_{\epsilon}|0\rangle \quad \text { e } \quad S_{\mathcal{F}}^{-}|0\rangle=0
$$

onde os parâmetros $a_{0}$ e $\zeta_{\epsilon}$ serão determinados posteriormente. Notamos apenas que, ao usarmos a definição (6.2.1) e tomarmos o limite $\epsilon \rightarrow 0$, obtemos uma relação entre tais parâmetros:

$$
\zeta_{\epsilon}=\frac{a_{0}}{\delta_{\epsilon}(0)}
$$

Além disso, ao considerarmos o operador de Casimir (6.2.11), inferimos uma relação entre os parâ- 
metros $\zeta_{\epsilon}$ e $\kappa_{\epsilon}$ :

$$
\zeta_{\epsilon}^{2}-\zeta_{\epsilon}=\kappa_{\epsilon}
$$

\subsubsection{Hamiltoniano $\mathcal{F}$-regularizado}

Com o introdução dos campos $\mathcal{F}$-regularizados, todos os produtos estão propriamente definidos, e podemos prosseguir com a construção das demais cargas conservadas. Naturalmente, o próximo passo a ser considerado consiste em rescrever o Hamiltoniano (6.1.14) em termos dos campos regularizados $S_{\mathcal{F}}$ :

$$
\mathcal{H}=\frac{\eta(\epsilon)}{4} \int d x\left[-2 \partial_{x} S_{\mathcal{F}}^{3}(x) \partial_{x} S_{\mathcal{F}}^{3}(x)+\partial_{x} S_{\mathcal{F}}^{+}(x) \partial_{x} S_{\mathcal{F}}^{-}(x)+\partial_{x} S_{\mathcal{F}}^{-}(x) \partial_{x} S_{\mathcal{F}}^{+}(x)\right]
$$

onde $\eta(\epsilon)$ é um parâmetro arbitrário que será fixado posteriormente ${ }^{17}$. Mostramos, a seguir, que o mesmo conjunto de estados de $n$ partículas:

$$
\left|f_{n}\right\rangle=\int \prod_{i=1}^{n} d x_{i} f_{n}\left(x_{1}, \ldots, x_{n}\right) \prod_{j=1}^{n} S^{+}\left(x_{j}\right)|0\rangle,
$$

fornece uma representação para a álgebra $\mathfrak{s u}(1,1)$ dos operadores escritos em termos dos campos $S_{\mathcal{F}}$. Assim como na consideração anterior, as funções $f_{n}\left(x_{1}, \ldots, x_{n}\right)$ são contínuas, simétricas no conjunto $\left\{x_{n}\right\}$ e decrescem suficientemente rápido no infinito para que a integral (6.2.19) esteja bem definida.

A ação do Hamiltoniano (6.2.18) sobre o vácuo ferromagnético fornece a energia do vácuo:

$$
\mathcal{H}|0\rangle=\left.\frac{\eta(\epsilon) \zeta(\epsilon)}{2} \int d x d z \partial_{x} \partial_{z} \mathcal{K}_{\epsilon}(x, y, z)\right|_{x=y}|0\rangle \equiv h_{0}(\epsilon)|0\rangle,
$$

enquanto que a ação sobre o estado com uma partícula:

$$
\left[\mathcal{H}-h_{0}(\epsilon)\right]\left|f_{1}\right\rangle \stackrel{\epsilon \rightarrow 0}{\longrightarrow}-\frac{a_{0} \eta(\epsilon)}{\delta^{2}(0)} \int d x \partial_{x}^{2} f_{1}(x) S^{+}(x)|0\rangle .
$$

Introduzimos o símbolo $\delta(0)$ para denotar $\lim _{\epsilon \rightarrow 0} \delta_{\epsilon}(0)$. Já para o mais complicado setor com duas partículas, podemos demonstrar que:

$$
\begin{aligned}
{\left[\mathcal{H}-h_{0}(\epsilon)\right]\left|f_{2}\right\rangle } & \stackrel{\epsilon \rightarrow 0}{\longrightarrow}-\frac{a_{0} \eta(\epsilon)}{\delta^{2}(0)} \int d x d y \triangle_{2} f_{2}(x, y) S^{+}(x) S^{+}(y)|0\rangle+ \\
& +\eta(\epsilon)\left[\frac{a_{0}}{\delta^{2}(0)}-\frac{1}{2 \delta(0)}\right] \int d x\left[\left(\partial_{x}-\partial_{y}\right) f_{2}(x, y)\right]_{y=x-\epsilon}^{y=x+\epsilon} S^{+}(x) S^{+}(x)|0\rangle- \\
& -\left.\frac{\eta(\epsilon)}{\delta^{2}(0)} \int d x \partial_{x} \partial_{y} f_{2}(x, y)\right|_{x=y} S^{+}(x) S^{+}(x)|0\rangle
\end{aligned}
$$

\footnotetext{
${ }^{17}$ De fato, poderíamos partir de uma forma ainda mais geral para o Hamiltoniano:

$$
\mathcal{H}=\frac{1}{4} \int d x\left[-2 \eta_{1}(\epsilon) \partial_{x} S_{\mathcal{F}}^{3}(x) \partial_{x} S_{\mathcal{F}}^{3}(x)+\eta_{2}(\epsilon) \partial_{x} S_{\mathcal{F}}^{+}(x) \partial_{x} S_{\mathcal{F}}^{-}(x)+\eta_{3}(\epsilon) \partial_{x} S_{\mathcal{F}}^{-}(x) \partial_{x} S_{\mathcal{F}}^{+}(x)\right]
$$

onde os parâmetros $\eta_{i}(\epsilon), i=1,2,3$ são arbitrários. Entretanto, a condição de diagonalização requer que fixemos $\eta_{1}(\epsilon)=\eta_{2}(\epsilon)=\eta_{3}(\epsilon) \equiv \eta(\epsilon)$, de forma que obtemos o Hamiltoniano na forma (6.2.18).
} 
Logo, se utilizarmos as condições de compatibilidade (6.1.16), inferimos o valor do parâmentro $a_{0}$ :

$$
a_{0}=1+\frac{\delta(0)}{2}
$$

É fácil compreender esse resultado se considerarmos o operador de Casimir ${ }^{18}$ (6.2.11). Utilizando as relaç̃̃es (6.2.17) obtemos o parâmentro $\kappa_{\epsilon}$ no limite $\epsilon \rightarrow 0$ :

$$
\mathcal{C}_{\mathcal{F}}=S_{\mathcal{F}}^{3}(x) S_{\mathcal{F}}^{3}(x)-\frac{1}{2}\left[S_{\mathcal{F}}^{+}(x) S_{\mathcal{F}}^{-}(x)+S_{\mathcal{F}}^{-}(x) S_{\mathcal{F}}^{+}(x)\right]=-1 / 4
$$

Portanto, construímos uma representação de $\mathfrak{s u}(1,1)$ que de fato possui spin $s=1 / 2$. Isso se deve ao fato de que introduzimos os operadores $S_{\mathcal{F}}^{i}$ de tal forma que em cada ponto fixo $x$, a álgebra $\mathfrak{s u}(1,1)$ que formam no limite $\epsilon \rightarrow 0$ (6.2.6) está bem definida. É importante ressaltar que, diferentemente do processo usual de discretização, que envolve a introdução dos operadores discretos $S_{n}$ e a construção de representações não-integráveis, isto é, com spin $s \neq 1 / 2,1, \ldots$, o nosso método só utiliza representações integráveis.

Podemos, também, fixar o valor de $\eta(\epsilon)$, escolhendo o seu comportamento limítrofe:

$$
\lim _{\epsilon \rightarrow 0} \eta(\epsilon)=\frac{\delta^{2}(0)}{a_{0}} E_{0}
$$

onde $E_{0}$ é alguma constante finita arbitrária. Por consegüinte, as expressões (6.2.20b) e (6.2.20c) assumem a seguinte forma:

$$
\begin{aligned}
& \left(\mathcal{H}-h_{0}(\epsilon)\right)\left|f_{1}\right\rangle \stackrel{\epsilon \rightarrow 0}{\longrightarrow}-E_{0} \int d x \partial_{x}^{2} f_{1}(x) S^{+}(x)|0\rangle, \\
& \left(\mathcal{H}-h_{0}(\epsilon)\right)\left|f_{2}\right\rangle \stackrel{\epsilon \rightarrow 0}{\longrightarrow}-E_{0} \int d x d y \triangle_{2} f_{2}(x, y) S^{+}(x) S^{+}(y)|0\rangle .
\end{aligned}
$$

Conseqüentemente, é possível reproduzir a construção das extensões auto-adjuntas para o setor com duas partículas de [44], conforme discutimos ao final da seção 6.1. De uma forma mais geral, podemos demonstrar a validade de relações similares a (6.1.16) e (6.1.17) para o Hamiltoniano $\mathcal{F}$-regularizado (6.2.18), de forma que a construção geral das extensões auto-adjuntas de [44] no setor com $n$ partículas também se aplica sem nenhuma complicação adicional para o Hamiltoniano (6.2.18).

Finalmente, notamos que tanto o Hamiltoniano quanto o operador de Casimir $\mathcal{F}$-regularizados podem ser escritos de uma forma significativamente simplificada, ao empregarmos o traço e o operador de $\operatorname{Lax} \mathcal{L}_{\mathcal{F}}$ :

$$
\begin{aligned}
& \mathcal{C}=-\frac{\lambda^{2}}{2} \int d x \operatorname{tr}\left[\mathcal{L}_{\mathcal{F}} \cdot \mathcal{L}_{\mathcal{F}}\right] \\
& \mathcal{H}=h_{0}+\frac{\lambda^{2}}{4} \int d x \eta(\epsilon) \operatorname{tr}\left[\partial_{x} \mathcal{L}_{\mathcal{F}}(\lambda, x) \cdot \partial_{x} \mathcal{L}_{\mathcal{F}}(\lambda, x)\right]
\end{aligned}
$$

É importante frisar que isso não teria sido possível sem a introdução dos campos $\mathcal{F}$-regularizados. Portanto, estabelecemos o resultado significativo de que a formas do Hamiltoniano e do operador

\footnotetext{
${ }^{18}$ Enfatizamos que, de fato, trata-se de um operador de Casimir apenas no limite $\epsilon \rightarrow 0$.
} 
de Casimir quânticos regularizados (6.2.24) essencialmente coincidem com os respectivos invariantes fundamentais clássicos em (4.2.32), com os campos clássicos substituídos pelos campos regularizados $S_{\mathcal{F}}^{i}$. Resta-nos, pois, apenas obter o terceiro invariante fundamental em termos dos operadores $\mathcal{F}$ regularizados e mostrar que ele coincide com a sua contraparte clássica. Ademais, mostraremos que ele pode também ser escrito em termos do traço do operador de Lax quântico.

\subsubsection{Espectro}

Na seção anterior, obtivemos uma expressão bem definida para o Hamiltoniano quântico (6.2.24b) e deduzimos o seu espectro através da diagonalização direta. Nesta seção, mostramos que o espectro de (6.2.24b) também pode ser inferido por intermédio do MEIQ. Dessa forma, estabelecemos uma conexão explícita entre os dois métodos ao obtermos as relações de comutação (6.1.27).

Primeiramente, precisamos encontrar o operador $\mathcal{M}_{2}(x ; \lambda)$ que satisfaz a relação:

$$
i\left[\mathcal{H} ; \mathcal{L}_{\mathcal{F}}(x ; \lambda)\right]=\partial_{x} \mathcal{M}_{2}(x ; \lambda)+\left[\mathcal{M}_{2}(x ; \lambda) ; \mathcal{L}_{\mathcal{F}}(x ; \lambda)\right]
$$

Não é difícil calcular o lado esquerdo de (6.2.25):

$$
i\left[\mathcal{H}, \mathcal{L}_{\mathcal{F}}(x ; \lambda)\right] \stackrel{\epsilon \rightarrow 0}{\longrightarrow}-\frac{\eta(\epsilon) \lambda}{2 \delta_{\epsilon}(0)} \partial_{x}\left[\mathcal{L}_{\mathcal{F}}(x ; \lambda), \partial_{x} \mathcal{L}_{\mathcal{F}}(x ; \lambda)\right] .
$$

De fato, usando a relação (6.2.15) é fácil constatar que o operador $\mathcal{M}_{2}(\lambda, x)$ possui a seguinte forma:

$$
\mathcal{M}_{2}(x ; \lambda)=\alpha_{2} \mathcal{L}_{\mathcal{F}}(x ; \lambda)+\beta_{2}\left[\mathcal{L}_{\mathcal{F}}(x ; \lambda), \partial_{x} \mathcal{L}_{\mathcal{F}}(x ; \lambda)\right],
$$

com os coeficientes $\alpha_{2}$ e $\beta_{2}$ dados por:

$$
\alpha_{2}=\frac{1+4 \kappa_{\epsilon}}{2 \lambda \delta_{\epsilon}(0)} \quad \text { e } \quad \beta_{2}=-\frac{\lambda}{2 \delta_{\epsilon}(0)}
$$

Finalmente, da equação (6.2.25) obtemos:

$$
i\left[\mathcal{H} ; T_{x_{-}}^{x_{+}}(\lambda)\right]=\mathcal{M}_{2}\left(x_{+} ; \lambda\right) T_{x_{-}}^{x_{+}}(\lambda)-T_{x_{-}}^{x_{+}}(\lambda) \mathcal{M}_{2}\left(x_{-} ; \lambda\right),
$$

onde naturalmente a matriz de monodromia é definida com respeito ao operador de Lax regularizado $\mathcal{L}_{\mathcal{F}}$. Se decompusermos $\mathcal{M}_{2}(x ; \lambda)$ da seguinte forma:

$$
\mathcal{M}_{2}(x ; \lambda)=\sigma_{+} \mathcal{M}_{2}^{+}(x ; \lambda)+\sigma_{-} \mathcal{M}_{2}^{-}(x ; \lambda)+\sigma_{3} \mathcal{M}_{2}^{3}(x ; \lambda),
$$

onde

$$
\begin{aligned}
& \mathcal{M}_{2}^{+}(\lambda, x)=\frac{\alpha_{2}}{i \lambda} S_{\mathcal{F}}^{+}(x)-\frac{2 \beta_{2}}{\lambda^{2}}\left[S_{\mathcal{F}}^{+}(x) \partial_{x} S_{\mathcal{F}}^{3}(x)-\partial_{x} S_{\mathcal{F}}^{+}(x) S_{\mathcal{F}}^{3}(x)\right], \\
& \mathcal{M}_{2}^{-}(\lambda, x)=\frac{i \alpha_{2}}{\lambda} S_{\mathcal{F}}^{-}(x)+\frac{2 \beta_{2}}{\lambda^{2}}\left[S_{\mathcal{F}}^{3}(x) \partial_{x} S_{\mathcal{F}}^{-}(x)-\partial_{x} S_{\mathcal{F}}^{3}(x) S_{\mathcal{F}}^{-}(x)\right], \\
& \mathcal{M}_{2}^{3}(\lambda, x)=\frac{i \alpha_{2}}{\lambda} S_{\mathcal{F}}^{3}(x)+\frac{\beta_{2}}{\lambda^{2}}\left[S_{\mathcal{F}}^{+}(x) \partial_{x} S_{\mathcal{F}}^{-}(x)-\partial_{x} S_{\mathcal{F}}^{+}(x) S_{\mathcal{F}}^{-}(x)\right],
\end{aligned}
$$


então a equação (6.2.25) é reduzida ao seguinte conjunto de relações de comutação para os elementos da matriz de monodromia (6.1.7):

$$
\begin{array}{r}
i\left[\mathcal{H} ; A_{x_{-}}^{x_{+}}(\lambda)\right]=\mathcal{M}_{2}^{+}\left(x_{+} ; \lambda\right) C_{x_{-}}^{x_{+}}(\lambda)+\mathcal{M}_{2}^{3}\left(x_{+} ; \lambda\right) A_{x_{-}}^{x_{+}}(\lambda)-A_{x_{-}}^{x_{+}}(\lambda) \mathcal{M}_{2}^{3}\left(x_{-} ; \lambda\right)-B_{x_{-}}^{x_{+}}(\lambda) \mathcal{M}_{2}^{-}\left(x_{-} ; \lambda\right), \\
i\left[\mathcal{H} ; B_{x_{-}}^{x_{+}}(\lambda)\right]=\mathcal{M}_{2}^{+}\left(x_{+} ; \lambda\right) D_{x_{-}}^{x_{+}}(\lambda)+\mathcal{M}_{2}^{3}\left(x_{+} ; \lambda\right) B_{x_{-}}^{x_{+}}(\lambda)-A_{x_{-}}^{x_{+}}(\lambda) \mathcal{M}_{2}^{+}\left(x_{-} ; \lambda\right)+B_{x_{-}}^{x_{+}}(\lambda) \mathcal{M}_{2}^{3}\left(x_{-} ; \lambda\right), \\
i\left[\mathcal{H} ; C_{x_{-}}^{x_{+}}(\lambda)\right]=\mathcal{M}_{2}^{-}\left(x_{+} ; \lambda\right) A_{x_{-}}^{x_{+}}(\lambda)-\mathcal{M}_{2}^{3}\left(x_{+} ; \lambda\right) C_{x_{-}}^{x_{+}}(\lambda)-D_{x_{-}}^{x_{+}}(\lambda) \mathcal{M}_{2}^{-}\left(x_{-} ; \lambda\right)-C_{x_{-}}^{x_{+}}(\lambda) \mathcal{M}_{2}^{3}\left(x_{-} ; \lambda\right), \\
i\left[\mathcal{H} ; D_{x_{-}}^{x_{+}}(\lambda)\right]=\mathcal{M}_{2}^{-}\left(x_{+} ; \lambda\right) B_{x_{-}}^{x_{+}}(\lambda)-\mathcal{M}_{2}^{3}\left(x_{+} ; \lambda\right) D_{x_{-}}^{x_{+}}(\lambda)+D_{x_{-}}^{x_{+}}(\lambda) \mathcal{M}_{2}^{3}\left(x_{-} ; \lambda\right)-C_{x_{-}}^{x_{+}}(\lambda) \mathcal{M}_{2}^{+}\left(x_{-} ; \lambda\right) .
\end{array}
$$

A passagem para o intervalo infinito é feita com a introdução da matriz de monodromia reduzida $T_{\infty}(\lambda)$ como o limite:

$$
T_{\infty}(\lambda)=\lim _{x_{ \pm} \rightarrow \pm \infty}\left[e\left(-x_{+} ; \lambda\right) T_{x_{-}}^{x_{+}}(\lambda) e\left(x_{-} ; \lambda\right)\right] \quad \text { onde } \quad e(x ; \lambda)=e^{\frac{i \sigma_{3}}{\lambda} x} .
$$

Então, multiplicando ambos os lados da equação (6.2.32) por $e\left(-x_{+}, \lambda\right)$ pela esquerda e $e\left(x_{-}, \lambda\right)$ pela direita, tomando o limite $x_{ \pm} \rightarrow \pm \infty$ e notando que

$$
S_{\mathcal{F}}^{3}(x) \stackrel{x \rightarrow \pm \infty}{\longrightarrow} 1 \text { e } S_{\mathcal{F}}^{ \pm}(x) \stackrel{x \rightarrow \pm \infty}{\longrightarrow} 0,
$$

concluímos que no limite $\epsilon \rightarrow 0$ :

$$
\begin{aligned}
& {\left[\mathcal{H} ; A_{\infty}(\lambda)\right]=0,} \\
& {\left[\mathcal{H} ; B_{\infty}(\lambda)\right]=\frac{2 \alpha_{2} \eta(\epsilon)}{\lambda} B_{\infty}(\lambda),} \\
& {\left[\mathcal{H} ; C_{\infty}(\lambda)\right]=-\frac{2 \alpha_{2} \eta(\epsilon)}{\lambda} C_{\infty}(\lambda),} \\
& {\left[\mathcal{H} ; D_{\infty}(\lambda)\right]=0 .}
\end{aligned}
$$

Portanto, provamos as relações (6.1.27), das quais o espectro do Hamiltoniano segue. Mostramos, pois, como fazer uma conexão entre o nosso esquema de regularização, a diagonalização direta e o MEIQ.

\subsubsection{Cargas Conservadas Superiores}

Nesta seção, construímos a versão quântica do invariante cúbico de (4.2.32), e mostramos que, similarmente ao Hamiltoniano e ao operador de Casimir, a densidade correspondente pode ser expressa em termos do traço do produto de operadores de Lax quânticos. Com isso concluímos que todos os três invariantes fundamentais quânticos possuem tal forma, e que coincidem com as suas contrapartes clássicas (4.2.32). Portanto, qualquer outra carga conservada deve ser decomposta em termos de tais invariantes fundamentais, e, por isso, escrita como um produto dos campos $\mathcal{L}_{\mathcal{F}}$ e suas 
derivadas espaciais. Finalmente, fornecemos uma prescrição geral para se obter os operadores $\mathcal{M}_{m}$, que estabelece a conexão com o MEIQ.

A expressão final para o invariante cúbico quântico regularizado, $\mathcal{Q}_{3}$, é a seguinte:

$$
\mathcal{Q}_{3}=i \frac{c_{0}(\epsilon) \lambda^{3}}{2} \int d x \operatorname{tr}\left[\mathcal{L}_{\mathcal{F}} \cdot \partial_{x} \mathcal{L}_{\mathcal{F}} \cdot \partial_{x}^{2} \mathcal{L}_{\mathcal{F}}\right]
$$

onde a constante $c_{0}(\epsilon)$ será determinada mais adiante. Para deduzi-la, partimos do seguinte polinômio cúbico geral:

$$
\begin{aligned}
\mathcal{Q}_{3}=i c_{0}(\epsilon) \int d x & {\left[a_{1} S_{\mathcal{F}}^{3} \partial_{x} S_{\mathcal{F}}^{+} \partial_{x}^{2} S_{\mathcal{F}}^{-}+a_{2} S_{\mathcal{F}}^{3} \partial_{x} S_{\mathcal{F}}^{-} \partial_{x}^{2} S_{\mathcal{F}}^{+}+a_{3} S_{\mathcal{F}}^{+} \partial_{x} S_{\mathcal{F}}^{3} \partial_{x}^{2} S_{\mathcal{F}}^{-}+\right.} \\
& \left.+a_{4} S_{\mathcal{F}}^{+} \partial_{x} S_{\mathcal{F}}^{-} \partial_{x}^{2} S_{\mathcal{F}}^{3}+a_{5} S_{\mathcal{F}}^{-} \partial_{x} S_{\mathcal{F}}^{3} \partial_{x}^{2} S_{\mathcal{F}}^{+}+a_{6} S_{\mathcal{F}}^{-} \partial_{x} S_{\mathcal{F}}^{+} \partial_{x}^{2} S_{\mathcal{F}}^{3}\right]
\end{aligned}
$$

onde os coeficientes $a_{i}, i=1, \ldots, 6$ são arbitrários e serão fixados posteriormente a partir da condição de diagonalização. É fácil constatar, usando a álgebra (6.2.4), que uma permutação arbitrária das derivadas leva exatamente à mesma expressão que (6.2.36). Então, após cálculos longos e tediosos, porém, sem maiores complicações técnicas, podemos demonstrar que o vetor (6.1.3) diagonaliza o operador $\mathcal{Q}_{3}$, se duas condições forem satisfeitas:

1. Os coeficientes relativos $a_{i}, i=1, \ldots, 6$ são:

$$
a_{1}=-1, \quad a_{2}=1, \quad a_{3}=1, \quad a_{4}=-1, \quad a_{5}=-1 \quad \text { e } \quad a_{6}=1 ;
$$

2. Para cancelar os termos de fronteira é necessária a imposição das condições de compatibilidade (6.1.16), bem como o uso da expressão (6.2.21) para o parâmetro $a_{0}$.

Assim, com os coeficientes relativos determinados e os termos de fronteira eliminados, podemos coletar os termos remanescentes em uma forma compacta:

$$
\mathcal{Q}_{3}=i c_{0}(\epsilon) \int d x \epsilon^{i j k}\left(S_{\mathcal{F}}\right)_{i} \partial_{x}\left(S_{\mathcal{F}}\right)_{j} \partial_{x}^{2}\left(S_{\mathcal{F}}\right)_{k}
$$

Ao expressarmos os campos $\left(S_{\mathcal{F}}\right)_{i}$ em termos do operador $\mathcal{L}_{\mathcal{F}}(x ; \lambda)$, obtemos o resultado (6.2.35). Fixando a função $c_{0}(\epsilon)$ pelo seu valor no limite $\epsilon \rightarrow 0$,

$$
\lim _{\epsilon \rightarrow 0} c_{0}(\epsilon)=\frac{\delta^{3}(0)}{4 a_{0}^{2}} Q_{0}
$$

onde $Q_{0}$ é alguma constante finita arbitrária, podemos, por exemplo, calcular a ação de $\mathcal{Q}_{3}$ sobre o estado com duas partículas:

$$
\mathcal{Q}_{3}\left|f_{2}\right\rangle \stackrel{\epsilon \rightarrow 0}{\longrightarrow}-i Q_{0} \int d y_{1} d y_{2}\left(\partial_{y_{1}}^{3}+\partial_{y_{2}}^{3}\right) f_{2}\left(y_{1}, y_{2}\right) S^{+}\left(y_{1}\right) S^{+}\left(y_{2}\right)|0\rangle .
$$

A partir dos resultados obtidos nas últimas seções, a saber:

1. Os três invariantes fundamentais quânticos possuem a mesma forma que suas contrapartes clássicas, quando escritos em termos dos campos $\mathcal{F}$-regularizados; 
2. As três cargas conservadas quânticas fundamentais podem ser escritos como o traço sobre um produto do operador de Lax e suas derivadas espaciais;

podemos reivindicar que qualquer integral do movimento pode ser decomposta em termos de tais invariantes e suas derivadas espaciais.

No que resta desta seção, desenvolvemos uma prescrição geral para a obtenção dos operadores $\mathcal{M}_{M}(x ; \lambda)$ correspondentes, e, assim, proporcionamos uma conexão explícita com o MEIQ. Posto que qualquer carga conservada pode ser escrita em termos do operador $\mathcal{L}_{\mathcal{F}}(x ; \lambda)$, é conveniente considerar a relação:

$$
\left[\mathcal{L}_{\mathcal{F}}^{(1)}\left(x, \lambda_{1}\right),{ }_{\mathcal{L}}^{(2)}\left(y, \lambda_{2}\right)\right]=\int d z K_{\epsilon}(x, y, z)\left[r\left(\lambda_{1}, \lambda_{2}\right), \stackrel{(1)}{\mathcal{L}_{\mathcal{F}}}\left(z, \lambda_{1}\right)+\stackrel{(2)}{\mathcal{L}} \mathcal{F}_{\left(z, \lambda_{2}\right)}\right]
$$

onde a matriz $r$ possui a seguinte forma:

$$
r\left(\lambda_{1}, \lambda_{2}\right)=\frac{i}{2\left(\lambda_{1}-\lambda_{2}\right)}\left(\mathbb{1}_{2} \otimes \mathbb{1}_{2}+\sum_{a} \sigma_{a} \otimes \sigma_{a}\right) .
$$

Note que, em todas as expressões finais, ao tomarmos o limite $\lambda_{1} \rightarrow \lambda_{2}=\lambda$, a singularidade $\frac{1}{\lambda_{1}-\lambda_{2}}$ é cancelada. Assim, por exemplo, para o Hamiltoniano, inferimos:

$$
\left[\mathcal{H}, \mathcal{L}_{\mathcal{F}}\left(y ; \lambda_{2}\right)\right]=\stackrel{(1)}{\operatorname{tr}} \int d x\left[\stackrel{(1)}{\mathcal{H}}\left(x ; \lambda_{1}\right), \stackrel{(2)}{\mathcal{L}_{\mathcal{F}}}\left(y ; \lambda_{2}\right)\right]
$$

que no limite $\epsilon \rightarrow 0$ e $\lambda_{1} \rightarrow \lambda_{2}$ fornece:

$$
i\left[\mathcal{H}, \mathcal{L}_{\mathcal{F}}(y ; \lambda)\right]=-\frac{\lambda \eta(\epsilon)}{2 \delta_{\epsilon}(0)} \partial_{y}\left[\mathcal{L}_{\mathcal{F}}, \partial_{y} \mathcal{L}_{\mathcal{F}}\right]
$$

levando ao resultado anterior (6.2.25-6.2.28). Similarmente, usando (6.2.39), podemos deduzir o operador $\mathcal{M}_{m}$ correspondente à qualquer carga, embora se torne um processo incrivelmente tediosos conforme consideramos ordens mais altas. Por exemplo, para $\mathcal{Q}_{3}$, um cálculo similar resulta na seguinte expressão:

$$
\left[\mathcal{Q}_{3}, \mathcal{L}_{\mathcal{F}}(y ; \lambda)\right]=\frac{i}{2} \partial_{y}\left[\wedge\left(\mathcal{L}_{\mathcal{F}}\right)\right]
$$

onde $\Lambda\left(\mathcal{L}_{\mathcal{F}}\right)$ é alguma função do operador $\mathcal{L}_{\mathcal{F}}(y ; \lambda)$.

\subsection{Extensões Auto-Adjuntas}

O teste final da consistência da presente construção consiste em verificar se os operados são autoadjuntos. A construção das extensões auto-adjuntas para o Hamiltoniano foi feita originalmente em [44] ${ }^{19}$. Nesta seção, generalizá-la-emos para o caso do terceiro invariante fundamental $\mathcal{Q}_{3}$. Consideramos em detalhe o setor de duas partículas com o produto escalar dado por (6.1.19), e no final tecemos alguns comentários sobre o caso geral, com $n$ partículas.

${ }^{19}$ Para os detalhes relevantes, vide seção 6.1 . 
Introduzimos a ação de $\mathcal{Q}_{3}$ sobre $\left|f_{2}\right\rangle$ como:

$$
\mathcal{Q}_{3}\left|f_{n}\right\rangle=\left(\begin{array}{c}
\hat{i}_{3} f_{1}(x) \\
-\triangle_{3} f_{2}(x, y)
\end{array}\right)
$$

com $\triangle_{3}=\omega \sum_{i=1}^{n} \partial_{i}^{3}$, onde $n$ denota a dimensão apropriada, neste caso, $n=2$, e $\omega \in \mathbb{C} \backslash \mathbb{R}$. Simetria com respeito ao produto escalar (6.1.19),

$$
\left\langle\mathcal{Q}_{3} \tilde{g}_{2} \mid f_{2}\right\rangle=\left\langle\tilde{g}_{2} \mid \mathcal{Q}_{3} f_{2}\right\rangle,
$$

requer

$$
\begin{aligned}
\frac{1}{2} \int d x \tilde{g}_{1}^{*}(x) \hat{i}_{3} f_{1}(x) & -\iint_{x_{1} \neq x_{2}} d x d y \tilde{g}_{2}^{*}(x, y) \triangle_{3} f_{2}(x, y)= \\
& =\frac{1}{2} \int d x\left[\hat{i}_{3} \tilde{g}_{1}(x)\right]^{*} f_{1}(x)-\iint_{x_{1} \neq x_{2}} d x d y\left[\triangle_{3} \tilde{g}_{2}(x, y)\right]^{*} f_{2}(x, y) .
\end{aligned}
$$

Então, calculamos

$$
\begin{aligned}
\iint_{x_{1} \neq x_{2}} d x d y \tilde{g}_{2}^{*}(x, y) \triangle_{3} f_{2}(x, y) & =\iint_{x_{1} \neq x_{2}} d x d y\left[\triangle_{3} \tilde{g}_{2}(x, y)\right]^{*} f_{2}(x, y)+ \\
& +\omega \int d x\left[\tilde{g}_{2}^{*}(x, y)\left(\partial_{x}^{2}-\partial_{y}^{2}\right) f_{2}(x, y)+\left(\partial_{x}^{2}-\partial_{y}^{2}\right) \tilde{g}_{2}^{*}(x, y) f_{2}(x, y)-\right. \\
& \left.-\partial_{x} \tilde{g}_{2}^{*}(x, y) \partial_{x} f_{2}(x, y)+\partial_{y} \tilde{g}_{2}^{*}(x, y) \partial_{y} f_{2}(x, y)\right]_{y=x-\epsilon^{\prime}}^{y=x+\epsilon}
\end{aligned}
$$

que é equivalente a

$$
\begin{aligned}
& \frac{3}{2} \int d x\left\{\tilde{g}_{2}^{*}(x, y)\left[\omega\left(\partial_{x}^{2}-\partial_{y}^{2}\right)\right] f_{2}(x, y)\right\}_{y=x-\epsilon}^{y=x+\epsilon}-\iint_{x_{1} \neq x_{2}} d x d y \tilde{g}_{2}^{*}(x, y) \triangle_{3} f_{2}(x, y)= \\
& \frac{3}{2} \int d x\left\{\left[\omega\left(\partial_{x}^{2}-\partial_{y}^{2}\right) \tilde{g}_{2}(x, y)\right]^{*} f_{2}(x, y)\right\}_{y=x-\epsilon}^{y=x+\epsilon}-\iint_{x_{1} \neq x_{2}} d x d y\left[\triangle_{3} g_{2}(x, y)\right]^{*} f_{2}(x, y) .
\end{aligned}
$$

Comparando com (6.3.2) inferimos:

$$
\hat{i}_{3} f_{1}(x)=3\left[\omega\left(\partial_{x}^{2}-\partial_{y}^{2}\right) f_{2}(x, y)\right]_{y=x-\epsilon}^{y=x+\epsilon}+\hat{i}_{3}^{\prime} f_{1}(x),
$$

onde $\hat{i}_{3}^{\prime}$ é algum operador auto-adjunto em $\mathcal{L}^{2}(\mathbb{R}, d x)$. Em seguida, a condição de que o espaço gerado pelos vetores $\left|f_{2}\right\rangle$ seja fechado sob a ação de $\mathcal{Q}_{3}$ permite que fixemos a forma de $\hat{i}_{3}^{\prime}$. Assim,

$$
\begin{aligned}
-\left.\triangle_{3} f_{2}(x, y)\right|_{x=y} & =\hat{i}_{3} f_{1} \\
& =3\left[\omega\left(\partial_{x}^{2}-\partial_{y}^{2}\right) f_{2}(x, y)\right]_{y=x-\epsilon}^{y=x+\epsilon}+\hat{i}_{3}^{\prime} f_{1}(x) \\
\Rightarrow-\omega \partial_{x}^{3} f_{2}(x, x) & =3 \omega\left(\partial_{x}+\partial_{y}\right)\left[\left.\left(\partial_{x}-\partial_{y}\right) f_{2}(x, y)\right|_{y=x-\epsilon} ^{y=x+\epsilon}-\left.\partial_{x} \partial_{y} f_{2}(x, y)\right|_{x=y}\right]+\hat{i}_{3}^{\prime} f_{1}(x),
\end{aligned}
$$


que com o uso das condições de compatibilidade (6.1.16) para $n=2$ :

$$
\left[\partial_{x} f_{2}(x, y)-\partial_{y} f_{2}(x, y)\right]_{y=x-\epsilon}^{y=x+\epsilon}=\left.\partial_{x} \partial_{y} f_{2}(x, y)\right|_{x=y},
$$

implica

$$
\hat{i}_{3}^{\prime} f_{1}(x)=-\omega \partial_{x}^{3} f_{2}(x, x)
$$

Logo, podemos concluir que

$$
\mathcal{Q}_{3}\left|f_{2}\right\rangle=\left(\begin{array}{c}
3 \omega\left[\left(\partial_{x}^{2}-\partial_{y}^{2}\right) f_{2}(x, y)\right]_{y=x-\epsilon}^{y=x+\epsilon}-\omega \partial_{x}^{3} f_{2}(x, x) \\
-\triangle_{3} f_{2}(x, y)
\end{array}\right)
$$

é um operador auto-adjunto.

É importante ressaltar que (6.3.6) não impõem nenhuma condição adicional sobre o espaço de Hilbert, sendo uma mera consequiência das condições de compatibilidade (6.1.16), que definem nosso espaço de Hilbert. Esse resultado pode ser generalizado para o setor geral de $n$-partículas com o uso da fatorabilidade da matriz $S$, que simplesmente expressa a equivalência exata entre as condições de compatibilidade para o caso geral, com $n$ partículas, e para o caso com apenas duas. 

Parte III

O Modelo de Alday-Arutyunov-Frolov 



\section{Capítulo 7}

\section{O Modelo de Alday-Arutyunov-Frolov}

"Ein Fachmann ist ein Mann, der einige der gröbsten Fehler kennt, die man in dem betreffenden Fach machen kann und der sie deshalb zu vermeiden versteht."

Werner Heisenberg

O modelo de Alday-Arutyunov-Frolov (AAF) surge como um truncamento consistente das equações de movimento da supercorda em $A d S_{5} \times S^{5}$ ao subsetor $\mathfrak{s u ( 1 | 1 )}$ [43]. Para tanto é necessária a imposição do calibre uniforme, que corresponde à identificação da coordenada temporal global do espaço de Anti de Sitter $\left(A d S_{5}\right)$ com o tempo da folha-mundo, bem como a igualar o momento de uma das coordenadas angulares de $S^{5}$ à carga $J$ do grupo $U(1)$ correspondente. Após a fixação do calibre uniforme permanecem apenas os graus de liberdade fermiônicos, que podem ser então combinados em um único férmion de Dirac $\psi$, e a ação assume uma forma manifestamente invariante de Lorentz.

O modelo de AAF é classicamente integrável, uma vez que o par de Lax para o modelo sigma de cordas completo também admite uma redução consistente para o setor $\mathfrak{s u}(1 \mid 1)$ [43]. Muito embora a integrabilidade quântica do modelo ainda não tenha sido estabelecida, as equações de Bethe foram deduzidas a partir do conhecimento da matriz $S$ para o espalhamento de duas partículas e da hipótese de espalhamento fatorável em [35].

Neste capítulo, revisamos a dedução do modelo de AAF seguindo o artigo original [43] e o artigo de revisão [13]. Faz-se necessário pois, uma breve recapitulação de alguns fatos sobre a álgebra superconforme $\mathfrak{p s u}(2,2 \mid 4)$ e da construção da Lagrangeana para o modelo sigma de cordas.

\subsection{A Supercorda em $A d S_{5} \times S^{5}$ como um Modelo Sigma}

A teoria de supercordas em $A d S_{5} \times S^{5}$ pode ser descrita como um modelo sigma não-linear cujo espaço alvo é o seguinte conjunto quociente:

$$
\frac{P S U(2,2 \mid 4)}{S O(4,1) \times S O(5)}
$$


O supergrupo $\operatorname{PSU}(2,2 \mid 4)$ contém o subgrupo bosônico $S U(2,2) \times S U(4)$, localmente isomórfico a $S O(4,2) \times S O(6)$ e cujo quociente com respeito ao grupo $S O(4,1) \times S O(5)$ fornece uma representação para a variedade $A d S_{5} \times S^{5}$ com o grupo das transformações locais de Lorentz dado por $S O(4,1) \times S O(5)$. O supergrupo $P S U(2,2 \mid 4)$ fornece as isometrias do superespaço $\operatorname{AdS}_{5} \times S^{5}$. Dessa forma a ação da teoria de supercordas surge como a soma da ação desse modelo sigma não-linear com o termo topológico de Wess-Zumino, que garante a simetria $\kappa$.

\subsubsection{A Álgebra Superconforme}

Considere a superálgebra $\mathfrak{s l}(4 \mid 4)$ sobre o corpo dos complexos. Como uma álgebra de matrizes, $\mathfrak{s l}(4 \mid 4)$ é gerada por matrizes $8 \times 8$ que podem ser escritas em termos de blocos $4 \times 4$ :

$$
M=\left(\begin{array}{cc}
m & \theta \\
\eta & n
\end{array}\right) .
$$

Tais matrizes devem possuir supertraço nulo:

$$
\operatorname{str} M=\operatorname{tr} m-\operatorname{tr} n=0 .
$$

A superálgebra $\mathfrak{s l}(4 \mid 4)$ possui uma estrutura $\mathbb{Z}_{2}$-graduada, isto é, as matrizes $m$ e $n$ são pares, enquanto que $\theta$ e $\eta$, ímpares ${ }^{1}$.

Já a superálgebra $\mathfrak{s u}(2,2 \mid 4)$ é uma forma real não-compacta de $\mathfrak{s l}(4 \mid 4)$, obtida a partir da identificação $M^{\star}=M$ em $\mathfrak{s l}(4 \mid 4)$, onde $M^{\star}=-H M^{\dagger} H^{-1}$ é a involução de Cartan e o adjunto de uma supermatriz $M$ é definido como $M^{\dagger}=\left(M^{t}\right)^{*}$. Assim, uma matriz $M \in \mathfrak{s u}(2,2 \mid 4)$ deve satisfazer além da condição de supertraço nulo (7.1.3), a seguinte condição de realidade:

$$
H M+M^{\dagger} H=0 .
$$

É conveniente fixarmos a forma da matrix Hermiteana $H$ :

$$
H=\left(\begin{array}{cc}
\Sigma & 0 \\
0 & -\mathbb{1}_{4}
\end{array}\right) \text {, }
$$

onde

$$
\Sigma=\left(\begin{array}{cc}
\mathbb{1}_{2} & 0 \\
0 & -\mathbb{1}_{2}
\end{array}\right) .
$$

Notamos que para qualquer elemento ímpar $\theta$ a conjugação atua como uma anti-involução antilinear:

$$
(c \theta)^{*}=\bar{c} \theta^{*}, \quad \theta^{* *}=\theta, \quad\left(\theta_{1} \theta_{2}\right)^{*}=\theta_{2}^{*} \theta_{1}^{*},
$$

que garante, em particular, que $\left(M_{1} M_{2}\right)^{\dagger}=M_{2}^{\dagger} M_{1}^{\dagger}$, ou seja, que supermatrizes anti-hermiteanas formam uma álgebra de Lie.

\footnotetext{
${ }^{1}$ Que podem ser consideradas como variáveis anticomutantes de Grassmann.
} 
A condição (7.1.4) implica:

$$
m^{\dagger}=-\Sigma m \Sigma^{-1}, \quad n^{\dagger}=-n, \quad \eta^{\dagger}=\Sigma \theta .
$$

De modo que, $m$ e $n$ geram as subálgebras unitárias $\mathfrak{u}(2,2)$ e $\mathfrak{u}(4)$, respectivamente. A álgebra $\mathfrak{s u}(2,2 \mid 4)$ também contém o gerador do grupo $\mathfrak{u}(1), i \mathbb{1}$, dado que esse também satisfaz a condição de realidade (7.1.4) e possui supertraço nulo. Portanto, a subálgebra bosônica de $\mathfrak{s u}(2,2 \mid 4)$ é:

$$
\mathfrak{s u}(2,2) \oplus \mathfrak{s u}(4) \oplus \mathfrak{u}(1)
$$

Finalmente, defini-se a superálgebra $\mathfrak{p s u}(2,2 \mid 4)$ como a álgebra quociente de $\mathfrak{s u}(2,2 \mid 4)$ sobre esse fator $\mathfrak{u}(1)$. Nota-se que tal álgebra não possui realização em termos de supermatrizes $8 \times 8$.

\subsubsection{Graduação $\mathbb{Z}_{4}$}

Considere a operação de supertransposição:

$$
M^{s t}=\left(\begin{array}{cc}
m^{t} & -\eta^{t} \\
\theta^{t} & n^{t}
\end{array}\right)
$$

Claramente, a operação $M \rightarrow-M^{\text {st }}$ define um automorfismo de quarta ordem em $\mathfrak{s l}(4 \mid 4)$, em particular, o mapa:

$$
\Omega: \mathfrak{s l}(4 \mid 4) \rightarrow \mathfrak{s l}(4 \mid 4), \quad M \rightarrow \Omega(M)=-\mathcal{K} M^{s t} \mathcal{K}^{-1},
$$

obtido através de uma transformação de similaridade com respeito a matriz $\mathcal{K}=\operatorname{diag}(K, K)$ onde 2 :

$$
K=\Gamma_{2}^{S O(4,1)} \Gamma_{4}^{S O(4,1)}=\left(\begin{array}{cccc}
0 & -1 & 0 & 0 \\
1 & 0 & 0 & 0 \\
0 & 0 & 0 & -1 \\
0 & 0 & 1 & 0
\end{array}\right)
$$

também define um automorfismo de quarta ordem, cuja ação sobre o produto de dois elementos $M_{1}, M_{2} \in \mathfrak{s l}(4,4)$ satisfaz:

$$
\Omega\left(M_{1} M_{2}\right)=-\Omega\left(M_{2}\right) \Omega\left(M_{1}\right) .
$$

A existência de um automorfismo de quarta ordem permite que equipemos a superálgebra de Lie $\mathfrak{s l}(4,4)$ com uma estrutura graduada do tipo $\mathbb{Z}_{4}$. Denotando $\mathscr{G}=\mathfrak{s l}(4,4)$, definimos:

$$
\mathscr{G}^{(k)}=\left\{M \in \mathscr{G}: \Omega(M)=i^{k} M\right\},
$$

de forma que, como espaço vetorial, $\mathscr{G}$ pode ser decomposto como uma soma direta de subespaços graduados:

$$
\mathscr{G}=\mathscr{G}^{(0)} \oplus \mathscr{G}^{(1)} \oplus \mathscr{G}^{(2)} \oplus \mathscr{G}^{(3)},
$$

onde $\left[\mathscr{G}^{(k)}, \mathscr{G}^{(l)}\right] \subset \mathscr{G}^{(k+l)}$ módulo $\mathbb{Z}_{4}$. Estamos particularmente interessados na projeção de uma

\footnotetext{
${ }^{2}$ Vide apêndice B para a definição das matrizes gama.
} 
matriz qualquer $M \in \mathscr{G}$ nos subespaços $\mathscr{G}^{(k)}$,

$$
M^{(k)}=\frac{1}{4}\left(M+i^{3 k} \Omega(M)+i^{2 k} \Omega^{2}(M)+i^{k} \Omega^{3}(M)\right) .
$$

Não é difícil de constatar que as projeções $M^{(0)}$ e $M^{(2)}$ são pares, enquanto que as projeções $M^{(1)}$ e $M^{(3)}$ são ímpares.

Queremos estender a decomposição (7.1.11) para matrizes em $\mathfrak{s u}(2,2 \mid 4)$, para tanto precisamos entender como o mapa $\Omega$ se relaciona com a involução de Cartan. Embora $[K, \Sigma]=0$, em geral, temos que $\left(M^{s t}\right)^{\dagger} \neq\left(M^{\dagger}\right)^{\text {st }}$; logo, tem-se que a ação de $\Omega$ (anti)comuta com a involução de Cartan:

$$
\Omega(M)^{\dagger}=\left\{\begin{aligned}
\Omega\left(M^{\dagger}\right), & \text { M par } \\
-\Omega\left(M^{\dagger}\right), & \text { M ímpar }
\end{aligned}\right.
$$

Suponha, pois, que $M \in \mathfrak{s u}(2,2 \mid 4)$, de forma que as expressões (7.1.13) podem ser rescritas de uma forma mais compacta:

$$
\Omega(M)^{\dagger}=\Upsilon \Omega(M) \Upsilon^{-1}=-(\Upsilon H) \Omega(M)(\Upsilon H)^{-1},
$$

onde introduzimos a hipercarga $\Upsilon=\operatorname{diag}\left(\mathbb{1}_{4},-\mathbb{1}_{4}\right)$ e podemos calcular facilmente o adjunto de $M^{(k)}$ :

$$
M^{(k) \dagger}=-\frac{1}{4} H\left[M+i^{k} \Upsilon \Omega(M) \Upsilon^{-1}+i^{2 k} \Omega^{2}(M)+i^{3 k} \Upsilon \Omega^{3}(M) \Upsilon^{-1}\right] H^{-1}
$$

Notando que $\Upsilon \Omega(M) \Upsilon^{-1}=\Omega^{3}(M)$, concluímos que $M^{(k)}=-H M^{(k) \dagger} H^{-1}$, e conseqüentemente, $M^{(k)} \in \mathfrak{s u}(2,2 \mid 4), \forall k$. Portanto qualquer matriz $M \in \mathfrak{s u}(2,2 \mid 4)$ pode ser decomposta unicamente na soma (7.1.11) com cada $M^{(k)} \in \mathfrak{s u}(2,2 \mid 4)$.

A subálgebra bosônica $\mathfrak{s u}(2,2) \oplus \mathfrak{s u}(4) \oplus \mathfrak{u}(1) \subset \mathfrak{s u}(2,2 \mid 4)$ é decomposta por $\Omega$ na soma direta de dois elementos graduados $\mathscr{G}^{(0)}$ e $\mathscr{G}^{(2)}$, cujos elementos são da forma:

$$
\begin{aligned}
M^{(0)} & =\frac{1}{2}\left(\begin{array}{cc}
m-K m^{t} K^{-1} & 0 \\
0 & n-K n^{t} K^{-1}
\end{array}\right) . \\
M^{(2)} & =\frac{1}{2}\left(\begin{array}{cc}
m+K m^{t} K^{-1} & 0 \\
0 & n+K n^{t} K^{-1}
\end{array}\right) .
\end{aligned}
$$

Assim, temos que $\mathscr{G}^{(0)}=\mathfrak{s o}(4,1) \oplus \mathfrak{s o}(5) \subset \mathfrak{s u}(2,2) \oplus \mathfrak{s u}(4)$, enquanto que os elementos de $\mathscr{G}^{(2)}$ geram o complemento ortogonal às álgebras de Lie $\mathfrak{s o}(4,1)$ e $\mathfrak{s o}(5)$ em $\mathfrak{s u}(2,2) \oplus \mathfrak{s u}(4)$, ou seja, $\mathscr{G}^{(2)}$ contém os geradores da álgebra de Lie correspondentes ao conjunto quociente $\frac{S U(2,2) \times S U(4)}{S O(4,1) \times S O(5)}=A d S_{5} \times S^{5}$. Notamos que o elemento central $i \mathbb{1} \in \mathfrak{s u}(2,2 \mid 4)$ também pertence à projeção $\mathscr{G}^{(2)}$.

Finalmente, para completarmos a discussão da decomposição $\mathbb{Z}_{4}$ de $\mathfrak{s u}(2,2 \mid 4)$, exibimos as pro- 
jeções ímpares:

$$
\begin{aligned}
& M^{(1)}=\frac{1}{2}\left(\begin{array}{cc}
0 & \theta-i K \eta^{t} K^{-1} \\
\eta+i K \theta^{t} K^{-1} & 0
\end{array}\right), \\
& M^{(3)}=\frac{1}{2}\left(\begin{array}{cc}
0 & \theta+i K \eta^{t} K^{-1} \\
\eta-i K \theta^{t} K^{-1} & 0
\end{array}\right) .
\end{aligned}
$$

\subsubsection{A Supercorda de Green-Schwarz como um Conjunto Quociente}

Consideramos uma corda fechada propagando no espaço $A d S_{5} \times S^{5}$, cuja folha-mundo é parametrizada por coordenadas $\tau$ e $\sigma$ e constitui um cilindro de circunferência $2 r$, com $\sigma \in[0 ; 2 r]$, onde $r$ é uma constante arbitrária, que fixamos da maneira canônica, $r=\pi$. É conveniente considerarmos a tensão da corda $g$ adimensional, que para o caso de $A d S_{5} \times S^{5}$ pode ser expressa em termos do raio $R$ de $S^{5}$ e de $\alpha^{\prime}$ :

$$
g=\frac{R^{2}}{2 \pi \alpha^{\prime}}
$$

no contexto da correspondência AdS/CFT, tem-se a seguinte relação com a constante de 't Hooft, $\lambda$ :

$$
g=\frac{\sqrt{\lambda}}{2 \pi} \Rightarrow \sqrt{\lambda}=\frac{R^{2}}{\alpha^{\prime}}
$$

Logo, a ação da supercorda assume a seguinte forma:

$$
S=\int d \tau \int_{0}^{2 \pi} \frac{d \sigma}{2 \pi} \mathscr{L}
$$

Seja $\mathfrak{g} \in \operatorname{SU}(2,2 \mid 4)$ e considere a seguinte 1 -forma com valores em $\mathfrak{s u}(2,2 \mid 4)$ :

$$
A=-\mathfrak{g}^{-1} d \mathfrak{g}=A^{(0)}+A^{(1)}+A^{(2)}+A^{(3)},
$$

onde exibimos a decomposição $\mathbb{Z}_{4}$ da corrente $A$ de acordo com (7.1.11). Claramente, a corrente $A$ satisfaz a condição de curvatura nula por construção:

$$
d A-A \wedge A=0 .
$$

A densidade Lagrangeana para a supercorda em $A d S^{5} \times S^{5}$ pode ser escrita como:

$$
\mathscr{L}=-\frac{\sqrt{\lambda}}{2} \gamma^{\alpha \beta} \operatorname{str}\left(A_{\alpha}^{(2)} A_{\beta}^{(2)}\right)-\kappa \epsilon^{\alpha \beta}\left(A_{\alpha}^{(1)} A_{\beta}^{(3)}\right)
$$

que é a soma de um termo cinético com um termo de Wess-Zumino. A simetria kappa requer que $\kappa= \pm \sqrt{\lambda} / 2$, notamos também que apenas sob essa condição a Lagrangeana (7.1.21) define um modelo classicamente integrável. A convenção adotada em (7.1.21) é a seguinte: $\epsilon^{\tau \sigma}=1$ e $\gamma^{\alpha \beta}=h^{\alpha \beta} \sqrt{-h}$ é a combinação invariante de Weyl da métrica na folha-mundo com det $\gamma=-1$.

Precisamos ainda justificar que a Lagrangeana (7.1.21) descreve uma corda no conjunto quociente (7.1.1). Para isso seja $\mathfrak{h} \in S O(4,1) \times S O(5)$ e considere a transformação induzida por esse elemento: 
$\mathfrak{g} \rightarrow \mathfrak{g h}$. Sob essa transformação a 1-forma (7.1.19):

$$
A \rightarrow \mathfrak{h}^{-1} A \mathfrak{h}-\mathfrak{h}^{-1} d \mathfrak{h} \Rightarrow A^{(0)} \rightarrow \mathfrak{h}^{-1} A^{(0)} \mathfrak{h}-\mathfrak{h}^{-1} d \mathfrak{h}, \quad A^{(1,2,3)} \rightarrow \mathfrak{h}^{-1} A^{(1,2,3)} \mathfrak{h} .
$$

É fácil constatar que a componente $A^{(0)}$ sofre uma transformação de calibre, enquanto que todas as outras componentes são levadas em suas representações adjuntas.

Por construção, a Lagrangeana (7.1.21) depende apenas do elemento do grupo g. Contudo, sob uma multiplicação à direita de $\mathfrak{g}$ por um elemento local ${ }^{3} \mathfrak{h} \in S O(4,1) \times S O(5)$, as componentes homogêneas $A^{(1)}, A^{(2)}$ e $A^{(3)}$ sofrem apenas uma transformação de similaridade, que deixa a Lagrangeana (7.1.21) invariante. Portanto, tal Lagrangeana depende apenas do conjunto quociente $\frac{S U(2,2 \mid 4)}{S O(4,1) \times S O(5)}$.

Resta apenas eliminarmos o fator $\mathfrak{u}(1) \subset \mathfrak{s u}(2,2 \mid 4)$, para isso relembramos que seu gerador, i1 aparece na projeção $A^{(2)}$ e notamos que como resultado de uma multiplicação à direita de $\mathfrak{g}$ por um

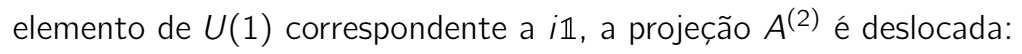

$$
A^{(2)} \rightarrow A^{(2)}+c \cdot i \mathbb{1}
$$

Dado que o supertraço de tanto $A^{(2)}$ como da matriz identidade são nulos, temos que tal transformação deixa a Lagrangeana (7.1.21) invariante. Conseqüentemente, além de $\mathfrak{s o}(4,1) \times \mathfrak{s o}(5)$, existe uma simetria $\mathfrak{u}(1)$ induzida pelo elemento central $i \mathbb{1}$. Tal simetria pode ser utilizada para eliminar a parte de traço de $A^{(2)}$ através de uma transformação de calibre. Dessa forma, no que se segue tomamos $A^{(2)}$ sem traço, o que pode ser visto como uma fixação de calibre para tais transformações $\mathfrak{u}(1)$. Portanto, tem-se que o grupo de simetrias globais de (7.1.21) coincide com PSU(2,2|4).

\subsubsection{Parametrização do Conjunto Quociente}

Naturalmente existem inúmeras maneiras de se parametrizar um elemento do conjunto quociente (7.1.1) e todas elas devem estar relacionadas entre si por alguma redefinição dos campos. Contudo, para os nosso propósitos a seguinte parametrização se mostra mais conveniente:

$$
\mathfrak{g}=g(\theta, \eta) g(x, y)
$$

onde $g(x, y)$ descreve a imersão de $A d S_{5} \times S^{5}$ em $S U(2,2) \times S U(4)$, enquanto que $g(\theta, \eta)$ é responsável por incorporar os 32 graus de liberdade fermiônicos. Tomamos:

$$
g(x, y)=g(x) g(y)=e^{\frac{1}{2}\left(x_{a} \Gamma_{a}^{S O(4,1)}\right)} e^{\frac{1}{2}\left(x_{b} \Gamma_{b}^{S O(5)}\right)},
$$

onde as coordenadas $x_{a}$ parametrizam $A d S_{5}$ e as $y_{b}, S^{5}$, as matrizes $\Gamma_{a}^{S O(4,1)}$ e $\Gamma_{b}^{S O(5)}$ estão definidas no Apêndice B. Deixamos subentendido que $g(x, y)$ é uma matriz bloco diagonal $8 \times 8$, com o bloco $4 \times 4$ superior correspondendo a $g(x)$, e o inferior, a $g(y)$.

$$
{ }^{3} \mathfrak{h}=\mathfrak{h}(\tau, \sigma)
$$


Já a matriz fermiônica assume a seguinte forma:

$$
g(\theta, \eta)=\exp \left(\begin{array}{cccccccc}
0 & 0 & 0 & 0 & \eta^{5} & \eta^{6} & \eta^{7} & \eta^{8} \\
0 & 0 & 0 & 0 & \eta^{1} & \eta^{2} & \eta^{3} & \eta^{4} \\
0 & 0 & 0 & 0 & \theta^{1} & \theta^{2} & \theta^{3} & \theta^{4} \\
0 & 0 & 0 & 0 & \theta^{5} & \theta^{6} & \theta^{7} & \theta^{8} \\
\eta_{5} & \eta_{1} & -\theta_{1} & -\theta_{5} & 0 & 0 & 0 & 0 \\
\eta_{6} & \eta_{2} & -\theta_{2} & -\theta_{6} & 0 & 0 & 0 & 0 \\
\eta_{7} & \eta_{3} & -\theta_{3} & -\theta_{7} & 0 & 0 & 0 & 0 \\
\eta_{8} & \eta_{4} & -\theta_{4} & -\theta_{8} & 0 & 0 & 0 & 0
\end{array}\right),
$$

onde $\theta^{i}$ e $\eta^{i}$ são $8+8$ férmions complexos que satisfazem a seguinte regra de conjugação: $\theta^{i *}=\theta_{i}$ e $\eta^{i *}=\eta_{i}$

A parametrização do elemento bosônico do conjunto quociente que escolhemos em (7.1.23) é feita em termos de $5+5$ coordenadas livres $x_{a}$ e $y_{b}$. Entretanto, para fins de implementação das simetrias conforme e $R$ é mais conveniente trabalhar com um conjunto de $6+6$ coordenadas vinculadas que descrevem a imersão de $A d S_{5}$ em $\mathbb{R}^{4,2}$ e $S^{5}$ em $\mathbb{R}^{6}$. Nesse caso os representantes de $A d S_{5}$ e de $S^{5}$, $g_{a}(v)$ e $g_{s}(u)$, são dados pelas matrizes:

$$
\begin{gathered}
g_{a}(v)=\left(\begin{array}{cccc}
0 & -i v_{5}-v_{6} & v_{1}-i v_{4} & -i v_{2}-v_{3} \\
i v_{5}+v_{6} & 0 & -i v_{2}+v_{3} & v_{1}+i v_{4} \\
-v_{1}+i v_{4} & i v_{2}-v_{3} & 0 & i v_{5}-v_{6} \\
i v_{2}+v_{3} & -v_{1}-i v_{4} & -i v_{5}+v_{6} & 0
\end{array}\right), \\
g_{s}(u)=\left(\begin{array}{cccc}
0 & -i u_{5}-u_{6} & -i u_{1}-u_{4} & -u_{2}+i u_{3} \\
i u_{5}+u_{6} & 0 & -u_{2}-i u_{3} & -i u_{1}+u_{4} \\
i u_{1}+u_{4} & u_{2}+i u_{3} & 0 & i u_{5}-u_{6} \\
u_{2}-i u_{3} & i u_{1}-u_{4} & -i u_{5}+u_{6} & 0
\end{array}\right) .
\end{gathered}
$$

As novas coordenadas satisfazem os vínculos:

$$
\begin{aligned}
-1 & =v_{1}^{2}+v_{2}^{2}+v_{3}^{2}+v_{4}^{2}-v_{5}^{2}-v_{6}^{2}, \\
1 & =u_{1}^{2}+u_{2}^{2}+u_{3}^{2}+u_{4}^{2}+u_{5}^{2}+u_{6}^{2},
\end{aligned}
$$

que garantem que $g_{a}(v) \in S U(2,2)$ e $g_{s}(u) \in S U(4,4)$. Notamos que nas coordenadas $(u, v)$ as transformações conforme e da simetria $R$ atuam de forma linear, ao contrário do que acontece com as coordenadas $(x, y)$. Uma relação entre essas duas descrições do conjunto quociente pode ser obtida ao considerarmos que os elementos $g_{a}(v) \in S U(2,2) / S O(4,1)$ e $g_{s}(u) \in S U(4) / S O(5)$ podem ser escritos como:

$$
g_{a}(v)=g(x) K g(x)^{t}, \quad g_{s}(u)=g(y) K g(y)^{t},
$$

onde $g(x) \in S U(2,2)$ e $g(y) \in S U(4)$ são dadas por (7.1.23). Além do mais as seguintes relações 
são satisfeitas:

$$
\begin{gathered}
x_{a}=\frac{|x|}{\sinh |x|} v_{a}, \quad|x|=\operatorname{arccosh} v_{6}, \quad|x|^{2}=x_{1}^{2}+x_{2}^{2}+x_{3}^{2}+x_{4}^{2}-x_{5}^{2}, \\
y_{a}=\frac{|y|}{\sin |y|} u_{a}, \quad|y|=\arccos u_{6}, \quad|y|^{2}=y_{1}^{2}+y_{2}^{2}+y_{3}^{2}+y_{4}^{2}+y_{5}^{2} .
\end{gathered}
$$

\subsubsection{Simetrias Globais}

Para podermos identificar o truncamento consistente ao setor $\mathfrak{s u}(1 \mid 1)$ precisamos analisar as simetrias globais mais atentamente. A ação do grupo de isometrias $\operatorname{PSU}(2,2 \mid 4)$ sobre um elemento do conjunto quociente é realizada por multiplicação pela esquerda:

$$
G \mathfrak{g}=\mathfrak{g}^{\prime} \mathfrak{h},
$$

onde $G \in P S U(2,2 \mid 4)$, $\mathfrak{g}$ e $\mathfrak{g}^{\prime}$ são representantes do conjunto quociente antes e depois da ação do grupo de isometrias, respectivamente, e $\mathfrak{h} \in S O(4,1) \times S O(5)$ é uma transformação de compensação. Consideraremos apenas transformações infinitesimais, ou seja, aquelas geradas pela álgebra de Lie $\mathfrak{p s u}(2,2 \mid 4)$.

\section{Transformações Conformes de Campos Bosônicos}

Considere o elemento do conjunto quociente que descreve o espaço de Anti de Sitter, $g(x)$. Claramente, pela discussão anterior ${ }^{4} g(x) \in S U(2,2) / S O(4,1)$ e deve satisfazer:

$$
K g(x)^{t} K^{-1}=g(x)
$$

Uma transformação conforme infinitesimal atuando sobre tal $g(x)$ deve ser da seguinte forma:

$$
\delta g(x)=\Phi g(x)-g(x) \Phi_{c}
$$

onde $\Phi \in \mathfrak{s u}(2,2)$ faz o papel do parâmetro de uma transformação infinitesimal e $\Phi_{c} \in \mathfrak{s o}(4,1) \subset$ $\mathfrak{s u}(2,2)$ é uma transformação de compensação, que satisfaz ${ }^{5}$ :

$$
K \Phi_{c}^{t} K^{-1}=-\Phi_{c}
$$

A transformação de compensação $\Phi_{c}$ é determinada a partir de $\Phi$ ao impormos que $\delta g(x) \in$ SU(2,2)/SO(4,1), ou seja, que $\delta g(x)$ também satisfaça a condição (7.1.30). Em particular, para determinarmos a regra de transformação em termos das coordenadas $v$ não precisamos desse fator de compensação, pois ao utilizarmos (7.1.27) em conjunto com (7.1.32), obtemos:

$$
\delta g_{a}(v)=\Phi g_{a}(v)+g_{a}(v) \Phi^{t}
$$

O operador de dilatação é identificado com o gerador das translações $\Phi=\frac{\xi}{2} \Gamma_{5}^{(S O(4,1))}$ da coor-

\footnotetext{
${ }^{4}$ Vide componente $M^{(2)} \in \mathscr{G}^{(2)}$ na discussão sobre decomposição $\mathbb{Z}_{4}$, equação (7.1.16b).

${ }^{5}$ Vide componente $M^{(0)} \in \mathscr{G}^{(0)}$ na discussão sobre decomposição $\mathbb{Z}_{4}$, equação (7.1.16a).
} 
denada global temporal de $A d S, t$, que pode ser expressa em termos das coordenadas $v_{5}$ e $v_{6}$, vide equação (7.1.26a),

$$
e^{i t}=i v_{5}+v_{6}
$$

De forma que o grupo $U(1)$ responsável apenas pelas rotações de $v_{5}$ e $v_{6}$ corresponde às translações em $t, t \rightarrow t^{\prime}=t+\xi$.

Em contrapartida, a rotação de $\mathfrak{s o}(4) \subset \mathfrak{s u}(2,2)$ que atua linearmente sobre $v_{1}, \ldots, v_{4}$, mas que não afeta as direções $v_{5}$ e $v_{6}$ é induzida pela seguinte matriz:

$$
\Phi_{\mathfrak{s o}(4)}=\left(\begin{array}{cccc}
i \xi_{1} & \alpha_{1}+i \beta_{1} & 0 & 0 \\
-\alpha_{1}+i \beta_{1} & -i \xi_{1} & 0 & 0 \\
0 & 0 & i \xi_{3} & \alpha_{6}+i \beta_{6} \\
0 & 0 & -\alpha_{6}+i \beta_{6} & -i \xi_{3}
\end{array}\right)
$$

que é a soma direta de dois $\mathfrak{s u}(2)$.

\section{Simetria $R$ para Campos Bosônicos}

Considere o elemento do conjunto quociente que descreve a esfera pentadimensional, $g(y)$. Claramente, $g(y) \in S U(4) / S O(5)$, de forma que uma análise similar à da simetria conforme se aplica ao caso da simetria $R$. Os seguintes escalares:

$$
Z_{1}=u_{4}+i u_{1}, \quad Z_{2}=u_{2}+i u_{3}, \quad Z_{3}=u_{6}+i u_{5}
$$

desempenham um papel central no truncamento consistente ao setor $\mathfrak{s u}(1 \mid 1)$. Cada um desses escalares $Z_{i}, i=1,2,3$, carrega uma unidade de carga com respeito a um $\mathfrak{u}(1) \subset \mathfrak{s u}(4)$ gerado, respectivamente, pelas matrizes:

$$
\begin{aligned}
& \Phi_{1}=\frac{1}{2}\left(\begin{array}{cccc}
1 & 0 & 0 & 0 \\
0 & -1 & 0 & 0 \\
0 & 0 & 1 & 0 \\
0 & 0 & 0 & -1
\end{array}\right), \Phi_{2}=\frac{1}{2}\left(\begin{array}{cccc}
-1 & 0 & 0 & 0 \\
0 & 1 & 0 & 0 \\
0 & 0 & 1 & 0 \\
0 & 0 & 0 & -1
\end{array}\right) \\
& \Phi_{3}=\frac{1}{2}\left(\begin{array}{cccc}
1 & 0 & 0 & 0 \\
0 & 1 & 0 & 0 \\
0 & 0 & -1 & 0 \\
0 & 0 & 0 & -1
\end{array}\right)=\frac{1}{2} \Gamma_{5}^{S O(5)} .
\end{aligned}
$$

e é neutro com respeito aos outros dois. Além do mais, notamos que as $\mathfrak{u}(1)$ geradas por $\Phi_{1}$ e $\Phi_{2}$ são subálgebras de $\mathfrak{s o}(4)=\mathfrak{s u}(2) \times \mathfrak{s u}(2)$, responsável por rodar somente as coordenadas $u_{1}, \ldots, u_{4}$ e gerado por:

$$
\Phi_{\mathfrak{s o}(4)}=\left(\begin{array}{cccc}
i \xi_{1} & \alpha_{1}+i \beta_{1} & 0 & 0 \\
-\alpha_{1}+i \beta_{1} & -i \xi_{1} & 0 & 0 \\
0 & 0 & i \xi_{3} & \alpha_{6}+i \beta_{6} \\
0 & 0 & -\alpha_{6}+i \beta_{6} & -i \xi_{3}
\end{array}\right)
$$




\section{Transformações Conformes e Simetria $R$ para Campos Fermiônicos}

Considere o elemento fermiônico do conjunto quociente $g(\theta, \eta) \equiv e^{\ominus}$ e denote o elemento bosônico simplesmente por $g$. A ação infinitesimal do grupo de simetria sobre os férmions pode ser obtida a partir da expressão para a variação de um elemento arbitrário do conjunto quociente:

$$
\delta_{\Phi}\left(E^{\ominus} g\right)=\Phi e^{\ominus} g-e^{\ominus} g \Phi_{c}
$$

onde $\Phi_{c}$ denota novamente uma transformação de compensação, que, em geral, depende de $\Phi, \Theta$ e g. Considerando que uma transformação conforme infinitesimal para campos bosônicos obedece à (7.1.31), concluímos:

$$
\delta_{\phi} \Theta=[\Phi, \Theta]
$$

Utilizando a parametrização do elemento fermiônico do conjunto quociente (7.1.24), podemos escrever a matriz $\Theta$ como uma matriz bloco antidiagonal. Similarmente, podemos também agrupar as transformações conformes e de simetria $R$ para a parte bosônica como uma matriz bloco diagonal.

$$
\Theta=\left(\begin{array}{cc}
0 & \tilde{\Psi} \\
\psi & 0
\end{array}\right), \quad \Phi=\left(\begin{array}{cc}
\Phi_{a} & 0 \\
0 & \Phi_{s}
\end{array}\right) .
$$

Dessa forma, (7.1.39) implica:

$$
\delta_{\phi} \Psi=\Phi_{s} \psi-\psi \Phi_{a}, \quad \delta_{\phi} \tilde{\psi}=\Phi_{a} \tilde{\Psi}-\tilde{\Psi} \Phi_{s}
$$

de onde concluímos que as colunas de $\Psi$ se transformam na representação fundamental de $\mathfrak{s u ( 4 ) ,}$ enquanto que as colunas de $\tilde{\Psi}$, na representação fundamental de $\mathfrak{s u}(2,2)$.

A forma da matriz de dilatação, $\Phi=\frac{\xi}{2} \Gamma_{5}^{S O(4,1)}$ juntamente com (7.1.41) permite que concluamos que a carga dos $\eta^{i}$ com respeito ao operador de dilatações é $\frac{1}{2}$, enquanto que a dos $\theta^{i}$ é $-\frac{1}{2}$.

\section{Transformações de Supersimetria}

Para completarmos a nossa discussão sobre simetrias globais, exibimos as transformações de supersimetria infinitesimais com respeito a um parâmetro fermiônico $\epsilon$ (compreendendo 32 supersimetrias):

$$
\begin{aligned}
\delta_{\epsilon} g & =\frac{1}{2}[\epsilon, \Theta] g-g \Phi_{c}, \\
\delta_{\epsilon} \Theta & =\epsilon,
\end{aligned}
$$

onde novamente denotamos o elemento bosônico do conjunto quociente por $g$ e a transformação de compensação $\Phi_{c} \in \mathfrak{s o}(4,1) \times \mathfrak{s o}(5)$. 


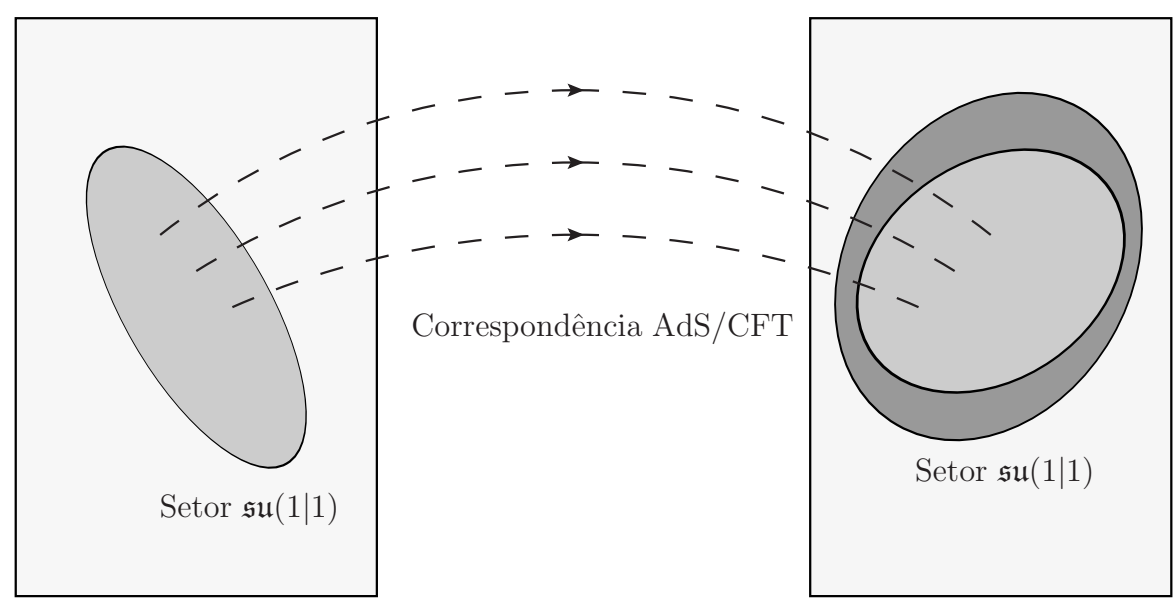

$\mathcal{N}=4 \mathrm{SYM}$

Supercorda em $A d S_{5} \times S^{5}$

Figura 7.1: Definição do setor $\mathfrak{s u}(1 \mid 1)$ na teoria de Supercordas a partir do setor $\mathfrak{s u}(1 \mid 1)$ na teoria de calibre dual.

\subsection{O Setor $\mathfrak{s u}(1 \mid 1)$ da Teoria de Supercordas}

A definição do setor $\mathfrak{s u}(1 \mid 1)$ da teoria de supercordas em $A d S_{5} \times S^{5}$ é feita de tal forma que ele seja o menor setor a conter todos os estados duais aos operadores contidos no setor $\mathfrak{s u}(1 \mid 1)^{6}$ da teoria de calibre dual ${ }^{7}$. Vide figura 7.1.

O setor $\mathfrak{s u}(1 \mid 1)$ da $\mathcal{N}=4$ SYM contém os operadores compostos da forma:

$$
\operatorname{tr}\left(\Psi^{M} Z^{J-\frac{M}{2}}\right)+\cdots
$$

de acordo com a linguagem $\mathcal{N}=1$, o operador $Z$ corresponde a um dos três supercampos escalares complexos, enquanto $\Psi$, à componente de maior peso de $\psi_{\alpha}$, o gaugino do multipleto vetorial. 0 campo $\Psi_{\alpha}$ se transforma como um espinor sob uma das subálgebras $\mathfrak{s u}(2)$ da álgebra de Lorentz $\mathfrak{s u}(2,2)$ e é neutro com respeito à outra. Os campos $Z$ e $\Psi$ carregam cargas 1 e $1 / 2$ sob o subgrupo $U(1) \subset S U(4)$ gerado por $\Phi_{3}(7.1 .36 \mathrm{~b})$.

É natural supormos que os escalares complexos (7.1.35) sejam duais aos supercampos escalares da teoria de calibre. Conseqüentemente, a redução ao setor $\mathfrak{s u}(1 \mid 1)$ requer, em particular, que $Z_{1}=$ $Z_{2}=0$. Além do mais, demandamos que $v_{i}=0, i=1,2,3,4$, de forma que apenas as coordenadas que correspondem ao tempo global de AdS, $v_{5}$ e $v_{6}$ são não-nulas. A álgebra da simetria bosônica residual é:

$$
\mathfrak{s o}(4) \times \mathfrak{s o}(4)=\underbrace{\mathfrak{s u}(2) \times \mathfrak{s u}(2)}_{A d S_{5}} \times \underbrace{\mathfrak{s u}(2) \times \mathfrak{s u}(2)}_{S^{5}} .
$$

Substituindo $\Phi=\mathbb{1}_{4} \otimes \Phi_{\mathfrak{s o}(4)}$ na regra de transformação para férmions (7.1.39) verificamos que, com respeito a simetria residual, os $8+8$ férmions complexos originais são decompostos da seguinte

\footnotetext{
${ }^{6} \mathrm{O}$ setor $\mathfrak{s u}(1 \mid 1)$ da $\mathcal{N}=4$ SYM é fechado, ou seja, constitui um subespaço invariante de operadores compostos sobre o qual a ação do operador de dilatação é fechada.

${ }^{7}$ Via correspondência AdS/CFT.
} 
maneira:

$$
(2,1 ; 2,1) \oplus(2,1 ; 1,2) \oplus(1,2 ; 2,1) \oplus(1,2 ; 1,2) .
$$

Por exemplo, a matriz fermiônica carregando a representação irredutível $(2,1 ; 1,2)$ é:

$$
\Theta_{(2,1 ; 1,2)}=\left(\begin{array}{cc|cc||cc|cc}
0 & 0 & 0 & 0 & 0 & 0 & \eta^{7} & \eta^{8} \\
0 & 0 & 0 & 0 & 0 & 0 & \eta^{3} & \eta^{4} \\
\hline 0 & 0 & 0 & 0 & 0 & 0 & 0 & 0 \\
0 & 0 & 0 & 0 & 0 & 0 & 0 & 0 \\
\hline \hline 0 & 0 & 0 & 0 & 0 & 0 & 0 & 0 \\
0 & 0 & 0 & 0 & 0 & 0 & 0 & 0 \\
\hline \eta_{7} & \eta_{3} & 0 & 0 & 0 & 0 & 0 & 0 \\
\eta_{8} & \eta_{4} & 0 & 0 & 0 & 0 & 0 & 0
\end{array}\right) .
$$

As equações de movimento da supercorda admitem uma redução consistente a esse setor ${ }^{8}$, que é governado por duas simetrias $\mathfrak{s u ( 2 ) , ~ u m a ~ a d v i n d a ~ d a ~ a ́ l g e b r a ~ d e ~ L o r e n t z ~ e ~ o u t r a ~ d a ~ s i m e t r i a ~} R$. Não obstante, um truncamento a um subconjunto ainda menor de férmions existe, correspondendo a imposição das condições:

$$
\eta^{4}=\eta^{7}=\eta_{4}=\eta_{7}=0 \quad \text { ou } \quad \eta^{3}=\eta^{8}=\eta_{3}=\eta_{8}=0
$$

Notamos, contudo, que não existe uma redução consistente que mantenha apenas um férmion, por exemplo $\eta^{8}$, não-nulo. O motivo advém do termo de Wess-Zumino, que contém um acoplamento cúbico de dois férmions com um único campo holomórfico $Z=Z_{3}$. Ao fixarmos $\eta^{4}=0$, quebramos a álgebra de Lorentz $\mathfrak{s u}(2)$ a um $\mathfrak{u}(1)$, sob o qual o campo $Z$ é neutro e os férmions $\eta^{3}$ e $\eta^{8}$ carregam cargas opostas. Portanto, existe um acoplamento cúbico invariante do tipo:

$$
e^{i t} Z \eta_{3} \eta_{8}
$$

com possíveis derivadas em $\tau$ e $\sigma$ atuando sobre os campos fermiônicos. Em vista desse acoplamento cúbico é inconsistente impor que $\eta_{3}=0$, dado que a equação de movimento para esse campo implicará um vínculo não-linear envolvendo $Z$ e $\eta_{8}$.

Dessa forma, consideraremos a redução que mantém apenas dois férmions complexos não-nulos, $\eta^{3}$ e $\eta^{8}$ como a definição do setor $\mathfrak{s u}(1 \mid 1)$ da teoria de supercordas. É importante frisar que tal truncamento consistente não coincide com os subsetores fechados da teoria de calibre dual, dado que possuem um número diferente de graus de liberdade ${ }^{9}$.

\footnotetext{
${ }^{8}(2,1 ; 1,2)$.

${ }^{9}$ Há dois férmions complexos $\eta^{3}$ e $\eta^{8}$ no truncamento da teoria de supercordas, enquanto apenas um, $\Psi$, no setor $\mathfrak{s u}(1 \mid 1)$ da teoria de calibre dual.
} 


\subsubsection{A Lagrangeana do Modelo de Alday-Arutyunov-Frolov}

Para obtermos a Lagrangeana do modelo reduzido além de impormos as condições discutidas anteriormente:

$$
\begin{aligned}
\text { Bósons: } & \left\{\begin{array} { r l } 
{ A d S _ { 5 } : } & { v _ { 1 } = v _ { 2 } = v _ { 3 } = v _ { 4 } = 0 } \\
{ S ^ { 5 } : } & { Z _ { 1 } = Z _ { 2 } = 0 \Rightarrow u _ { 1 } = u _ { 2 } = u _ { 3 } = u _ { 4 } = 0 ; }
\end{array} \text { Férmions: } \left\{\begin{array}{l}
\theta_{1}=\theta_{2}=\theta_{3}=\theta_{4}=\theta_{5}=\theta_{7}=\theta_{8}=0 \\
\eta_{1}=\eta_{2}=\eta_{4}=\eta_{5}=\eta_{6}=\eta_{7}=0
\end{array}\right.\right.
\end{aligned}
$$

na Lagrangeana (7.1.21), precisamos fixar a liberdade de reparametrização de (7.1.21). Isso é feito através do calibre uniforme, que consiste em identificar a coordenada $\tau$ da folha-mundo com 0 tempo global de AdS, $t$, e distribuir a única componente não-nula do momento angular em $S^{5}$, $J=J_{3}$, homogeneamente ao longo da corda:

$$
\tau=t, \quad p_{\phi}=J_{3},
$$

onde $\phi$ é uma variável angular ao longo de um dos $S^{1} \subset S^{5}$. De acordo com as nossas definições:

$$
i v_{5}+v_{6}=e^{i t}, \quad Z=i u_{5}+u_{6}=e^{i \phi} .
$$

Dado que consideramos cordas fechadas os campos são funções periódicas de $\sigma \in[0,2 \pi]$, em particular:

$$
\phi(2 \pi)-\phi(0)=2 \pi m, \quad m \in \mathbb{Z}
$$

O número de enovelamento ${ }^{10}, m$, representa o número de voltas que a corda descreve ao redor do círculo parametrizado por $\phi$.

Finalmente, substituindo em (7.1.21) as condições (7.2.3) e (7.2.4), obtemos:

$$
\begin{aligned}
\mathscr{L} & =\frac{\sqrt{\lambda}}{2} \gamma^{\tau \tau}\left[\dot{t}^{2}-\dot{\phi}^{2}+\frac{i}{2}(\dot{t}+\dot{\phi}) \zeta_{\tau}\right]+\frac{\sqrt{\lambda}}{2} \gamma^{\sigma \sigma}\left[t^{\prime 2}-\phi^{\prime 2}+\frac{i}{2}\left(t^{\prime}+\phi^{\prime}\right) \zeta_{\sigma}\right]+ \\
& +\sqrt{\lambda} \gamma^{\tau \sigma}\left[\dot{t} t^{\prime}-\dot{\phi} \phi^{\prime}+\frac{i}{4}(\dot{t}+\dot{\phi}) \zeta_{\sigma}+\frac{i}{4}\left(t^{\prime}+\phi^{\prime}\right) \zeta_{\tau}\right]+\mathscr{L}_{W z},
\end{aligned}
$$

onde denotamos as derivadas com respeito a $\tau$ e $\sigma$ por um ponto e por uma linha, respectivamente; além do mais introduzimos a seguinte notação para os férmions:

$$
\begin{aligned}
& \zeta_{\tau}=\eta_{i} \dot{\eta}^{i}+\eta^{i} \dot{\eta}_{i}, \\
& \zeta_{\sigma}=\eta_{i} \eta^{i^{\prime}}+\eta^{i} \eta_{i}^{\prime},
\end{aligned}
$$

com $i=3$, 8. A Lagrangeana de Wess-Zumino contém termos da forma $e^{i(t+\phi)}$, ausentes na parte cinética, mas que podem ser eliminados por meio da transformação:

$$
\eta_{3,8}=e^{-\frac{i}{2}(t+\phi)} \vartheta_{3,8}, \quad \eta^{3,8}=e^{\frac{i}{2}(t+\phi)} \vartheta^{3,8}
$$

\footnotetext{
${ }^{10}$ Winding number.
} 
Notamos que os férmions originais $\eta$ carregavam uma carga sob as simetrias $\mathfrak{u}(1)$ de translação em $t$ e $\phi$, contudo, as novas variáveis $\vartheta$ são neutras. Observamos também que os férmions $\vartheta$ são (anti)periódicos se $m$ for (ím)par. Levando em conta a transformação (7.2.9), a Lagrangeana (7.2.7) assume a seguinte forma:

$$
\begin{aligned}
\mathscr{L} & =\frac{\sqrt{\lambda}}{2} \gamma^{\tau \tau}\left[\dot{t}^{2}-\dot{\phi}^{2}+\frac{i}{2}(\dot{t}+\dot{\phi}) \zeta_{\tau}-\frac{1}{2}(\dot{t}+\dot{\phi})^{2} \wedge\right]+ \\
& +\frac{\sqrt{\lambda}}{2} \gamma^{\sigma \sigma}\left[t^{\prime 2}-\phi^{\prime 2}+\frac{i}{2}\left(t^{\prime}+\phi^{\prime}\right) \zeta_{\sigma}-\frac{1}{2}\left(t^{\prime}+\phi^{\prime}\right)^{2} \wedge\right]+ \\
& +\sqrt{\lambda} \gamma^{\tau \sigma}\left[\dot{t} t^{\prime}-\dot{\phi} \phi^{\prime}+\frac{i}{4}(\dot{t}+\dot{\phi}) \zeta_{\sigma}+\frac{i}{4}\left(t^{\prime}+\phi^{\prime}\right) \zeta_{\tau}-\frac{1}{2}(\dot{t}+\dot{\phi})\left(t^{\prime}+\phi^{\prime}\right) \wedge\right]+\mathscr{L}_{W Z}
\end{aligned}
$$

enquanto que o termo de Wess-Zumino:

$$
\mathscr{L}_{W Z}=\frac{\kappa}{2} \Omega_{\tau}\left(t^{\prime}+\phi^{\prime}\right)-\frac{\kappa}{2} \Omega_{\sigma}(\dot{t}+\dot{\phi})
$$

e usamos as seguintes notações:

$$
\begin{aligned}
& \zeta_{\tau}=\vartheta_{i} \dot{\vartheta}^{i}+\vartheta^{i} \dot{\vartheta}_{i}, \quad \Omega_{\tau}=\vartheta_{3} \dot{\vartheta}_{8}+\vartheta_{8} \dot{\vartheta}_{3}-\vartheta^{3} \dot{\vartheta}^{8}-\vartheta^{8} \dot{\vartheta}^{3}, \quad \Lambda=\vartheta_{i} \vartheta^{i}, \\
& \zeta_{\sigma}=\vartheta_{i} \vartheta^{i^{\prime}}+\vartheta^{i} \vartheta_{i}{ }^{\prime}, \quad \Omega_{\sigma}=\vartheta_{3} \vartheta_{8}{ }^{\prime}+\vartheta_{8} \vartheta_{3}{ }^{\prime}-\vartheta^{3} \vartheta^{8^{\prime}}-\vartheta^{8} \vartheta^{3^{\prime}} .
\end{aligned}
$$

O próximo passo consiste em identificar os verdadeiros graus de liberdade físicos, o que faremos ao construir a formulação Hamiltoniana do modelo reduzido. Denotando por $p_{t}$ e $p_{\phi}$ os momentos canonicamente conjugados a $t$ e $\phi$, obtidos de (7.2.10), rescrevemos a Lagrangeana em termos das coordenadas do espaço de fase:

$$
\begin{aligned}
\mathscr{L} & =p_{t} \dot{t}+p_{\phi} \dot{\phi}+\frac{i}{4}\left(p_{t}-p_{\phi}\right) \zeta_{\tau}+\frac{\kappa}{2}\left(t^{\prime}+\phi^{\prime}\right) \Omega_{\tau}- \\
& -\frac{1}{\gamma^{\tau \tau} \sqrt{\lambda}}\left\{\frac{1}{4}\left(p_{t}-p_{\phi}\right)\left[p_{t}(2+\Lambda)+p_{\phi}(2-\Lambda)+2 \kappa \Omega_{\sigma}\right]+\right. \\
& \left.+\frac{\lambda}{4}\left(t^{\prime}+\phi^{\prime}\right)\left[t^{\prime}(2-\Lambda)-\phi^{\prime}(2+\Lambda)+i \zeta_{\sigma}\right]\right\}+ \\
& +\frac{\gamma^{\tau \sigma}}{\gamma^{\tau \tau}}\left[p_{t} t^{\prime}+p_{\phi} \phi^{\prime}+\frac{i}{4}\left(p_{t}-p_{\phi}\right) \zeta_{\sigma}+\frac{\kappa}{2}\left(t^{\prime}+\phi^{\prime}\right) \Omega_{\sigma}\right],
\end{aligned}
$$

onde as componentes da métrica na folha-mundo entram como multiplicadores de Lagrange.

Ao impormos o calibre uniforme (7.2.4) algumas das equações de movimento que seguem de (7.2.13) transformam-se em vínculos, que devem ser resolvidos para encontrarmos os verdadeiros graus de liberdade. Notamos que não introduzimos ainda os momentos canonicamente conjugados aos férmions, visto que eles não estão diretamente envolvidos na nossa escolha de calibre e, portanto, podem ser tratados após termos resolvido todos os vínculos bosônicos.

Ao variarmos (7.2.13) com respeito a $\gamma^{\tau \sigma}$, obtemos uma equação para $\phi^{\prime}$ :

$$
\phi^{\prime}=-i \frac{p_{t}-p_{\phi}}{4 p_{\phi}+2 \kappa \Omega_{\sigma}} \zeta_{\sigma}
$$


enquanto que a variação com respeito a $\gamma^{\tau \tau}$ fornece uma equação para $p_{t}$, cujas soluções são:

$$
\begin{aligned}
& p_{t}=p_{\phi}, \\
& p_{t}=-\frac{p_{\phi}(2-\Lambda)+2 \kappa \Omega_{\sigma}}{2+\Lambda} .
\end{aligned}
$$

A variável $p_{t}$, conjugada ao tempo global de AdS, $t$, corresponde à densidade de energia espaçotemporal da corda: $p_{t}=\mathscr{H}$. Em particular, como impomos o calibre uniforme $(t=\tau)$, a carga de Noether associada às translações do tempo global deve corresponder à Hamiltoniana $H$ para os graus de liberdade físicos:

$$
H=\int_{0}^{2 \pi} \frac{d \sigma}{2 \pi} \mathscr{H} .
$$

Selecionamos a segunda solução, equação (7.2.15b), dado que ela leva ao limite bosônico correto $p_{t}=-p_{\phi}$, ou seja, $\mathscr{H}=J$. Logo, a densidade Hamiltoniana assume a seguinte forma:

$$
\mathscr{H}=\frac{J(2-\Lambda)+2 \kappa \Omega_{\sigma}}{2+\Lambda} .
$$

Notamos que a densidade Hamiltoniana (7.2.17) não contém derivadas temporais dos campos fermiônicos.

Substituindo a solução para $p_{t}(7.2 .15 \mathrm{~b})$ em (7.2.14) obtemos:

$$
\phi^{\prime}=\frac{i \zeta_{\sigma}}{2+\Lambda}
$$

Integrando sobre $\sigma$ e considerando (7.2.6), obtemos o vínculo:

$$
\nu=i \int_{0}^{2 \pi} \frac{\zeta_{\sigma}}{2+\Lambda}=m
$$

que corresponde à condição de nivelamento ${ }^{11}$ imposta aos estados físicos da teoria. O campo $\phi$ é, por sinal, não-físico, sua evolução temporal pode ser obtida ao variarmos (7.2.10) com respeito a $p_{\phi}$ :

$$
\dot{\phi}=\frac{2-\Lambda+i \zeta_{\tau}}{2+\Lambda}
$$

Claramente as equações (7.2.18) e (7.2.20) determinam o campo $\phi$ em termos das coordenadas fermiônicas. Logo, ao impormos a condição de calibre uniforme e resolvermos os vínculos, concluímos que os graus de liberdade desse setor são carregados somente por férmions.

Podemos resolver as equações de movimento para $\phi$ e $p_{t}$ para obtermos a métrica da folha-mundo:

$$
\begin{aligned}
\gamma^{\tau \tau} & =\frac{i}{2 \sqrt{\lambda}} \frac{\lambda \zeta_{\sigma}^{2}+4\left(2 J+\kappa \Omega_{\sigma}\right)^{2}}{\left(\zeta_{\tau}-4 i\right)\left(2 J+\kappa \Omega_{\sigma}\right)-\kappa \zeta_{\sigma} \Omega_{\tau}}, \\
\gamma^{\tau \sigma} & =\frac{i}{2 \sqrt{\lambda}} \frac{\lambda \zeta_{\sigma}\left(\zeta_{\tau}-4 i\right)+4 \kappa\left(2 J+\kappa \Omega_{\sigma}\right) \Omega_{\tau}}{\left(\zeta_{\tau}-4 i\right)\left(2 J+\kappa \Omega_{\sigma}\right)-\kappa \zeta_{\sigma} \Omega_{\tau}} .
\end{aligned}
$$

Finalmente, substituindo as soluções dos vínculos em (7.2.10) obtemos a Lagrangeana no calibre

\footnotetext{
${ }^{11}$ Level-matching condition.
} 
uniforme:

$$
\mathscr{L}=-J-\frac{i J \zeta_{\tau}+2 \kappa \Omega_{\sigma}-2 J \Lambda}{2+\Lambda}-\frac{i \kappa}{2} \frac{\zeta_{\tau} \Omega_{\sigma}-\zeta_{\sigma} \Omega_{\tau}}{2+\Lambda}
$$

\subsubsection{A Hamiltoniana e a Estrutura de Poisson do Modelo de Alday-Arutyunov- Frolov}

A Lagrangeana (7.2.22) define uma teoria invariante de Lorentz para um férmion de Dirac em uma folha-mundo bidimensional. Para tornarmos tal fato explícito, introduzimos um espinor (de Dirac) complexo de duas componentes $\psi$, ao combinarmos os férmions $\vartheta_{3}$ e $\vartheta_{8}$ da seguinte forma:

$$
\psi=\left(\begin{array}{l}
\vartheta_{3} \\
\vartheta^{8}
\end{array}\right) .
$$

Utilizando as matrizes de Dirac $\rho^{0}$ e $\rho^{1}$ (B.3.2) definimos o espinor de Dirac conjugado $\bar{\psi}=\psi^{\dagger} \rho^{0}$ e rescrevemos as quantidades fermiônicas:

$$
\begin{array}{ll}
\zeta_{\tau}=\bar{\psi} \rho^{0} \partial_{0} \psi-\partial_{0} \bar{\psi} \rho^{0} \psi, & \Omega_{\tau}=-i\left(\bar{\psi} \rho^{1} \partial_{0} \psi-\partial_{0} \bar{\psi} \rho^{1} \psi\right), \quad \Lambda=\bar{\psi} \psi \\
\zeta_{\sigma}=\bar{\psi} \rho^{0} \partial_{1} \psi-\partial_{1} \bar{\psi} \rho^{0} \psi, & \Omega_{\sigma}=-i\left(\bar{\psi} \rho^{1} \partial_{1} \psi-\partial_{1} \bar{\psi} \rho^{1} \psi\right),
\end{array}
$$

com $\tau=x^{0}$ e $\sigma=x^{1}$. Dessa forma a Lagrangeana (7.2.22) assume a seguinte forma:

$$
\begin{aligned}
\mathscr{L} & =-J-\frac{i J}{2}\left(\bar{\psi} \rho^{0} \partial_{0} \psi-\partial_{0} \bar{\psi} \rho^{0} \psi\right)+i \kappa\left(\bar{\psi} \rho^{1} \partial_{1} \psi-\partial_{1} \bar{\psi} \rho^{1} \psi\right)+J \bar{\psi} \psi+ \\
& +\frac{i J}{4}\left(\bar{\psi} \rho^{0} \partial_{0} \psi-\partial_{0} \bar{\psi} \rho^{0} \psi\right) \bar{\psi} \psi-\frac{i \kappa}{2}\left(\bar{\psi} \rho^{1} \partial_{1} \psi-\partial_{1} \bar{\psi} \rho^{1} \psi\right) \bar{\psi} \psi-\frac{J}{2}(\bar{\psi} \psi)^{2}+ \\
& +\frac{\kappa}{2} \epsilon^{\alpha \beta}\left(\bar{\psi} \partial_{\alpha} \psi \bar{\psi} \rho^{5} \partial_{\beta} \psi-\partial_{\alpha} \bar{\psi} \psi \partial_{\beta} \bar{\psi} \rho^{5} \psi\right)+\frac{\kappa}{8} \epsilon^{\alpha \beta}(\bar{\psi} \psi)^{2} \partial_{\alpha} \bar{\psi} \rho^{5} \partial_{\beta} \psi
\end{aligned}
$$

onde $\rho^{5}=\rho^{0} \rho^{1}$ e o tensor de Levi-Civita é tal que $\epsilon^{01}=\epsilon_{10}=1$. Através da seguinte transformação:

$$
\psi \rightarrow \psi+\frac{1}{4} \psi(\bar{\psi} \psi), \quad \bar{\psi} \rightarrow \bar{\psi}+\frac{1}{4} \bar{\psi}(\bar{\psi} \psi)
$$

podemos simplificar drasticamente a Lagrangeana (7.2.25)

$$
\begin{aligned}
\mathscr{L} & =-J-\frac{i J}{2}\left(\bar{\psi} \rho^{0} \partial_{0} \psi-\partial_{0} \bar{\psi} \rho^{0} \psi\right)+i \kappa\left(\bar{\psi} \rho^{1} \partial_{1} \psi-\partial_{1} \bar{\psi} \rho^{1} \psi\right)+J \bar{\psi} \psi+ \\
& +\frac{\kappa}{2} \epsilon^{\alpha \beta}\left(\bar{\psi} \partial_{\alpha} \psi \bar{\psi} \rho^{5} \partial_{\beta} \psi-\partial_{\alpha} \bar{\psi} \psi \partial_{\beta} \bar{\psi} \rho^{5} \psi\right)-\frac{\kappa}{8} \epsilon^{\alpha \beta}(\bar{\psi} \psi)^{2} \partial_{\alpha} \bar{\psi} \rho^{5} \partial_{\beta} \psi
\end{aligned}
$$

Finalmente, ao fazermos a seguinte mudança na coordenada $\sigma$ da folha-mundo:

$$
\sigma \rightarrow-\frac{2 \kappa}{J} \sigma
$$


podemos agrupar o termo cinético em uma forma invariante de Lorentz:

$$
\begin{aligned}
\mathscr{L} & =-J\left[1+\frac{i}{2}\left(\bar{\psi} \rho^{\alpha} \partial_{\alpha} \psi-\partial_{\alpha} \bar{\psi} \rho^{\alpha} \psi\right)-\bar{\psi} \psi+\right. \\
& \left.+\frac{1}{4} \epsilon^{\alpha \beta}\left(\bar{\psi} \partial_{\alpha} \psi \bar{\psi} \rho^{5} \partial_{\beta} \psi-\partial_{\alpha} \bar{\psi} \psi \partial_{\beta} \bar{\psi} \rho^{5} \psi\right)-\frac{1}{16} \epsilon^{\alpha \beta}(\bar{\psi} \psi)^{2} \partial_{\alpha} \bar{\psi} \rho^{5} \partial_{\beta} \psi\right] .
\end{aligned}
$$

Claramente, a Lagrangeana (7.2.29) define uma teoria de um férmion de Dirac interagente em duas dimensões. É interessante notar que os férmions espaçotemporais da supercorda de Green-Schwarz original são combinados em espinores bidimensionais na folha-mundo, de uma forma similar à relação entre à formulações no cone-de-luz da supercorda de NSR e GS.

Nesse ponto, precisamos enfatizar uma diferença significativa presente no último termo de interação da Lagrangeana (7.2.29) quando comparada à Lagrangeana (5.5) do trabalho original [43]. O fator adicional de $\frac{1}{2}$ que aparece em nossa Lagrangeana é crucial, como veremos no próximo capítulo, para a integrabilidade quântica do modelo, e não foi considerado em [43].

O próximo passo consiste em encontrar a Hamiltoniana e a estrutura de Poisson do modelo de AAF. Para isso é conveniente que consideremos a Lagrangeana (7.2.27), dado que ela naturalmente apresenta a seguinte estrutura $\mathscr{L}=\mathscr{L}_{\text {cinética }}-\mathscr{H}$, de onde facilmente lemos:

$$
\mathscr{H}=J-i \kappa\left(\bar{\psi} \rho^{1} \partial_{1} \psi-\partial_{1} \bar{\psi} \rho^{1} \psi\right)-J \bar{\psi} \psi
$$

que corresponde a densidade Hamiltoniana para um férmion de Dirac bidimensional massivo. O termo cinético contém as derivadas temporais e define a estrutura de Poisson do modelo:

$$
\begin{aligned}
\left\{\psi_{i}(\sigma), \psi_{j}\left(\sigma^{\prime}\right)\right\} & =-\frac{i \kappa}{2 J^{2}}\left(\psi_{k} \psi_{l}\right)^{\prime} \delta_{i j} \epsilon_{k l} \delta\left(\sigma-\sigma^{\prime}\right)+\ldots \\
\left\{\psi_{i}(\sigma), \psi_{j}^{*}\left(\sigma^{\prime}\right)\right\} & =\left[\frac{i \delta_{i j}}{J}+\frac{i \kappa}{J^{2}}\left(\epsilon_{i k} \delta_{j l} \psi_{l}^{\prime} \psi_{k}^{*}+\epsilon_{j k} \delta_{i l} \psi_{k} \psi_{l}^{* \prime}\right)\right] \delta\left(\sigma-\sigma^{\prime}\right)+\ldots
\end{aligned}
$$

onde $\psi_{i}, i=1,2$ são as componentes do espinor de Dirac (7.2.23) e $\epsilon_{12}=1$. Os parênteses de Poisson possuem uma expressão altamente não-trivial, estendendo-se até a oitava ordem nos férmions $\psi$ e suas derivadas espaciais $\psi^{\prime}$, a expressão completa pode ser encontrada no apêndice $E$ de [43]. Notamos, contudo, que a estrutura de Poisson completa ainda é ultralocal e pode ser levada à forma canônica através de uma redefinição não-linear das variáveis fermiônicas, no entanto, a forma da Hamiltoniana nesse caso, torna-se notadamente mais complicada.

\subsubsection{A Conexão de Lax}

Nesta seção testamos a consistência do truncamento da teoria de supercordas em $A d S_{5} \times S^{5}$ ao setor $\mathfrak{s u}(1 \mid 1)$, ao verificarmos que a conexão de Lax para o modelo completo em $\mathfrak{p s u}(2,2 \mid 4)$ pode ser consistentemente reduzida para uma conexão de Lax codificando as equações de movimento para os campos físicos no setor $\mathfrak{s u}(1 \mid 1)$. Considere, pois, a conexão de Lax bidimensional [32]:

$$
\mathcal{L}_{\alpha}=I_{0} A_{\alpha}^{(0)}+I_{1} A_{\alpha}^{(2)}+I_{2} \gamma_{\alpha \beta} \epsilon^{\beta \rho} A_{\rho}^{(2)}+I_{3} Q_{\alpha}^{+}+I_{4} Q_{\alpha}^{-},
$$


onde $l_{i}$ são constantes e $Q^{ \pm}=A^{(1)} \pm A^{(3)}$. A conexão $\mathcal{L}$ deve satisfazer a condição de curvatura nula:

$$
\partial_{\alpha} \mathcal{L}_{\beta}-\partial_{\beta} \mathcal{L}_{\alpha}-\left[\mathcal{L}_{\alpha}, \mathcal{L}_{\beta}\right]=0
$$

como conseqüência das equações de movimento e da condição de curvatura nula de $A_{\alpha}$. A condição (7.2.33) também determina as constantes $l_{i}$ :

$$
l_{0}=1, \quad l_{1}=\frac{1+x^{2}}{1-x^{2}}, \quad l_{2}=s_{1} \frac{2 x}{1-x^{2}}, \quad l_{3}=\frac{s_{2}}{\sqrt{1-x^{2}}}, \quad l_{4}=s_{3} \frac{x}{\sqrt{1-x^{2}}}
$$

onde $x$ é o parâmetro espectral, $s_{2}^{2}=s_{3}^{2}=1$ e $s_{1} \pm s_{2} s_{3}=0$ para $\kappa= \pm \frac{\sqrt{\lambda}}{2}$. Por simplicidade fixamos $\kappa=\frac{\sqrt{\lambda}}{2}$.

Considerando então a redução ao setor $\mathfrak{s u}(1 \mid 1)$, na qual mantemos apenas o férmion $\psi$ não-nulo e expressamos a métrica da folha-mundo $\gamma^{\alpha \beta}$ e os campos não-físicos $t$ e $\phi$ em termos de $\psi$ por meio das condições de calibre e dos vínculos. Encontramos as seguintes correntes bosônicas para o modelo truncado ${ }^{12}$ :

$$
\begin{aligned}
& A_{\sigma}^{(0)}=\frac{1}{4}(1+\bar{\psi} \psi)\left(\bar{\psi} \psi^{\prime}-\bar{\psi}^{\prime} \psi\right) \square, \\
& A_{\tau}^{(0)}=\left[\frac{1}{4}(1+\bar{\psi} \psi)(\bar{\psi} \dot{\psi}-\dot{\bar{\psi}} \psi)+\frac{i}{2} \bar{\psi} \rho^{0} \psi\right] \square, \\
& A_{\sigma}^{(2)}=\frac{1}{8} \zeta_{\sigma} \rrbracket, \\
& A_{\tau}^{(2)}=\frac{1}{8}\left(\zeta_{\tau}+2 i \bar{\psi} \psi-4 i\right) \rrbracket,
\end{aligned}
$$

onde $\mathbb{V}=\operatorname{diag}(1,-1,0,0 ; 0,0,-1,1)$ e $\mathbb{J}=\operatorname{diag}(1,1,0,0 ; 0,0,-1,-1)$. As matrizes ímpares $Q_{\alpha}^{ \pm}$são antidiagonais e podem ser escritas como:

$$
Q_{\alpha}^{+}=\left[A_{\alpha}^{(2)}, \Theta\right]-\partial_{\alpha} \Theta, \quad Q_{\alpha}^{-}=\left[A_{\alpha}^{(2)}, \hat{\Theta}\right]+\partial_{\alpha} \hat{\Theta}
$$

com

$$
\begin{aligned}
& \Theta=\left(1+\frac{1}{4} \bar{\psi} \psi\right)\left(\begin{array}{cccc}
0 & 0 & 0 & \psi_{2} \\
0 & 0 & \psi_{1}^{*} & 0 \\
0 & \psi_{1} & 0 & 0 \\
\psi_{2}^{*} & 0 & 0 & 0
\end{array}\right), \\
& \hat{\Theta}=i\left(1+\frac{1}{4} \bar{\psi} \psi\right)\left(\begin{array}{cccc}
0 & 0 & 0 & -\psi_{1} \\
0 & 0 & -\psi_{2}^{*} & 0 \\
0 & \psi_{2} & 0 & 0 \\
\psi_{1}^{*} & 0 & 0 & 0
\end{array}\right) .
\end{aligned}
$$

\footnotetext{
${ }^{12}$ Usamos as mesmas variáveis que na Lagrangeana (7.2.27).
} 
Já o termo proporcional à métrica da folha-mundo assume a seguinte forma:

$$
\begin{aligned}
& \gamma_{\tau \beta} \epsilon^{\beta \rho} A_{\rho}^{(2)}=\frac{\sqrt{\lambda}}{8 J} \zeta_{\sigma} \rrbracket, \\
& \gamma_{\sigma \beta} \epsilon^{\beta \rho} A_{\rho}^{(2)}=\frac{i}{4 \sqrt{\lambda}}(J+\mathscr{H}) \downarrow .
\end{aligned}
$$

Finalmente, ao substituirmos todas as componentes devidamente reduzidas na conexão de Lax, verificamos que a condição de curvatura nula (7.2.33) é satisfeita como uma consequiência das equações de movimento para os férmions obtidas a partir da Lagrangeana (7.2.27). Dessa forma não só se mostrou a consistência do truncamento ao setor $\mathfrak{s u}(1 \mid 1)$, como se demonstrou que o modelo de AAF, definido pela Lagrangeana (7.2.27), é classicamente integrável. 



\section{Capítulo 8}

\section{Fatorabilidade da Matriz S}

"I wish it need not have happened in my time," said Frodo. "So do I," said Gandalf, "and so do all who live to see such times. But that is not for them to decide. All we have to decide is what to do with the time that is given us."

J.R.R.Tolkien, The Lord of the Rings - The Fellowship of the Ring

O modelo de Alday-Arutyunov-frolov (AAF) constitui um exemplo especialmente interessante no contexto dos modelos classicamente integráveis por ser o primeiro modelo puramente fermiônico nãotrivial, dotado de uma estrutura altamente não-linear e singular quando comparado com o exemplo usual, o modelo de Thirring. Essas singularidades advêm da presença de derivadas na Hamiltoniana de interação, resultando, do ponto de vista da mecânica quântica, em cargas conservadas e, em particular, numa Hamiltoniana, altamente singulares, que dificultam sua análise por meio dos métodos usuais. O modelo de Landau-Lifshitz $[37,44,107]$ é um outro exemplo representativo dessas dificuldades, por meio do qual foi entendido que para a correta aplicação do método do espalhamento inverso quântico é necessário um estudo detalhado dessas singularidades e dos operadores quânticos envolvidos, demandando também a concomitante reconstrução do espaço de Hilbert físico.

Conforme discutido no capítulo anterior, o modelo de AAF herda a integrabilidade clássica da teoria de cordas em $A d S_{5} \times S^{5}$, uma vez que o par de Lax do modelo sigma de cordas completo admite a mesma redução consistente ao setor $\mathfrak{s u}(1 \mid 1)$. Posteriormente, conjecturou-se a integrabilidade quântica do modelo de AAF em [35], onde as equações de Bethe foram deduzidas a partir do conhecimento da matriz $S$ para duas partículas e da hipótese de espalhamento fatorável. Nesse caso o modelo de AAF foi tratado como uma teoria de campos quântica em duas dimensões e sua matriz $S$ obtida por métodos perturbativos.

Apesar de o método do espalhamento inverso ser a única técnica confiável para se contemplar todos os efeitos não-perturbativos de um modelo integrável, ele ainda não foi desenvolvido para o modelo de AAF, em virtude de sua natureza altamente singular e não-linear. Dessa forma, a abordagem perturbativa constitui a única forma disponível de se estudar a integrabilidade quântica e de se obter as equações de Bethe. É claro, no entanto, que os métodos perturbativos apresentam algumas deficiências e, em particular, informações sobre o regime não-perturbativo podem ser perdidas. Não obstante, é bem sabido que isso não acontece na maioria dos casos, e os resultados oriundos 
de cálculos perturbativos concordam com os obtidos através do método do espalhamento inverso quântico.

Embora exista uma formulação alternativa do modelo de AAF [43], na qual a estrutura de Poisson é trivializada por meio de uma redefinição dos campos, mesmo que ao preço de sua Hamiltoniana se tornar extremamente complicada, ambas as abordagens são assoladas pelos problemas usuais encontrados na quantização de modelos integráveis contínuos. Essas dificuldades refletem a natureza singular do produto de operadores definidos em um mesmo ponto e podem ser resolvidas, em princípio, por meio da discretização do modelo. Contudo, essas técnicas, quando aplicáveis, levam a resultados significativamente complicados.

Uma outra possibilidade consiste em encontrar uma escolha de calibre ${ }^{1}$ que linearize as equações de movimento. De fato, se o modelo de cordas truncado ao setor $\mathfrak{s u}(1 \mid 1)$ tiver a sua invariância de calibre fixada por meio do calibre do cone-de-luz uniforme [116], ele se reduz à teoria para férmions massivos livres em duas dimensões. Assim, a quantização é trivial e o espectro pode ser facilmente obtido. Todavia, não é claro se a mudança de calibre leva a modelos quanticamente equivalentes, uma vez que a redução da teoria de cordas ao setor $\mathfrak{s u}(1 \mid 1)$ quebra a invariância conforme, necessária para a equivalência quântica de dois calibres distintos. Conseqüentemente, é de fundamental importância que se considere a quantização do modelo de AAF no calibre uniforme, e se compare o espectro dessa forma obtido com o advindo da ação livre.

Nesse capítulo, examinamos a integrabilidade quântica do modelo de AAF no calibre uniforme ao analisarmos a fatorabilidade de sua matriz $S$, seguindo a exposição original [42]. Utilizamos a mesma abordagem empregada para o tratamento do modelo de Landau-Lifshitz no capítulo 5 e consideramos o espalhamento de três partículas no contexto de uma teoria quântica de campos. É importante salientar que uma condição necessária para um espalhamento fatorável é a ausência de interações genuínas envolvendo três ou mais partículas. A princípio, esse não é o caso para o modelo de AAF, dado que ele contém explicitamente um vértice de interação entre três corpos. De fato, mostramos que mesmo em primeira ordem não-trivial, a fatorabilidade da matriz $S$ só pode ser constatada se contribuições de ordens mais altas foram devidamente levadas em consideração.

Notamos que para a nossa abordagem, é conveniente atribuir dimensões canônicas de massa aos campos, originalmente adimensionais, do modelo de AAF. Dessa forma, somos levados naturalmente à introdução de duas constantes de acoplamento, uma para cada vértice de interação. Ao considerarmos tais constantes de acoplamento independentes, analisamos um modelo mais geral e encontramos uma condição necessária para a sua integrabilidade quântica. Essa condição efetivamente reduz o número de constantes de acoplamento para um, em perfeita concordância com o modelo de AAF.

\subsection{O Modelo de AAF como uma Teoria Quântica de Campos}

Devido a forma altamente não-trivial dos parênteses de Poisson (7.2.31), que envolve termos de até oitava ordem nos férmions e suas derivadas espaciais, não é uma tarefa simples quantizar a teoria definida pela Lagrangeana (7.2.27) diretamente através do método do espalhamento inverso quântico. No entanto, como estamos interessados em examinar a integrabilidade quântica do modelo

\footnotetext{
${ }^{1}$ Lembre-se que para deduzirmos o modelo de AAF no capítulo 7 precisamos impor o calibre uniforme (7.2.4).
} 
de AAF, podemos considerá-lo como uma teoria quântica de campos e analisar a fatorabilidade de sua matriz $S$. Nessa seção, introduzimos todo o ferramental necessário para o cômputo das matrizes $S$ para o espalhamento de duas e três partículas.

Seja a ação do modelo de AAF definida a partir da Lagrangeana (7.2.27):

$$
S=\int d \tau \int_{0}^{2 \pi} \frac{d \sigma}{2 \pi} \mathscr{L}_{(7.2 .27)}
$$

e considere a transformação de coordenadas (7.2.28),

$$
S=\frac{\kappa}{\pi J} \int d \tau \int_{-\frac{\pi J}{\kappa}}^{0} d \sigma \mathscr{L}_{(7.2 .29)}
$$

Da forma que está definido na Lagrangeana (7.2.29), o termo cinético possui um sinal negativo global em comparação com a convenção usual. Existem duas forma equivalentes de se desenvolver a teoria perturbativa nesse caso. Uma delas consiste em lidar diretamente com a convenção de sinais de (7.2.29), mas nesse caso é necessário maior cuidado com a expansão em modos, pois a energia é negativa definida. Em outras palavras, os operadores de partículas e antipartículas estão trocados com respeito a convenção usual, adotada nos livros-texto. Alternativamente, é possível modificar a Lagrangeana de modo a tornar o termo cinético, bem como a energia, positivos definidos, ao se considerar a transformação $\mathscr{L} \rightarrow(-1) \mathscr{L}$. Classicamente, essa transformação não altera a dinâmica do modelo. Contudo, quanticamente a situação é mais complicada, primeiramente porque o propagador adquire um sinal negativo global adicional e, em segundo lugar, os pólos são trocados, assim como os estados correspondendo a energias positivas e negativas, em comparação com a primeira abordagem. Conforme afirmamos, ambas as abordagens são equivalentes, mas não podem ser misturadas, e aparentemente, esse é o caso de [35], levando, pois, à obtenção da matriz $S$ inversa. Preferimos proceder da segunda maneira, fazendo com que o termo cinético seja positivo ao multiplicarmos a Lagrangeana clássica por $(-1)$.

Adicionalmente, para tornar o limites de integração positivos, redefinimos a variável de integração:

$$
\sigma \rightarrow \sigma+\frac{2 \pi J}{\sqrt{\lambda}}
$$

Podemos também desprezar o termo constante presente em (7.2.29), posto que sua remoção significa apenas uma translação da origem da escala de energia. Além do mais, fixamos ${ }^{2} \kappa=\frac{\sqrt{\lambda}}{2}$ e mudamos a base das matrizes de Dirac através da transformação de similaridade (B.3.3),

$$
\begin{aligned}
S & =\frac{\sqrt{\lambda}}{2 \pi} \int d \tau \int_{0}^{\frac{2 \pi J}{\sqrt{\lambda}}} d \sigma\left[\frac{i}{2}\left(\bar{\psi} \gamma^{\alpha} \partial_{\alpha} \psi-\partial_{\alpha} \bar{\psi} \gamma^{\alpha} \psi\right)-\bar{\psi} \psi+\right. \\
& \left.+\frac{1}{4} \epsilon^{\alpha \beta}\left(\bar{\psi} \partial_{\alpha} \psi \bar{\psi} \gamma^{3} \partial_{\beta} \psi-\partial_{\alpha} \bar{\psi} \psi \partial_{\beta} \bar{\psi} \gamma^{3} \psi\right)-\frac{1}{16} \epsilon^{\alpha \beta}(\bar{\psi} \psi)^{2} \partial_{\alpha} \bar{\psi} \gamma^{3} \partial_{\beta} \psi\right]
\end{aligned}
$$

Notando que,

$$
\frac{i}{2}\left(\bar{\psi} \gamma^{\alpha} \partial_{\alpha} \psi-\partial_{\alpha} \bar{\psi} \gamma^{\alpha} \psi\right)=i \bar{\psi} \not \partial \psi-\frac{i}{2} \partial_{\alpha}\left(\bar{\psi} \gamma^{\alpha} \psi\right)
$$

\footnotetext{
${ }^{2}$ Equivalentemente, poderíamos, em vez de multiplicar a Lagrangeana por $(-1)$, escolher $\kappa=-\frac{\sqrt{\lambda}}{2}$.
} 
podemos simplificar a densidade de Lagrangeana presente em (8.1.4) ao desprezarmos tal derivada total.

Até o dado momento, trabalhamos apenas com as quantidades adimensionais obtidas diretamente da teoria de cordas, entretanto, é conveniente para nossa análise atribuir dimensões canônicas de massa aos campos. Para isso consideramos a seguinte transformação de coordenadas:

$$
x^{\alpha} \rightarrow y^{\alpha}=\frac{\sqrt{\lambda}}{2 \pi} x^{\alpha}, \quad \text { com } \quad x^{0}=\tau, \quad x^{1}=\sigma,
$$

sob a qual a ação (8.1.4) assume a forma:

$$
\begin{aligned}
S & =\int d y^{0} \int_{0}^{\lrcorner} d y^{1}\left[i \bar{\psi} \not \partial-\frac{2 \pi}{\sqrt{\lambda}} \bar{\psi} \psi+\frac{\sqrt{\lambda}}{8 \pi} \epsilon^{\alpha \beta}\left(\bar{\psi} \partial_{\alpha} \psi \bar{\psi} \gamma^{3} \partial_{\beta} \psi-\partial_{\alpha} \bar{\psi} \psi \partial_{\beta} \bar{\psi} \gamma^{3} \psi\right)-\right. \\
& \left.-\frac{\sqrt{\lambda}}{32 \pi} \epsilon^{\alpha \beta}(\bar{\psi} \psi)^{2} \partial_{\alpha} \bar{\psi} \gamma^{3} \partial_{\beta} \psi\right],
\end{aligned}
$$

enquanto que a dependência dos campos fermiônicos nas coordenadas adimensionais originais da folha-mundo é simplesmente $\psi=\psi\left(\frac{\sqrt{\lambda}}{2 \pi} \tau,-\frac{\lambda}{2 \pi J} \sigma+J\right)$. Identificando o parâmetro na frente de $\bar{\psi} \psi$ como a massa da teoria:

$$
m=\frac{2 \pi}{\sqrt{\lambda}}
$$

obtemos:

$$
\begin{aligned}
S & =\int d y^{0} \int_{0}^{J} d y^{1}\left[i \bar{\psi} \not \partial \psi-m \bar{\psi} \psi+\frac{\epsilon^{\alpha \beta}}{4 m}\left(\bar{\psi} \partial_{\alpha} \psi \bar{\psi} \gamma^{3} \partial_{\beta} \psi-\partial_{\alpha} \bar{\psi} \psi \partial_{\beta} \bar{\psi} \gamma^{3} \psi\right)-\right. \\
& \left.-\frac{\epsilon^{\alpha \beta}}{16 m}(\bar{\psi} \psi)^{2} \partial_{\alpha} \bar{\psi} \gamma^{3} \partial_{\beta} \psi\right] .
\end{aligned}
$$

Consideramos então, as dimensões dos diversos objetos presentes em (8.1.9). Primeiramente, notamos que $\lambda$ é a constante de t'Hooft e, por conseguinte,

$$
\sqrt{\lambda}=\frac{R^{2}}{\alpha^{\prime}} \Rightarrow \lambda \sim \frac{1}{l_{s}^{2}}
$$

onde $I_{s}$ é o comprimento da corda, de forma que com a transformação (8.1.6), a dimensão de massa canônica atribuída às coordenadas é: $[y]=-1$. Assim, ao demandarmos que a ação (8.1.9) seja adimensional, obtemos a partir do termo cinético e de massa que: $[\psi]=\frac{1}{2}$ e $[m]=1$. Claramente,

$$
[m]=1 \Rightarrow\left[\frac{2 \pi}{\sqrt{\lambda}}\right]=1 \Rightarrow[\lambda]=-2,
$$

mostrando que, ao menos no que tange aos termos cinéticos e de massa, a atribuição de dimensões de massa que fizemos é consistente.

No entanto, ao analisarmos as Lagrangeanas de interação, verificamos que tais objetos possuem dimensões extras:

- Interação de Duas Partículas: $\mathscr{L}_{\text {lnt }}^{(2)} \sim m^{-1}(\bar{\psi} \partial \psi)^{2} \Rightarrow\left[\mathscr{L}_{\text {lnt }}^{(2)}\right]=3>2$. Logo, há uma dimensão de massa adicional! 

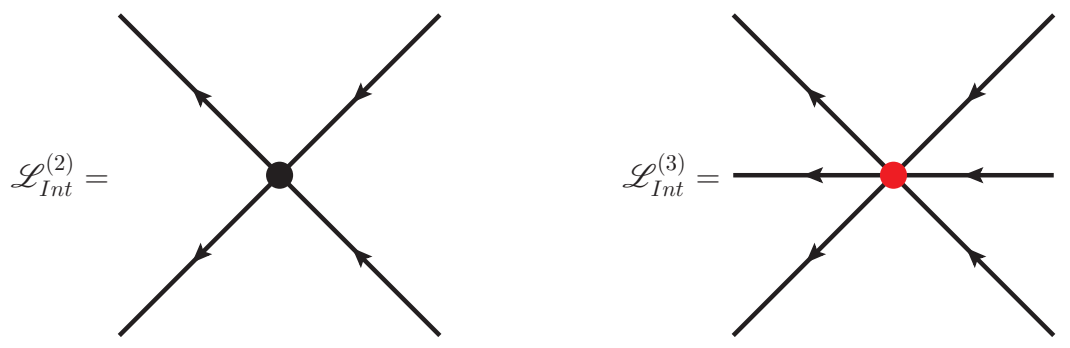

Figura 8.1: Vértices de interação na representação dos momentos.

- Interação de Três Partículas: $\mathscr{L}_{\text {lnt }}^{(3)} \sim m^{-1}(\bar{\psi} \psi)^{2} \partial \bar{\psi} \partial \psi \Rightarrow\left[\mathscr{L}_{\text {Int }}^{(3)}\right]=4>2$. Logo, há duas dimensões de massa adicionais!

Uma possível solução dessa inconsistência corresponde à introdução de constantes de acoplamento nos vértices de duas e três partículas, $g_{2}$ e $g_{3}$, respectivamente, de forma que as Lagrangeanas de interação tenham as dimensões de massa apropriadas. Naturalmente, $\left[g_{2}\right]=-1$ e $\left[g_{3}\right]=-2$. Conseqüentemente, a ação (8.1.9) adquire a seguinte forma:

$$
\begin{aligned}
S & =\int d y^{0} \int_{0}^{J} d y^{1}\left[i \bar{\psi} \not \partial \psi-m \bar{\psi} \psi+\frac{g_{2}}{4 m} \epsilon^{\alpha \beta}\left(\bar{\psi} \partial_{\alpha} \psi \bar{\psi} \gamma^{3} \partial_{\beta} \psi-\partial_{\alpha} \bar{\psi} \psi \partial_{\beta} \bar{\psi} \gamma^{3} \psi\right)-\right. \\
& \left.-\frac{g_{3}}{16 m} \epsilon^{\alpha \beta}(\bar{\psi} \psi)^{2} \partial_{\alpha} \bar{\psi} \gamma^{3} \partial_{\beta} \psi\right] .
\end{aligned}
$$

É essencial notar que nesse estágio as duas constantes de acoplamento não são independentes, posto que advêm diretamente do modelo de AAF que contém apenas um parâmetro, $\lambda$. Não obstante, é interessante relaxar essa condição e, dessa forma, considerar um modelo mais geral no qual as duas constantes de acoplamento são independentes. Essa generalização é deveras conveniente para a análise perturbativa, pois permite que mantenhamos controle sobre a contribuição de cada vértice. Para futura conveniência, introduzimos a seguinte notação para os vértices de interação, vide figura 8.1:

$$
\begin{aligned}
\mathscr{L}_{\text {Livre }} & =i \bar{\psi} \not \psi-m \bar{\psi} \psi, \\
\mathscr{L}_{\text {Int }}^{(2)} & =\mathbb{G}_{a c, b d}^{\alpha \beta}\left(\bar{\psi}^{a} \partial_{\alpha} \psi^{b} \bar{\psi}^{c} \partial_{\beta} \psi^{d}-\partial_{\alpha} \bar{\psi}^{a} \psi^{b} \partial_{\beta} \bar{\psi}^{c} \psi^{d}\right), \\
\mathscr{L}_{\text {Int }}^{(3)} & =\mathbb{H}_{a c e, b d f}^{\alpha \beta} \bar{\psi}^{a} \psi^{b} \bar{\psi}^{c} \psi^{d} \partial_{\alpha} \bar{\psi}^{e} \partial_{\beta} \psi^{f},
\end{aligned}
$$

onde definimos as matrizes

$$
\begin{aligned}
\mathbb{G}_{a c, b d}^{\alpha \beta} & :=\frac{g_{2}}{8 m} \epsilon^{\alpha \beta} \mathbb{P}, \quad \text { com } \quad \mathbb{P}:=\mathbb{1}_{2} \otimes \gamma^{3}-\gamma^{3} \otimes \mathbb{1}_{2}, \\
\mathbb{H}_{a c e, b d f}^{\alpha \beta} & :=-\frac{g_{3}}{16 m} \epsilon^{\alpha \beta} \mathbb{Q}_{a c e, b d f} \quad \text { com } \quad \mathbb{Q}:=\mathbb{1}_{2} \otimes \mathbb{1}_{2} \otimes \gamma^{3} .
\end{aligned}
$$

Portanto,

$$
S=\int d y^{0} \int_{0}^{J} d y^{1}\left(\mathscr{L}_{\text {Livre }}+\mathscr{L}_{\text {lnt }}^{(2)}+\mathscr{L}_{\text {lnt }}^{(3)}\right)
$$


O modelo generalizado de $A A F$, cuja dinâmica é descrita pela ação (8.1.13), está definido em um cilindro: $-\infty<y^{0}<+\infty$ e $0 \leq y^{1} \leq J$, de sorte que seus campos fermiônicos $\psi(y)$ devem satisfazer condições de contorno periódicas. Tais propriedades são um mero reflexo da teoria de supercordas do tipo IIB, da qual o modelo de AAF surge como um truncamento consistente ao setor $\mathfrak{s u}(1 \mid 1)$ no calibre uniforme. Em particular, uma configuração física correspondente a uma corda fechada deve satisfazer a condição de nivelamento, que é equivalente a anulação do momento total na folha-mundo. Conforme discutido anteriormente, a quantização exata do modelo de AAF é uma tarefa altamente não-trivial, e o fato de o modelo estar definido em um cilindro só a torna ainda mais complicada. A maneira usual de se lidar com esse problema no contexto da teoria de cordas consiste em considerar o limite de descompactificação [13], onde tomamos o limite do raio $J$ desse cilindro indo para o infinito, enquanto mantemos a tensão da corda fixa. Dessa forma passamos de uma teoria definida em um cilindro para uma definida no plano, onde a noção de estados assintóticos está bem definida, pois as condições de contorno periódicas são substituídas pela condição de que os campos devem se anular identicamente no infinito, e podemos aplicar trivialmente todo o arcabouço ferramental da teoria de campos.

No que se segue estudamos o modelo generalizado de AAF no limite de descompactificação e examinamos a sua integrabilidade quântica. De uma forma mais concreta, consideramos a seguinte transformação de coordenadas ${ }^{3}$ :

$$
y^{1} \rightarrow y^{1}-\frac{J}{2}
$$

de forma que a ação (8.1.13) pode ser escrita como:

$$
S=\int d y^{0} \int_{-\frac{J}{2}}^{\frac{J}{2}} d y^{1}\left(\mathscr{L}_{\text {Livre }}+\mathscr{L}_{\text {lnt }}^{(2)}+\mathscr{L}_{\text {lnt }}^{(3)}\right) \stackrel{\jmath \rightarrow \infty}{\longrightarrow} \int d^{2} y\left(\mathscr{L}_{\text {Livre }}+\mathscr{L}_{\text {lnt }}^{(2)}+\mathscr{L}_{\text {lnt }}^{(3)}\right) .
$$

\subsubsection{Quantização do Campo de Dirac}

O próximo passo consiste em quantizar a teoria livre descrita por (8.1.11a), ou seja, o campo de Dirac massivo, cujas equações de movimento são as equações de Dirac e sua conjugada em duas dimensões:

$$
i \not \partial \psi-m \psi=0 \quad \text { e } \quad i \partial_{\alpha} \bar{\psi} \gamma^{\alpha}+m \bar{\psi}=0 .
$$

Os momentos canonicamente conjugados ${ }^{4}$ às variáveis $\psi$ e $\bar{\psi}$ :

$$
\Pi_{\psi}=\frac{\partial \mathscr{L}_{\text {Livre }}}{\partial \partial_{0} \psi}=-i \psi^{\dagger}, \quad \Pi_{\bar{\psi}}=\frac{\partial \mathscr{L}_{\text {Livre }}}{\partial \partial_{0} \bar{\psi}}=0,
$$

claramente indicam que lidamos com uma teoria vinculada, cujos vínculos primários são:

$$
\phi^{a \dagger}=\Pi_{\psi}^{a}+i \psi^{a \dagger} \approx 0, \quad \rho^{a}=\Pi_{\bar{\psi}}^{a} \approx 0
$$

Para podermos prosseguir com a quantização canônica, precisamos obter a descrição Hamiltoniana da teoria vinculada, o que pode ser feito por intermédio do método de Dirac $[117,118]$. A densidade

\footnotetext{
${ }^{3}$ Alternativamente, poderíamos ter considerado a transformação $\sigma \rightarrow \sigma+\frac{\pi J}{\sqrt{\lambda}}$ no lugar de (8.1.3), para obter (8.1.15) diretamente.

${ }^{4}$ Nossa convenção é tomar as derivadas fermiônicas (anticomutantes) pela esquerda.
} 
Hamiltoniana canônica é dada por:

$$
\begin{aligned}
\mathscr{H}_{\text {Can }} & =-\Pi_{\psi}^{a} \partial_{0} \psi^{a}+\partial_{0} \psi^{a \dagger} \Pi_{\bar{\psi}}^{a}-\mathscr{L}_{\text {Livre }} \\
& =-i \bar{\psi} \gamma^{1} \partial_{1} \psi+m \bar{\psi} \psi,
\end{aligned}
$$

levando naturalmente a $H_{C a n}=\int d x^{1} \mathscr{H}_{C a n}$. A partir da Hamiltoniana canônica, definimos a Hamiltoniana primária ao incorporarmos os vínculos (8.1.18) por intermédio de multiplicadores de Lagrange fermiônicos, $\xi^{a}$ e $\lambda^{a \dagger}$ :

$$
H_{P}=H_{C a n}+\int d x^{1}\left(\phi^{a \dagger} \xi^{a}+\lambda^{a \dagger} \rho^{a}\right)
$$

Os parênteses de Poisson em tempos iguais não-nulos são:

$$
\left\{\psi^{a}(x) ; \Pi_{\psi}^{b}\left(x^{\prime}\right)\right\}=\left\{\psi^{a \dagger}(x) ; \Pi_{\bar{\psi}}^{b}\left(x^{\prime}\right)\right\}=\delta^{a b} \delta\left(x-x^{\prime}\right)
$$

Calculemos, pois, a evolução temporal dos vínculos primários:

$$
\begin{aligned}
\partial_{0} \phi^{a^{\dagger}} & \approx\left\{\phi^{a \dagger} ; H_{P}\right\} \approx\left(i \partial_{1} \bar{\psi} \gamma^{1}+m \bar{\psi}\right)^{a}+i \lambda^{a \dagger}, \\
\partial_{0} \rho^{a} & \approx\left\{\rho^{a} ; H_{P}\right\} \approx-\left[\gamma^{0}\left(-i \gamma^{1} \partial_{1}+m\right) \psi+i \xi\right]^{a} .
\end{aligned}
$$

Demandando que os vínculos sejam constantes do movimento, determinamos os multiplicadores de Lagrange:

$$
\lambda^{a \dagger}=\left(-\partial_{1} \bar{\psi} \gamma^{1}+i m \bar{\psi}\right)^{a} \quad \text { e } \quad \xi^{a}=\left[\gamma^{0}\left(\gamma^{1} \partial_{1}+i m\right) \psi\right]^{a} .
$$

Nesse caso, a imposição da independência temporal dos vínculos não introduz nenhum vínculo novo, apenas determina os multiplicadores de Lagrange, indicando, portanto, que os vínculos (8.1.18) são de segunda classe. Substituindo (8.1.23) em (8.1.20) obtemos:

$$
H_{P}=\int d x^{1}\left[\Pi_{\psi} \gamma^{0}\left(\gamma^{1} \partial_{1} \psi+i m \psi\right)+\left(-\partial_{1} \bar{\psi} \gamma^{1}+i m \bar{\psi}\right) \Pi_{\bar{\psi}}\right]
$$

Os parênteses de Poisson para os vínculos podem ser convenientemente escritos em forma matricial, ao agruparmos os vínculos da seguinte forma: $\Phi^{A}=\left(\phi^{a \dagger}, \rho^{a}\right)$,

$$
\begin{aligned}
C\left(x, x^{\prime}\right) & =\left(\begin{array}{cc}
\left\{\phi^{a \dagger}(x) ; \phi^{b^{\dagger}}\left(x^{\prime}\right)\right\} & \left\{\phi^{a \dagger}(x) ; \rho^{b}\left(x^{\prime}\right)\right\} \\
\left\{\rho^{a}(x) ; \phi^{b^{\dagger}}\left(x^{\prime}\right)\right\} & \left\{\rho^{a}(x) ; \rho^{b}\left(x^{\prime}\right)\right\}
\end{array}\right) \\
& =-i\left(\begin{array}{cc}
0 & \delta^{a b} \\
\delta^{a b} & 0
\end{array}\right) \delta\left(x-x^{\prime}\right) .
\end{aligned}
$$

Portanto, possibilitando a construção dos parênteses de Dirac entre quaisquer duas variáveis dinâmicas:

$$
\left\{F(x) ; G\left(x^{\prime}\right)\right\}_{D}=\left\{F(x) ; G\left(x^{\prime}\right)\right\}-\int d z \int d z^{\prime}\left\{F(x) ; \Phi^{A}(z)\right\} C_{A B}^{-1}\left(z, z^{\prime}\right)\left\{\Phi^{B}\left(z^{\prime}\right) ; G\left(x^{\prime}\right)\right\}
$$




$$
\begin{aligned}
& =\left\{F(x) ; G\left(x^{\prime}\right)\right\}-i \int d z\left[\left\{F(x) ; \Pi_{\psi}^{a}(z)+i \psi^{a \dagger}(z)\right\}\left\{\Pi_{\bar{\psi}}^{a}(z) ; G\left(x^{\prime}\right)\right\}+\right. \\
& \left.+\left\{F(x) ; \Pi_{\bar{\psi}}^{a}(z)\right\}\left\{\Pi_{\psi}^{a}(z)+i \psi^{a \dagger}(z) ; G\left(x^{\prime}\right)\right\}\right] .
\end{aligned}
$$

Lembrando que os vínculos podem ser igualados a zero dentro dos parênteses de Dirac, obtemos:

$$
\begin{gathered}
\left\{\psi^{a}(x) ; \psi^{b}\left(x^{\prime}\right)\right\}_{D}=0, \\
\left\{\psi^{a \dagger}(x) ; \psi^{b^{\dagger}}\left(x^{\prime}\right)\right\}_{D}=0, \\
\left\{\psi^{a}(x) ; \psi^{b^{\dagger}}\left(x^{\prime}\right)\right\}_{D}=-i \delta^{a b} \delta\left(x-x^{\prime}\right) .
\end{gathered}
$$

A partir dos parênteses de Dirac (8.1.27) introduzimos as relações de anticomutação canônicas:

$$
\begin{aligned}
\left\{\psi^{a}(x) ; \psi^{b}\left(x^{\prime}\right)\right\} & =0, \\
\left\{\psi^{a \dagger}(x) ; \psi^{b^{\dagger}}\left(x^{\prime}\right)\right\} & =0, \\
\left\{\psi^{a}(x) ; \psi^{b^{\dagger}}\left(x^{\prime}\right)\right\} & =\delta^{a b} \delta\left(x-x^{\prime}\right) .
\end{aligned}
$$

Além do mais, dado que utilizamos os parênteses de Dirac, podemos ignorar os vínculos e, assim, a Hamiltoniana da teoria livre assume a forma:

$$
H=\int d x^{1}\left(-i \bar{\psi} \gamma^{1} \partial_{1} \psi+m \bar{\psi} \psi\right)
$$

Ao resolvermos as equações de movimento (8.1.16), verificamos que as soluções de onda plana (F.1.5) fornecem uma base completa para o espaço dos espinores de duas componentes, de forma que podemos expandir o operador de Dirac e seu adjunto:

$$
\begin{aligned}
& \psi(x)=\int \frac{d p_{1}}{2 \pi}\left[a\left(p_{1}\right) u(p) e^{-i p \cdot x}+b\left(-p_{1}\right) v(-p) e^{i p \cdot x}\right] \\
& \bar{\psi}(x)=\int \frac{d p_{1}}{2 \pi}\left[a^{\dagger}\left(p_{1}\right) \bar{u}(p) e^{i p \cdot x}+b^{\dagger}\left(-p_{1}\right) \bar{v}(-p) e^{-i p \cdot x}\right]
\end{aligned}
$$

onde $p_{0}=\omega(p)$. Invertendo as relações (8.1.30a) e (8.1.30b) e utilizando (8.1.28), obtemos as relações canônicas de anticomutação para os osciladores:

$$
\begin{aligned}
\left\{a\left(k_{1}\right) ; a^{\dagger}\left(p_{1}\right)\right\} & =2 \pi \delta\left(k_{1}-p_{1}\right), \\
\left\{b\left(-k_{1}\right) ; b^{\dagger}\left(-p_{1}\right)\right\} & =2 \pi \delta\left(k_{1}-p_{1}\right) .
\end{aligned}
$$

Podemos rescrever a Hamiltoniana (8.1.29) em termos dos osciladores $a\left(p_{1}\right)$ e $b\left(p_{1}\right):{ }^{5}$

$$
H=\int \frac{d p_{1}}{2 \pi} p_{0}\left[a^{\dagger}\left(p_{1}\right) a\left(p_{1}\right)-b^{\dagger}\left(p_{1}\right) b\left(p_{1}\right)\right]
$$

\footnotetext{
${ }^{5}$ Note que a Hamiltoniana (8.1.32) não é positiva definida, fato que poderia ser imediatamente remediado através da redefinição usual: $b^{\dagger} \rightarrow \tilde{b}$ e $b \rightarrow \tilde{b}^{\dagger}$, que simplesmente troca o papel dos osciladores $b$ e $b^{\dagger}$. Naturalmente, em virtude dessa redefinição os estados com energia negativa são eliminados.
} 
com $p_{0}=\omega(p)$.

O fato do modelo de AAF constituir uma teoria relativística implica que seu propagador usual não é puramente retardado, logo, é impossível efetuar o cálculo da matriz $S$ da mesma forma que para o modelo de Landau-Lifshitz, onde tal fato foi crucial para controlar as correções quânticas e assim possibilitar a soma sobre todos os diagramas de Feynman. Contudo, podemos utilizar a mesma técnica empregada por [119] para contornar essa dificuldade. A idéia consiste em quantizar a teoria com respeito a um pseudovácuo, no lugar do verdadeiro estado fundamental. O pseudovácuo é, por definição, o estado aniquilado pelo operador de Dirac:

$$
\psi(x)|0\rangle=0
$$

Portanto, todos os níveis de antipartículas são deixados vazios e a matriz $S$ de excitações sobre o pseudovácuo pode ser calculada através dos mesmos métodos empregados para teorias nãorelativísticas. Podemos interpretar esse procedimento como a presença de um potencial químico infinito atuando sobre o sistema. Finalmente, a partir dessa matriz $S$ "nua" podemos obter as equações de Bethe, cujas soluções permitem o preenchimento do mar de Dirac e conseqüente reconstrução do verdadeiro estado fundamental [22]. É importante ressaltar que o preenchimento do mar de Dirac deve modificar drasticamente o espectro e a matriz $S$. Claramente, (8.1.33) demanda que:

$$
a\left(p_{1}\right)|0\rangle=b\left(p_{1}\right)|0\rangle=0 \Rightarrow H|0\rangle=0 .
$$

Definindo os estados de pseudopartículas como:

$$
a^{\dagger}\left(p_{1}\right)|0\rangle=|p\rangle, \quad b^{\dagger}\left(p_{1}\right)|0\rangle=|\tilde{p}\rangle,
$$

verificamos que:

$$
H|p\rangle=p_{0}|p\rangle, \quad H|\tilde{p}\rangle=-p_{0}|\tilde{p}\rangle,
$$

ou seja, os operadores $a^{\dagger}\left(p_{1}\right)\left[a\left(p_{1}\right)\right]$ e $b^{\dagger}\left(p_{1}\right)\left[b\left(p_{1}\right)\right]$ criam [aniquilam] excitações com momento $p_{1} \mathrm{e}$ energia $p_{0}=+\omega(p)$ e $p_{0}=-\omega(p)$, respectivamente. Portanto os operadores a e $a^{\dagger}$ (respectivamente, $b$ e $b^{\dagger}$ ) podem ser vistos como operadores de aniquilação e criação para pseudopartículas com energia positiva (negativa). O vácuo físico $|\Omega\rangle$ é então obtido a partir de $|0\rangle$ ao se excitar todos os modos de energia negativa, ou seja, ao se preencher o mar de Dirac.

Finalmente, calculamos o propagador com respeito ao pseudovácuo (8.1.33):

$$
\begin{aligned}
D\left(x-x^{\prime}\right) & =\left\langle 0\left|T \psi(x) \bar{\psi}\left(x^{\prime}\right)\right| 0\right\rangle=\theta\left(x_{0}-x_{0}^{\prime}\right)\left\langle 0\left|\psi(x) \bar{\psi}\left(x^{\prime}\right)\right| 0\right\rangle \\
& =\theta\left(x_{0}-x_{0}^{\prime}\right) \int \frac{d p_{1}}{2 \pi} \frac{1}{2 p_{0}}\left[(\not p+m) e^{-i p \cdot\left(x-x^{\prime}\right)}+(\not p-m) e^{+i p \cdot\left(x-x^{\prime}\right)}\right] \\
& =(i \not \partial+m) \int \frac{d^{2} p}{4 \pi^{2}} \frac{i e^{-i \cdot\left(x-x^{\prime}\right)}}{\left[p_{0}+\omega(p)+i \varepsilon\right]\left[p_{0}-\omega(p)+i \varepsilon\right]},
\end{aligned}
$$

onde $d^{2} p=d p_{0} d p_{1}$. Notando que no limite $\varepsilon \rightarrow 0$ :

$$
\left[p_{0}+\omega(p)+i \varepsilon\right]\left[p_{0}-\omega(p)+i \varepsilon\right]=p^{2}-m^{2}+2 i \varepsilon p_{0}+\varepsilon^{2} \rightarrow p^{2}-m^{2}+2 i \varepsilon p_{0}
$$




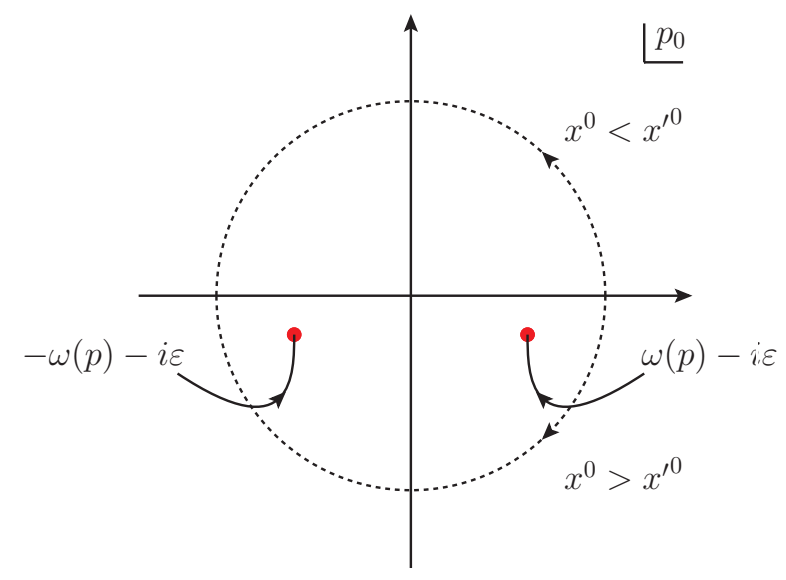

Figura 8.2: Prescrição de pólos para o propagador do modelo de AAF.

concluímos que:

$$
D\left(x-x^{\prime}\right)=(i \not \partial+m) \int \frac{d^{2} p}{4 \pi^{2}} \frac{i e^{-i p \cdot\left(x-x^{\prime}\right)}}{p^{2}-m^{2}+2 i \varepsilon p_{0}} .
$$

Dado que ambos os pólos estão localizados no semiplano inferior, vide figura 8.2, podemos utilizar o mesmo argumento empregado no caso do modelo de Landau-Lifshitz, para concluir que o laço fechado de propagadores orientados de uma mesma maneira se anula. Por consegüinte, todas as consequiências do teorema 5.1.1 são válidas para o modelo de AAF.

\subsubsection{Espalhamento de Pseudopartículas no Modelo de AAF}

No que se segue, estaremos interessados no cálculo da matriz $S$ para o espalhamento de duas e três pseudopartículas. Por simplicidade, consideraremos apenas o espalhamento de pseudopartículas com energia positiva (excitações sobre o pseudovácuo criadas por $a^{\dagger}$ ), posto que o espalhamento envolvendo partículas com energia negativa (criadas por $b^{\dagger}$ ) pode ser obtido facilmente por intermédio de uma continuação analítica a rapidezes $\operatorname{complexas} \theta$,

$$
p_{0}=m \cosh \theta \text { e } p_{1}=m \sinh \theta \text {, se }\left\{\begin{array}{ll}
\theta=\alpha \in \mathbb{R} & \Rightarrow p_{0} \in \mathbb{R}_{+} \text {e } p_{1} \in \mathbb{R} \\
\theta=i \pi-\alpha, \alpha \in \mathbb{R} & \Rightarrow p_{0} \in \mathbb{R}_{-} \text {e } p_{1} \in \mathbb{R}
\end{array} .\right.
$$

Conseqüentemente, as pseudopartículas com energia positiva são apropriadamente descritas por rapidezes reais $\theta=\alpha \in \mathbb{R}$, enquanto que as com energia negativa são naturalmente parametrizadas por rapidezes imaginárias $\theta=i \pi-\alpha, \alpha \in \mathbb{R}$. Dado que a matriz $S$ é uma função meromórfica das rapidezes [24,25], ela descreve simultaneamente o espalhamento de ambos os tipos de pseudopartículas. Portanto, podemos, sem perda de generalidade, considerar que os estados externos são compostos 


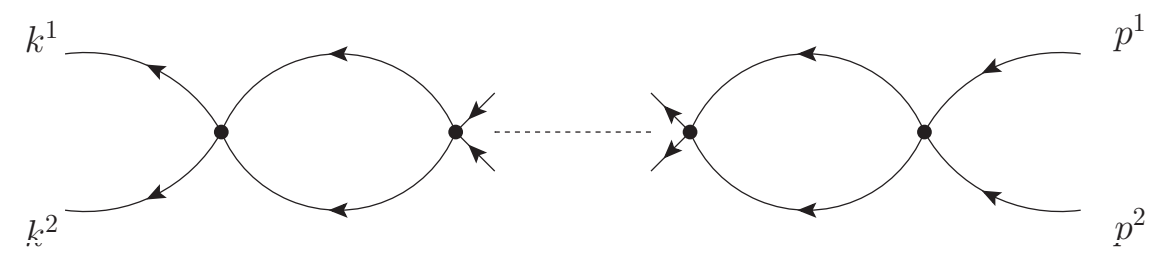

Figura 8.3: Diagrama tipo bolha para o espalhamento de duas pseudopartículas.

por apenas pseudopartículas na camada de massa positiva:

$$
|\mathbf{p}\rangle=\prod_{i=1}^{n} a^{\dagger}\left(p_{1}^{i}\right)|0\rangle, \quad\langle\mathbf{k}|=\langle 0| \prod_{i=1}^{n} a\left(k_{1}^{i}\right) .
$$

\subsection{Espalhamento de Duas Pseudopartículas}

A matriz S para o espalhamento de duas pseudopartículas foi originalmente obtida em [35], contudo tal análise apresenta diversas inconsistências, cuja conseqüência mais notável foi a dedução da matriz $S$ invertida. Dentre elas, duas são especialmente importantes para o presente cálculo. Primeiramente, em [35], apenas uma constante de acoplamento foi introduzida para ambos os vértices de interação, o que não é correto, posto que $g_{2}$ e $g_{3}$ possuem diferentes dimensões de massa, levando assim a uma análise dimensional equivocada. Em segundo lugar, ressaltamos que o sinal do termo cinético da ação (4.3) considerada por [35] não é consistente com sua expansão de modos (4.5) e (4.6). Evidentemente, essa é causa da inversão da matriz S. Logo, uma dedução alternativa baseada em técnicas usuais da teoria quântica de campos [24,41] é imperativa.

O fato de o propagador ser puramente retardado implica que a matriz $S$ para o espalhamento de duas pseudopartículas pode ser obtida a partir da soma de diagramas tipo bolhas (e suas torções espinoriais) descritos na figura 8.3. Portanto, precisamos apenas considerar a contribuição do termo de interação entre duas partículas $\mathscr{L}_{\text {lnt }}^{(2)}$. Sem perda de generalidade, supomos que os momentos incidentes estão na camada de massa e ordenados: $p_{1}^{1}>p_{1}^{2}$. No que se segue, sempre denotaremos os momentos espaçotemporais como $p_{\mu}^{i}$, onde $\mu=0,1$ é o índice de Lorentz e $i=1, \ldots, n$, o rótulo da pseudopartícula. A matriz $S$ entre duas pseudopartículas é determinada através da relação

$$
\left\langle k^{1} k^{2}|\hat{S}| p^{1} p^{2}\right\rangle=S\left(p^{1}, p^{2}\right) \delta_{-}^{(2)}\left(p^{1}, p^{2} ; k^{1}, k^{2}\right),
$$

onde denotamos:

$$
\delta_{ \pm}^{(2)}\left(p^{1}, p^{2} ; k^{1}, k^{2}\right)=4 \pi^{2}\left[\delta\left(k_{1}^{1}-p_{1}^{1}\right) \delta\left(k_{1}^{2}-p_{1}^{2}\right) \pm \delta\left(k_{1}^{1}-p_{1}^{2}\right) \delta\left(k_{1}^{2}-p_{1}^{1}\right)\right]
$$


A amplitude de espalhamento é dada por:

$$
\begin{aligned}
\left\langle k^{1} k^{2}|\hat{S}| p^{1} p^{2}\right\rangle & =\left\langle k^{1} k^{2}\left|e^{i \int \mathscr{L}_{\text {lnt }}^{(2)} d^{2} x}\right| p^{1} p^{2}\right\rangle \\
& =\left\langle k^{1} k^{2} \mid p^{1} p^{2}\right\rangle+i\left\langle k^{1} k^{2}\left|T \int \mathscr{L}_{\text {lnt }}^{(2)} d^{2} x\right| p^{1} p^{2}\right\rangle- \\
& -\frac{1}{2}\left\langle k^{1} k^{2}\left|T\left(\int \mathscr{L}_{\text {lnt }}^{(2)} d^{2} x\right)^{2}\right| p^{1} p^{2}\right\rangle+\cdots
\end{aligned}
$$

O termo de não-espalhamento é facilmente calculado:

$$
\left\langle k^{1} k^{2} \mid p^{1} p^{2}\right\rangle=\delta_{-}^{(2)}\left(p^{1}, p^{2} ; k^{1}, k^{2}\right) .
$$

Já no nível de árvore, precisamos considerar:

$$
\begin{aligned}
\left.\left\langle k^{1} k^{2}|\hat{S}| p^{1} p^{2}\right\rangle\right|_{g_{2}} & =i\left\langle k^{1} k^{2}\left|T \int \mathscr{L}_{\operatorname{lnt}}^{(2)} d^{2} x\right| p^{1} p^{2}\right\rangle \\
& =-i \mathbb{G}_{a c, b d}^{\alpha \beta}\left\langle k^{1} k^{2}\left|\int d^{2} x\left(\bar{\psi}^{a} \bar{\psi}^{c} \partial_{\alpha} \psi^{b} \partial_{\beta} \psi^{d}-\partial_{\alpha} \bar{\psi}^{a} \partial_{\beta} \bar{\psi}^{c} \psi^{b} \psi^{d}\right)\right| p^{1} p^{2}\right\rangle
\end{aligned}
$$

Nesse caso, podemos calcular todas as integrais para obter a contribuição do nível de árvore fora da camada de massa ${ }^{6}$ :

$$
\begin{aligned}
\left.\left\langle k^{1} k^{2}|\hat{S}| p^{1} p^{2}\right\rangle\right|_{g_{2}} & =-\frac{i g_{2}}{8 m}\left\{k^{2} \times k^{1}\left(\bar{U}_{12}^{k}+\bar{U}_{21}^{k}\right) \mathbb{P}\left(U_{12}^{p}-U_{21}^{p}\right)\right. \\
& \left.+p^{2} \times p^{1}\left(-\bar{U}_{12}^{k}+\bar{U}_{21}^{k}\right) \mathbb{P}\left(U_{12}^{p}+U_{21}^{p}\right)\right\} 4 \pi^{2} \delta^{(2)}\left(k^{1}+k^{2}-p^{1}-p^{2}\right) .
\end{aligned}
$$

onde introduzimos a notação conveniente para os biespinores:

$$
\bar{U}_{i j}^{k}=\bar{u}\left(k^{i}\right) \otimes \bar{u}\left(k^{j}\right), \quad U_{i j}^{p}=u\left(p^{i}\right) \otimes u\left(p^{j}\right),
$$

e denotamos: $\epsilon^{\alpha \beta} p_{\alpha}^{i} p_{\beta}^{j}=p^{i} \times p^{j}$

Tomando as pseudopartículas espalhadas também na camada de massa, podemos utilizar a identidade:

$$
4 \pi^{2} \delta^{(2)}\left(k^{1}+k^{2}-p^{1}-p^{2}\right)=\left|\frac{p_{0}^{1} p_{0}^{2}}{p^{2} \times p^{1}}\right| \delta_{+}^{(2)}\left(p^{1}, p^{2} ; k^{1}, k^{2}\right),
$$

em conjunto com o fato de que para a nosso ordenamento dos momentos incidentes $p^{2} \times p^{1}>0$, para escrever:

$$
\left.\left\langle k^{1} k^{2}|\hat{S}| p^{1} p^{2}\right\rangle\right|_{g_{2}}=-\frac{i g_{2}}{2 m} p_{0}^{1} p_{0}^{2} \bar{U}_{21}^{p} \mathbb{P} U_{12}^{p} \delta_{-}^{(2)}\left(p^{1}, p^{2} ; k^{1}, k^{2}\right) .
$$

Finalmente, calculamos o produto espinorial

$$
\bar{U}_{21}^{p} \mathbb{P} U_{12}^{p}=\frac{p^{2} \times p^{1}}{p_{0}^{1} p_{0}^{2}}
$$

\footnotetext{
${ }^{6}$ Mais precisamente, as pseudopartículas espalhadas estão fora da camada de massa; porém, como não é necessário o uso da hipótese de partículas incidentes na camada de massa para se obter (8.2.6), podemos dizer sem prejuízo que a amplitude está fora da camada de massa.
} 
para obter a amplitude de nível de árvore na camada de massa:

$$
\left.\left\langle k^{1} k^{2}|\hat{S}| p^{1} p^{2}\right\rangle\right|_{g_{2}}=-\frac{i g_{2}}{2 m}\left(p^{2} \times p^{1}\right) \delta_{-}^{(2)}\left(p^{1}, p^{2} ; k^{1}, k^{2}\right) .
$$

O cálculo da amplitude de 1-laço é significativamente mais intrincado por possuir mais termos a serem considerados. A saber, precisamos computar:

$$
\begin{aligned}
\left.\left\langle k^{1} k^{2}|\hat{S}| p^{1} p^{2}\right\rangle\right|_{g_{2}^{2}} & =-\frac{1}{2}\left\langle k^{1} k^{2}\left|T\left(\int \mathscr{L}_{\text {lnt }}^{(2)} d^{2} x\right)^{2}\right| p^{1} p^{2}\right\rangle \\
& =-\frac{1}{2} \mathbb{G}_{a c, b d}^{\alpha \beta} \mathbb{G}_{e g, f h}^{\gamma \delta}\left\langle k^{1} k^{2}\right| T \int d^{2} x d^{2} y\left[\bar{\psi}^{a} \bar{\psi}^{c} \partial_{\alpha} \psi^{b} \partial_{\beta} \psi^{d} \bar{\phi}^{e} \bar{\phi}^{g} \partial_{\gamma} \phi^{f} \partial_{\delta} \phi^{h}-\right. \\
& -\bar{\psi}^{a} \bar{\psi}^{c} \partial_{\alpha} \psi^{b} \partial_{\beta} \psi^{d} \partial_{\gamma} \bar{\phi}^{e} \partial_{\delta} \bar{\phi}^{g} \phi^{f} \phi^{h}-\partial_{\alpha} \bar{\psi}^{a} \partial_{\beta} \bar{\psi}^{c} \psi^{b} \psi^{d} \bar{\phi}^{e} \bar{\phi}^{g} \partial_{\gamma} \phi^{f} \partial_{\delta} \phi^{h}+ \\
& \left.+\partial_{\alpha} \bar{\psi}^{a} \partial_{\beta} \bar{\psi}^{c} \psi^{b} \psi^{d} \partial_{\gamma} \bar{\phi}^{e} \partial_{\delta} \bar{\phi}^{g} \phi^{f} \phi^{h}\right]\left|p^{1} p^{2}\right\rangle
\end{aligned}
$$

onde, para maior clareza, optamos por denotar $\psi \equiv \psi(x)$ e $\phi \equiv \psi(y)$. Expandindo o produto temporalmente ordenado, percebemos que apenas termos com duas contrações do tipo

\section{$\overrightarrow{\psi \bar{\phi}} \overline{\psi \phi}, \quad$ ou $\quad \bar{\psi} \phi \bar{\psi} \phi$}

contribuem. Todos os outros termos se anulam, seja porque seu produto interno

$$
\left\langle k^{1} k^{2}\left|f\left(a, a^{\dagger}\right)\right| p^{1} p^{2}\right\rangle=0 \quad \text { ou } \quad\left\langle k^{1} k^{2}|g(\psi, \bar{\psi})| p^{1} p^{2}\right\rangle \propto \theta\left(x^{0}-y^{0}\right) \theta\left(y^{0}-x^{0}\right)=0
$$

com $f\left(a, a^{\dagger}\right)$ e $g(\psi, \bar{\psi})$ sendo duas funções arbitrárias. Logo,

$$
\begin{aligned}
\left.\left\langle k^{1} k^{2}|\hat{S}| p^{1} p^{2}\right\rangle\right|_{g_{2}^{2}} & =2\left(\frac{g_{2}}{8 m}\right)^{2}\left[\left(k^{2} \times k^{1}\right)\left(p^{2} \times p^{1}\right)\left(\bar{U}_{12}^{k}+\bar{U}_{21}^{k}\right) \mathbb{P} I_{0}\left(p^{1}, p^{2}\right) \mathbb{P}\left(U_{12}^{p}+U_{21}^{p}\right)-\right. \\
& -\left(p^{2} \times p^{1}\right)\left(\bar{U}_{12}^{k}-\bar{U}_{21}^{k}\right) \mathbb{P} I_{1}\left(p^{1}, p^{2}\right) \mathbb{P}\left(U_{12}^{p}+U_{21}^{p}\right)- \\
& -\left(k^{2} \times k^{1}\right)\left(\bar{U}_{12}^{k}+\bar{U}_{21}^{k}\right) \mathbb{P} I_{1}\left(p^{1}, p^{2}\right) \mathbb{P}\left(U_{12}^{p}-U_{21}^{p}\right)+ \\
& \left.+\left(\bar{U}_{12}^{k}-\bar{U}_{21}^{k}\right) \mathbb{P} I_{2}\left(p^{1}, p^{2}\right) \mathbb{P}\left(U_{12}^{p}-U_{21}^{p}\right)\right] 4 \pi^{2} \delta^{(2)}\left(k^{1}+k^{2}-p^{1}-p^{2}\right),
\end{aligned}
$$

onde as integrais $l_{i}\left(p^{1}, p^{2}\right), i=0,1,2$ estão definidas no apêndice $G$ pelas equações (G.0.2).

As relações de completeza (F.2.3), juntamente com o ordenamento escolhido para os momentos incidentes e a identidade para momentos na camada de massa:

$$
\frac{\left(p^{2} \times p^{1}\right)\left(p_{0}^{1}+p_{0}^{2}\right)}{\left(p_{1}^{1}-p_{1}^{2}\right)\left(p^{1}+p^{2}\right)^{2}}=\frac{1}{2}
$$

levam à seguinte relação central entre a amplitude de 1-laço e a amplitude de nível de árvore fora da camada de massa ${ }^{7}(8.2 .6)$

$$
\left.\left\langle k^{1} k^{2}|\hat{S}| p^{1} p^{2}\right\rangle\right|_{g_{2}^{2}}=\left.2\left(p^{2} \times p^{1}\right)\left(\frac{-i g_{2}}{8 m}\right)\left\langle k^{1} k^{2}|\hat{S}| p^{1} p^{2}\right\rangle\right|_{g_{2}} .
$$

Posto que as pseudopartículas espalhadas estão fora da camada de massa, a equação (8.2.14) sugere

\footnotetext{
${ }^{7}$ De fato, aqui consideramos apenas as pseudopartículas espalhadas fora da camada de massa.
} 
que podemos considerar a amplitude de espalhamento de 1-laço como sendo o vértice de interação na representação dos momentos com os momentos incidentes $p^{1}$ e $p^{2}$ na camada de massa, multiplicado por alguma função desse par de momentos e da constante de acoplamento. Portanto, a amplitude de espalhamento com $n$ laços corresponde ao produto de $n$ desses vértices modificados:

$$
\begin{aligned}
\left.\left\langle k^{1} k^{2}|\hat{S}| p^{1} p^{2}\right\rangle\right|_{g_{2}^{n+1}} & =\left.\left[2\left(p^{2} \times p^{1}\right)\left(\frac{-i g_{2}}{8 m}\right)\right]^{n}\left\langle k^{1} k^{2}|\hat{S}| p^{1} p^{2}\right\rangle\right|_{g_{2}} \\
& \stackrel{\text { on-shell }}{=} 2\left(-\frac{i g_{2}}{4 m}\left(p^{2} \times p^{1}\right)\right)^{n+1} \delta_{-}\left(p^{1}, p^{2} ; k^{1}, k^{2}\right),
\end{aligned}
$$

claramente, no último passo, tomamos as pseudopartículas espalhadas $k^{1}$ e $k^{2}$ na camada de massa.

Podemos, pois, obter a amplitude de espalhamento completa:

$$
\begin{aligned}
\left\langle k^{1} k^{2}|\hat{S}| p^{1} p^{2}\right\rangle & =\left\langle k^{1} k^{2} \mid p^{1} p^{2}\right\rangle+\left.\sum_{n=1}^{\infty}\left\langle k^{1} k^{2}|\hat{S}| p^{1} p^{2}\right\rangle\right|_{g_{2}^{n}} \\
& =\frac{1-\frac{i g_{2}}{4 m} p^{2} \times p^{1}}{1+\frac{i g_{2}}{4 m} p^{2} \times p^{1}} \delta_{-}\left(p^{1}, p^{2} ; k^{1}, k^{2}\right),
\end{aligned}
$$

cuja comparação com (8.2.1) permite a dedução da matriz $S$ para o espalhamento de duas pseudopartículas

$$
S\left(p^{1}, p^{2}\right)=\frac{1-\frac{i g_{2}}{4 m} p^{2} \times p^{1}}{1+\frac{i g_{2}}{4 m} p^{2} \times p^{1}}
$$

É fundamental notar que a nossa matriz $S$ é o inverso da obtida por [35].

\subsection{Espalhamento de Três Pseudopartículas}

Nessa seção, analisamos a matriz $S$ para o setor de três pseudopartículas com o intuito de verificar sua fatorabilidade em termos de matrizes $S$ para o espalhamento de duas pseudopartículas, e assim sondar a integrabilidade quântica do modelo. Antes de estudarmos em detalhe as amplitudes de espalhamento entre três pseudopartículas, conforme fizemos na seção anterior para o setor com apenas duas pseudopartículas, achamos conveniente examinar sua expressão advinda do argumento de fatorabilidade, a saber,

$$
\begin{aligned}
S\left(p^{1}, p^{2}, p^{3}\right) & =S\left(p^{1}, p^{2}\right) S\left(p^{1}, p^{3}\right) S\left(p^{2}, p^{3}\right) \\
& =\frac{1-\frac{i g_{2}}{4 m} p^{2} \times p^{1}}{1+\frac{i g_{2}}{4 m} p^{2} \times p^{1}} \cdot \frac{1-\frac{i g_{2}}{4 m} p^{3} \times p^{1}}{1+\frac{i g_{2}}{4 m} p^{3} \times p^{1}} \cdot \frac{1-\frac{i g_{2}}{4 m} p^{3} \times p^{2}}{1+\frac{i g_{2}}{4 m} p^{3} \times p^{2}} \\
& =1+2 \sum_{n=1}^{3}\left[\left(-\frac{i g_{2}}{4 m}\right)\left(p^{2} \times p^{1}+p^{3} \times p^{1}+p^{3} \times p^{2}\right)\right]^{n}+ \\
& +2\left(\frac{i g_{2}}{4 m}\right)^{3}\left(p^{2} \times p^{1}+p^{3} \times p^{1}\right)\left(p^{2} \times p^{1}+p^{3} \times p^{2}\right)\left(p^{3} \times p^{1}+p^{3} \times p^{2}\right)+ \\
& +O\left(g_{2}^{4}\right) .
\end{aligned}
$$


No que se segue, calculamos a amplitude de espalhamento para três pseudopartículas e mostramos que a condição necessária para integrabilidade quântica (8.3.1) é satisfeita na primeira ordem nãotrivial em $g_{2}$ e $g_{3}$, contanto que uma relação entre as constantes de acoplamento seja verdadeira.

\subsubsection{Cálculo Diagramáticos}

Nesse caso, devemos considerar a Lagrangeana de interação completa

$$
\mathscr{L}_{\text {lnt }}=\mathscr{L}_{\text {Int }}^{(2)}+\mathscr{L}_{\text {lnt }}^{(3)}
$$

dado que tanto o estado inicial quanto o final envolvem três pseudopartículas:

$$
|\mathbf{p}\rangle=\left|p^{1} p^{2} p^{3}\right\rangle=a^{\dagger}\left(p_{1}^{1}\right) a^{\dagger}\left(p_{1}^{2}\right) a^{\dagger}\left(p_{1}^{3}\right)|0\rangle \quad \text { e } \quad\langle\mathbf{k}|=\left\langle k^{1} k^{2} k^{3}\right|=\langle 0| a\left(k_{1}^{3}\right) a\left(k_{1}^{2}\right) a\left(k_{1}^{1}\right) .
$$

De forma análoga à seção anterior, também supomos sem perda de generalidade que as pseudopartículas incidentes estão na camada de massa e possuem seus momentos ordenados: $p_{1}^{1}>p_{1}^{2}>p_{1}^{3}$. A analiticidade nas constantes de acoplamento da amplitude de espalhamento implica:

$$
\langle\mathbf{k}|\hat{S}| \mathbf{p}\rangle=\langle\mathbf{k} \mid \mathbf{p}\rangle+\left.\langle\mathbf{k}|\hat{S}| \mathbf{p}\rangle\right|_{g}+\left.\langle\mathbf{k}|\hat{S}| \mathbf{p}\rangle\right|_{g^{2}}+\cdots
$$

onde $g$ denota $g_{1}$ ou $g_{2}$. O termo de não-espalhamento é facilmente computado:

$$
\langle\mathbf{k} \mid \mathbf{p}\rangle=3 !(2 \pi)^{3} \mathcal{A}_{p}\left[\delta\left(k^{1}-p^{1}\right) \delta\left(k^{2}-p^{2}\right) \delta\left(k^{3}-p^{3}\right)\right] .
$$

Nesse ponto, mostra-se conveniente introduzir o operador de antissimetrização, ou simplesmente, antissimetrizador, definido por:

$$
\mathcal{A}_{q}[f(\mathbf{q})]:=\frac{1}{3 !} \sum_{A} \operatorname{sign}(A) f(A[\mathbf{q}])
$$

com a soma sobre todas as permutações $\left(A_{1}, A_{2}, A_{3}\right)$ de $(1,2,3)$, cujo sinal é dado por $\operatorname{sign}(A)$, o vetor $A[\mathbf{q}]:=\left(q^{A_{1}}, q^{A_{2}}, q^{A_{3}}\right)$ e $f(\mathbf{q})$ é uma função arbitrária dos momentos.

Para o nível de árvore precisamos calcular:

$$
\left.\langle\mathbf{k}|\hat{S}| \mathbf{p}\rangle\right|_{g}=i\left\langle\mathbf{k}\left|T \int d^{2} x\left(\mathscr{L}_{\text {Int }}^{(2)}+\mathscr{L}_{\text {lnt }}^{(3)}\right)\right| \mathbf{p}\right\rangle,
$$

conduzindo-nos à amplitude de nível de árvore fora da camada de massa,

$$
\begin{aligned}
\left.\langle\mathbf{k}|\hat{S}| \mathbf{p}\rangle\right|_{g} & =(3 !)^{2} \mathcal{A}_{p, k}\left[\frac{i g_{2}}{8 m}\left(k^{2} \times k^{1}+p^{2} \times p^{1}\right) \bar{U}_{12}^{k} \mathbb{P} U_{21}^{p} 2 \pi \delta\left(k^{3}-p^{3}\right)-\right. \\
& \left.-\frac{i g_{3}}{16 m}\left(k^{3} \times p^{3}\right) \bar{U}_{123}^{k} \mathbb{Q} U_{123}^{p}\right] 4 \pi^{2} \delta^{(2)}(\mathbf{k}-\mathbf{p}),
\end{aligned}
$$

onde introduzimos a notação quase auto-evidente para os triespinores:

$$
\bar{U}_{i j l}^{k}=\bar{u}\left(k^{i}\right) \otimes \bar{u}\left(k^{j}\right) \otimes \bar{u}\left(k^{\prime}\right), \quad U_{i j l}^{p}=u\left(p^{i}\right) \otimes u\left(p^{j}\right) \otimes u\left(p^{\prime}\right) .
$$


Para o primeiro termo de (8.3.6) podemos proceder exatamente da mesma forma que no caso envolvendo apenas duas pseudopartículas, ou seja, impomos que as pseudopartículas espalhadas estejam também na camada de massa de forma a poder empregar a identidade (8.2.7) e usar que, para a nossa escolha de ordenamento para os momentos iniciais,

$$
p^{i} \times p^{j}>0, \text { se } i>j,
$$

em conjunto com (8.2.9). Finalmente, concluímos que:

$$
\begin{aligned}
\left.\langle\mathbf{k}|\hat{S}| \mathbf{p}\rangle\right|_{g} & =-\frac{i g_{2}}{2 m}\left[p^{2} \times p^{1}+p^{3} \times p^{1}+p^{3} \times p^{2}\right]\langle\mathbf{k} \mid \mathbf{p}\rangle- \\
& -\frac{i g_{3}}{16 m}(3 !)^{2} \mathcal{A}_{p, k}\left[k^{3} \times p^{3} \bar{U}_{123}^{k} \mathbb{Q} U_{123}^{p}\right] 4 \pi^{2} \delta^{(2)}(\mathbf{k}-\mathbf{p}) .
\end{aligned}
$$

É fundamental notar que não existe uma identidade similar a (8.2.7) envolvendo três momentos. Logo, não é possível calcular o produto espinorial contido no segundo termo de (8.3.6) para reduzi-lo a uma expressão proporcional a $\langle\mathbf{k} \mid \mathbf{p}\rangle$. Relembrando que para um modelo integrável, a matriz $S$ deve ser da forma:

$$
\langle\mathbf{k}|\hat{S}| \mathbf{p}\rangle=S(\mathbf{p})\langle\mathbf{k} \mid \mathbf{p}\rangle,
$$

fica claro que é impossível deduzir a expressão (8.3.1) na aproximação do nível de árvore, de fato, nem mesmo escrever a matriz $S$ na forma (8.3.8) é factível. E embora tal obstáculo pareça impedir a demonstração da integrabilidade quântica para o modelo generalizado de AAF, esse não é caso, pois, conforme vimos no capítulo 5, esse tipo de dificuldade também aparece no modelo mais simples de Landau-Lifshitz, onde mostramos que tais termos problemáticos ${ }^{8}$ são cancelados por certas contribuições advindas de amplitudes de espalhamento de ordem mais alta em $g$.

Dessa forma, consideramos a amplitude de 1-laço e procuramos a contribuição necessária para cancelar o segundo termo de (8.3.6) e, conseqüentemente tornar a matriz $S$ fatorável no nível de árvore.

$$
\begin{aligned}
\left.\langle\mathbf{k}|\hat{S}| \mathbf{p}\rangle\right|_{g^{2}} & =-\frac{1}{2}\left\langle\mathbf{k}\left|T\left[\int d^{2} x\left(\mathscr{L}_{\text {lnt }}^{(2)}+\mathscr{L}_{\text {Int }}^{(3)}\right)\right]^{2}\right| \mathbf{p}\right\rangle \\
& =-\frac{1}{2}\langle\mathbf{k}| T \int d^{2} x d^{2} y\left[\mathscr{L}_{\text {lnt }}^{(2)}(x) \mathscr{L}_{\text {Int }}^{(2)}(y)+\mathscr{L}_{\text {Int }}^{(2)}(x) \mathscr{L}_{\text {Int }}^{(3)}(y)+\mathscr{L}_{\text {Int }}^{(3)}(x) \mathscr{L}_{\text {lnt }}^{(2)}(y)+\right. \\
& \left.+\mathscr{L}_{\text {lnt }}^{(3)}(x) \mathscr{L}_{\text {lnt }}^{(3)}(y)\right]|\mathbf{p}\rangle \\
& \equiv V_{1}^{(2)}(\mathbf{k}, \mathbf{p})+V_{2}^{(2)}(\mathbf{k}, \mathbf{p})+V_{3}^{(2)}(\mathbf{k}, \mathbf{p})+V_{4}^{(2)}(\mathbf{k}, \mathbf{p})
\end{aligned}
$$

Claramente, esse cancelamento só pode ocorrer entre diagramas com a mesma ordem em $\hbar .{ }^{9}$ De fato, todos os diagramas no nível de árvore são proporcionais a $\hbar^{0}$, portanto, já nesse estágio, podemos considerar apenas o primeiro termo da expansão (8.3.9), $V_{1}^{(2)}(\mathbf{k}, \mathbf{p})$, visto que ele é o único a possuir

\footnotetext{
${ }^{8}$ Como o segundo termo de (8.3.6).

${ }^{9}$ Consideramos uma expansão no número de laços, que corresponde à expansão nas potências de $\hbar$. Logo, não é possível nenhum cancelamento entre diagramas com um diferente número de laços. Não obstante, ressaltamos que, no caso em consideração, existem duas constantes de acoplamento distintas $g_{2}$ e $g_{3}$, cujas dimensões são tais que o segundo diagrama da figura 8.1 e o primeiro da figura 8.4 são exatamente da mesma ordem, e, por isso, podem se cancelar mutuamente. Assim, devemos considerar todos os diagramas de uma dada ordem em $\hbar^{n}$.
} 


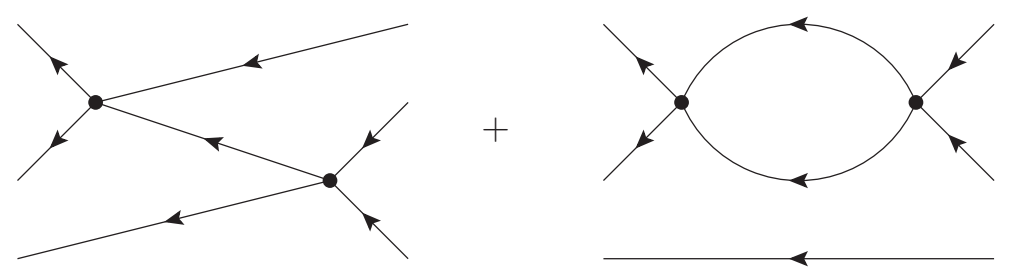

Figura 8.4: Diagramas de Feynman para a amplitude de espalhamento a 1-laço $V_{1}^{(2)}(\mathbf{k}, \mathbf{p})$. O primeiro gráfico corresponde ao valor principal da contribuição oriunda dos termos com apenas uma contração, enquanto que o segundo, à soma das contribuições das funções delta dos termos com apenas uma contração com as contribuições advindas do termo com duas contrações.

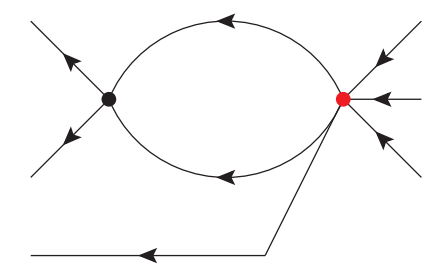

$(a)$

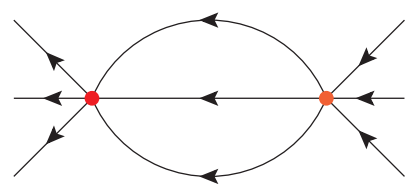

(b)

Figura 8.5: Gráficos de Feynman correspondentes às amplitudes de 1-laço: $(a) V_{2}^{(2)}(\mathbf{k}, \mathbf{p})$ e $V_{3}^{(2)}(\mathbf{k}, \mathbf{p})$, (b) $V_{4}^{(2)}(\mathbf{k}, \mathbf{p})$.

termos de ordem zero em $\hbar$. Vide figuras 8.4 e 8.5 para os gráficos de Feynman correspondentes.

A análise do produto temporalmente ordenado segue nas mesmas linhas do caso envolvendo apenas duas pseudopartículas, porquanto apenas o vértice de interação entre duas pseudopartículas contribui para $V_{1}^{(2)}$. Contudo, dado que os estados iniciais e finais envolvem três pseudopartículas, os termos com apenas uma contração também contribuem. Portanto precisamos levar em consideração as contribuições dos termos contendo apenas as seguintes contrações:

$$
\overrightarrow{\psi \vec{\phi}}, \bar{\psi} \phi, \vec{\psi} \bar{\phi} \vec{\psi}, \text { ou } \bar{\psi} \phi \bar{\psi} \phi
$$

Os termos com duas contrações contribuem da seguinte forma:

$$
\begin{aligned}
V_{1(2 C)}^{(2)}(\mathbf{k}, \mathbf{p}) & =\frac{1}{2}\left(-\frac{3 ! g_{2}}{4 m}\right)^{2} \mathcal{A}_{p, k}\left\{\overline { U } _ { 1 2 } ^ { k } \mathbb { P } \left[\left(p^{2} \times p^{1}\right)\left(k^{2} \times k^{1}\right) I_{0}\left(p^{1}, p^{2}\right)+\left(k^{2} \times k^{1}-\right.\right.\right. \\
- & \left.\left.\left.p^{2} \times p^{1}\right) I_{1}\left(p^{1}, p^{2}\right)-I_{2}\left(p^{1}, p^{2}\right)\right] \mathbb{P} U_{21}^{p} 8 \pi^{3} \delta^{(2)}\left(k^{1}+k^{2}-p^{1}-p^{2}\right) \delta\left(k^{3}-p^{3}\right)\right\}
\end{aligned}
$$

com as integrais $I_{0}\left(p^{1}, p^{2}\right), I_{1}\left(p^{1}, p^{2}\right)$ e $I_{2}\left(p^{1}, p^{2}\right)$ definidas no apêndice $G$. Novamente, ao usarmos as relações de completeza (F.2.3) e impormos que as pseudopartículas espalhadas estejam na camada de massa, podemos utilizar a identidade (8.2.13) conjuntamente com o nosso ordenamento dos 
momentos incidentes para concluir:

$$
V_{1(2 C)}^{(2)}(\mathbf{k}, \mathbf{p})=2\left(-\frac{i g_{2}}{4 m}\right)^{2}\left[\left(p^{2} \times p^{1}\right)^{2}+\left(p^{3} \times p^{1}\right)^{2}+\left(p^{3} \times p^{2}\right)^{2}\right]\langle\mathbf{k} \mid \mathbf{p}\rangle
$$

Já o cálculo dos termos contendo apenas uma contração é significativamente mais complicado

$$
\begin{aligned}
& V_{1(1 C)}^{(2)}(\mathbf{k}, \mathbf{p})=\left(-\frac{3 ! g_{2}}{4 m}\right)^{2} \mathbb{P}_{a c, b d} \mathbb{P}_{e g, f h} \mathcal{A}_{p, k}\left\{\left[-\left(p^{1} \times p^{2}\right)\left(k^{2} \times k^{3}\right) I+\epsilon^{\alpha \beta}\left(\left(p^{1} \times p^{2}\right)+\right.\right.\right. \\
& \left.\left.\left.+\left(k^{2} \times k^{3}\right) k_{\beta}^{1}\right) I_{\alpha}-\epsilon^{\alpha \beta} \epsilon^{\gamma \delta} k_{\beta}^{1} p_{\delta}^{3} I_{\alpha \gamma}\right]^{f a} \bar{u}^{c}\left(k_{1}^{1}\right) \bar{u}^{e}\left(k_{1}^{2}\right) \bar{u}^{g}\left(k_{1}^{3}\right) u^{b}\left(p_{1}^{1}\right) u^{d}\left(p_{1}^{2}\right) u^{h}\left(p_{1}^{3}\right)\right\}
\end{aligned}
$$

com as integrais $I, I_{\alpha}$ e $I_{\alpha \gamma}$ definidas no apêndice $G$ pelas equações (G.0.3). A propriedade fundamental das integrais supracitadas é que todas elas podem ser decompostas na soma de um termo envolvendo somente funções delta com outro compreendendo valores principais (v.p.):

$$
\underbrace{\frac{\Delta+m}{4 \omega(\Delta)} 2 \pi\left[\delta\left(\Delta_{0}-\omega(\Delta)\right)-\delta\left(\Delta_{0}+\omega(\Delta)\right)\right]}_{\text {termo de delta }}+\underbrace{\frac{i(\Delta+m)}{\Delta^{2}-m^{2}}}_{\text {termo de v.p. }},
$$

onde introduzimos a combinação conveniente de momentos $\Delta \equiv p^{1}+p^{2}-k^{1}$.

Essa decomposição da contribuição dos termos com uma única contração assume um papel central na discussão subseqüente. Como veremos, a contribuição do termo contendo as funções delta em (8.3.13) a (8.3.12) se combinará com (8.3.11) para fornecer a matriz $S$ fatorável a 1-laço, enquanto que a contribuição advinda dos termos de valor principal em (8.3.13) cancelará a contribuição oriunda da Lagrangeana de interação para três pseudopartículas no nível de árvore, que impedia a fatoração da matriz $S$ nessa ordem. Substituindo as integrais $I_{,} I_{\alpha}$ e $I_{\alpha \gamma}$, simplificamos severamente a expressão (8.3.12),

$$
\begin{aligned}
V_{1(1 C)}^{(2)}(\mathbf{k}, \mathbf{p}) & =\left(-\frac{3 ! i g_{2}}{4 m}\right)^{2} \mathcal{A}_{p, k}\left\{( p ^ { 2 } \times p ^ { 1 } + \Delta \times k ^ { 1 } ) ( k ^ { 3 } \times k ^ { 2 } + \Delta \times p ^ { 3 } ) \left[\frac{i}{\Delta^{2}-m^{2}}+\right.\right. \\
& \left.\left.+\frac{2 \pi}{4 \omega(\Delta)}\left(\delta\left(\Delta_{0}-\omega(\Delta)\right)-\delta\left(\Delta_{0}+\omega(\Delta)\right)\right)\right] \bar{U}_{123}^{k} M(\Delta) U_{213}^{p}\right\} 4 \pi^{2} \delta^{(2)}(\mathbf{k}-\mathbf{p})
\end{aligned}
$$

onde introduzimos

$$
\begin{aligned}
M(q):=\gamma^{3} \otimes(\phi l+m) \otimes \gamma^{3}-\gamma^{3} \otimes \gamma^{3}(\phi l+m) \otimes \mathbb{1}_{2}- \\
-\mathbb{1}_{2} \otimes(\not l+m) \gamma^{3} \otimes \gamma^{3}+\mathbb{1}_{2} \otimes \gamma^{3}(\phi+m) \gamma^{3} \otimes \mathbb{1}_{2} .
\end{aligned}
$$

A contribuição do termo contendo as funções delta é mais fácil de examinar, uma vez que tais funções implementam a condição de camada de massa para as pseudopartículas:

$$
\underbrace{\delta\left(\Delta_{0}-\omega(\Delta)\right)}_{\text {Energia Positiva }}-\underbrace{\delta\left(\Delta_{0}+\omega(\Delta)\right)}_{\text {Energia Negativa }} .
$$

Dado que, por hipótese, lidamos apenas com pseudopartículas com energia positiva, a condição 
$\Delta_{0}+\omega(\Delta)=0$ não pode ser satisfeita para nenhum par de momentos $\mathbf{p}$ e $\mathbf{k}$, portanto podemos desconsiderar a segunda função delta acima. Podemos rescrever convenientemente a função delta remanescente:

$$
\delta\left(\Delta_{0}-\omega(\Delta)\right)=\frac{p_{0}^{1} p_{0}^{2}}{\left|p^{2} \times p^{1}\right|}\left[\delta\left(k_{1}^{1}-p_{1}^{1}\right)+\delta\left(k_{1}^{1}-p_{1}^{2}\right)\right],
$$

dessa forma, a função delta correspondente à conservação de energia e momento totais pode ser simplificada, permitindo o uso da identidade (8.2.7), que em conjunto com o nosso ordenamento para os momentos iniciais, torna trivial o cálculo dos produtos espinoriais. Após longos e tediosos, porém, diretos cálculos, a contribuição do termo contendo as funções delta é reduzida à seguinte forma:

$$
4\left(-\frac{i g_{2}}{4 m}\right)^{2}\left[\left(p^{2} \times p^{1}\right)\left(p^{3} \times p^{1}\right)+\left(p^{2} \times p^{1}\right)\left(p^{3} \times p^{2}\right)+\left(p^{3} \times p^{2}\right)\left(p^{3} \times p^{1}\right)\right]\langle\mathbf{k} \mid \mathbf{p}\rangle .
$$

Podemos, pois, facilmente combinar as equações (8.3.11) e (8.3.17), obtendo:

$$
\begin{aligned}
V_{1}^{(2)}(\mathbf{k}, \mathbf{p}) & =2\left(-\frac{i g_{2}}{4 m}\right)^{2}\left[\left(p^{2} \times p^{1}\right)+\left(p^{3} \times p^{2}\right)+\left(p^{3} \times p^{1}\right)\right]^{2}\langle\mathbf{k} \mid \mathbf{p}\rangle+ \\
& +i\left(-\frac{3 ! i g_{2}}{4 m}\right)^{2} \mathcal{A}_{k, p}\left\{\frac{\left[p^{2} \times p^{1}+\Delta \times k^{1}\right]\left[k^{3} \times k^{2}+\Delta \times p^{3}\right]}{\Delta^{2}-m^{2}} \bar{U}_{123}^{k} \mathbb{M}(\Delta) U_{213}^{p}\right\} \\
& .4 \pi^{2} \delta^{(2)}(\mathbf{k}-\mathbf{p})
\end{aligned}
$$

Claramente, o primeiro termo de (8.3.18) corresponde a toda a contribuição necessária para se ter uma matriz $S$ fatorável a 1-laço (8.3.1). Assim da mesma forma que para o modelo de LandauLifshitz, vide capítulo 5, os termos remanescentes da amplitude de espalhamento a 1-laço (8.3.9), $V_{i}^{(2)}(\mathbf{k}, \mathbf{p}), i=2,3,4$, devem ser devidamente cancelados por contribuições de ordem mais alta, para que tenhamos uma matriz $S$ fatorável.

\subsection{Continuidade das Amplitudes de Espalhamento}

Antes de prosseguirmos com a demonstração da fatorabilidade da matriz $S$ em primeira ordem, uma digressão sobre uma sutileza que aparece durante o cálculo das integrais $I, I_{\alpha}$ and $I_{\alpha \gamma}$ [vide (8.3.12)] é necessária. Ressaltamos que tal sutileza está intimamente relacionada com a demonstração formal da fatorabilidade da matriz $S$ em modelos quânticos contínuos $[24,25,100]$. Considere uma dessas integrais, por exemplo:

$$
\begin{aligned}
& I=\iint d^{2} x d^{2} y e^{-i x \cdot \Delta-i y \cdot \tilde{\Delta}} \int \frac{d^{2} q}{4 \pi^{2}} e^{i q \cdot(x-y)} D(q) \\
& =(\Delta+m)\left\{\frac{2 \pi}{4 \omega(\Delta)}\left[\delta\left(\Delta_{0}-\omega(\Delta)\right)-\delta\left(\Delta_{0}+\omega(\Delta)\right)\right]+\frac{i}{\Delta^{2}-m^{2}}\right\} 4 \pi^{2} \delta^{(2)}(\mathbf{k}-\mathbf{p}) .
\end{aligned}
$$




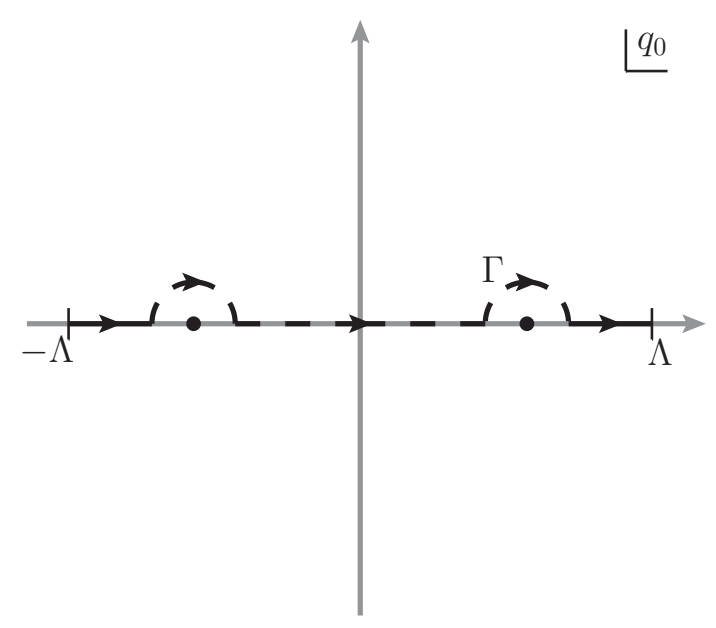

Figura 8.6: Contorno de integração sobre $q_{0}$.

Rigorosamente, o resultado em (8.4.1) só é válido se $\Delta_{0}-\omega(\Delta) \neq 0$. De fato, esse é o caso para a situação usual, onde só há um pólo na reta real, e escrevemos formalmente:

$$
\frac{1}{x \pm i 0}=\mp i \pi \delta(x)+\text { v.p. }\left(\frac{1}{x}\right)
$$

Entretanto, uma característica peculiar do modelo que consideramos é que, apesar de termos efetuado a quantização com respeito ao pseudovácuo (8.1.33), implicando um propagador puramente retardado, ainda precisamos levar em conta ambos os pólos do propagador quando calculamos a integral (8.4.1).

Consideremos, pois, as etapas cruciais de tal cálculo ${ }^{10}$. Em primeiro lugar, o contorno de integração sobre $q_{0}$ precisa ser dividido na união de três segmentos:

$$
(-\infty,-\omega(q)-\epsilon) \bigcup(-\omega(q)+\epsilon, \omega(q)-\epsilon) \bigcup(\omega(q)+\epsilon,+\infty),
$$

e em dois semicírculos ao redor dos pólos $\pm \omega(q)$, vide figura 8.6. A integração sobre a união dos segmentos produz o que chamamos de valor principal, ao passo que a integração sobre as semicircunferências resulta nas funções delta presentes em (8.4.1). Uma análise cuidadosa do termo de valor principal mostra que a integral típica que precisamos considerar é da seguinte forma:

$$
\int_{0}^{\infty} d x \sin (x \eta) \operatorname{si}(x \epsilon)=\left\{\begin{array}{rl}
-\frac{\pi}{2 \eta}, & \eta^{2}>\epsilon^{2} \\
0, & \eta^{2}<\epsilon^{2}
\end{array} .\right.
$$

onde $\eta \equiv \Delta_{0}-\omega(\Delta)$, e si $(x)$ é o seno integral (vide, por examplo, (6.252) de [120]). O resultado (8.4.1) só é válido para $\eta \neq 0$ e, conseqüentemente, $\eta^{2}>\epsilon^{2}$, posto que devemos considerar o limite $\epsilon \rightarrow 0$. Ao notarmos que o ponto $\eta=0$ corresponde à condição de integrabilidade, a saber, que $\circ$ conjunto dos momentos iniciais coincide com o dos momentos finais, concluímos que o resultado

\footnotetext{
${ }^{10}$ Para maiores detalhes, vide o cálculo completo exibido no apêndice G.2.
} 
(8.4.1) só é verdadeiro se os momentos não estiverem no ponto de integrabilidade.

No entanto, se $\eta=0$, ou seja, quando os momentos satisfazem a condição de integrabilidade, a integral (8.4.2) se anula, levando, pois, a um resultado necessariamente diferente de (8.4.1). Esse comportamento é um tanto quanto perturbador, uma vez que, a princípio, esperamos a continuidade da amplitude de espalhamento nos momento externos. Muito embora cada um desses termos não seja necessariamente contínuo individualmente, a continuidade nos momentos externos deve ser restabelecida quando a contribuição de todos os diagramas de uma dada ordem em $\hbar$ à amplitude de espalhamento for considerada. Essa singularidade artificial que aparece no ponto $\eta=0$ é devida unicamente à forma com que lidamos com a amplitude de espalhamento, ao forçarmos sua separação em termos das integrais (G.0.3).

Esse ponto especial torna a análise severamente mais complicada, porque introduz um número ainda maior de permutações a serem consideradas. De uma forma mais concreta, se tomarmos uma dada condição de integrabilidade, correspondendo a uma escolha particular dos conjuntos de momentos iniciais e finais, precisamos escrever explicitamente todos os termos presentes em (8.3.12) e examinar separadamente os subconjuntos determinados pela validade, ou não, dessa condição de integrabilidade. Toda essa complicação pode ser, no entanto, evitada, se empregarmos distribuições de pacotes de onda localizados para descrever as partículas envolvidas no espalhamento. Assim, ao considerarmos tais pacotes de onda, introduzimos um fator de amortecimento nas integrais, impedindo que o ponto $\eta=0$ seja atingido e, de fato, não precisamos sequer levá-lo em conta. Logo, concluímos que o resultado $(8.4 .1)^{11}$ é correto e podemos utilizá-lo para provar a fatorabilidade da matriz $S$. Notamos ademais que dessa forma a nossa análise está em completa consonância com a demonstração formal da fatorabilidade da matriz $S$ para modelos integráveis quânticos, que requer a consideração de pacotes de onda.

Para concluir essa discussão, comentamos que esse tipo de dificuldade não aparece em modelos mais simples, tal como o modelo de Landau-Lifshitz estudado no capítulo 5. Isso se deve ao fato de que o propagador do modelo de Landau-Lifshitz possui apenas um pólo, e, dessa forma, o caso problemático $\eta=0$ não contribui, porque na condição de integrabilidade o termo de valor principal é trivialmente zero. Entretanto, no modelo de AAF a presença de dois pólos leva a uma contribuição não-nula do termo de valor principal em $\eta=0$, porém, conforme explicado acima, o uso de pacotes de onda demanda que a contribuição dos termos de valor principal levem a uma função contínua nos momentos externos. Conseqüentemente, o caso $\eta=0$ não assume nenhum papel na discussão posterior.

\subsection{Fatoração da Matriz $S$}

Nessa seção, demonstramos a fatorabilidade da matriz $S$ em primeira ordem não-trivial ao mostrar que os segundos termos de (8.3.7) e (8.3.18) se cancelam mutuamente. A estratégia empregada consiste em expressar tais termos como funções das rapidezes, para assim tornar possível o cálculo dos produtos espinoriais sem precisar recorrer a uma identidade como (8.2.7) e, posteriormente,

\footnotetext{
${ }^{11}$ Bem como as outras integrais em (G.0.3).
} 
efetuar todas as antissimetrizações necessárias. Sejam as rapidezes:

$$
p_{0}^{i}=m \cosh \theta_{i}, p_{1}^{i}=m \sinh \theta_{i}, k_{0}^{i}=m \cosh \eta_{i}, k_{1}^{i}=m \sinh \eta_{i}, i=1,2,3 .
$$

A contribuição não-integrável no nível de árvore é facilmente computada:

$$
-\frac{i g_{3}}{16 m}(3 !)^{2} \mathcal{A}_{p, k}\left[k^{3} \times p^{3} \bar{U}_{123}^{k} \mathbb{Q} U_{123}^{p}\right]=-i g_{3} m \cosh \left(\sum_{i=1}^{3} \frac{\eta_{i}-\theta_{i}}{2}\right) F(\eta, \theta),
$$

onde introduzimos a seguinte função das rapidezes:

$$
F(\eta, \theta)=\frac{\sinh \left(\frac{\eta_{1}-\eta_{2}}{2}\right) \sinh \left(\frac{\eta_{1}-\eta_{3}}{2}\right) \sinh \left(\frac{\eta_{2}-\eta_{3}}{2}\right) \sinh \left(\frac{\theta_{1}-\theta_{2}}{2}\right) \sinh \left(\frac{\theta_{1}-\theta_{3}}{2}\right) \sinh \left(\frac{\theta_{2}-\theta_{3}}{2}\right)}{\sqrt{\cosh \eta_{1} \cosh \eta_{2} \cosh \eta_{3} \cosh \theta_{1} \cosh \theta_{2} \cosh \theta_{3}}} .
$$

O próximo passo é menos trivial e consiste em reduzir o segundo termo em (8.3.18) ao oposto de (8.5.1). Primeiramente, rescrevemos o argumento do antissimetrizador em termos das rapidezes:

$$
\frac{\left[p^{2} \times p^{1}+\Delta \times k^{1}\right]\left[k^{3} \times k^{2}+\Delta \times p^{3}\right]}{\Delta^{2}-m^{2}} \bar{U}_{123}^{k} M(\Delta) U_{213}^{p}=-\frac{8 m^{3} G(\eta, \theta)}{\sqrt{\prod_{i=1}^{3} \cosh \eta_{i} \cosh \theta_{i}}}
$$

com

$$
\begin{array}{r}
G(\eta, \theta)=\cosh \left(\frac{\eta_{2}-\eta_{3}}{2}\right) \cosh \left(\frac{\theta_{1}-\theta_{2}}{2}\right) \cosh \left(\frac{\eta_{2}-\theta_{3}}{2}\right) \operatorname{coth}\left(\frac{\eta_{1}-\theta_{1}}{2}\right) . \\
\cdot \sinh \left(\frac{\eta_{1}-\theta_{2}}{2}\right) \sinh \left(\frac{\eta_{3}-\theta_{3}}{2}\right) \sinh \left(\frac{\eta_{2}-\eta_{3}-\theta_{2}+\theta_{3}}{2}\right) .
\end{array}
$$

Em seguida, em vez de antissimetrizarmos diretamente a expressão acima, mostra-se mais conveniente rescrever $G(\eta, \theta)$ de forma a minimizar o número de funções hiperbólicas dependendo apenas da diferença de um $\eta \operatorname{com}$ um $\theta$. Obtemos, pois, a seguinte decomposição:

$$
G(\eta, \theta)=\frac{1}{4} \sum_{i=1}^{8} A_{i}(\eta, \theta)
$$

com os fatores $A_{i}(\eta, \theta)$ definidos no apêndice $H$ pelas equações (H.0.1). Naturalmente, podemos aplicar o antissimetrizador a cada $A_{i}(\eta, \theta)$ separadamente. Surpreendentemente,

$$
\mathcal{A}_{\theta, \eta}\left[A_{i}(\eta, \theta)\right]=0, \quad \text { para } i=2,4,5,7 \quad \text { e } \quad \mathcal{A}_{\theta, \eta}\left[A_{6}(\eta, \theta)\right]=\mathcal{A}_{\theta, \eta}\left[A_{8}(\eta, \theta)\right] .
$$

Fornecemos as expressões explícitas para os termos não-nulos no apêndice $H$, equações (H.0.2). Por consegüinte, a expressão para $G(\eta, \theta)$ é simplificada consideravelmente ao considerarmos sua antissimetrização:

$$
\mathcal{A}_{\theta, \eta}[G(\eta, \theta)]=\frac{1}{4} \mathcal{A}_{\theta, \eta}\left[A_{1}(\eta, \theta)+A_{3}(\eta, \theta)+2 A_{6}(\eta, \theta)\right],
$$

porém, ainda não corresponde ao oposto de (8.5.1).

Isso, entretanto, não é inesperado, pois ainda não impusemos a condição de que tanto o momento 
quanto a energia total devem ser conservados a (8.5.7). De fato, ao restringirmos os momentos $p^{i}$ e $k^{i}$ a essa subvariedade, através da implementação da função delta $\delta^{(2)}(\mathbf{k}-\mathbf{p})$, verificamos que ambos os termos $\mathcal{A}_{\theta, \eta}\left[A_{3}(\eta, \theta)\right]$ e $\mathcal{A}_{\theta, \eta}\left[A_{6}(\eta, \theta)\right]$ se anulam identicamente. A forma mais simples de demonstrar esse comportamento consiste em considerar os momentos no cone-de-luz:

$$
p_{ \pm}^{i}=e^{ \pm \theta} \quad \text { e } \quad k_{ \pm}^{i}=e^{ \pm \eta} \quad(i=1,2,3)
$$

para os quais a condição de camada de massa assume uma forma conveniente:

$$
p_{-}^{i}=\frac{1}{p_{+}^{i}} \quad \text { e } \quad k_{-}^{i}=\frac{1}{k_{+}^{i}} .
$$

Proporcionando, pois, não só uma forma adequada de se escrever $\mathcal{A}_{\theta, \eta}\left[A_{3}(\eta, \theta)\right]$ e $\mathcal{A}_{\theta, \eta}\left[A_{6}(\eta, \theta)\right]$, dado que dependem apenas de funções hiperbólicas, mas também de implementar a conservação de momento e energia totais sem introduzir relações não-lineares entre os momentos. Dessa forma, após longas e tediosas contas, encontramos:

$$
\mathcal{A}_{\theta, \eta}\left[A_{3}(\eta, \theta)\right] \delta^{(2)}(\mathbf{k}-\mathbf{p})=0 \quad \text { e } \quad \mathcal{A}_{\theta, \eta}\left[A_{6}(\eta, \theta)\right] \delta^{(2)}(\mathbf{k}-\mathbf{p})=0
$$

Logo, temos que:

$$
\begin{aligned}
(3 !)^{2} \mathcal{A}_{\theta, \eta}[G(\eta, \theta)] \delta^{(2)}(\mathbf{k}-\mathbf{p}) & =\frac{1}{4}(3 !)^{2} \mathcal{A}_{\theta, \eta}\left[A_{1}(\eta, \theta)-A_{3}(\eta, \theta)\right] \delta^{(2)}(\mathbf{k}-\mathbf{p}) \\
= & 2 \cosh \left(\sum_{i=1}^{3} \frac{\eta_{i}-\theta_{i}}{2}\right) \sinh \left(\frac{\eta_{1}-\eta_{2}}{2}\right) \sinh \left(\frac{\eta_{1}-\eta_{3}}{2}\right) . \\
& \cdot \sinh \left(\frac{\eta_{2}-\eta_{3}}{2}\right) \sinh \left(\frac{\theta_{1}-\theta_{2}}{2}\right) \sinh \left(\frac{\theta_{1}-\theta_{3}}{2}\right) . \\
& \cdot \sinh \left(\frac{\theta_{2}-\theta_{3}}{2}\right) \delta^{(2)}(\mathbf{k}-\mathbf{p}) .
\end{aligned}
$$

E finalmente, obtemos:

$$
\begin{aligned}
i\left(-\frac{3 ! i g_{2}}{4 m}\right)^{2} \mathcal{A}_{k, p} & \left\{\frac{\left[p^{2} \times p^{1}+\Delta \times k^{1}\right]\left[k^{3} \times k^{2}+\Delta \times p^{3}\right]}{\Delta^{2}-m^{2}} \bar{U}_{123}^{k} M(\Delta) U_{213}^{p}\right\} 4 \pi^{2} \delta^{(2)}(\mathbf{k}-\mathbf{p})= \\
& =i g_{2}^{2} m \cosh \left(\sum_{i=1}^{3} \frac{\eta_{i}-\theta_{i}}{2}\right) F(\eta, \theta) 4 \pi^{2} \delta^{(2)}(\mathbf{k}-\mathbf{p}) .
\end{aligned}
$$

Ao notarmos que também há um fator de $4 \pi \delta^{(2)}(\mathbf{k}-\mathbf{p})$ multiplicando o termo (8.5.1), concluímos que o termo que impedia a fatoração da matriz $\mathbf{S}$ no nível de árvore (8.5.1) é realmente cancelado pela contribuição advinda do valor principal dos diagramas com contrações simples a 1-laço (8.5.10), contanto que a seguinte relação entre as constantes de acoplamento seja satisfeita:

$$
g_{2}^{2}=g_{3}
$$

Esse é um dos resultados centrais de nossa análise, e fornece a condição necessária para a integrabilidade quântica do modelo de AAF. Ressaltamos que a condição (8.5.11) está em plena concordância 
com as dimensões de massa que atribuímos às constantes de acoplamento $g_{2}$ e $g_{3}$, bem como com a ação clássica (8.1.10). De fato, com (8.5.11) podemos ir da ação (8.1.10) com constantes de acoplamento de volta a sua contraparte sem dimensões de massa, (8.1.7), com os coeficientes corretos.

Portanto, se denotarmos $g=g_{2}$, a amplitude de espalhamento para três pseudopartículas assume a seguinte forma:

$$
\langle\mathbf{k}|\hat{S}| \mathbf{p}\rangle=\left\{1+2 \sum_{n=1}^{2}\left[\left(-\frac{i g}{4 m}\right)\left(p^{2} \times p^{1}+p^{3} \times p^{1}+p^{3} \times p^{2}\right)\right]^{n}\right\}\langle\mathbf{k} \mid \mathbf{p}\rangle+O\left(g^{3}\right),
$$

onde incluímos as amplitudes a 1-laço, $V_{i}^{(2)}(\mathbf{k}, \mathbf{p}), i=2,3,4$, em $O\left(g^{3}\right)$, pois

$$
V_{2}^{(2)}(\mathbf{k}, \mathbf{p}) \sim V_{3}^{(2)}(\mathbf{k}, \mathbf{p}) \sim g_{2} g_{3} \sim g^{3} \quad \text { e } \quad V_{4}^{(2)}(\mathbf{k}, \mathbf{p}) \sim g_{3}^{2} \sim g^{4}
$$

Claramente, a expressão (8.5.12) é da forma (8.3.8), permitindo, pois, a extração da matriz $S$ no setor de três pseudopartículas a 1-laço:

$$
S\left(p^{1}, p^{2}, p^{3}\right)=1+2 \sum_{n=1}^{2}\left[\left(-\frac{i g}{4 m}\right)\left(p^{2} \times p^{1}+p^{3} \times p^{1}+p^{3} \times p^{2}\right)\right]^{n}+O\left(g^{3}\right) .
$$

Comparando (8.5.13) com (8.3.1), que obtivemos unicamente a partir do conhecimento da matriz $S$ para duas pseudopartículas, verificamos que (8.5.13) corresponde à matriz $S$ para três pseudopartículas fatorável em segunda ordem em $g$. Conseqüentemente, constatamos que mesmo para se ter a fatorabilidade da matriz $S$ apenas no nível de árvore, é imperativo que se considere amplitudes de ordem mais alta, uma vez que elas proporcionam as contribuições responsáveis pelo cancelamento dos termos não-integráveis de ordem mais baixa.

\subsection{Comentários Finais}

Não é difícil ver que esse notável esquema de cancelamentos seria completamente arruinado, se não tivéssemos corrigido o fator de $\frac{1}{2}$ perdido em [43] e a inconsistência entre os sinais de [35], porquanto esses foram fundamentais para garantir o ajuste preciso entre os termos de interação necessário para a fatorabilidade da matriz $S$. A retificação dos sinais presentes na ação (8.1.10) apresenta outras conseqüências significativas, a mais proeminente sendo a dedução do inverso da matriz $S$ proposta por [35].

Interessantemente, nossa matriz $S$ no setor de duas partículas (8.2.17), quando escrita em termos de rapidezes:

$$
S\left(\theta_{1}, \theta_{2}\right)=\frac{1-\frac{i m g_{2}}{4} \sinh \left(\theta_{1}-\theta_{2}\right)}{1+\frac{i m g_{2}}{4} \sinh \left(\theta_{1}-\theta_{2}\right)}
$$

é deveras similar à matriz $S$ para o espalhamento de duas partículas no modelo de Thirring:

$$
S_{\text {Thirring }}\left(\theta, \theta^{\prime}\right)=\frac{1-\frac{i g}{2} \tanh \frac{\theta-\theta^{\prime}}{2}}{1+\frac{i g}{2} \tanh \frac{\theta-\theta^{\prime}}{2}}
$$


cuja Lagrangeana,

$$
\mathcal{L}_{\text {Thirring }}=\bar{\psi}(i \not \partial-m) \psi-\frac{g_{T}}{2} \bar{\psi} \gamma^{\mu} \psi \bar{\psi} \gamma_{\mu} \psi
$$

é consideravelmente mais simples do que a do modelo de AAF (8.1.10), que além de possuir um segundo termo de interação, envolvendo três partículas, apresenta acoplamentos derivativos, que sinalizam uma maior singularidade da teoria.

Estamos finalmente em posição para escrever as equações de Bethe corretas ${ }^{12}$ para o modelo de AAF:

$$
e^{i J m \sinh \theta_{i}}=\prod_{k \neq i} \frac{1+\frac{i m g_{2}}{4} \sinh \left(\theta_{i}-\theta_{k}\right)}{1-\frac{i m g_{2}}{4} \sinh \left(\theta_{i}-\theta_{k}\right)}
$$

que constituem o primeiro passo para a análise dos estados ligados e excitados, uma vez que suas soluções permitem o preenchimento do mar de Dirac e conseqüente reconstrução do verdadeiro estado fundamental, bem como a obtenção da matriz $S$ física.

Para concluir nossa discussão sobre a integrabilidade quântica do modelo de AAF, fazemos uma comparação mais detalhada com a análise e os resultados de [35]. Com esse intuito, retornamos à ação original do modelo de AAF (8.1.2) e consideramos a transformação: ${ }^{13}$

$$
\tau \rightarrow-\tau, \quad \sigma \rightarrow-\sigma, \quad \rho^{\alpha} \rightarrow-\rho^{\alpha},
$$

Dessa forma obtemos:

$$
\begin{aligned}
S & =\frac{\sqrt{\lambda}}{2 \pi} \int d \tau \int_{0}^{\frac{2 \pi J}{\sqrt{\lambda}}} d \sigma\left[\frac{i}{2}\left(\bar{\chi} \gamma^{\alpha} \partial_{\alpha} \chi-\partial_{\alpha} \bar{\chi} \gamma^{\alpha} \chi\right)-\bar{\chi} \chi-\right. \\
& \left.-\frac{1}{4} \epsilon^{\alpha \beta}\left(\bar{\chi} \partial_{\alpha} \chi \bar{\chi} \gamma^{3} \partial_{\beta} \chi-\partial_{\alpha} \bar{\chi} \chi \partial_{\beta} \bar{\chi} \gamma^{3} \chi\right)-\frac{1}{16} \epsilon^{\alpha \beta}(\bar{\chi} \chi)^{2} \partial_{\alpha} \bar{\chi} \gamma^{3} \partial_{\beta} \chi\right],
\end{aligned}
$$

onde denotamos $\chi(\tau, \sigma) \equiv \psi(-\tau,-\sigma)$.

A ação (8.6.6) difere da que examinamos (8.1.4) pelo sinal do termo quártico e corresponde essencialmente à considerada por [35], escrita em termos dos campos $\chi(\tau, \sigma)$. Portanto, essas duas ações já são diferentes classicamente. Uma inspeção cuidadosa revela que a matriz $S$ de [35], de fato, corresponde ao espalhamento de partículas descritas pelos campos $\chi(\tau, \sigma)$ com a dinâmica fornecida pela ação (8.6.6), e não em termos dos campos originais, $\psi(\tau, \sigma)$. Podemos facilmente constatar através da expansão em modos de ambos os campos, que a transição de $\chi(\tau, \sigma)$ para $\psi(\tau, \sigma)$ corresponde a troca dos operadores descrevendo partículas e antipartículas $a^{\dagger} \longleftrightarrow b^{\dagger}$, visto que a transformação (8.6.5) envolve inversão temporal. Posto dessa forma, não é surpreendente que a matriz $S$ obtida em [35] seja o inverso de (8.6.1), e para fazermos uma conexão direta entre os dois resultados, precisamos fazer $g_{2} \rightarrow-g_{2}$. Finalmente, é importante enfatizar que a condição de integrabilidade quântica (8.5.11) é invariante sob a transformação: $g_{2} \rightarrow-g_{2}$, e conseqüentemente, ambos os modelos (8.1.10) e (8.6.6) são integráveis.

\footnotetext{
${ }^{12}$ Notamos que as equações de Bethe para o modelo de AAF, originalmente deduzidas por [35], estão incorretas, uma vez que foram obtidas a partir do inverso da matriz $S$.

${ }^{13}$ Nesse caso também desprezamos o termo constante, fixamos $\kappa=\frac{\sqrt{\lambda}}{2}$ e mudamos a base das matrizes de Dirac através da transformação de similaridade (B.3.3) .
} 



\section{Conclusão}

"Ao vencedor, as batatas."

Machado de Assis - Quincas Borba

Nesta tese, motivados pelas dificuldades encontradas no processo de quantização da teoria de supercordas do tipo IIB em $A d S_{5} \times S^{5}$, estudamos a integrabilidade quântica de modelos que surgem de seus subsetores. Naturalmente, esse é um problema cujo interesse ultrapassa as fronteiras da teoria de cordas, sendo extremamente relevante no contexto da correspondência AdS/CFT, e no desenvolvimento de métodos de quantização para sistemas (integráveis) contínuos. Os dois modelos considerados na presente tese, o modelo de Landau-Lifshitz (LL) e o modelo de Alday-ArutyunovFrolov (AAF), fornecem exemplos representativos das dificuldades associadas com a aplicação do método do espalhamento inverso para a análise da integrabilidade quântica em modelo contínuos, relacionadas com a natureza singular de suas interações. Notadamente, não é possível utilizar as identidades de traço, de forma que obter o conjunto de cargas quânticas conservadas em involução, expressas na forma de integrais de densidades locais, constitui, em geral, um problema de formidável complexidade.

Contudo, existe uma abordagem alternativa para investigar a integrabilidade quântica de um dado modelo, que consiste em considerá-lo como uma teoria de campos, e, através de métodos perturbativos, estudar a fatorabilidade de sua matriz $S$. Verificamos em ambos os modelos de LL e de AAF a presença do mesmo mecanismo intrincado de cancelamentos necessário para garantir que a matriz $S$ seja fatorável, mesmo na primeira ordem não-trivial. Esse mecanismo deve ser responsável por garantir a fatorabilidade da matriz $S$ em todas as ordens, mas, devido à complicada análise diagramática envolvida, acreditamos que o problema de generalizar a nossa construção para ordens superiores exija um formalismo alternativo. Além disso, dada as naturezas díspares desses modelos, um é bosônico, enquanto o outro é fermiônico, só para citar uma, é razoável esperar que esse mecanismo esteja presente em diversos outros modelos integráveis.

Um ponto sutil de nossa análise, e que merece ser investigado posteriormente com mais cuidado é a renormalização dos modelos de LL e AAF, em particular, no que concerne às constantes de acoplamento. No contexto do modelo de LL, precisamos introduzi-las para manter controle sobre a ordem perturbativa de nossos cálculos, e, ao seu final, fixá-las como a unidade para garantir a fatorabilidade da matriz $S$, o que fizemos sem considerar os efeitos da renormalização, que são importantes para validar essa hipótese. Similarmente, no modelo de AAF a renormalização pode afetar a relação entre as duas constantes de acoplamento, que também é fundamental para a fatorabilidade 
da matriz $S$. Ademais, o procedimento de renormalização do modelo do ponto de vista da teoria de campos está relacionado com regularização das singularidades que surgem no contexto do MEIQ, ao considerarmos a álgebra dos operadores locais. Logo, esse tipo de análise levaria ao estabelecimento de um relação explícita entre os dois métodos.

O nosso estudo do modelo de AAF também mostrou em que sentido a integrabilidade quântica fornece um teste de consistência, revelando alguns erros na literatura prévia. Em particular, a matriz $S$ correta é o inverso da originalmente obtida por [35]. Assim, uma nova análise dos estados ligados e com energia negativa se faz necessária.

Uma das formas de se evitar as dificuldades inerentes à abordagem perturbativa, consiste em quantizar o sistema diretamente. Estudamos esse problema no exemplo do modelo de LL, porém apenas no caso $^{14} \mathfrak{s u}(1,1)$, e propusemos um novo método de quantização para modelos integráveis contínuos que não depende de nenhum esquema de regularização. Explicamos também como obter as identidades de traço quânticas e estabelecemos uma conexão explícita com o MEIQ. É importante ressaltar que o nosso método se baseia em diversos aspectos da integrabilidade, aparentemente distintos, mas intrinsecamente interrelacionados. Primeiramente, a diagonalização das cargas requer a regularização dos operadores, levando a uma álgebra altamente não-local, que, contudo, corresponde apenas a representações integráveis. Em segundo lugar, a natureza singular do modelo de LL também demanda a construção do espaço de Hilbert estendido. Assim, a regularização dos operadores, sujeita à integrabilidade quântica, em conjunto com a construção das extensões auto-adjuntas levam a inúmeras conseqüências importantes, como, por exemplo, a fatorabilidade da matriz $S$, a natureza da representação e a relação com o MEIQ.

Em suma, apesar de o MEIQ ser um procedimento relativamente direto, a presença de singularidades requer uma análise mais cuidadosa do produto operatorial e de sua regularização, bem como a construção das extensões auto-adjuntas. Muito embora, essa abordagem matematicamente correta seja, em geral, ignorada para a vasta maioria dos modelos físicos, ela é essencial para o modelo de LL e vários outros, como, por exemplo, o modelo de AAF. Acreditamos que o nosso método seja diretamente aplicável para a quantização de tais modelos. Em particular, seria extremamente interessante obter os espaços de Hilbert estendidos para os modelo de de AAF e de LL (su(2)). Não esperamos, todavia, que a construção dos operadores auto-adjuntos e suas correspondentes extensões coincidam, uma vez que carregam um número de graus de liberdade diferente. Além do mais, é uma questão crucial entender se essas duas extensões podem ser acomodadas em um espaço maior, que englobe tanto os graus de liberdade bosônicos, quanto os fermiônicos. Esse é um passo importantíssimo para a compreensão do espaço de Hilbert completo para a supercorda em $A d S_{5} \times S^{5}$.

\footnotetext{
${ }^{14}$ Notamos, entretanto, que o caso relevante no contexto da teoria de cordas e, conseqüentemente, da correspondência AdS/CFT é o su(2).
} 
Parte IV

Apêndices 



\section{Apêndice A}

\section{Algumas Demonstrações}

"Nobody is my name. My father and mother call me Nobody, as do all the others who are my companions."

Odysseus, in his tale

Richmond Lattimore, The Odyssey of Homer

Neste apêndice reunimos as demonstrações de alguns teoremas apresentados ao longo do texto.

\section{A.1 Demonstração do Teorema de Darboux}

Nesta seção, demonstramos o teorema de Darboux (teorema 2.2.6), seguindo a demonstração exibida em [46].

Teorema (2.2.6). Em qualquer variedade simplética $\left(\Gamma_{2 n}, \omega\right)$ é possível introduzir, localmente ao redor de um ponto $m_{0}$, coordenadas canônicas $\xi=\left(p_{i}, q_{i}\right)$ tais que $\omega=d p^{i} \wedge d q_{i}$. Além do mais, é possivel escolher $p_{1}$ como qualquer função $H$ em $\Gamma_{2 n}$ tal que $d H\left(m_{0}\right) \neq 0$.

Demonstração: A demonstração será feita por indução em $n$ na dimensão do espaço de fase $\Gamma_{2 n}$. Começamos com o caso $n=1$ e consideramos a construção das coordenadas $p_{1}$ e $q_{1}$.

Seja $m_{0} \in \Gamma_{2 n}$, considere um sistema de coordenadas $y \in \mathbb{R}^{2 n}$ ao redor de $m_{0}$ e defina $p_{1}(y)=H(y)$. Podemos supor sem perda de generalidade que $p_{1}\left(m_{0}\right)=0$. Introduzimos, então, o campo vetorial Hamiltoniano associado a $p_{1}, X_{p_{1}}$, que é não-nulo na vizinhança de $m_{0}$ considerada. Em seguida, considere a hipersuperfície $\Sigma_{0}$ passando por $m_{0}$, mas que não seja tangente a $X_{p_{1}}$, ou seja, $\Sigma_{0}$ é transversal a $X_{p_{1}}$, conforme a figura A.1. Queremos definir $q_{1}$ de forma que $\left\{p_{1}, q_{1}\right\}=1$ e $q_{1} \mid \Sigma_{0}=0$. Portanto, para qualquer trajetória do fluxo gerado por $X_{p_{1}}$, temos que $\dot{q}_{1}=\left\{p_{1}, q_{1}\right\}=1$ e, conseqüentemente, $q_{1}(y)-q_{1}(z)=q_{1}(y)=t$ com $z$ sendo o ponto onde a trajetória intercepta $\Sigma_{0}$, ou seja, $q_{1}(z)=0$. Pela hipótese de transversalidade, podemos definir, para qualquer $y$ suficientemente próximo a $\Sigma_{0}, q_{1}(y)$ como o tempo necessário para se ir de $z \in \Sigma_{0}$ a esse y pela trajetória gerada pelo fluxo de $X_{p_{1}}$. Assim, a vizinhança de $m_{0}$ é folheada tanto por hipersuperfícies $\Sigma_{t} \operatorname{com} q_{1}(y)=t$, quanto por hipersuperfícies $\Phi_{s}$ onde $p_{1}(y)=s$. 


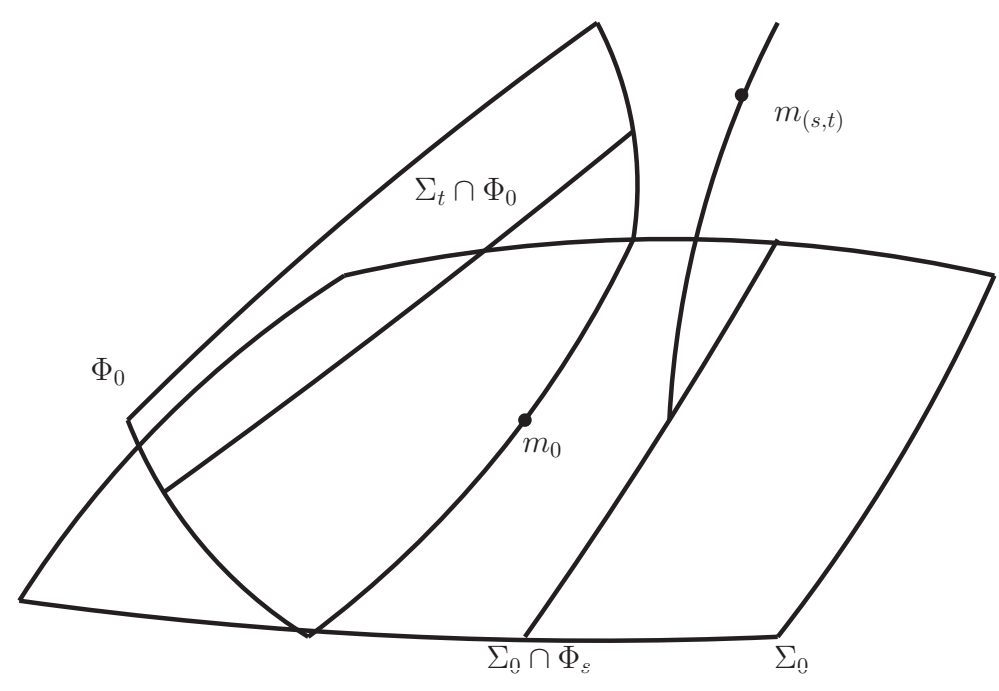

Figura A.1: Folheação do espaço de fase pelas hipersuperfícies $\Sigma_{t}$ e $\Phi_{s}$.

Mostramos a seguir que é possível resolver simultaneamente:

$$
\partial_{s} y=-X_{q_{1}}(y) \text { e } \partial_{t} y=X_{p_{1}}(y)
$$

e desse modo podemos escrever $y=y(s, t, z)$ com $z \in \Sigma_{0} \cap \Phi_{0}$. Claramente, para qualquer função $f(y)$ temos que

$$
\partial_{s} f=-X_{q_{1}} f=\left\{-q_{1}, f\right\} \quad \text { e } \partial_{t} f=X_{p_{1}} f=\left\{p_{1}, f\right\},
$$

e usando a identidade de Jacobi obtemos:

$$
\partial_{s}\left(\partial_{t} f\right)-\partial_{t}\left(\partial_{s} f\right)=-\left\{q_{1},\left\{p_{1}, f\right\}\right\}+\left\{p_{1},\left\{q_{1}, f\right\}\right\}=\left\{\left\{p_{1}, q_{1}\right\}, f\right\}=0,
$$

pois $\left\{p_{1}, q_{1}\right\}=1$.

Como $X_{p_{1}} p_{1}=\left\{p_{1}, p_{1}\right\}=0$ temos que $X_{p_{1}}$ é tangente a qualquer $\Phi_{s}$, em particular, a $\Phi_{0}$; analogamente, $X_{q_{1}}$ é tangente a qualquer $\Sigma_{t}$ e, em particular, a $\Sigma_{0}$. Dessa forma, ambos são transversos a hipersuperfície definida por $\Sigma_{0} \cap \Phi_{0}$ e independentes, pois $\left\{p_{1}, q_{1}\right\}=\omega\left(X_{p_{1}}, X_{q_{1}}\right)=$ $1 \neq 0$. Finalmente, para qualquer vetor $V$ tangente a $\Sigma_{0} \cap \Phi_{0}$ temos que $\omega\left(V, X_{p_{1}}\right)=\omega\left(V, X_{q_{1}}\right)=0$ porque $\omega\left(V, X_{p_{1}}\right)=V \cdot p_{1}, \omega\left(V, X_{q_{1}}\right)=V \cdot q_{1}$ e $\left.p_{1}\right|_{\Sigma_{0} \cap \Phi_{0}}=\left.q_{1}\right|_{\Sigma_{0} \cap \Phi_{0}}=0$ são constantes. Concluímos, pois, que a restrição de $\omega$ a $\Sigma_{0} \cap \Phi_{0}$ é não-degenerada.

Com o término da construção para $n=1$, podemos usar a hipótese de indução para construir as coordenadas canônicas $p_{i}$ e $q_{i}$ com $i \geq 2$ na variedade simplética com dimensão $(2 n-2), \Sigma_{0} \cap \Phi_{0}$. Estendemos essas coordenadas como funções sobre $\Gamma_{2 n}$ ao fixarmos $p_{i}(y)=p_{i}(z)$ e $q_{i}(y)=q_{i}(z)$ para $i \geq 2$ e $y=y\left(p_{1}, q_{1}, z\right)$ com $z \in \Sigma_{0} \cap \Phi_{0}$. Implicando sua constância ao longo dos fluxos $X_{p_{1}} \mathrm{e}$ $X_{q_{1}}$, de forma que:

$$
\left\{p_{1}, p_{i}\right\}=\left\{p_{1}, q_{i}\right\}=\left\{q_{1}, p_{i}\right\}=\left\{q_{1}, q_{i}\right\}=0 .
$$

Resta mostrar que a forma simplética $\omega$ em $\Gamma_{2 n}$ é $\tilde{\omega}=\sum_{i=1}^{n} d p_{i} \wedge d q_{i}$. Fazemos isso inicialmente 
para qualquer ponto $p_{1}=q_{1}=0$ de $\Sigma_{0} \cap \Phi_{0}$. Claramente, qualquer vetor $V$ tangente a $\Gamma_{2 n}$ nesse ponto pode ser decomposto como a soma de um vetor $V_{1}$ contido no espaço gerado por $X_{p_{1}}$ e $X_{q_{1}}$, com um vetor $V_{2}$ tangente a $\Sigma_{0} \cap \Phi_{0}$.

$$
\begin{aligned}
\omega\left(V_{1}+V_{2}, W_{1}+W_{2}\right) & =\omega\left(V_{1}, W_{1}\right)+\omega\left(V_{1}, W_{2}\right)+\omega\left(V_{2}, W_{1}\right)+\omega\left(V_{2}, W_{2}\right) \\
& =\left(d p_{1} \wedge d q_{1}\right)\left(V_{1}, W_{1}\right)+\left(\sum_{i=2}^{n} d p_{i} \wedge d q_{i}\right)\left(V_{2}, W_{2}\right)
\end{aligned}
$$

pois $\omega\left(V_{1}, W_{2}\right)=\omega\left(V_{2}, W_{1}\right)=0$, para ver que $\omega\left(V_{1}, W_{1}\right)=\left(d p_{1} \wedge d q_{1}\right)\left(V_{1}, W_{1}\right)$, basta considerar $V_{1}=X_{p_{1}}$ e $W_{1}=X_{q_{1}}$ e notar que $\omega\left(X_{p_{1}}, X_{q_{1}}\right)=\left\{p_{1}, q_{1}\right\}=1$, bem como $\left(d p_{1} \wedge d q_{1}\right)\left(X_{p_{1}}, X_{q_{1}}\right)=$ $\left\{p_{1}, q_{1}\right\}=1$; finalmente, para $\omega\left(V_{2}, W_{2}\right)$ usamos a hipótese de indução. Agora, para mostrar que essa igualdade é válida para todo $\Gamma_{2 n}$, precisamos considerar a evolução Hamiltoniana $U_{(s, t)}$ sob os fluxos de $X_{p_{1}}$ e $X_{q_{1}}$. Em coordenadas, temos

$$
\left(p_{1}, q_{1}, p_{2}, q_{2}, \ldots, p_{n}, q_{n}\right) \mapsto\left(p_{1}+s, q_{1}+t, p_{2}, q_{2}, \ldots, p_{n}, q_{n}\right) .
$$

De onde vemos que a forma $\tilde{\omega}$ é trivialmente invariante. Pelo outro lado, a forma simplética $\omega$ também é invariante sob a evolução Hamiltoniana. Como mostramos que $\omega=\tilde{\omega}$ para $p_{1}=q_{1}=0$, temos que tais formas coincidem em todos os pontos.

\section{A.2 Propriedades Analíticas da Matriz de Transição para o Mo- delo NLS}

Nesta seção, demonstramos a proposição 2.6.3 e o corolário 2.6.4. Para isso precisamos de alguns resultados auxiliares que permitam uma melhor compreensão das propriedades analíticas da matriz de transição (e de monodromia) do modelo NLS com condições de contorno quasiperiódicas. Como primeiro passo, consideramos o problema linear fundamental dado pela equação diferencial (2.6.2) e o rescrevemos como uma equação integral.

Lema A.2.1. As equações integrais

e

$$
T(x, y ; \lambda)=E(x-y ; \lambda)+\int_{y}^{x} d z T(x, z ; \lambda) L_{x}^{0}(z) E(z-y ; \lambda)
$$

$$
T(x, y ; \lambda)=E(x-y ; \lambda)+\int_{y}^{x} d z E(x-z ; \lambda) L_{x}^{0}(z) T(z, y ; \lambda)
$$

com $y \leq x$ e $E(x ; \lambda)=e^{\lambda L_{x}^{\frac{1}{x} x}}$ são equivalentes ao problema linear auxiliar (2.6.2) para o modelo NLS com condições de contorno quasiperiódicas. 
Demonstração: Derivemos (A.2.1a) com respeito a $x$

$$
\begin{aligned}
\partial_{x} T(x, y ; \lambda) & =\lambda L_{x}^{1} E(x-y ; \lambda)+\left.T(x, z ; \lambda) L_{x}^{0}(z) E(z-y ; \lambda)\right|_{z=x}+ \\
& +\int_{y}^{x} d z \partial_{x} T(x, z ; \lambda) L_{x}^{0}(z) E(z-y ; \lambda) \\
& =\lambda L_{x}^{1} E(x-y ; \lambda)+\mathbb{1}_{2} L_{x}^{0}(x) E(x-y ; \lambda)+\int_{y}^{x} d z L_{x}(x ; \lambda) T(x, z ; \lambda) L_{x}^{0}(z) E(z-y ; \lambda) \\
& =L_{x}(x ; \lambda)\left[E(x-y ; \lambda)+\int_{y}^{x} d z T(x, z ; \lambda) L_{x}^{0}(z) E(z-y ; \lambda)\right] .
\end{aligned}
$$

Ademais, é claro que $\left.T(x, y ; \lambda)\right|_{x=y}=E(0 ; \lambda)=\mathbb{1}_{2}$. Já para (A.2.1b), derivamos com respeito a $y$

$$
\begin{aligned}
\partial_{y} T(x, y ; \lambda) & =-\lambda L_{x}^{1} E(x-y ; \lambda)-\left.E(x-z ; \lambda) L_{x}^{0}(z) T(z, y ; \lambda)\right|_{z=y}+ \\
& +\int_{y}^{x} d z E(x-z ; \lambda) L_{x}^{0}(z) \partial_{y} T(z, y ; \lambda) \\
& =-\lambda L_{x}^{1} E(x-y ; \lambda)-E(x-y ; \lambda) L_{x}^{0}(y) \mathbb{1}_{2}-\int_{y}^{x} d z E(x-z ; \lambda) L_{x}^{0}(z) T(z, y ; \lambda) L_{x}(y) \\
& =-\left[E(x-y ; \lambda)+\int_{y}^{x} d z E(x-z ; \lambda) L_{x}^{0}(z) T(z, y ; \lambda)\right] L_{x}(y) .
\end{aligned}
$$

A função $E(x ; \lambda)$ é a solução do problema linear fundamental $(2.6 .2) \operatorname{com} L_{x}^{0}(x)=0$ e satisfaz a seguinte propriedade.

Lema A.2.2. A seguinte relação é válida:

$$
E(x ; \lambda) L_{x}^{0}(y)=L_{x}^{0}(y) E(-x ; \lambda)
$$

Demonstração: Calculemos:

$$
\begin{aligned}
E(x ; \lambda) L_{x}^{0}(y) & =\sum_{n=0}^{\infty}\left(\frac{\lambda}{2 i} \gamma^{3} x\right)^{n} i \sqrt{g}\left[\psi(y) \gamma^{+}-\bar{\psi}(y) \gamma^{-}\right] \\
& =i \sqrt{g}\left[\psi(y) \gamma^{+}-\bar{\psi}(y) \gamma^{-}\right] \sum_{n=0}^{\infty}\left(\frac{-\lambda}{2 i} \gamma^{3} x\right)^{n} \\
& =L_{x}^{0}(y) E(-x ; \lambda),
\end{aligned}
$$

onde usamos que $\gamma^{3} \gamma^{ \pm}=-\gamma^{ \pm} \gamma^{3}$.

As equações (A.2.1) são equações integrais de Volterra, logo suas iterações são absolutamente convergentes. De fato, ao usarmos (A.2.2) podemos coletar todos os fatores $E(\cdot ; \lambda)$ em um dos lados do produto com $L_{x}^{0}$ e usar a propriedade de superposição de $E(x ; \lambda)$, para obter o seguinte lema. 
Lema A.2.3. As equações integrais de Volterra (A.2.1) podem ser representadas da seguinte forma:

$$
\begin{aligned}
& T(x, y ; \lambda)=E(x-y ; \lambda)+\int_{2 y-x}^{x} d z K(x, y, z) E(z-y ; \lambda), \\
& T(x, y ; \lambda)=E(x-y ; \lambda)+\int_{y}^{2 x-y} d z E(x-z ; \lambda) \tilde{K}(x, y, z),
\end{aligned}
$$

com os núcleos $K$ e $\tilde{K}$ satisfazendo

$$
\begin{aligned}
& K(x, y, z)=\frac{1}{2} L_{x}^{0}\left(\frac{x+z}{2}\right)+\int_{y}^{\frac{x+z}{2}} d s K(x, s, 2 s-z) L_{x}^{0}(s) \quad \text { com } \quad y \leq \frac{x+z}{2} \leq x, \\
& \tilde{K}(x, y, z)=\frac{1}{2} L_{x}^{0}\left(\frac{y+z}{2}\right)+\int_{\frac{y+z}{2}}^{x} d s L_{x}^{0}(s) \tilde{K}(s, y, 2 s-z) \quad \text { com } \quad y \leq \frac{y+z}{2} \leq x .
\end{aligned}
$$

Demonstração: Claramente, se substituirmos

$$
K(x, y, \tilde{z})=\frac{1}{2} T\left(x, \frac{x+\tilde{z}}{2} ; \lambda\right) L_{x}^{0}\left(\frac{x+\tilde{z}}{2}\right) E\left(x-\frac{x+\tilde{z}}{2} ; \lambda\right)
$$

em (A.2.3a) e considerarmos a mudança de variável de integração $z=\frac{x+\tilde{z}}{2}$, obtemos (A.2.1a). Resta mostrarmos que (A.2.4a) é verdadeira, para tanto substituímos (A.2.3a) em (A.2.1a):

$$
\begin{aligned}
\int_{2 y-x}^{x} d z K(x, y, z) E(z-y ; \lambda) & =\int_{y}^{x} d z E(x-z ; \lambda) L_{x}^{0}(z) E(z-y ; \lambda)+ \\
& +\int_{y}^{x} d z \int_{2 z-x}^{x} d s K(x, z, s) E(s-z ; \lambda) L_{x}^{0}(z) E(z-y ; \lambda) \\
& =\int_{y}^{x} d z L_{x}^{0}(z) E(2 z-x-y ; \lambda)+ \\
& +\int_{y}^{x} d z \int_{2 z-x}^{x} d s K(x, z, s) L_{x}^{0}(z) E(2 z-s-y ; \lambda) .
\end{aligned}
$$

Considerando a transformação $2 z-x=\tilde{z}$ na integral simples presente no lado direito e a transformação $(2 z-s=\tilde{z}, z=\tilde{s})$ na integral dupla, obtemos:

$$
\begin{aligned}
& =\frac{1}{2} \int_{2 y-x}^{x} d \tilde{z} L_{x}^{0}\left(\frac{x+\tilde{z}}{2}\right) E(\tilde{z}-y ; \lambda)+ \\
& +\int_{2 y-x}^{x} d \tilde{z} \int_{y}^{\frac{x+\tilde{z}}{2}} d \tilde{s} K(x, \tilde{s}, 2 \tilde{s}-\tilde{z}) L_{x}^{0}(\tilde{s}) E(\tilde{z}-y ; \lambda) \\
& =\int_{2 y-x}^{x} d \tilde{z}\left\{\frac{1}{2} L_{x}^{0}\left(\frac{x+\tilde{z}}{2}\right)+\right. \\
& \left.+\int_{y}^{\frac{x+\tilde{z}}{2}} d \tilde{s} K(x, \tilde{s}, 2 \tilde{s}-\tilde{z}) L_{x}^{0}(\tilde{s})\right\} E(\tilde{z}-y ; \lambda)
\end{aligned}
$$

Analogamente, ao substituirmos

$$
\tilde{K}(x, y \tilde{z})=\frac{1}{2} E\left(\tilde{z}-\frac{\tilde{z}+y}{2} ; \lambda\right) L_{x}^{0}\left(\frac{\tilde{z}+y}{2}\right) T\left(\frac{\tilde{z}+y}{2}, y ; \lambda\right)
$$


em (A.2.3b) e considerarmos a mudança de variável de integração $z=\frac{\tilde{z}+y}{2}$, obtemos (A.2.1b). Resta mostrarmos que (A.2.4b) é verdadeira, para tanto substituímos (A.2.3b) em (A.2.1b):

$$
\begin{aligned}
\int_{y}^{2 x-y} d z E(x-z ; \lambda) \tilde{K}(x, y, z) & =\int_{y}^{x} d z E(x+y-2 z ; \lambda) L_{x}^{0}(z)+ \\
& +\int_{y}^{x} d z \int_{y}^{2 z-y} d s E(x+s-2 z ; \lambda) L_{x}^{0}(z) \tilde{K}(z, y, s) \\
& =\int_{y}^{2 x-y} d \tilde{z} E(x-\tilde{z} ; \lambda)\left\{\frac{1}{2} L_{x}^{0}\left(\frac{\tilde{z}+y}{2}\right)+\right. \\
& \left.+\int_{\frac{\tilde{z}+y}{2}}^{x} d \tilde{s} L_{x}^{0}(\tilde{s}) \tilde{K}(\tilde{s}, y, 2 \tilde{s}-\tilde{z})\right\},
\end{aligned}
$$

onde usamos as seguintes mudanças de variáveis $2 z-y=\tilde{z}$ e $(2 z-s=\tilde{z}, z=\tilde{s})$ nas integrais simples e dupla presentes no lado direito, respectivamente.

Finalmente, como os argumentos que usamos são reversíveis, estabelecemos que as equações integrais (A.2.1) são equivalentes às (A.2.4).

Considere que $\psi(x)$ e $\bar{\psi}(x)$ são limitados em $-L \leq x \leq L$, ou seja, se $\|\cdot\|$ denota uma norma matricial, temos que existe um $C_{1} \in \mathbb{R}_{+}$tal que

$$
C_{1}=\max _{-L \leq x \leq L}\left\|L_{x}^{0}(x)\right\| .
$$

Dessa forma, podemos demonstrar o seguinte resultado.

Lema A.2.4. Os núcleos $K$ e $\tilde{K}$ são absolutamente integráveis em $2 y-x \leq z \leq x$ e $y \leq z \leq 2 x-y$, respectivamente.

Demonstração: Defina:

$$
\phi(x, y)=\int_{2 y-x}^{x} d z\|K(x, y, z)\|
$$

Claramente,

$$
\begin{aligned}
\int_{2 y-x}^{x} d z K(x, y, z) & =\frac{1}{2} \int_{2 y-x}^{x} d z L_{x}^{0}\left(\frac{x+z}{2}\right)+\int_{2 y-x}^{x} d z \int_{y}^{\frac{x+z}{2}} d s K(x, s, 2 s-z) L_{x}^{0}(s) \\
& =\int_{y}^{x} d z L_{x}^{0}(z)+\int_{y}^{x} d z \int_{2 z-x}^{x} d s K(x, z, s) L_{x}^{0}(z) .
\end{aligned}
$$

Portanto,

$$
\phi(x, y) \leq \int_{y}^{x} d z\left\|L_{x}^{0}(z)\right\|+\int_{y}^{x} d z\left\|L_{x}^{0}(z)\right\|\|\phi(x, y)\| \leq \exp \left[\int_{y}^{x} d z\left\|L_{x}^{0}(z)\right\|\right]-1 \leq e^{C_{1}(x-y)}-1 .
$$

Para $\tilde{K}$ introduzimos:

$$
\tilde{\phi}(x, y)=\int_{y}^{2 x-y} d z\|\tilde{K}(x, y, z)\|
$$

e procedemos exatamente da mesma maneira. 
Uma conseqüência imediata do lema A.2.4 é que $\|T(x, y ; \lambda)\|$ é limitado. Para vermos isso, consideramos

$$
\|T(x, y ; \lambda)\| \leq\|E(x-y ; \lambda)\|+\int_{2 y-x}^{x} d z\|E(z-y ; \lambda)\|\|K(x, y, z)\|,
$$

e notamos que $L_{x}^{1}$ é limitado em $-L \leq x \leq x$, por consegüinte existe um $C_{2} \in \mathbb{R}_{+}$tal que

$$
C_{2}=\max _{-L \leq x \leq L}\|E(x ; \lambda)\|
$$

Logo,

$$
\|T(x, y ; \lambda)\| \leq C_{2}+C_{2} \phi(x, y) \leq C_{2}\left(1+e^{i C_{1}(x-y)}-1\right) \leq C_{2} e^{2 i L C_{1}} .
$$

Em virtude das equações (A.2.4), os núcleos $K$ e $\tilde{K}$ possuem as mesmas propriedades de continuidade e diferenciabilidade que os campos $\psi(x)$ e $\bar{\psi}(x)$, portanto, se $\psi(x), \bar{\psi}(x) \in \mathscr{C}^{\infty}(\mathbb{R})$, inferimos que os núcleos $K$ e $\tilde{K}$ são infinitamente diferenciáveis com respeito a cada um de seus argumentos. Conseqüentemente, podemos usar a equação diferencial satisfeita por $E(x ; \lambda)$ :

$$
\partial_{x} E(x ; \lambda)=\lambda L_{x}^{1} E(x ; \lambda) \quad \text { com } \quad E(0 ; \lambda)=\mathbb{1}_{2}
$$

para integrar as equações (A.2.3) por partes. Obtemos, pois, a expansão assintótica de $T(x, y ; \lambda)$ (2.6.16) para um parâmetro espectral real e grande. Dessa forma, estabelecemos a validade da proposição 2.6.3.

Proposição (2.6.3). Seja o modelo NLS com condições de contorno quasiperiódicas, se $\psi(x), \bar{\psi}(x) \in$ $\mathscr{C}^{\infty}(\mathbb{R})$, então a matriz de transição $T(x, y ; \lambda)$ é uma função inteira e admite a seguinte expansão assintótica para $\lambda \in \mathbb{R}, \lambda \gg 0$,

$$
T(x, y ; \lambda)=E(x-y ; \lambda)+\sum_{n=1}^{\infty} \frac{T_{n}(x, y)}{\lambda^{n}} E(x-y ; \lambda)+\sum_{n=1}^{\infty} \frac{\tilde{T}_{n}(x, y)}{\lambda^{n}} E(y-x ; \lambda)+O\left(|\lambda|^{-\infty}\right),
$$

$\operatorname{com} E(x ; \lambda)=e^{\lambda L \frac{1}{x} x}$

Resta mostrarmos a veracidade do corolário 2.6.4, para isso notamos que a propriedade involução satisfeita pela matriz de transição (2.6.7) é extendida aos núcleos $K$ e $\tilde{K}$. Claramente,

$$
\gamma^{0} \bar{E}(x ; \bar{\lambda}) \gamma^{0}=\gamma^{0} \exp \left[\frac{i \lambda}{2} \gamma^{3} x\right] \gamma^{0}=\exp \left[\frac{\lambda}{2 i} \gamma^{3} x\right]=E(x ; \lambda)
$$

Assim, usando esse resultado juntamente com a involução da matriz de transição:

$$
\gamma^{0} \bar{T}(x, y ; \bar{\lambda}) \gamma^{0}=T(x, y ; \lambda)
$$

concluímos que as equações (A.2.3) implicam:

$$
\gamma^{0} \bar{K}(x, y, z) \gamma^{0}=K(x, y, z) \text { e } \gamma^{0} \overline{\tilde{K}}(x, y, z) \gamma^{0}=\tilde{K}(x, y, z)
$$


De onde inferimos que os núcleos $K$ e $\tilde{K}$ admitem uma decomposição da forma (2.6.17):

$$
K=\left(\begin{array}{cc}
\alpha & \epsilon \bar{\beta} \\
\beta & \bar{\alpha}
\end{array}\right) \quad \text { e } \quad \tilde{K}=\left(\begin{array}{cc}
\tilde{\alpha} & \epsilon \overline{\tilde{\beta}} \\
\tilde{\beta} & \overline{\tilde{\alpha}}
\end{array}\right) .
$$

As representações integrais (A.2.3) determinam as relações entre os núcleos escalares $\alpha, \beta$ e $\tilde{\alpha}, \tilde{\beta}$, respectivamente. Para estabelece-las, usamos os seguintes fatos:

$$
\begin{aligned}
E(x ; \lambda)\left(\begin{array}{cc}
\alpha & 0 \\
0 & \bar{\alpha}
\end{array}\right) & =\left(\begin{array}{cc}
\alpha & 0 \\
0 & \bar{\alpha}
\end{array}\right) E(x ; \lambda), \\
E(x ; \lambda)\left(\begin{array}{cc}
0 & \epsilon \bar{\beta} \\
\beta & 0
\end{array}\right) & =\left(\begin{array}{cc}
0 & \epsilon \bar{\beta} \\
\beta & 0
\end{array}\right) E(-x ; \lambda),
\end{aligned}
$$

para levar $E(z-y ; \lambda)$ à esquerda de $K(x, y, z)$ em (A.2.3a) e portanto obter uma representação da forma (A.2.3b). Logo,

$$
\int_{2 y-x}^{x} d z\left(\begin{array}{cc}
\alpha e^{\frac{\lambda}{2 i}(z-y)} & \epsilon \bar{\beta} e^{\frac{i \lambda}{2}(z-y)} \\
\beta e^{\frac{\lambda}{2 i}(z-y)} & \bar{\alpha} e^{\frac{i \lambda}{2}(z-y)}
\end{array}\right)=\int_{y}^{2 x-y} d z\left(\begin{array}{cc}
\tilde{\alpha} e^{\frac{\lambda}{2 i}(x-z)} & \epsilon \overline{\tilde{\beta}} e^{\frac{\lambda}{2 i}(x-z)} \\
\tilde{\beta} e^{\frac{i \lambda}{2}(x-z)} & \overline{\tilde{\alpha}} e^{\frac{i \lambda}{2}(x-z)}
\end{array}\right),
$$

de onde extraímos:

$$
\begin{aligned}
& \alpha(x, y, z)=\tilde{\alpha}(x, y, x+y-z), \\
& \beta(x, y, z)=\tilde{\beta}(x, y, x-y+z) .
\end{aligned}
$$

Finalmente, ao considerarmos o limite $x \rightarrow L$ e $y \rightarrow-L$ em (A.2.3), obtemos equações integrais para a matriz de monodromia.

$$
\begin{aligned}
& T_{L}(\lambda)=E(2 L ; \lambda)+\int_{-2 L}^{2 L} d x K(L,-L, x-L) E(x ; \lambda), \\
& T_{L}(\lambda)=E(2 L ; \lambda)+\int_{-2 L}^{2 L} d x E(x ; \lambda) \tilde{K}(L,-L, L-x) .
\end{aligned}
$$

Tais equações implicam o seguinte comportamento para os coeficientes de transição $a_{L}(\lambda)$ e $b_{L}(\lambda)$ :

$$
\begin{aligned}
& a_{L}(\lambda)=e^{-i \lambda L}+\int_{-L}^{L} d x \alpha_{L}(x) e^{-i \lambda x}, \\
& b_{L}(\lambda)=\int_{-L}^{L} d x \beta_{L}(x) e^{i \lambda x}
\end{aligned}
$$

onde

$$
\begin{aligned}
& \alpha_{L}(x)=2 \alpha(L,-L, 2 x-L)=2 \tilde{\alpha}(L,-L, L-2 x), \\
& \beta_{L}(x)=2 \beta(L,-L, 2 x-L)=2 \tilde{\beta}(L,-L, L+2 x) .
\end{aligned}
$$

Portanto as funções inteiras $a_{L}(\lambda)$ e $b_{L}(\lambda)$ são exponenciais do tipo $L$ e, se $\psi(x), \bar{\psi}(x) \in \mathscr{C}^{\infty}(\mathbb{R})$, admitem as expansões assintóticas (2.6.18). Demonstramos, pois, o corolário 2.6.4. 
Corolário 1 (2.6.4). Seja o modelo NLS com condições de contorno quasiperiódicas, se $\psi(x), \bar{\psi}(x) \in$ $\mathscr{C}^{\infty}(\mathbb{R})$, então $a_{L}(\lambda)$ e $b_{L}(\lambda)$ são funções inteiras com as seguintes expansões assintóticas para $\lambda \in \mathbb{R}$, $\lambda \gg 0$,

$$
\begin{aligned}
& a_{L}(\lambda)=e^{-i \lambda L}+e^{-i \lambda L} \sum_{n=1}^{\infty} \frac{a_{n}}{\lambda^{n}}+e^{i \lambda L} \sum_{n=1}^{\infty} \frac{\tilde{a}_{n}}{\lambda^{n}}+O\left(|\lambda|^{-\infty}\right), \\
& b_{L}(\lambda)=e^{-i \lambda L} \sum_{n=1}^{\infty} \frac{b_{n}}{\lambda^{n}}+e^{i \lambda L} \sum_{n=1}^{\infty} \frac{\tilde{b}_{n}}{\lambda^{n}}+O\left(|\lambda|^{-\infty}\right) .
\end{aligned}
$$

\section{A.3 Admissibilidade do Funcional Gerador das Quantidades Con- servadas para o Modelo de Schrödinger Não-Linear}

Nesta seção, demonstramos que o funcional gerador das quantidades conservadas (2.6.13):

$$
\zeta(\lambda)=\operatorname{tr}\left[T_{L}(\lambda) Q(\theta)\right]
$$

é um funcional admissível no espaço de fase $\Gamma_{L, \theta}$. Para isso consideramos a matriz de transição $T(x, y ; \lambda)$ com $-L<y<x<L$, e notamos que a equação integral de Volterra (A.2.1a) fornece uma expansão em $\psi(z)$ e $\bar{\psi}(z)$ da forma (2.4.9) para a matriz de transição. Calculamos em seguida as derivadas funcionais de $T(x, y ; \lambda)$ com respeito a $\psi(z)$ e $\bar{\psi}(z)$ com $y<z<x$, utilizando a variação do problema linear auxiliar (2.6.2),

$$
\partial_{x}[\delta T(x, y ; \lambda)]=\delta L_{x}(x ; \lambda) T(x, y ; \lambda)+\left.L_{x}(x ; \lambda) \delta T(x, y ; \lambda) \quad \operatorname{com} \quad \delta T(x, y ; \lambda)\right|_{x=y}=0
$$

cuja solução é

$$
\delta T(x, y ; \lambda)=\int_{y}^{x} d z T(x, z ; \lambda) \delta L_{x}(z ; \lambda) T(z, y ; \lambda)
$$

e usamos que

$$
\delta L_{x}(z ; \lambda)=i \sqrt{g}\left[\delta \psi(z) \gamma^{+}-\delta \bar{\psi}(z) \gamma^{-}\right]
$$

para obter:

$$
\begin{aligned}
& \frac{\delta T(x, y ; \lambda)}{\delta \psi(z)}=i \sqrt{g} T(x, z ; \lambda) \gamma^{+} T(z, y ; \lambda) \\
& \frac{\delta T(x, y ; \lambda)}{\delta \bar{\psi}(z)}=-i \sqrt{g} T(x, z ; \lambda) \gamma^{-} T(z, y ; \lambda)
\end{aligned}
$$

Note que para $z$ pertencente ao domínio fundamental, mas fora do intervalo $(y, x)$, as derivadas funcionais (A.3.5) se anulam. Conseqüentemente, elas são descontínuas e os elementos da matriz de transição não são funcionais admissíveis.

O próximo passo consistem em tomar o limite $x \rightarrow L$ e $y \rightarrow-L$ em (A.3.5), para obtermos as derivadas funcionais da matriz de monodromia, que são claramente contínuas em $z \in(-L, L)$. 
Considerando os limites $z \rightarrow L-0$ e $z \rightarrow L+0$, inferimos:

$$
\begin{aligned}
& \left.\frac{\delta T_{L}(\lambda)}{\delta \psi(z)}\right|_{z=L}=i \sqrt{g} \gamma^{+} T_{L}(\lambda) \text { e }\left.\frac{\delta T_{L}(\lambda)}{\delta \bar{\psi}(z)}\right|_{z=L}=-i \sqrt{g} \gamma^{-} T_{L}(\lambda), \\
& \left.\frac{\delta T_{L}(\lambda)}{\delta \psi(z)}\right|_{z=-L}=i \sqrt{g} T_{L}(\lambda) \gamma^{+} \quad \text { e }\left.\frac{\delta T_{L}(\lambda)}{\delta \bar{\psi}(z)}\right|_{z=-L}=-i \sqrt{g} T_{L}(\lambda) \gamma^{-},
\end{aligned}
$$

que mostram que os elementos da matriz de monodromia também não são funcionais admissíveis. Continuamos multiplicando as equações (A.3.6) por $Q(\theta)$ pela direita e usando que:

$$
Q(\theta) \gamma^{\mp} Q^{-1}(\theta)=e^{ \pm i \theta} \gamma^{\mp}
$$

para obtermos as condições de quasiperiodicidade para $\zeta(\lambda)$

$$
\begin{aligned}
& \left.\frac{\delta \zeta(\lambda)}{\delta \psi(z)}\right|_{z=L}=\left.e^{-i \theta} \frac{\delta \zeta(\lambda)}{\delta \psi(z)}\right|_{z=-L}, \\
& \left.\frac{\delta \zeta(\lambda)}{\delta \bar{\psi}(z)}\right|_{z=L}=\left.e^{i \theta} \frac{\delta \zeta(\lambda)}{\delta \bar{\psi}(z)}\right|_{z=-L} .
\end{aligned}
$$

A condição de quasiperiodicidade também é válida para as derivadas em $z$ das derivadas funcionais de $\zeta(\lambda)$, para mostrarmos isso consideramos a derivada em $z$ das equações (A.3.5):

$$
\begin{aligned}
& \partial_{z}\left(\frac{\delta T(x, y ; \lambda)}{\delta \psi(z)}\right)=i \sqrt{g} T(x, z ; \lambda)\left[\gamma^{+} ; L_{x}(z ; \lambda)\right] T(z, y ; \lambda), \\
& \partial_{z}\left(\frac{\delta T(x, y ; \lambda)}{\delta \bar{\psi}(z)}\right)=-i \sqrt{g} T(x, z ; \lambda)\left[\gamma^{-} ; L_{x}(z ; \lambda)\right] T(z, y ; \lambda) .
\end{aligned}
$$

e repetimos os mesmos argumentos, notando apenas que a quasiperiodicidade de $L_{x}(z ; \lambda)$ demanda que:

$$
L_{x}(L ; \lambda)=Q^{-1}(\theta) L_{x}(-L ; \lambda) Q(\theta)
$$

para concluir que:

$$
\begin{aligned}
& \left.\partial_{z}\left(\frac{\delta \zeta(\lambda)}{\delta \psi(z)}\right)\right|_{z=L}=\left.e^{-i \theta} \partial_{z}\left(\frac{\delta \zeta(\lambda)}{\delta \psi(z)}\right)\right|_{z=-L}, \\
& \left.\partial_{z}\left(\frac{\delta \zeta(\lambda)}{\delta \bar{\psi}(z)}\right)\right|_{z=L}=\left.e^{i \theta} \partial_{z}\left(\frac{\delta \zeta(\lambda)}{\delta \bar{\psi}(z)}\right)\right|_{z=-L}
\end{aligned}
$$

As condições de quasiperiodicidade (A.3.7) e (A.3.9) em conjunto com a continuidade e diferenciabilidade das derivadas funcionais de $\zeta(\lambda)$ com respeito a $\psi(z)$ e $\bar{\psi}(z)$ para $-L<z<L$ possibilitam a extensão dessas funções para todo o eixo real de uma forma suave e quasiperiódica:

$$
\frac{\delta \zeta(\lambda)}{\delta \bar{\psi}(z+2 L)}=e^{i \theta} \frac{\delta \zeta(\lambda)}{\delta \bar{\psi}(z)} \quad \text { e } \frac{\delta \zeta(\lambda)}{\delta \psi(z+2 L)}=e^{-i \theta} \frac{\delta \zeta(\lambda)}{\delta \psi(z)} .
$$

Assim, se $\psi(z)$ e $\bar{\psi}(z)$ forem infinitamente diferenciáveis, as derivadas funcionais $\frac{\delta \zeta(\lambda)}{\delta \psi(z)}$ e $\frac{\delta \zeta(\lambda)}{\delta \bar{\psi}(z)}$ também são funções infinitamente diferenciáveis e quasiperiódicas em todo o eixo real. Ademais, $\zeta(\lambda)$ é um funcional analítico real, cuja expansão do tipo (2.4.9) é uma conseqüência direta da 
A.3 Admissibilidade do Funcional Gerador das Quantidades Conservadas para o Modelo de Schrödinger Não-Linear

expansão do funcional $\operatorname{tr}[T(x, y ; \lambda) Q(\theta)]$ no limite $x \rightarrow L$ e $y \rightarrow-L$. Dessa forma, concluímos que $\zeta(\lambda)$ é um funcional admissível em $\Gamma_{L, \theta}$. 



\section{Apêndice $B$}

\section{Matrizes Gama}

"Young man, in mathematics you don't understand things. You just get used to them."

John von Neumann

Neste apêndice coletamos as matrizes gama utilizadas em diversas partes do texto. Denotamos por $\mathbb{1}_{n}$ a matriz unitária $n \times n$.

\section{B.1 $S O(4,1)$}

Seja a álgebra de Clifford $S O(4,1)$ :

$$
\Gamma_{a}^{S O(4,1)} \Gamma_{b}^{S O(4,1)}+\Gamma_{b}^{S O(4,1)} \Gamma_{a}^{S O(4,1)}=2 \eta_{a b} \mathbb{1}_{5}, \quad a, b=1, \ldots, 5
$$

onde $\eta=\operatorname{diag}(1,1,1,1,-1)$. Uma possível realização dessa álgebra é dada pelas seguintes matrizes:

$$
\begin{array}{ll}
\Gamma_{1}^{S O(4,1)}=\left(\begin{array}{cccc}
0 & 0 & 0 & 1 \\
0 & 0 & -1 & 0 \\
0 & -1 & 0 & 0 \\
1 & 0 & 0 & 0
\end{array}\right), & \Gamma_{2}^{S O(4,1)}=\left(\begin{array}{cccc}
0 & 0 & i & 0 \\
0 & 0 & 0 & -i \\
-i & 0 & 0 & 0 \\
0 & i & 0 & 0
\end{array}\right), \\
\Gamma_{3}^{S O(4,1)}=\left(\begin{array}{cccc}
0 & 0 & 1 & 0 \\
0 & 0 & 0 & 1 \\
1 & 0 & 0 & 0 \\
0 & 1 & 0 & 0
\end{array}\right), & \Gamma_{4}^{S O(4,1)}=\left(\begin{array}{cccc}
0 & 0 & 0 & -i \\
0 & 0 & -i & 0 \\
0 & i & 0 & 0 \\
i & 0 & 0 & 0 \\
0 & i & 0 & 0 \\
0 & 0 & -i & 0 \\
0 & 0 & 0 & -i
\end{array}\right), \\
\Gamma_{5}^{S O(4,1)} & =
\end{array}
$$


Além disso, as matrizes $\Gamma_{a}^{S O(4,1)}$ também pertencem à álgebra de Lie $\mathfrak{s u}(2,2)$ dado que elas satisfazem à relação:

$$
\Sigma \Gamma_{a}^{S O}(4,1)+\Gamma_{a}^{S O}(4,1) \dagger \Sigma=0, \quad \Sigma=\operatorname{diag}(1,1,-1,-1) .
$$

Ademais, notamos que as matrizes $\Gamma_{a}^{S O(4,1)}$ obedecem a relação:

$$
K\left(\Gamma_{a}^{S O}(4,1)\right)^{t} K^{-1}=\Gamma_{a}^{S O(4,1)}
$$

onde $K=\Gamma_{2}^{S O(4,1)} \Gamma_{4}^{S O(4,1)}$.

\section{B.2 SO(5)}

Seja a álgebra de Clifford SO(5):

$$
\Gamma_{a}^{S O(5)} \Gamma_{b}^{S O(5)}+\Gamma_{b}^{S O(5)} \Gamma_{a}^{S O(5)}=2 \delta_{a b} \mathbb{1}_{5}, \quad a, b=1, \ldots, 5 .
$$

Uma possível realização dessa álgebra é dada pelas seguintes matrizes:

$$
\begin{aligned}
& \Gamma_{1}^{S O(5)}=\left(\begin{array}{cccc}
0 & 0 & 0 & -1 \\
0 & 0 & 1 & 0 \\
0 & 1 & 0 & 0 \\
-1 & 0 & 0 & 0
\end{array}\right), \quad \Gamma_{2}^{S O(5)}=\left(\begin{array}{cccc}
0 & 0 & -i & 0 \\
0 & 0 & 0 & i \\
i & 0 & 0 & 0 \\
0 & -i & 0 & 0
\end{array}\right), \\
& \Gamma_{3}^{S O(5)}=\left(\begin{array}{cccc}
0 & 0 & -1 & 0 \\
0 & 0 & 0 & -1 \\
-1 & 0 & 0 & 0 \\
0 & -1 & 0 & 0
\end{array}\right), \quad \Gamma_{4}^{S O(5)}=\left(\begin{array}{cccc}
0 & 0 & 0 & i \\
0 & 0 & i & 0 \\
0 & -i & 0 & 0 \\
-i & 0 & 0 & 0
\end{array}\right) \text {, } \\
& \Gamma_{5}^{S O(5)}=\left(\begin{array}{cccc}
1 & 0 & 0 & 0 \\
0 & 1 & 0 & 0 \\
0 & 0 & -1 & 0 \\
0 & 0 & 0 & -1
\end{array}\right) \text {. }
\end{aligned}
$$

Além do mais, todas as matrizes $\Gamma_{a}^{S O(5)}$ são Hermiteanas, de forma que $i \Gamma_{a}^{S O(5)} \in \mathfrak{s u}(4)$. A relação (B.1.3) também é satisfeita pelas matrizes $\Gamma_{a}^{S O(5)}$.

\section{B.3 $S O(1,1)$}

Seja a álgebra de Clifford $S O(1,1)$ :

$$
\gamma^{\mu} \gamma^{\nu}+\gamma^{\nu} \gamma^{\mu}=\eta^{\mu \nu} \mathbb{1}_{2}, \quad \mu, \nu=0,1
$$


onde $\eta=\operatorname{diag}(1,-1)$ é a métrica plana de Minkowsky bidimensional. Uma possível realização dessa álgebra é dada pelas seguintes matrizes:

$$
\rho^{0}=\left(\begin{array}{cc}
-1 & 0 \\
0 & 1
\end{array}\right), \quad \rho^{1}=\left(\begin{array}{cc}
0 & i \\
i & 0
\end{array}\right), \quad \rho^{5}=\rho^{0} \rho^{1},
$$

Outra realização de interesse pode ser obtida através da transformação de similaridade:

$$
\gamma^{\mu}=M \rho^{\mu} M^{-1}, \quad M=\frac{1}{\sqrt{2}}\left(\begin{array}{cc}
1 & -i \\
-1 & -i
\end{array}\right)
$$

que fornece:

$$
\gamma^{0}=\left(\begin{array}{ll}
0 & 1 \\
1 & 0
\end{array}\right), \quad \gamma^{1}=\left(\begin{array}{cc}
0 & -1 \\
1 & 0
\end{array}\right), \quad \gamma^{3}=\gamma^{0} \gamma^{1}
$$





\section{Apêndice C}

\section{Equivalência de Calibre entre o LL e o NLS}

"Der Wissenschaftler findet seine Belohnung in dem, was Poincaré die Freude am Verstehen nennt, nicht in den Anwendungsmöglichkeiten seiner Erfindung."

Albert Einstein

Neste apêndice ilustramos a noção de equivalência de calibre entre dois modelos, ao relacionarmos o modelo de LL $\mathfrak{s u}(2)$ com o modelo NLS através de suas correntes planas [90]. Dois sistemas de equações não-lineares (integráveis pelo MEIC) são ditas equivalentes de calibre, se suas conexões de Lax estão definidas em um mesmo fibrado vetorial e são dedutíveis, uma a partir da outra, por transformações de calibre independentes do parâmetro espectral (2.5.8) com $h(x, t) \in G L(k, \mathbb{C})$. Relembremos as conexões de Lax para o NLS (2.5.9):

$$
\begin{aligned}
& L_{x}^{N L S}=L_{x}^{0}+\lambda L_{x}^{1} \quad \text { e } \quad L_{t}^{N L S}=L_{t}^{0}-\lambda L_{x}^{0}-\lambda^{2} L_{x}^{1} \\
& \quad \operatorname{com} L_{x}^{0}=i \sqrt{g}\left(\psi \sigma_{-}-\bar{\psi} \sigma_{+}\right), \quad L_{x}^{1}=\frac{\sigma_{3}}{2 i} \text { e } L_{t}^{0}=i g \sigma_{3}|\psi|^{2}-\sqrt{g}\left(\partial_{x} \psi \sigma_{-}+\partial_{x} \bar{\psi} \sigma_{+}\right),
\end{aligned}
$$

e para o $L L \mathfrak{s u}(2)$ :

$$
\begin{array}{r}
L_{x}^{L L}=\frac{\lambda}{2 i} \mathbb{S} \text { e } \quad L_{t}^{L L}=\left[\frac{i \lambda^{2}}{2} \mathbb{S}+\frac{\lambda}{2}\left(\partial_{x} \mathbb{S}\right) \mathbb{S}\right], \\
\operatorname{com} \mathbb{S}=S^{i} \sigma_{i}, \quad \mathbb{S}=\mathbb{S}^{\dagger} \text { e } \mathbb{S}^{2}=\mathbb{1}_{2},
\end{array}
$$

onde usamos as identidades $\sigma_{ \pm}=\gamma^{\mp}$ e $\sigma_{3}=\gamma^{3}$.

As expressões (C.0.1) deixam claro que as conexões de Lax para ambos os modelos possuem os mesmos pólos, embora a conexão de Lax para o NLS apresente termos constantes, ausentes na conexão do LL. No que se segue, queremos construir um mapa inversível:

$$
\Omega(x, t): \mathbf{S}(x) \rightarrow(\psi(x), \bar{\psi}(x)),
$$

com $\mathbb{S}(x)$ uma solução de (4.1.9b) e $\psi(x)$ de (2.4.5). 
Comecemos construindo a inversa, $\Omega^{-1}(x, t)$, para isso seja $\psi(x, t)$ uma solução da equação de Schrödinger não-linear (2.4.5). Conseqüentemente, as matrizes $L_{x}^{N L S}$ e $L_{t}^{N L S}$ (C.0.1a) satisfazem a condição de curvatura nula. Defina, então, $\Omega(x, t)$ como a matriz unitária satisfazendo o sistema

$$
\begin{aligned}
& \partial_{x} \Omega=L_{x}^{0} \Omega, \\
& \partial_{t} \Omega=L_{t}^{0} \Omega .
\end{aligned}
$$

Logo, a transformação de calibre induzida por $\Omega^{-1}(x, t)$ (2.5.8) assume a seguinte forma:

$$
\begin{aligned}
& \tilde{L}_{x}^{N L S}=-\Omega^{-1} \partial_{x} \Omega+\Omega^{-1} L_{x}^{N L S} \Omega, \\
& \tilde{L}_{t}^{N L S}=-\Omega^{-1} \partial_{t} \Omega+\Omega^{-1} L_{t}^{N L S} \Omega .
\end{aligned}
$$

Usando (C.0.1a) e (C.0.2a), concluímos

$$
\tilde{L}_{x}^{N L S}=-\Omega^{-1} L_{x}^{0} \Omega+\Omega^{-1}\left(L_{x}^{0}+\lambda L_{x}^{1}\right) \Omega=\frac{\lambda}{2 i} \Omega^{-1} \sigma_{3} \Omega=L_{x}^{L L},
$$

se

$$
\mathbb{S}=\Omega^{-1} \sigma_{3} \Omega
$$

Por outro lado,

$$
\tilde{L}_{t}^{N L S}=-\Omega^{-1} L_{t}^{0} \Omega+\Omega^{-1}\left(L_{t}^{0}-\lambda L_{x}^{0}-\lambda^{2} L_{x}^{1}\right) \Omega=\frac{i \lambda^{2}}{2} \mathbb{S}-\lambda \Omega^{-1} \partial_{x} \Omega .
$$

Precisamos expressar $\Omega^{-1} \partial_{x} \Omega$ em termos de $\mathbb{S}$, notamos, pois, que ao diferenciarmos (C.0.5) com respeito a $x$,

$$
\partial_{x} \mathbb{S}=\left[\mathbb{S}, \Omega^{-1} \partial_{x} \Omega\right] .
$$

Ademais, como $\Omega^{-1} \partial_{x} \Omega=\Omega^{-1} L_{x}^{0} \Omega$ e $\sigma_{3}$ anticomuta com $L_{x}^{0}$, temos que:

$$
0=\left\{\sigma_{3}, L_{x}^{0}\right\}=\Omega^{-1} \sigma_{3} \Omega \Omega^{-1} L_{x}^{0} \Omega+\Omega^{-1} L_{x}^{0} \Omega \Omega^{-1} \sigma_{3} \Omega=\left\{S, \Omega^{-1} \partial_{x} \Omega\right\} .
$$

Portanto,

$$
\partial_{x} \mathbb{S}=\left[\mathbb{S}, \Omega^{-1} \partial_{x} \Omega\right]=\Omega^{-1} \partial_{x} \Omega \mathbb{S} \quad \Rightarrow \quad \Omega^{-1} \partial_{x} \Omega=-\frac{1}{2}\left(\partial_{x} \mathbb{S}\right) \mathbb{S} .
$$

Levando-nos a conclusão:

$$
\tilde{L}_{t}^{N L S}=\frac{i \lambda^{2}}{2} \mathbb{S}+\frac{\lambda}{2}\left(\partial_{x} \mathbb{S}\right) \mathbb{S} .
$$

Logo, contruímos uma matriz $\mathbb{S}(x)$ a partir da solução $\psi(x)$ da equação não-linear de Schrödinger que satisfaz a equação de Landau-Lifshitz.

O próximo passo é mostrar que essa transformação é de fato inversível. Para tanto, supomos agora que $\mathbb{S}$ é uma solução da equação de Landau-Lifshitz (4.1.9b). Reduzindo-a a sua forma diagonal por intermédio de uma matriz unitária $\Omega(x, t)$, isto é,

$$
\mathbb{S}=\Omega^{-1} \sigma_{3} \Omega,
$$


a qual pode ser escolhida da seguinte forma

$$
\sigma_{3} \partial_{x} \Omega \Omega^{-1}+\partial_{x} \Omega \Omega^{-1} \sigma_{3}=0
$$

Tal condição implica que a matriz anti-hermiteana $\partial_{x} \Omega \Omega^{-1}$ possui diagonal principal nula. Assim, podemos fixar:

$$
L_{x}^{0}=\partial_{x} \Omega \Omega^{-1}
$$

o que define os campos $\psi(x)$ e $\bar{\psi}(x)$ a partir de um dado $\mathbb{S}(x)$. Similarmente, consideramos a transformação de calibre induzida por $\Omega$ :

$$
\begin{aligned}
& \tilde{L}_{x}^{L L}=\partial_{x} \Omega \Omega^{-1}+\Omega L_{x}^{L L} \Omega^{-1}=L_{x}^{0}+\frac{\lambda}{2 i} \sigma_{3}=L_{x}^{N L S}, \\
& \tilde{L}_{t}^{L L}=\partial_{t} \Omega \Omega^{-1}+\Omega L_{t}^{L L} \Omega^{-1}=\partial_{t} \Omega \Omega^{-1}+\frac{i \lambda^{2}}{2} \Omega \mathbb{S} \Omega^{-1}+\frac{\lambda}{2} \Omega \partial_{x} \mathbb{S} \mathbb{S} \Omega^{-1} .
\end{aligned}
$$

Notando que

$$
\Omega \partial_{x} S \mathbb{S}^{-1} \Omega^{-1}=-\partial_{x} \Omega \Omega^{-1} \sigma_{3}^{2}+\sigma_{3} \partial_{x} \Omega \Omega^{-1} \sigma_{3}=-2 \partial_{x} \Omega \Omega^{-1}=-2 L_{x}^{0}
$$

Já para expressarmos $\partial_{t} \Omega \Omega^{-1}$ em termos de $\psi$ e $\bar{\psi}$, precisamos utilizar a condição de curvatura nula (C.0.1a) satisfeita por $L_{x}^{L L}$ e $L_{t}^{L L}$, que fornece um polinômio de terceiro grau em $\lambda$. Em particular, do coeficiente de $\lambda^{1}$, obtemos:

$$
\partial_{x} L_{x}^{0}+\frac{1}{2 i}\left[\sigma_{3}, \partial_{t} \Omega \Omega^{-1}\right]=0
$$

Ao verificarmos que

$$
\left[\sigma_{3}, \sigma_{3} \partial_{x} L_{x}^{0}\right]=2 \partial_{x} L_{x}^{0}
$$

somos levados à conclusão

$$
\partial_{t} \Omega \Omega^{-1}=-i \sigma_{3} \partial_{x} L_{x}^{0}+i c(x, t) \sigma_{3}
$$

onde $c(x, t)$ é uma função real arbitrária. Para determinarmos o coeficiente $c(x, t)$, considerarmos o coeficiente de $\lambda^{0}$ :

$$
\partial_{t} L_{x}^{0}-\partial_{x}\left(\partial_{t} \Omega \Omega^{-1}\right)+\left[L_{x}^{0}, \partial_{t} \Omega \Omega^{-1}\right]=0
$$

e, ao notarmos que

$$
\left[L_{x}^{0}, \partial_{t} \Omega \Omega^{-1}\right]=i g \partial_{x}|\psi|^{2} \sigma_{3}+i c\left(\psi \sigma_{-}+\bar{\psi} \sigma_{+}\right)
$$

inferimos a partir de sua parte diagonal, a seguinte relação:

$$
\partial_{x}\left(i g|\psi|^{2}-i c\right)=0
$$

Combinando (C.0.11) com (C.0.12), obtemos:

$$
\partial_{t} \Omega \Omega^{-1}=-i \sigma_{3} \partial_{x} L_{x}^{0}+o g|\psi|^{2} \sigma_{3}+i \tilde{c}(t) \sigma_{3}=L_{t}^{0}+i \tilde{c}(t) \sigma_{3}
$$


com $\tilde{c}(t)$ sendo uma função real. Finalmente, usando a arbitrariedade remanescente na definição de $\Omega$ em (C.0.8), podemos fazer a transformação:

$$
\Omega \rightarrow e^{i \tilde{C}(t) \sigma_{3}} \Omega \quad \text { com } \quad \tilde{C}(t) \quad \text { tal que } \quad \partial_{t} \tilde{C}(t)=\tilde{C}(t)
$$

para eliminarmos o termo proporcional a $\tilde{c}(t)$ em $\partial_{t} \Omega \Omega^{-1}$. Logo,

$$
\tilde{L}_{t}^{L L}=\frac{i \lambda^{2}}{2} \sigma_{3}-\lambda_{x}^{0}+L_{t}^{0}=L_{t}^{N L S},
$$

e, portanto, o $\psi(x, t)$, que definimos a partir de uma solução arbitrária $\mathbf{S}(x, t)$ da equação de LandauLifshitz, deve satisfazer a equação não-linear de Schrödinger. 


\section{Apêndice D}

\section{Integrais Relevantes para o Modelo de Landau-Lifshitz}

"It is important to do everything with enthusiasm, it embellishes life enormously."

Lev Davidovich Landau

Seja $D\left(x-x^{\prime}\right)$ o propagador (5.1.16) para o modelo de Landau-Lifshitz (LL) com respeito ao estado fundamental (5.1.15) no espaço das coordenadas e denote por $D(q)$ o propagador no espaço dos momentos:

$$
D(q)=\frac{i}{q^{0}-q^{1^{2}}+i \epsilon}
$$

Para o cálculo da amplitude de espalhamento a 1-ciclo no setor de duas partículas, as seguintes integrais no espaço dos momentos são relevantes:

$$
\begin{aligned}
& I_{0}\left(p_{1}, p_{2}\right)=\int \frac{d^{2} q}{4 \pi^{4}} D(q) D\left(p_{1}+p_{2}-q\right)=\iota_{0}\left(p_{1}, p_{2}\right), \\
& I_{1}\left(p_{1}, p_{2}\right)=\int \frac{d^{2} q}{4 \pi^{4}} i q^{1} i\left(p_{1}^{1}+p_{2}^{1}-q^{1}\right) D(q) D\left(p_{1}+p_{2}-q\right)=\iota_{1}\left(p_{1}, p_{2}\right)+D_{1}, \\
& I_{2}\left(p_{1}, p_{2}\right)=\int \frac{d^{2} q}{4 \pi^{4}} q^{12}\left(p_{1}^{1}+p_{2}^{1}-q^{1}\right)^{2} D(q) D\left(p_{1}+p_{2}-q\right)=\iota_{2}\left(p_{1}, p_{2}\right)+D_{2},
\end{aligned}
$$

onde $D_{1}$ e $D_{2}$ denotam as partes divergentes da segunda e terceira integrais e $\iota_{n}\left(p_{1}, p_{2}\right)$ é a seguinte função:

$$
\iota_{n}\left(p_{1}, p_{2}\right)=\frac{(-1)^{n}}{2} \frac{\left[p_{1}^{1} p_{2}^{1}-\frac{1}{2}\left(p_{1}^{0}-{p_{1}^{1}}^{2}\right)-\frac{1}{2}\left(p_{2}^{0}-p_{2}^{1^{2}}\right)\right]^{n}}{\sqrt{\left(p_{1}^{1}-p_{2}^{1}\right)^{2}+2\left(p_{1}^{0}-p_{1}^{1^{2}}\right)+2\left(p_{2}^{0}-p_{2}^{12}\right)}}
$$

É interessante notar que no limite onde os momentos $p_{1}$ e $p_{2}$ estão na camada de massa, isto é, $p_{i}^{0}=p_{i}^{1^{2}}$, podemos simplificar significativamente (D.0.3):

$$
\iota_{n}\left(p_{1}, p_{2}\right)=\frac{(-1)^{n}}{2} \frac{\left(p_{1}^{1} p_{2}^{1}\right)^{n}}{\left|p_{1}^{1}-p_{2}^{1}\right|} .
$$


Já para o setor de três partículas, as integrais relevantes para o cálculo da amplitude de espalhamento em segunda ordem são as seguintes:

$$
\begin{aligned}
I_{a}\left(p_{1}, p_{2}\right) & =\int \frac{d q}{q^{2}-\frac{1}{4}\left(p_{1}-p_{2}\right)^{2}-i \epsilon}=\frac{2 \pi i}{\left|p_{1}-p_{2}\right|}, \\
I_{b}\left(p_{1}, p_{2}, p_{3}\right) & =\int \frac{d q}{q^{2}-\frac{1}{4}\left(p_{1}+p_{2}\right)^{2}-p_{3}\left(p_{1}+p_{2}\right)+p_{1} p_{2}+p_{1} p_{3}+p_{2} p_{3}-i \epsilon}=\frac{2 \pi i}{\left|p_{1}-p_{2}\right|}, \\
I_{c}\left(p_{1}, p_{2}, p_{3}\right) & =\iint \frac{d q_{1} d q_{2}}{q_{1}^{2}+q_{2}^{2}+q_{1} q_{2}-\left(q_{1}+q_{2}\right) \sum p+\left(p_{1} p_{2}+p_{1} p_{3}+p_{2} p_{3}\right)-i \epsilon} \\
& =-\frac{4 \pi}{\sqrt{3}}\left[\frac{i \pi}{2}-\frac{1}{2} \log Q^{2}+\frac{1}{2} \lim _{R \rightarrow \infty} \log \left(R^{2}-Q^{2}\right)\right]
\end{aligned}
$$

onde denotamos por $Q^{2}$ a seguinte quantidade:

$$
Q^{2}=\frac{4}{6}\left[\left(p_{1}-p_{2}\right)^{2}+\left(p_{1}-p_{3}\right)^{2}+\left(p_{2}-p_{3}\right)^{2}\right]
$$

É importante ressaltar que os resultados exibidos em (D.0.5) são válidos para momentos na camada de massa ${ }^{1}$.

\section{D.1 Integrais para o Espalhamento de Duas Partículas: Deta- Ihes Computacionais}

Nesta seção, descrevemos as principais etapas para o cálculo das integrais (D.0.2), no exemplo representativo da integral $I_{0}\left(p_{1}, p_{2}\right)$, dada por (D.0.2a). Ressaltamos que a presença dos fatores $q^{1}\left(p_{1}^{1}+p_{2}^{1}-q^{1}\right)$, conquanto aumente o grau de divergência superficial, não introduz maiores complicações técnicas. Substituindo a expressão para o propagador (5.1.16) em (D.0.2a):

$$
I_{0}\left(p_{1}, p_{2}\right)=\int \frac{d^{2} q}{4 \pi^{2}} \frac{i}{q^{0}-q^{1^{2}}+i \epsilon} \frac{i}{\left(p_{1}^{0}+p_{2}^{0}-q^{0}\right)-\left(p_{1}^{1}+p_{2}^{1}-q^{1}\right)^{2}+i \epsilon},
$$

verificamos a existência de dois pólos simples com respeito à integração sobre $q^{0}$ :

$$
q_{1}^{0}=p_{1}^{0}+p_{2}^{0}-\left(p_{1}^{1}+p_{2}^{1}-q^{1}\right)^{2}+i \epsilon \text { e } \quad q_{2}^{0}=q^{1^{2}}-i \epsilon .
$$

Fechando o contorno de integração no semiplano superior, conforme esboçado na figura D.1, obtemos:

$$
I_{0}\left(p_{1}, p_{2}\right)=\int \frac{d q^{1}}{2 \pi} \frac{i}{p_{1}^{0}+p_{2}^{0}-q^{1^{2}}-\left(p_{1}^{1}+p_{2}^{1}-q^{1}\right)^{2}+2 i \epsilon} .
$$

\footnotetext{
${ }^{1}$ Vide as identidades (5.3.9) e a discussão seguinte.
} 


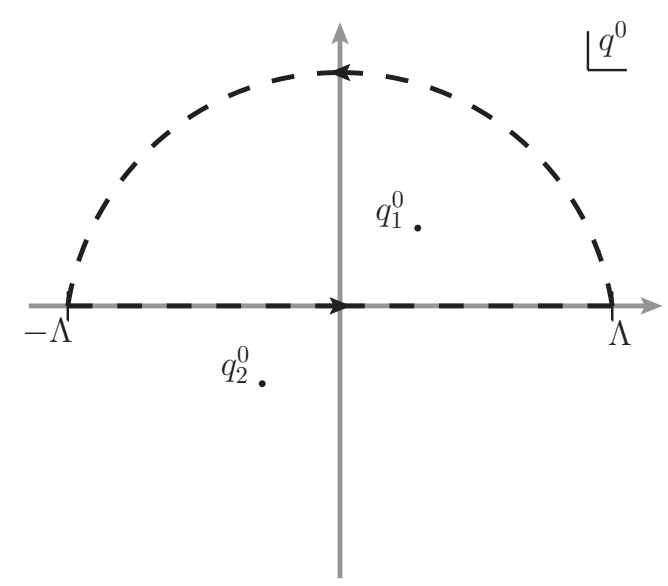

Figura D.1: Disposição dos pólos em $q^{0}$ de (D.1.1) e contorno de integração.

Podemos rescrever o denominador de (D.1.2) da seguinte maneira:

$$
\begin{aligned}
p_{1}^{0}+p_{2}^{0}-q^{1^{2}}-\left(p_{1}^{1}+p_{2}^{1}-q^{1}\right)^{2}+2 i \epsilon & =\left(p_{1}^{0}-p_{1}^{1^{2}}\right)+\left(p_{2}^{0}-p_{2}^{1^{2}}\right)- \\
& -2\left[\left(q^{1}-\frac{p_{1}^{1}+p_{2}^{1}}{2}\right)^{2}-\frac{1}{4}\left(p_{1}^{1}-p_{2}^{2}\right)^{2}-i \epsilon\right] .
\end{aligned}
$$

De forma que mudarmos a variável de integração:

$$
q^{1} \rightarrow q+\frac{p_{1}^{1}+p_{2}^{1}}{2}
$$

obtemos:

$$
I_{0}\left(p_{1}, p_{2}\right)=\frac{1}{2 i} \int \frac{d q}{2 \pi} \frac{1}{q^{2}-\frac{1}{4}\left[\left(p_{1}^{1}-p_{2}^{2}\right)^{2}+2\left(p_{1}^{0}-p_{1}^{1^{2}}\right)+2\left(p_{2}^{0}-p_{2}^{1^{2}}\right)\right]-i \epsilon} .
$$

Introduzindo ${ }^{2}$,

$$
M^{2}=\frac{1}{4}\left[\left(p_{1}^{1}-p_{2}^{2}\right)^{2}+2\left(p_{1}^{0}-p_{1}^{1^{2}}\right)+2\left(p_{2}^{0}-p_{2}^{1^{2}}\right)\right]
$$

concluímos que

$$
I_{0}\left(p_{1}, p_{2}\right)=\frac{1}{2 i} \int \frac{d q}{2 \pi} \frac{1}{q+M+\frac{i \epsilon}{2 M}} \frac{1}{q-M-\frac{i \epsilon}{2 M}} .
$$

Claramente, a estrutura de pólos de (D.1.5) é semelhante a de (D.1.1) com respeito a integração sobre $q$, admitindo a mesma representação gráfica, vide figura D.1. De sorte que, ao fecharmos o contorno de integração no semiplano superior demonstramos a validade de (D.0.2a).

\footnotetext{
${ }^{2}$ Note que devido ao ordenamento dos momentos incidentes $p_{1}>p_{2}$ e ao fato de que tais momentos estão na camada de massa, fica claro que $M^{2}$ é uma quantidade positiva e não-nula.
} 


\section{D.2 Integrais para o Espalhamento de Três Partículas: Detalhes Computacionais}

A integral (D.0.5a) pode ser trivialmente calculado por meio do teorema de resíduos de uma maneira extremamente semelhante ao cálculo de (D.1.5), pois devido ao ordenamento dos momentos incidentes $p_{i}-p_{j} \neq 0$, se $i \neq j$. Já a integral (D.0.5b) pode ser reduzida a (D.0.5a), ao usarmos as identidades (5.3.9) para simplificarmos seu denominador da seguinte forma:

$$
q^{2}-\frac{1}{4}\left(p_{1}+p_{2}\right)^{2}-p_{3}\left(p_{1}+p_{2}\right)+p_{1} p_{2}+p_{1} p_{3}+p_{2} p_{3}=q^{2}-\frac{1}{4}\left(p_{1}+p_{2}\right)^{2}+p_{1} p_{2}=q^{2}-\frac{1}{4}\left(p_{1}-p_{2}\right)^{2} .
$$

A integral restante, $I_{c}\left(p_{1}, p_{2}, p_{3}\right)$, é a única do conjunto (D.0.5) a propiciar alguma real dificuldade, no que se segue exibimos um método para seu cálculo. Nossa primeira meta é reduzi-la a uma integral dupla no plano complexo sobre um par de variáveis conjugadas, para isso precisaremos empregar uma seqüência de transformações de variáveis. Notamos, inicialmente, que seu denominador pode ser rescrito da seguinte forma:

$q_{1}^{2}+q_{2}^{2}+q_{1} q_{2}-\left(q_{1}+q_{2}\right) \sum p+\left(p_{1} p_{2}+p_{1} p_{3}+p_{2} p_{3}\right)-i \epsilon=\left(q_{1}+q_{2}\right)\left[\frac{3}{4}\left(q_{1}+q_{2}\right)-\sum p\right]+\frac{1}{4}\left(q_{1}-q_{2}\right)$

Tornando a transformação de variáveis:

$$
q_{+}=q_{1}+q_{2} \quad \text { e } \quad q_{-}=q_{1}-q_{2} \quad \text { com } \quad d q_{1} d q_{2}=-\frac{1}{2} d q_{+} d q_{-}
$$

uma escolha natural. Com isso, obtemos:

$$
I_{c}\left(p_{1}, p_{2}, p_{3}\right)=-\frac{1}{2} \iint \frac{d q_{+} d q_{-}}{\frac{3}{4} q_{+}\left(q_{+}-\frac{4}{3} \sum p\right)+\frac{1}{4} q_{-}^{2}+\left(p_{1} p_{2}+p_{1} p_{3}+p_{2} p_{3}\right)-i \epsilon} .
$$

Se, além disso, transladarmos $q_{+}$,

$$
q_{+} \rightarrow q_{+}+\frac{2}{3} \sum p
$$

e notarmos ${ }^{3}$ que

$$
-\frac{1}{3}\left(\sum p\right)^{2}+\left(p_{1} p_{2}+p_{1} p_{3}+p_{2} p_{3}\right)=-\frac{1}{6}\left[\left(p_{1}-p_{2}\right)^{2}+\left(p_{1}-p_{3}\right)^{2}+\left(p_{2}-p_{3}\right)^{2}\right]=-\frac{1}{4} Q^{2},
$$

inferimos que:

$$
I_{c}\left(p_{1}, p_{2}, p_{3}\right)=-2 \iint \frac{d q_{+} d q_{-}}{3 q_{+}^{2}+q_{-}^{2}-Q^{2}-i \epsilon} .
$$

A seguir, introduzimos o par de variáveis complexas:

$$
a=\sqrt{3} q_{+}+i q_{-} \quad \text { e } \quad \bar{a}=\sqrt{3} q_{+}-i q_{-} \quad \text { com } \quad d q_{+} d q_{-}=\frac{i}{2 \sqrt{3}} d a d \bar{a}
$$

que levam a integral $I_{c}$ à seguinte forma:

$$
I_{c}\left(p_{1}, p_{2}, p_{3}\right)=\frac{1}{i \sqrt{3}} \iint_{\mathbb{C}} \frac{d a d \bar{a}}{|a|^{2}-Q^{2}-i \epsilon} .
$$

\footnotetext{
${ }^{3}$ Vide, (D.0.6) para a definição de $Q^{2}$.
} 


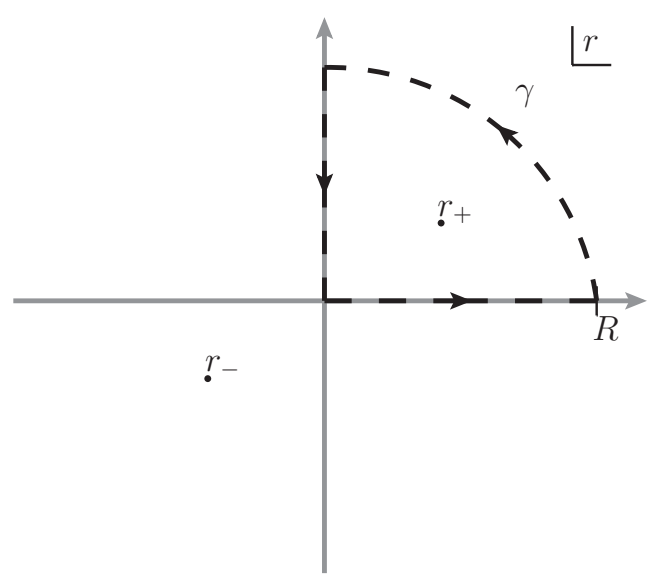

Figura D.2: Caminho de integração $\gamma$ para a integral radial.

Finalmente notando que qualquer número complexo pode ser expresso na forma polar $a=r e^{i \theta}$, concluímos que:

$$
I_{c}\left(p_{1}, p_{2}, p_{3}\right)=-\frac{2}{\sqrt{3}} \int_{0}^{\infty} d r \int_{0}^{2 \pi} d \theta \frac{r}{r^{2}-Q^{2}-i \epsilon} .
$$

A integração angular é trivial, de forma que resta-nos considerar a integração radial. Como o integrando radial possui apenas dois pólos simples:

$$
r_{ \pm}= \pm\left(Q+\frac{i \epsilon}{Q}\right)
$$

podemos efetuar a integração sobre $r$ ao considerarmos o contorno:

$$
\gamma=\left\{\begin{array}{ll}
r=x, & x: 0 \mapsto R \\
r=R e^{i \theta}, & \theta: 0 \mapsto \frac{\pi}{2}, \\
r=i y, & y: R \mapsto 0
\end{array},\right.
$$

que esboçamos na figura D.2. Finalmente, notando que devemos nos aproximar do corte de ramo do logaritmo por cima, isto é,

$$
\lim _{r \rightarrow-1+0} \log r=i \pi+2 n \pi i
$$

concluímos que:

$$
\int_{0}^{R} \frac{d r r}{r^{2}-Q^{2}-i \epsilon}=\frac{i \pi}{2}-\frac{1}{2} \log Q^{2}+\frac{1}{2} \log \left(R^{2}-Q^{2}\right)-n \pi i .
$$

De forma que ao fixarmos $n=0$, obtemos o resultado procurado (D.0.5c). 



\title{
Apêndice $E$
}

\section{Diagramas de Feynman Adicionais para o Modelo de Landau-Lifshitz}

\author{
"If I were forced to sum up in one sentence what the Copenhagen interpretation says \\ to me, it would be 'Shut up and calculate!"
}

David Mermin

Neste apêndice, desenhamos os diagramas de Feynman remanescentes para a amplitude de espaIhamento de segunda ordem com as derivadas explicitamente distribuídas. Notamos, contudo, que a soma sobre diagramas com a mesma topologia, mas com diferentes distribuições das derivadas no momentos externos, é deixada implícita.

Os dois diagramas esboçados na figura 5.11, que correspondem, respectivamente, a cada um dos termos finitos presentes na equação (5.3.8a), são representados, com as derivadas explicitamente distribuídas, pelas figuras 5.12 e E.1.

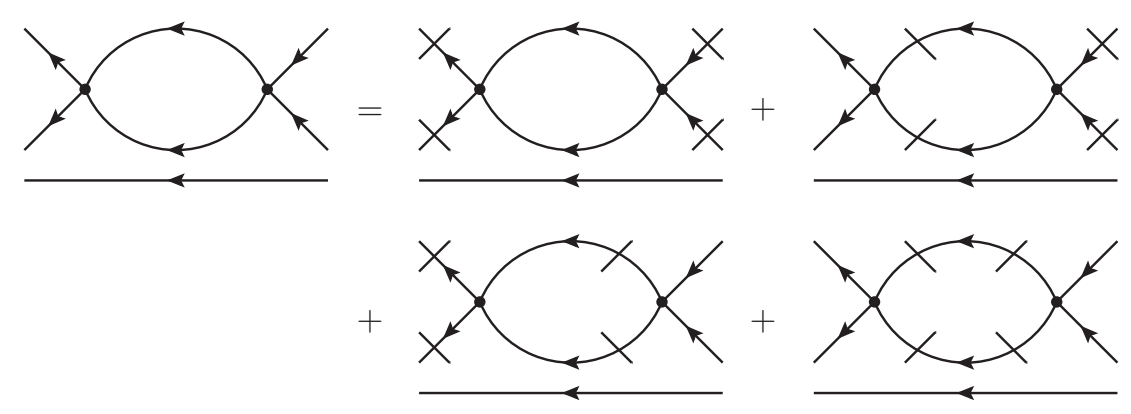

Figura E.1: Diagramas de Feynman para o segundo termo da equação (5.3.8a) com as derivadas explicitamente colocadas.

A lista de figuras seguinte contém os diagramas de Feynman completos, correspondentes às 


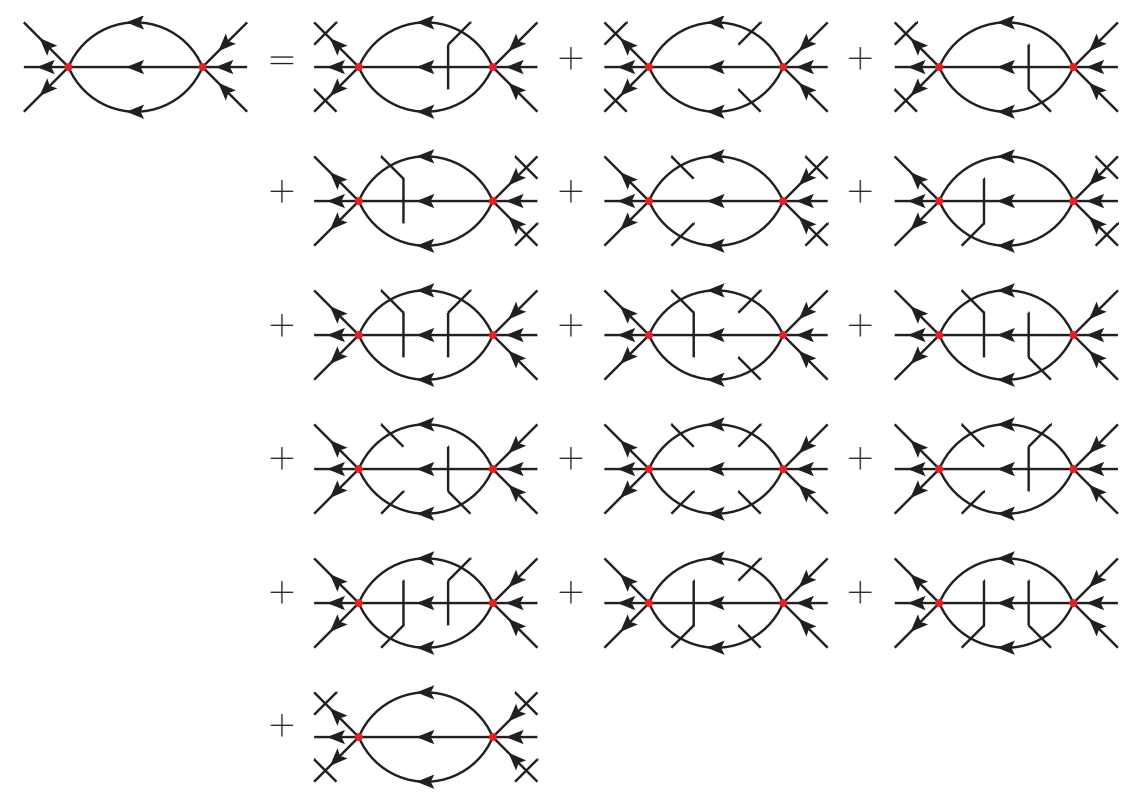

Figura E.2: Diagramas de Feynman proporcionais a $g_{2}^{2}$, correspondendo à equação (5.3.8b).

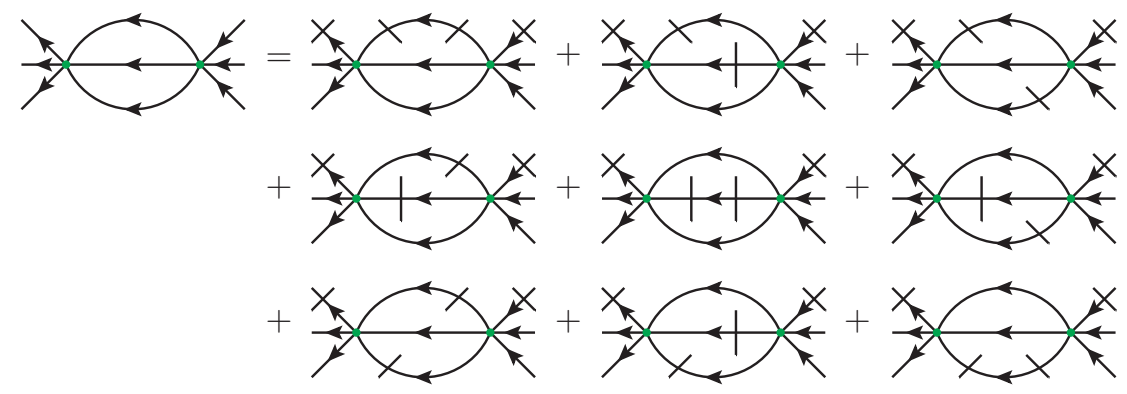

Figura E.3: Diagramas de Feynman proporcionais a $g_{3}^{2}$, correspondendo à equação (5.3.8c).

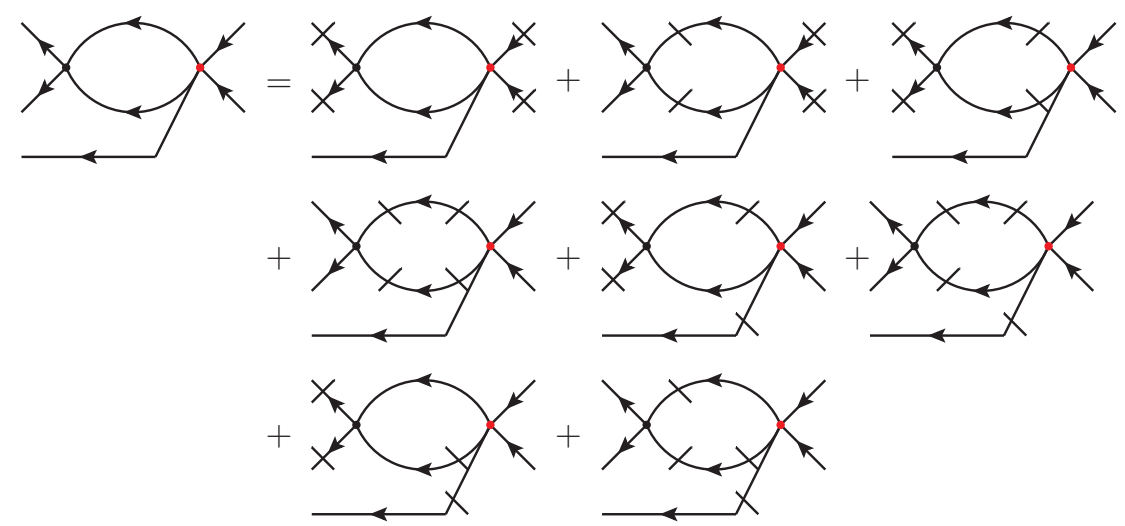

Figura E.4: Diagramas de Feynman proporcionais a $g_{1} g_{2}$, correspondendo à equação (5.3.8d). Por simplicidade omitimos os diagramas obtidos por reversão temporal. 


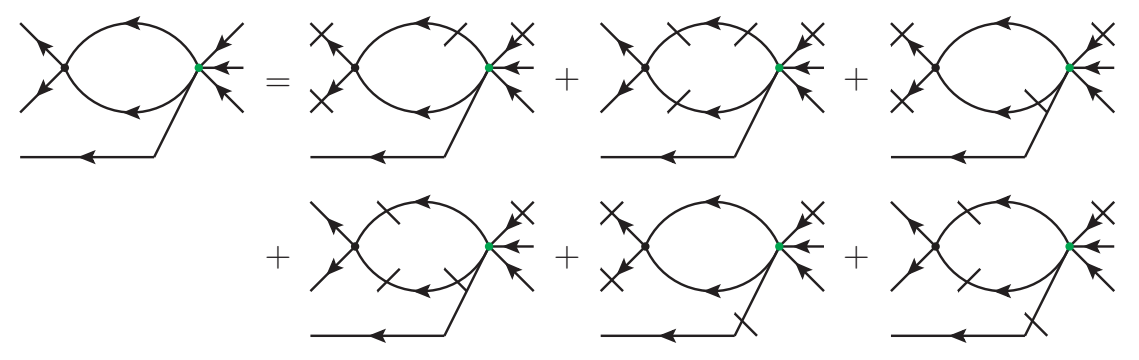

Figura E.5: Diagramas de Feynman proporcionais a $g_{1} g_{3}$, correspondendo à equação (5.3.8e). Por simplicidade omitimos os diagramas obtidos por reversão temporal.

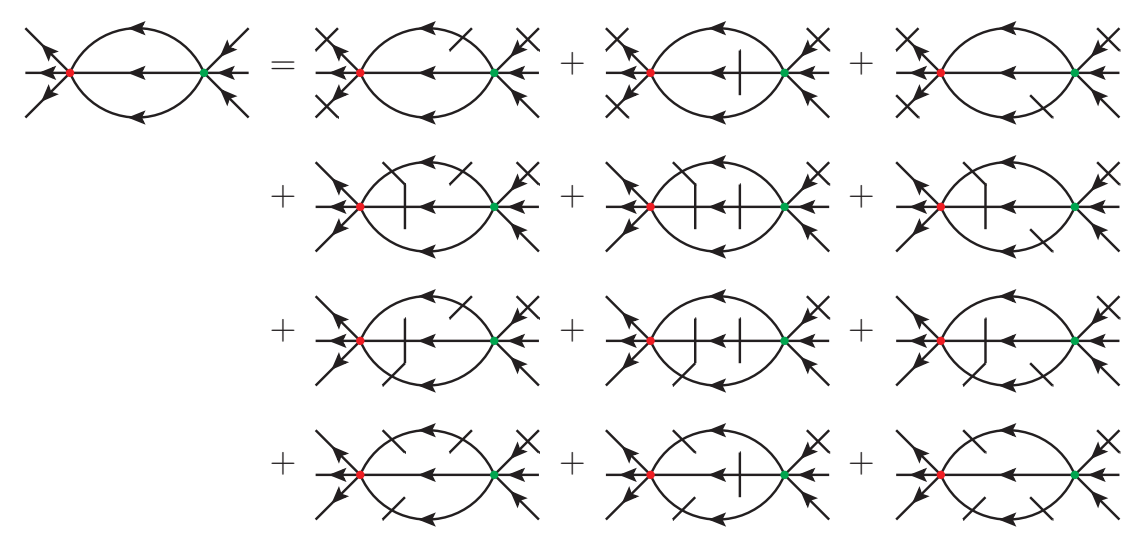

Figura E.6: Diagramas de Feynman proporcionais a $g_{2} g_{3}$, correspondendo à equação (5.3.8f). Por simplicidade omitimos os diagramas obtidos por reversão temporal.

equações (5.3.8b-5.3.8f). 



\section{Apêndice $\mathbf{F}$}

\section{Equação de Dirac em Duas Dimensões}

"Es gibt keinen Gott und Dirac ist sein Prophet."

Wolfgang Ernst Pauli

Neste apêndice estudamos as equações de Dirac em duas dimensões (8.1.16) para deduzirmos as soluções de onda plana e as relações de completeza. Considere a equação de Dirac:

$$
(i \not \supset-m) \psi(x)=0
$$

e a multiplique pela esquerda por $(i \not \partial+m)$,

$$
\left(i \gamma^{\mu} \partial_{\mu}+m\right)\left(i \gamma^{\nu} \partial_{\nu}+m\right) \psi(x)=0 \Rightarrow\left(-\gamma^{\mu} \gamma^{\nu} \partial_{\mu} \partial_{\nu}-m^{2}\right) \psi(x)=0
$$

Claramente, podemos escrever a combinação $\gamma^{\mu} \gamma^{\nu}$ como a soma de um tensor simétrico com um antissimétrico, além do mais, por $\partial_{\mu} \partial_{\nu}$ constituir um tensor simétrico, concluímos que a combinação antissimétrica de $\gamma^{\mu} \gamma^{\nu}$ não deve contribuir, deixando-nos apenas com:

$$
\left[-\frac{1}{2}\left(\gamma^{\mu} \gamma^{\nu}+\gamma^{\nu} \gamma^{\mu}\right) \partial_{\mu} \partial_{\nu}-m^{2}\right] \psi(x)=0
$$

A equação (F.0.2) se reduz à equação de Klein-Gordon, se

$$
\left\{\gamma^{\mu} ; \gamma^{\nu}\right\}=2 \eta^{\mu \nu} \mathbb{1}_{2}
$$

ou seja, se $\gamma^{\mu}$ for um elemento da álgebra de Clifford $S O(1,1)$, em particular as matrizes gama (B.3.4) fornecem uma representação dessa álgebra. 


\section{F.1 Soluções de Onda Plana}

Seja a solução de onda plana:

$$
\psi_{a}(x)=e^{-i p \cdot x} u_{a}(p), \quad \text { onde } \quad p \cdot x=\eta^{\mu \nu} p_{\mu} x_{\nu}
$$

Substituindo tal Ansatz em (F.0.1), obtemos:

$$
(\not p-m) u_{a}(p)=0
$$

essa equação possui uma solução não-trivial, se e somente se,

$$
\operatorname{det}(\not p-m)=0 \Rightarrow m^{2}-p^{2}=0
$$

ou seja, se e somente se, o momento $p$ estiver na camada de massa, resolvendo-a para a energia, obtemos:

$$
p_{0}= \pm \sqrt{p_{1}^{2}+m^{2}}=: \pm \omega(p)
$$

As soluções de energia positiva e negativa de (F.1.2), já devidamente normalizadas, são:

$$
u_{+}(p)=\left(\begin{array}{c}
\sqrt{\frac{\omega(p)-p_{1}}{2 \omega(p)}} \\
\sqrt{\frac{\omega(p)+p_{1}}{2 \omega(p)}}
\end{array}\right), \quad u_{-}(p)=\left(\begin{array}{c}
\sqrt{\frac{\omega(p)+p_{1}}{2 \omega(p)}} \\
-\sqrt{\frac{\omega(p)-p_{1}}{2 \omega(p)}}
\end{array}\right),
$$

onde $u_{+}(p)=u_{+}\left(p_{0}, p_{1}\right)$ e $u_{-}(p)=u_{-}\left(-p_{0}, p_{1}\right)$. Logo, as soluções de onda plana da equação de Dirac em duas dimensões são:

$$
\begin{array}{ll}
\psi_{+}(x)=e^{-i p \cdot x} u(p), & \text { com } \quad(\not p-m) u(p)=0, \\
\psi_{-}(x)=e^{i p \cdot x} v(-p), & \text { com } \quad(\not p+m) v(-p)=0,
\end{array}
$$

onde introduzimos a notação auxiliar: $u(p):=u_{+}(p)$ e $v(p):=u_{-}\left(p_{0}, p_{1}\right)$.

\section{F.2 Relações de Ortonormalização e Completeza}

As soluções $u_{+}(p)$ e $u_{-}(p)$ (F.1.4) satisfazem as seguintes relações de ortonormalização:

$$
\begin{array}{ll}
u_{+}^{\dagger}(p) u_{+}(p)=1, & u_{-}^{\dagger}(p) u_{-}(p)=1 \\
u_{+}^{\dagger}(p) u_{-}(p)=0, & u_{-}^{\dagger}(p) u_{+}(p)=0
\end{array}
$$

bem como,

$$
\begin{array}{ll}
\bar{u}_{+}(p) u_{+}(p)=\frac{m}{\omega(p)}, & \bar{u}_{-}(p) u_{-}(p)=-\frac{m}{\omega(p)}, \\
\bar{u}_{+}(p) u_{-}(p)=\frac{p_{1}}{\omega(p)}, & \bar{u}_{-}(p) u_{+}(p)=\frac{p_{1}}{\omega(p)},
\end{array}
$$

onde os espinores conjugados são definidos da maneira usual: $\bar{u}_{ \pm}=u_{ \pm}^{\dagger} \gamma^{0}$. 
Finalmente, as relações de completeza para os espinores $u(p)$ e $v(p)$ são:

$$
u(p) \bar{u}(p)=\frac{\not p+m}{2 \omega(p)} \quad \text { e } \quad v(-p) \bar{v}(-p)=\frac{\not p-m}{2 \omega(p)} .
$$





\section{Apêndice G}

\section{Integrais Relevantes para o Modelo de Alday-Arutyunov-Frolov}

"It's one of the great tragedies of life - something always changes."

House [Hugh Laurie], House, M.D.

Seja $D\left(x-x^{\prime}\right)$ o propagador (8.1.36) do modelo de Alday-Arutyunov-Frolov (AAF) com respeito ao pseudovácuo (8.1.33) no espaço das coordenadas e denote por $D(q)$ o propagador no espaço dos momentos:

$$
D(q)=\frac{i(\phi+m)}{q^{2}-m^{2}+2 i \epsilon q_{0}} .
$$

Para o cômputo da amplitude de espalhamento a 1-ciclo no setor de duas pseudopartículas, as seguintes integrais no espaço dos momentos são relevantes:

$$
\begin{aligned}
I_{0}\left(p^{1}, p^{2}\right) & =\int \frac{d^{2} q}{4 \pi^{2}} D(q) \otimes D\left(p^{1}+p^{2}-q\right) \\
& =\frac{p_{0}^{1}+p_{0}^{2}}{4\left|p_{1}^{1}-p_{1}^{2}\right|\left(p^{1}+p^{2}\right)^{2}}\left[\left(\not p^{1}+m\right) \otimes\left(\not p^{2}+m\right)+\left(\not p^{2}+m\right) \otimes\left(\not p^{1}+m\right)\right]+D_{0}, \\
I_{1}\left(p^{1}, p^{2}\right) & =\int \frac{d^{2} q}{4 \pi^{2}}\left[\left(p^{1}+p^{2}-q\right) \times q\right] D(q) \otimes D\left(p^{1}+p^{2}-q\right) \\
& =\frac{p^{2} \times p^{1}\left(p_{0}^{1}+p_{0}^{2}\right)}{4\left|p_{1}^{1}-p_{1}^{2}\right|\left(p^{1}+p^{2}\right)^{2}}\left[\left(\not p^{1}+m\right) \otimes\left(\not p^{2}+m\right)-\left(\not p^{2}+m\right) \otimes\left(\not p^{1}+m\right)\right]+D_{1}, \\
I_{2}\left(p^{1}, p^{2}\right) & =\int \frac{d^{2} q}{4 \pi^{2}}\left[\left(p^{1}+p^{2}-q\right) \times q\right]^{2} D(q) \otimes D\left(p^{1}+p^{2}-q\right) \\
& =\frac{\left(p^{2} \times p^{1}\right)^{2}\left(p_{0}^{1}+p_{0}^{2}\right)}{4\left|p_{1}^{1}-p_{1}^{2}\right|\left(p^{1}+p^{2}\right)^{2}}\left[\left(\not p^{1}+m\right) \otimes\left(\not p^{2}+m\right)+\left(\not p^{2}+m\right) \otimes\left(\not p^{1}+m\right)\right]+D_{2},
\end{aligned}
$$

onde $D_{i}, i=0,1,2$ representam suas partes divergentes. As integrais (G.0.2) reaparecem no setor de três pseudopartículas, mais precisamente, na consideração das contribuições com duas contrações de $V_{1}^{(2)}(\mathbf{k}, \mathbf{p})$, pois, conforme explicamos anteriormente, a análise envolvida é essencialmente a mesma requerida para o cálculo da amplitude de espalhamento a 1-ciclo envolvendo apenas duas pseudopartículas. No entanto, a análise dos termos contendo apenas uma contração de $V_{1}^{(2)}(\mathbf{k}, \mathbf{p})$ envolve 
também a consideração das seguintes integrais:

$$
\begin{aligned}
I & =\iint d^{2} x d^{2} y e^{-i x \cdot \Delta-i y \cdot \tilde{\Delta} \int} \frac{d^{2} q}{4 \pi^{2}} e^{i q \cdot(x-y)} D(q) \\
& =(\Delta+m)\left\{\frac{2 \pi}{4 \omega(\Delta)}\left[\delta\left(\Delta_{0}-\omega(\Delta)\right)-\delta\left(\Delta_{0}+\omega(\Delta)\right)\right]+\frac{i}{\Delta^{2}-m^{2}}\right\} 4 \pi^{2} \delta^{(2)}(\mathbf{k}-\mathbf{p}) \\
I_{\alpha} & =\iint d^{2} x d^{2} y e^{-i x \cdot \Delta-i y \cdot \tilde{\Delta}} \int \frac{d^{2} q}{4 \pi^{2}} e^{i q \cdot(x-y)} q_{\alpha} D(q) \\
& =\Delta_{\alpha}(\Delta+m)\left\{\frac{2 \pi}{4 \omega(\Delta)}\left[\delta\left(\Delta_{0}-\omega(\Delta)\right)-\delta\left(\Delta_{0}+\omega(\Delta)\right)\right]+\frac{i}{\Delta^{2}-m^{2}}\right\} 4 \pi^{2} \delta^{(2)}(\mathbf{k}-\mathbf{p}) \\
I_{\alpha \gamma} & =\iint d^{2} x d^{2} y e^{-i x \cdot \Delta-i y \cdot \tilde{\Delta}} \int \frac{d^{2} q}{4 \pi^{2}} e^{i q \cdot(x-y)} q_{\alpha} q_{\gamma} D(q) \\
& =\Delta_{\alpha} \Delta_{\gamma}(\Delta+m)\left\{\frac{2 \pi}{4 \omega(\Delta)}\left[\delta\left(\Delta_{0}-\omega(\Delta)\right)-\delta\left(\Delta_{0}+\omega(\Delta)\right)\right]+\frac{i}{\Delta^{2}-m^{2}}\right\} 4 \pi^{2} \delta^{(2)}(\mathbf{k}-\mathbf{p})
\end{aligned}
$$

onde empregamos as seguintes combinações dos momentos $\Delta=p^{1}+p^{2}-k^{1}$ e $\tilde{\Delta}=p^{3}-k^{2}-k^{3}$.

\section{G.1 Integrais para o Espalhamento de Duas Pseudopartículas: Detalhes Computacionais}

Nesta seção, descrevemos as etapas principais para o cálculo das integrais (G.0.2), no exemplo representativo da integral $I_{0}\left(p^{1}, p^{2}\right)$, dada por (G.0.2a). Notamos que a presença dos fatores $\left(p^{1}+p^{2}-q\right) \times q$ nas outras duas integrais (G.0.2b) e (G.0.2c), conquanto aumente o grau de divergência superficial, não introduz maiores complicações técnicas. Substituindo a expressão para o propagador (G.0.1) em (G.0.2a):

$$
I_{0}\left(p^{1}, p^{2}\right)=\int \frac{d^{2} q}{4 \pi^{2}} \frac{i(\not l+m)}{q^{2}-m^{2}+2 i \epsilon q_{0}} \otimes \frac{i\left(\not p^{1}+\not p^{2}-\not \phi+m\right)}{\left(p^{1}+p^{2}-q\right)^{2}-m^{2}+2 i \epsilon\left(p_{0}^{1}+p_{0}^{2}-q_{0}\right)},
$$

verificamos a existência de quatro pólos com respeito a integração sobre $d q_{0}$ :

$$
q_{0}^{(1 \pm)}= \pm \omega(q)-i \epsilon \quad \text { e } \quad q_{0}^{2 \pm}=p_{0}^{1}+p_{0}^{2} \pm \tilde{\omega}(q)+i \epsilon,
$$

onde introduzimos

$$
\tilde{\omega}(q):=\sqrt{\left(p_{1}^{1}+p_{1}^{2}-q_{1}\right)^{2}-m^{2}} .
$$

Podemos integrar (G.1.1) por resíduos, fechando o contorno de integração no semiplano complexo inferior, conforme figura G.1, precisamos apenas calcular os resíduos dos pólos mais simples $q_{0}^{(1 \pm)}$. 


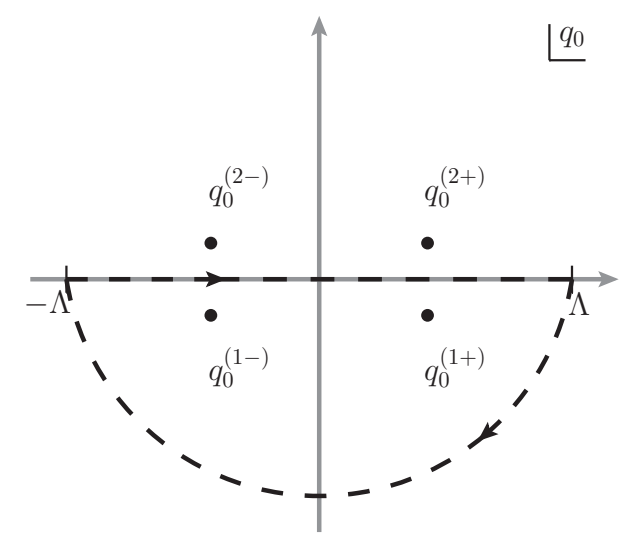

Figura G.1: Disposição dos pólos em $q_{0}$ de (G.1.1) e contorno de integração.

Portanto

$$
\begin{aligned}
I_{0}\left(p^{1}, p^{2}\right) & =\frac{i}{8 \pi} \int \frac{d q_{1}}{\omega}\left\{\frac{-\omega^{2} \gamma^{0} \otimes \gamma^{0}+\omega\left(\gamma^{0} \otimes \alpha-\beta \otimes \gamma^{0}\right)+\beta \otimes \alpha}{\left[\left(\omega-p_{0}^{1}\right)\left(\omega-p_{0}^{2}\right)-\left(q_{1}-p_{1}^{1}\right)\left(q_{1}-p_{1}^{2}\right)+2 i \epsilon\left(p_{0}^{1}+p_{0}^{2}-\omega\right)\right]}+\right. \\
& \left.+\frac{\omega^{2} \gamma^{0} \otimes \gamma^{0}+\omega\left(\gamma^{0} \otimes \alpha-\beta \otimes \gamma^{0}\right)-\beta \otimes \alpha}{\left[\left(\omega+p_{0}^{1}\right)\left(\omega+p_{0}^{2}\right)-\left(q_{1}-p_{1}^{1}\right)\left(q_{1}-p_{1}^{2}\right)+2 i \epsilon\left(p_{0}^{1}+p_{0}^{2}+\omega\right)\right]}\right\}
\end{aligned}
$$

onde, por simplicidade, denotamos $\omega \equiv \omega(q)$ e introduzimos as combinações:

$$
\alpha:=\left(p_{0}^{1}+p_{0}^{2}\right) \gamma^{0}+\left(p_{1}^{1}+p_{1}^{2}-q_{1}\right) \gamma^{1}+m \text { e } \beta:=q_{1} \gamma^{1}+m
$$

Usando a hipótese de que os momentos incidentes estão na camada de massa positiva, temos que:

$$
\omega^{2}-p_{0}^{i^{2}}=q^{1^{2}}-p_{1}^{i^{2}}, \quad i=1,2 ; \quad \text { e } \quad 2\left(m^{2}+p^{1} \cdot p^{2}\right)=\left(p^{1}+p^{2}\right)^{2},
$$

e podemos simplificar a expressão (G.1.4),

$$
\begin{aligned}
& =i \int \frac{d q_{1}}{4 \pi}\left\{\frac{-\omega^{2}\left(p_{0}^{1}+p_{0}^{2}\right) \gamma^{0} \otimes \gamma^{0}+\left[\omega^{2}+p_{0}^{1} p_{0}^{2}-\left(q_{1}-p_{1}^{1}\right)\left(q_{1}-p_{1}^{2}\right)\right]\left(\gamma^{0} \otimes \alpha-\beta \otimes \gamma^{0}\right)}{-\left(q_{1}-p_{1}^{1}\right)\left(q_{1}-p_{1}^{2}\right)\left(p^{1}+p^{2}\right)^{2}+4 i \epsilon\left(p_{0}^{1}+p_{0}^{2}\right)\left[p_{0}^{1} p_{0}^{2}-\left(q_{1}-p_{1}^{1}\right)\left(q_{1}-p_{1}^{2}\right)\right]}+\right. \\
& \left.+\frac{\left(p_{0}^{1}+p_{0}^{2}\right) \beta \otimes \alpha}{-\left(q_{1}-p_{1}^{1}\right)\left(q_{1}-p_{1}^{2}\right)\left(p^{1}+p^{2}\right)^{2}+4 i \epsilon\left(p_{0}^{1}+p_{0}^{2}\right)\left[p_{0}^{1} p_{0}^{2}-\left(q_{1}-p_{1}^{1}\right)\left(q_{1}-p_{1}^{2}\right)\right]}\right\}
\end{aligned}
$$

O próximo passo é integrar sobre $q_{1}$, para isso precisamos identificar os pólos de (G.1.6). Claramente, temos apenas dois pólos, e eles são da forma $q_{1}=p_{1}^{i}+\epsilon^{i}$. Não é difícil verificar que:

$$
\epsilon^{1}=\frac{4 i \epsilon p_{0}^{1} p_{0}^{2}\left(p_{0}^{1}+p_{0}^{2}\right)}{\left(p_{1}^{1}-p_{1}^{2}\right)\left(p^{1}+p^{2}\right)^{2}} \quad \text { e } \quad \epsilon^{2}=-\epsilon^{1}
$$

Como também supomos que os momentos incidentes estão ordenados, temos que os denominadores de $\epsilon^{1}$ e $\epsilon^{2}$ não podem se anular, e conseqüentemente, podemos redefini-los, tomando cuidado apenas 
com os sinais relativos, para escrever:

$$
I_{0}\left(p^{1}, p^{2}\right)=\int \frac{d q_{1}}{4 i \pi} \frac{f\left(q_{1}\right)}{\left(p^{1}+p^{2}\right)^{2}\left(q_{1}-p_{1}^{1}-i \varepsilon\right)\left(q_{1}-p_{1}^{2}+i \varepsilon\right)},
$$

onde denotamos o numerador de (G.1.6) por $f\left(q_{1}\right)$. É fácil constatar que $f\left(q_{1}\right)$ é um polinômio de segundo grau em $q_{1}$. Logo, a integral que precisamos calcular em (G.1.7) corresponde a:

$$
\int d q \frac{a q^{2}+b q+c}{\left(q-p_{1}^{1}-i \varepsilon\right)\left(q-p_{1}^{2}+i \varepsilon\right)}=\int d q \frac{a q^{2}+b q+c}{\left(q-P_{+}\right)^{2}-P_{-}^{2}-2 i \varepsilon P_{-}}
$$

onde

$$
P_{+}:=\frac{p_{1}^{1}+p_{1}^{2}}{2} \text { e } \quad P_{-}:=\frac{p_{1}^{1}-p_{1}^{2}}{2} .
$$

Redefinindo a variável de integração, $x=q-P_{+}$, obtemos:

$$
\begin{aligned}
\int d q \frac{a q^{2}+b q+c}{\left(q-p_{1}^{1}-i \varepsilon\right)\left(q-p_{1}^{2}+i \varepsilon\right)} & =\left[a\left(P_{-}{ }^{2}+P_{+}{ }^{2}\right)+b P_{+}+c\right] \int \frac{d x}{\left(x-P_{-}-i \varepsilon\right)\left(x+P_{-}+i \varepsilon\right)}+ \\
& +a \int d x
\end{aligned}
$$

Naturalmente a prescrição de pólos para a primeira integral em (G.1.9) depende do sinal de $P_{-}$. Neste ponto poderíamos invocar o nosso ordenamento para os momentos iniciais, contudo é conveniente mantermos uma maior generalidade na presente discussão. Dessa forma temos que:

$$
\int \frac{d x}{\left(x-P_{-}-i \varepsilon\right)\left(x+P_{-}+i \varepsilon\right)}=\frac{i \pi}{\left|P_{-}\right|}
$$

cuja substituição em (G.1.9) permite que concluamos que:

$$
\begin{aligned}
I_{0}\left(p^{1}, p^{2}\right) & =i \pi \frac{\left(a p_{1}^{12}+b p_{1}^{1}+c\right)+\left(a p_{1}^{22}+b p_{1}^{2}+c\right)}{\left|p_{1}^{1}-p_{1}^{2}\right|}+2 a \lim _{\wedge \rightarrow \infty} \wedge \\
& =\frac{f\left(p_{1}^{1}\right)+f\left(p_{1}^{2}\right)}{4\left|p_{1}^{1}-p_{1}^{2}\right|\left(p^{1}+p^{2}\right)^{2}}+D_{0},
\end{aligned}
$$

onde $D_{0}$ representa a parte divergente de $I_{0}\left(p^{1}, p^{2}\right)$. Finalmente, ao calcularmos:

$$
\begin{aligned}
& f\left(p_{1}^{1}\right)=\left(p_{0}^{1}+p_{0}^{2}\right)\left(\not \not^{1}+m\right) \otimes\left(\not p^{2}+m\right), \\
& f\left(p_{1}^{2}\right)=\left(p_{0}^{1}+p_{0}^{2}\right)\left(\not \not^{2}+m\right) \otimes\left(\not p^{1}+m\right),
\end{aligned}
$$

obtemos o resultado desejado (G.0.2a).

\section{G.2 Integrais para o Espalhamento de Três Pseudopartículas: Detalhes Computacionais}

O cálculo das integrais (G.0.3) é consideravelmente mais complicado, mas exibe propriedades interessantes que são fundamentais para a demonstração da fatorabilidade da matriz $S$. Nesta 


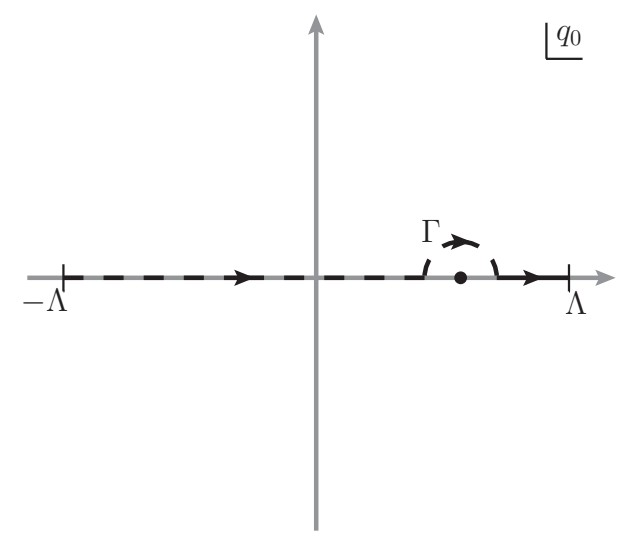

Figura G.2: Caminho de integração $\Gamma$ para o primeiro termo de (G.2.3).

seção, descrevemos os passos principais necessários para sua avaliação. Faremos isso no exemplo mais simples, a integral I, dada por (G.0.3a), notamos, entretanto, que as outras duas integrais, (G.0.3b) e (G.0.3c), podem ser calculadas precisamente da mesma forma, e os fatores extras de momento não introduzem sérias complicações técnicas.

Introduzindo coordenadas tipo cone-de-luz: $x=x_{+}+x_{-}$e $y=x_{+}-x_{-}$, a integral (G.0.3a) adquire a seguinte forma:

$$
I=4 \iint d^{2} x_{+} d^{2} x_{-} e^{-i x_{+} \cdot(\Delta+\tilde{\Delta})} e^{-i x_{-} \cdot(\Delta-\tilde{\Delta})} \int \frac{d^{2} q}{4 \pi^{2}} e^{2 i q \cdot x_{-}} \frac{i(\not l+m)}{q^{2}-m^{2}+2 i \epsilon q_{0}} .
$$

Podemos integrar sobre $x_{+}$, e assim obter a função delta, $\delta^{(2)}(\Delta+\tilde{\Delta})=\delta^{(2)}(\mathbf{k}-\mathbf{p})$, representando a conservação global de momento e energia. Fazendo a transformação de variáveis $x_{-} \rightarrow-\frac{1}{2} x \mathrm{e}$ usando a decomposição exata do propagador:

$$
\frac{1}{q^{2}-m^{2}+2 i \epsilon q_{0}}=\frac{1}{2 \omega(q)}\left[\frac{1}{q_{0}-\omega(q)+i \epsilon}-\frac{1}{q_{0}+\omega(q)+i \epsilon}\right]
$$

obtemos:

$$
I=\int d^{2} x e^{i x \cdot \Delta} \int \frac{d^{2} q}{4 \pi^{2}} \frac{i(\not d+m) e^{-i x \cdot q}}{2 \omega(q)}\left[\frac{1}{q_{0}-\omega(q)+i \epsilon}-\frac{1}{q_{0}+\omega(q)+i \epsilon}\right] 4 \pi^{2} \delta^{(2)}(\mathbf{k}-\mathbf{p}) .
$$

Para efetuar a integração sobre $q_{0}$ no primeiro termo de (G.2.3), consideramos o seguinte caminho:

$$
\Gamma=\left\{\begin{array}{ll}
q_{0}, & q_{0} \in[-\Lambda, \omega(q)-\epsilon) \cup(\omega(q)+\epsilon, \Lambda], \\
q_{0}-\omega(q)=\epsilon e^{-i \theta}, & \theta \in[0, \pi] \quad\left(C_{\epsilon}\right)
\end{array},\right.
$$

esboçado na figura G.2. Logo, 


$$
\begin{aligned}
\int \frac{d q_{0}}{2 \pi} \frac{(\phi+m) e^{-i x^{0} q_{0}}}{q_{0}-\omega(q)+i \epsilon} & =\lim _{\substack{\Lambda \rightarrow \infty \\
\epsilon \rightarrow 0}}\left\{\left[\int_{-\Lambda}^{\omega(q)-\epsilon}+\int_{\omega(q)+\epsilon}^{\wedge}\right] \frac{d q_{0}}{2 \pi} \frac{(\phi+m) e^{-i x^{0} q_{0}}}{q_{0}-\omega(q)}+\right. \\
& \left.+\frac{1}{2 \pi}\left[(\phi+m) e^{-i x^{0} q_{0}}\right]_{q_{0}=\omega(q)} \int_{C_{\epsilon}} \frac{d q_{0}}{q_{0}-\omega(q)}\right\} \\
& =f \frac{d q_{0}}{2 \pi} \frac{(\phi+m) e^{-i x^{0} q_{0}}}{q_{0}-\omega(q)}-\frac{i}{2}\left[(\phi+m) e^{-i x^{0} q_{0}}\right]_{q_{0}=\omega(q)}
\end{aligned}
$$

correspondendo à soma de um valor principal (v.p.) com uma função delta que proporciona a decomposição (8.3.13). O segundo termo de (G.2.3) pode ser calculado exatamente da mesma maneira. Portanto,

$$
\begin{aligned}
I & =\int d^{2} x e^{i x \cdot \Delta} \int \frac{d q_{1}}{2 \pi} \frac{i e^{-i x^{1} q_{1}}}{2 \omega(q)}\left\{-\frac{i}{2}\left[\left.(\phi q+m) e^{-i x^{0} q_{0}}\right|_{q_{0}=\omega(q)}-\left.(\phi+m) e^{-i x^{0} q_{0}}\right|_{q_{0}=-\omega(q)}\right]+\right. \\
& \left.+f \frac{d q_{0}}{2 \pi}(\phi+m) e^{-i x^{0} q_{0}}\left[\frac{1}{q_{0}-\omega(q)}-\frac{1}{q_{0}+\omega(q)}\right]\right\} 4 \pi^{2} \delta^{(2)}(\mathbf{k}-\mathbf{p}) .
\end{aligned}
$$

Conforme afirmado acima, os termos presentes na primeira linha de (G.2.6) contribuem para as funções delta na decomposição (8.3.13). Para chegarmos a essa conclusão, precisamos somente agrupar as exponenciais e notar que podemos impunemente trocar a ordem das integrações, por consegüinte obtemos uma função delta a partir da integração sobre $x$. Mais precisamente,

$$
\begin{array}{r}
\int d^{2} x e^{i x \cdot \Delta} \int \frac{d q_{1}}{2 \pi} \frac{i e^{-i x^{1} q_{1}}}{2 \omega(q)}\left\{-\frac{i}{2}\left[\left.(\not \phi+m) e^{-i x^{0} q_{0}}\right|_{q_{0}=\omega(q)}-\left.(\not \phi+m) e^{-i x^{0} q_{0}}\right|_{q_{0}=-\omega(q)}\right]\right\}= \\
=\frac{\Delta+m}{4 \omega(\Delta)} 2 \pi\left[\delta\left(\Delta_{0}-\omega(\Delta)\right)-\delta\left(\Delta_{0}+\omega(\Delta)\right)\right] .
\end{array}
$$

Obter a contribuição do valor principal requer mais trabalho. Primeiramente, denote o valor principal da integral (G.2.6) simplesmente por / e note que:

$$
\begin{aligned}
(\phi+m)\left[\frac{1}{q_{0}-\omega(q)}-\frac{1}{q_{0}+\omega(q)}\right] & =\gamma^{0} \omega(q)\left[\frac{1}{q_{0}-\omega(q)}+\frac{1}{q_{0}+\omega(q)}\right]+ \\
& +\left(q_{1} \gamma^{1}+m\right)\left[\frac{1}{q_{0}-\omega(q)}-\frac{1}{q_{0}+\omega(q)}\right]
\end{aligned}
$$

Portanto, ao usarmos as identidades:

$$
\begin{aligned}
& f \frac{d q_{0}}{2 \pi} e^{-i x^{0} q_{0}}\left[\frac{1}{q_{0}-\omega(q)}-\frac{1}{q_{0}+\omega(q)}\right]=-\frac{2}{\pi} \sin \left(\omega(q) x^{0}\right) \int_{\epsilon}^{\infty} \frac{d z}{z} \sin \left(x^{0} z\right), \\
& f \frac{d q_{0}}{2 \pi} e^{-i x^{0} q_{0}}\left[\frac{1}{q_{0}-\omega(q)}+\frac{1}{q_{0}+\omega(q)}\right]=-\frac{2 i}{\pi} \cos \left(\omega(q) x^{0}\right) \int_{\epsilon}^{\infty} \frac{d z}{z} \sin \left(x^{0} z\right),
\end{aligned}
$$


onde deixamos o limite $\epsilon \rightarrow 0$ implícito, podemos escrever:

$$
\begin{aligned}
I & =\int d^{2} x e^{i x \cdot \Delta} \int \frac{d q_{1}}{2 \pi} \frac{i e^{-i x^{1} q_{1}}}{2 \omega(q)}\left\{-\frac{2 i}{\pi} \gamma^{0} \omega(q) \cos \left(\omega(q) x^{0}\right)-\frac{2}{\pi}\left(q_{1} \gamma^{1}+m\right) \sin \left(\omega(q) x^{0}\right)\right\} \\
& \cdot \int_{\epsilon}^{\infty} \frac{d z}{z} \sin \left(x^{0} z\right) 4 \pi \delta^{(2)}(\mathbf{k}-\mathbf{p}) \\
& =\frac{i}{8 \pi^{2}} \int d^{2} x \int d q_{1} \frac{e^{i x^{1}\left(\Delta_{1}-q_{1}\right)}}{\omega(q)}\left[\int_{-\infty}^{-\epsilon}+\int_{\epsilon}^{\infty}\right] \frac{d z}{z}\left\{\left[\omega(q) \gamma^{0}-q_{1} \gamma^{1}-m\right] e^{i x^{0}\left[\Delta_{0}+\omega(q)-z\right]}+\right. \\
& \left.+\left[\omega(q) \gamma^{0}+q_{1} \gamma^{1}+m\right] e^{i x^{0}\left[\Delta_{0}-\omega(q)-z\right]}\right\} 4 \pi^{2} \delta^{(2)}(\mathbf{k}-\mathbf{p})
\end{aligned}
$$

Em seguida, trocamos a ordem das integrações para podermos avaliar primeiramente as integrais sobre $x^{1}$ e $x^{0}$. A integral com respeito a $d x^{1}$ fatora e claramente leva a uma função delta, que pode, consequientemente, ser usada para calcular a integral sobre $q_{1}$, estabelecendo que $q_{1}=\Delta_{1}$. Já a análise envolvendo a integração sobre $x^{0}$ é mais delicada e exige mais cuidado. Nesse caso, o limite $\epsilon \rightarrow 0$ assume um papel fundamental, uma vez que remove o ponto $z=0$ do domínio de integração, expurgando, pois, a singularidade proporcional a $\frac{1}{z}$. Logo, podemos proceder como antes, ou seja, integrar sobre $x^{0}$ para obter uma função delta, que pode, por sua vez, ser usada para efetuar a integração sobre $z$. Portanto, concluímos que:

$$
\begin{aligned}
I & =\frac{i}{2 \omega(\Delta)}\left\{\frac{\omega(\Delta) \gamma^{0}-\Delta_{1} \gamma^{1}-m}{\Delta_{0}+\omega(\Delta)}+\frac{\omega(\Delta) \gamma^{0}+\Delta_{1} \gamma^{1}+m}{\Delta_{0}-\omega(\Delta)}\right\} 4 \pi^{2} \delta^{(2)}(\mathbf{k}-\mathbf{p}) \\
& =\frac{i(\Delta+m)}{\Delta^{2}-m^{2}} 4 \pi^{2} \delta^{(2)}(\mathbf{k}-\mathbf{p}) .
\end{aligned}
$$

Finalmente, ao somarmos ambas contribuições (G.2.7) e (G.2.11), obtemos o resultado desejado, (G.0.3a).

Todavia, é crucial ressaltar que uma análise cuidadosa acerca da nulidade do fator $\Delta_{0} \pm \omega(\Delta)$ é de fundamental importância nesse contexto, dado que ela pode implicar a existência de divergências ou descontinuidades nas amplitudes de espalhamento. A avaliação do caso com o sinal positivo é significativamente mais simples, pois, uma vez que lidamos apenas com pseudopartículas com energia positiva, ao impormos a condição de camada de massa, verificamos que tal polinômio não possui raízes reais. Claramente, o mesmo não acontece com o polinômio advindo de $\Delta_{0}-\omega(\Delta)$, que pode ser resolvido por exemplo para $k^{1}$, implicando que $k^{1}=p^{1}$ ou $k^{1}=p^{2}$. E apesar de ainda podermos efetuar as integrações sobre $x^{0}$ e $z$ como fizemos anteriormente, mesmo que $\Delta_{0}-$ $\omega(\Delta)=0$, o resultado é obviamente diferente de (G.2.11). De fato, ele muda de tal maneira a evitar quaisquer divergências oriundas da anulação do denominador. Dessa forma, resta apenas a questão sobre a continuidade das amplitudes de espalhamento conforme nos aproximamos do ponto de integrabilidade, mas, segundo a discussão presente na seção 8.4, esse problema pode ser desconsiderado, ao empregarmos distribuições de pacotes de onda localizadas, pois nesse caso a condição $\Delta_{0}-\omega(\Delta)=0$ nunca é satisfeita. 



\section{Apêndice H}

\section{Expressões Relevantes para a \\ Demonstração da Fatorabilidade da \\ Matriz S no Modelo de \\ Alday-Arutyunov-Frolov}

"And if your head explodes with dark forebodings too

I'll see you on the dark side of the moon"

Pink Floyd - Brain Damage

Neste apêndice reunimos todas as fórmulas adicionais necessárias para a demonstração do cancelamento entre as contribuições espúrias que impediram a fatoração da matriz $S$. Os fatores $A_{i}(\eta, \theta)$ presentes na decomposição (8.5.5) de $G(\eta, \theta)$ são:

$$
\begin{aligned}
A_{1}(\eta, \theta) & =\cosh \left(\frac{\eta_{2}-\eta_{3}}{2}\right) \cosh \left(\frac{\theta_{1}-\theta_{2}}{2}\right) \cosh \left(\frac{\theta_{2}-\theta_{3}}{2}\right) \operatorname{csch}\left(\frac{\eta_{1}-\theta_{1}}{2}\right) \sinh \left(\frac{\eta_{2}-\eta_{3}}{2}\right) . \\
& \cdot \sinh \left(\frac{2 \eta_{1}-\theta_{1}-\theta_{2}}{2}\right) \sinh \left(\frac{\eta_{2}+\eta_{3}-2 \theta_{3}}{2}\right), \\
A_{2}(\eta, \theta) & =-\cosh \left(\frac{\eta_{2}-\eta_{3}}{2}\right) \cosh \left(\frac{\theta_{1}-\theta_{2}}{2}\right) \cosh \left(\frac{\theta_{2}-\theta_{3}}{2}\right) \operatorname{csch}\left(\frac{\eta_{1}-\theta_{1}}{2}\right) \cdot \\
& \cdot \sinh ^{2}\left(\frac{\eta_{2}-\eta_{3}}{2}\right) \sinh \left(\frac{2 \eta_{1}-\theta_{1}-\theta_{2}}{2}\right), \\
A_{3}(\eta, \theta) & =\cosh \left(\frac{\eta_{2}-\eta_{3}}{2}\right) \cosh \left(\frac{\theta_{1}-\theta_{2}}{2}\right) \cosh \left(\frac{\theta_{2}-\theta_{3}}{2}\right) \operatorname{csch}\left(\frac{\eta_{1}-\theta_{1}}{2}\right) \sinh \left(\frac{\eta_{2}-\eta_{3}}{2}\right) . \\
& \cdot \sinh \left(\frac{\theta_{1}-\theta_{2}}{2}\right) \sinh \left(\frac{\eta_{2}+\eta_{3}-2 \theta_{3}}{2}\right), \\
A_{4}(\eta, \theta) & =-\cosh \left(\frac{\eta_{2}-\eta_{3}}{2}\right) \cosh \left(\frac{\theta_{1}-\theta_{2}}{2}\right) \cosh \left(\frac{\theta_{2}-\theta_{3}}{2}\right) \operatorname{csch}\left(\frac{\eta_{1}-\theta_{1}}{2}\right) \cdot \\
& \cdot \sinh ^{2}\left(\frac{\eta_{2}-\eta_{3}}{2}\right) \sinh \left(\frac{\theta_{1}-\theta_{2}}{2}\right),
\end{aligned}
$$




$$
\begin{aligned}
A_{5}(\eta, \theta) & =-\cosh ^{2}\left(\frac{\eta_{2}-\eta_{3}}{2}\right) \cosh \left(\frac{\theta_{1}-\theta_{2}}{2}\right) \operatorname{csch}\left(\frac{\eta_{1}-\theta_{1}}{2}\right) \sinh \left(\frac{\theta_{2}-\theta_{3}}{2}\right) . \\
& \cdot \sinh \left(\frac{2 \eta_{1}-\theta_{1}-\theta_{2}}{2}\right) \sinh \left(\frac{\eta_{2}+\eta_{3}-2 \theta_{3}}{2}\right), \\
A_{6}(\eta, \theta) & =\cosh ^{2}\left(\frac{\eta_{2}-\eta_{3}}{2}\right) \cosh \left(\frac{\theta_{1}-\theta_{2}}{2}\right) \operatorname{csch}\left(\frac{\eta_{1}-\theta_{1}}{2}\right) \sinh \left(\frac{\eta_{2}-\eta_{3}}{2}\right) . \\
& \cdot \sinh \left(\frac{\theta_{2}-\theta_{3}}{2}\right) \sinh \left(\frac{2 \eta_{1}-\theta_{1}-\theta_{2}}{2}\right), \\
A_{7}(\eta, \theta) & =-\cosh ^{2}\left(\frac{\eta_{2}-\eta_{3}}{2}\right) \cosh \left(\frac{\theta_{1}-\theta_{2}}{2}\right) \operatorname{csch}\left(\frac{\eta_{1}-\theta_{1}}{2}\right) \sinh \left(\frac{\theta_{1}-\theta_{2}}{2}\right) . \\
& \sinh \left(\frac{\theta_{2}-\theta_{3}}{2}\right) \sinh \left(\frac{\eta_{2}+\eta_{3}-2 \theta_{3}}{2}\right), \\
A_{8}(\eta, \theta) & =\cosh \left(\frac{\eta_{2}-\eta_{3}}{2}\right) \cosh \left(\frac{\theta_{1}-\theta_{2}}{2}\right) \operatorname{csch}\left(\frac{\eta_{1}-\theta_{1}}{2}\right) \sinh \left(\frac{\eta_{2}-\eta_{3}}{2}\right) . \\
\cdot & \sinh \left(\frac{\theta_{1}-\theta_{2}}{2}\right) \sinh \left(\frac{\theta_{2}-\theta_{3}}{2}\right) .
\end{aligned}
$$

Abaixo fornecemos as expressões explícitas para os termos não-nulos obtidos através da ação do antissimetrizador nos fatores $A_{i}(\eta, \theta)$ :

$$
\begin{aligned}
(3 !)^{2} \mathcal{A}_{\theta, \eta}\left[A_{1}(\eta, \theta)\right] & =\frac{1}{4}\left\{\operatorname{csch}\left[\frac{\eta_{1}-\theta_{1}}{2}\right] \operatorname{csch}\left[\frac{\eta_{1}-\theta_{2}}{2}\right] \operatorname{csch}\left[\frac{\eta_{1}-\theta_{3}}{2}\right] \sinh \left(\eta_{2}-\eta_{3}\right) \cdot\right. \\
& \cdot\left[3 \sinh \left(\frac{2 \eta_{1}-\eta_{2}-\eta_{3}}{2}\right)-\sinh \left(\frac{4 \eta_{1}+\eta_{2}+\eta_{3}-2 \theta_{1}-2 \theta_{2}-2 \theta_{3}}{2}\right)-\right. \\
& -\sinh \left(\frac{4 \eta_{1}-\eta_{2}-\eta_{3}-2 \theta_{1}}{2}\right)-\sinh \left(\frac{4 \eta_{1}-\eta_{2}-\eta_{3}-2 \theta_{2}}{2}\right)- \\
& -\sinh \left(\frac{4 \eta_{1}-\eta_{2}-\eta_{3}-2 \theta_{3}}{2}\right)+\sinh \left(\frac{2 \eta_{1}+\eta_{2}+\eta_{3}-2 \theta_{1}-2 \theta_{2}}{2}\right)+ \\
& +\sinh \left(\frac{2 \eta_{1}+\eta_{2}+\eta_{3}-2 \theta_{1}-2 \theta_{3}}{2}\right)+\sinh \left(\frac{2 \eta_{1}+\eta_{2}+\eta_{3}-2 \theta_{2}-2 \theta_{3}}{2}\right)+ \\
& +\sinh \left(\frac{2 \eta_{1}-\eta_{2}-\eta_{3}+2 \theta_{1}-2 \theta_{2}}{2}\right)+\sinh \left(\frac{2 \eta_{1}-\eta_{2}-\eta_{3}-2 \theta_{1}+2 \theta_{2}}{2}\right)+ \\
& +\sinh \left(\frac{2 \eta_{1}-\eta_{2}-\eta_{3}+2 \theta_{1}-2 \theta_{3}}{2}\right)+\sinh \left(\frac{2 \eta_{1}-\eta_{2}-\eta_{3}-2 \theta_{1}+2 \theta_{3}}{2}\right)+ \\
& \left.+\sinh \left(\frac{2 \eta_{1}-\eta_{2}-\eta_{3}+2 \theta_{2}-2 \theta_{3}}{2}\right)+\sinh \left(\frac{2 \eta_{1}-\eta_{2}-\eta_{3}-2 \theta_{2}+2 \theta_{3}}{2}\right)\right]+
\end{aligned}
$$




$$
\begin{aligned}
& +\operatorname{csch}\left[\frac{\eta_{2}-\theta_{1}}{2}\right] \operatorname{csch}\left[\frac{\eta_{2}-\theta_{2}}{2}\right] \operatorname{csch}\left[\frac{\eta_{2}-\theta_{3}}{2}\right] \sinh \left(\eta_{1}-\eta_{3}\right) \cdot \\
& \cdot\left[3 \sinh \left(\frac{\eta_{1}-2 \eta_{2}+\eta_{3}}{2}\right)+\sinh \left(\frac{\eta_{1}+4 \eta_{2}+\eta_{3}-2 \theta_{1}-2 \theta_{2}-2 \theta_{3}}{2}\right)-\right. \\
& -\sinh \left(\frac{\eta_{1}-4 \eta_{2}+\eta_{3}-2 \theta_{1}}{2}\right)-\sinh \left(\frac{\eta_{1}-4 \eta_{2}+\eta_{3}-2 \theta_{2}}{2}\right)- \\
& -\sinh \left(\frac{\eta_{1}-4 \eta_{2}+\eta_{3}-2 \theta_{3}}{2}\right)-\sinh \left(\frac{\eta_{1}+2 \eta_{2}+\eta_{3}-2 \theta_{1}-2 \theta_{2}}{2}\right)- \\
& -\sinh \left(\frac{\eta_{1}+2 \eta_{2}+\eta_{3}-2 \theta_{1}-2 \theta_{3}}{2}\right)-\sinh \left(\frac{\eta_{1}+2 \eta_{2}+\eta_{3}-2 \theta_{2}-2 \theta_{3}}{2}\right)+ \\
& +\sinh \left(\frac{\eta_{1}-2 \eta_{2}+\eta_{3}+2 \theta_{1}-2 \theta_{2}}{2}\right)+\sinh \left(\frac{\eta_{1}-2 \eta_{2}+\eta_{3}-2 \theta_{1}+2 \theta_{2}}{2}\right)+ \\
& +\sinh \left(\frac{\eta_{1}-2 \eta_{2}+\eta_{3}+2 \theta_{1}-2 \theta_{3}}{2}\right)+\sinh \left(\frac{\eta_{1}-2 \eta_{2}+\eta_{3}-2 \theta_{1}+2 \theta_{3}}{2}\right)+ \\
& \left.+\sinh \left(\frac{\eta_{1}-2 \eta_{2}+\eta_{3}+2 \theta_{2}-2 \theta_{3}}{2}\right)+\sinh \left(\frac{\eta_{1}-2 \eta_{2}+\eta_{3}-2 \theta_{2}+2 \theta_{3}}{2}\right)\right]- \\
& -\operatorname{csch}\left[\frac{\eta_{3}-\theta_{1}}{2}\right] \operatorname{csch}\left[\frac{\eta_{3}-\theta_{2}}{2}\right] \operatorname{csch}\left[\frac{\eta_{3}-\theta_{3}}{2}\right] \sinh \left(\eta_{1}-\eta_{2}\right) \cdot \\
& \cdot\left[3 \sinh \left(\frac{\eta_{1}+\eta_{2}-2 \eta_{3}}{2}\right)+\sinh \left(\frac{\eta_{1}+\eta_{2}+4 \eta_{3}-2 \theta_{1}-2 \theta_{2}-2 \theta_{3}}{2}\right)-\right. \\
& -\sinh \left(\frac{\eta_{1}+\eta_{2}-4 \eta_{3}+2 \theta_{1}}{2}\right)-\sinh \left(\frac{\eta_{1}+\eta_{2}-4 \eta_{3}+2 \theta_{2}}{2}\right)- \\
& -\sinh \left(\frac{4 \eta_{1}+\eta_{2}-4 \eta_{3}+2 \theta_{3}}{2}\right)-\sinh \left(\frac{\eta_{1}+\eta_{2}+2 \eta_{3}-2 \theta_{1}-2 \theta_{2}}{2}\right)- \\
& -\sinh \left(\frac{\eta_{1}+\eta_{2}+2 \eta_{3}-2 \theta_{1}-2 \theta_{3}}{2}\right)-\sinh \left(\frac{\eta_{1}+\eta_{2}+2 \eta_{3}-2 \theta_{2}-2 \theta_{3}}{2}\right)+ \\
& +\sinh \left(\frac{\eta_{1}+\eta_{2}-2 \eta_{3}+2 \theta_{1}-2 \theta_{2}}{2}\right)+\sinh \left(\frac{\eta_{1}+\eta_{2}-2 \eta_{3}-2 \theta_{1}+2 \theta_{2}}{2}\right)+ \\
& +\sinh \left(\frac{\eta_{1}+\eta_{2}-2 \eta_{3}+2 \theta_{1}-2 \theta_{3}}{2}\right)+\sinh \left(\frac{\eta_{1}+\eta_{2}-2 \eta_{3}-2 \theta_{1}+2 \theta_{3}}{2}\right)+ \\
& \left.\left.+\sinh \left(\frac{\eta_{1}+\eta_{2}-2 \eta_{3}+2 \theta_{2}-2 \theta_{3}}{2}\right)+\sinh \left(\frac{\eta_{1}+\eta_{2}-2 \eta_{3}-2 \theta_{2}+2 \theta_{3}}{2}\right)\right]\right\} \text {. } \\
& \cdot \sinh \left(\frac{\theta_{1}-\theta_{2}}{2}\right) \sinh \left(\frac{\theta_{1}-\theta_{3}}{2}\right) \sinh \left(\frac{\theta_{2}-\theta_{3}}{2}\right) \text {, }
\end{aligned}
$$

$(3 !)^{2} \mathcal{A}_{\theta, \eta}\left[A_{3}(\eta, \theta)\right]=\frac{1}{4}\left\{-\operatorname{csch}\left(\frac{\eta_{3}-\theta_{1}}{2}\right) \operatorname{csch}\left(\frac{\eta_{3}-\theta_{2}}{2}\right) \operatorname{csch}\left(\frac{\eta_{3}-\theta_{3}}{2}\right) \sinh \left(\eta_{1}-\eta_{2}\right)\right.$.

$$
\begin{aligned}
& \cdot\left[\sinh \left(\frac{\eta_{1}+\eta_{2}-2 \eta_{3}}{2}\right)+\sinh \left(\frac{\eta_{1}+\eta_{2}-2 \theta_{1}}{2}\right)+\sinh \left(\frac{\eta_{1}+\eta_{2}-2 \theta_{2}}{2}\right)+\right. \\
& +\sinh \left(\frac{\eta_{1}+\eta_{2}-2 \theta_{3}}{2}\right)-\sinh \left(\frac{\eta_{1}+\eta_{2}+2 \eta_{3}-2 \theta_{1}-2 \theta_{2}}{2}\right)- \\
& -\sinh \left(\frac{\eta_{1}+\eta_{2}+2 \eta_{3}-2 \theta_{1}-2 \theta_{3}}{2}\right)-\sinh \left(\frac{\eta_{1}+\eta_{2}+2 \eta_{3}-2 \theta_{2}-2 \theta_{3}}{2}\right)+ \\
& +\sinh \left(\frac{\eta_{1}+\eta_{2}-2 \theta_{1}-2 \theta_{2}+2 \theta_{3}}{2}\right)+\sinh \left(\frac{\eta_{1}+\eta_{2}-2 \theta_{1}+2 \theta_{2}-2 \theta_{3}}{2}\right)+ \\
& \left.+\sinh \left(\frac{\eta_{1}+\eta_{2}+2 \theta_{1}-2 \theta_{2}-2 \theta_{3}}{2}\right)\right]+
\end{aligned}
$$




$$
\begin{aligned}
& +\operatorname{csch}\left(\frac{\eta_{2}-\theta_{1}}{2}\right) \operatorname{csch}\left(\frac{\eta_{2}-\theta_{2}}{2}\right) \operatorname{csch}\left(\frac{\eta_{2}-\theta_{3}}{2}\right) \sinh \left(\eta_{1}-\eta_{3}\right) \text {. } \\
& \cdot\left[\sinh \left(\frac{\eta_{1}-2 \eta_{2}+\eta_{3}}{2}\right)+\sinh \left(\frac{\eta_{1}+\eta_{3}-2 \theta_{1}}{2}\right)+\sinh \left(\frac{\eta_{1}+\eta_{3}-2 \theta_{2}}{2}\right)+\right. \\
& +\sinh \left(\frac{\eta_{1}+\eta_{3}-2 \theta_{3}}{2}\right)-\sinh \left(\frac{\eta_{1}+2 \eta_{2}+\eta_{3}-2 \theta_{1}-2 \theta_{2}}{2}\right)- \\
& -\sinh \left(\frac{\eta_{1}+2 \eta_{2}+\eta_{3}-2 \theta_{1}-2 \theta_{3}}{2}\right)-\sinh \left(\frac{\eta_{1}+2 \eta_{2}+\eta_{3}-2 \theta_{2}-2 \theta_{3}}{2}\right)+ \\
& +\sinh \left(\frac{\eta_{1}+\eta_{3}-2 \theta_{1}-2 \theta_{2}+2 \theta_{3}}{2}\right)+\sinh \left(\frac{\eta_{1}+\eta_{3}-2 \theta_{1}+2 \theta_{2}-2 \theta_{3}}{2}\right)+ \\
& \left.+\sinh \left(\frac{\eta_{1}+\eta_{3}+2 \theta_{1}-2 \theta_{2}-2 \theta_{3}}{2}\right)\right]+ \\
& +\operatorname{csch}\left(\frac{\eta_{1}-\theta_{1}}{2}\right) \operatorname{csch}\left(\frac{\eta_{1}-\theta_{2}}{2}\right) \operatorname{csch}\left(\frac{\eta_{1}-\theta_{3}}{2}\right) \sinh \left(\eta_{2}-\eta_{3}\right) \cdot \\
& \cdot\left[\sinh \left(\frac{2 \eta_{1}-\eta_{2}-\eta_{3}}{2}\right)-\sinh \left(\frac{\eta_{2}+\eta_{3}-2 \theta_{1}}{2}\right)-\sinh \left(\frac{\eta_{2}+\eta_{3}-2 \theta_{2}}{2}\right)-\right. \\
& -\sinh \left(\frac{\eta_{2}+\eta_{3}-2 \theta_{3}}{2}\right)+\sinh \left(\frac{2 \eta_{1}+\eta_{2}+\eta_{3}-2 \theta_{1}-2 \theta_{2}}{2}\right)+ \\
& +\sinh \left(\frac{2 \eta_{1}+\eta_{2}+\eta_{3}-2 \theta_{1}-2 \theta_{3}}{2}\right)+\sinh \left(\frac{2 \eta_{1}+\eta_{2}+\eta_{3}-2 \theta_{2}-2 \theta_{3}}{2}\right)- \\
& -\sinh \left(\frac{\eta_{2}+\eta_{3}-2 \theta_{1}-2 \theta_{2}+2 \theta_{3}}{2}\right)-\sinh \left(\frac{\eta_{2}+\eta_{3}-2 \theta_{1}+2 \theta_{2}-2 \theta_{3}}{2}\right)- \\
& \left.\left.-\sinh \left(\frac{\eta_{2}+\eta_{3}+2 \theta_{1}-2 \theta_{2}-2 \theta_{3}}{2}\right)\right]\right\} \text {. } \\
& \cdot \sinh \left(\frac{\theta_{1}-\theta_{2}}{2}\right) \sinh \left(\frac{\theta_{1}-\theta_{3}}{2}\right) \sinh \left(\frac{\theta_{2}-\theta_{3}}{2}\right) \text {, } \\
& (3 !)^{2} \mathcal{A}_{\theta, \eta}\left[A_{6}(\eta, \theta)\right]=\frac{1}{4}\left\{\operatorname{csch}\left(\frac{\eta_{2}-\eta_{3}}{2}\right) \operatorname{csch}\left(\frac{\eta_{1}-\theta_{1}}{2}\right) \operatorname{csch}\left(\frac{\eta_{1}-\theta_{2}}{2}\right) \operatorname{csch}\left(\frac{\eta_{1}-\theta_{3}}{2}\right) .\right. \\
& \cdot \sinh ^{2}\left(\eta_{2}-\eta_{3}\right)\left[\sinh \left(\eta_{1}-\eta_{2}\right)+\sinh \left(\eta_{1}-\eta_{3}\right)\right]- \\
& -\operatorname{csch}\left(\frac{\eta_{1}-\eta_{3}}{2}\right) \operatorname{csch}\left(\frac{\eta_{2}-\theta_{1}}{2}\right) \operatorname{csch}\left(\frac{\eta_{2}-\theta_{2}}{2}\right) \operatorname{csch}\left(\frac{\eta_{2}-\theta_{3}}{2}\right) \text {. } \\
& \cdot \sinh ^{2}\left(\eta_{1}-\eta_{3}\right)\left[\sinh \left(\eta_{2}-\eta_{1}\right)+\sinh \left(\eta_{2}-\eta_{3}\right)\right]+ \\
& +\operatorname{csch}\left(\frac{\eta_{1}-\eta_{2}}{2}\right) \operatorname{csch}\left(\frac{\eta_{3}-\theta_{1}}{2}\right) \operatorname{csch}\left(\frac{\eta_{3}-\theta_{2}}{2}\right) \operatorname{csch}\left(\frac{\eta_{3}-\theta_{3}}{2}\right) \text {. } \\
& \left.\cdot \sinh ^{2}\left(\eta_{1}-\eta_{2}\right)\left[\sinh \left(\eta_{3}-\eta_{1}\right)+\sinh \left(\eta_{3}-\eta_{2}\right)\right]\right\} \text {. } \\
& \cdot \sinh \left(\frac{\theta_{1}-\theta_{2}}{2}\right) \sinh \left(\frac{\theta_{1}-\theta_{3}}{2}\right) \sinh \left(\frac{\theta_{2}-\theta_{3}}{2}\right) \text {. }
\end{aligned}
$$




\section{Referências Bibliográficas}

[1] M. B. Green, J. H. Schwarz, and E. Witten, Superstring theory I: Introduction. Cambridge Monographs on Mathematical Physics, 480 p., 1987.

[2] M. B. Green, J. H. Schwarz, and E. Witten, Superstring theory II: Loop Amplitudes, Anomalies and Phenomenology. Cambridge Monographs on Mathematical Physics, 608 p., 1987.

[3] J. Polchinski, String theory. Vol. 1: An introduction to the bosonic string. Cambridge University Press, 402 p., 1998.

[4] J. Polchinski, String theory. Vol. 2: Superstring theory and beyond. Cambridge University Press, 531 p., 1998.

[5] K. Becker, M. Becker, and J. Schwarz, String theory and M-theory: a modern introduction. Cambridge University Press, 2007.

[6] G. 't Hooft, "A planar diagram theory for strong interactions," Nucl. Phys. B72 (1974) 461.

[7] J. Polchinski, "Dirichlet Branes and Ramond-Ramond Charges," Physical Review Letters 75 (Dec., 1995) 4724-4727, arXiv: hep-th/9510017.

[8] E. Witten, "Small Instantons in String Theory," Nucl. Phys. B460 (1996) 541-559, hep-th/9511030.

[9] J. M. Maldacena, "The large N limit of superconformal field theories and supergravity," Adv. Theor. Math. Phys. 2 (1998) 231-252, hep-th/9711200.

[10] O. Aharony, S. S. Gubser, J. M. Maldacena, H. Ooguri, and Y. Oz, "Large N field theories, string theory and gravity," Phys. Rept. 323 (2000) 183-386, arXiv:9905111 [hep-th].

[11] E. D'Hoker and D. Z. Freedman, "Supersymmetric gauge theories and the AdS/CFT correspondence," hep-th/0201253.

[12] G. T. Horowitz and J. Polchinski, "Gauge / gravity duality," gr-qc/0602037.

[13] G. Arutyunov and S. Frolov, "Foundations of the $A d S_{5} \times S^{5}$ Superstring. Part I," J. Phys. A42 (2009) 254003, 0901.4937.

[14] G. 't Hooft, "Dimensional reduction in quantum gravity," arXiv9:310026 [gr-qc]. 
[15] J. D. Bekenstein, "Entropy bounds and black hole remnants," Phys. Rev. D49 (1994) 1912-1921, arXiv:9307035 [gr-qc].

[16] R. R. Metsaev and A. A. Tseytlin, "Type IIB superstring action in $A d S_{5} \times S^{5}$ background," Nucl. Phys. B533 (1998) 109-126, hep-th/9805028.

[17] D. Berenstein, J. M. Maldacena, and H. Nastase, "Strings in flat space and pp waves from N = 4 super Yang Mills," JHEP 04 (2002) 013, hep-th/0202021.

[18] N. Beisert, C. Kristjansen, and M. Staudacher, "The dilatation operator of N = 4 super yang-mills theory," Nucl. Phys. B664 (2003) 131-184, arXiv:0303060 [hep-th].

[19] N. Beisert et al., "Review of AdS/CFT Integrability: An Overview," arXiv:1012.3982 [hep-th].

[20] L. D. Faddeev and L. A. Takhtajan, Hamiltonian Methods in the Theory of Solitons. Springer Series in Soviet Mathematics, 592 p., 1987.

[21] L. D. Faddeev, "Integrable models in (1+1)-dimensional quantum field theory," Recent advances in Field Theory and Statistical Mechanics (1982). Proc. of Summer School of Theoretical Physics, Les Houches, France, Aug 2 - Sep 10, eds. J.B. Zuber and R. Stora (North-Holland, Amsterdam, 1984).

[22] V. E. Korepin, N. M. Bogoliubov, and A. G. Izergin, Quantum Inverse Scattering Method and Correlation Functions. Cambridge Monographs on Mathematical Physics. Cambridge University Press, 1997.

[23] E. K. Sklyanin, "Quantum version of the method of inverse scattering problem," J. Sov. Math. 19 (1982) 1546-1596.

[24] P. Dorey, "Exact S matrices," hep-th/9810026.

[25] A. B. Zamolodchikov and A. B. Zamolodchikov, "Factorized S-matrices in two dimensions as the exact solutions of certain relativistic quantum field models," Annals Phys. 120 (1979) 253-291.

[26] J. A. Minahan and K. Zarembo, "The Bethe-ansatz for $\mathrm{N}=4$ super yang-mills," JHEP 03 (2003) 013, hep-th/0212208.

[27] N. Beisert, "The complete one-loop dilatation operator of N=4 Super Yang-Mills theory," Nucl. Phys. B676 (2004) 3-42, hep-th/0307015.

[28] V. A. Kazakov, A. Marshakov, J. A. Minahan, and K. Zarembo, "Classical / quantum integrability in AdS/CFT," JHEP 05 (2004) 024, hep-th/0402207.

[29] N. Gromov and V. Kazakov, "Asymptotic Bethe ansatz from string sigma model on $S^{3} \times R$," hep-th/0605026. 
[30] G. Arutyunov, S. Frolov, and M. Staudacher, "Bethe ansatz for quantum strings," JHEP 10 (2004) 016, arXiv:0406256 [hep-th].

[31] G. Arutyunov and M. Staudacher, "Matching higher conserved charges for strings and spins," JHEP 03 (2004) 004, arXiv: 0310182 [hep-th].

[32] I. Bena, J. Polchinski, and R. Roiban, "Hidden symmetries of the $A d S_{5} \times S^{5}$ superstring," Phys. Rev. D69 (2004) 046002, hep-th/0305116.

[33] L. D. Faddeev and N. Y. Reshetikhin, "Integrability of the principal chiral field model in (1+1)-dimension," Ann. Phys. 167 (1986) 227.

[34] A. Das, J. Maharana, A. Melikyan, and M. Sato, "The algebra of transition matrices for the $\operatorname{AdS}_{5} \times S^{5}$ superstring," JHEP 12 (2004) 055, hep-th/0411200.

[35] T. Klose and K. Zarembo, "Bethe ansatz in stringy sigma models," J. Stat. Mech. 0605 (2006) P006, hep-th/0603039.

[36] A. G. Izergin and V. E. Korepin, "Lattice versions of quantum field theory models in two-dimensions," Nucl. Phys. B205 (1982) 401-413.

[37] A. Melikyan, A. Pinzul, and G. Weber, "Higher charges and regularized quantum trace identities in su(1,1) Landau-Lifshitz model," J. Math. Phys. 51 (2010), no. 12, 123501, arXiv: 1008.1054 [hep-th].

[38] H. B. Thacker, "Exact integrability in quantum field theory and statistical systems," Rev. Mod. Phys. 53 (1981) 253.

[39] H. B. Thacker, "Bethe's hypothesis and feynman diagrams: Exact calculation of a three body scattering amplitude by perturbation theory," Phys. Rev. D11 (1975) 838.

[40] V. Giangreco Marotta Puletti, T. Klose, and O. Ohlsson Sax, "Factorized world-sheet scattering in near-flat $A d S_{5} \times S^{5}$," Nucl. Phys. B792 (2008) 228-256, 0707. 2082.

[41] A. Melikyan, A. Pinzul, V. O. Rivelles, and G. Weber, "On S-matrix factorization of the Landau-Lifshitz model," JHEP 10 (2008) 002, arXiv:0808.2489 [hep-th].

[42] A. Melikyan, A. Pinzul, V. Rivelles, and G. Weber, "Quantum integrability of the Alday-Arutyunov-Frolov model," arXiv:1106.0512 [hep-th].

[43] L. F. Alday, G. Arutyunov, and S. Frolov, "New integrable system of 2 dim fermions from strings on $A d S_{5} \times S^{5}$," arXiv: 0508140 [hep-th].

[44] A. Melikyan and A. Pinzul, "On quantum integrability of the Landau-Lifshitz model," J. Math. Phys. 50 (2009) 103518, arXiv:0812.0188 [hep-th].

[45] V. I. Arnold, Mathematical methods of classical mechanics / V. I. Arnold ; translated by K. Vogtmann and A. Weinstein. Springer-Verlag, New York :, 1978. 
[46] O. Babelon, D. Bernard, and M. Talon, Introduction to Classical Integrable Systems. Cambridge University Press, 2003.

[47] M. Dunajski, Solitons, instantons, and twistors. Oxford Graduate Texts in Mathematics. Oxford University Press, 2010.

[48] L. D. Faddeev, "What is complete integrability in quantum mechanics," Proceedings of the Symposium Henri Poincaré, Brussels, 8-9 October 2004 (2004).

[49] J. Clemente-Gallardo and G. Marmo, "Towards a Definition of Quantum Integrability," International Journal of Geometric Methods in Modern Physics 6 (2009) 129-+, 0808.3819.

[50] J.-S. Caux and J. Mossel, "Remarks on the notion of quantum integrability," J.Stat.Mech. 1102 (2011) P02023, 1012.3587.

[51] H. Bethe, "Zur theorie der metalle,". 10.1007/BF01341708.

[52] C.-N. Yang and C. Yang, "Thermodynamics of one-dimensional system of bosons with repulsive delta function interaction," J.Math.Phys. 10 (1969) 1115-1122.

[53] T. C. Dorlas, "Orthogonality and completeness of the bethe ansatz eigenstates of the nonlinear schroedinger model," Communications in Mathematical Physics 154 (1993) 347-376. 10.1007/BF02097001.

[54] V. Tarasov, L. Takhtadzhyan, and L. Faddeev, "Local Hamiltonians for integrable quantum models on a lattice," Theoretical and Mathematical Physics 57 (1983), no. 2, 1059-1073.

[55] V. Tarasov, "Local Hamiltonians for integrable quantum models on a lattice. II," Theoretical and Mathematical Physics 61 (1984), no. 3, 1211-1215.

[56] A. Kundu and B. Basu-Mallick, "Construction of integrable quantum lattice models through Sklyanin like algebras," Modern Phys. Lett. A 7 (1992) 61-69.

[57] A. Kundu, "On sigma models with noncompact groups," Lett. Math. Phys. 6 (1982) 479-485.

[58] B. Davies, "Higher conservation laws for the quantum non-linear schrödinger equation," Physica A: Statistical Mechanics and its Applications 167 (1990), no. 2, 433 - 456.

[59] B. Sutherland, Beautiful models: 70 years of exactly solved quantum many-body problems. World Scientific, 2004.

[60] C.-N. Yang, "S matrix for the one-dimensional $\mathrm{N}$ body problem with repulsive or attractive delta function interaction," Phys.Rev. 168 (1968) 1920-1923.

[61] N. Gromov, V. Kazakov, K. Sakai, and P. Vieira, "Strings as multi-particle states of quantum sigma-models," Nucl. Phys. B764 (2007) 15-61, hep-th/0603043.

[62] M. Staudacher, "The factorized S-matrix of CFT/AdS," JHEP 05 (2005) 054, hep-th/0412188. 
[63] G. Arutyunov and S. Frolov, "On $A d S_{5} \times S^{5}$ string S-matrix," Phys. Lett. B639 (2006) 378-382, arXiv:0604043 [hep-th].

[64] R. A. Janik, "The $A d S_{5} \times S^{5}$ superstring worldsheet S-matrix and crossing symmetry," Phys. Rev. D73 (2006) 086006, hep-th/0603038.

[65] N. Beisert, "On the scattering phase for $A d S_{5} \times S^{5}$ strings," Mod. Phys. Lett. A22 (2007) 415-424, hep-th/0606214.

[66] S. Schafer-Nameki, "The algebraic curve of 1-loop planar N = 4 SYM," Nucl. Phys. B714 (2005) 3-29, hep-th/0412254.

[67] N. Beisert, "The su(2|3) dynamic spin chain," Nucl. Phys. B682 (2004) 487-520, hep-th/0310252.

[68] S. Bellucci and P. Y. Casteill, "Sigma model from SU(1,1|2) spin chain," Nucl. Phys. B741 (2006) 297-312, hep-th/0602007.

[69] M. Kruczenski, "Spin chains and string theory," Phys. Rev. Lett. 93 (2004) 161602, hep-th/0311203.

[70] M. Kruczenski, A. V. Ryzhov, and A. A. Tseytlin, "Large spin limit of $A d S_{5} \times S^{5}$ string theory and low energy expansion of ferromagnetic spin chains," Nucl. Phys. B692 (2004) 3-49, hep-th/0403120.

[71] R. Roiban, A. Tirziu, and A. A. Tseytlin, "Asymptotic Bethe ansatz S-matrix and Landau-Lifshitz type effective 2-d actions," J. Phys. A39 (2006) 13129-13169, hep-th/0604199.

[72] J. Stefanski, B., "Landau-Lifshitz sigma-models, fermions and the AdS/CFT correspondence," JHEP 07 (2007) 009, 0704.1460.

[73] A. Tirziu, "Quantum Landau-Lifshitz model at four loops: $1 / \mathrm{J}$ and $1 / \mathrm{J}^{* *} 2$ corrections to BMN energies," Phys. Rev. D73 (2006) 106001, hep-th/0601139.

[74] E. Fradkin, Field theories of condensed matter systems. Frontiers in physics. Addison-Wesley Pub. Co., 1991.

[75] A. Mikhailov, "A nonlocal poisson bracket of the sine-Gordon model," hep-th/0511069.

[76] A. Mikhailov and S. Schafer-Nameki, "Algebra of transfer-matrices and Yang-Baxter equations on the string worldsheet in $A d S_{5} \times S^{5}$," arXiv:0712.4278 [hep-th].

[77] J. M. Maillet, "New integrable canonical structures in two-dimensional models," Nucl. Phys. B269 (1986) 54.

[78] A. Duncan, H. Nicolai, and M. Niedermaier, "On the Poisson bracket algebra of monodromy matrices," Z. Phys. C46 (1990) 147. 
[79] A. Das, A. Melikyan, and M. Sato, "The algebra of flat currents for the string on $A d S_{5} \times S^{5}$ in the light-cone gauge," JHEP 11 (2005) 015, hep-th/0508183.

[80] A. Das, A. Melikyan, and V. O. Rivelles, "The S-matrix of the Faddeev-Reshetikhin Model, Diagonalizability and PT Symmetry," JHEP 09 (2007) 104, arXiv:0707.0511 [hep-th].

[81] G. Grignani, T. Harmark, and M. Orselli, "The SU(2) $\times \mathrm{SU}(2)$ sector in the string dual of $\mathrm{N}=6$ superconformal Chern-Simons theory," 0806. 4959.

[82] O. Aharony, O. Bergman, D. L. Jafferis, and J. Maldacena, " $\mathrm{N}=6$ superconformal Chern-Simons-matter theories, M2-branes and their gravity duals," 0806.1218.

[83] M. Lakshmanan, "Continuum spin system as an exactly solvable dynamical system," Physics Letters A $6 \mathbf{1}$ (1977), no. 1,.

[84] H. Fogedby, "Magnon-Soliton Phase Shift Analysis of the Classical Continuous Heisenberg Chain," Physica Scripta 22 (1980) 404-405.

[85] H. Fogedby, "Solitons and magnons in the classical Heisenberg chain," Journal of Physics A: Mathematical and General 13 (1980), no. 4, 1467-1499.

[86] H. Fogedby, "The spectrum of the continuous isotropic quantum Heisenberg chain: quantum solitons as magnon bound states," Journal of Physics C: Solid State Physics 13 (1980), no. 9, L195-L200.

[87] M. Lakshmanan, K. Porsezian, and M. Daniel, "Effect of discreteness on the continuum limit of the Heisenberg spin chain," Physics letters. A 133 (1988), no. 9, 483-488.

[88] L. A. Takhtajan, "Integration of the Continuous Heisenberg Spin Chain Through the Inverse Scattering Method," Phys. Lett. A64 (1977) 235-237.

[89] E. K. Sklyanin, "On complete integrability of the Landau-Lifshitz equation," Preprint LOMI E3-79, Leningrad (in russian) (1979).

[90] V. E. Zakharov and L. A. Takhtajan, "Equivalence of the nonlinear Schrodinger equation and the equation of a Heisenberg ferromagnet," Theor. Math. Phys. 38 (1979) 17-23.

[91] A. Jevicki and N. Papanicolaou, "Semiclassical spectrum of the continuous heisenberg spin chain," Ann. Phys. 120 (1979) 107.

[92] E. K. Sklyanin, "Quantization of the continuous Heisenberg ferromagnet," Lett. Math. Phys. 15 (1988) 357-368.

[93] A. G. Izergin and V. E. Korepin, "The lattice quantum sine-gordon model," Lett. Math. Phys. 5 (1981) 199-205.

[94] P. P. Kulish and E. K. Sklyanin, "Quantum spectral transform method. Recent developments," Lect. Notes Phys. 151 (1982) 61-119. 
[95] T. Klose, T. McLoughlin, R. Roiban, and K. Zarembo, "Worldsheet scattering in AdS(5) $x$ S**5," JHEP 0703 (2007) 094, hep-th/0611169.

[96] T. Klose and K. Zarembo, "Reduced sigma-model on AdS(5) $\times \mathrm{S}^{* *}$ 5: One-loop scattering amplitudes," JHEP 0702 (2007) 071, hep-th/0701240.

[97] T. Klose, T. McLoughlin, J. A. Minahan, and K. Zarembo, "World-sheet scattering in $A d S_{5} \times S^{5}$ at two loops," arXiv:0704.3891 [hep-th].

[98] P. P. Kulish, "Factorization of the Classical and Quantum s Matrix and Conservation Laws," Theor. Math. Phys. 26 (1976) 132.

[99] I. V. Cherednik, "On a method of constructing factorized s matrices in elementary functions," Theor. Math. Phys. 43 (1980) 356-358.

[100] S. J. Parke, "Absence of particle production and factorization of the S-matrix in $1+1$ dimensional models," Nucl. Phys. B174 (1980) 166.

[101] M. Karowski and P. Weisz, "Exact Form-Factors in (1+1)-Dimensional Field Theoretic Models with Soliton Behavior," Nucl. Phys. B139 (1978) 455.

[102] M. Karowski, "An Exact Relativistic s Matrix in (1+1)-Dimensions: The On-Shell Solution of the Massive Thirring Model and the Quantum Sine-Gordon Equation,". Presented at Int. School of Subnuclear Physics, Erice, Italy, Jul 23 - Aug 10, 1977.

[103] M. Karowski, "Exact S matrices and form factors in (1+1)-dimensional field theoretic models with soliton behavior," Phys. Rept. 49 (1979) 229-237.

[104] H. B. Thacker, "Many body scattering processes in a one-dimensional boson system," Phys. Rev. D14 (1976) 3508.

[105] C.-N. Yang, "Some exact results for the many body problems in one dimension with repulsive delta function interaction," Phys. Rev. Lett. 19 (1967) 1312-1314.

[106] B. Zhao, "Quantum continuous Heisenberg ferromagnetic chain," Physics Letters A 92 (1982), no. 1,.

[107] A. Melikyan and A. Pinzul, "Thermodynamics of the quantum su(1,1) Landau-Lifshitz model," J. Stat. Mech. 1006 (2010) P06007, arXiv:1001.5450 [hep-th].

[108] A. G. Izergin and V. E. Korepin, "A lattice model related to the nonlinear Schroedinger equation," Dokl. Akad. Nauk Ser. Fiz. 259 (1981) 76-79.

[109] L. D. Faddeev and A. Y. Volkov, "Quantum inverse scattering method on a spacetime lattice," Theor. Math. Phys. 92 (1992) 837-842.

[110] H. E. Boos, V. E. Korepin, and F. A. Smirnov, "Connecting lattice and relativistic models via conformal field theory," math-ph/0311020. 
[111] A. G. Izergin, V. E. Korepin, and F. A. Smirnov, "Trace formulas for the quantum nonlinear Schrödinger equation," Theor. Math. Phys. 48 (1981) 773-776.

[112] E. K. Sklyanin, "Some algebraic structures connected with the Yang-Baxter equation," Funct. Anal. Appl. 16 (1982) 263-270.

[113] E. K. Sklyanin, "Some algebraic structures connected with the Yang-Baxter equation. Representations of quantum algebras," Funct. Anal. Appl. 17 (1983) 273-284.

[114] S. Albeverio, R. Hoegh-Krohn, D. Testard, and A. Vershik, "Factorial representation of path groups," J.Funct.Anal 51 (1983) 115-131. CPT-82-PE-1421.

[115] R. Jackiw, Diverse topics in theoretical and mathematical physics. Advanced Series in Mathematical Physics. World Scientific, 1995.

[116] G. Arutyunov and S. Frolov, "Uniform light-cone gauge for strings in $A d S_{5} \times S^{5}$ : Solving

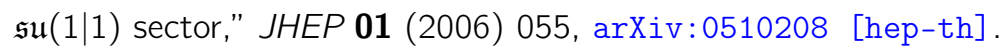

[117] P. A. M. Dirac, Lectures on Quantum Mechanics. Belfer Graduate School of Science, Yeshiva University, New York, 1964.

[118] A. Das, Lectures on Quantum Field Theory. Hackensack, USA: World Scientific, 2008.

[119] H. Bergknoff and H. B. Thacker, "Method for solving the massive Thirring model," Phys. Rev. Lett. 42 (1979) 135.

[120] I. S. Gradshteyn and I. M. Ryzhik, Table of Integrals, Series, and Products. Academic Press, 5th ed., 1994. 


\section{Índice Remissivo}

$\delta_{ \pm}^{(2)}, 130,199$

Ansatz de Bethe Algébrico, 77

Antissimetrizador, 203

Calibre uniforme, 181

Campo vetorial Hamiltoniano, 17

Cargas conservadas, 14, 33

Involução, 46, 71

Quânticas, 73

Condição de curvatura nula, 29, 173, 186

Condições de contorno

Decaimento rápido no infinito, 26

Quasiperiódicas, 26

Conexão de Lax, 28

Coordenadas de ângulo e ação, 22

Decomposição $\mathbb{Z}_{4}, 171$

Dirac

Equação de, 194, 249

Espinor de, 250

Hamiltoniana de, 196

Operador de, 196

Parênteses de, 196

Solução de onda plana, 250

Equação de Yang-Baxter, 44, 91

Equações de Bethe, 79

Estrutura simplética, 17

Identidade de Jacobi, 14, 16, 43

Integrabilidade

de Liouville, 19

de Sutherland, 96

Involução de Cartan, 170

Matriz
$R, 72,76$

$r, 43,69$

de monodromia, 32, 75

de Pauli, 112

de permutação, 42

de transição, 31, 32

Discreta, 69

Modelo de Alday-Arutyunov-Frolov, 169

Ação, 191, 193, 194

Biespinor, 200

Constantes de acoplamento, 193, 211

Equações de Bethe, 213

Hamiltoniana, 185

Lagrangeana, 184, 185, 193

Limite de descompactificação, 194

Massa, 192

Matriz M(q), 206

Matriz $S$

para duas pseudopartículas, 199, 202, 212

para três pseudopartículas, 202, 212

Parênteses de Poisson, 185

Produto vetorial, 200

Propagador, 198, 253

Pseudopartículas, 199

Triespinor, 203

Vértices de interação, 193

Modelo de Landau-Lifshitz

Álgebra regularizada, 154, 155

Ação, 124, 125, 129

Campos regularizados, 154

Cargas conservadas, 115, 118, 150

Fundamentais, 119, 159, 162

Involução, 120

Condição de camada de massa, 131 
Condições de compatibilidade, 150

Conexão de Lax, 112, 235

Equações de movimento

Anisotrópicas, 111

Isotrópicas, 112

Extensões auto-adjuntas, 151, 163

Hamiltoniana

Anisotrópica, 111

de interação, 130

Isotrópica, 112

Hamiltoniano, 150, 158, 159, 161

Identidades de traço, 115, 118, 149

Lagrangeana, 132

Livre, 126

Matriz

S para duas partículas, 130, 132

S para três partículas, 138, 139, 141

$r, 120,163$

de monodromia, 147, 160

de monodromia reduzida, 114, 161

de transição, 114

Operador de Lax, 155

Operador de Casimir, 156, 157, 159

Operador de Lax, 147

Parênteses de Poisson, 111, 119

Problema linear fundamental, 113

Propagador, 127, 239

Soluções de Jost, 114

Vértice de interação, 130

Vínculo, 111, 124, 156, 157

Modelo de Schrödinger não-linear

Ansatz de Bethe coordenado, 64, 65

Cargas conservadas, 41

Quânticas, 90

Conexão de Lax, 35, 235

Discreto, 73

Equação de movimento, 25, 61

Linearizada, 54

Equações de Bethe, 64, 66

Hamiltoniana, 27

Hamiltoniano, 61
Identidades de traço, 41, 55, 89

Lagrangeana, 97, 132

Matriz

$R, 74,83$

S, 106

$r, 44,120$

de monodromia, 35

de monodromia reduzida, 52

de transição, 32, 80

Par de Lax, 29

Parênteses de Poisson, 27

Regras de Feynman, 97, 98

Soluções de Jost, 52

Modelo de Thirring

Matriz S, 212

Ação, 213

Operador

de antissimetrização, 203

de simetrização, 99

Ordenamento normal, 80, 90

Par de Lax, 28

Parênteses de Poisson, 14, 15, 24, 42

Degenerados, 16

Fundamentais, 43

Discretos, 69

Problema de Riemann-Hilbert, 55

Problema linear fundamental, 28

Discreto, 69

Produto de Sklyanin, 148, 156

Produto tensorial, 42

Pseudovácuo, 61, 127, 197

Rearranjo normal de fatores operacionais ::, 81

Relações

de anticomutação canônicas, 196

de comutação canônicas, 61, 126, 146

de comutação fundamentais, 72

Representação de Lax, 28

Simetrizador, 99

Supercorda 
Ação, 173

Conexão de Lax, 185

Conjunto quociente em $A d S_{5} \times S^{5}, 169$

Lagrangeana em $A d S_{5} \times S^{5}, 173$

Supertraço, 170

Supertransposição, 171

Teorema

de Arnold-Liouville, 23

de Darboux, 18, 219

de Liouville, 20

de não-renormalização, 127

de Sokhotsky-Weierstrass, 106, 139, 208

Termo de Wess-Zumino, 124, 125

Ultralocalidade, 43

Vácuo Ferromagnético, 146

Vácuo ferromagnético, 150, 157

Variedade

de Poisson, 16

simplética, 17 